LBNL-63187

\title{
A Measurement of the Rate of Muon Capture in Hydrogen Gas and Determination of the Proton's Induced Pseudoscalar Coupling $g_{P}$
}

\author{
Thomas Ira Banks \\ Ph.D. Thesis \\ Department of Physics \\ University of California, Berkeley \\ Berkeley, CA 94720 \\ and \\ Nuclear Science Division \\ Ernest Orlando Lawrence Berkeley National Laboratory \\ Berkeley, CA 94720
}

July 2007

This work was supported by the Director, Office of Science, Office of Basic Energy Sciences, Materials Sciences and Engineering Division, of the U.S. Department of Energy under Contract Nos. DE-FG02-00ER41129 and DE-AC02-05CH11231. 
A Measurement of the Rate of Muon Capture in Hydrogen Gas and Determination of the Proton's Induced Pseudoscalar Coupling $g_{P}$

\author{
Copyright 2007 \\ by
}

Thomas Ira Banks

The U.S. Department of Energy has the right to use this document for any purpose whatsoever, including the right to reproduce all or any part thereof. 


\begin{abstract}
A Measurement of the Rate of Muon Capture in Hydrogen Gas and Determination of the Proton's Induced Pseudoscalar Coupling $g_{P}$ by

Thomas Ira Banks

Doctor of Philosophy in Physics

University of California, Berkeley

Professor Stuart J. Freedman, Chair
\end{abstract}

This dissertation describes a measurement of the rate of nuclear muon capture by the proton, performed by the MuCap Collaboration using a new technique based on a time projection chamber operating in ultraclean, deuterium-depleted hydrogen gas at room temperature and $1 \mathrm{MPa}$ pressure. The hydrogen target's low gas density of $1 \%$ compared to liquid hydrogen is key to avoiding uncertainties that arise from the formation of muonic molecules. The capture rate was obtained from the difference between the $\mu^{-}$ disappearance rate in hydrogen — as determined from data collected in the experiment's first physics run in fall 2004 - and the world average for the $\mu^{+}$decay rate. After combining the results of my analysis with the results from another independent analysis of the 2004 data, the muon capture rate from the hyperfine singlet ground state of the $\mu p$ atom is found to be $\Lambda_{S}=725.0 \pm 17.4 \mathrm{~s}^{-1}$, from which the induced pseudoscalar coupling of the nucleon, $g_{P}\left(q^{2}=-0.88 m_{\mu}^{2}\right)=7.3 \pm 1.1$, is extracted. This result for $g_{P}$ is consistent with theoretical predictions that are based on the approximate chiral symmetry of QCD. 
Professor Stuart J. Freedman Dissertation Committee Chair 
To my family

I cannot do it; yet I'll hammer it out.

-Shakespeare, Richard II 5.5.5 


\section{Contents}

List of figures $\quad$ vi

List of tables $\quad x$

Glossary of terms xii

1 Introduction $\quad 1$

1.1 Muon capture and $g_{P} \ldots \ldots \ldots \ldots \ldots \ldots$

1.2 Previous measurements of muon capture in hydrogen . . . . . . . . . . 5

1.3 The MuCap experiment . . . . . . . . . . . . . . . . . . 11

1.4 Situation overview . . . . . . . . . . . . . . . . . . 14

2 Theory 17

2.1 Historical overview of $g_{P} \ldots \ldots \ldots \ldots \ldots \ldots$

2.2 Chiral perturbation theory and $g_{P} \ldots \ldots \ldots \ldots \ldots$

2.3 Treatments of muon capture . . . . . . . . . . . . . . . . . . 20

2.4 Radiative corrections to muon capture . . . . . . . . . . . . . . . . . . . . . 23

2.5 Implications of a muon capture measurement . . . . . . . . . . . . . 24

3 Muon kinetics in hydrogen $\quad 28$

3.1 Atomic capture, thermalization, and diffusion . . . . . . . . . . . 29

3.2 Formation of $p \mu p$ molecules . . . . . . . . . . . . . . . . . . 34

3.3 Impurities . . . . . . . . . . . . . . . . . . . . . 37

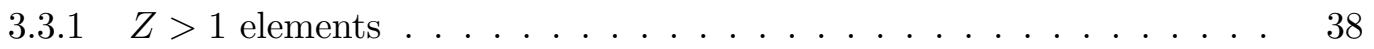

3.3.2 Deuterium . . . . . . . . . . . . . . . . . 40

3.4 Analytic description of full $\mu^{-}$kinetics . . . . . . . . . . . . . . 47

3.5 Kinetics of $\mu^{+} \ldots \ldots \ldots \ldots \ldots \ldots \ldots$

$\begin{array}{lll}4 & \text { Experimental apparatus } & \mathbf{5 7}\end{array}$

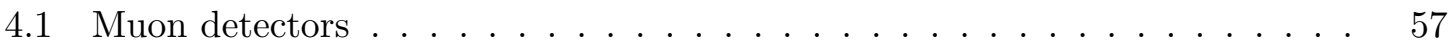

4.2 Electron detectors . . . . . . . . . . . . . . . . . 64

4.3 Hydrogen gas system . . . . . . . . . . . . . . . . . . 67

4.4 Data acquisition (DAQ) system and electronics . . . . . . . . . . 68 
$\begin{array}{lll}5 & 2004 \text { experimental run (Run8) } & \mathbf{7 1}\end{array}$

5.1 History . . . . . . . . . . . . . . . . . . . . . . 71

5.2 Beamline description . . . . . . . . . . . . . . . . . . . 73

5.3 Run chronology . . . . . . . . . . . . . . . . . . 76

5.4 Apparatus performance . . . . . . . . . . . . . . . 80

5.4 Muon detectors . . . . . . . . . . . . . . . . 80

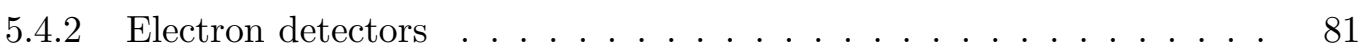

5.4 .3 DAQ and Electronics . . . . . . . . . . . . . . 83

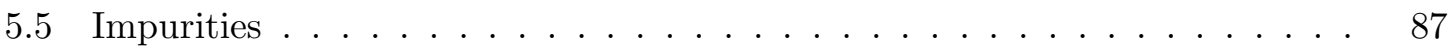

$5.5 .1 \quad Z>1$ elements and CHUPS . . . . . . . . . . 87

5.5 .2 Deuterium . . . . . . . . . . . . . . . . . . 88

5.6 Summary . . . . . . . . . . . . . . . . . . . . . 90

$\begin{array}{llr}6 & \text { Data analysis } & 91\end{array}$

6.1 Infrastructure . . . . . . . . . . . . . . . . . . . . . 91

6.1 .1 Blinding procedures . . . . . . . . . . . . . . . . . 91

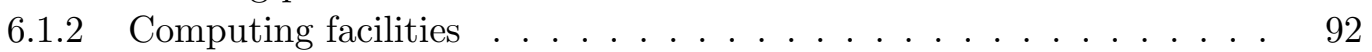

6.1 .3 Software . . . . . . . . . . . . . . . . . 93

6.1.4 Organization of data processing . . . . . . . . . . . . . 95

6.2 Data preprocessing . . . . . . . . . . . . . . . . . 96

6.3 Muon detector analysis . . . . . . . . . . . . . . . . . . . . . . . 99

$6.3 .1 \mu \mathrm{SC}(\& \mu \mathrm{SCA}) \ldots \ldots \ldots \ldots \ldots \ldots$

$6.3 .2 \mu \mathrm{PC} 1 \ldots \ldots \ldots \ldots \ldots \ldots$

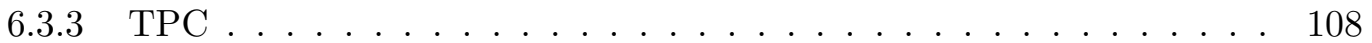

6.3.4 Coincidences . . . . . . . . . . . . . . . . . . 113

6.4 Electron detector analysis . . . . . . . . . . . . . . . . 118

$6.4 .1 \mathrm{ePC} 1 \ldots \ldots \ldots \ldots \ldots \ldots$

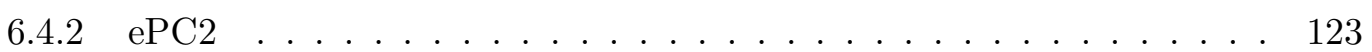

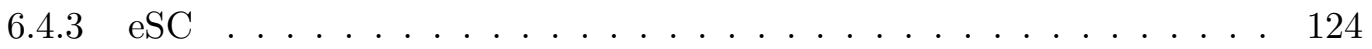

6.4.4 Coincidences . . . . . . . . . . . . . . . . . . 127

6.5 Muon lifetime studies and consistency checks . . . . . . . . . . . . 131

6.5.1 The lifetime histogram . . . . . . . . . . . . . . . . . . . . 131

6.5.2 Fitting function . . . . . . . . . . . . . . . . 135

6.5.3 Lifetime alignment . . . . . . . . . . . . . . . . . . . . . 138

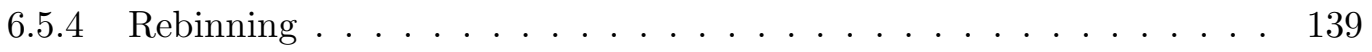

6.5 .5 Fit range . . . . . . . . . . . . . . . . . . . . . 143

6.5.6 Adjustments for cosmics . . . . . . . . . . . . . . . . . . . . . 148

6.5.7 The impact parameter . . . . . . . . . . . . . . . . 154

6.5.8 Magnetic field effects on the impact parameter . . . . . . . . . . . 157

6.5.9 Data selection . . . . . . . . . . . . . . . . . . . . . 159

6.5 .10 Special data cuts . . . . . . . . . . . . . . . . . . . . . . . . . . . . . . . . . . . 162

6.5 .11 Lifetime vs. time . . . . . . . . . . . . . . . . . 170

6.5 .12 Lifetime vs. space . . . . . . . . . . . . . . . . . . . 170

$6.5 .13 \mathrm{TPC}$ fiducial cuts . . . . . . . . . . . . . . . . . . . 180

6.5.14 Lifetime vs. muon entrance detector treatment (the pileup veto) . . 187 
6.5.15 Lifetime vs. electron detector treatment . . . . . . . . . . . . . . 196

6.5.16 Unique-electron condition . . . . . . . . . . . . . . . . . . 199

6.6 Determination of the (blinded) muon disappearance rate(s) . . . . . . . 201

6.6.1 Uncorrected rates . . . . . . . . . . . . . . . . . . . . 203

$6.6 .2 \mu+p$ scatter correction . . . . . . . . . . . . . 206

$6.6 .3 Z>1$ impurity correction . . . . . . . . . . . . . . . . . 218

6.6.4 Deuterium correction . . . . . . . . . . . . . . . 234

6.6 .5 Impact cut correction . . . . . . . . . . . . . . . . . . 241

6.6.6 Uncertainty from muon entrance detector inefficiencies . . . . . . . . 244

7 Determination of the muon capture rate $\Lambda_{S} \quad 246$

7.1 Unblinding of the DAQ clock frequency . . . . . . . . . . . . . 246

7.2 Self-consistent determination of $\Lambda_{S} \ldots \ldots \ldots \ldots \ldots \ldots \ldots$

$7.2 .1 \quad p \mu p$ correction . . . . . . . . . . . . . . . . 251

7.2.2 Subtraction of the free muon decay rate $\lambda_{\mu^{+}} \ldots \ldots \ldots . \ldots 254$

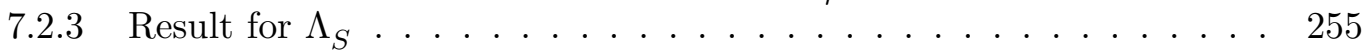

7.2 .4 Error summary . . . . . . . . . . . . . . . . 257

7.3 Final consistency checks . . . . . . . . . . . . . . . 257

7.3.1 Lifetime fits using the full kinetics function . . . . . . . . . . 257

7.3.2 CAEN clock and beam RF beating studies . . . . . . . . . . . 262

7.3.3 $\mu^{+}$lifetime measurement . . . . . . . . . . . . . 264

8 Conclusions $\quad \mathbf{2 7 2}$

8.1 Comparison of Berkeley and Illinois results for $\Lambda_{S} \ldots \ldots \ldots \ldots$. . . . . . . . . . 272

8.2 Determination of $g_{P} \ldots \ldots \ldots \ldots \ldots \ldots \ldots \ldots$

8.3 Remaining questions and possibilities . . . . . . . . . . . 278

8.4 Outlook . . . . . . . . . . . . . . . . . . 281

$\begin{array}{ll}\text { A Muon capture kinematics } & 283\end{array}$

$\begin{array}{ll}\text { B Muon capture calculations } & 285\end{array}$

$\begin{array}{ll}\text { C } \mu p \text { diffusion in hydrogen gas } & 287\end{array}$

D Calculation of the hydrogen gas density in Run8 291

E Effects of $\mu d$ diffusion on the lifetime spectrum $\quad 294$

F $\mu^{+}$precession frequencies in hydrogen gas 296

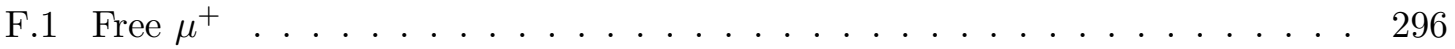

F.2 Muonium "triplet" . . . . . . . . . . . . . . . . . . . . . . 297

F.3 Muonium "singlet" . . . . . . . . . . . . . . . . . . . . . . . . . 298 
G Technical specifications: detectors, electronics, and software $\quad 300$

G.1 General detector channel / parameter assignments . . . . . . . . . . . . . 300

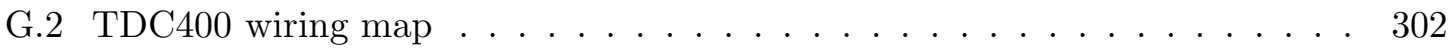

G.3 $\mu \mathrm{SC} \operatorname{logic} \ldots \ldots \ldots \ldots \ldots \ldots$

G.4 TPC . . . . . . . . . . . . . . . . . . . 304

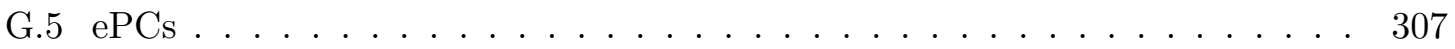

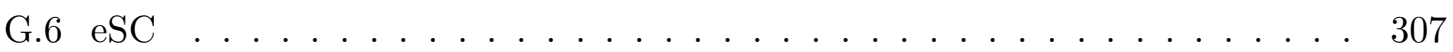

G.7 Berkeley analysis modules - listing . . . . . . . . . . . . 310

H Smearing of the lifetime spectrum due to detector response 311

I Error calculations for muon disappearance rate shifts associated $\begin{array}{lr}\text { with } \mu+p \text { scatters } & 314\end{array}$

$\begin{array}{ll}\text { Bibliography } & 317\end{array}$ 


\section{List of figures}

1.1 Feynman diagram of nuclear muon capture in terms of fundamental fermions

1.2 Chronological plot of $g_{P}$ determinations from muon capture experiments in hydrogen . . . . . . . . . . . . . . . . . . . . 14

1.3 Summary of experimental constraints on $g_{P}$ vs. $\lambda_{\mathrm{op}} \ldots \ldots \ldots \ldots$

2.1 Feynman diagrams of pion field effects in muon capture . . . . . . . . . . 18

2.2 Feynman diagram of ordinary muon capture as a contact interaction . . . . 21

3.1 Fractional population of $\mu p$ hyperfine singlet atoms vs. time . . . . . . . . 31

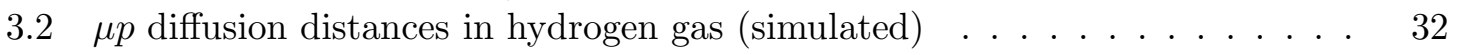

$3.3 \mu p$ and $p \mu p$ populations vs. time for different hydrogen gas densities . . . . 37

3.4 Scattering cross sections for $\mu p+\mathrm{H}_{2}$ and $\mu d+\mathrm{H}_{2}$, as a function of collisional energy . . . . . . . . . . . . . . . . . . . . 42

3.5 $\mu d$ diffusion distances in hydrogen gas (simulated), for two deuterium con-

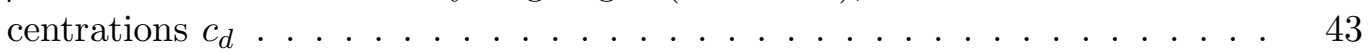

3.6 Flowchart of $\mu^{-}$kinetics in hydrogen . . . . . . . . . . . . . . . . 48

$3.7 \mathrm{Mu}$ triplet precession in the MuCap lifetime spectrum . . . . . . . . . 55

3.8 Free $\mu^{+}$precession signal $\ldots \ldots \ldots \ldots \ldots \ldots$

4.1 Cross-sectional diagram of the MuCap detector . . . . . . . . . . . 58

4.2 Schematic drawing of the entrance muon detector assembly . . . . . . . . 60

4.3 Photograph of the TPC mounted on the pressure vessel, viewed from above 61

4.4 Photograph of the TPC mounted on the pressure vessel, viewed from below 61

4.5 The pressure vessel and $\mu^{+} \mathrm{SR}$ magnet . . . . . . . . . . 63

4.6 Photograph of the outer electron proportional chamber, ePC2 . . . . . . . 65

4.7 CAD drawings of the eSC hodoscope assembly and components . . . . . . 66

5.1 PSI Experimental Hall floor plan . . . . . . . . . . . . . . . . . . . 74

5.2 Overhead photograph of the $\pi \mathrm{E} 3$ area and MuCap detectors . . . . . . . . 77

5.3 The PSI $\pi$ E3 beamline area . . . . . . . . . . . . . . . . . . 78

5.4 The $\pi \mathrm{E} 3$ area enclosed within a climate-controlled tent . . . . . . . . . 78

5.5 The pressure vessel assembly . . . . . . . . . . . . . . 78

5.6 The nested ePC chambers . . . . . . . . . . . . . . . 78 
5.7 Photograph of the $2004 \mathrm{MuCap}$ apparatus, installed in the $\pi \mathrm{E} 3$ area at PSI

5.8 Screen capture of the muon detectors' online display page . . . . . . . . . 84

5.9 Screen capture of the ePC1 online display page . . . . . . . . . . . 84

5.10 Statistics accumulation vs. time during the 2004 run $\ldots \ldots \ldots$. . . . . 87

5.11 CHUPS schematic diagram . . . . . . . . . . . . . . 89

5.12 Photograph of the CHUPS purification and recirculation system in 2004 . . 89

6.1 Temporal autocorrelation plots of the $\mu \mathrm{SC}$ copy1 signal . . . . . . . . . . 101

6.2 Temporal autocorrelation plots of the $\mu \mathrm{SC}$ copy 1 and random source signals 103

6.3 Temporal autocorrelation plots of the $\mu \mathrm{SC}$ copy 1 signal, and the union of all $\mu \mathrm{SC}$ signals . . . . . . . . . . . . . . . . . . 104

6.4 Temporal autocorrelations of raw $\mu \mathrm{PC} 1$ signals $\ldots \ldots \ldots \ldots$. . . . . . . . 107

6.5 Temporal autocorrelations of processed $\mu \mathrm{PC} 1$ signals . . . . . . . . . . . 107

6.6 Profiles of hit clusters in the $\mu \mathrm{PC} 1 \mathrm{X}$ amd Y planes . . . . . . . . . . . 109

6.7 2D plot of $\mu \mathrm{PC} 1 \mathrm{X}$ - and Y-plane coincidences . . . . . . . . . . . . . 109

6.8 Bragg curve for muons stopping in hydrogen . . . . . . . . . . . . . . 110

6.9 A good muon stop in the TPC, viewed in the Illinois event display . . . . . 111

6.10 Time differences between $\mu \mathrm{SC}$ and $\mu \mathrm{PC} 1$ hits . . . . . . . . . . . . 114

6.11 The TPC drift distribution of muon stops . . . . . . . . . . . . . 115

6.12 Diagram of the TPC detector regions . . . . . . . . . . . . . 116

6.13 Cartoon diagram of the ePC1 detector geometry . . . . . . . . . . . . 120

6.14 Three-plane coincidences in the ePC1 detector . . . . . . . . . . . . . . 122

6.15 Three-plane coincidences in the ePC2 detector . . . . . . . . . . . . . . 123

6.16 Alignment of the upstream and downstream signal times in the inner eSC gondolas . . . . . . . . . . . . . . . . . . 126

6.17 Alignment of signal times in the inner and outer eSC gondolas . . . . . . 126

6.18 The numbering scheme for the eSC gondolas, and the number of fourfold coincidences in each gondola . . . . . . . . . . . . . . . 127

$6.19(\phi, z)$ plots of coincidences in all three electron detectors $(\mathrm{ePC} 1+\mathrm{ePC} 2+\mathrm{eSC}) 130$

6.20 Muon lifetime spectra, created using the TPC data . . . . . . . . . . . . . . 132

6.21 Alignment of the eSC lifetime histograms . . . . . . . . . . . . . . 139

6.22 CAEN interpolator distributions . . . . . . . . . . . . . . . . 140

6.23 Fit results from scan over the lifetime spectra's 32-rebin boundary placement 142

6.24 Fit start time scan results . . . . . . . . . . . . . . . . . . . 145

6.25 Fit stop time scan results . . . . . . . . . . . . . . . . . 146

6.26 Lifetime spectra backgrounds observed in the eSC gondolas . . . . . . . . 149

6.27 Angular distribution of throughgoing cosmics . . . . . . . . . . . . . 151

6.28 Accidental background from cosmics . . . . . . . . . . . . . . . 152

6.29 Run8 impact parameter distribution and its Cartesian components . . . . . 155

$6.30 \mu^{-}$disappearance rate $\lambda$ vs. impact parameter cut . . . . . . . . . . . 157

6.31 Magnetic field contribution to the impact parameter distribution . . . . . . 159

6.32 Example of a chamberwide ePC2 spark . . . . . . . . . . . . . . 164

6.33 Example of a simultaneous chamberwide spark in $\mathrm{ePC} 1$ and $\mathrm{ePC} 2 \ldots \ldots 5$

6.34 Local sparks in the ePC2 anode plane . . . . . . . . . . . . . . . 165

6.35 Fitted rate versus chronological run group . . . . . . . . . . . . . . 171 
6.36 Fitted $\mu^{-}$disappearance rate vs. eSC $z$ position . . . . . . . . . . . .

6.37 Fitted $\mu^{-}$disappearance rate vs. eSC gondola (illustration of the "gondola effect") . . . . . . . . . . . . . . . . . . 173

6.38 Schematic diagram of the detectors in cross section . . . . . . . . . . . 174

$6.392 \mathrm{D}$ tomography plot of electron scattering materials in and around the pressure vessel . . . . . . . . . . . . . . . . . . . . . . 177

6.40 Monte Carlo reproduction of the $\mu d$-diffusion-driven "gondola effect" . . . . 178

6.41 Diffusion-driven gondola effect in upper/lower TPC regions . . . . . . . . . 179

6.42 Illustration of the TPC fiducial cut boundaries . . . . . . . . . . . . . . 184

6.43 Fits to scans over the TPC fiducial cut criteria . . . . . . . . . . . . 185

6.44 Concentric shells of muon stops, and fit results . . . . . . . . . . . . . 187

6.45 Monte Carlo reproduction of the TPC-related distortion in the non-pileupprotected lifetime spectrum . . . . . . . . . . . . . . . 188

6.46 Effects of muon pileup protection on the Prod-50 TPC drift distribution . . 189

6.47 Simulation of the effects of a time-dependent inefficiency in the muon entrance detectors . . . . . . . . . . . . . . . . . . . . . . 191

$6.48 \mu \mathrm{SC}$ time-independent inefficiency vs. run number during Run8 . . . . . . . 192

6.49 The per-run and cumulative time-independent inefficiencies in the Prod-50 data . . . . . . . . . . . . . . . . . . 193

6.50 Example of a "unique-electron" lifetime spectrum . . . . . . . . . . . . . 200

6.51 Example of a $\mu+p$ scatter event in the TPC . . . . . . . . . . . . 207

6.52 Fitted $\mu^{-}$disappearance rate of $\mu+p$ scatter events, vs. eSC gondola . . . 210

6.53 Fitted $\mu^{-}$disappearance rate of $\mu+p$ scatter events, start time scan . . . . 210

6.54 Simulated $\mu+p$ scatter fractions as a function of the proton recoil energy . 214

6.55 Angular distributions of $\mu+p$ scatters in the SRIM data, for several different recoil energy lower cutoffs . . . . . . . . . . . . . . . . . . . 215

6.56 Comparison of the angular distributions of $\mu+p$ scatters in SRIM data and the Run8 data . . . . . . . . . . . . . . . . . . . 216

6.57 Example of a probable muon capture by a $Z>1$ impurity . . . . . . . . . 220

6.58 TPC $Z>1$ capture yield vs. time during Run8 . . . . . . . . . . . . 222

$6.59 Z>1$ impurity capture time distributions . . . . . . . . . . . . . . 225

6.60 Fit to a $Z>1$ impurity capture time distribution . . . . . . . . . . . . 226

6.61 Monte-Carlo-generated $Z>1$ impurity correction distribution . . . . . . . . 233

6.62 Schematic illustration of the deuterium zero-extrapolation correction . . . . 235

6.63 Fitted $\mu^{-}$disappearance rate vs. impact parameter cut, for three different Run8 deuterium concentrations . . . . . . . . . . . . . . . . . . 240

7.1 Simulated lifetime spectra involving magnified CAEN interpolator nonuniformities . . . . . . . . . . . . . . . . .

7.2 Fits to simulated lifetime spectra involving magnified CAEN interpolator nonuniformities . . . . . . . . . . . . . . . . 265

7.3 Diagram of $\mu^{+}$spin rotation geometries in MuCap . . . . . . . . . . 267

7.4 $\mu^{+} \mathrm{SR}$ angular frequencies observed in different regions of the TPC, illustrating $B$ field nonuniformity . . . . . . . . . . . . . . 268

7.5 Fitted $\lambda_{\mu^{+}}$vs. impact parameter cut . . . . . . . . . . . . 271 
8.1 Summary of constraints on $g_{P}$ vs. $\lambda_{\mathrm{op}} \ldots \ldots \ldots \ldots \ldots \ldots \ldots$

C.1 Simulated distribution of thermal $\mu p$ radial diffusion distances at the time of $\mu$ decay . . . . . . . . . . . . . . . . . . 290

G.1 Schematic diagram of the $\mu \mathrm{SC}$ electronics setup . . . . . . . . . . . 305

G.2 Schematic drawing of the TPC and pressure vessel in sideview . . . . . . 306

H.1 Plot of the lifetime "turn-on" function . . . . . . . . . . . . . . . . 313 


\section{List of tables}

1.1 The weak nucleonic charged-current form factors, 2007 values . . . . . . . . 3

1.2 Previous measurements of nuclear muon capture in hydrogen . . . . . . . 8

2.1 Theoretical predictions for $g_{P}, \Lambda_{S}$, and $\Lambda_{T} \ldots \ldots \ldots \ldots$

$3.1 \mu^{-}$nuclear capture rates in the $\mu p$ atom and $p \mu p$ molecule . . . . . . . . 35

$3.2 p \mu p$ formation rates and the $p \mu p$ ortho $\rightarrow$ para transition rate . . . . . . 36

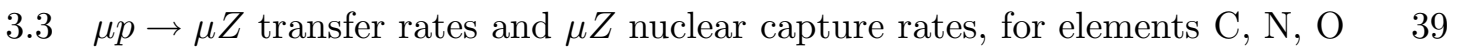

3.4 Survey of $\mu^{+}$lifetime measurements . . . . . . . . . . . 53

5.1 Breakdown of muon acceptance fractions . . . . . . . . . . . . . . 85

6.1 Run8 gas fills of interest in the $\mu^{-}$analysis $\ldots \ldots \ldots$. . . . . . . . 160

6.2 Fitted $\mu^{-}$disappearance rates for different $\mu \mathrm{SC}$ problem periods . . . . . . 194

6.3 Fitted $\mu^{-}$disappearance rates for different electron detector treatments . . 197

6.4 Results of fits to "unique-electron" lifetime spectra and standard spectra . . 202

6.5 The $\mu^{-}$disappearance rates prior to corrections . . . . . . . . . . . . 205

6.6 Statistics on the $\mu+p$ scatter events identified in the Prod-50 and CalibNat data . . . . . . . . . . . . . . . . . . . 209

6.7 Fitted $\mu^{-}$disappearance rates for different $\mu+p$ scatter inclusion/exclusion conditions, Prod-50 fill . . . . . . . . . . . . . . . . 211

6.8 Fitted $\mu^{-}$disappearance rates for different $\mu+p$ scatter inclusion/exclusion conditions, CalibNat fill . . . . . . . . . . . . . . . . . 212

6.9 The $\mu^{-}$disappearance rates after correcting for $\mu+p$ scatters . . . . . . 218

$6.10 Z>1$ impurity capture yields from the Berkeley analysis software . . . . . 224

6.11 The $\mu^{-}$disappearance rates after correcting for $Z>1$ impurities . . . . . . 234

6.12 Mass spectrometry measurements of Run8 deuterium concentrations . . . . 239

6.13 The $\mu^{-}$disappearance rates after correcting for deuterium . . . . . . . . . 242

6.14 The $\mu^{-}$disappearance rates after correcting for the $120 \mathrm{~mm}$ impact cut . . 244

6.15 The $\mu^{-}$disappearance rates after adding an error to account for uncertainties in the effects of muon entrance detector inefficiencies . . . . . . . . . . . 245

7.1 The $\mu^{-}$disappearance rates after unblinding the Run8 DAQ clock frequency. 248 
7.2 The $\mu^{-}$disappearance rates after correcting for $p \mu p$ effects $\ldots \ldots$. . . . 253

$7.3 \Lambda_{S}$ results after subtracting $\lambda_{0}$ from the $\mu^{-}$disappearance rates . . . . . . 255

7.4 Final values and errors for $\Lambda_{S}$, after enlarging the uncertainties by the "sensitivity" scaling factor . . . . . . . . . . . . . . . . . 255

7.5 Error summary . . . . . . . . . . . . . . . . . . . 258

$7.6 \Lambda_{S}$ values from fits using a function that includes $p \mu p$ kinetics . . . . . . . 261

$8.1 \Lambda_{S}$ results from the Berkeley and Illinois analyses . . . . . . . . . . . 273

G.1 Correspondence between detector channel and parameter number . . . . . . 300

G.2 Diagram of the wiring of TPC channel inputs to the TDC400s _ . . . . 303

G.3 Technical specifications for the ePC chambers. . . . . . . . . . . . . 308 


\section{Glossary of terms}

ADC Analog-to-Digital Converter

ADW Adler, Dothan, and Wolfenstein, the first to identify a correction to the GoldbergerTreiman $(\mathrm{G}-\mathrm{T})$ formula for $g_{P}$

bar A non-SI unit of pressure. 1 bar $=10^{5}$ Pascal $=0.98692 \mathrm{~atm}$.

barrack The portable, trailer-sized building that houses most of the MuCap electronics and computers, and from which shift personnel monitor the experiment

CalibN2 A calibration run performed during the 2004 data collection period (Run8), in which the hydrogen gas in the TPC was doped with nitrogen of concentration (by number) $c_{N} \approx 11 \mathrm{ppm}$

CalibNat A calibration run performed during the 2004 data collection period (Run8), in which the hydrogen gas in the TPC was doped with deuterium of concentration (by number) $c_{d} \approx 120 \mathrm{ppm}$; the gas also exhibited an elevated $Z>1$ impurity level during this fill.

CAEN Manufacturer of power supplies and electronics for nuclear physics experiments. CAEN V767 multihit TDC modules are used for the fast muon and electron timing measurements in MuCap. 
CAMAC Computer Automated Measurement And Control, an internationally accepted system of modular electronics and data handling based upon a group of standards adopted in the early 1970s by the U.S. NIM and European ESONE Committees. CAMAC electronics are widely used in particle and nuclear physics research.

ChPT Chiral Perturbation Theory

CHUPS Circulating Hydrogen Ultra-high Purification System, designed by PNPI (Gatchina) to continuously clean the hydrogen gas of $Z>1$ impurities

COMET COMpressor for Electron Tracks, a system of electronics modules developed by UCL for efficient readout, compression, and transfer of the large volume of data from the ePC detectors

COMP COMPressor, an electronics module that is part of the COMET system

CPLD Complex Programmable Logic Device. The DAQ logic was implemented using PSI-designed LB500 CPLD modules.

CVC Conserved Vector Current hypothesis

CVS Concurrent Versions System, a version control system widely used in software development

DAQ Data acquisition system

EL, EH, EVH "Energy Low," "Energy High," and "Energy Very High" TPC signal discrimination thresholds

ePC electron Proportional Chamber. MuCap uses two cylindrical, concentric ePC detectors to track decay electron trajectories. 
eSC electron Scintillating Counter, a cylindrical hodoscope which provides the fast electron timing signal for the MuCap lifetime measurement

FADC Flash Analong-to-Digital Converter, used to digitize the shape of signal pulses in time over a short time period

$\boldsymbol{g}_{\boldsymbol{P}}$ The weak nucleonic charged-current induced pseudoscalar coupling, whose determination is the primary motivation for the MuCap experiment

GEANT GEometry ANd Tracking, a software platform designed to simulate the passage of elementary particles through matter using Monte Carlo methods. GEANT was originally developed at CERN in 1974 for the study of high energy physics experiments, and has undergone numerous revisions in the time since.

G-T Goldberger-Treiman, the first to obtain a formula for $g_{P}$

gondola One of the sixteen eSC scintillating element pairs

HBChPT Heavy Baryon Chiral Perturbation Theory, the modern theroretical framework used to make predictions for the value of $g_{P}$

$\Lambda_{\boldsymbol{S}}$ The nuclear muon capture rate from the $\mu p$ hyperfine singlet ground state. The immediate goal of the MuCap experiment is to precisely measure $\Lambda_{S}$ and thereby make an unambiguous determination of the pseudoscalar form factor $g_{P}$.

$\boldsymbol{\Lambda}_{\boldsymbol{T}}$ The nuclear muon capture rate from the $\mu p$ hyperfine triplet ground state

$\Lambda_{Z}$ The nuclear muon capture rate from a $\mu Z$ atom, where $Z$ is an element of $Z>1$. The value of $\Lambda_{Z}$ increases as $\sim Z^{4}$.

$\boldsymbol{\Lambda}_{\boldsymbol{d}}$ The nuclear muon capture rate from the $\mu d$ atom, where $d$ is a deuteron 
$\boldsymbol{\Lambda}_{\mathbf{o m}}$ The nuclear muon capture rate from the ortho state of the $p \mu p$ molecule

$\boldsymbol{\Lambda}_{\mathbf{p m}}$ The nuclear muon capture rate from the para state of the $p \mu p$ molecule

$\lambda_{\mathbf{0}}$ The free, or "vacuum," muon decay rate, which is assumed to be equivalent to the $\mu^{+}$ decay rate, $\lambda_{\mu^{+}}$, by the $C P T$ theorem

$\boldsymbol{\lambda}_{\boldsymbol{\mu}^{+}}$The $\mu^{+}$decay rate

$\lambda_{\text {op }}$ The transition rate from the ortho state of the $p \mu p$ molecule to its para state

$\boldsymbol{\lambda}_{\boldsymbol{p} \boldsymbol{d}}$ The muon transfer rate from a proton to a deuteron, $\mu p+d \rightarrow p+\mu d$, normalized to liquid hydrogen density and atomic concentration

$\lambda_{p Z}$ The muon transfer rate from a proton to an element $Z>1, \mu p+Z \rightarrow p+\mu Z$, normalized to liquid hydrogen density and atomic concentration

$\lambda_{d Z}$ The muon transfer rate from a deuteron to an element $Z>1, \mu d+Z \rightarrow d+\mu Z$, normalized to liquid hydrogen density and atomic concentration

$\lambda_{\text {of }}$ The formation rate of ortho-state $p \mu p$ molecules, normalized to liquid hydrogen density

$\lambda_{\mathbf{p f}}$ The formation rate of para-state $p \mu p$ molecules, normalized to liquid hydrogen density

$\lambda_{p \mu p}$ The total $p \mu p$ formation rate, $\lambda_{p \mu p}=\lambda_{\text {of }}+\lambda_{\text {pf }}$, where $\lambda_{\text {pf }} \ll \lambda_{\text {of }}$

LEC Low Energy Constants, the experimental inputs to chiral perturbation theory

$\mathbf{L H}_{2}$ Liquid hydrogen

MBAS MuCap Berkeley Analysis Software

Merlin Linux-based parallel computing cluster at PSI, used extensively in Berkeley's analysis of the 2004 data 
MIAS MuCap Illinois Analysis Software

MIDAS Maximum Integration Data Acquisition System, a DAQ system commonly used in medium-sized nuclear physics experiments; MuCap uses the MIDAS framework for both DAQ and data analysis.

Michel electron A commonly encountered term for the electron produced by muon decay, $\mu \rightarrow e+\bar{\nu}_{e}+\nu_{\mu}$

MQL Muon Query Language, a high-level language specially designed for the MuCap data analysis

Mu Muonium, the bound state of a postive muon and an electron $\left(\mu^{+} e^{-}\right)$

$\boldsymbol{\mu E} 4$ PSI beamline used by MuCap until its closure in early 2004

$\boldsymbol{\mu S C}$ muon Scintillating Counter, the MuCap detector which provides the fast timing signal for the muon arrival, and the start time for the muon lifetime measurement

$\boldsymbol{\mu S C A}$ muon Scintillating Anti-Counter, a veto counter for scattered muons

$\boldsymbol{\mu P C}$ muon Proportional Chamber, a multiwire detector used to measure the position and time of muon passage

$\boldsymbol{\mu}^{+} \mathbf{S R}$ muon Spin Rotation

MWPC Multiwire Proportional Chamber

MySQL Database management system utilized in the offline data analysis

NIM Nuclear Instrument Module, the oldest and simplest international standard for modular electronics in experimental physics, created in 1969 
NLO, NNLO, ... Next-to-Leading Order, Next-to-Next-to-Leading Order, etc., (used in ChPT expansions)

OMC Ordinary muon capture, $\mu^{-}+p \rightarrow n+\nu_{\mu}$

PCAC Partially Conserved Axial Current hypothesis

PDG Particle Data Group

$\phi$ Atomic density of hydrogen, relative to liquid hydrogen $\left(\rho_{\mathrm{LH}_{2}}=4.25 \times 10^{22} \mathrm{~cm}^{-3}\right.$ at $300 \mathrm{~K})$

$\pi$ E3 PSI beamline used by MuCap for data collection since 2004

PNPI Petersburg Nuclear Physics Institute, Gatchina, St. Petersburg, Russia

Prod-50 The main body of data collected in fall 2004 (Run8), in which the hydrogen gas contained low levels of elemental and isotopic impurities, and the TPC operated at a voltage of $\approx 5 \mathrm{kV}$. The Prod-50 data forms the basis for the experimental results presented in this dissertation.

protium Isotopically purified hydrogen (i.e. depleted of deuterium)

PSI Paul Scherrer Institut, Villigen, Switzerland; a national research facility where the MuCap experiment is conducted.

QCD Quantum chromodynamics

RMC Radiative muon capture, $\mu^{-}+p \rightarrow n+\nu_{\mu}+\gamma$

ROOT Data analysis package developed at CERN in the mid-1990s and widely used in modern nuclear and particle physics experiments 
Run8 The fall 2004 data collection period, numbered according to to MuCap's internal run counting scheme. The Run8 data analysis is the subject of this dissertation.

Run9 The fall 2005 data collection period, numbered according to MuCap's internal run counting scheme

Run10 The spring 2006 data collection period, numbered according to MuCap's internal run counting scheme

SM Standard Model of particle physics

SSE "Small-scale expansion," an approach in chiral perturbation theory which includes $\Delta(1232)$ effects

SRIM "Stopping and Range of Ions in Matter," a collection of proprietary software packages which calculate features of ion transport in matter. MuCap has used the eponymous SRIM program to simulate $\mu+p$ scattering and stopping in hydrogen.

TDC Time-to-Digital Converter

TDC400 TDC module custom-designed by the PSI electronics group for readout of the MuCap TPC data; approximately twelve such modules were built, and at least seven are needed to completely instrument the TPC.

TPC Time Projection Chamber

TRIUMF TRI-University Meson Facility, a Canadian national laboratory for particle and nuclear physics research, located on the campus of the University of British Columbia

UCB University of California, Berkeley

UCL Université Catholique de Louvain à Louvain-La-Neuve, Belgium 
UIUC University of Illinois at Urbana-Champaign

VME VERSAmodule Eurocard, a nonproprietary high-speed data bus standard, released in 1981 and still widely used in industry and nuclear electronics instrumentation

VME64 A more recent VME standard, approved by the American National Standards Institute (ANSI) in 1995 


\section{Acknowledgments}

The measurement described in this dissertation is the product of years of tireless effort by the many members of the MuCap Collaboration. Below is a list of those who contributed to the development, execution, and/or data analysis of the experiment ${ }^{1}$; I extend my thanks to them all, and my apologies to anyone whom I may have inadvertantly omitted.

- University of California, Berkeley, and LBNL: T.A. Case, K.M. Crowe, S.J. Freedman, B. Gartner, F.E. Gray, B. Lauss, and J. Meyer;

- University of Illinois at Urbana-Champaign: S.M. Clayton, D.B. Chitwood, P.T. Debevec, D.W. Hertzog, P. Kammel², B. Kiburg, S. Knaack, C.J.G. Onderwater, C.S. Özben, and P. Winter;

- Paul Scherrer Institute (Switzerland): P. Dick, A. Dijksman, J. Egger, D. Fahrni, M. Hildebrandt, A. Hofer, V. Markushin, L. Meier, F. Mulhauser, C. Petitjean², and R. Schmidt;

- Petersburg Nuclear Physics Institute (Russia): V.A. Andreev, A.A. Fetisov, V.A. Ganzha, V.I. Jatsoura, P.A. Kravtsov, A.G. Krivshich, E.M. Maev, O.E. Maev, G.E. Petrov, S. Sadetsky, G.N. Schapkin, G.G. Semenchuk, M.A. Soroka, A.A. Vasilyev, A.A. Vorobyov, and M.E. Vznuzdaev;

- Université Catholique de Louvain (Belgium): L. Bonnet, J. Deutsch, J. Govaerts, D. Michotte, and R. Prieels;

- University of Kentucky: T. Gorringe, M. Ojha, V. Tishchenko, and P. Zolnierczuk;

\footnotetext{
${ }^{1}$ The institution affiliation is that at the time of the individual's participation.

${ }^{2}$ Spokesperson
} 
- Boston University: R.M. Carey, K.R. Lynch, and J. Paley;

- Technische Universität München (Germany): F.J. Hartmann;

\section{- Institute of Nuclear Physics, Polish Academy of Sciences (Poland):}

\section{A. Adamczak.}

I am also grateful to the technical staff of the collaborating institutions for their vital contributions, notably to the PSI staff for delivering beam of excellent quality. I would like to express my appreciation for the many years of camaraderie and cooperation we at Berkeley have shared with our close friends at Illinois.

On a more personal level, I wish to thank my advisor, Professor Stuart Freedman, for giving me the opportunity to participate in this project, and for his help in editing my dissertation. I am indebted to my Berkeley colleagues, Drs. Tom Case, Frederick Gray, and Bernhard Lauss, for their invaluable assistance over the past few years. Without their patient help, this dissertation would not have been possible.

I would like to thank the past and present members of the Berkeley Weak Interactions Group for creating a collegial atmosphere of vitality and enthusiasm of which I was glad to be a part. Thanks are also due to the Weak Interactions Group's administrative assistant, Melissa Barclay, and to the staff of the Berkeley physics department, both for their good cheer and for helping me to navigate through the Cal bureaucracy.

Thanks to Andrzej Adamczak for generously allowing me to reproduce his figures in my dissertation.

Funding for the experiment was provided in part by the U.S. National Science Foundation, the U.S. Department of Energy and CRDF, PSI, and the Russian Academy of Sciences. My own tuition and research expenses were supported by the U.S. Department of Energy under Contract No. DE-FG02-00ER41129, though some auxiliary DOE 
support was also provided through LBNL via Contract No. DE-AC02-05CH11231. Special thanks are due to Dr. Brad Tippens, the DOE Program Manager for Medium Energy Nuclear Physics, for graciously extending financial support for MuCap at Berkeley beyond the original allocation, in large part so that I might finish my Ph.D. studies.

Finally, I would like to thank my family and friends for helping me to make it through the long, hard pull of graduate school. 


\section{Chapter 1}

\section{Introduction}

This dissertation describes a precise measurement of the rate $\Lambda_{S}$ of the semileptonic weak interaction process of nuclear muon capture by the proton,

$$
\mu^{-}+p \rightarrow n+\nu_{\mu}
$$

from the hyperfine singlet ground state of the $\mu p$ atom. The data set under consideration was collected in fall 2004 as part of an ongoing experimental effort by the MuCap Collaboration [1] at Switzerland's Paul Scherrer Institut (PSI), where the project carries the official designation $\mathrm{R}-97-05[2,3]$. The measurement described herein represents the collaboration's first experimental result after many years of planning and development, beginning in 1996 .

MuCap was motivated by a desire to resolve the uncertainty surrounding the value of $g_{P}$, the weak nucleonic charged-current induced pseudoscalar coupling constant, a parameter that describes in part how the strongly interacting substructure of the nucleon affects its participation in weak interactions. This chapter explains the connection between $g_{P}$ and muon capture, surveys previous experimental results on the subject, and describes the basic principles and first results of the MuCap experiment. 


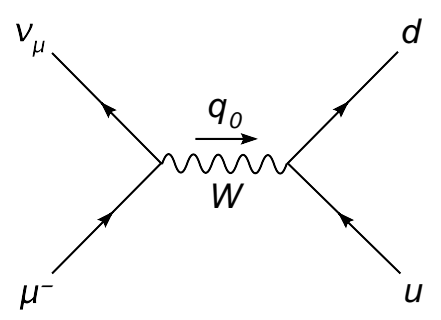

Figure 1.1: Tree-level Feynman diagram of nuclear muon capture in terms of fundamental fermions. The flow of time is upwards, as a negatively charged muon and an up quark exchange a virtual $W$ boson to produce a muon neutrino and a down quark. The transferred four-momentum is $q_{0} \equiv p_{\mu}-p_{\nu_{\mu}}$. (Diagram created using JaxoDraw [4].)

\subsection{Muon capture and $g_{P}$}

In the Standard Model, the weak interaction at a fundamental level involves fermion currents with so-called $V-A$ ("vector minus axial-vector") structure, $\gamma_{\alpha}\left(1-\gamma_{5}\right)$. Nuclear muon capture is a weak interaction process, and it can be described in terms of fundamental fermions by the Lagrangian

$$
\mathcal{L}=\frac{G_{F} V_{u d}}{\sqrt{2}}\left[\bar{d} \gamma^{\alpha}\left(1-\gamma_{5}\right) u\right]\left[\bar{\nu}_{\mu} \gamma_{\alpha}\left(1-\gamma_{5}\right) \mu\right]
$$

where an up quark $(u)$ is converted into a down quark $(d)$, a muon $(\mu)$ turns into a muon neutrino $\left(\nu_{\mu}\right)$, and both particle currents exhibit $V-A$ structure. The coefficient $G_{F}$ in Equation 1.2 is the Fermi weak coupling constant, and $V_{u d}$ is the appropriate element from the Cabibbo-Kobayashi-Maskawa matrix. The tree-level Feynman diagram for the capture process is pictured in Figure 1.1. The momentum transfer in muon capture is fixed at $q_{0}^{2}=-0.88 m_{\mu}^{2}$ (see Appendix A), so $\left|q_{0}\right|$ is always much less than the mass $m_{W}=80 \mathrm{GeV}$ of the charged weak vector boson $W$ which mediates the interaction. This simplifies the details of the $W$ propagator depicted in Figure 1.1, and muon capture can be effectively treated as a four-fermion contact (i.e. pointlike) interaction to an excellent approximationa presupposition implicit in the use of $G_{F}$ in Equation 1.2. 


\begin{tabular}{clc}
\hline \hline Form factor & Name & Value at $q_{0}^{2}=-0.88 m_{\mu}^{2}$ \\
\hline$g_{V}$ & Fermi, or vector & $0.976 \pm 0.001$ \\
$g_{M}$ & Weak magnetism, or tensor & $3.583 \pm 0.003$ \\
$g_{S}$ & Induced scalar & 0 \\
$g_{A}$ & Gamov-Teller, or axial vector & $1.247 \pm 0.004$ \\
$g_{T}$ & Weak electricity, or induced tensor & 0 \\
$g_{P}$ & Induced pseudoscalar & $7.3 \pm 1.1$ \\
\hline \hline
\end{tabular}

Table 1.1: The weak nucleonic charged-current form factors, and their 2007 values at the momentum transfer characteristic of muon capture. The value for $g_{P}$ is the first result from the MuCap Collaboration [7], as described in this dissertation, while the remaining nonzero form factors are from [8].

Although the preceding elementary description of muon capture is appealingly straightforward, it is not entirely accurate. Muon capture by a proton involves nucleons rather than isolated quarks, and the simple $V-A$ quark current in Equation 1.2 must be replaced with a more complicated expression that accounts for effects arising from the spatial extent and strongly interacting internal structure of nucleons. The most general, Lorentz-covariant form of the matrix element for the charged nucleonic current is [5]

$$
\begin{aligned}
\left\langle n\left|J^{\alpha}\right| p\right\rangle=V_{u d} \bar{\psi}_{n}[ & g_{V}\left(q^{2}\right) \gamma^{\alpha}+\frac{i g_{M}\left(q^{2}\right)}{2 m_{N}} \sigma^{\alpha \beta} q_{\beta}+\frac{g_{S}\left(q^{2}\right)}{m_{\mu}} q^{\alpha} \\
& \left.\quad-g_{A}\left(q^{2}\right) \gamma^{\alpha} \gamma_{5}-\frac{i g_{T}\left(q^{2}\right)}{2 m_{N}} \sigma^{\alpha \beta} q_{\beta} \gamma_{5}-\frac{g_{P}\left(q^{2}\right)}{m_{\mu}} q^{\alpha} \gamma_{5}\right] \psi_{p},
\end{aligned}
$$

where $\psi_{p}$ and $\psi_{n}$ are the four-component wavefunctions of the proton and neutron, $m_{N}$ is the mean nucleon mass, $m_{\mu}$ is the muon mass $^{1}$, and the six real, nonnegative form factors $g_{i}$ are listed in Table 1.1. The form factors are all functions of $q^{2}$, where $q$ is the fourmomentum exchanged in the process under consideration.

If the strong interaction were absent and nucleons were point particles, the nucleonic current in Equation 1.3 would revert to the simple $V-A$ form depicted in Equation 1.2,

\footnotetext{
${ }^{1}$ The appearance of the lepton mass $m_{\mu}$ in the nucleonic current in Equation 1.3 is a convenient convention that is commonly used in the field of muon capture, following reference [6]. Some authors use $G_{P} / 2 m_{N}=g_{P} / m_{\mu}$ instead.
} 
and only the vector form factor $\left(g_{V} \rightarrow 1\right)$ and axial form factor $\left(g_{A} \rightarrow 1\right)$ would remain nonzero. In reality, the weak nucleonic vertex is "dressed" by the strong interactions, and the other four form factors $-g_{M}, g_{S}, g_{T}$, and $g_{P}$-are said to be "induced" by the strongly interacting quark substructure of the nucleon. According to reference [9],

this parameterization of the nuclear matrix element [...] in terms of form factors ${ }^{2}$ is only a phenomenological representation of our ignorance of the microscopic nonperturbative quark dynamics, [but] this approach to nuclear muon capture allows nevertheless for explicit predictions independent of the details of nuclear models, relying only on the results of other experiments and the power of symmetry principles.

Assuming $\mu-e$ universality [11], the form factors $g_{V}$ and $g_{M}$ are known from their electromagnetic counterparts in elastic electron-nucleon scattering experiments, via the Conserved Vector Current (CVC) hypothesis [12]. The CVC-based values for $g_{V}$ and $g_{M}$ have also been tested directly in weak processes at low energies, using superallowed nuclear beta decays for $g_{V}$ [13], and beta decays in mirror and isospin triplet nuclei (in particular, the $A=12$ triad) for $g_{M}$ [14-17]. The value of form factor $g_{A}(0)$ has been determined from beta decay measurements [18], and its $q^{2}$-dependence has been determined from (quasi)elastic (anti)neutrino scattering on protons [19] and charged pion electroproduction on protons [20-22]; see references $[22,23]$ for reviews. The so-called "second-class" form factors $g_{S}$ and $g_{T}$ [24] are believed to be zero, or at least negligibly small, according to arguments involving CVC and G-parity invariance $[25,26]$, and the experimental upper bounds on their values indicate that they can indeed be safely ignored [27-29]. This leaves the pseudoscalar form factor $g_{P}$, which has long been the least well known of the nucleonic charged-current form factors. Despite efforts spanning the last forty years, experimental determinations of $g_{P}$ were often controversial and model dependent, with uncertainties as

\footnotetext{
${ }^{2}$ The form factor parameterization of nuclear currents is commonly referred to as the "elementary particle model" of nuclear electroweak processes - particularly when heavier elements are involved-because the nucleus is treated as an elementary particle [10].
} 
large as $50 \%$.

In principle, $g_{P}$ participates in any weak interaction process that involves the nucleonic charged current in Equation 1.3. This includes beta decay, muon capture, and neutrino-initiated processes. However, the momentum transfer in beta decay is too small for $g_{P}$ to have an appreciable effect ${ }^{3}$, and neutrino processes are notoriously difficult to detect. Consequently, muon capture provides the best opportunity for measuring the pseudoscalar coupling $g_{P} \equiv g_{P}\left(q_{0}^{2}\right)$. For reviews on the relationship between muon capture and $g_{P}$, see references $[5,6,23,30-34]$. It should be noted that pion electroproduction is another potential source of information on $g_{P}[21,23]$, but the connection is controversial and model dependent [5].

Muon capture thus provides an excellent opportunity to probe the weak axial current of the nucleon and to rigorously test our basic understanding of low-energy strong interaction physics. The modern field of chiral perturbation theory, which is based upon the approximate chiral symmetries of quantum chromodynamics (QCD), currently yields precise $(2-3 \%)$ predictions for the value of $g_{P}$. Consequently, like the axial vector coupling $g_{A}, g_{P}$ can be regarded as a testing ground for nonperturbative realizations of quantum chromodynamics, and experimental input from muon capture measurements is essential.

\subsection{Previous measurements of muon capture in hydrogen}

Nuclear muon capture can take place within any nucleus, but experiments involving high- $Z$ nuclei are difficult to interpret because the physics of interest is obscured by the complexity of the nuclear environment. To cleanly extract $g_{P}$ from a capture measurement,

\footnotetext{
${ }^{3}$ The magnitude of $g_{P}$ is actually $\approx 1.5$ times larger in beta decay than in muon capture, but in beta decay the effect of the form factor is suppressed by the low momentum transfer in coefficient $q$, as well as by the multiplying $\gamma_{5}$ matrix (see Equation 1.3). The larger momentum transfer in muon capture enhances the magnitude of the pseudoscalar term by the muon-electron mass ratio $m_{\mu} / m_{e} \sim 200$.
} 
it is desirable to minimize the uncertainties due to nuclear structure as much as possible. Muon capture by a free proton - that is, a proton not bound within a nucleus - is therefore attractive because of its theoretical simplicity. However, muon capture in hydrogen is challenging to measure, for several reasons:

- Muons stopped in hydrogen form neutral $\mu p$ atoms, which in turn tend to form $p \mu p$ molecules or to surrender their muons to any isotopic or elemental impurities that are encountered in the course of diffusion. Each of these muonic systems has a characteristic nuclear capture rate, and they all contribute to the measured capture rate to some degree.

- Due to the $V-A$ structure of the weak interaction, the muon capture rate is sensitive to the hyperfine spin state of the atomic or molecular system from which capture takes place.

- Capture from the $\mu p$ atomic hyperfine singlet ground state is rare, with a branching ratio of only $0.16 \%$. Most muons disappear via decay.

- The final-state capture particles are neutral and difficult to detect. Furthermore, muon capture suffers from the related disadvantage that a practical measurement only provides a single number - the capture rate - in contrast to processes such as beta decay which offer a wealth of additional observables in the form of energy spectra, polarization correlations, etc.

The basic experimental situation can thus be summarized as follows. In the ideal case, there are two disappearance channels available for negatively charged muons in hydrogen:

$$
\begin{aligned}
\mu^{-} & \rightarrow e^{-}+\bar{\nu}_{e}+\nu_{\mu} \\
\mu^{-}+p & \rightarrow n+\nu_{\mu} .
\end{aligned}
$$


The $\mu^{-}$decay channel in Process 1.4 proceeds at a constant rate which is close to $\lambda_{0}$, the "free," or "vacuum," muon decay rate, regardless of what state the muon is in. The value of $\lambda_{0}$ has been determined to $\approx 10 \mathrm{ppm}$ precision from experimental studies of $\mu^{+}$decay, which is used because decay is the only disappearance channel available for positive muons ${ }^{4}$. The single capture channel presented in Equation 1.5 is an oversimplification, because in reality there exist many possible capture pathways, such as from $\mu p$ atoms, $p \mu p$ molecules, and $\mu X$ atoms, where $X$ represents an isotopic impurity such as deuterium or an elemental impurity such as oxygen. Muon capture therefore proceeds at a rate $\Lambda_{\mathrm{c}}$ which in general includes contributions from multiple capture pathways, and whose exact value depends upon the particular experimental conditions under study. The total capture rate is also generally a function of time, $\Lambda_{\mathrm{c}}=\Lambda_{\mathrm{c}}(t)$, because the occupation numbers of the various muonic states change with time in a manner determined by the density and purity of the hydrogen gas.

Results from previous measurements of muon capture in hydrogen are listed in Table 1.2, along with their corresponding determinations of $g_{P}$. The variations in the capture rate $\left(\Lambda_{\mathrm{c}}\right)$ values in Table 1.2 are primarily due to differences in the hydrogen target densities that were used; in liquid hydrogen most captures take place in $p \mu p$ molecules, while in gaseous hydrogen most captures proceed from the hyperfine singlet ground state of the $\mu p$ atom.

The muon capture experiments before 1981 used either bubble chambers [35-37] or scintillating neutron detectors [38-41] to identify capture events that occurred after muons were stopped in a hydrogen target. Capture events were recognized from the unique signature generated by the monoenergetic, $5.2 \mathrm{MeV}$ final-state neutrons (see Appendix A). By counting the number of muons that underwent nuclear capture, $N_{\text {captures, }}$ as well as either the number of muons that decayed, $N_{\text {decays }}$, or the total number of stopped muons,

\footnotetext{
${ }^{4}$ It is worth noting that most $\mu^{-}$particle properties are actually known from $\mu^{+}$experiments and the reasonable assumption of $C P T$ invariance [34].
} 


\begin{tabular}{|c|c|c|c|c|c|c|c|c|c|}
\hline Year & Location & Process & $\mathrm{H}_{2}$ & Exp. method & $\Lambda_{\mathrm{c}}\left(\mathrm{s}^{-1}\right)$ & $\delta \Lambda_{\mathrm{c}} / \Lambda_{\mathrm{c}}$ & $g_{P}\left(-0.88 m_{\mu}^{2}\right)$ & $\delta g_{P} / g_{P}$ & Ref. \\
\hline 1962 & Chicago & $\mathrm{OMC}$ & liquid & bubble chamber & $428 \pm 85$ & $20 \%$ & $18.7 \pm 8.2$ & $44 \%$ & {$[35,36]$} \\
\hline 1962 & CERN & $\mathrm{OMC}$ & liquid & bubble chamber & $450 \pm 50$ & $11 \%$ & $16.4 \pm 4.9$ & $30 \%$ & {$[37]$} \\
\hline 1962 & Columbia & $\mathrm{OMC}$ & liquid & neutron counters & $515 \pm 85$ & $17 \%$ & $6.3 \pm 8.7$ & $100 \%$ & {$[38]$} \\
\hline 1963 & Columbia & $\mathrm{OMC}$ & liquid & neutron counters & $464 \pm 42$ & $9 \%$ & $11.4 \pm 4.2$ & $37 \%$ & {$[39]$} \\
\hline 1969 & CERN & $\mathrm{OMC}$ & gas & neutron counters & $651 \pm 57$ & $9 \%$ & $11.0 \pm 3.8$ & $35 \%$ & {$[40]$} \\
\hline 1974 & Dubna & $\mathrm{OMC}$ & gas & neutron counters & $686 \pm 88$ & $13 \%$ & $8.7 \pm 5.7$ & $66 \%$ & {$[41]$} \\
\hline \multirow[t]{2}{*}{1981} & Saclay $^{\dagger}$ & $\mathrm{OMC}$ & liquid & muon lifetime & $460 \pm 20$ & $4 \%$ & $7.9 \pm 3.0$ & $38 \%$ & {$[42,43]$} \\
\hline & Saclay $\ddagger$ & & & & $435 \pm 17$ & $4 \%$ & $10.6 \pm 2.7$ & $25 \%$ & {$[5]$} \\
\hline \multirow[t]{2}{*}{2007} & PSI (MuCap) & $\mathrm{OMC}$ & gas & muon lifetime & $725 \pm 17$ & $2 \%$ & $7.3 \pm 1.1$ & $15 \%$ & {$[7]$} \\
\hline & & & & & $R_{\gamma}$ & $\delta R_{\gamma} / R_{\gamma}$ & & & \\
\hline 1998 & TRIUMF & $\mathrm{RMC}$ & liquid & photon counters & $\begin{array}{c}(2.10 \pm 0.21) \\
\times 10^{-8}\end{array}$ & $10 \%$ & $\begin{array}{r}12.2 \pm 0.9 \\
\pm 0.4\end{array}$ & $8 \%$ & {$[44]$} \\
\hline
\end{tabular}

$\dagger$ using the $1981 \mu^{+}$lifetime

$\ddagger$ using the $2003 \mu^{+}$lifetime

Table 1.2: Previous measurements of nuclear muon capture in hydrogen $\left(\mathrm{H}_{2}\right)$, and the corresponding determinations of $g_{P}$ at the characteristic momentum transfer $q_{0}^{2}=-0.88 m_{\mu}^{2}$. All of the ordinary muon capture (OMC) experiments published a capture rate $\Lambda_{\mathrm{c}}$, while the TRIUMF radiative muon capture (RMC) result was given as the partial branching ratio $R_{\gamma}$ for capture events where a photon was radiated with an energy $k_{\gamma}>60 \mathrm{MeV}$. Excepting MuCap, all $g_{P}$ values are taken from reference [5], where they were derived from the published capture data using 2003 parameter values and certain assumptions about the occupation numbers of the atomic and molecular muonic states in each experiment. It should be mentioned that the quoted precisions $\delta g_{P} / g_{P}$ do not tell the entire story, because some of the errors do not accurately reflect the sizable uncertainty in the $p \mu p$ kinetics rates which are needed for extracting $g_{P}$ from the capture rate, as will be discussed in Section 1.4. For comparison, chiral perturbation theory predicts $g_{P}\left(-0.88 m_{\mu}^{2}\right)=8.26 \pm 0.23[23]$. 
$N_{\text {stops }}$, these experiments were able to calculate an effective, time-integrated capture rate $\Lambda_{\mathrm{c}}$ according to the basic, branching-ratio-based formula

$$
\Lambda_{\mathrm{c}}=\lambda_{0}\left(\frac{N_{\text {captures }}}{N_{\text {decays }}}\right)=\lambda_{0}\left(\frac{N_{\text {captures }}}{N_{\text {stops }}-N_{\text {captures }}}\right) .
$$

The neutron scintillator experiments had the additional capability of looking at the time distribution of captures, whereas the bubble chamber experiments could not. The precisions of the measurements were limited by statistics and/or the calibration accuracy of the neutron detection efficiencies. A detailed survey of these early experiments can be found in reference [32].

The 1981 Saclay experiment [42] adopted a novel approach: instead of attempting to observe capture events directly — which requires confronting the difficult task of detecting capture neutrons - they looked for the effects of capture on the time spectrum of decay electrons, $N_{e}(t)$, where

$$
N_{\text {decays }}=\int_{0}^{\infty} N_{e}(t) d t
$$

The total $\mu^{-}$disappearance rate in liquid hydrogen,

$$
\Lambda_{\mathrm{tot}}=\lambda_{0}+\Lambda_{\mathrm{c}}=\frac{1}{\tau_{\mu^{-}}}
$$

was determined by extracting the $\mu^{-}$lifetime $\tau_{\mu^{-}}$from the approximately exponential $N_{e}(t)$ spectrum, and the muon capture rate was then obtained by subtracting off the value of $\lambda_{0}$. (In fact, the Saclay investigators also performed their own determination of $\lambda_{0}$ by measuring the time spectrum of $\mu^{+}$decays.) Prior to MuCap, the Saclay muon capture rate determination was the most precise of any experiment, but the extraction of $g_{P}$ is clouded by the complex physics of muonic molecules in liquid hydrogen, as will be discussed shortly. 
In 1998 an experiment at TRIUMF measured the partial branching ratio for radiative muon capture (RMC) in hydrogen,

$$
\mu^{-}+p \rightarrow n+\nu_{\mu}+\gamma
$$

which differs from ordinary muon capture (OMC) in that a final-state photon is produced. RMC presents an experimental challenge because it is an exceedingly rare process in hydrogen: the partial branching ratio that was measured,

$$
R_{\gamma}=\frac{N_{\text {captures }}^{\mathrm{RMC}}\left(E_{\gamma}>60 \mathrm{MeV}\right)}{N_{\text {stops }}},
$$

is of order $10^{-8}$, far smaller than the OMC $\mu p$ hyperfine singlet branching ratio of $\sim 10^{-3}$. The suppression of RMC is due in part to the fact that the emitted photon carries away angular momentum, which has the effect of swapping the hyperfine singlet and triplet OMC likelihoods [45]; a crude estimate using the hyperfine triplet OMC rate $\Lambda_{T}$ predicts an RMC branching ratio of $\sim \alpha \Lambda_{T} / \lambda_{0} \sim 10^{-7}$. RMC is nevertheless appealing because it is a more sensitive probe of $g_{P}$, due to the fact that the process does not have a fixed momentum transfer and can therefore explore energy regions where $g_{P}$ has a larger effect. Furthermore, the TRIUMF experiment was less sensitive to molecular effects than the Saclay OMC experiment. The relatively precise RMC result for $g_{P}$ exceeds predictions by $4.2 \sigma[44]$, and despite intense theoretical scrutiny the discrepancy remains unresolved.

One of the best determinations of $g_{P}$ comes from a 1998 experiment investigating $\mathrm{OMC}$ in ${ }^{3} \mathrm{He}$ [46]. Compared to capture in hydrogen, the ${ }^{3} \mathrm{He} \rightarrow{ }^{3} \mathrm{H}$ capture process offers the advantages of a relatively high rate and an absence of complications from atomic and molecular effects. The authors of reference [46] measured the rate of the reaction $\mu^{-}+{ }^{3} \mathrm{He} \rightarrow{ }^{3} \mathrm{H}+\nu_{\mu}$ to $0.3 \%$ precision, reporting $\Lambda_{3 \mathrm{He}}^{\exp }=1496 \pm 4 \mathrm{~s}^{-1}$. This experimental result is in agreement with the theoretical predictions $\Lambda_{3}^{\mathrm{th}} \mathrm{He}=1497 \pm 21 \mathrm{~s}^{-1}$ [47] and $\Lambda_{3 \mathrm{He}}^{\mathrm{th}}=1485 \pm 8 \mathrm{~s}^{-1}[48]$, which were obtained using two quite different approaches (see 
the reviews in references $[5,8])$. From $\Lambda_{3 \mathrm{He}}^{\mathrm{exp}}$, the value $g_{P}\left(-0.954 m_{\mu}^{2}\right)=8.53 \pm 1.54$ was extracted, which implies $g_{P}\left(-0.88 m_{\mu}^{2}\right)=8.77 \pm 1.58$ [5]. Although this result is precise to $18 \%$, the complexity of the many-body ${ }^{3} \mathrm{He}$ system is a source of some unease. Moreover, very recent calculations which include radiative corrections to $\mathrm{OMC}$ in ${ }^{3} \mathrm{He}$ report sizable modifications to the theoretical estimates for $\Lambda_{3 \mathrm{He}}^{\mathrm{th}}$ above, giving $1537 \pm 22 \mathrm{~s}^{-1}$ and $1506 \pm 12 \mathrm{~s}^{-1}$, respectively [8].

\subsection{The MuCap experiment}

The aim of the MuCap experiment is to measure the rate of ordinary muon capture in hydrogen, in a manner that avoids many of the uncertainties and ambiguities encountered by previous experiments and which enables a more reliable determination of $g_{P}$. MuCap uses the so-called "lifetime," or "disappearance," technique to determine the OMC rate in gaseous hydrogen, the same technique first employed by the Saclay experiment to determine the $\mathrm{OMC}$ rate in liquid hydrogen. Muons are stopped in a hydrogen target, and surrounding detectors monitor the emission of decay electrons. Each muon's arrival time $t_{\mu}$ and decay time $t_{e}$ are recorded, enabling the construction of the elapsed-time spectrum $\Delta t=t_{e}-t_{\mu}$. The ensemble of decay events is prepared one at a time, and the resulting experimental observable is the time spectrum of decay electrons $N_{e}(t)$, which is proportional to the surviving muon population $N_{\mu}(t)$ :

$$
N_{e}(t)=-\left.\frac{d N_{\mu}}{d t}\right|_{\text {decay }}=\lambda_{0} N_{\mu}(t) .
$$

A full solution for $N_{\mu}(t)$ requires a complete treatment of the complicated kinetics of muons in hydrogen. However, in MuCap we use an ultrapure, deuterium-depleted, low-density hydrogen gas target in which most muons are restricted to the hyperfine singlet ground 
state of the $\mu p$ atom. In this case,

$$
\Lambda_{\mathrm{c}}(t) \approx \Lambda_{S}
$$

and, to a good approximation, the $\mu^{-}$survival probability in hydrogen follows a simple exponential,

$$
N_{\mu^{-}}(t) \propto e^{-\left(\lambda_{0}+\Lambda_{S}\right) t}
$$

The total muon disappearance rate $\left(\lambda_{0}+\Lambda_{S}\right)$ can therefore be extracted from a best fit to the measured lifetime spectrum $N_{e}(t)$, fitting to the function

$$
N(t)=N e^{-\lambda t}+B
$$

where $N$ is an overall scaling factor and $B$ accounts for accidental background. The $\mu p$ singlet capture rate $\Lambda_{S}$ is then obtained by

$$
\Lambda_{S}=\lambda-\lambda_{0}
$$

It is worth noting that, when using this method, the radiative muon capture channel contributes to effective $\mu^{-}$disappearance rate $\lambda$, and hence to the deduced capture rate $\Lambda_{S}$. However, the branching ratio for RMC is miniscule compared to OMC, $\left(\Lambda_{\mathrm{RMC}} / \Lambda_{S}\right) \sim 10^{-5}$, so the RMC contribution can be safely ignored.

Although this approach is simple in principle, in practice it presents formidable challenges. The $\mu p$ hyperfine singlet capture rate [8]

$$
\Lambda_{S} \approx 710 \mathrm{~s}^{-1}
$$

is much smaller than the free muon decay rate [49]

$$
\lambda_{0}=455,162.2 \pm 4.4 \mathrm{~s}^{-1}
$$

which means that, according to the experimental prescription in Equation 1.13, MuCap obtains a relatively small number, $\Lambda_{S}$, by taking the difference between two relatively large, 
experimentally determined numbers, $\lambda$ and $\lambda_{0}$. Moreover, the simple, single-channel $\mu^{-}$ nuclear capture model $\Lambda_{\mathrm{c}}(t) \approx \Lambda_{S}$ is not completely accurate, and captures by impurities and $p \mu p$ molecules must also be taken into account. The experimental challenges therefore lie in gathering sufficient statistics to resolve the $\mu^{-}$lifetime in hydrogen to high precision, and in minimizing, controlling, and correcting for systematic effects.

To accomplish this task, we use an ultrapure, low-density hydrogen gas target to suppress unwanted effects from $p \mu p$ molecules and from isotopic and elemental impurities. Under these conditions, $96 \%$ of all captures proceed from the $\mu p$ singlet state, and the ambiguities that can arise from the presence of additional $\mu^{-}$disappearance channels are largely avoided. In order to clearly identify which muons stop in the low-density gas - and thereby avoid contributions from muon stops in other materials - we employ a new technique, which is to use a hydrogen-gas-filled time projection chamber as an active target. The hydrogen target is surrounded by an array of electron tracking detectors which provide a suite of methods for suppressing accidentals and correcting systematic effects. The detectors, electronics, and computer systems are designed to make the collection of high statistics in a reasonable time period possible. In fall 2004 , we recorded $\approx 1.6 \times 10^{9}$ good decay events, sufficient for a $2.4 \%$ precision determination of $\Lambda_{S}$ and a $15 \%$ determination of $g_{P}$. Our capture rate measurement, $\Lambda_{S}=725.0 \pm 17.4 \mathrm{~s}^{-1}$, is consistent within $1 \sigma$ with radiative-corrected theoretical predictions [8], and the ensuing determination of $g_{P}\left(q^{2}=-0.88 m_{\mu}^{2}\right)=7.3 \pm 1.1$ agrees with the predictions of modern chiral perturbation theory. In the time since 2004 we have collected more than $10^{10}$ decay events in total, which should ultimately enable a $1 / \sqrt{10^{10}}=10 \mathrm{ppm}$ precision measurement of the $\mu^{-}$lifetime in hydrogen, thus determining $\Lambda_{S}$ to within $1 \%$ and the pseudoscalar form factor $g_{P}$ to within $7 \%$. It is worth noting that $\mu^{+}$decay events are also collected with the same apparatus, as a control on the $\mu^{-}$ measurement. 


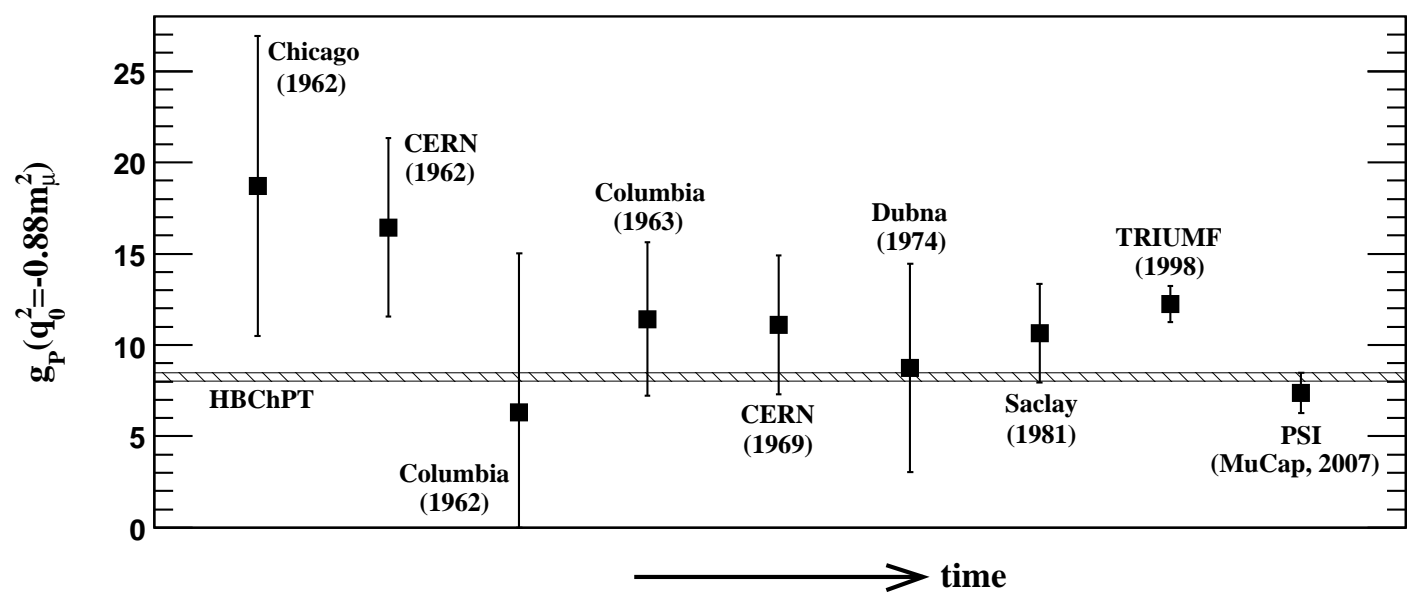

Figure 1.2: Chronology of $g_{P}$ determinations from muon capture experiments in hydrogen, as listed in Table 1.2. It should be emphasized that the $g_{P}$ values presented here are the 2003 updated values from reference [5], and therefore they are not necessarily the same as the $g_{P}$ values that might have been reported in the original publications. The hatched band indicates the $g_{P}=8.26 \pm 0.23$ region predicted by heavy baryon chiral perturbation theory (HBChPT) [23].

\subsection{Situation overview}

Historically, all previous ordinary muon capture (OMC) measurements yielded $g_{P}$ values consistent with theoretical expectations, and the more recent TRIUMF radiative muon capture (RMC) result was the only source of disagreement. However, when the OMC results for hydrogen were reinterpreted with the 2003 Particle Data Group (PDG) $\mu^{+}$lifetime, the world average for $g_{P}^{\mathrm{OMC}}$ increased by roughly $1 \sigma$. The updated $g_{P}^{\mathrm{OMC}}$ value was still consistent with theory, but its central value was actually closer to the anomalous RMC result $[5,50]$. In contrast, the 2007 MuCap result for $g_{P}$ in Table 1.2 is more consistent with theoretical predictions than with the most recent experimental results. The $g_{P}$ determinations in Table 1.2 are plotted in Figure 1.2 in chronological order, to illustrate the evolution of experimental precision and the spread in values.

Figure 1.2 does not tell the entire story, however, because some of the quoted 


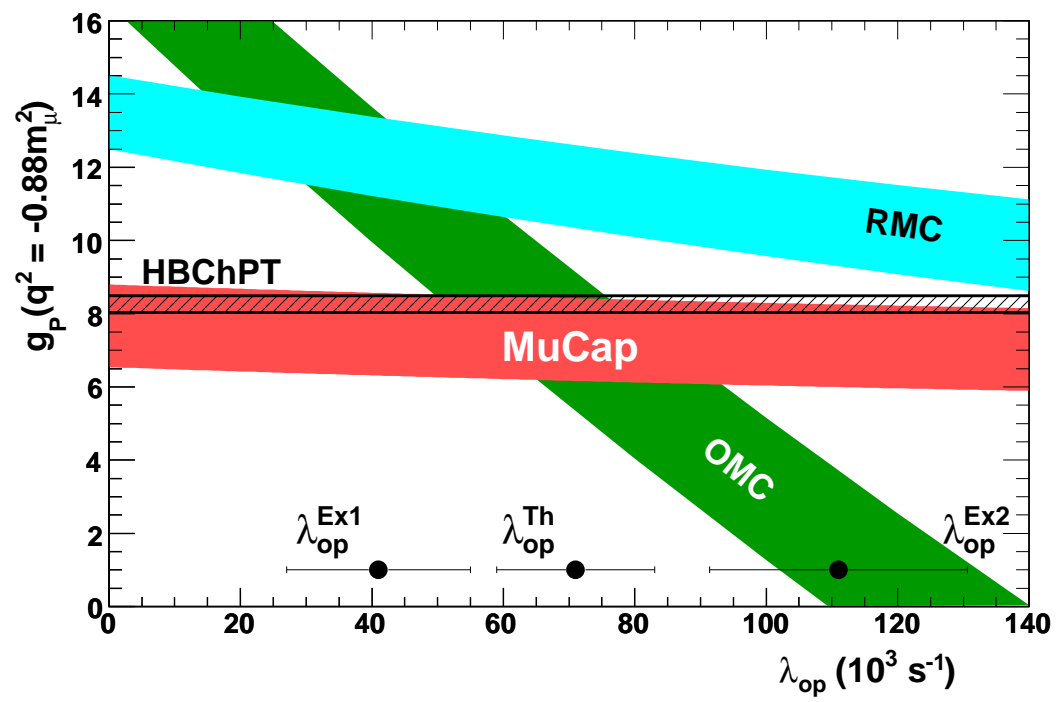

Figure 1.3: Experimental and theoretical determinations of $g_{P}$, presented vs. the ortho-para transition rate $\lambda_{\mathrm{op}}$ in the $p \mu p$ molecule. The value for $g_{P}$ predicted by heavy baryon chiral perturbation theory (HBChPT) is nearly identical to earlier current-algebra predictions involving the partially conserved axial current (PCAC). The most precise previous OMC experiment [42] and the lone RMC experiment [44] both depend significantly on the value of $\lambda_{\mathrm{op}}$, which itself is poorly known due to mutually inconsistent experimental ( $\lambda_{\mathrm{op}}^{\mathrm{Ex}}[43]$, $\left.\lambda_{\mathrm{op}}^{\mathrm{Ex} 2}[51]\right)$ and theoretical $\left(\lambda_{\mathrm{op}}^{\mathrm{Th}}[52]\right)$ results. In contrast, the MuCap result for $g_{P}$ is nearly independent of molecular effects.

precisions do not reflect the uncertainty in the values of the parameters describing $p \mu p$ kinetics, which have a strong influence on the interpretation of muon capture measurements. In fact, the different experiments used a variety of molecular values when extracting $g_{P}$ from their capture results, and thus a simple comparison of the final $g_{P}$ values is potentially misleading. A more informative plot is presented in Figure 1.3, where $g_{P}$ is plotted versus $\lambda_{\mathrm{op}}$, the transition rate from the ortho hyperfine state of the $p \mu p$ molecule to its para state. The two most precise previous $g_{P}$ determinations - the Saclay OMC and TRIUMF RMC experiments - were carried out using liquid hydrogen targets, in which muon capture proceeds predominantly from the ortho and para states of $p \mu p$ molecules. As a result, those experiments measured an effective capture rate that is a combination of two different molecular capture rates, and interpretation of their results sensitively depends upon the specifics 
of muonic molecular chemistry in hydrogen - in particular, on the ortho $\rightarrow$ para state transition rate $\lambda_{\mathrm{op}}$. Alas, this quantity is poorly known, owing to the significant disagreement among the theoretical and experimental determinations of its value. Prior to the advent of MuCap, the situation presented in Figure 1.3 was one of confusion. Modern studies within the context of chiral perturbation theory had reached an accuracy of $2-3 \%$ on $g_{P}$ which corroborated earlier current-algebra-based predictions, so the theoretical description was considered reliable, making a precise experiment a significant test of the theory. The experimental situation, however, was tenuous: the best OMC result was compromised by ambiguities from muon molecular physics, and the RMC result was completely inconsistent with expectations. It proved to be impossible to simultaneously reconcile the RMC and Saclay OMC results with the theoretical prediction in a fashion that was consistent with the general understanding of muon chemistry in liquid hydrogen $[5,50]$. This state of affairs is reflected in the lack of a common intersection of the OMC, RMC, and theory bands in Figure 1.3.

The first MuCap result for $g_{P}$, which is also presented in Figure 1.3, does not suffer from the sensitivity to molecular effects that afflicted previous experiments. The MuCap error band is relatively flat because the low-density hydrogen gas target suppresses molecular effects on the measured capture rate. MuCap's precise, unambiguous determination of $g_{P}$ appears to confirm modern theoretical predictions, and does not support the large deviation implied by the $\mathrm{RMC}$ result. The MuCap result thus affirms the validity of the chiral symmetry principles that currently guide our understanding of strong interaction physics in the low-energy regime, and therefore suggests that the inconsistencies in the earlier experimental OMC and RMC $g_{P}$ results originated from an incorrect or incomplete understanding of muon chemistry in hydrogen. Future MuCap results are expected to reduce the width of the $g_{P}$ error band by a factor of two, to within the precision goal of $7 \%$. 


\section{Chapter 2}

\section{Theory}

This chapter surveys the development of theoretical descriptions of $g_{P}$ and muon capture over the past half century, examines modern theoretical models, and discusses the implications of a precise muon capture measurement.

\section{$2.1 \quad$ Historical overview of $g_{P}$}

A prediction for $g_{P}$ was first offered in 1958 by Goldberger and Treiman [53]. Using dispersion relation techniques, they were able to express $g_{P}$ in terms of the muon mass $m_{\mu}$, the pion mass $m_{\pi}$, the momentum transfer $q$, the pion decay constant $f_{\pi}$, and the pion-nucleon-nucleon coupling constant $g_{\pi N N}\left(q^{2}\right)$ :

$$
g_{P}\left(q^{2}\right)=\frac{2 m_{\mu} f_{\pi} g_{\pi N N}\left(q^{2}\right)}{m_{\pi}^{2}-q^{2}}
$$

A similarly derived expression for the axial form factor, the so-called "Goldberger-Treiman relation" [54]

$$
g_{A}(0)=\frac{f_{\pi} g_{\pi N N}}{m_{N}}
$$




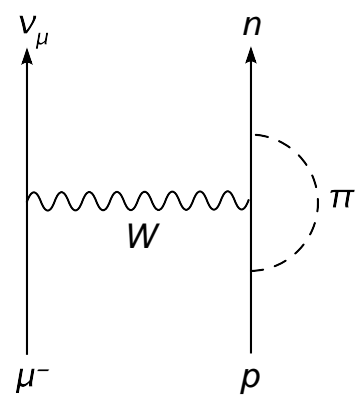

(a)

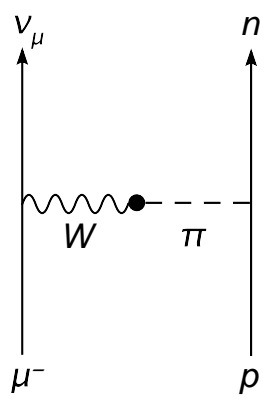

(b)

Figure 2.1: Feynman diagrams of pion field contributions to the axial form factors in muon capture. The pion-nucleon vertex correction in (a) contributes to the axial vector form factor $g_{A}$, while the pion pole process in (b) contributes to the pseudoscalar form factor $g_{P}$. (Diagrams created using JaxoDraw [4].)

was more readily testable using contemporary experimental data, and its surprising success attracted a great deal of attention. Although the calculations leading to Equation 2.2 were only approximate, the resulting simple formula yielded results accurate to within $10 \%$ and revealed unexpected connections between the strong and weak interactions [55].

The subsequent current algebra program of the 1960s developed in large part out of efforts to explain the accuracy of Equation 2.2. The Goldberger-Treiman (G-T) expressions came to be understood from a new perspective as a consequence of the fundamental notion of the partially conserved axial current (PCAC) and its relation to the pion field $[56,57]$ (Figure 2.1). Furthermore, the current algebra approach enabled Adler and Dothan as well as Wolfenstein (ADW) to identify a small correction to the G-T expression for $g_{P}[58,59]$,

$$
g_{P}\left(q^{2}\right)=\frac{2 m_{\mu} f_{\pi} g_{\pi N N}\left(q^{2}\right)}{m_{\pi}^{2}-q^{2}}-\frac{1}{3} g_{A}(0) m_{\mu} m_{N}\left\langle r_{A}^{2}\right\rangle
$$

where $\left\langle r_{A}^{2}\right\rangle$ is the mean-square axial radius of the nucleon.

PCAC, together with the conserved vector current (CVC) hypothesis [60,61], formed the backbone of the current algebra program and pointed the way toward the mod- 
ern gauge theory structure of electroweak interactions in the Standard Model. CVC and PCAC were also the historical precursors for the chiral symmetries of quantum chromodynamics (QCD), which govern many modern theoretical approaches toward $g_{P}$ and muon capture.

\subsection{Chiral perturbation theory and $g_{P}$}

In principle, all of the nucleon's induced form factors should be derivable from the fundamental quark-gluon QCD Lagrangian. In practice this has not been accomplished, and low-energy effective field theories such as chiral perturbation theory $(\mathrm{ChPT})^{1}$ presently provide the best opportunity for studying $g_{P}$. ChPT incorporates QCD symmetries into a systematic, low-energy expansion that can be applied to processes where the external momenta are small compared to the chiral symmetry breaking scale, $\Lambda_{\chi} \sim m_{N} \sim 1 \mathrm{GeV}$. As with any effective theory, the ChPT Lagrangian contains parameters which cannot be derived from ChPT itself, but must be fixed by experiment [62]. In ChPT these are the so-called low-energy constants, or LECs.

ChPT was originally formulated to describe the interactions of light mesons [63], but it can be applied to baryons without invalidating the small-momentum expansion [64]. In this latter context ChPT is commonly known as heavy baryon chiral perturbation theory (HBChPT), and this area of study has experienced great advances in recent years. In HBChPT, pions are identified as the (approximate) Goldstone bosons of spontaneously broken chiral symmetry in the strong interactions, and this has implications for the nucleonic matrix elements. Namely, the HBChPT expansion rapidly converges to reproduce the PCAC result in Equation 2.3, while enabling the systematic calculation of corrections. The

\footnotetext{
${ }^{1} \mathrm{ChPT}$ is sometimes described as "nonperturbative" QCD. This potentially confusing terminology refers to the fact that the effective chiral Lagrangian is not a perturbation to-i.e. cannot be derived from-the fundamental QCD Lagrangian.
} 
leading term in the HBChPT expansion is the original pion-pole-dominated G-T expression for $g_{P}$, followed by the ADW term at one-loop order in the pion field $[62,65]$. Recent HBChPT calculations have confirmed that the PCAC expression remains accurate to twoloop order in the pion field for low momentum transfers [66]. Using present-day values for the input parameters, the pseudoscalar coupling is predicted to be $g_{P}=8.26 \pm 0.23$ [23].

There exist numerous approaches to ChPT, such as the application of chiral Ward identities [65], HBChPT [62,67], and the "small-scale expansion" (SSE), which explicitly includes $\Delta(1232)$ effects [68]. More recently, $g_{P}$ has been calculated in manifestly Lorentzinvariant HBChPT [69,70], made possible through the use of either "infrared regularization" (IR) [71] or "extended-on-mass-shell" (EOMS) [72] renormalization schemes. These renormalization methods - whose formalism has been developed only in the last few yearsavoid the power counting breakdown that was identified [73] to afflict earlier relativistic HBChPT treatments. A recent and comprehensive review of modern ChPT methods can be found in reference [74].

It should be emphasized, however, that all the ChPT approaches listed above give nearly identical results in terms of observables $[5,75,76]$ and therefore they do not offer any new predictions beyond the PCAC expression for $g_{P}$ in Equation 2.3. Rather, ChPT is attractive because it is founded on more fundamental principles than PCAC, and because it provides a framework for systematic study.

\subsection{Treatments of muon capture}

Ordinary muon capture (OMC) provides the best experimental means for determining $g_{P}$, so it is worth reviewing theoretical treatments of this weak interaction process. Muon capture is typically described using either the standard, established techniques of 


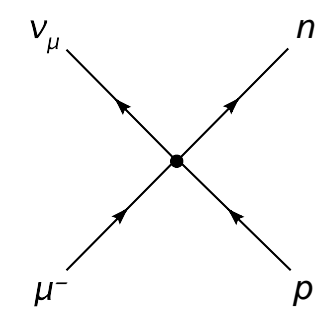

Figure 2.2: Tree-level Feynman diagram of ordinary muon capture as a Fermi contact interaction. (Diagram created using JaxoDraw [4].)

time-dependent perturbation theory, or the more modern techniques of chiral perturbation theory.

The earliest theoretical studies of muon capture were performed by Primakoff, who did extensive work on OMC in hydrogen [31] and (along with collaborators) other light nuclei $[10,77]$. In the Primakoff approach to OMC in hydrogen, the essential physics is determined by the general weak nucleonic current introduced in Equation 1.3, which is phenomenological in nature. The momentum transfer in OMC is small enough that the capture process can be treated as a Fermi contact interaction (Figure 2.2), and standard time-dependent perturbation theory (TDPT) techniques allow one to write the differential capture rate as

$$
d \Lambda_{\mathrm{c}}=\frac{\delta\left(E_{f}-E_{i}\right) d^{3} \vec{p}_{\nu}}{(2 \pi)^{2}}|A|^{2}
$$

where the transition amplitude $A$ is given by

$$
\begin{aligned}
& A=\frac{G_{F} V_{u d}}{\sqrt{2}} \int \bar{\psi}_{n}(\vec{x})\left[g_{V} \gamma^{\alpha}+\frac{i g_{M}}{2 m_{N}} \sigma^{\alpha \beta} q_{\beta}-g_{A} \gamma^{\alpha} \gamma_{5}-\frac{g_{P}}{m_{\mu}} q^{\alpha} \gamma_{5}\right] \psi_{p}(\vec{x}) \\
& \bar{\psi}_{\nu}(\vec{x}) \gamma_{\alpha}\left(1-\gamma_{5}\right) \psi_{\mu}(\vec{x}) \cdot d^{3} x
\end{aligned}
$$

After some tedious algebra [78], one finds that the $\mathrm{OMC}$ rate is highly sensitive to the $\mu p$ atom's hyperfine spin state. This spin sensitivity is a direct consequence of the weak interaction's maximally-parity-violating $V-A$ character, which was first predicted by Bernstein, Lee, Yang, and Primakoff in 1958 [79]. As discussed in Chapter 1, the MuCap experiment 
is concerned with $\mathrm{OMC}$ from the $\mu p$ hyperfine singlet state, and the corresponding capture rate $\Lambda_{S}$ can be written as a function of the form factors of the general hadronic current, $\Lambda_{S}=\Lambda_{S}\left(g_{V}, g_{M}, g_{A}, g_{P}\right)$. The formula for $\Lambda_{S}$, which is provided in Appendix B, can be rearranged so that $g_{P}$ is written in terms of the singlet capture rate and the other three better-known form factors,

$$
g_{P}=g_{P}\left(\Lambda_{S}, g_{V}, g_{M}, g_{A}\right)
$$

This phenomenological expression is useful from an experimental standpoint, because it clearly shows how a capture measurement would provide the missing ingredient needed to determine $g_{P}$. In contrast, the complementary but less transparent $\mathrm{ChPT}$ approaches typically seek to express the form factors in terms of weak, strong, and low-energy constants from various experiments,

$$
\left.g_{P}\right|_{\mathrm{ChPT}}=g_{P}\left(f_{\pi}, g_{\pi N N}, b_{i j}\right)
$$

There have been many OMC treatments in the TDPT style described above, and they have generally obtained results similar to those of Primakoff (see, e.g., references $[9$, $45,80])$. As long as modern values are used for the input parameters, these standard phenomenological approaches consistently yield $\Lambda_{S}$ values in the range $688-695 \mathrm{~s}^{-1}$. In all of these calculations, $g_{P}$ is the only missing piece of the puzzle, so it has typically been fixed at its PCAC value in order to estimate the capture rate. It should be emphasized that the numerical value for $\Lambda_{S}$ can vary according to the exact values of the inputs, whether directly (e.g. through $g_{A}$ ) or indirectly (through $g_{P}$, which depends upon $g_{\pi N N}$ ).

Muon capture has also been studied within the context of chiral perturbation theory. Many such analyses stop at the point of writing down the relevant matrix elements and transition amplitudes, and do not provide any actual numbers for $\Lambda_{S}[62,65,75]$. This is also true of the very recent application of manifestly Lorentz-invariant, or "relativistic," HBChPT to muon capture [81]. However, the authors of references [67] and [82] carried out 
ChPT calculations to NNLO and obtained $\Lambda_{S}$ values of $695 \mathrm{~s}^{-1}$ and $687.4 \mathrm{~s}^{-1}$, respectively. The $1.5 \%$ difference in these two results is due in part to the use of different input values (e.g. for $\left.g_{\pi N N}\right)$, but there is also a more fundamental discrepancy involved, which can perhaps be attributed to different phase space treatments (see the discussion in [67]). It is worth noting that $\mathrm{HBChPT}$ was applied in reference [67], while the small-scale expansion (SSE) of ChPT was applied in reference [82]. In the latter case, the authors claim that they obtained very similar results from a HBChPT analysis of their own, and that the two approaches only differ slightly in the NNLO contribution.

Just as is the case with $g_{P}$, the ChPT treatments of muon capture do not provide any new numbers for the capture rates, and the $\Lambda_{S}$ values obtained using ChPT are quite consistent with earlier treatments using standard time-dependent perturbation theory. ChPT merely offers a alternative way of describing the underlying physics.

\subsection{Radiative corrections to muon capture}

The various theoretical treatments of muon capture in hydrogen described in the preceding section all produce similar results for $\Lambda_{S}$, but they have in common one significant omission: they do not take electroweak radiative corrections into account. Radiative corrections (RC) arise from two sources, which factorize when applied to any capture rate formula [8]:

$$
\Lambda_{S}^{\prime}=\Lambda_{S}\left(1+\mathrm{RC}_{1}+\mathrm{RC}_{2}\right)
$$

The first correction, $\mathrm{RC}_{1}$, is universal to all semileptonic weak charged current amplitudes that are normalized in terms of the Fermi constant $G_{F}$, itself obtained from measurements of the free muon lifetime. The correction, which arises from quantum loop effects and is of order $\mathcal{O}(\alpha)$, is well-established, having been developed long ago to describe radiative 
modifications to neutron and nuclear $\beta$-decay [83-86]. More recently, new methods were

developed to compute "hadronic effects on electroweak radiative corrections to low-energy weak interaction semileptonic processes" [87], and when these are applied to muon capture in hydrogen, the effect is found to be $2.4(4) \%$ [8].

The second radiative correction, $\mathrm{RC}_{2}$, arises from $\mathrm{QED}$ corrections to the wavefunction of the muonic atom. These modifications are dominated by vacuum polarization effects on the Coulombic interactions of the $\mu p$ bound state, as first discussed by Goldman [88]. The magnitude of $\mathrm{RC}_{2}$ is of order $\mathcal{O}(\alpha / \pi)$, and it was recently calculated to produce a $0.4 \%$ correction to the capture rate in hydrogen [8]. This value is slightly smaller than the $0.7 \%$ correction originally computed by Goldman.

The two radiative corrections add to produce a multiplicative factor of $\left(1+\mathrm{RC}_{1}+\right.$ $\left.\mathrm{RC}_{2}\right)=1.028(2)$ when applied to muon capture in hydrogen, thereby increasing the predicted value of the capture rate $\Lambda_{S}$ from $692(3) \mathrm{s}^{-1}$ to $712(4) \mathrm{s}^{-1}$ [8]. This is a significant development in the theoretical description of muon capture, and thus very important for any precision comparison between theory and experiment. Moreover, this new treatment improves the theoretical precision on $\Lambda_{S}$ to less than $1 \%$, which is competitive with the anticipated level of precision of the final MuCap result.

Theoretical predictions for the hyperfine singlet muon capture rate $\Lambda_{S}$, the hyperfine triplet muon capture rate $\Lambda_{T}$, and the induced pseudoscalar coupling $g_{P}$ are summarized in Table 2.1.

\subsection{Implications of a muon capture measurement}

The properties of the muon and the weak interaction are well established, and it is unlikely that muon capture experiments will produce any surprises in those areas. Instead, 


\begin{tabular}{|c|c|c|c|c|c|c|c|}
\hline Year & Approach (authors) & $g_{\pi N N}\left(q^{2}\right)$ & $g_{A}(0)$ & $g_{P}\left(-0.88 m_{\mu}^{2}\right)$ & $\Lambda_{S}\left(\mathrm{~s}^{-1}\right)$ & $\Lambda_{T}\left(\mathrm{~s}^{-1}\right)$ & Ref. \\
\hline 1958 & Dispersion relations $(\mathrm{G}-\mathrm{T})$ & & 1.24 & $9.92\left(=8 g_{A}\right)$ & & & {$[53]$} \\
\hline 1959 & TDPT (Primakoff) & & 1.21 & $9.68\left(=8 g_{A}\right)$ & 636 & 13 & {$[31]$} \\
\hline 1964 & TDPT (Opat) & & 1.22 & $9.76\left(=8 g_{A}\right)$ & 634 & 13.3 & {$[45]$} \\
\hline $1966 / 70$ & $\operatorname{PCAC}(\mathrm{G}-\mathrm{T}+\mathrm{ADW})$ & & 1.18 & $\mathrm{n} / \mathrm{a}$ & & & {$[58,59]$} \\
\hline 1972 & $\begin{array}{c}\text { TDPT: w/o rad. corr. } \\
\text { w/rad. corr. }\end{array}$ & & 1.24 & & $\begin{array}{l}665 \\
669.5(5)\end{array}$ & $\begin{array}{l}12.5 \\
12.6\end{array}$ & {$[88]$} \\
\hline 1975 & TDPT (Primakoff) & & $1.24(1)$ & & $664(20)$ & $11.9(7)$ & [89] \\
\hline 1994 & QCD Chiral Ward identities & ${ }^{\dagger} 13.31(34)$ & $1.2573(28)$ & $8.44(23)$ & & & {$[65]$} \\
\hline 1997 & $\mathrm{ChPT}$ & $\dagger^{\dagger} 13.0(1)$ & $1.2601(25)$ & $8.21(9)$ & & & {$[62]$} \\
\hline 2000 & ChPT and generalized TDPT & $\star 13.37(9)$ & $1.2670(35)$ & $8.475(76)$ & $688.4(3.8)$ & $12.01(12)$ & {$[9]$} \\
\hline 2000 & $\begin{array}{l}\text { ChPT, NLO } \\
\text { ChPT, NNLO }\end{array}$ & ${ }^{\star} 13.4$ & 1.267 & & $\begin{array}{l}722 \\
695\end{array}$ & $\begin{array}{l}12.2 \\
11.9\end{array}$ & {$[67]$} \\
\hline 2001 & $\begin{array}{l}\text { ChPT, SSE, NLO } \\
\text { ChPT, SSE, NNLO } \\
\text { ChPT, SSE, NNLO }\end{array}$ & $\begin{array}{l}\star 13.10(35) \\
\star 13.4\end{array}$ & $1.2673(35)$ & $8.26(23)$ & $\begin{array}{l}711 \\
687.4 \\
681.9\end{array}$ & $\begin{array}{l}14.0 \\
12.9\end{array}$ & {$[23,82]$} \\
\hline 2002 & PCAC & $\dagger 13.05(8)$ & $1.2670(35)$ & 8.23 & & & {$[5]$} \\
\hline 2003 & ChPT, two-loop & $\star 13.2(2)$ & & $8.3(2)$ & & & [66] \\
\hline 2006 & Lorentz-invariant $\mathrm{HBChPT}$ & ${ }^{\dagger} 13.21\left({ }_{-0.05}^{+0.11}\right)$ & $1.2695(29)$ & $8.29\left({ }_{-0.13}^{+0.24}\right)(0.52)$ & & & {$[70]$} \\
\hline 2007 & $\begin{array}{c}\text { TDPT: w/o rad. corr. } \\
\text { w/rad. corr. }\end{array}$ & $\ddagger_{13.05(20)}$ & $1.272(2)$ & $8.2(2)$ & $\begin{array}{l}692(3) \\
712(4)\end{array}$ & & {$[8]$} \\
\hline
\end{tabular}

$\dagger$ Evaluated at the momentum transfer of the pion pole, $q^{2}=m_{\pi}^{2}$

$\ddagger$ Evaluated at the momentum transfer of OMC, $q^{2}=-0.88 m_{\mu}^{2}$

* The momentum transfer of the evaluation was not explicitly specified.

Table 2.1: Historical survey of theoretical predictions for $g_{P}$ and the atomic OMC rates $\Lambda_{S}$ and $\Lambda_{T}$, from a variety of treatments. The capture rates are calculated using either time-dependent perturbation theory (TDPT) or chiral perturbation theory (ChPT), while the $g_{P}$ values are calculated using either PCAC or ChPT. The capture rates are sensitive to $g_{A}(0)$, while the dominant uncertainty in $g_{P}$ comes from $g_{\pi N N}$; hence, the input values are explicitly provided. The more recent predictions for $\Lambda_{S}$ tend to be higher than earlier estimates due to updates in the value of $g_{A}(0)$. Excluding the latest development regarding radiative corrections, most of the differences among the recent $\Lambda_{S}$ and $g_{P}$ estimates can be attributed to slight variations in the inputs. 
muon capture is best viewed as a means of testing our understanding of nuclear structure, as a precision measurement of muon capture by the proton provides an excellent opportunity to probe the weak axial current of the nucleon and determine $g_{P}$.

ChPT makes apparently robust predictions for $g_{P}$ at the $2-3 \%$ level, with the primary uncertainty coming from the present knowledge of $g_{\pi N N}$. A $2 \% g_{P}$ measurement would cleanly distinguish the pion pole contribution from the ADW correction [65], although it should be emphasized that MuCap does not plan to reach that level of accuracy. Nonetheless, a muon capture measurement to $1 \%$ precision as planned by MuCap would determine $g_{P}$ to $7 \%$, and stringently test the predictions of ChPT and hence our understanding of chiral symmetries in low-energy, effective QCD [23]. Prior to MuCap, it was unclear whether the controversy surrounding $g_{P}$ was due to limitations in the understanding of muon chemistry in hydrogen, or in the understanding of the fundamental strong interaction dynamics. MuCap's recent $15 \%$ experimental determination of $g_{P}$ supports existing theoretical predictions, and thus implies that something is amiss in the interpretation of the results of the lone radiative muon capture experiment [44].

A measurement of muon capture in hydrogen provides a single number - the capture rate - which can in turn be used to extract one other number. First and foremost, the measured capture rate is used to test the PCAC/ChPT prediction for $g_{P}$. If the experimentally determined $g_{P}$ value proves to be consistent with theoretical expectations - which is currently the case with MuCap - there are two alternative courses of action: one could use the experimental $g_{P}$ result to infer a value for $g_{\pi N N}$, or one could fix $g_{P}$ at its PCAC value and use the capture rate to constrain the second-class form factors $g_{S}$ and $g_{T}[9]$.

An OMC singlet rate measurement can thus potentially comment on possible physics beyond the Standard Model. First, as mentioned above, muon capture can establish strict limits on the magnitude of the second-class form factors, particularly the scalar 
coupling [90]. An OMC measurement of $1 \%$ precision would produce a factor-of-three improvement in constraints on the second-class form factors, beyond the limits achieved by a previous ${ }^{3}$ He capture measurement [46]. However, these new limits would still be an order of magnitude above the maximum expected range of values. Second, a capture measurement could provide helicity constraints on right-handed gauge bosons by increasing the lower bound. The tensor helicity is the most competitive candidate, since it is the most sensitive to a capture result. Third, it has been pointed out that muon capture treatments generally make three assumptions about the structure of the hadronic weak current:

- Lorentz covariance.

- The discrete symmetry of time-reversal invariance holds; consequently, the form factors are real-valued.

- Exact isospin conservation; consequently, there are no second-class currents.

Furthermore, the leptonic current has always been assumed to possess a pure $V-A$ structure. In 2000, Govaerts and Lucio-Martinez performed capture rate calculations within a far more general formalism: they assumed Lorentz covariance only, thereby allowing for the possibility of six imaginary form factors in both the leptonic and hadronic currents [9]. Their results are useful for considering how muon capture measurements could potentially probe physics beyond the Standard Model, and they also provide a more detailed examination of the scenarios described above.

The implications of the first MuCap result [7] on constraints for $g_{\pi N N}$, the secondclass form factors, or physics beyond the Standard Model have yet to be assessed, and we eagerly await the outcome of future work on this subject. 


\section{Chapter 3}

\section{Muon kinetics in hydrogen}

When fast-moving muons come to a stop in hydrogen gas, they are subject to a number of complex processes which can confound the interpretation of a lifetime measurement. The potential for experimental complications is especially acute with negative muons, as they tend to migrate among numerous atomic and molecular states which have distinct nuclear capture rates. Furthermore, trace amounts of elemental or isotopic impurities in the hydrogen target can produce large, time-dependent distortions in the $\mu^{-}$lifetime spectrum. In order to make a meaningful capture rate measurement, a detailed understanding of muon behavior in impurity-doped hydrogen is essential. Fortunately, a great deal is already known about such behavior, in large part from many years of muon-catalyzed fusion $(\mu \mathrm{CF})$ research [91]. This chapter describes muon kinetics in hydrogen gas for both $\mu^{-}$and $\mu^{+}$, as well as how the MuCap experiment minimizes and corrects for contributions from unwanted channels. 


\subsection{Atomic capture, thermalization, and diffusion}

When a high-velocity, negatively charged muon enters a hydrogen target, it undergoes the following sequence: deceleration, atomic (i.e. electromagnetic) capture, deexcitation, thermalization, diffusion, and then possibly molecular formation or exchange to other elements (i.e. impurities). The muon can decay at any time during this progression, but nuclear capture can only occur after the atomic bound state has been formed.

The slowing down and atomic capture of muons in hydrogen has been studied extensively, both theoretically [92-96] and experimentally [97,98]. The deceleration of fast muons occurs primarily via multiple Coulomb scattering from atomic electrons. For muon velocities that are large compared to atomic electron velocities, $v_{\mu} \gg \alpha c$, the Born approximation is applicable and the average energy loss per unit time is well described by the Bethe-Bloch formula. At intermediate-to-low muon velocities the Born approximation is no longer valid, and different ionization mechanisms come into play [93]. Atomic capture in the Coulomb field of a proton occurs once the muon has slowed to kinetic energy $\varepsilon \leq 17 \mathrm{eV}[99]$; for a muon with an incoming momentum of $20 \mathrm{MeV} / c$, typical for MuCap, this happens within a few nanoseconds. At this point the muon dissociates an $\mathrm{H}_{2}$ molecule (a process requiring $5 \mathrm{eV}$ ) and forms a muonic hydrogen atom in a highly excited Rydberg state,

$$
\mu+\mathrm{H}_{2} \rightarrow(p p \mu e) \rightarrow(\mu p)^{*}+\mathrm{H}
$$

As a rough rule-of-thumb, the $(\mu p)^{*}$ atom is created with a kinetic energy of $1 \mathrm{eV}$ and principal quantum number $n=\sqrt{m_{\mu}^{\text {reduced }} / m_{e}} \approx 14$, corresponding to the size and energy of the original electronic orbital. The average $n$ value is actually somewhat higher due to the $n^{-3}$ distribution of initial populations for energy levels $n \geq 14$ [99], and molecular models predict that the initial distribution is peaked at $n=12$ [96].

Once formed, the excited $(\mu p)^{*}$ atom immediately undergoes deexcitation. The 
generally accepted description of deexcitation mechanisms in exotic hydrogen atoms is known as the "standard cascade model" [99-102]. According to this model, deexcitation to the $\mu p$ ground state proceeds via radiative transitions, external Auger effect, Coulomb interactions, and Stark mixing, where the last three processes involve collisions with surrounding matter. The exact cascade details are largely irrelevant for MuCap, however, since the entire process transpires in less than a nanosecond [32].

When $\mu p$ atoms reach their $1 s$ ground state, the hyperfine spin states are assumed to be populated in a statistical $F_{+}: F_{-}=3: 1$ ratio, where $F_{+}$and $F_{-}$represent the upper triplet $(F=1)$ and lower singlet $(F=0)$ hyperfine states, respectively ${ }^{1}$. The $(\mu p)_{1 s}$ atoms exhibit a wide range of initial kinetic energies, covering $10^{-3} \mathrm{eV}$ to $10^{2} \mathrm{eV}$ [103], with a mean kinetic energy between $1-5 \mathrm{eV}$ [104]. Experimental results indicate that the initial $\mu p$ energy distribution has two Maxwell components $[105,106]$ : roughly half of the atoms are thermal $\left(\varepsilon \sim 0.04 \mathrm{eV}\right.$ in $\mathrm{H}_{2}$ gas at $\left.300 \mathrm{~K}\right)$ while the other half are epithermal, with high energies up to tens of eV due to acceleration from Coulomb deexcitations [102]. The "hot" $\mu p\left(F_{+}\right)$atoms have large scattering cross sections and are rapidly decelerated by collisions with neighboring $\mathrm{H}_{2}$ molecules, via both elastic scattering, charge- (or isotopic-) exchange interactions, and spin-flip collisions (see [103,107] and the review in reference [108]). Within tens of nanoseconds the average $\mu p\left(F_{+}\right)$kinetic energy drops below the hyperfine splitting energy $\Delta E_{\mu p}^{\mathrm{hfs}}=0.182 \mathrm{eV}$, at which point the $F_{-} \rightarrow F_{+}$spin-flip transition is energetically forbidden and the triplet spin state is rapidly depopulated at the rate $\phi \lambda_{10}$, where $\phi$ is the gas density relative to liquid hydrogen $\left(n_{\mathrm{LH}_{2}} \equiv 4.25 \times 10^{22}\right.$ atoms $\left./ \mathrm{cm}^{3}\right)$ and $\lambda_{10} \approx$ $1.7 \times 10^{10} \mathrm{~s}^{-1}$ is the theoretically predicted $F_{+} \rightarrow F_{-}$transition rate in liquid hydrogen $[109$, 110]; it should be noted that although this value for $\lambda_{10}$ is not controversial, no strong experimental constraints exist. In MuCap we use $\mathrm{H}_{2}$ gas at pressure 10 bar $(1 \mathrm{MPa})$ and

\footnotetext{
${ }^{1}$ The $\mu p$ singlet and triplet hyperfine states are also sometimes denoted in shorthand as $\uparrow \downarrow$ and $\uparrow \uparrow$, respectively, in the literature.
} 


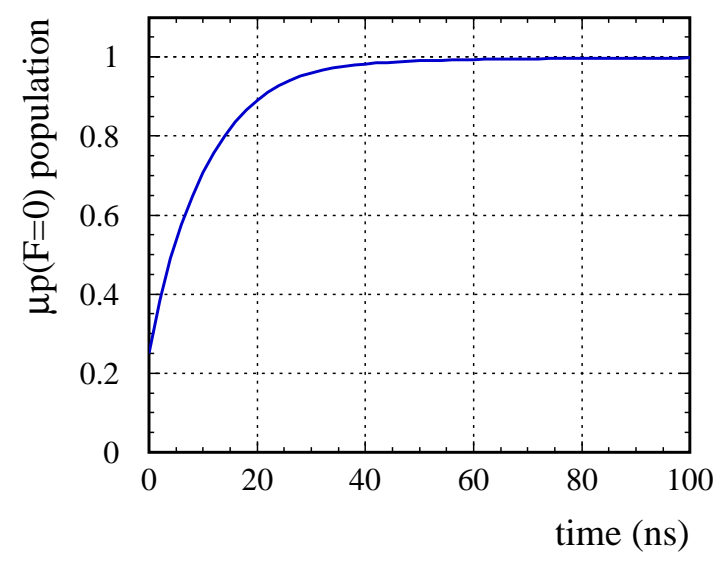

Figure 3.1: The theoretically predicted fractional population of $\mu p$ hyperfine singlet $(F=0)$ atoms vs. time in pure $\mathrm{H}_{2}$ gas at $T=300 \mathrm{~K}$ and $\phi=0.01$. The rapid rise in the singlet population is due to the depopulation of the $\mu p$ hyperfine triplet $(F=1)$ state. (This plot provided courtesy of A. Adamczak.)

room temperature $300 \mathrm{~K}$, in which case $\phi \approx 0.01$, the effective lifetime of thermalized $\mu p\left(F_{+}\right)$ atoms is $\sim 6 \mathrm{~ns}$, and the fraction of muons in the triplet state is below $1 \%$ roughly $50 \mathrm{~ns}$ after the muon stop [104] (see Figure 3.1). Beyond that point in time the triplet state is effectively emptied, and has little or no effect on the measured capture rate. This state of affairs is highly desirable, because the nuclear capture rate from the triplet state, $\Lambda_{T} \approx 12 \mathrm{~s}^{-1}$, is dramatically different from the capture rate from the singlet state, $\Lambda_{S} \approx 680 \mathrm{~s}^{-1}$. By ensuring that virtually no captures proceed from the $\mu p$ triplet state, we greatly simplify the interpretation of experimental results ${ }^{2}$.

Thermal effects are relevant not only as the mechanism for depopulation of the $\mu p$ hyperfine triplet state, but also when considering the diffusion of $\mu p$ atoms away from the muon's original stopping point $[105,111]$. Diffusion during the $\mu p$ epithermal stage is in the sub-millimeter range, and most diffusion takes place after the atom has completely thermalized. (Thermalization of $\mu p\left(F_{-}\right)$atoms occurs approximately $400 \mathrm{~ns}$ after atomic

\footnotetext{
${ }^{2}$ It is interesting to note that although $\Lambda_{T}$ is far more difficult to experimentally isolate and measure than $\Lambda_{S}, \Lambda_{T}$ is actually more sensitive to $g_{P}$.
} 


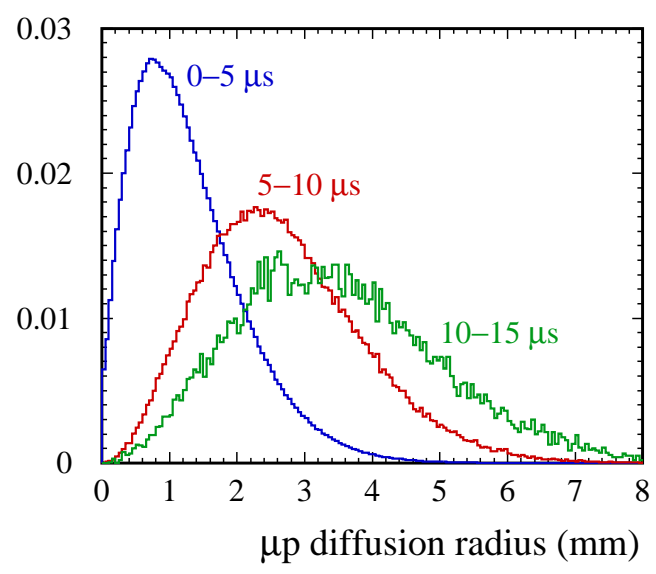

(a)

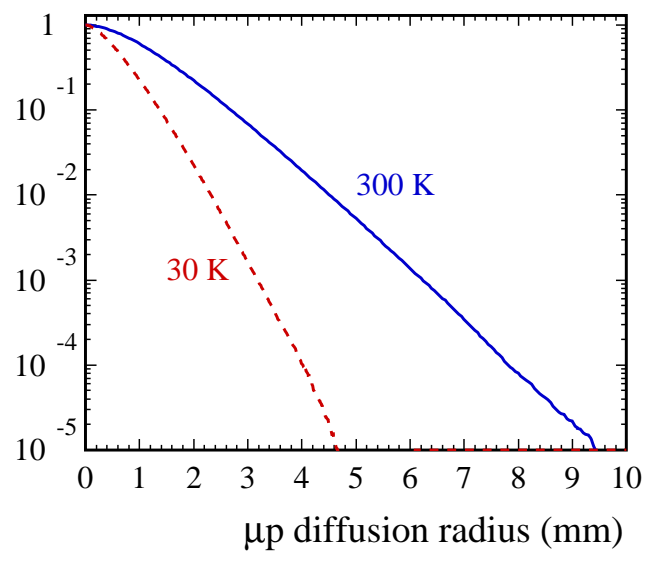

(b)

Figure 3.2: (a) Simulated radial distributions of $\mu p$ atoms at the time of $\mu^{-}$decay, for three successive decay time intervals, in $\mathrm{H}_{2}$ gas at temperature $T=300 \mathrm{~K}$ and density $\phi=0.01$. The $\mu p$ atoms are assumed to have started with energies drawn from the double-Maxwell energy distribution described in the text. The diffusion distributions have been rescaled for presentation purposes, because the statistics at later times are reduced by the constant loss to decay at rate $e^{-\lambda_{0} t}$. (b) Simulated fraction of $\mu^{-}$decays outside of a given diffusion radius from the point of $\mu p$ formation, for decay times $\Delta t \leq 20 \mu \mathrm{s}$, density $\phi=0.01$, and $\mathrm{H}_{2}$ temperatures $T=30$ and $300 \mathrm{~K}$. (Plots provided courtesy of A. Adamczak.)

capture, much later than for $\mu p\left(F_{+}\right)$atoms, which completely thermalize within $\left.50 \mathrm{~ns}.\right)$ Numerical estimates and Monte Carlo simulations have demonstrated that the mean thermal diffusion range for $\mu p$ atoms in hydrogen gas at pressure 10 bar and temperature $300 \mathrm{~K}$ is about $1 \mathrm{~mm}$, although the diffusion tail can reach up to a few $\mathrm{mm}[104,112,113]$ (see Figures 3.2(a),(b)). Some simple analytical diffusion estimates are presented in Appendix C.

Diffusion is a potential problem for MuCap for two reasons: (1) the muon can drift into surrounding detector materials, where it will transfer to $Z>1$ nuclei which have a much higher nuclear capture rate than hydrogen, and (2) if the muon drifts too far away from its original stopping point, the decay event cannot be reconstructed and will be lost to the analysis. Both of these processes produce time-dependent distortions in the muon 
lifetime spectrum which elevate the effective muon disappearance rate above its value in the $\mu p$ singlet state. Fortunately, in hydrogen gas at pressure 10 bar and temperature $300 \mathrm{~K}$, the typical $\mu p$ diffusion distance is only $\approx 1 \mathrm{~mm}$, which leads to a relatively small effect on the muon lifetime spectrum.

The muon is almost completely depolarized in the course of $(\mu p)^{*}$ deexcitation $[114$, 115], and, provided that external magnetic fields are small compared to the hyperfine field of the unpolarized proton, any residual muon polarization is eliminated in the formation of the $\mu p$ singlet ground state. As a result, the angular distribution of decay electrons emitted from the $\mu p$ singlet ground state is isotropic. It should also be noted that the $\mu^{-}$decay rate is altered by the muon's involvement in a $\mu p$ bound state, a phenomenon first identified in 1951 by Porter and Primakoff [116]. The bound state $\mu^{-}$decay rate is commonly written as the product of the $\mu^{+}$decay rate and the so-called "Huff factor" Q [117]:

$$
\lambda_{\mu^{-}}^{\text {decay }}=Q \lambda_{\mu^{+}}^{\text {decay }}
$$

For light elements $[118,119]$

$$
Q=1-\frac{1}{2}(Z \alpha)^{2}-0.06(Z \alpha)^{2}\left(\frac{m_{\mu}}{m_{N}}\right)
$$

where $\alpha$ is the fine structure constant, $Z$ is the atomic number of the nucleus, $m_{\mu}$ is the muon mass, and $m_{N}$ is the nucleon mass. The $\mu p$ atom's bound state modification to the $\mu^{-}$decay rate is then

$$
\begin{aligned}
\Delta \lambda_{\mu^{-}}^{\text {decay }} & =(Q-1) \lambda_{\mu^{+}}^{\text {decay }} \\
& \simeq-\frac{1}{2}\left(\frac{1}{137}\right)^{2}\left(455,162 \mathrm{~s}^{-1}\right) \\
& \approx-12 \mathrm{~s}^{-1}
\end{aligned}
$$

This offset in the decay rate was taken into account by the Saclay group in their experimental determinations of the nuclear capture rates in $\mu p[42]$ and $\mu d[120]$. 


\section{$3.2 \quad$ Formation of $p \mu p$ molecules}

Once the $\mu p$ atom reaches its hyperfine singlet ground state, subsequent $\mu^{-}$kinetics in pure hydrogen gas are governed by the comparatively slower processes of $p \mu p$ molecule formation. The $p \mu p$ molecules are formed in collisions between the electrically neutral $\mu p$ atom and $\mathrm{H}_{2}$ molecules, via Auger emission,

$$
\mu p+\mathrm{H}_{2} \rightarrow p \mu p+e+\mathrm{H}
$$

Protons are fermions, so the total wavefunction of the nuclear system in the $p \mu p$ molecule must be antisymmetric. Consequently, the $p \mu p$ molecule is formed in either an antisymmetric, excited "ortho" rotational state with orbital momentum $J=1$ and symmetric nuclear spin $I=1$, or in a rotationally symmetric "para" state with antisymmetric total spin, $(J=0, I=0)$. Transitions between the two nuclear states are strictly forbidden in a nonrelativistic approximation, but spin flips from the ortho to para state do occur due to relativistic components in the $p \mu p$ wavefunction [52]; see reference [5] for a detailed discussion of the relevant physics.

The formation of $p \mu p$ molecules is significant because the ortho and para states have characteristic nuclear capture rates that are quite different from the $\mu p$ singlet state's nuclear capture rate (see Table 3.1). Any measurement of muon capture in hydrogen inevitably receives contributions from both atomic and molecular capture channels, and the contributions are not easily disentangled. In order to extract a particular capture rate from the combined capture rate that is observed, some external information must be used, or assumptions made, about the formation and nuclear capture rates of the other channels under the given experimental conditions.

Determining the $p \mu p$ formation rate is not straightforward. Experiments generally measure the total formation rate $\phi \lambda_{p \mu p}=\phi\left(\lambda_{\text {of }}+\lambda_{\text {pf }}\right)$, where $\lambda_{\text {of }}$ is the dominant ortho 


\begin{tabular}{|c|c|c|c|}
\hline State & Capture rate & Theoretical prediction $\left(\mathrm{s}^{-1}\right)$ & Ref. \\
\hline$(\mu p)_{1 s \uparrow \downarrow}$ (singlet) & $\Lambda_{S}$ & $688.4 \pm 3.8$ & {$[9]$} \\
\hline$(\mu p)_{1 s \uparrow \uparrow}($ triplet $)$ & $\Lambda_{T}$ & $12.01 \pm 0.12$ & {$[9]$} \\
\hline$(p \mu p)_{\uparrow \uparrow}$ (ortho) & $\Lambda_{\mathrm{om}}=2 \gamma_{\mathrm{o}}\left(\frac{3}{4} \Lambda_{S}+\frac{1}{4} \Lambda_{T}\right)$ & $524 . \quad \pm 3$ & \\
\hline$(p \mu p)_{\uparrow \downarrow}($ para $)$ & $\Lambda_{\mathrm{pm}}=2 \gamma_{\mathrm{p}}\left(\frac{1}{4} \Lambda_{S}+\frac{3}{4} \Lambda_{T}\right)$ & 207. $\quad \pm 1$ & \\
\hline
\end{tabular}

Table 3.1: The nuclear capture rates from the different spin states of the atomic $\mu p$ and molecular $p \mu p$ systems. The "molecular overlap factors" $\gamma_{\mathrm{o}}$ and $\gamma_{\mathrm{p}}$ were introduced as a means of expressing the molecular capture rates in terms of the atomic capture rates [121124]. The overlap values have been calculated to be $2 \gamma_{\mathrm{o}}=1.009 \pm 0.001$ and $2 \gamma_{\mathrm{p}}=1.143 \pm$ $0.001[52,125]$, although relativistic effects might modify these numbers by $1-2 \%$ [126]. The $p \mu p$ capture rates in the table were calculated from the $\mu p$ capture rates using the indicated formulas.

state formation rate and $\lambda_{\text {pf }}$ is the comparatively small para state formation rate, both normalized to liquid hydrogen density. There exist a variety of theoretical predictions and experimental results for $\lambda_{\text {of }}$, covering the range $1.8-3.9 \times 10^{6} \mathrm{~s}^{-1}$ (see e.g. the review in reference [127]), but no direct experimental information for $\lambda_{\text {pf }}$ exists. The most recent experimental [127] and theoretical [128] values for $\lambda_{\text {of }}$ are in disagreement, as can be seen in Table 3.2 .

There is also significant experimental and theoretical disagreement over the value of the ortho $\rightarrow$ para transition rate $\lambda_{\mathrm{op}}$. Unlike molecular formation, the ortho $\rightarrow$ para transition is not an explicitly density-dependent process; that is, $\lambda_{\mathrm{op}}$ is not multiplied by the density coefficient $\phi$ when determining the molecular transition rate. The reason is that the ortho $\rightarrow$ para transition takes place within molecular complexes such as $\left.[(p \mu p) p e]^{+}\right]$and $[(p \mu p) p e e]$, rather than in isolated $p \mu p$ molecules, and the formation of such molecular clusters is extremely fast, of order $10^{13} \mathrm{~s}^{-1}$ at $\phi=1[52,129]$. The role of molecular complexes also makes $\lambda_{\text {op }}$ difficult to calculate. To date there have been two measurements of $\lambda_{\mathrm{op}}[43,51]$ and one theoretical calculation of its value [52]. The three numbers are mutually 


\begin{tabular}{crlrcc}
\hline \hline Quantity & Theory $\left(\mathrm{s}^{-1}\right)$ & Ref. & Experiment $\left(\mathrm{s}^{-1}\right)$ & $\mathrm{H}_{2}$ target & Ref. \\
\hline$\lambda_{\text {of }}$ & $1.8 \times 10^{6}$ & {$[128]$} & $(1.89 \pm 0.20) \times 10^{6}$ & liquid & {$[130]$} \\
& & & $(2.55 \pm 0.18) \times 10^{6}$ & liquid & {$[131]$} \\
& & & $(2.34 \pm 0.17) \times 10^{6}$ & gas & {$[132]$} \\
$\lambda_{\text {pf }}$ & & & $(3.12 \pm 0.18) \times 10^{6}$ & solid & {$[127]$} \\
$\lambda_{\text {op }}$ & $(7.1 \pm 1.2) \times 10^{3}$ & {$[128]$} & & & \\
& & & $(11.1 \pm 1.9) \times 10^{4}$ & liquid & {$[51]$} \\
\hline \hline
\end{tabular}

Table 3.2: Experimental and theoretical values for the $p \mu p$ formation rates $\lambda_{\text {of }}$ and $\lambda_{\text {pf }}$, and for the transition rate $\lambda_{\mathrm{op}}$ from the $p \mu p$ molecule's ortho state to its para state.

inconsistent and there is no reason to favor one result over the others. A summary of $p \mu p$ formation and transition rates is provided in Table 3.2.

Our general experimental strategy for dealing with molecular formation is to create conditions that restrict the muon to the $\mu p$ hyperfine singlet ground state as much as possible. Monte Carlo simulations have indicated that an $\mathrm{H}_{2}$ density of $\sim 1 \%$ of liquid hydrogen (i.e. $\phi=0.01$ ) is an optimal compromise between competing interests: In 10 bar $\mathrm{H}_{2}$ gas at $300 \mathrm{~K}$, the hyperfine triplet $\mu p$ state depopulates quickly and $p \mu p$ molecules form slowly (see Figure 3.3), and roughly $96 \%$ of nuclear captures proceed from the $\mu p$ singlet state. Meanwhile, the target remains sufficiently dense to stop incoming muons and prevent any large-scale $\mu p$ diffusion. Under these circumstances, MuCap is able to largely avoid the molecular uncertainties that have clouded the interpretation of the most precise experimental OMC result [42], which was performed in liquid hydrogen. Monte Carlo simulations have also been used to explore the sensitivity of the fitted lifetime to the molecular rates and the fit range [2]. Results suggest that the present uncertainties in the $p \mu p$ rates - primarily $\lambda_{\text {of }}$ and $\lambda_{\text {op }}$-should contribute an error of roughly $1 \%$ to a $\Lambda_{S}$ measurement. 

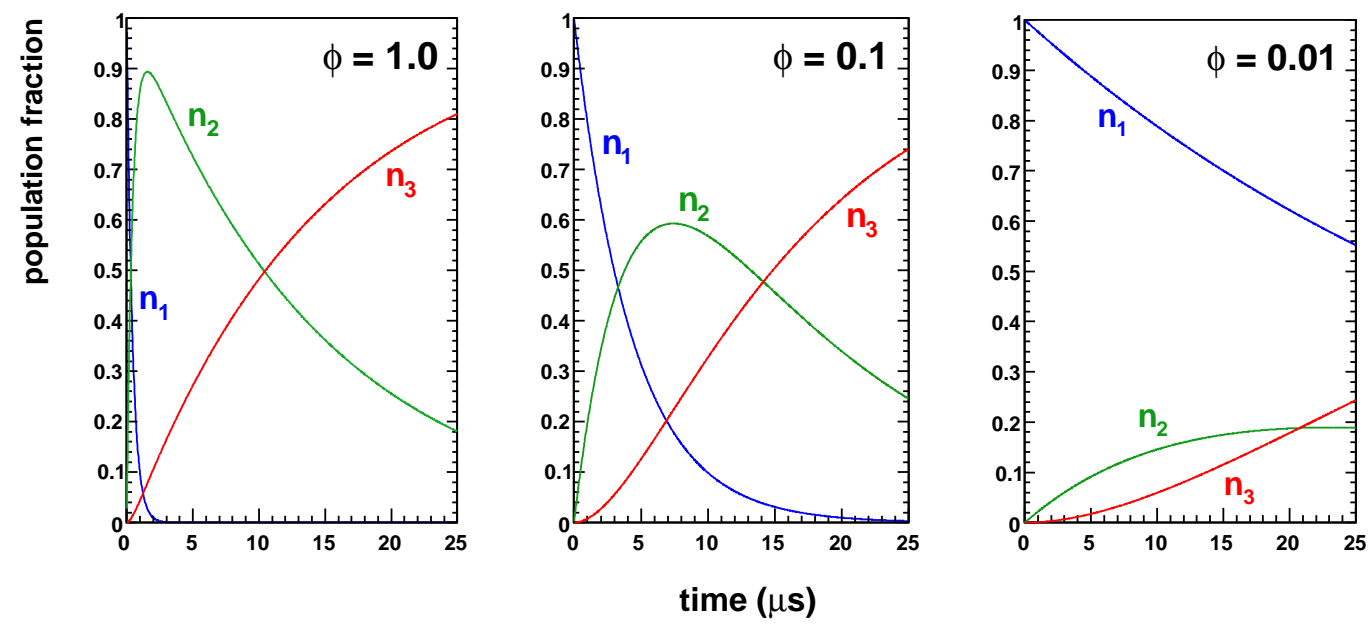

Figure 3.3: The relative populations of $\mu p$ hyperfine singlet atoms $\left(n_{1}\right), p \mu p$ ortho molecules $\left(n_{2}\right)$, and $p \mu p$ para molecules $\left(n_{3}\right)$ as a function of time, for three different $\mathrm{H}_{2}$ gas densities $\phi$. The occupation numbers were calculated using kinetics parameter values $\lambda_{\text {of }}=2.3 \times 10^{6} \mathrm{~s}^{-1}$, $\lambda_{\text {pf }}=7.4 \times 10^{3} \mathrm{~s}^{-1}$, and $\lambda_{\mathrm{op}}=6.9 \times 10^{4} \mathrm{~s}^{-1}$, as determined from Table 3.2. To better illustrate the population changes over time, the $n_{i}$ have been multiplied by the factor $\mathrm{e}^{\lambda_{0} t}$ to eliminate the constant loss component from decay.

\subsection{Impurities}

Hydrogen gas impurities pose the largest threat to a precision $\mu^{-}$lifetime measurement, because some degree of contamination is inevitable, and even trace amounts of impurities - which can be extremely difficult to measure accurately - have the potential to produce large distortions in the lifetime spectrum. Muons in the $\mu p$ system will preferentially (and in the case of $Z>1$ elements, irreversibly) transfer to any impurities that are encountered in the course of diffusion, so impurities have the potential to generate disproportionate effects. 


\subsection{1 $\quad Z>1$ elements}

Muon transfer from the proton to heavier elements,

$$
\mu p+Z \rightarrow \mu Z+p
$$

is a complex subject which still has many open questions; see e.g. the reviews [113,133-136]. However, the basic features of the transfer process are well understood. When a $\mu p$ atom collides with a heavier element, the small, neutral $\mu p$ can penetrate deep into the other atom's electron cloud. There the $\mu p$ atom is polarized by the Coulomb field of the larger nucleus, and the muon quickly transfers to the heavier element because of its stronger binding energy. The actual transfer rate depends upon a variety of factors, including the $\mu p$ atom's kinetic energy, angle of incidence, etc. There is also strong evidence that some elements (e.g. oxygen [137]) have complicated, energy-dependent transfer rates, although the reasons are not well understood and remain controversial. Nevertheless, it is generally possible to write an effective $\mu p \rightarrow \mu Z$ transfer rate as

$$
\Lambda_{p Z}=\phi c_{Z} \lambda_{p Z}
$$

where $\phi$ is the atomic gas density relative to liquid hydrogen, $c_{Z}$ is the atomic concentration (by number) of the elemental impurity, and $\lambda_{p Z}$ is the "normalized" impurity transfer rate in liquid hydrogen.

Of course, the fundamental reason why $\mu^{-}$transfer to heavier elements is a concern is because of the resulting increase in the nuclear capture rate. The capture rate $\Lambda_{Z}$ in nuclei scales roughly as $Z^{4}$, so $\Lambda_{Z}$ quickly outpaces $\Lambda_{S}$ with increasing $Z$. A compendium of muon nuclear capture rates for a wide range of elements can be found in reference [138]. The $\mu p \rightarrow \mu Z$ transfer rates and $\mu Z$ nuclear capture rates for the common hydrogen gas contaminants carbon, nitrogen, and oxygen are provided in Table 3.3. 


\begin{tabular}{|c|c|c|c|c|c|c|}
\hline \multirow[t]{2}{*}{ Element } & \multicolumn{4}{|c|}{$\begin{array}{l}\text { Transfer rate, } \lambda_{p Z} \\
\qquad\left(\times 10^{10} \mathrm{~s}^{-1}\right)\end{array}$} & \multirow[t]{2}{*}{$\begin{array}{l}\text { Capture rate, } \Lambda_{Z} \\
\qquad\left(\times 10^{6} \mathrm{~s}^{-1}\right)\end{array}$} & \multirow[t]{2}{*}{ Ref. } \\
\hline & Theory & Ref. & Expt. & Ref. & & \\
\hline $\mathrm{C}$ & & & 9.5 & [139] & $0.0388(5)$ & [138] \\
\hline \multirow[t]{2}{*}{$\mathrm{N}$} & 5.2 & [140] & $3.4(7)$ & [141] & $0.0693(8)$ & [138] \\
\hline & 3.0 & [142] & & & & \\
\hline \multirow[t]{2}{*}{$\mathrm{O}$} & 7.77 & [140] & $8.5(2)$ & [137] & $0.1026(6)$ & [138] \\
\hline & 4.42 & [142] & & & & \\
\hline
\end{tabular}

Table 3.3: The normalized $\mu p \rightarrow \mu Z$ transfer rates at room temperature, and the $\mu Z$ nuclear capture rates, for the common hydrogen gas contaminants carbon, nitrogen, and oxygen. Note that the theoretical values for $\lambda_{p Z}$ are quite different. Reference [140] gives the impression that everything has been solved and that theory and experiment are in agreement, but the authors of reference [142] claim that the calculations in [140] ignored the effects of electron screening, which are especially significant for oxygen.

Analytic and phenomenological Monte Carlo simulations have been used to investigate the sensitivity of the $\mu^{-}$lifetime to $Z>1$ contamination in hydrogen gas $[2,113]$. As a result of these studies, the design goal was established for MuCap to use an ultraclean hydrogen target with impurity concentrations $c_{Z}$ below $10 \mathrm{ppb}$, which can be achieved with standard methods. Under such clean conditions, $Z>1$ impurities will not affect a $\mu^{-}$ lifetime measurement. Unfortunately, to date we have not been able to achieve such high purity levels due to outgassing in the apparatus. Consequently, we are forced to perform a correction to the measured $\mu^{-}$disappearance rate, to compensate for the effects of the $Z>1$ impurities that are present. The impurity level can be monitored in situ by directly observing capture reactions in our hydrogen target, as well as by regular gas sampling and chromatographic analyses. Information from both methods is ultimately used to inform the correction, which will be described in detail in Section 6.6.3. It should be noted that the atomic capture of muons by elements of $Z>1$ also produces unique X-ray signatures [136] which in principle could provide information about the gas impurity content [143]. However, impurity transfers occur so infrequently in our purified target that their X-rays are buried 
in beam bremsstrahlung background, so the technique is not considered feasible.

It should be noted that $Z>1$ capture effects can also appear due to muon stops in the experimental apparatus. In fact, this is the primary motivation for our use of a time projection chamber as an active gas target: it enables us to identify the muons that stopped in the hydrogen gas, and to discard the muons that might have stopped in $Z>1$ detector materials. However, some muon stops in detector materials are still inadvertantly accepted due to inefficiencies in the muon detectors, which will ultimately force us to enlarge the error on the final result for $\Lambda_{S}$.

\subsubsection{Deuterium}

When a $\mu p$ atom encounters a deuteron, the muon preferentially transfers to the larger nucleus

$$
\mu p+d \rightarrow p+\mu d
$$

because of the $\mu d$ system's $5 \%$ larger reduced mass and correspondingly larger (that is, more negative) binding energy. This isotopic exchange process has an effective rate

$$
\Lambda_{p d}=\phi c_{d} \lambda_{p d}
$$

where $\phi$ is the hydrogen density relative to liquid hydrogen, $c_{d}$ is the deuterium concentration (by number) in atomic units, and $\lambda_{p d}$ is the proton-deuteron transfer rate normalized to liquid hydrogen. There exist two inconsistent experimental results for $\lambda_{p d}$ in gaseous hydrogen at room temperature: $\lambda_{p d}=(1.43 \pm 0.13) \times 10^{10} \mathrm{~s}^{-1}$ [130] and $\lambda_{p d}=(0.84 \pm 0.13) \times 10^{10} \mathrm{~s}^{-1}[144]$, the former of which is consistent with the theoretical prediction $\lambda_{p d}(T=300 \mathrm{~K})=1.6 \times 10^{10} \mathrm{~s}^{-1}[145]$. The $\mu d$ nuclear capture rate $\Lambda_{d}$ has likewise yet to be conclusively determined. Of the two most recent experimental results for $\Lambda_{d}, 470 \pm 29 \mathrm{~s}^{-1}[120]$ and $409 \pm 40 \mathrm{~s}^{-1}$ [146], only the latter measurement is compatible 
with the latest theoretical predictions, one of which is in the range $397-400 \mathrm{~s}^{-1}$ [147], the other being $386 \pm 5 \mathrm{~s}^{-1}$ [148]; see also reference [34] for a historical survey of experimental and theoretical $\Lambda_{d}$ values. It is worth noting that more recent theoretical calculations of $\Lambda_{d}$ were reported in reference [149], but no explicit value for $\Lambda_{d}$ was given. The authors argue that there is insufficient information to constrain the value of $\Lambda_{d}$; the parameter $L_{1 A}$ would be needed to do so. In fact, the theoretical community is divided about the legitimacy of the so-called "hybrid" approach employed in reference [148], which allows for numerical predictions for $\Lambda_{d}$ in the absence of $L_{1 A}$ information.

Despite the uncertainty surrounding the precise value of $\Lambda_{d}$, its probable range of values is sufficiently close to the $\mu p$ singlet capture rate $\Lambda_{S}$ that, for ppm-level deuterium concentrations, $\Lambda_{d}$ alters the muon disappearance rate by very little. For example, for $c_{d}=1 \mathrm{ppm}$, the effective disappearance rate is pulled down by at most $0.3 \mathrm{~s}^{-1}$ because of the difference in isotopic capture rates. A far more serious problem arises from $\mu d$ diffusion, due to the existence of a Ramsauer-Townsend ( $\mathrm{R}-\mathrm{T})$ minimum in the elastic scattering of $\mu d$ atoms by $\mathrm{H}_{2}$ molecules, for collisions in the energy range $2-30 \mathrm{eV}$. The transfer process in Equation 3.2 releases $135 \mathrm{eV}$, of which $45 \mathrm{eV}$ is imparted to the $\mu d$ atom [150]. Thus, in the course of its subsequent thermalization, the $\mu d$ ineluctably passes through the $\mathrm{R}-\mathrm{T}$ minimum where the $\mu d+\mathrm{H}_{2}$ cross section is reduced by more than two orders of magnitude compared to $\mu p+\mathrm{H}_{2}$ (see Figure 3.4, as well as [151] and references therein). As a result, the surrounding $\mathrm{H}_{2}$ gas is rendered quasi-transparent to $\mu d$ atoms, which can easily diffuse several centimeters away from the muon's stopping point, as illuatrated in Figure 3.5. Consequently, the diffusion concerns which are deemed negligible for $\mu p$ atoms cannot be ignored for $\mu d$ atoms - namely, conveyance to $Z>1$ detector materials, and diffusion outside of an electron cut radius surrounding the muon stop.

Let us examine how $\mu d$ diffusion can affect decay event reconstruction and thus 


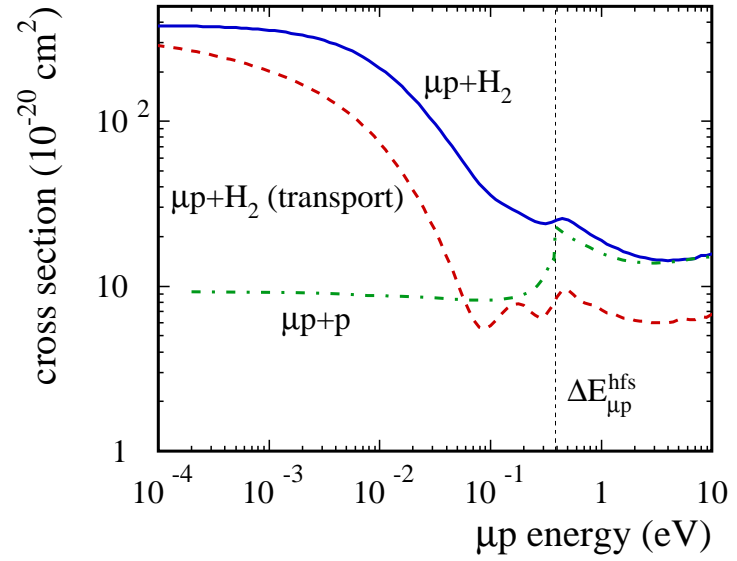

(a)

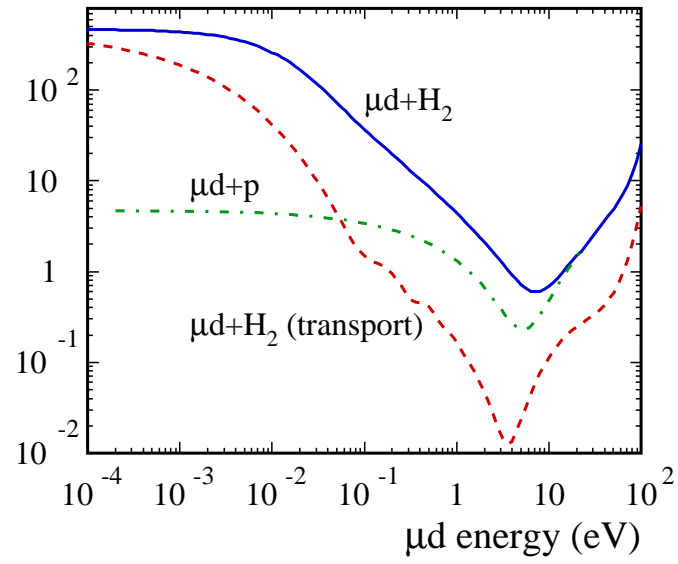

(b)

Figure 3.4: (a) The cross sections for spin-conserving scattering of $\mu p(F=0)$ atoms from ground-state $\mathrm{H}_{2}$ molecules and from protons, as a function of the collisional energy in the laboratory system. The $\mu p+p$ cross sections have been scaled up by a factor of two from their actual values in order to facilitate comparison with the more accurate $\mu p+\mathrm{H}_{2}$ cross sections, which take into account (1) the rotational and vibrational internal degrees of freedom of the target molecule, and (2) the presence of electrons, which dominate $\mu p$ scattering at the lowest energies. Note that the nuclear and molecular cross sections converge at higher scattering energies, where the effects of molecular binding and electron screening disappear. The so-called "transport" cross section, defined as $\sigma_{\text {trans }}=\int d \Omega(1-\cos \theta)(d \sigma(\theta) / d \Omega)$, shows the effects of the anisotropic nature of $\mu p+\mathrm{H}_{2}$ scattering; of the three curves, it is the most accurate model of reality. The $\mu p$ hyperfine transition energy threshold $\Delta E_{\mu p}^{\mathrm{hfs}}$ is indicated. (b) The cross sections for spin-conserving scattering of $\mu d$ atoms from groundstate $\mathrm{H}_{2}$ molecules and protons. As in (a), the $\mu d+p$ cross sections have been doubled for comparison with the $\mu d+\mathrm{H}_{2}$ cross sections. The deep Ramsauer-Townsend minimum between $2-30 \mathrm{eV}$ is clearly visible, where the cross sections for $\mu d$ scattering are much lower than for $\mu p$ scattering. (Plots provided courtesy of A. Adamczak.) 


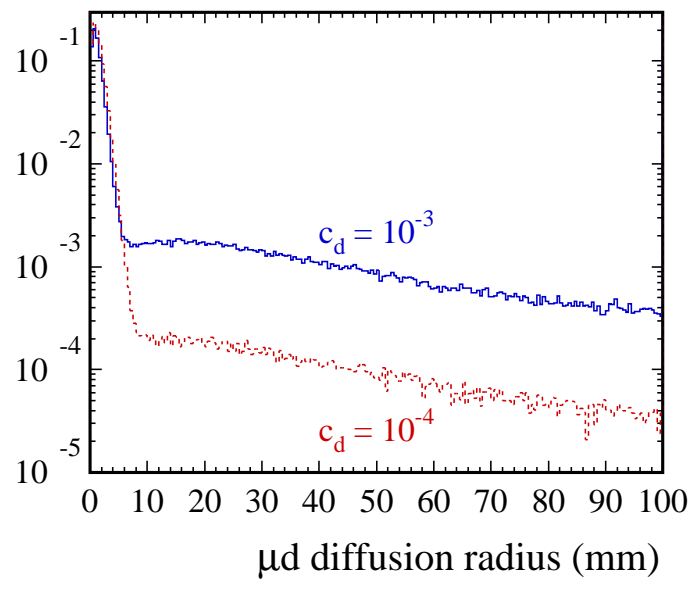

Figure 3.5: Simulated fraction of $\mu^{-}$decays outside of a given diffusion radius from the point of $\mu d$ formation in $\mathrm{H}_{2}$ gas, for decay times $\Delta t \leq 20 \mu$ s and $\mathrm{H}_{2}$ gas temperature $T=300 \mathrm{~K}$ and density $\phi=0.01$, and for deuterium concentrations of $c_{d}=1000$ and $100 \mathrm{ppm}$. Note the existence of an extended $\mu d$ diffusion tail, which is not present in the $\mu p$ radial diffusion plot in Figure 3.2b. (Plot provided courtesy of A. Adamczak.)

the lifetime spectrum. To simplify things, we ignore $\mu d$ nuclear capture and assume that $\mu p$ diffusion distances are negligible. In this case the muon lifetime spectrum ${ }^{3} N_{\mu}(t)$ is the sum of two terms, the first being the probability that the muon did not transfer to a deuteron, and the second involving the probability $p\left(r, t^{\prime}\right)$ that the muon transferred to a deuteron at time $t^{\prime}$ but remains within a radius $r$ of the muon stop at decay time $t[3]$ :

$$
N_{\mu}(t)=e^{-\left(\lambda_{0}+\Lambda_{p d}\right) t}\left[1+\Lambda_{p d} \int_{0}^{t} p\left(r, t^{\prime}\right) e^{\Lambda_{p d} t^{\prime}} d t^{\prime}\right] .
$$

(See Appendix E for the derivation of this formula.) Notice that the muon's effective disappearance rate is sensitive to the value of the electron cut radius $r$ : in the limit $r \rightarrow 0$, $p\left(r, t^{\prime}\right) \rightarrow 0$, all transferred muons are immediately lost, and the disappearance rate increases from $\lambda_{0}$ to $\lambda_{0}+\Lambda_{p d}$; in the opposite limit as $r \rightarrow \infty, p\left(r, t^{\prime}\right) \rightarrow 1$ and $\mu d$ diffusion has no effect - the muon disappearance rate is simply the decay rate $\lambda_{0}$. Practically speaking, the results from an actual experimental cut on the muon/electron radial vertex will

\footnotetext{
${ }^{3}$ Recall from Equation 1.10 that the measured time spectrum of muon decay electrons, $N_{e}(t)$, is proportional to the surviving muon population, $N_{\mu}(t)$.
} 
lie somewhere between these two extremes. It is important to note that the integral in Equation 3.3 is nonanalytic because the parent function $g\left(r^{\prime}, t^{\prime}\right)$ in

$$
p\left(r, t^{\prime}\right)=\int_{0}^{r} g\left(r^{\prime}, t^{\prime}\right) d r^{\prime}
$$

involves some complicated combination of Maxwell-Boltzmann thermal statistics and energydependent scattering effects, the precise details of which are often unknown. Consequently, one must resort to Monte Carlo simulations to make quantitative estimates of the $\mu d$ diffusion-driven lifetime effects that are formally expressed by Equation 3.3.

Monte Carlo simulations are also useful for estimating the effects from $\mu d$ diffusion into $Z>1$ materials, where the muon is subjected to much higher capture rates than in the hydrogen gas $[3,152]$. However, a credible correction for $\mu d$-related effects cannot rely on computer simulations, but must ultimately come from real data. One means of accomplishing this is to measure the muon lifetime at two different deuterium concentrations, $c_{d 1}$ and $c_{d 2}$, and extrapolate the results to $c_{d}=0$. This sort of zero-extrapolation procedure is valid for measurements involving deuterium concentrations $c_{d} \leq 250 \mathrm{ppm}$ [153], where $\mu d+d$ scattering is negligible and $\mu d$ diffusion is completely determined by $\mu d+p$ scattering and $p \mu d$ molecule formation. In this regime, the $p\left(r, t-t^{\prime}\right)$ term in Equation 3.3 is effectively independent of $c_{d}$, and the resulting radial cut dependence has a fixed shape that scales linearly with $c_{d}$. One advantageous feature of the zero-extrapolation technique is that if $c_{d 2}$ is sufficiently high, the statistical requirements for the second measurement point are dramatically reduced by the long lever arm $c_{d 2} / c_{d 1}[3]$.

Of course, in order to perform a zero-extrapolation, the deuterium concentration in the hydrogen gas target must be precisely determined for both of the measurement points. There are four possible methods for ascertaining $c_{d}$ :

1. External mass spectrometry measurements of gas samples. The problem with this 
approach is the ever-present danger of contamination during the sampling and measurement process, which raises concerns about the reliability of the results.

2. Direct observation of $\mu d$ diffusion, by looking for events where the decay electron track is displaced by several millimeters from the muon's stopping point. Unfortunately, this method is impractical in MuCap because of the extensive electron scattering from the experimental apparatus (primarily the aluminum pressure vessel), which significantly worsens the muon/electron vertex resolution.

3. Observation of muon-catalyzed $p$ - $d$ fusion events, either by detecting the $5.3 \mathrm{MeV}$ muon emitted in the process $p d \mu \rightarrow{ }^{3} \mathrm{He}+\mu^{-}$(15\% of all fusions), or by detecting the $5.5 \mathrm{MeV} \gamma$ ray emitted in the radiative version, $p d \mu \rightarrow \mu^{3} \mathrm{He}+\gamma(85 \%$ of all fusions) $[154,155]$. Unfortunately, the use of fusion events is problematic for several reasons. First, both reaction channels have relatively low rates under standard MuCap experimental conditions, because the low gas density of $\phi=0.01$ and the generally low deuterium concentration suppress the initial formation of $\mu d$ atoms, and the low density also suppresses the subsequent formation of the $p \mu d$ molecules which are the precursors to $p$ - $d$ fusion. For example, for $c_{d}=1 \mathrm{ppm}$ and $\phi=0.01$, the total fusion yield is only $\sim 13 \mathrm{ppm}$ (see references $[155,156]$ for the numbers and formulas leading to this estimate). Furthermore, separating the nonradiative fusion event signature from background in our target is difficult (because of the large $\mu d$ diffusion distances) and extremely sensitive to detector performance (the time projection chamber must operate above $5 \mathrm{kV}$ to register the outgoing muon track), while detecting the radiative fusion channel would require extensive additions and modifications to the experimental apparatus [156-158].

4. Analysis of how the measured $\mu^{-}$disappearance rate changes as the spatial cuts and 
fit methods are varied. The muon/electron vertex cut is especially useful in this regard, because small variations in the cut radius can produce large variations in the observed disappearance rate, due to the time-dependent term in Equation 3.3 that describes radial $\mu d$ diffusion. It should also be possible to glean similar information by studying the effects of variations in the fitted time interval, or in the fiducial cut on the muon stopping distribution. Neither of these latter techniques is very robust, however, because other processes (such as $p \mu p$ formation, in the case of the fit time interval) can produce effects similar to those from $\mu d$, and disentangling the myriad contributions is highly nontrivial. Of course, for all of these methods, it is in principle only possible to obtain a ratio of the deuterium concentrations from two sets of data, by comparing the analysis results of each. Care is therefore needed to guard against using the same information for both the deuterium concentration determination and the zero extrapolation.

The particular $c_{d}$ measurement technique that is selected depends largely upon the experimental exigencies of a particular run period. In our experience, approaches 1 and 4 have proved to be the most feasible, and they will be discussed in greater detail in Section 6.6.4.

Our strategy for dealing with deuterium is to suppress the deuterium level as much as possible for the primary data collection, and to use zero-extrapolation techniques to correct for all effects from any remaining contamination. Of course, this approach requires that we also perform a "calibration" run, wherein we collect data using hydrogen gas that contains an elevated level of deuterium. We use isotopically pure hydrogen ("protium") depleted of deuterium to $\approx 1 \mathrm{ppm}$, more than 100 times below its natural abundance of $\sim 150 \mathrm{ppm}$. Deuterium levels of a few ppm are actually tolerable, although they necessitate increasingly larger corrections to the lifetime measurement, with corresponding increases in the systematic uncertainty. If purity levels of $c_{d} \leq 0.3 \mathrm{ppm}$ can be achieved, the systematic 
uncertainty from deuterium becomes negligble.

Finally, it should be noted that the muons in $\mu d$ atoms will preferentially transfer to $Z>1$ impurities - a behavior which is not true for the muons that have formed $p \mu p$ molecules. The $\mu d \rightarrow \mu Z$ exchange is described in the same fashion as the completely analogous process of $\mu p \rightarrow \mu Z$ transfer:

$$
\Lambda_{d Z}=\phi c_{Z} \lambda_{d Z}
$$

where $\lambda_{d Z}$ is the "normalized" transfer rate in liquid deuterium. The experimental value for $\lambda_{d Z}$ in nitrogen, $\lambda_{d \mathrm{~N}}=(14.5 \pm 0.2) \times 10^{10} \mathrm{~s}^{-1}$ [159], is in good agreement with the lone theoretical prediction, $\lambda_{d \mathrm{~N}}=14.8 \times 10^{10} \mathrm{~s}^{-1}$ [140]; of course, this theoretical value should be regarded with some caution in view of the objections that have been raised about the author's methods, as discussed in Table 3.3. For oxygen, the value $\lambda_{d \mathrm{O}}=(5.5 \pm 0.6) \times$ $10^{10} \mathrm{~s}^{-1}$ was measured in $\mathrm{SO}_{2}[160]$, and the value $\lambda_{d \mathrm{O}}=(6.3 \pm 0.5) \times 10^{10} \mathrm{~s}^{-1}$ was measured in $\mathrm{D}_{2}+\mathrm{O}_{2}$ [161]; a theoretical calculation predicts $\lambda_{d \mathrm{O}}=6.6 \times 10^{10} \mathrm{~s}^{-1}[140]$.

\subsection{Analytic description of full $\mu^{-}$kinetics}

This section presents a simplified analytic formulation of $\mu^{-}$kinetics in a hydrogen gas target containing deuterium and a single impurity of $Z>1$, though the calculations can be expanded in a straightforward manner to describe the presence of multiple $Z>1$ impurities. The kinetics model, illustrated in Figure 3.6, includes all of the major processes described in the preceding sections. It should be noted that some second-order processes, such as $p$ - $d$ fusion and subsequent muon recycling, have not been included.

In keeping with prior notation, let $n_{1}$ be the fraction of muons in $\mu p$ hyperfine singlet atoms, $n_{2}$ the fraction in $p \mu p$ ortho molecules, $n_{3}$ the fraction in $p \mu p$ para molecules, $n_{4}$ the fraction in $\mu d$ atoms, and $n_{5}$ the fraction in $\mu Z$ atoms. We can safely ignore the 


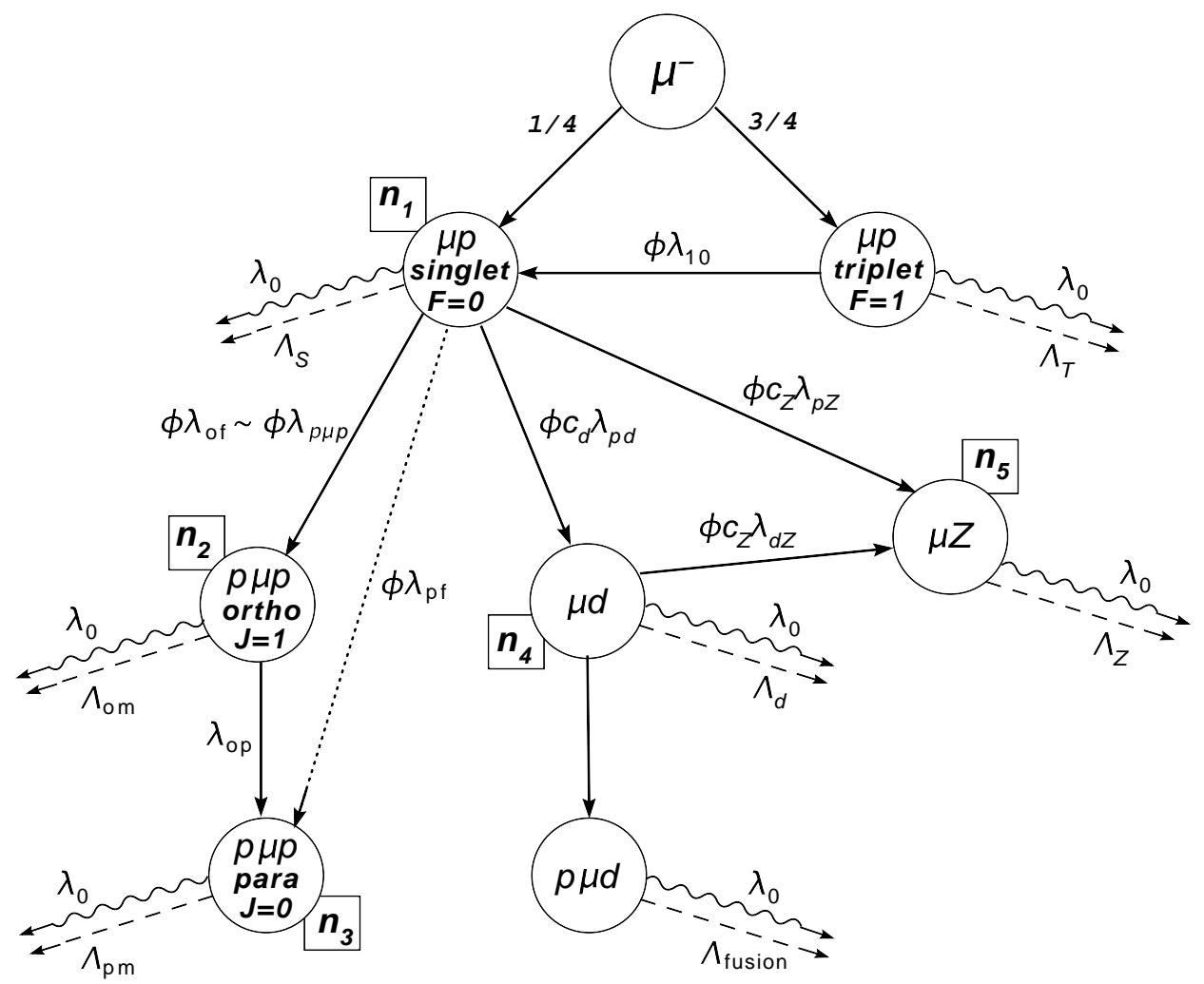

Figure 3.6: Flowchart of $\mu^{-}$kinetics in hydrogen gas containing deuterium and a single $Z>1$ impurity. As indicated, a muon can disappear from each state either via decay $\left(\lambda_{0}\right)$ or via nuclear capture $\left(\Lambda_{\mathrm{X}}\right)$. The $p \mu d$ fusion channel is pictured for the sake of completeness, though it is not included in the calculations in the text.

$\mu p$ hyperfine triplet state, since it is effectively empty during the times of interest. The evolution of the muon populations in time is described by the following set of coupled, linear, first-order differential equations

$$
\begin{aligned}
\frac{d n_{1}}{d t} & =-\lambda_{1} n_{1} \\
\frac{d n_{2}}{d t} & =\left(\phi \lambda_{\text {of }}\right) n_{1}-\lambda_{2} n_{2} \\
\frac{d n_{3}}{d t} & =\left(\phi \lambda_{\text {pf }}\right) n_{1}+\lambda_{\text {op }} n_{2}-\lambda_{3} n_{3} \\
\frac{d n_{4}}{d t} & =\left(\phi c_{d} \lambda_{p d}\right) n_{1}-\lambda_{4} n_{4} \\
\frac{d n_{5}}{d t} & =\left(\phi c_{Z} \lambda_{p Z}\right) n_{1}+\left(\phi c_{Z} \lambda_{d Z}\right) n_{4}-\lambda_{5} n_{5}
\end{aligned}
$$


where the disappearance rates $\lambda_{i}$ of the five species are

$$
\begin{aligned}
& \lambda_{1}=\lambda_{0}+\Lambda_{S}+\left(\phi \lambda_{\mathrm{of}}\right)+\left(\phi \lambda_{\mathrm{pf}}\right)+\left(\phi c_{d} \lambda_{p d}\right)+\left(\phi c_{Z} \lambda_{p Z}\right) \\
& \lambda_{2}=\lambda_{0}+\Lambda_{\mathrm{om}}+\lambda_{\mathrm{op}} \\
& \lambda_{3}=\lambda_{0}+\Lambda_{\mathrm{pm}} \\
& \lambda_{4}=\lambda_{0}+\Lambda_{d}+\left(\phi c_{Z} \lambda_{d Z}\right) \\
& \lambda_{5}=\lambda_{0}+\Lambda_{Z} .
\end{aligned}
$$

Note how the presence of molecules and impurities leads to the appearance of the muon loss terms $\Lambda_{\mathrm{om}}, \Lambda_{\mathrm{pm}}, \Lambda_{d}$, and $\Lambda_{Z}$, in addition to the underlying $\mu p$ extinction rate $\lambda_{0}+\Lambda_{S}$ which MuCap seeks to measure. For the sake of simplicity, I have ignored the bound-state modifications to the vacuum decay rate $\lambda_{0}$; when accuracy is important, the Huff factor $Q$ can easily be reinstated (i.e., $\lambda_{0} \rightarrow Q \lambda_{0}$ ).

In the MuCap hydrogen gas target, impurity concentrations are extremely low, the $\mu p$ triplet state depopulates quickly, and $p \mu p$ molecules form slowly, so we can safely assume that all muons start out in the $\mu p$ singlet state. In this case, $n_{1}(0)=1$ and $n_{i}(0)=0$ for $i \neq 1$, and the analytic solutions to Equations 3.4 are

$$
\begin{aligned}
n_{1}(t)= & e^{-\lambda_{1} t} \\
n_{2}(t)= & \left(\frac{\phi \lambda_{\mathrm{of}}}{\lambda_{1}-\lambda_{2}}\right)\left[e^{-\lambda_{2} t}-e^{-\lambda_{1} t}\right] \\
n_{3}(t)= & \left(\frac{\phi \lambda_{\mathrm{pf}}}{\lambda_{1}-\lambda_{3}}\right)\left[e^{-\lambda_{3} t}-e^{-\lambda_{1} t}\right]+ \\
& \left(\frac{\phi \lambda_{\mathrm{of}} \lambda_{\mathrm{op}}}{\lambda_{1}-\lambda_{2}}\right)\left[\left(\frac{e^{-\lambda_{3} t}-e^{-\lambda_{2} t}}{\lambda_{2}-\lambda_{3}}\right)-\left(\frac{e^{-\lambda_{3} t}-e^{-\lambda_{1} t}}{\lambda_{1}-\lambda_{3}}\right)\right] \\
n_{4}(t)= & \left(\frac{\phi c_{d} \lambda_{p d}}{\lambda_{1}-\lambda_{4}}\right)\left[e^{-\lambda_{4} t}-e^{-\lambda_{1} t}\right] \\
n_{5}(t)= & \left(\frac{\phi c_{Z} \lambda_{p Z}}{\lambda_{1}-\lambda_{5}}\right)\left[e^{-\lambda_{5} t}-e^{-\lambda_{1} t}\right]+ \\
& \left(\frac{\left(\phi c_{d} \lambda_{p d}\right)\left(\phi c_{Z} \lambda_{d Z}\right)}{\lambda_{1}-\lambda_{4}}\right)\left[\left(\frac{e^{-\lambda_{5} t}-e^{-\lambda_{4} t}}{\lambda_{4}-\lambda_{5}}\right)-\left(\frac{e^{-\lambda_{5} t}-e^{-\lambda_{1} t}}{\lambda_{1}-\lambda_{5}}\right)\right] .
\end{aligned}
$$


Referring back to Equation 1.10, the time spectrum of decay electrons that is observed by the detectors can then be written as

$$
N_{e}(t)=\lambda_{0} N_{\mu}(t)=\lambda_{0} \sum_{i=1}^{5} n_{i}(t)=\lambda_{0} \sum_{i=1}^{5} c_{i} e^{-\lambda_{i} t},
$$

where I have introduced the coefficients $c_{i}$ :

$$
\begin{aligned}
c_{1}= & 1-\left(\frac{\phi \lambda_{\mathrm{of}}}{\lambda_{1}-\lambda_{2}}\right)\left[1-\left(\frac{\lambda_{\mathrm{op}}}{\lambda_{1}-\lambda_{3}}\right)\right]-\left(\frac{\phi \lambda_{\mathrm{pf}}}{\lambda_{1}-\lambda_{3}}\right) \\
& \quad-\left(\frac{\phi c_{d} \lambda_{p d}}{\lambda_{1}-\lambda_{4}}\right)\left[1-\left(\frac{\phi c_{Z} \lambda_{d Z}}{\lambda_{1}-\lambda_{5}}\right)\right]-\left(\frac{\phi c_{Z} \lambda_{p Z}}{\lambda_{1}-\lambda_{5}}\right) \\
c_{2}= & \left(\frac{\phi \lambda_{\mathrm{of}}}{\lambda_{1}-\lambda_{2}}\right)\left[1-\left(\frac{\lambda_{\mathrm{op}}}{\lambda_{2}-\lambda_{3}}\right)\right] \\
c_{3}= & \left(\frac{\phi \lambda_{\mathrm{pf}}}{\lambda_{1}-\lambda_{3}}\right)+\left(\frac{\phi \lambda_{\mathrm{of}}}{\lambda_{1}-\lambda_{3}}\right)\left(\frac{\lambda_{\mathrm{op}}}{\lambda_{2}-\lambda_{3}}\right) \\
c_{4}= & \left(\frac{\phi c_{d} \lambda_{p d}}{\lambda_{1}-\lambda_{4}}\right)\left[1-\left(\frac{\phi c_{Z} \lambda_{d Z}}{\lambda_{4}-\lambda_{5}}\right)\right] \\
c_{5}= & \left(\frac{\phi c_{Z} \lambda_{p Z}}{\lambda_{1}-\lambda_{5}}\right)+\left(\frac{\phi c_{d} \lambda_{p d}}{\lambda_{1}-\lambda_{5}}\right)\left(\frac{\phi c_{Z} \lambda_{d Z}}{\lambda_{4}-\lambda_{5}}\right) .
\end{aligned}
$$

Using these expressions it is possible to calculate the lifetime spectrum's effective decay rate from the first moment of the muon population's time distribution,

$$
\lambda_{\text {eff }}=\frac{\int_{0}^{\infty} N_{e}(t) d t}{\int_{0}^{\infty} t N_{e}(t) d t}=\frac{\int_{0}^{\infty} N_{\mu}(t) d t}{\int_{0}^{\infty} t N_{\mu}(t) d t}=\frac{\sum_{i=1}^{5}\left(\frac{c_{i}}{\lambda_{i}}\right)}{\sum_{i=1}^{5}\left(\frac{c_{i}}{\lambda_{i}^{2}}\right)} .
$$

Equation 3.9 is the analytic version of Peierls' method [162], a technique that was used by the Saclay experiment [42] to extract the effective muon lifetime $\tau$ from a decay spectrum histogrammed into $K$ bins,

$$
\tau=\frac{\sum_{i=1}^{K} n_{i} t_{i}}{\sum_{i=1}^{K} n_{i}}
$$

where $t_{i}$ is the central time of the $i^{\text {th }}$ bin and $n_{i}$ is its content. In MuCap we rely instead on best-fit techniques to analyze the experimental lifetime spectra, but the analytic formulation in Equation 3.9 remains extremely useful for several reasons. First, the analytic framework lends itself to Taylor expansion in small parameters, and thereby enables us to perform 
sensitivity studies on how the muon disappearance rate is affected by variations in the kinetics parameters [153]. Second, the first moment formula can be used to study the systematic effects that arise from the use of a simple exponential fitting function over a finite time range [2]. In practice, the first moment formula will be important when determining $\Lambda_{S}$, as it enables the calculation of a "correction" that compensates for the effects of muon capture in $p \mu p$ molecules on the fitted disappearance rate.

\subsection{Kinetics of $\boldsymbol{\mu}^{+}$}

In MuCap, we regard a $\mu^{+}$lifetime measurement in hydrogen as a valuable control on our $\mu^{-}$lifetime measurement, so we require a detailed understanding of $\mu^{+}$behavior in hydrogen. The kinetics of $\mu^{+}$in hydrogen are considerably simpler than for $\mu^{-}$because the $\mu^{+}$does not migrate among different states or undergo any nuclear reactions. However, polarization effects must be considered when measuring the $\mu^{+}$lifetime, as will be discussed below.

When a fast-moving $\mu^{+}$enters a hydrogen target, it decelerates due to multiple Coulomb scattering with electrons, in similar fashion as the $\mu^{-}$[163]. By the time the $\mu^{+}$ has slowed to a kinetic energy of $200 \mathrm{eV}$, roughly $60 \%$ of the $\mu^{+}$will have captured an unpolarized electron and formed muonium $(\mathrm{Mu})$, a $\mu^{+} e^{-}$bound state which behaves like a light, radioactive hydrogen isotope. The exact details of $\mathrm{Mu}$ formation and thermalization are somewhat complex and not completely understood, but the essential features are (1) the $\mathrm{Mu}$ atoms are thermalized within a nanosecond after the muon's arrival, and (2) the Mu atoms preserve a nonzero fraction of the initial $\mu^{+}$polarization [164]. Muonium diffusion is negligible because the $\mathrm{Mu}$ atom presents a large scattering cross section. The stopped $\mu^{+}$which do not form Mu are considered "free," although there is some evidence that 
they participate in the formation of molecular ions. Like $\mathrm{Mu}$, these free $\mu^{+}$retain some fraction of the initial polarization. Unlike $\mathrm{Mu}$, however, the $\mu^{+}$have a net charge and are therefore propelled upwards by the drift field of the time projection chamber. It is difficult to estimate the exact field-induced drift velocity of $\mu^{+}$in hydrogen, due to the fact that (to our knowledge) no $\mu^{+}+\mathrm{H}_{2}$ cross sections are available. By using the known cross sections for $\mathrm{H}^{+}+\mathrm{H}_{2}$ and scaling the mass from $\mathrm{H}^{+}$to $\mu^{+}$, Dr. Malte Hildebrandt has estimated the $\mu^{+}$ diffusion speed in our time projection chamber to be $\approx 0.08 \mathrm{~mm} / \mu \mathrm{s}$ [165], which is roughly thirty times smaller than the diffusion speed of $\mu p$ atoms in the same hydrogen target (see Appendix C). The hypothesis of a small $\mu^{+}$diffusion scale is empirically supported by the results from analysis of our $\mu^{+}$data, which are discussed in Section 7.3.3.

Every $\mu^{+}$eventually disappears via decay,

$$
\mu^{+} \rightarrow e^{+} \nu_{e}+\bar{\nu}_{\mu}
$$

a process that proceeds at constant rate $\lambda_{0}=1 / \tau_{\mu^{+}}$, regardless of whether or not the $\mu^{+}$has formed Muonium [166]. The best $\mu^{+}$lifetime $\left(\tau_{\mu^{+}}\right)$measurements to date were performed by four experiments which used fast scintillation techniques. Their measurements and the most recent world averages are summarized in Table 3.4. The first three of these experiments achieved comparable, statistics-limited precisions of $\sim 30 \mathrm{ppm}$, and their combined $18 \mathrm{ppm}$ world average stood for more than 20 years. The fourth and ongoing experiment, MuLan, recently announced a first result of precision $11 \mathrm{ppm}$. It is worth noting that there are currently three experimental efforts underway to improve upon the muon lifetime: the R77 experiment at the RIKEN-RAL muon facility in the UK aims for a precision of 10ppm [170], while two separate efforts at PSI, FAST [171] and MuLan $[49,172]$, aim to determine $\tau_{\mu^{+}}$ to within $1 \mathrm{ppm}$.

The persistence of $\mu^{+}$polarization in hydrogen is of relevance in lifetime mea- 


\begin{tabular}{lcllcl}
\hline \hline Exp. location & Year & \multicolumn{2}{c}{$\tau_{\mu^{+}}\left(10^{-6} \mathrm{~s}\right)$} & $\delta \tau / \tau$ & Ref. \\
\hline Dubna & 1974 & $2.19711 \pm 0.00008$ & $36 \mathrm{ppm}$ & {$[167]$} \\
Saclay & 1984 & $2.197078 \pm 0.000073$ & $33 \mathrm{ppm}$ & {$[168]$} \\
TRIUMF & 1984 & $2.19695 \pm 0.00006$ & $27 \mathrm{ppm}$ & {$[169]$} \\
PSI (MuLan) & 2007 & $2.197013 \pm 0.000024$ & $11 \mathrm{ppm}$ & {$[49]$} \\
\hline PDG avg. & 2006 & $2.19703 \pm 0.00004$ & $18 \mathrm{ppm}$ & {$[18]$} \\
PDG+MuLan avg. & 2007 & $2.197019 \pm 0.000021$ & $9.6 \mathrm{ppm}$ & {$[49]$} \\
\hline \hline
\end{tabular}

Table 3.4: The four most precise $\mu^{+}$lifetime measurements to date, and the most recent world averages. The 2006 Particle Data Group (PDG) world average for the muon lifetime includes some additional experimental contributions that are not presented here, and the PDG average does not include the recent MuLan result. The "PDG+MuLan" average was calculated by the MuLan Collaboration, by combining their result with the 2006 PDG value.

surements because of the combined effects of two phenomena: (1) the $\mu^{+}$spin precesses in time according to the strength of the ambient magnetic field, and (2) the decay positron is preferentially emitted in the direction of the $\mu^{+}$spin, due to parity violation in the weak interaction. The differential probability for the positron to be emitted with normalized energy $\omega=E / E_{\max }=E /\left(\frac{1}{2} m_{\mu}\right)$ at an angle $\theta$ with respect to the muon's spin is [163]

$$
\frac{d P}{d \omega d \Omega}=\frac{\omega^{2}}{2 \pi}[(3-2 \omega)-(1-2 \omega) \cos \theta]
$$

and integrating Equation 3.10 over the range of possible energies $\omega$ gives

$$
\frac{d P}{d \Omega}=\int_{0}^{1} \frac{d P}{d \omega d \Omega} d \omega \propto 1+\frac{1}{3} \cos \theta .
$$

Equation 3.11 reveals that the angular emission spectrum of positrons is asymmetic and peaked in the direction of the muon's spin. This behavior, when coupled with the precession or relaxation of the residual $\mu^{+}$polarization, can turn geometric variations in detector acceptance into time-dependent effects in the observed lifetime spectrum. The extent of the distortion is determined by numerous experimental factors, such as the degree of beam polarization, energy dependence in the detector efficiencies, the detector's solid angle coverage, and the alignment of the muon spin with the experimental axis of symmetry. 
In transverse field experiments like MuCap, a weak external magnetic field $B$ is applied perpendicular to the muon polarization in order to exercise some control over the precession of the muon spin. As a result, the observed $\mu^{+}$lifetime distribution is an exponential modulated by $\mu^{+}$spin $\operatorname{rotation}^{4}\left(\mu^{+} \mathrm{SR}\right)$,

$$
f(t)=N e^{-\lambda_{0} t}[1+S(t)]+B
$$

Here $N$ is a scaling factor, $\lambda_{0}$ is the muon's vacuum decay rate, $S(t)$ describes the $\mu^{+} \mathrm{SR}$ effects, and $B$ is a time-independent background term. Positive muons in $\mathrm{H}_{2}$ exhibit three characteristic precession frequencies: $13.5 \mathrm{kHz} /$ gauss for free muons, $1.4 \mathrm{MHz} /$ gauss for the $\mathrm{Mu}$ "triplet" state, and $4.5 \mathrm{GHz}$ for the $\mathrm{Mu}$ "singlet" state (see Appendix F for derivations of these rates). The fast $4.5 \mathrm{GHz} \mathrm{Mu}$ singlet precession rate corresponds to an oscillation period of $0.22 \mathrm{~ns}$, which is too small to be resolved by the MuCap electronics (which have $1.25 \mathrm{~ns}$ time resolution) and therefore averages out. The Mu triplet's slower $1.4 \mathrm{MHz} /$ gauss rotation is observable but short-lived, relaxing away within $\sim 200 \mathrm{~ns}$ in MuCap's 50 gauss magnetic field (see Figure 3.7); moreover, it effectively disappears when the spectra from all of the electron detectors are added together. Thus only the free $\mu^{+}$precession signal is generally visible in the typical MuCap lifetime fit range of 100-24000 ns, and $S(t)$ takes the form

$$
S(t)=A(t) \cos \left(\omega_{\mu} t+\phi\right)
$$

As shown in Figure 3.8, the sinusoidal $\mu^{+} \mathrm{SR}$ signal relaxes with time, primarily due to inhomogeneities in the magnetic field [164]. Consequently, the function $A(t)$ usually describes an exponential or Gaussian envelope.

MuCap takes a phenomenological approach to fitting the $\mu^{+}$lifetime: Any unknown details about the degree of beam polarization, alignment between the longitudinal

\footnotetext{
${ }^{4}$ For a comprehensive review of the field of muon spin rotation, see reference [173].
} 


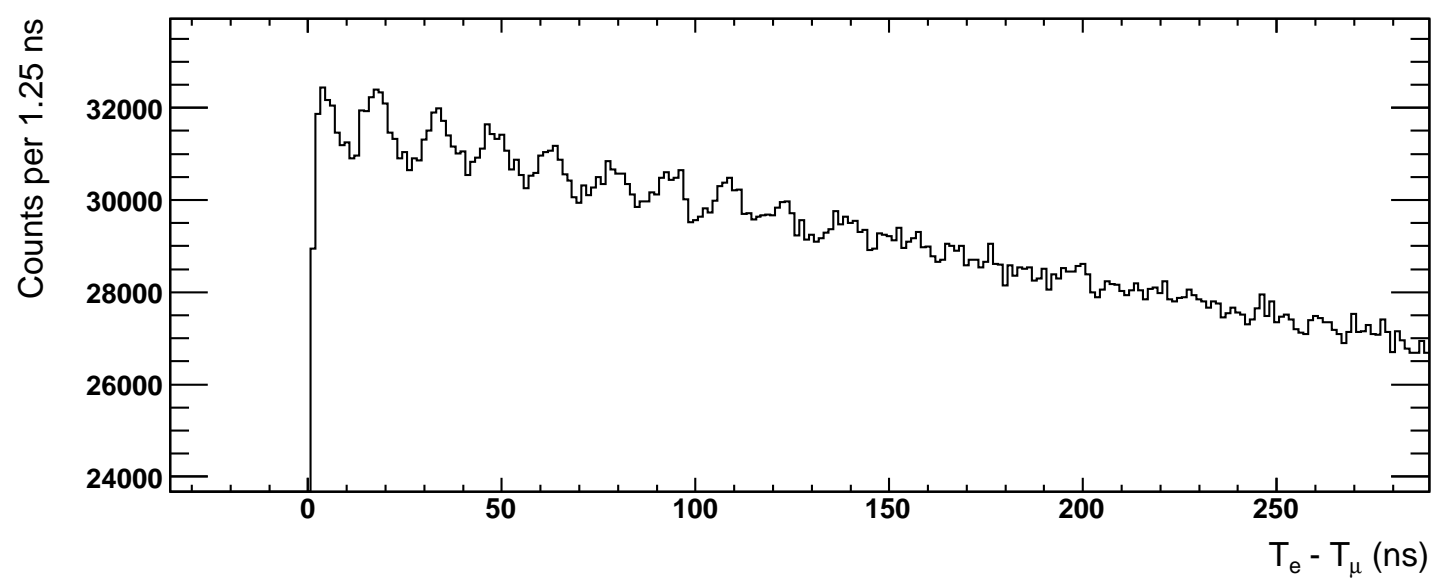

Figure 3.7: The $\mu^{+}$lifetime spectrum observed by one of the MuCap electron detectors (eSC "gondola" no. 9), zoomed in to early times to reveal the oscillation peaks produced by $\mathrm{Mu}$ "triplet" ( $\uparrow$ ) precession. The observed oscillation period of roughly $15 \mathrm{~ns}$ is consistent with the value expected in our $\approx 50$ gauss transverse magnetic field, as the calculations in Appendix F predict $\tau_{\mathrm{Mu} \uparrow}=[(1.4 \mathrm{MHz} / \text { gauss })(50 \text { gauss })]^{-1} \approx 14$ ns. Note that the oscillations appear to have completely relaxed away within $200 \mathrm{~ns}$.

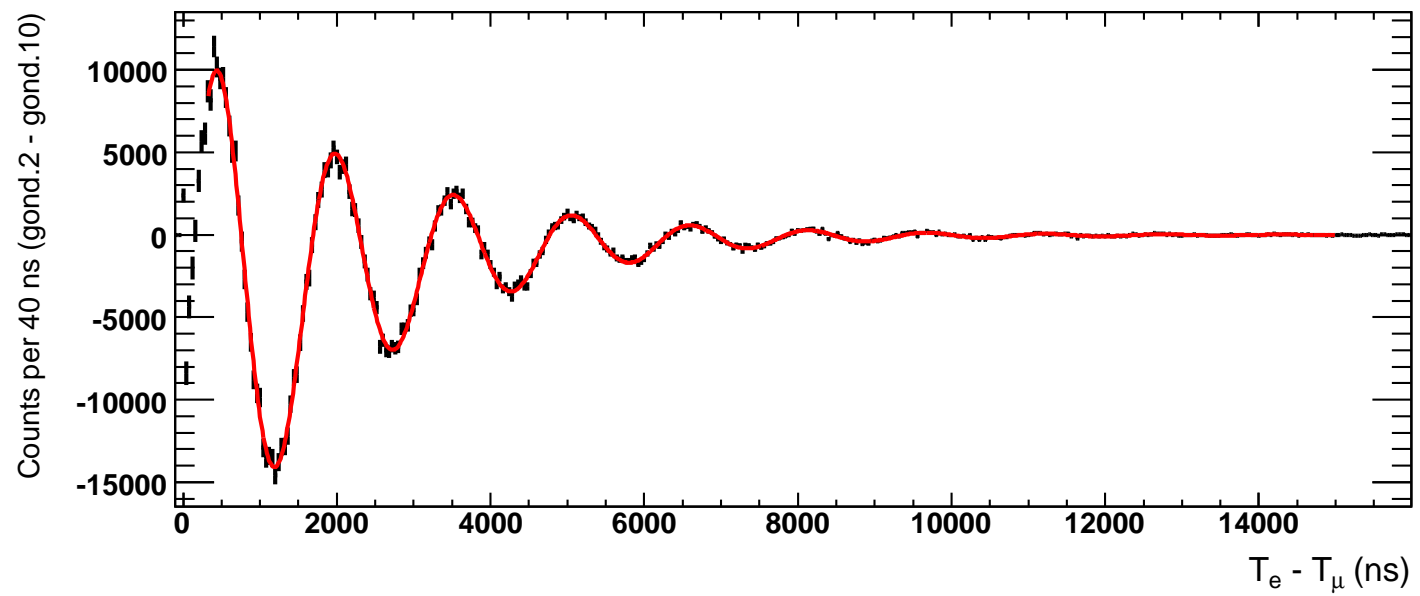

Figure 3.8: The free $\mu^{+}$precession signal observed in the $\approx 50$ gauss transverse magnetic field used in MuCap. The precession signal was isolated by subtracting the lifetime spectra from diametrically opposed electron detectors (in this case, eSC gondolas no. 2 and no. 10). The red line is a best-fit to the data points. The observed oscillation period of roughly $1.5 \mu \mathrm{s}$ is in agreement with the value predicted by the calculations in Appendix F, which give $\tau_{\mu^{+} \mathrm{SR}}=[(13.5 \mathrm{kHz} / \text { gauss })(50 \text { gauss })]^{-1} \approx 1.5 \mu \mathrm{s}$. 
beam polarization and the detector axis, effects from detector asymmetries, etc., are encapsulated in the parameters in Equation 3.13. The $\mu^{+} \mathrm{SR}$ signal is first isolated by subtracting counts in geometrically opposed detectors at angles $\phi$ and $\phi+\pi$, as shown in Figure 3.8; the signal is generally strongest in the detectors situated transverse to the magnetic field direction. The $\mu^{+} \mathrm{SR}$ precession and relaxation parameters are determined from this isolated signal and then fixed at their empirical values. After that, only the magnitude of the $\mu^{+} \mathrm{SR}$ oscillation is left free to vary in fits to the $\mu^{+}$lifetime data. This procedure reduces the potential for residual $\mu^{+} \mathrm{SR}$ components to distort the final lifetime fit. 


\section{Chapter 4}

\section{Experimental apparatus}

The fundamental purpose of the MuCap detectors is to (1) determine the arrival time and stopping position of muons which enter a protium gas target, and (2) measure the emission time and trajectory of subsequent decay electrons. The detectors, pictured in Figure 4.1, can therefore be naturally divided into two parts: the muon detectors and the electron detectors, which are deliberately decoupled to the largest extent possible in order to avoid spurious correlations between muon and electron measurements. This chapter describes the two detector groups and their supporting subsystems in detail.

\subsection{Muon detectors}

The muon entrance detector assembly is depicted in Figure 4.2. When muons arrive at the end of the beamline, they emerge from the beampipe through a $75-\mu \mathrm{m}$-thick Mylar vacuum window. The muons next encounter the muon Scintillating Anti-Counter, $\mu \mathrm{SCA}$, and a lead collimator. The $\mu \mathrm{SCA}$ and collimator both have 40-mm-diameter holes in their centers, through which most arriving muons pass unhindered; the $\mu \mathrm{SCA}$ and collimator are thus used to reject stray muons and to help sharpen the beam spot. The $\mu \mathrm{SCA}$ veto 


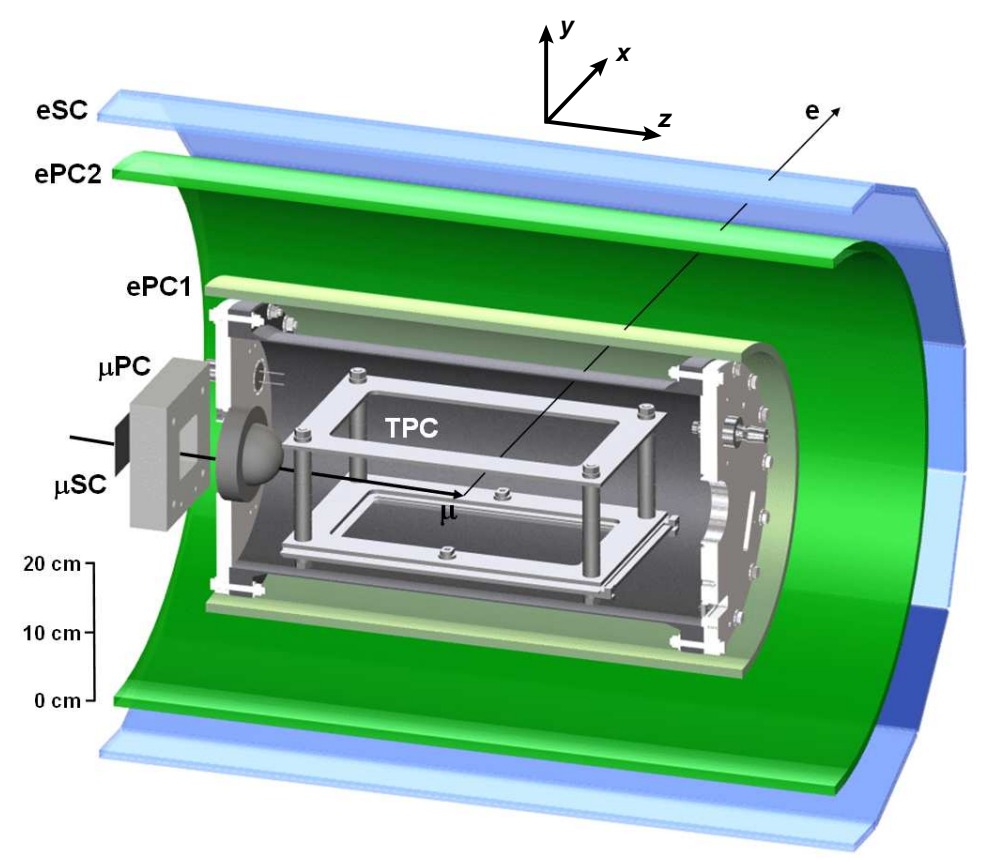

Figure 4.1: Cross-sectional diagram of the MuCap detector, with the main detector components labeled. The convention for the coordinate system is as indicated, although the coordinate origin actually lies at the center of the ePC cylinders, within the TPC.

counter is $5 \mathrm{~mm}$ thick to ensure $\approx 100 \%$ detection efficiency.

The next detector encountered by an arriving muon is the muon Scintillating Counter, $\mu \mathrm{SC}$, which provides the fast timing signal for the start of the muon lifetime measurement. On different occasions MuCap has used a 250- $\mu \mathrm{m}$-thick and a $500-\mu \mathrm{m}$-thick scintillator for the $\mu \mathrm{SC}$ detector; both thicknesses provide sufficient energy resolution to distinguish between muons and electrons, which is useful for maximizing the muon-toelectron ratio in the beam.

After passing through the $\mu \mathrm{SC}$, a muon next strikes the multiwire muon proportional chamber, $\mu \mathrm{PC} 1$, which records the $(x, y)$ position and time of the muon's passage. The $\mu \mathrm{PC} 1$ detector contains two anode planes and four cathode planes, sandwiched between two $50 \mu \mathrm{m}$ Mylar windows. The chamber is operated at $2.5 \mathrm{kV}$ and filled to 1 bar pressure 
with a gas mixture of $49.9 \%$ argon, $59.9 \% \mathrm{C}_{2} \mathrm{H}_{6}$, and $0.2 \%$ Freon; the total gas thickness is $35 \mathrm{~mm}$. Each anode plane contains 24 parallel wires with $2 \mathrm{~mm}$ spacing, and each wire acts as an independent detector, collecting electron avalanches that follow from the primary ionization generated by the throughgoing muon. One anode plane's wires are vertically oriented to provide $x$ information, while the other anode plane's wires are horizontally oriented to provide $y$ information. Together the two anode planes form a $5 \times 5 \mathrm{~cm}^{2}$-large wire grid with an effective $(x, y)$ spatial resolution of roughly three wires in each direction, or $36 \mathrm{~mm}^{2}$. Although $\mu \mathrm{PC} 1$ was intended for use in three-dimensional tracking of the incoming muon trajectories, it has been used primarily for beam tuning, and it provides valuable redundancy in basic muon detection, since its spatial resolution enables it to identify some of the muons that are missed by the $\mu \mathrm{SC}$.

As shown in Figure 4.2, we originally intended to include a second proportional chamber, $\mu \mathrm{PC} 2$, for enhanced muon tracking accuracy. However, doubts about the usefulness of $\mu \mathrm{PC} 1+\mu \mathrm{PC} 2$ tracking, as well as concerns about excessive scattering generated by $\mu \mathrm{PC} 2$, convinced us to abandon it for the 2004 experimental run. Further technical details about the $\mu \mathrm{PC} 2$ detector (which is essentially the same as the $\mu \mathrm{PC} 1$ detector) can be found in reference [174].

After passing through $\mu \mathrm{SCA}, \mu \mathrm{SC}$, and $\mu \mathrm{PC} 1$, muons reach the pressure vessel, an aluminum cylinder filled with ultrapure protium gas at 10 bar (1 $\mathrm{MPa}$ ) pressure and at ambient room temperature. Muons enter the pressure vessel through a beryllium window, a 0.5 -mm-thick hemispherical shell of radius $35 \mathrm{~mm}$ which was selected to minimize scattering. Approximately $65 \%$ of the incoming muons stop inside the sensitive volume of the time projection chamber (TPC), an ionizing drift detector of active volume $\Delta x \times \Delta y \times \Delta z=15 \times 12 \times 30 \mathrm{~cm}^{3}$ that sits at the center of the pressure vessel (see Figures 4.3, 4.4, and also the schematic drawing in appendix Figure G.2). The TPC is the 


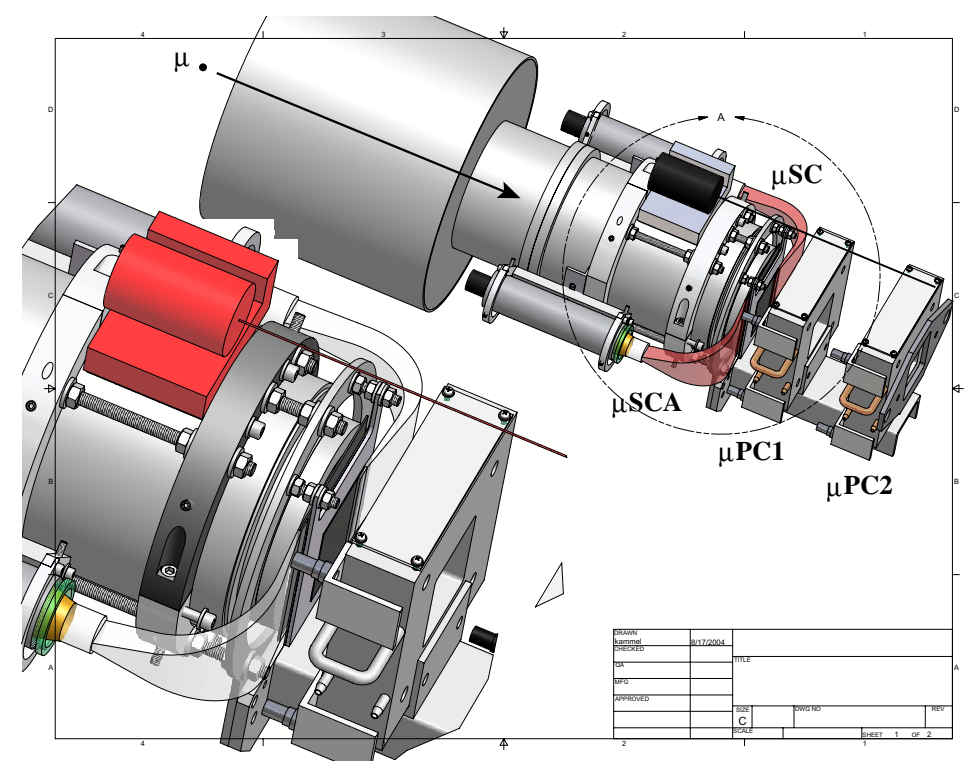

Figure 4.2: Drawing of the beampipe-supported entrance muon detector assembly, which consists of $\mu \mathrm{SCA}$, a lead collimator, $\mu \mathrm{SC}, \mu \mathrm{PC} 1$, and $\mu \mathrm{PC} 2$. Although $\mu \mathrm{PC} 2$ is pictured here, it was not included in the 2004 experimental run.

heart of the MuCap experiment: it is a versatile instrument that accurately tracks the incoming muon trajectories in three dimensions, and which can also register signals from exotic capture and fusion processes. The MuCap TPC was specially developed to meet the unique demands of the experiment, since there is little world experience with TPCs operating in high pressure hydrogen gas $[3,175]$. It is challenging to operate a TPC under such conditions because hydrogen is a difficult chamber gas, and because the high gas pressure requires voltages in the range $4.6-6.5 \mathrm{kV}$, which are higher than those used in conventional chambers. The TPC itself is constructed of ultraclean, low-outgassing, bakeable materials, and the entire pressure vessel and TPC system can baked up to $130^{\circ} \mathrm{C}$ to liberate impurities, which are pumped away under vacuum prior to filling with hydrogen.

The TPC operates in proportional mode, and its wide dynamic range makes it sensitive to a variety of muonic physics phenomena. The primary responsibility of the TPC is the unambiguous identification of muons that stop in the protium gas. If the chamber 


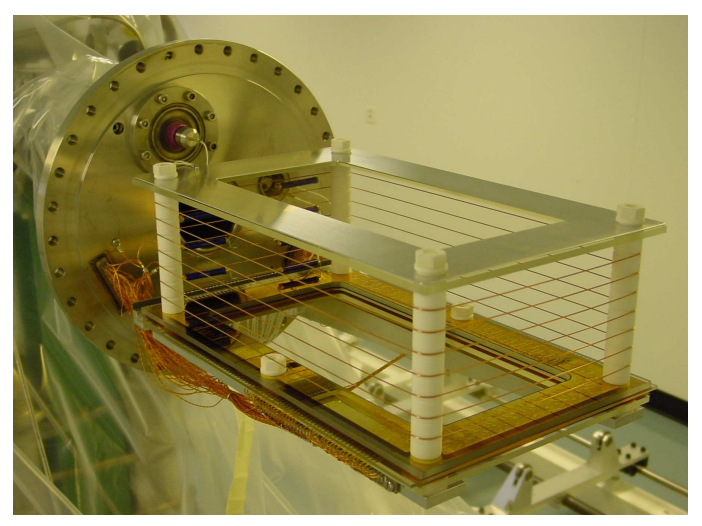

Figure 4.3: Photograph of the TPC mounted on the back flange of the pressure vessel, viewed from above.

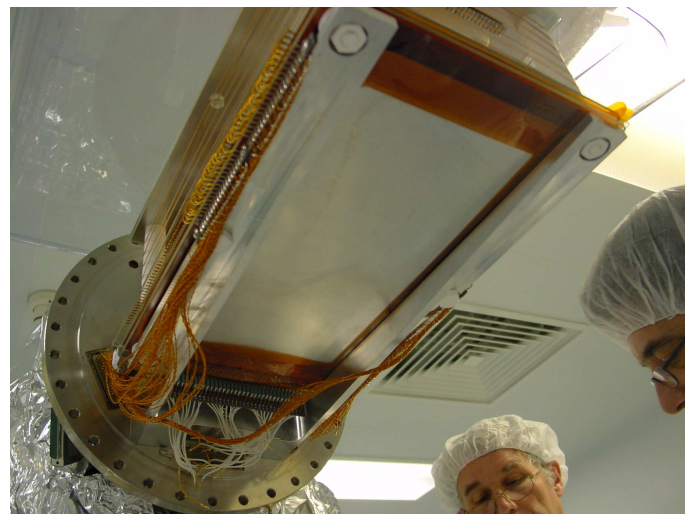

Figure 4.4: View from below the TPC, after a 1-mm-thick aluminum plate was installed underneath the multiwire proportional chamber (MWPC) region to prevent dust from accumulating on the wires.

were to operate at an amplifying voltage of $6.5 \mathrm{kV}$ as designed, it could also detect the minimum ionizing tracks from decay electrons, whose energy deposition is comparatively small. However, in 2004 the TPC's amplifying voltage did not exceed $\approx 5.2 \mathrm{kV}$, and most of the data was recorded at $\approx 5.0 \mathrm{kV}$ or slightly lower.

The TPC works as follows ${ }^{1}$ : When a charged particle passes through the hydrogen gas, it leaves behind a trail of ionization electrons and protons. If this occurs inside the sensitive volume of the TPC, the ions and electrons are pulled in opposite directions by the TPC's vertical, homogeneous electric "drift" field. The electrons migrate downwards along the $2 \mathrm{kV} / \mathrm{cm}$ low-field lines at a constant rate of roughly $0.55 \mathrm{~cm} / \mu \mathrm{s}$. When the electrons reach the bottom of the TPC, they encounter a high-field multiwire proportional chamber (MWPC) amplification region. For an $\approx 5 \mathrm{kV}$ MWPC field in hydrogen, the resulting signal avalanche amplifies the initial charges by a factor of $\approx 60$, and this information is recorded by a grid of wires in the $x z$ plane. There are 35 parallel cathode strips oriented

\footnotetext{
${ }^{1}$ See reference $[176]$ for a description of the principles of operation of multiwire proportional and drift chambers.
} 
in the $z$ direction to record $x$ position information, and there are 75 parallel anode wires oriented in the $x$ direction to record $z$ information. The $y$ position of the track relative to the MWPC grid is determined from the drift time, but another detector is needed to provide a reference time for when the ionization particles were created - in our case, the $\mu \mathrm{SC}$ supplies the necessary information. However, the TPC's entanglement of time with the $y$ dimension remains an inherently complex feature that requires care when analyzing data.

The TPC has modest timing and position resolution. Signals are recorded at $200 \mathrm{~ns}$ intervals, and the $x, y$, and $z$ position resolutions are $4 \mathrm{~mm}, 1.09 \mathrm{~mm}$, and $4 \mathrm{~mm}$, respectively. Additional information is available in the TPC signal strength, which is proportional to the energy deposited along the track of the ionizing particle. The TPC electronics are designed to record three signal levels: "Energy Low" (EL), "Energy High" (EH), and "Energy Very High" (EVH). The optimal settings for these signal levels vary with the hydrogen gas density, and they are tuned by eye using a graphical event display; typical settings are in the vicinity of $\mathrm{EL}=35 \mathrm{keV}, \mathrm{EH}=65 \mathrm{keV}$, and $\mathrm{EVH}=260 \mathrm{keV}$. The $\mathrm{EL}$ and $\mathrm{EH}$ thresholds are tuned so that each muon generates a trail of EL pixels along its ionizing track, terminating in an EH cluster at its stopping point, where there is a large Bragg peak in the energy deposition. The EVH threshold is generally set just above the $250 \mathrm{keV}$ high-energy tail of the Bragg peak in the muon stopping distribution, in order to register signals from high-energy processes - namely, nuclear muon capture by $Z>1$ impurities, which typically generate recoil nuclei in the energy range $200-350 \mathrm{keV}$. However, the $\mathrm{EH}$ threshold is also potentially useful in making impurity assessments, both elemental and isotopic. For instance, for TPC voltages above $5 \mathrm{kV}$, the byproducts of nonradiative muon-catalyzed $p$ - $d$ fusion [177],

$$
p d \mu \rightarrow{ }^{3} \mathrm{He}+\mu^{-}(5.3 \mathrm{MeV})
$$




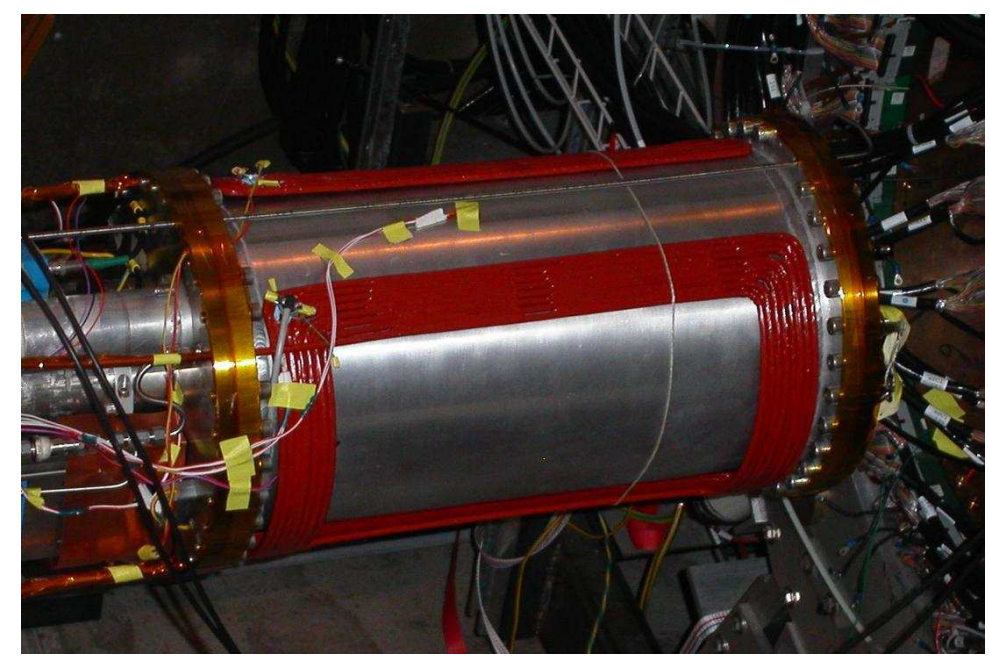

Figure 4.5: Photograph of the pressure vessel and saddle-coil $\mu^{+} \mathrm{SR}$ magnet (in red).

often generate a unique signature: the recoiling nucleus will trigger the EH threshold (at a relatively large distance from the muon stopping point, due to the rapid diffusion of the precursory $\mu d$ atom), while the outgoing 5.3 MeV "Alvarez muon" [178] leaves behind a trail of EL pixels leading away from the EH cluster. Such an observable is potentially useful because the frequency of fusion events is proportional to the deuterium concentration in the hydrogen gas. Thus, in addition to its basic three-dimensional tracking capabilities, the TPC in principle provides a means to measure and monitor impurities in the purified protium gas at the ppb level.

In order to control spin precession in the $\mu^{+}$lifetime measurements, we installed a paired saddle-coil magnet on the outside of the pressure vessel (Figure 4.5) to provide a weak transverse magnetic field. The magnet coils are wound from 0.25-in-diameter tubes, made of alloy 3003-O aluminum to minimize scattering of outgoing decay electrons. In 2004 the magnet's coils carried a 125 Amp current, which generated an $\approx 50$ gauss, or $5 \mathrm{mT}$, magnetic field within the TPC volume. The tubes are continuously cooled by water which circulates through their interior. 


\subsection{Electron detectors}

The times and trajectories of outgoing decay electrons are measured by a series of concentric cylindrical detectors surrounding the hydrogen $\operatorname{target}^{2}$ (see Figure 4.1). It is important to note that the decay electrons of interest emanate from inside the pressure vessel, where more than $90 \%$ of beam muons are stopped. Electrons must therefore pass through the pressure vessel wall, which is made of 4-mm-thick Anticorodal-110 aluminum in order to minimize scattering, before they can strike the surrounding electron detectors.

The first detectors encountered by an outgoing electron are two nested multiwire proportional chambers named ePC1 and ePC2. MuCap initially planned to use existing wire chambers from the decommissioned SINDRUM I apparatus at PSI [179] as ePC1 and ePC2. The spare and previously unused SINDRUM \#3 chamber became the ePC1 detector, and its dimensions constrained the design of the hydrogen pressure vessel. Unfortunately, the inherited SINDRUM \#5 chamber was severely damaged in storage and could not function as ePC2, so we were forced to build our own. A photograph of the new ePC2, which was constructed in 2003, is shown in Figure 4.6. Each ePC is actually comprised of three separate detectors: an anode wire drum is sandwiched between two cathode strip drums. The anodes run lengthwise along the chamber, and their $\approx 2 \mathrm{~mm}$ spacing gives the ePCs fine angular resolution. The two sets of cathode strips corkscrew in opposite directions around the $\mathrm{ePC}$ cylinder, and the strips have $\approx 6 \mathrm{~mm}$ spacing. When an anode wire and cathode strip fire in coincidence, their point of overlap provides the $(r, \phi, z)$ coordinates of the spot where the electron passed through the chamber. The ePCs have coarse time resolution $(\approx 30 \mathrm{~ns})$, but the two chambers together enable reconstruction of the electron's trajectory in three dimensions, which should point back to the stopping point of the parent

\footnotetext{
${ }^{2}$ As was noted in the previous section, if the TPC reaches a sufficiently high operating voltage it can also contribute information about decay electron trajectories. However, to date this has not been accomplished.
} 


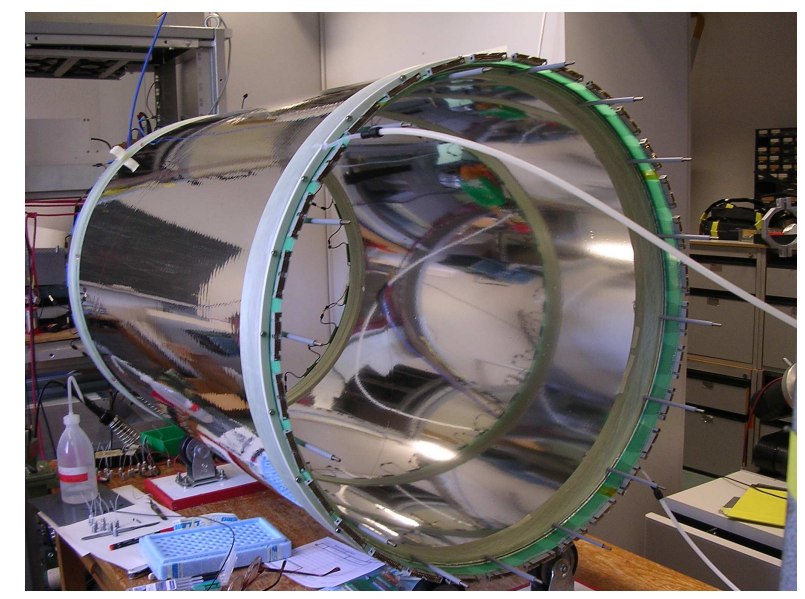

Figure 4.6: Photograph of the outer electron proportional chamber, ePC2.

muon. A full listing of the ePC1 and ePC2 technical specifications is given in appendix Table G.3.

The outermost electron detector is the electron scintillating counter (eSC), a hodoscope consisting of 16 layered scintillator pairs, or "gondolas" (Figure 4.7). Each scintillating layer is constructed from Bicron BC-404 plastic and measures $900 \times 150.5 \times 5 \mathrm{~mm}^{3}$; the $5 \mathrm{~mm}$ thickness ensures near $100 \%$ detection efficiency for decay electrons. Each layer is read out by two Photonis XP1911 photomultiplier tubes, one at each end; the double layering helps to suppress background. The eSC is designed to complement the capabilities of the ePCs: Whereas the ePCs provide good spatial but poor temporal resolution, the eSC does the opposite. Its angular segmentation and low $z$ resolution give the eSC poor overall spatial resolution, but it supplies fast timing information $(\approx 1.25 \mathrm{~ns}$ resolution $)$ for the "stop" signal of the muon lifetime measurement.

The electron detectors do not provide complete solid angle coverage. The fraction of solid angle covered by a cylinder of length $L$ and diameter $D$ is

$$
F_{\text {solid angle }}=\frac{L}{\sqrt{L^{2}+D^{2}}}
$$




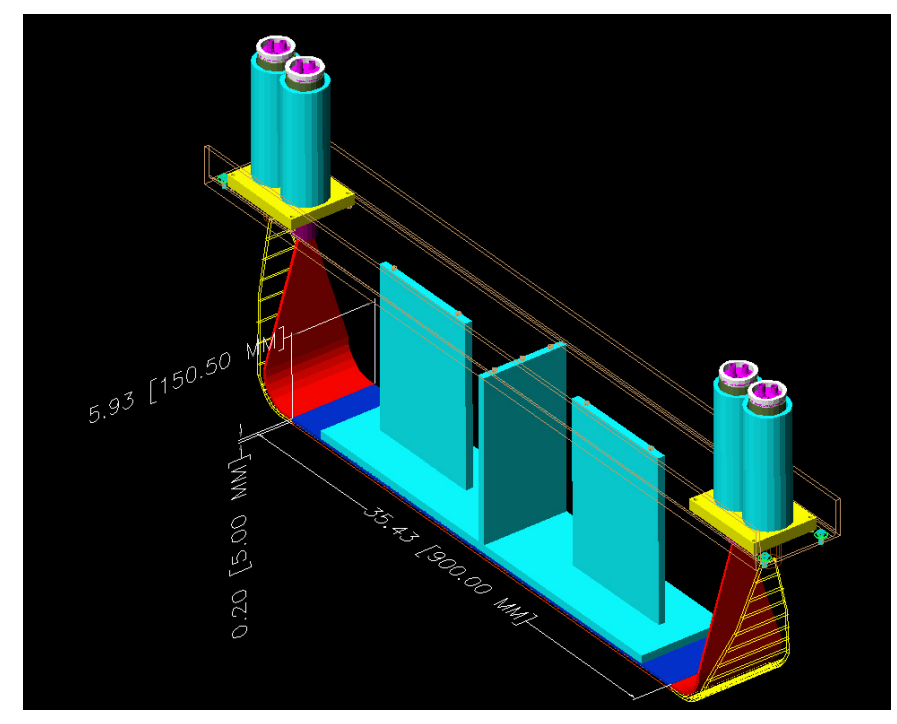

(a)

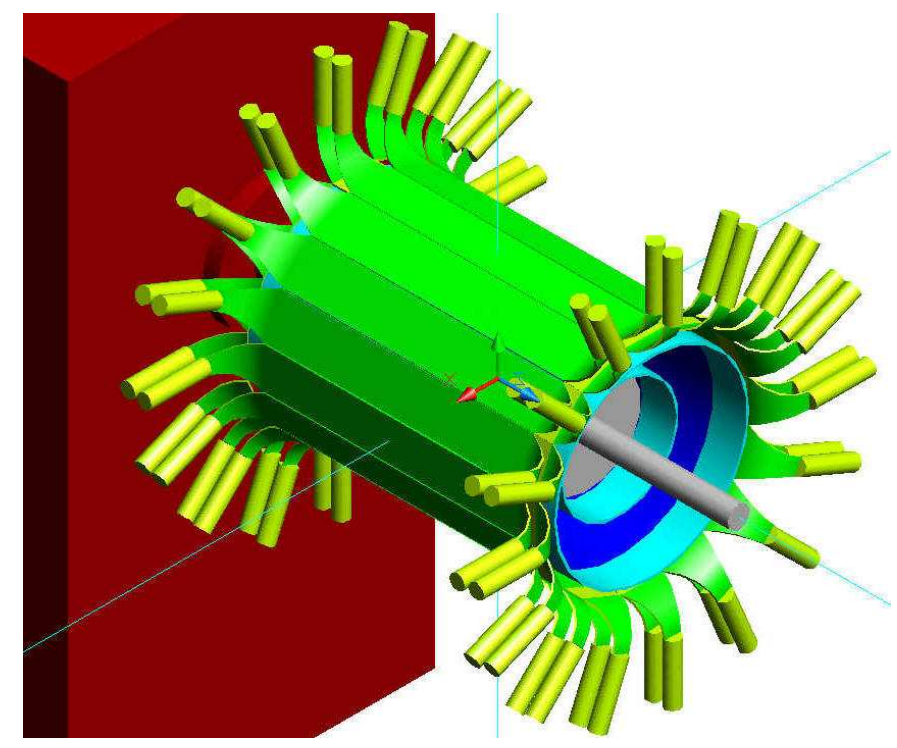

(b)

Figure 4.7: CAD drawings of (a) one of the sixteen eSC scintillator elements, or "gondolas," and (b) the assembled eSC. 
The nominal dimensions for ePC1, ePC2, and the eSC (see Appendices G.5 and G.6) yield coverage fractions of about $83 \%, 78 \%$, and $76 \%$, respectively. Although the eSC is the largest of the electron detectors, it is the one which limits the maximum solid angle coverage.

\subsection{Hydrogen gas system}

Prior to the 2003 experimental run, Dr. Claude Petitjean constructed a custom system for handling hydrogen gas [180]. In it, a Whatman Hydrogen Gas Generator produced protium gas from deuterium-depleted water via electrolysis, and the gas was stored in two hydride storage beds until needed. When the time came to record experimental data, we released the stored protium gas, passed it through a palladium filter to remove $Z>1$ impurities, and filled the gas into the pressure vessel to a pressure of 10 bar. Once the pressure vessel had been filled, it was sealed shut for subsequent data taking. The setup also contained an ultrahigh vacuum chamber and mass spectrometer for studying the composition of the protium gas in the pressure vessel.

The drawback of the 2003 setup was that the protium gas inside the pressure vessel did not remain clean. We performed two separate hydrogen gas fillings, and each time the TPC data revealed a steady, linear increase in the $Z>1$ impurity content of the hydrogen gas with time. This accumulation of impurities - hypothesized to arise from outgassing from materials inside the pressure vessel-poses serious systematics dangers to a precision $\mu^{-}$lifetime measurement.

To fix this problem, our colleagues at Gatchina developed the Circulating Hydrogen Ultrahigh Purification System (CHUPS) [181]. The purpose of CHUPS is to continuously recirculate and clean the hydrogen gas in the pressure vessel, removing $Z>1$ impurities as they appear. CHUPS was first integrated in the hydrogen system prior to the 
2004 experimental run. Dr. Petitjean's existing gas handling system continued to be used for the preparation of protium gas and the initial filling of the pressure vessel, but CHUPS was used to clean the gas during data taking.

\subsection{Data acquisition (DAQ) system and electronics}

MuCap utilizes a variety of different electronics modules to digitize and store its detector data:

- All TPC information is processed by PSI-designed, VME64-format TDC400 modules that were custom built for the MuCap experiment. The TDC400s record timestamped hit patterns from the TPC wires (48 bits for data, 16 bits for time) at the three digitized signal thresholds (EL, EH, EVH), in $200 \mathrm{~ns}$ intervals (i.e. at a $5 \mathrm{MHz}$ clock rate).

- In 2004, the central 16-wire TPC sector (i.e. anodes 33-48) was also instrumented with Struck DL401 flash ADC (FADC) VME modules with DL403 controllers. The FADCs provide analog information on the TPC anode signals, albeit at a limited data rate compared to the TDC400s, since the FADCs were only read out on rare occasions when the impurity capture trigger logic fired. Our intention was to use the analog data to assist in recognizing capture signatures from different impurity elements.

- The ePC signals are processed by custom-built multihit TDC modules which we refer to as "compressors." The compressors, or COMP modules, are part of the COMET system [182] designed by UC-Louvain to efficiently record the large volume of information generated by the ePCs. The compressors have a time resolution of $30 \mathrm{~ns}$.

- Signals in the remaining detectors - $\mu \mathrm{SC}, \mu \mathrm{SCA}, \mu \mathrm{PC} 1$, and $\mathrm{eSC}$ - are sent through 
LeCroy 4413 discriminators, and the discriminated hits are recorded primarily by CAEN V767 multihit TDC modules [183]. Separate CAEN modules are used for the muon and electron detectors to prevent cross talk. Although the CAENs have a fine time resolution of $1.25 \mathrm{~ns}$ when operated at $25 \mathrm{MHz}$, experience has shown that they suffer from a number of shortcomings. For instance, the CAEN modules' time resolution is effected by an internal 32-bin interpolator which subdivides the 40-nsperiod external clock signal, but the interpolator's binning is consistently nonuniform. The CAENs have also been known to occasionally "lose" hits from their memory registers when subjected to high data rates. To avoid losing information we record redundant copies of all critical signals.

- Copies of the eSC signals are also fanned out to LRS ADCs for diagnostic purposes, and to the compressors for redundancy.

- The status of the experimental setup is continuously monitored by a wide assortment of sensors and computers. Most of the slow control information, such as the detector voltages and temperatures, is written to tape alongside the detector data.

All of the electronics modules are housed in VME or CAMAC crates, which are stacked in racks. There are usually six or seven racks in total, some of which sit in the experimental area next to the detectors, while others are located in the nearby counting "barrack" where shift personnel monitor the experiment. All of the electronics modules' clock signals are derived from a common external source - a $100 \mathrm{MHz}$ signal (accurate to $10^{-8}$ ) generated by an Agilent E4400B ESG-A Series frequency synthesizer with the 1E5 High-Stability Time-Base Option [184] - to ensure that they operate synchronously.

Since March 2003, MuCap has used the Maximum Integration Data Acquisition System (MIDAS) [185] for its data acquisition (DAQ) needs. MIDAS is a combined DAQ 
and data analysis software framework that is well suited for medium-sized nuclear physics experiments, and it is the standard system used at PSI and TRIUMF.

The MuCap MIDAS-based DAQ operates in a minimum-bias mode, recording detector information during blocks of livetime. For each block, VMIC VMIVME-7740 single-board CPUs collect detector data from the electronics modules in their respective crates and send the data over a Gigabit Ethernet network to the primary DAQ computer, which in 2004 was a 64-bit system from Southwest Technologies with 1 GB of RAM and two AMD Opteron Processor 242 chips operating at $1.6 \mathrm{GHz}$. The DAQ computer assembles the data (a process referred to as "event-building") and analyzes a small fraction of it online so that shift operators can monitor detector performance and data quality in real time. The DAQ block cycle is coordinated by an LB500 CPLD (Complex Programmable Logic Device) module built by the PSI electronics group. The incoming data is losslessly compressed in real time using a Huffman coding algorithm $[186,187]$. Once the size of the accumulated data reaches a preset limit of 1.6 GB - usually after several thousand block cycles - the DAQ computer writes the data to a file on disk. Aside from the compression, the data is deliberately recorded raw and unprocessed so that subsequent offline analysis is not constrained by any preprocessing decisions. 


\section{Chapter 5}

\section{4 experimental run (Run8)}

The data presented in this dissertation was collected during October-November 2004 at PSI, a period designated within the MuCap Collaboration as "Run8" [188]. This chapter describes the experimental conditions of that run.

\subsection{History}

The MuCap experiment is conducted inside the PSI Experimental Hall, a cavernous building which houses a proton cyclotron and several secondary downstream beamlines. Like many of the facility's other users, the MuCap group does not have a permanent, dedicated experimental area. Instead we receive an annual allocation of beamtime - usually several weeks sometime between April and December - and the experimental setup must be assembled for the assigned run period and dismantled afterwards.

Consequently, MuCap has for several years followed an annual cycle of incremental improvement. During the spring and summer months, collaborators work to develop and test new components and software at their home institutions. Prior to the start of the assigned beamtime period, a large contingent converges at PSI, the new components are 
integrated into the experiment, and the setup undergoes testing.

In 2004 several critical improvements were made to the experimental setup:

- Refinements to the muon entrance detector assembly (Figure 4.2) and the installation of a new beryllium pressure vessel window produced two significant effects: (1) the muon detection efficiency improved, and (2) the muon stopping efficiency within the TPC target volume doubled from $30 \%$ to $60 \%$, primarily because of reductions in the scattering of beam muons.

- Construction and instrumentation of the outer electron wire chamber, ePC2, the last remaining major detector component, was completed. When combined with the existing inner electron wire chamber, ePC1, ePC2 enables full, three-dimensional decay electron tracking.

- The PNPI (Gatchina) group built the Circulating Hydrogen Ultrahigh Purification System (CHUPS) to continuously clean high- $Z$ impurities from the hydrogen gas target.

- New electronics and an improved data acquisition system increased the experiment's reliability, data throughput, and livetime. The new PSI-designed LB500 CPLD modules were an especially important addition, as they replaced the antiquated and undependable NIM logic that had previously coordinated DAQ operation.

For the first time since its inception in 1996, MuCap was ready to record high statistics in clean hydrogen with a complete detector. The goal of Run8 was to acquire enough data to make a useful measurement of $g_{P}$. 


\subsection{Beamline description}

There are several research facilities in the world which provide muon beams suitable for stopping in targets. These sites include PSI (Switzerland), RIKEN/RAL (England), KEK (Japan), TRIUMF (Canada), and JINR (Russia); other muon facilities at CERN (France), LANL (USA), and BNL (USA) are either not designed for stopping in targets or no longer have muon physics programs. Among the active beamlines, PSI is the most attractive because it offers the highest luminosity DC beam (by a factor of nearly twenty) at the low momenta $(30-35 \mathrm{MeV} / c)$ necessary for stopping muons in 10 bar hydrogen gas. Some facilities offer pulsed muon beams with higher instantaneous intensities, but the PSI beam is the only one that affords the opportunity to steadily accumulate high statistics.

The PSI ring cyclotron generates a $1.8 \mathrm{~mA}, 590 \mathrm{MeV}$ proton beam which is directed onto a 40-mm-thick spinning graphite target. The resulting collisions produce a shower of pions, muons, and electrons, and the downstream beamlines can be tuned to select specific types of particles from this secondary stream.

For several years MuCap utilized the $\mu \mathrm{E} 4$ beamline in the Experimental Hall (Figure 5.1). However, $\mu \mathrm{E} 4$ was closed in early 2004 for a major multiyear upgrade, so MuCap relocated to the $\pi \mathrm{E} 3$ beamline, which we had evaluated in 2003 and found suitable for our needs [189]. The only serious drawback of the $\pi \mathrm{E} 3$ area was the limited amount of space at the end of its beamline.

The $\pi$ E3 beam can be tuned to select either $\mu^{+}$or $\mu^{-}$particles within the momentum range 10-280 $\mathrm{MeV} / \mathrm{c}$; to stop muons in the center of our hydrogen target we generally operated in the range 32-34 MeV/c. Most of the concomitant, unwanted electrons in the beam were removed by a vertical separator and two pairs of beam slits. The $\mu^{+}$beam had 


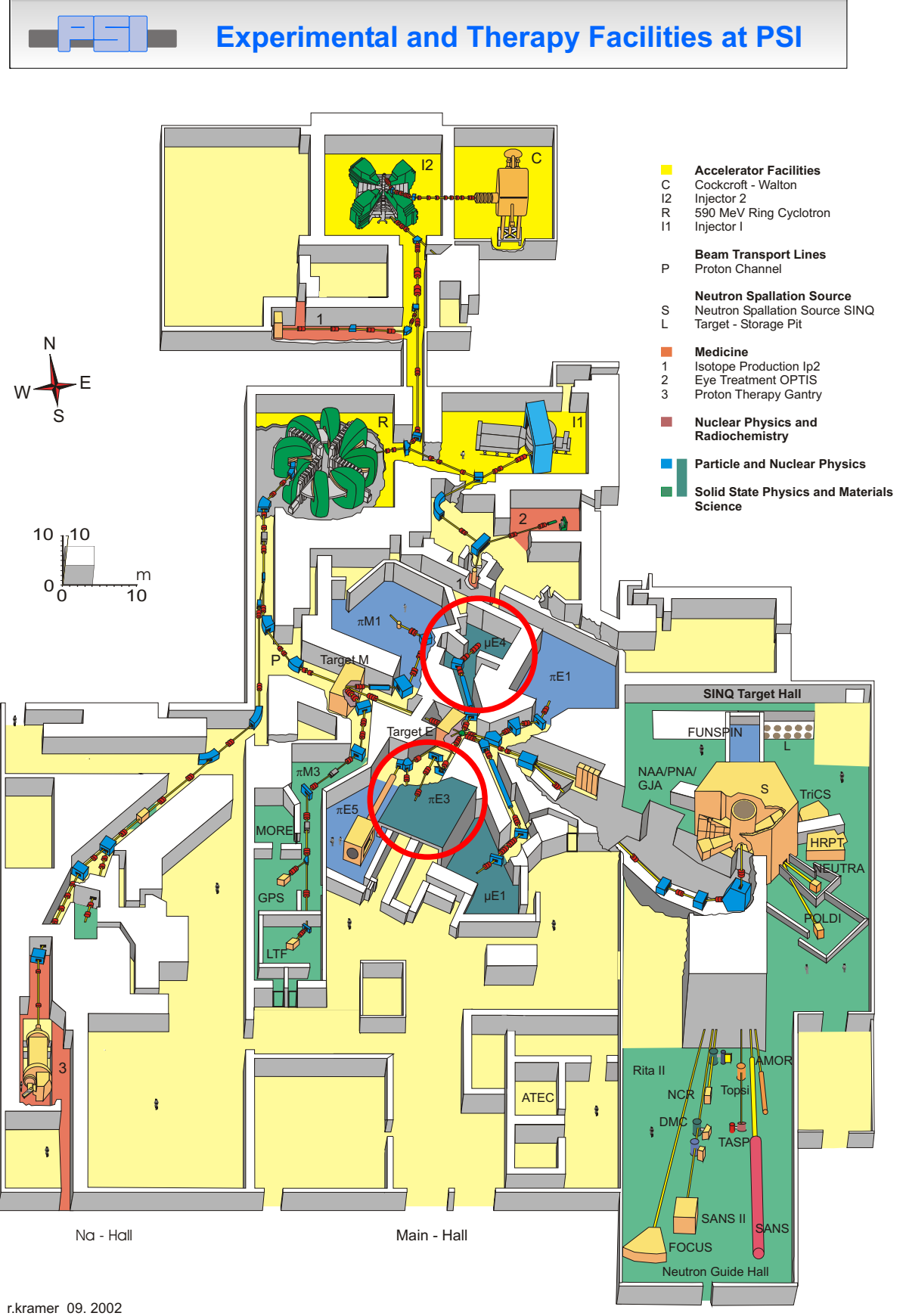

Figure 5.1: Floor plan of the PSI Experimental Hall. The cyclotron is the green segmented device in the northwest corner of the main building; the $\mu \mathrm{E} 4$ and $\pi \mathrm{E} 3$ beamline areas used by $\mathrm{MuCap}$ are circled in red. 
a net polarization which pointed downstream, while the $\mu^{-}$beam had a net polarization which pointed upstream, opposite the momentum vector. The $\pi \mathrm{E} 3 \mathrm{DC}$ beamline can provide a maximum $\mu^{+}$rate of $50 \mathrm{MHz}$ and a maximum $\mu^{-}$rate of $150 \mathrm{kHz}$, but we typically selected muon beam rates in the vicinity of $21 \mathrm{kHz}$. This particular choice was driven by the need to avoid muon-pileup-related distortions in the lifetime spectra that incorporate TPC information (see Section 6.5.14). To ensure the unique association of a muon entrance time with its TPC track, muon arrivals must be separated in time ("pileup protected") by an interval wider than the maximum TPC drift time. The choice of pileup protection time interval, $\pm T_{P P}$, determines the optimal beam rate. Consider a muon beam of rate $R_{b}$, where the muon arrival times follow a Poisson distribution. In this case the normalized distribution of waiting times between stochastic muon arrivals is

$$
W(t)=R_{b} e^{-R_{b} t}
$$

and the corresponding fraction of acceptable muon events, pileup-protected in time from $-T_{P P}$ to $+T_{P P}$, is

$$
F_{\text {accepted }}=\left(\int_{T_{P P}}^{\infty} W(t) d t\right)^{2}=e^{-2 R_{b} T_{P P}} .
$$

The rate at which pileup-protected events are accumulated is then

$$
R_{P P}=R_{b} \cdot F_{\text {accepted }}=R_{b} e^{-2 R_{b} T_{P P}}
$$

Setting the first derivative of $R_{P P}$ to zero, we find that the accumulation of pileup-protected events is maximized for a beam rate

$$
\left.R_{b}\right|_{\text {optimal }}=\frac{1}{2 T_{P P}} .
$$

For $T_{P P}$ equal to the TPC drift interval of $23.5 \mu \mathrm{s}$, the optimal muon beam rate is approximately $21 \mathrm{kHz}$. 


\subsection{Run chronology}

In September 2004 the MuCap detectors were moved into the $\pi \mathrm{E} 3$ area for the first time (Figure 5.2). The detectors were surveyed, precisely aligned, and then enclosed within a plastic tent (see Figures 5.3 and 5.4) to help provide the stable climate control essential for reliable operation of the ePC2 high-voltage cards. The pressure vessel and electron detector assemblies, shown in Figures 5.5 and 5.6, were mounted on separate platforms with customized rails so that they could be rolled apart for servicing. The completely assembled MuCap detector is shown in Figure 5.7.

After the detectors were in place, we began tuning the muon beam, producing protium gas for the target, and adjusting the electronics and data acquisition setup. On October 1, 2004, the pressure vessel was filled with deuterium-depleted hydrogen gas through a "Mr. Hydrogen" palladium filter to remove any high- $Z$ impurities, and production datataking commenced. One week later, CHUPS was connected to the TPC volume and began cleaning impurities from the hydrogen gas. Aside from some $\mu^{+}$measurement periods and brief CHUPS diagnostic tests, $\mu^{-}$data was recorded in this configuration until November 21 , 2004.

Near the end of the assigned beamtime, we performed several calibration measurements. We added small (10-120 ppm), known amounts of different impurities - such as nitrogen, oxygen, deuterium, and water - to the hydrogen gas, in order to investigate the effects of each. These calibration runs will be discussed in greater detail in Chapter 6; their information plays a crucial role in enabling us to perform corrections for the systematic effects of certain impurities. 


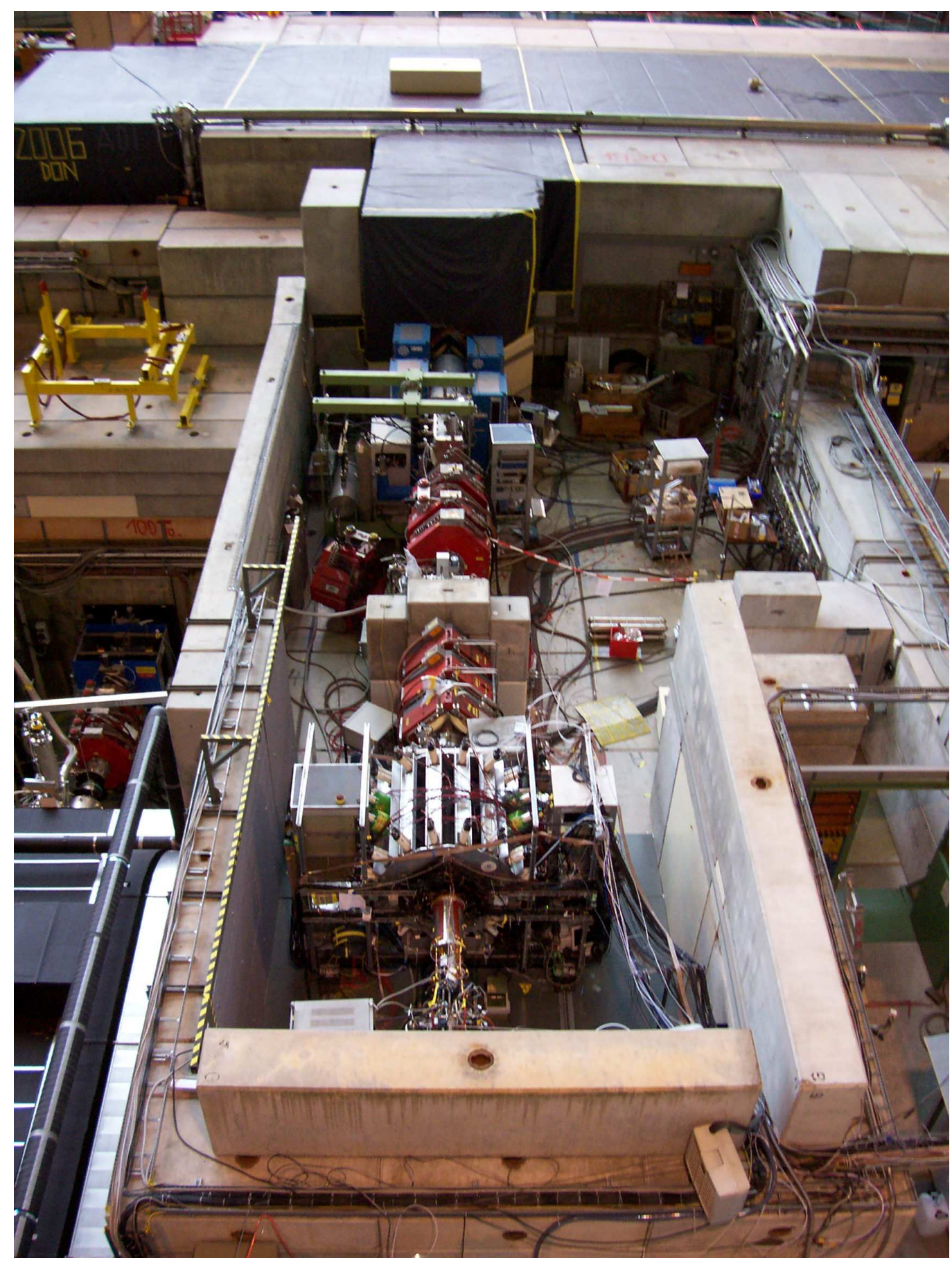

Figure 5.2: Overhead view of the $\pi \mathrm{E} 3$ area, with the MuCap detectors installed at the end of the beamline. This picture was actually taken in June 2006, but the experimental layout is essentially the same as in 2004. (Photograph by Peter Winter.) 

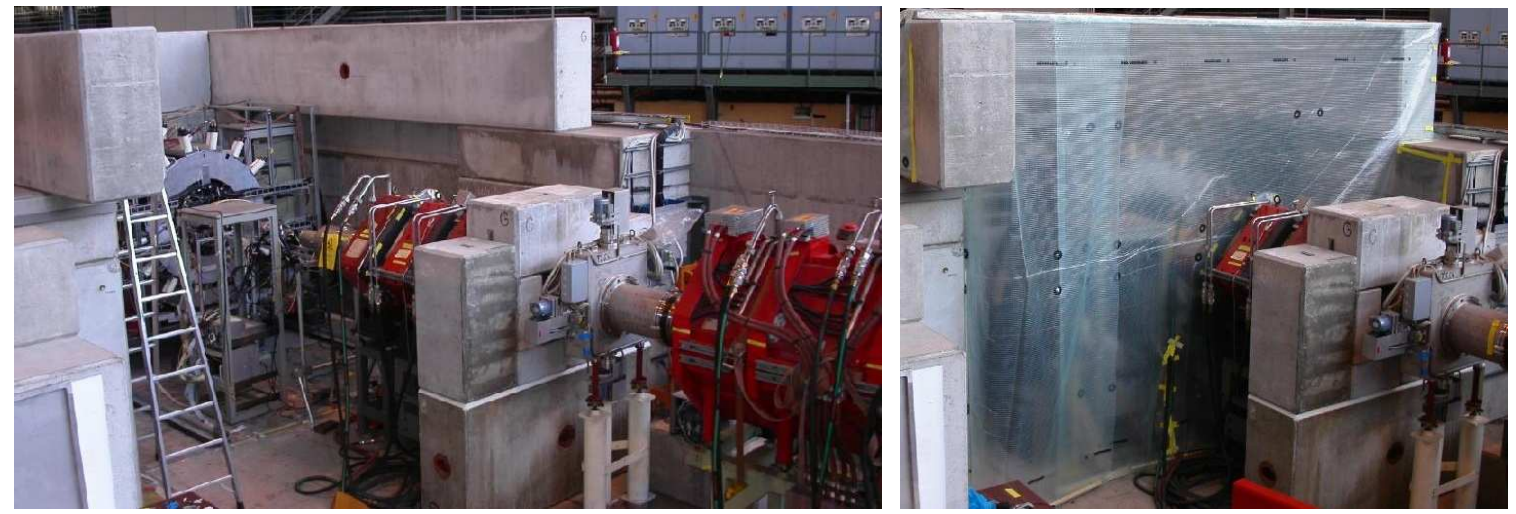

Figure 5.3: Photograph of the $\pi \mathrm{E} 3$ area, taken during the early stages of the 2004 MuCap move-in. The electron detector assembly is visible at the end of the beamline, on the left side of the picture.

Figure 5.4: Photograph of the $\pi \mathrm{E} 3$ area, taken after the $2004 \mathrm{MuCap}$ installation was complete. The entire experimental area has been covered with a plastic tent to facilitate climate control of the apparatus.

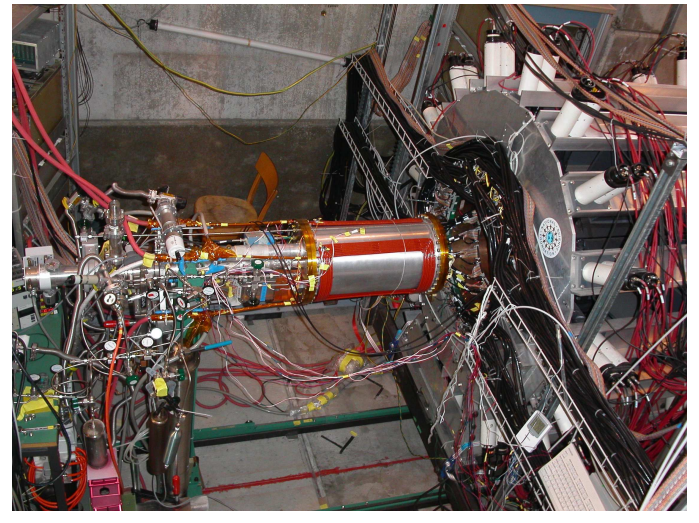

Figure 5.5: Overhead view of the pressure vessel assembly, rolled back from the electron detectors.

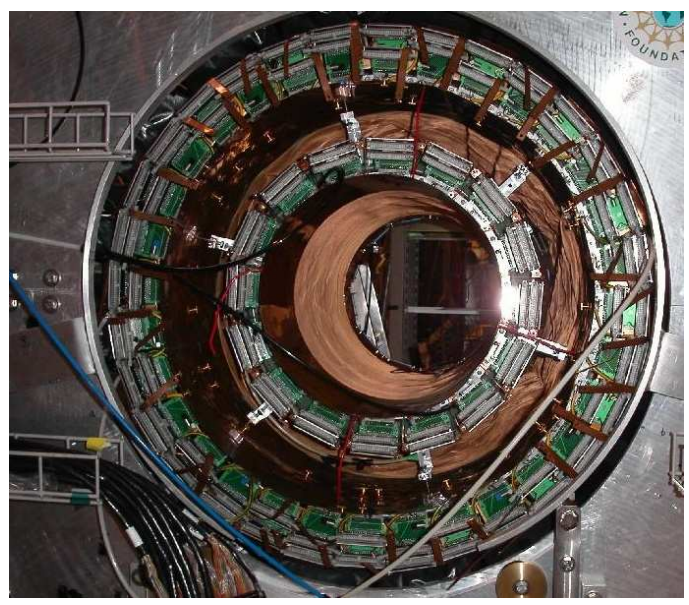

Figure 5.6: Close-up view of the anode instrumentation at the ends of the nested ePC chambers, inside the eSC support structure. 


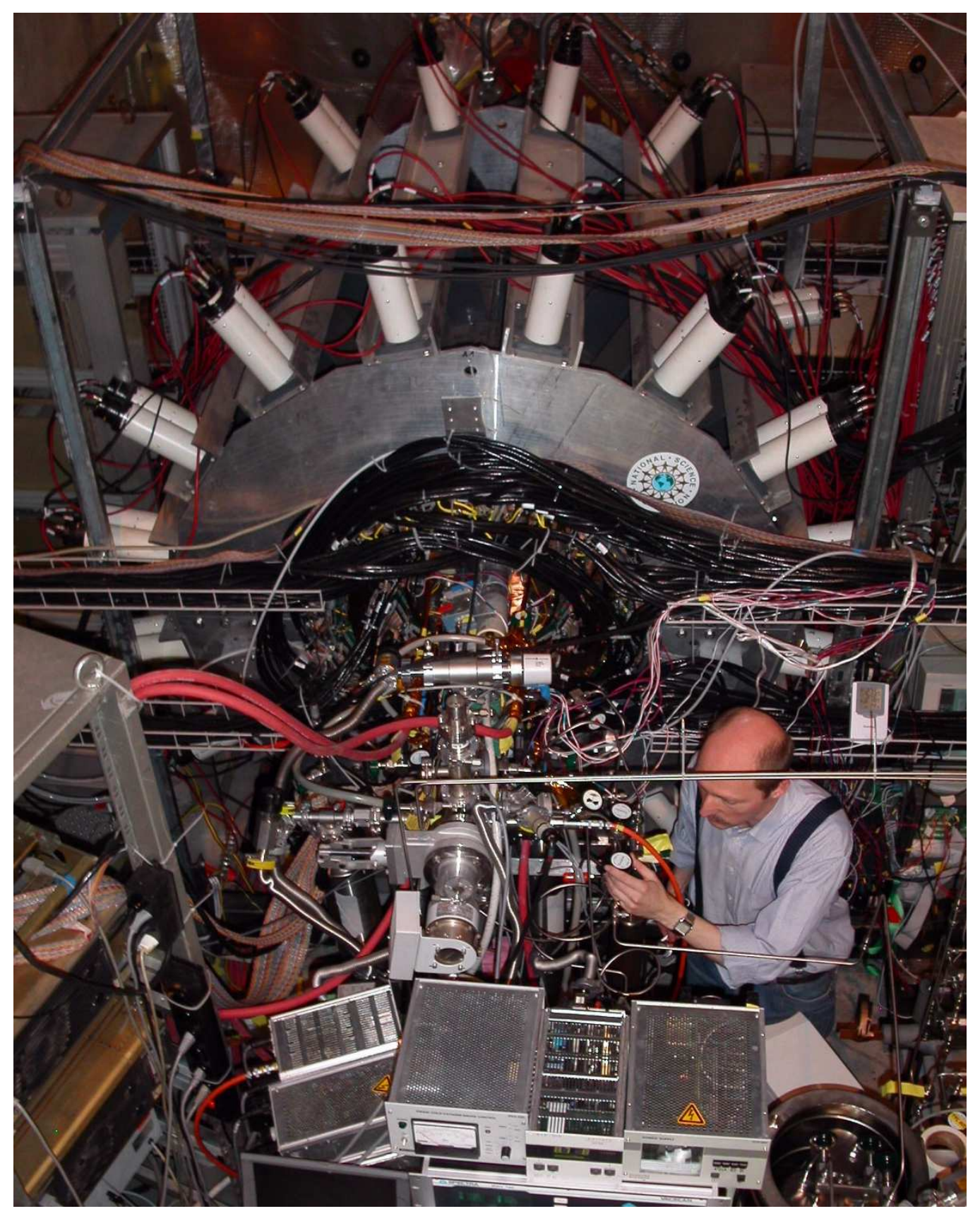

Figure 5.7: Photograph of the 2004 experimental setup in the $\pi \mathrm{E} 3$ area at PSI. The view is from downstream, looking towards the beamline (not visible). The apparatus being inspected in the foreground is part of the gas and pressure vessel system. The pressure vessel itself is hidden inside the electron detectors, whose supporting superstructure can be seen in the background, studded with the white photomultiplier tubes of the eSC. 


\subsection{Apparatus performance}

In general, the MuCap detectors, CHUPS, and the data acquisition system operated stably and reliably for the duration of the 2004 run. However, it is worth discussing the performance of the different subsystems in some detail.

\subsubsection{Muon detectors}

As previously mentioned in Section 4.1, the 2004 MuCap experimental configuration differed in some aspects from the original design plans. Several changes were made to the muon detectors to reduce scattering and range straggling along the muon beam path and thereby improve the muon stopping efficiency in the TPC. First, shortly before the run began, the PNPI group constructed a new $\mu \mathrm{PC} 1$ detector with thinner window materials and 25-micron, aluminized Mylar cathode foils in place of the wires that had previously been used. Second, $\mu \mathrm{PC} 2$ was removed from the entrance detector assembly because the advantages it offered as a redundant detector were outweighed by the additional muon scattering it produced. Third, a 0.5-mm-thick Beryllium pressure vessel window replaced the previous $100-\mu \mathrm{m}$-thick Havar window. These reductions in the material budget along the muon beam path more than doubled the fraction of muons that stopped in the TPC: the muon stopping fraction increased from $\approx 30 \%$ in 2003 to $\approx 62 \%$ in 2004 , doubling the good decay event rate.

Midway through the 2004 run, the 500- $\mu$ m-thick $\mu$ SC scintillator was replaced with a 250- $\mu$ m-thick scintillator wrapped in thin aluminized Mylar, to reduce scattering and increase the fraction of muons stopping in the TPC. In retrospect we should have kept the original $500 \mu \mathrm{m}$ scintillator, since it was better aligned and produced a larger signal. The replacement $250 \mu \mathrm{m}$ scintillator gave poorer muon/electron separation, was less well aligned, and provided only a marginal improvement in the in the stopping muon fraction. 
The TPC functioned well in the 2004 run as a stopping muon detector, but it continued to suffer from a chronic high-voltage problem. The TPC has never reached its design maximum of $6.5 \mathrm{kV}$ in hydrogen because hotspots, sparks, and voltage trips are common at settings above $5 \mathrm{kV}$. Most of the 2004 data was acquired with the TPC operating at $5 \mathrm{kV}$, and the maximum stable voltage deteriorated slightly over the course of the run from $5 \mathrm{kV}$ to $4.8 \mathrm{kV}$. A detailed chronology of TPC training and performance in 2003-2004 is available in reference [188]. Because of its lowered operating voltage in 2004, the TPC was unable to detect electron tracks, which require $6.5 \mathrm{kV}$, or "Alvarez muons" from $p d \mu$-fusion events (see Section 4.1), which require $5.4 \mathrm{kV}$. The latter capability would have been especially useful, since detecting Alvarez muons provides another method for determining the level of deuterium contamination in the protium gas.

The source of the TPC defects remains unclear. A variety of mechanisms have been

proposed - dust accumulation on the wires is favored - but the lack of worldwide experience with TPC operation in hydrogen gas has hindered efforts to identify and eliminate the problem.

Finally, it is worth noting that the $\mu^{+} \mathrm{SR}$ magnet remained energized for the duration of Run8, for the sake of maintaining stable running conditions during both $\mu^{-}$and $\mu^{+}$data taking.

\subsubsection{Electron detectors}

The ePC1 and eSC detectors were commissioned and thoroughly tested during the 2003 engineering run, so they were prepared for routine operation in 2004 . The readiness of the new ePC2 detector was more problematic: although ePC2 had been constructed and conditioned in late 2003, we were not able to devote resources to complete its preparation until spring of 2004. As such, we had only a few months to get the chamber instrumented 
and tested before the scheduled start of the run in September 2004.

Numerous problems plagued the ePC2 commissioning. Bad circuit board prints on the readout electronics led to imperfect connections, and special frame-mounted shunts had to be installed to circumvent the faulty circuitry. The ePC2 high-voltage cards were extremely sensitive to humidity, which led to excessive dark current and sparking at the nominal operating voltage. The cards were sprayed with a urethane conformal coating to protect against moisture, but that failed to eliminate the problem. We found that the best way to ensure stable ePC2 operation was to climate control its environment. Therefore, the entire MuCap apparatus and $\pi \mathrm{E} 3$ area were enclosed within a plastic tent (Figure 5.4) whose interior climate was maintained at a stable temperature and low humidity. In spite of its tight commissioning schedule and many obstacles, ePC2 was ready in time for the start of the 2004 run.

Once installed, the electron detectors operated without incident during the 2004 run and exhibited good efficiencies: the ePC1 three-plane efficiency was $\approx 92 \%$, and the ePC2 efficiency was $\approx 89 \%$. In addition to the climate control measures that were adopted, the insertion of thin bronze grounding meshes on the interior of each ePC cylinder also helped to reduce noise and pickup (see Figure 5.6).

The two electron proportional chambers were a tremendous improvement in the MuCap experimental capability, because ePC1 and ePC2 together provide invaluable electron tracking information. Ultimately, the $\mathrm{ePC}$ information is used to identify decay electron candidates, which contribute to the lifetime histograms of good, fiducial muon stops in the TPC. However, during the 2004 run, the ePC tracking information also enabled us to ascertain where many of the non-fiducial muons were stopping. By plotting the $z$ position of the points where ePC tracks passed closest to the experiment's $z$ axis, we were able to establish that a significant fraction $(\approx \% 25)$ of the muons that passed through the entrance 
detectors were stopping in the infrastructural materials inside the pressure vessel.

\subsubsection{DAQ and Electronics}

The muon and electron detector data were deliberately routed to different CAEN TDC modules, in order to minimize the potential for cross talk between the muon and electron measurements. Three CAENs were employed during Run8: two of them (\#0 and \#2) were dedicated to muon data, while the remaining module (\#1) was dedicated to electron data, although some ancillary muon-related signals were in fact routed into CAEN 1; see Appendix G.1.

The MuCap MIDAS-based DAQ system allowed us to continuously monitor data quality and detector performance during the 2004 run. The DAQ MIDAS status page displayed information about the flow of incoming data as it was collected from the different electronics crates, consolidated, and written to disk. The MIDAS Analyzer continually processed small samples of the incoming data stream (roughly $15 \%$ of the total), and posted the cumulative results from each data file in real time to a display monitored by shift personnel. This online display contained five pages: one for accumulated counts in all detector channels, one for the muon detectors, and one each for ePC1, ePC2, and the eSC. Sample screen captures of the muon detector and ePC1 pages are shown in Figures 5.8 and 5.9. The online display histograms were reset at the start of each new 1.6 GB MIDAS data file, which usually took 15 minutes to fill.

Several slow control displays monitored the status of various experimental subsystems such as the beam magnets, the wire chamber gas flow, the ePC and TPC high voltage history, and the temperature of various detectors. The slow control data was also written to disk in each runfile, alongside the detector data.

The addition of ePC2 nearly doubled the data volume. For a typical $21 \mathrm{kHz}$ beam 


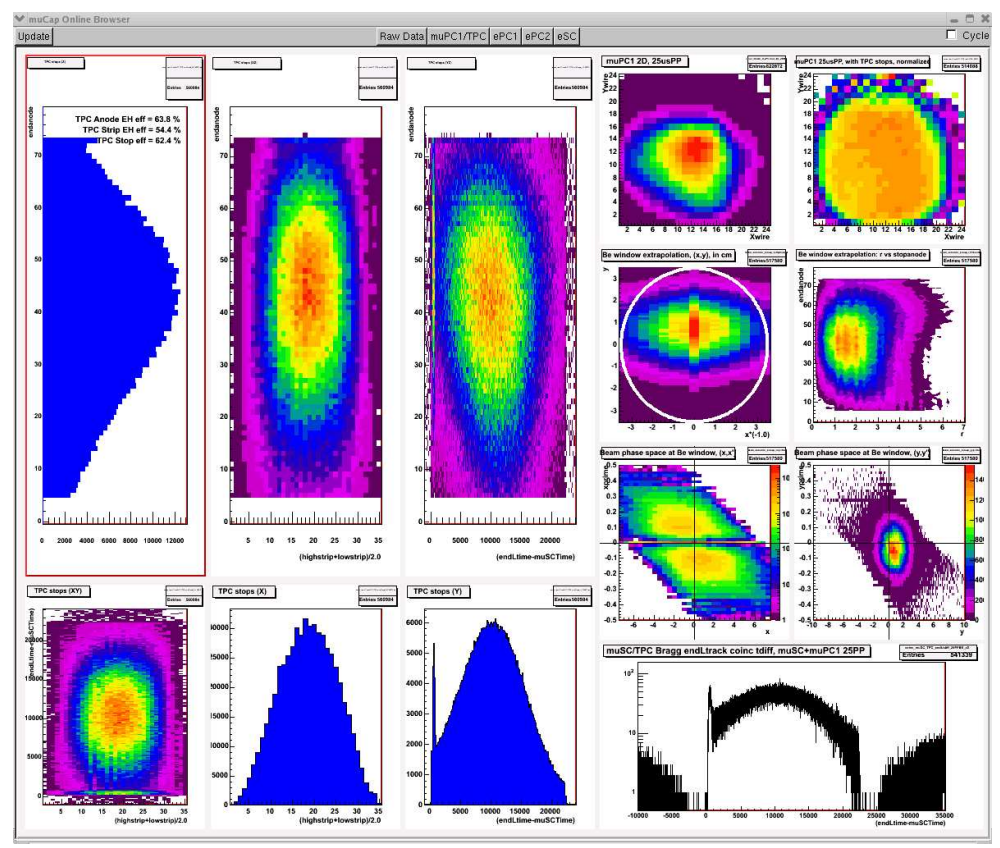

Figure 5.8: Screen capture of the 2004 online display page for the muon detectors. It shows the muPC1 hit profile and efficiency, the muon stopping distribution in the TPC from different perspectives, and the muon pileup-protected TPC drift distribution.

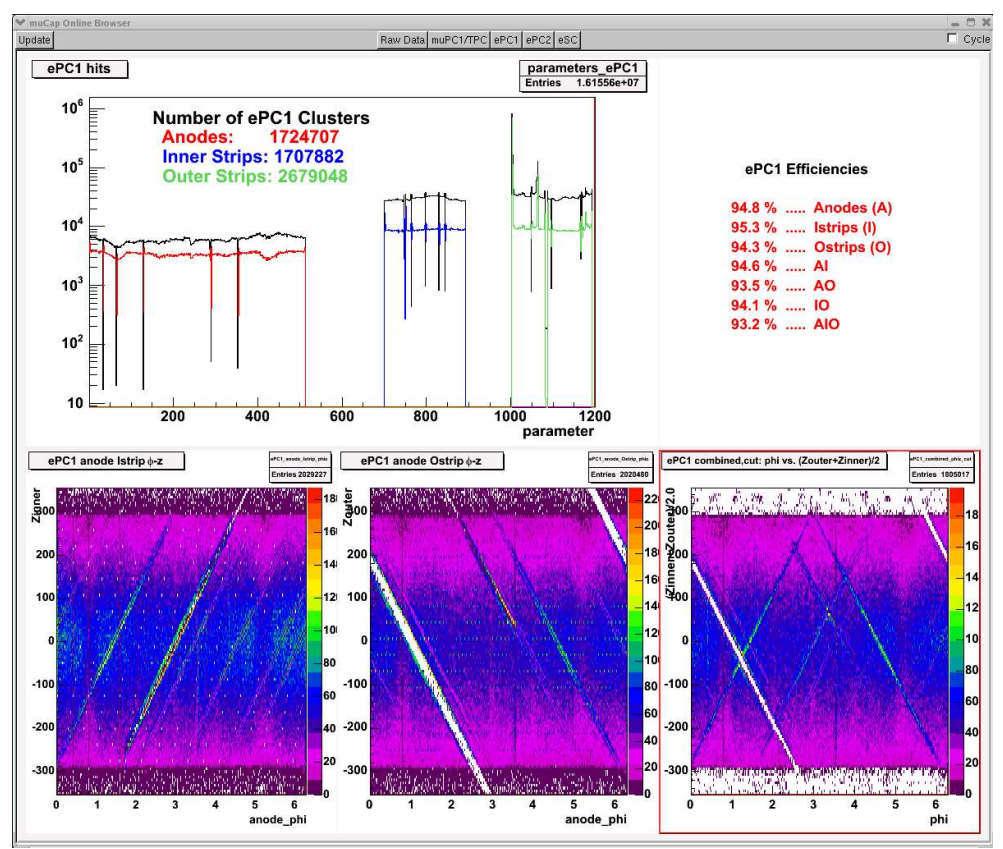

Figure 5.9: Screen capture of the online display page for ePC1. It shows the number of raw and clustered counts in each channel, as well as the efficiencies and $(\phi, z)$ data plots for different combinations of coincidences in the three ePC1 planes. 


\begin{tabular}{lc}
\hline \hline Source of Event Loss & Retention Fraction $\left(F_{i}\right)$ \\
\hline DAQ livetime & $91 \%$ \\
$\mu \mathrm{SC}$ & $\sim 100 \%$ \\
$\mu \mathrm{SCA}$ & $99.8 \%$ \\
$\mu \mathrm{PC} 1$ & $94.4 \%$ \\
$\mathrm{TPC}$ & $62 \%$ \\
Pileup Protection $(\mathrm{PP})$ & $e^{-2 R_{b} T_{P P} \sim 35 \%}$ \\
$\mathrm{ePC} 1$ & $92 \%$ \\
$\mathrm{ePC} 2$ & $89 \%$ \\
$\mathrm{eSC}$ & $\sim 100 \%$ \\
eDet solid angle coverage & $76 \%$ \\
\hline Total $\left(\prod F_{i}\right)$ & $11.6 \%$ \\
\hline \hline
\end{tabular}

Table 5.1: Breakdown of the acceptance fractions which contribute to the effective accumulation rate of good muon decay events. For the pileup protection estimate I have used a beam rate $R_{b}=21 \mathrm{kHz}$ and pileup protection interval $T_{P P}=25 \mu \mathrm{s}$.

the total incoming raw data rate from the 2004 detectors was close to $5 \mathrm{MB} / \mathrm{s}$. The raw data was losslessly compressed using a Huffman coding algorithm [186,187], which reduced the data size by a factor between 2.2-2.5. Copies of the compressed data were then written to two separate locations: the PSI archive (via ethernet) and to HP Ultrium LTO-2 tapes in an Overland Storage NEO 2000 tape library inside the electronics barrack. Over the course of the run we recorded roughly six terabytes of data in total, which filled $\sim 30$ LTO-2 tapes of 200GB native capacity each. After the run the LTO-2 tapes were shipped to UIUC, and their contents were copied to a local computing archive for storage and later analysis.

The DAQ livetime during normal data-taking was roughly $91 \%$; the primary limitation was in the readout of ePC data from the compressor modules. By multiplying the DAQ livetime fraction with the individual detector efficiencies and pileup constraints, we can estimate the probability that any given muon arrival will contribute to final lifetime statistics (Table 5.1). For a conservative muon pileup protection window $T_{P P}=25 \mu$ s and typical beam rate $R_{b}=21 \mathrm{kHz}$, the number of good, pileup-protected muon decay events 
recorded per day was

$$
\begin{aligned}
\left.R\right|_{\text {statistics }} & =R_{b} \cdot \prod_{i=1}^{n} F_{i} \\
& =(21 \mathrm{kHz}) \cdot(0.116)\left[\frac{86400 \mathrm{~s}}{\text { day }}\right] \\
& \approx 2 \times 10^{8} \frac{\text { muons }}{\text { day }} .
\end{aligned}
$$

In reality, the effective event rate was reduced by the testing overhead and delays which attend nearly any experiment. There were unavoidable pauses from scheduled beam-off days; when the beam was on, there were occasional lulls in the intensity, as is common in normal cyclotron operation. A few days were spent on diagnostic tests and equipment troubleshooting, and there were some DAQ crashes that prompted nearly daylong recovery periods, although nothing crippling. The biggest disruptions in data-taking came from a sequence of events outside of our control: At 18:39 on Saturday, October 23, 2004, a transformer on the PSI power lines failed, cutting off all power to the Experimental Hall. Power was restored a few hours later when electricity was routed through a backup transformer, only to suddenly shut off again at 10:39 on October 25 when the second transformer abruptly failed from overload. These two sudden and extended power outages created some equipment problems - primarily for CHUPS — but fortunately no lasting damage was done. The most serious consequence was in lost time, for it took several days to restore MuCap to its pre-outage working order.

We ultimately recorded $\approx 2 \times 10^{9}$ good $\mu^{-}$events and $\approx 2 \times 10^{8}$ good $\mu^{+}$events over a 3.5 -week period. Figure 5.10 shows the chronological accumulation of statistics during the 2004 production run; the solid blue line is the most significant, as it provides an estimate of the number of fully reconstructed, pileup-protected $\mu^{-}$decay events in clean hydrogen recorded over time. 


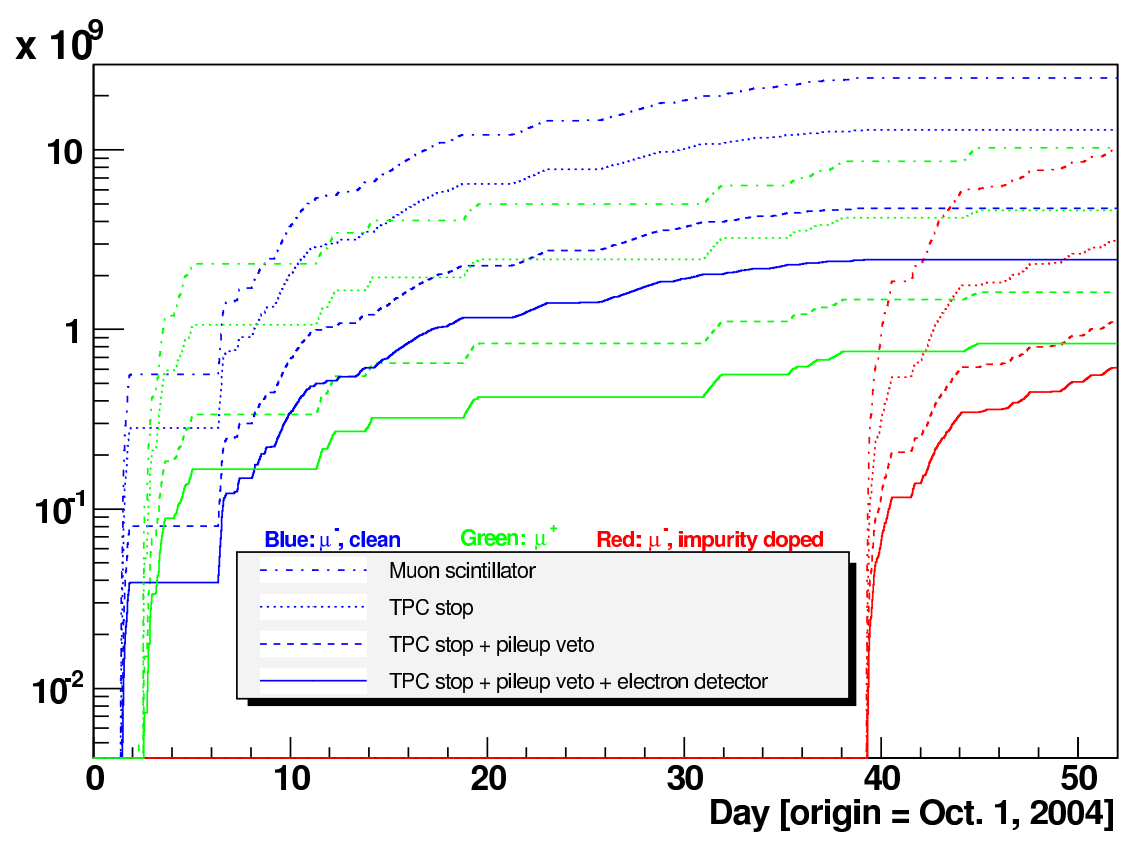

Figure 5.10: Statistics accumulation vs. time during the 2004 run.

\subsection{Impurities}

\subsection{1 $\quad Z>1$ elements and CHUPS}

Prior to the run, the TPC system was heated to $115^{\circ} \mathrm{C}$ under vacuum for several weeks to remove impurities. When we filled the pressure vessel with protium gas just prior to the start of data taking, we passed the gas through a "Mr. Hydrogen" palladium filter to remove $Z>1$ impurities. However, the palladium filter can only provide a one-time cleaning upon the initial filling. We therefore relied upon CHUPS for continued $Z>1$ impurity cleaning during subsequent operation.

The CHUPS system was developed in 2004 by the PNPI (Gatchina) group, and installed shortly before Run8. CHUPS is designed to continuously circulate and remove $Z>1$ impurities from the hydrogen gas, thereby maintaining an ultra-pure target during 
data taking [181]. The need for a gas cleaning system became apparent during the 2003 engineering run, when it was discovered that the concentration of $Z>1$ impurities in the hydrogen gas within the sealed pressure vessel steadily increased over time, presumably due to outgassing from the vessel walls.

CHUPS uses an adsorption cryopump system to circulate the hydrogen gas through a sequence of cryogenically cooled Zeolite filters, at a rate between 1-5 L/min, while maintaining a steady 10 bar pressure in the TPC. During Run8, a computer controlled the gas compression, purification, and return stages, but periodic liquid nitrogen refillings by the shift crew were necessary. The original CHUPS design (Figure 5.11) was modified over the course of Run8 to incorporate additional safety features, some of which proved extremely useful during two abrupt PSI power outages in late October 2004. The CHUPS installation in the $\pi \mathrm{E} 3$ area in 2004 is shown in Figure 5.12.

The MuCap design goals require that the usual vacuum contaminants - carbon, nitrogen, oxygen, and water-be reduced below $0.05 \mathrm{ppm}$, and CHUPS accomplished that task. CHUPS produced an exponential attenuation in the $Z>1$ impurity concentration immediately after being turned on, and ultimately achieved an equilibrium concentration (by number) of $c_{Z}<5 \times 10^{-8}$, as monitored by direct TPC detection of recoil nuclei from muon capture by impurities. This represented an order-of-magnitude improvement over the impurity levels observed in previous years.

\subsubsection{Deuterium}

The hydrogen gas used in Run7 (fall 2003) and Run8 (fall 2004) was produced from a single 5 liter supply of deuterium-depleted water, purchased in August 2002 from Ontario Power Generation, Inc., Canada. We used $2 \mathrm{~L}$ of the water for Run7, and an additional $1 \mathrm{~L}$ for Run8. The water was converted to hydrogen gas using a model 75-32 Whatman 


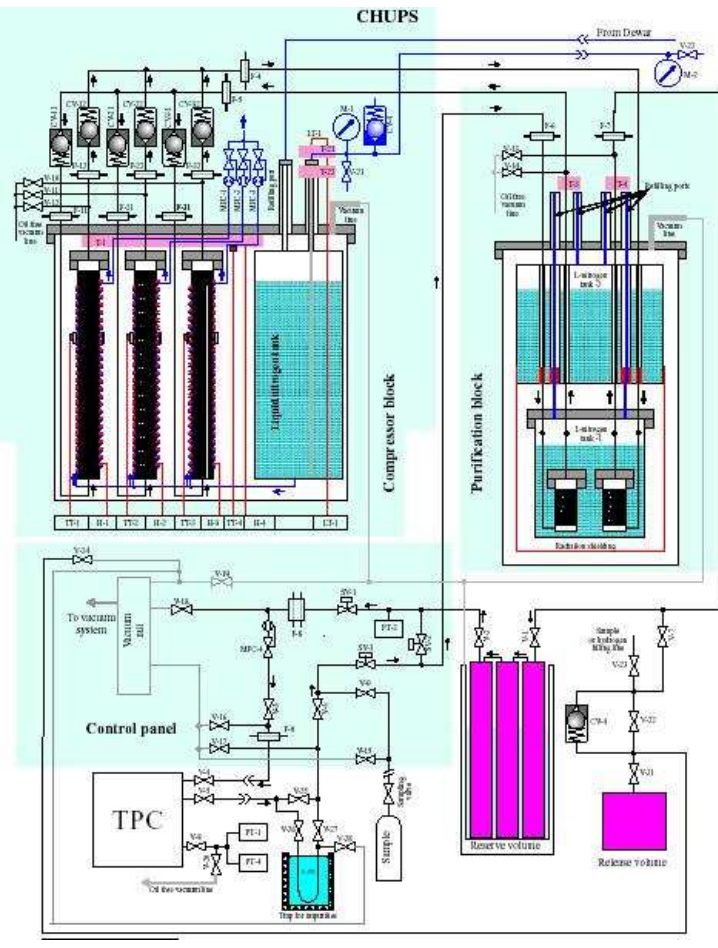

Figure 5.11: CHUPS schematic diagram, including the compressors, purifiers, release volumes, and valves.

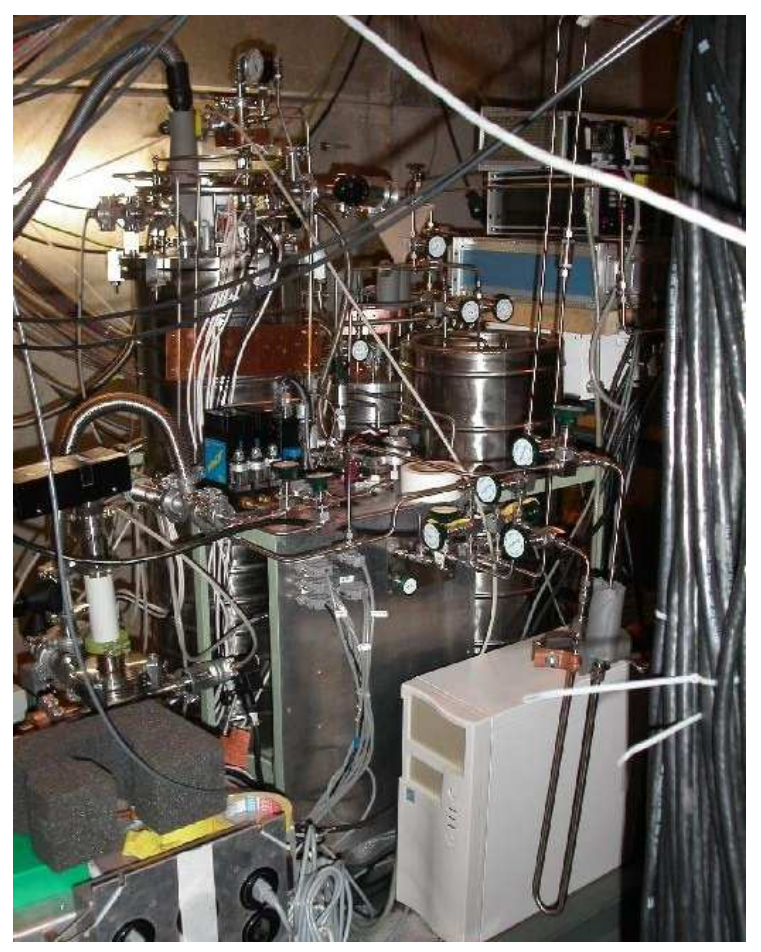

Figure 5.12: Photograph of the CHUPS purification and recirculation system in 2004 .

Hydrogen Gas Generator (provided courtesy of TRIUMF), which employs electrolysis in connection with a palladium electrode to remove $Z>1$ impurities. The resulting hydrogen gas was stored in pressure bottles (up to $10 \mathrm{~L}$ at 50 bar) and hydride beds until it was time to fill the gas into the experimental apparatus. In $2004, \approx 1500$ STP liters of protium were produced in total, just sufficient to load the TPC (400 L) and the CHUPS circulation system $(800 \mathrm{~L})$, and to establish a reserve for replacing gas that was sampled during the run.

It should be emphasized that there was no system in place during Run8 for removing deuterium from the hydrogen gas, so we had to rely on the low deuterium concentration of the initial filling. As will be discussed in Section 6.6.4, we believe that the deuterium concentration of the clean filling was roughly $1.5 \mathrm{ppm}$, based upon the results of both mass 
spectrometry measurements and data analysis. This residual deuterium level, though small, will necessitate a correction.

\subsection{Summary}

In 2004 MuCap recorded its first useful physics data, with a complete detector operating under sustained clean gas conditions. Approximately $2 \times 10^{9} \operatorname{good} \mu^{-}$decay events were collected, almost $25 \%$ of the MuCap design goal of $10^{10}$ events. The 2004 statistics are at least twice that of any previous muon capture experiment in hydrogen, and they were recorded under better experimental conditions. The MuCap apparatus enables a more direct interpretation of the results, and offers the capability of controlling systematic effects. 


\section{Chapter 6}

\section{Data analysis}

The bulk of the analysis of the 2004 data was performed in parallel and semiindependently by two graduate students: myself and Steven Clayton of the University of Illinois at Urbana-Champaign (UIUC). This chapter describes features common to the two analysis efforts - namely, precautions against bias, a shared software framework, and some shared code - and then delves into the details of my own analysis. In the final section I obtain several (blinded) values for the $\mu^{-}$disappearance rate in hydrogen.

\subsection{Infrastructure}

\subsubsection{Blinding procedures}

For most of the duration of the data analysis, we employed two blinding schemes to prevent psychological bias from affecting our results. The first was global in scope: The 2004 DAQ clock frequency was detuned within $1 \%$ from $100 \mathrm{MHz}$ (i.e. somewhere between 100 and 100.1 MHz) by PSI technician Dr. Malte Hildebrandt, and knowledge of the frequency setting was entrusted exclusively to Dr. Hildebrandt to Genna Petrov of PNPI. The true clock frequency was kept secret from all collaborators directly involved in the analysis, in 
order to prevent us from inadvertantly steering our results towards the expected answer. We were therefore forced to conduct the Run8 analysis in "pseudo time" where the DAQ clock frequency was assumed to be exactly $100 \mathrm{MHz}$. This allowed us to focus our efforts on consistency checks and lifetime studies, rather than on worrying about the palatability of the end results. The second blinding scheme was relative: Whenever Steven Clayton or I presented results at collaboration meetings or teleconferences, we each shifted our fitted rates by a secret offset of our own choosing, which served to prevent the two of us from artificially converging towards a common result. The relative blinding was lifted in May 2006, and the global clock blinding was lifted a few months later, in October 2006. The consequences of the global unblinding will be described in Section 7.1.

\subsubsection{Computing facilities}

For the most part, the Berkeley and Illinois analysis efforts used different computing facilities to process the 2004 data. On the Berkeley side, large-scale production passes were carried out on computers at PSI and managed remotely from California. The reasons for this seemingly inconvenient arrangement were twofold: (1) it enabled quick access to the Run8 data stored on the PSI archive, and (2) we could utilize PSI's Linux-based Merlin computer farm of 64 parallel AMD AthlonMP 1600+ processors [190], our usage of which was restricted only by the presence of other users. In light of these circumstances, we at Berkeley did not deem it worth the effort and expense to move tens of terabytes of Run8 data elsewhere and establish a new computing setup. We made ample use of the Merlin computing cluster, although in summer 2006 we began to transfer processing responsibilities to the three idle MuCap DAQ PCs at PSI, two of which had powerful dual AMD Opteron 270 CPUs that produced results faster than the Merlin cluster. Regardless

of exactly how the processing at PSI was accomplished, it was coordinated in real time by 
a MySQL database on an on-site MuCap DAQ PC, which maintained information about the status of processed jobs. The comparatively undemanding task of fitting the lifetime histograms produced by the large-scale data analyses was performed in Berkeley on PCs.

In contrast to Berkeley, Illinois has primarily used their own local computing resources for large-scale analysis processing. The UIUC analysis software was initially installed on a 20-CPU Linux farm at the UIUC Nuclear Physics Lab (NPL), but portions were later moved to the nearby National Center for Supercomputing Applications (NCSA). Illinois also has their own local copies of the 2004 data, obtained from LTOII tapes that were shipped from PSI to UIUC after the run, and whose contents were copied to the NCSA mass storage system (MSS).

\subsubsection{Software}

The MuCap DAQ, Monte Carlo, and analysis software is archived in a master CVS [191] repository named "[mucap]" [192] which is hosted on a Berkeley Weak Interactions Group computer [193]. The analysis-specific software is stored in the [mucap] subdirectory $\mathrm{mu} /$, which itself has subdirectories that contain the Berkeley and Illinois analysis code.

The Berkeley and Illinois analyses both utilize the MIDAS Analyzer framework [194], which is a natural and convenient choice since the MuCap DAQ is MIDAS-based (see Section 4.4). In order to explain how the MIDAS Analyzer works, it is necessary to first describe the structure of our raw data: Each of the 2004 runfiles is divided into blocks

of data collected during periods of DAQ livetime, and each block is subdivided into banks named according to the electronics module that received the data (TDC400s, CAEN V767s, COMET compressors, etc.). The MIDAS Analyzer uses a so-called "multistage" approach to process the data blocks: In each successive stage a software module processes one or more 
of the block's data banks, usually performing some specific task. The results of the module's operations are packaged into one or more new banks, which are passed downstream alongside existing banks. The initial analysis stages typically process and prepare the raw data, while later stages perform more complex coincidence operations and fill histograms.

One advantage of the multistage approach is that it allows for standard collaboration preprocessing stages, followed by customized individual studies. MuCap has adopted this strategy and integrated the Berkeley and Illinois analyses into a common Analyzer setup. Both analyses use the same set of modules to process the raw data and the $\mu \mathrm{SC}$ detector data, but subsequent processing is the province of the individual analysis efforts namely, the MuCap Berkeley Analysis Software (MBAS) [195] and the MuCap Illinois Analysis Software (MIAS) [196].

The MBAS modules are written in either $\mathrm{C}++$ or the Muon Query Language (MQL) [197], the latter of which is a high-level language created by Dr. Frederick Gray to compactly and efficiently form coincidences and create ROOT [198,199] "Ntuples" (i.e. data tables) and histograms from detector data. MQL also supports the m4 macro processor [200], which is useful for making MQL code even more compact. Some information about the experiment is available to the analysis modules through a MIDAS file known as the "online database," or ODB, which was also used in the management of the DAQ system during Run8.

It is worth mentioning that the data analysis software is supplemented by a variety of GEANT and custom-designed fast Monte Carlo programs, which are used to investigate various aspects of the MuCap experiment as needed. 


\subsubsection{Organization of data processing}

Analysis of raw Run8 data is a time-consuming process. For example, in MarchApril 2005 we used the Merlin cluster to perform a pass over the entire Run8 data set, and it took approximately six weeks to complete. We decided soon thereafter to subdivide the analysis operations into the following three stages to expedite future processing:

- Skimming: The raw data is "skimmed" with a minimal amount of processing to identify and save the sections of data which contain 25- $\mu$ s-pileup-protected muon arrival candidates. (For reasons to be explained in Sections 6.5.1 and 6.5.14, these are the only muons that are ultimately allowed to contribute to the lifetime histograms.) Data containing other exceptional events of interest - such as FADC triggers, or high multiplicities of hits in a detector ("sparks") — can also be saved in the skimming process. A full skimming pass over the Run8 data requires approximately two weeks.

- Ntuple production: We process the skimmed data files to produce tables (namely, ROOT "Ntuples") of muon arrivals and electron emissions, using a variety of detector treatments. The timescale for producing Ntuples from the Run8 data is approximately 1.5 weeks.

- Ntuple analysis: The muon and electron Ntuples are joined together to form lifetime histograms suitable for fitting. This is the most rapid of the processing stages, and only takes a few days to complete.

This subdivision of the analysis complicates the processing logistics and limits the amount of information available for study downstream, but it vastly improves the turnaround time for the most frequent steps - namely, detector processing (Ntuple production) and lifetime formation (Ntuple analysis) — and thus expedites analysis refinements. A list of the MBAS 
modules used for the skimming, Ntuple production, and Ntuple analysis stages can be found in Appendix G.7.

\subsection{Data preprocessing}

Before physics analysis of the detector information can proceed, the raw data must be processed and prepared into a more usable format. In particular, we must perform the low-level tasks of uncompression, quality checking, and time sorting of detector hits.

The first step is to uncompress the raw data by reversing the Huffman coding algorithm that was executed at the time the files were first written to disk (see Section 4.4). Next, we check for the appearance of duplicate data blocks. Normally each block within a file is unique and appears only once, but we occasionally encounter instances where a block is immediately repeated; for example, instead of the sequence $(A, B, C, \ldots)$, we observe $(A, B, B, C \ldots)$. The reason for this behavior remains unknown-a bug in MIDAS is suspected - but fortunately such duplication appears very rarely in the Run8 data, and when it does occur it is readily identified: we simply check each block's serial number, and if a block with the same number has already been processed, we discard the duplicate block.

We next process the data from the CAEN and COMP modules. Each datum from these modules corresponds to a single detector signal and carries a time stamp. We convert each datum into the form (parameter,time), where the detector parameter is determined from the one-to-one correspondence between the detector wires and the electronics channels (see appendix Table G.1), and where the time is a "global" time calculated relative the start of the block of DAQ live-time being processed. The complete list of detector hits during the live-time period is then sorted into a list by increasing time, and exported downstream in a bank named "HITS." 
Several diagnostic checks are performed in the course of processing and sorting the raw CAEN and COMP data. First, the CAEN data are inspected for error flags, which were generated either by the CAEN module itself or by the DAQ during data taking. The DAQintroduced error datums were implemented as a redundancy measure, because the CAENs themselves do not reliably produce the end-of-buffer (EOB) datums which report module errors. At the end of each block of live-time, the DAQ checked each CAEN module's TDC status for error codes, and inserted an imitation EOB if the module reported any internal errors. Error flags can appear for a variety of reasons, though most tend to be related to misbehavior in the TPC chips, such as unlocking of the interpolator's delay-locked loop. The second check on the CAEN data quality is to count the number of datums carrying a trailing edge flag. We recorded data in leading-edge-only mode, in which the CAEN TDCs assign each discriminated detector signal a single time corresponding to the leading edge of the input signal pulse. However, the CAENs occasionally produce datums containing a trailing edge flag, and sometimes the modules will spew trailing edge hits in large numbers, nearly all at once. Most of the time we simply ignore the appearance of occasional trailing edge hits, but if more than 100 trailing edges are encountered, the block is discarded.

Care is also required in calculating the times for the CAEN and COMP data, for several reasons: First, the raw data from both kinds of modules can exhibit backwardsgoing times. This is a rather common and natural feature of the CAEN, due to the manner in which data from its multiple data buffers are merged; in the COMP, this behavior is believed to indicate the loss of a single data word, which then throws off the synchronization. Consequently, no cuts are made on the CAEN data based upon backwards-going times (they are rather easily reordered), but if more than 1000 time-ordering errors are encountered in the COMP data, the block is discarded. A related issue that arises for the CAENs involves the role of their internal clock rollover. A backwards-going time in a CAEN can either 
indicate a harmless misordering of the data, or it can indicate a rollover of the CAEN's 20bit time counter. Although the high input data rates generally prevent this from becoming an issue, in order to eliminate any ambiguity in interpretation of rollovers and backwards data, and to ensure that no rollovers are missed, we fed a steady, relatively low-rate $2.5 \mathrm{kHz}$ (period $0.4 \mathrm{~ms}$ ) signal into each CAEN. Since the CAEN rollover period is $\approx 1.3 \mathrm{~ms}^{1}$, the $2.5 \mathrm{kHz}$ input signal guarantees that at least three hits will register within each rollover period. I should note that rollovers are not an issue for the COMPs, because their rollover period is nearly 2 seconds long (due to their coarser, $30 \mathrm{~ns}$ time resolution and 24-bit time counter), and the DAQ logic ended each block of live-time by $450 \mathrm{~ms}$ at the latest. The $2.5 \mathrm{kHz}$ CAEN signals - colloquially referred to as the "rollover signals" - are also used to check the synchronization of the three CAEN modules. If any discrepancies are observed in the rollover signal count for the three modules, the block is discarded. A more extensive examination of CAEN pathologies, and their potential effects on our lifetime measurement, is described in Section 6.5.10.

Many of the same considerations involved in the CAEN and COMP raw data processing also apply to the raw TDC400 data: if any time-ordering errors or DAQ-introduced error flags are encountered, then the data block is cut. (It should be noted that there are some lingering questions about whether the TDC400 error words were correctly formatted when the data was recorded during Run8; in any case, very few error such words are found.) The preparation of the TDC400 data is somewhat more elaborate than for the CAEN and COMP data: Instead of simply filling a table of (detector,time) entries, the TDC400 data is filled into a three-levels-deep, "stacked" array of TPC wire (anode and cathode) hits vs. time, where the three levels correspond to the three TPC thresholds. This structuring of

\footnotetext{
${ }^{1}$ The CAENs are driven by an external clock signal of frequency $25 \mathrm{MHz}$, and their internal 32-bit interpolator subdivides the $40 \mathrm{~ns}$ clock period into $1.25 \mathrm{~ns}$ bins. This means that rollovers occur at the rate $1 /\left(1.25 \mathrm{~ns} \times 2^{20}\right) \approx 763 \mathrm{~Hz}$, which corresponds to a period of $1.3 \mathrm{~ms}$.
} 
the data greatly facilitates the search for muon tracks.

Finally, two modules in the skimming stage cross-check the number of eSC hits recorded by the CAEN and COMP modules, and a block is discarded if there is an excessive number of mismatches.

\subsection{Muon detector analysis}

The function of the muon detectors is to measure the arrival time of beam muons and to identify those muons that stop in the hydrogen gas target. As described in Section 4.1, stochastically arriving beam muons pass through the detectors in the following sequence:

$$
\mu \rightarrow \mu \mathrm{SCA} \rightarrow \mu \mathrm{SC} \rightarrow \mu \mathrm{PC} 1 \rightarrow \mathrm{TPC}
$$

The $\mu \mathrm{SC}$ and TPC are the two essential muon detectors: the $\mu \mathrm{SC}$ provides the fast timing signal used in the lifetime histograms, and the TPC (in conjunction with the $\mu \mathrm{SC}$ ) measures the muon's trajectory in three dimensions, thereby enabling the identification of good muon stops. In the Run 8 analysis, the $\mu \mathrm{SCA}$ and $\mu \mathrm{PC} 1$ serve a practical, rather than a unique or critical, purpose: they assist the $\mu \mathrm{SC}$ by providing redundancy in detecting muon arrivals.

In this section I describe the manner in which I process the data from the individual muon detectors, and how the detector information is then joined together to identify good muon arrival candidates. Some of the more technical, software-related details of the data processing can be found in reference [201].

\subsection{1 $\mu \mathrm{SC}(\& \mu \mathrm{SCA})$}

When an arriving muon passes through the solid portion of the entrance scintillators, the resulting analog phototube signals are sent through a complex series of discrimina- 
tors and related electronics. A schematic diagram of the $\mu \mathrm{SC}$ signal processing setup which was implemented by Dr. Bernhard Lauss for Run8 can be found in appendix Figure G.1.

The $\mu \mathrm{SC}$ is a critical detector for our experiment, so we recorded several copies of its signal for redundancy. In the analysis we try to reconcile these parallel versions of the signal in a way that maximizes the pileup protection efficiency while protecting against spurious detector hits. Dr. Frederick Gray led the development of these somewhat complex algorithms for comparing $\mu \mathrm{SC}$ signals; it should be noted that the algorithms had to be modified slightly once data skimming was introduced.

First I will describe the procedures used to obtain the bank "MUSC" of good muon arrival times, which serve as the seed times (i.e. $t=0)$ for our lifetime measurement. There were two "direct" copies of the $\mu \mathrm{SC}$ signal: parameter 6001 (copy1) and parameter 6011 (copy2), which were sent to separate CAEN TDC modules in different electronics crates. These signals, along with the $\mu \mathrm{SCA}$ veto counter signal, are processed in the following sequence to obtain a table of muon arrival times:

1. Raw data selection of $\mu S C$ copy1 $\&$ copy2, $\mu S C A$ : Entries in the bank "HITS" with parameter numbers 6001 and 6011 are selected into $\mu \mathrm{SC}$ tables "copy1" and "copy2," respectively. A time offset of is added to the copy2 signal to align it with the copy 1 signal. Likewise, the $\mu \mathrm{SCA}$ signals labeled by parameter 6002 are selected into their own table and receive a timing offset to bring them into alignment with $\mu \mathrm{SC}$ copy 1 .

2. Afterpulse clustering of $\boldsymbol{\mu S C}$ copy $1 \&$ copy2, $\boldsymbol{\mu} \boldsymbol{S C A}$ : The $\mu \mathrm{SC}$ copy 1 and copy2 signals each have a inherent deadtime of rougly $29 \mathrm{~ns}$, so we impose an artificial deadtime (that is, a non-transitive, or non-updating, clustering) of exactly $29 \mathrm{~ns}$ on copy 1 and copy2 to eliminate the small amount of afterpulsing noise observed 


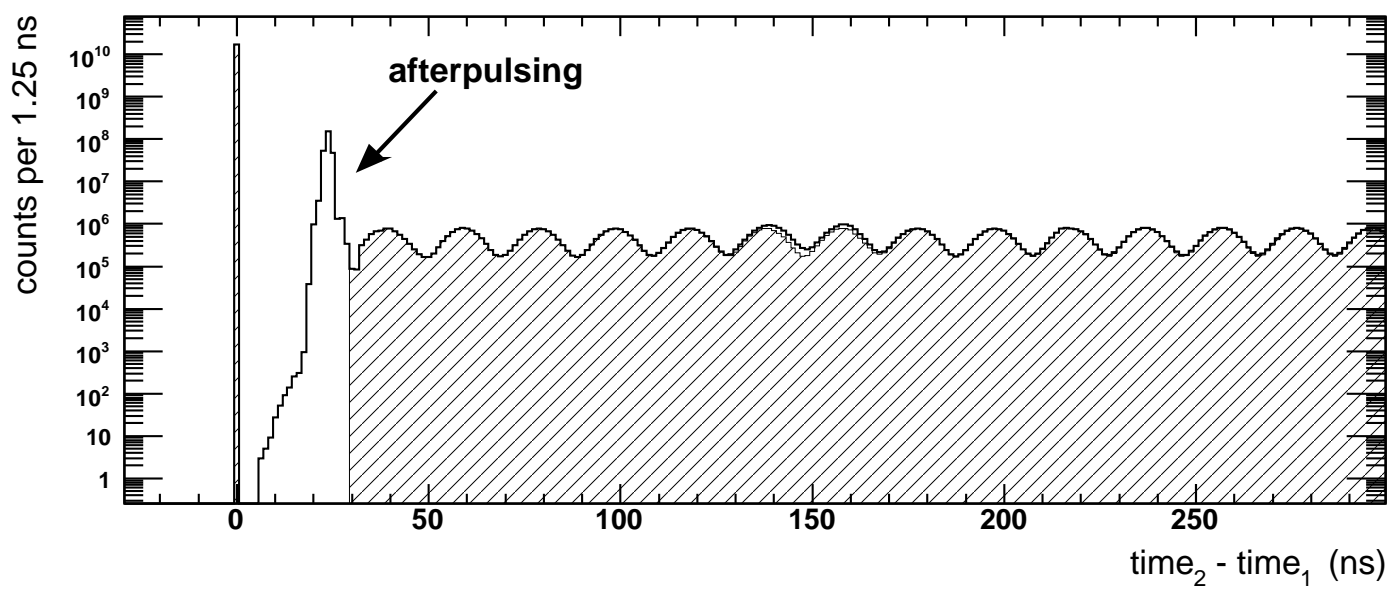

Figure 6.1: Autocorrelation plots of $\mu \mathrm{SC}$ copy1 signal times before (plain histogram) and after (hatched histogram) a $29 \mathrm{~ns}$ artificial deadtime is imposed in software to eliminate afterpulsing noise.

in their natural deadtime regions. The results are illustrated in Figure 6.1, where I have plotted the temporal autocorrelations of the copy1 signals before and after application of the $29 \mathrm{~ns}$ artificial deadtime. Autocorrelation histograms contain the time differences between all possible pairs of signals from a particular channel, and thus provide a useful tool for revealing hidden structure. A much longer artificial deadtime of $90 \mathrm{~ns}$ is applied to the $\mu \mathrm{SCA}$ veto counter's signal.

3. Formation of intersection of $\boldsymbol{\mu S C}$ copy1 $\&$ copy2: The two $\mu \mathrm{SC}$ copies are compared to one another, and only those copy1 hits which have a copy 2 partner within $\pm 7 \mathrm{~ns}$ (the half-width of their coincidence distribution) are accepted. If there are too many discrepancies between the two signals - namely, if there are more than three copy1 hits without a corresponding copy2 partner - then the block is flagged so that it can be cut later.

4. Imposition of $\boldsymbol{\mu S C A}$ veto: A veto using $\mu \mathrm{SCA}$ hits is applied to the copy1 hits which survived the copy1/copy2 intersection. 
5. Exported bank: The surviving table of copy1 hits is exported as the "MUSC" bank of good muon arrival times.

As Figure 6.1 reveals, there exists a pronounced periodicity in the $\mu \mathrm{SC}$ autocorrelation plots, a feature first observed in 2003 during Run7. At that time we suspected that the $\sim 50 \mathrm{MHz}$ modulations originated in the PSI cyclotron beam structure, but we could not rule out the possibility that the CAENs (or other electronics) were responsible, because the DAQ clock signal was derived from the cyclotron beam RF signal. To resolve the question, midway through Run8 we built a setup which directed a radioactive source onto the discarded $250 \mu \mathrm{m} \mu \mathrm{SC}$ scintillator paddle, and we fed the resulting stream of random hits into a CAEN module. If the random signal were to exhibit a $50 \mathrm{MHz}$ periodicity, we would know that the electronics were the culprit. Fortunately, as Figure 6.2 shows, the random source's autocorrelation plot is nicely flat - a feature confirmed by Fourier analysis - and we can confidently conclude that the PSI proton beam's RF structure is indeed responsible for the periodicity in the muon arrivals. In Section 7.3.2 I report the results of investigations into the potential systematic consequences of this phenomenon.

The second MIDAS bank produced in the $\mu \mathrm{SC}$ and $\mu \mathrm{SCA}$ analysis is "MUFP," which is intended for use in pileup protection (see sections 6.5.1 and 6.5.14 for discussions about the motivations for pileup protection and its efficacy). The MUFP bank is created in the following manner:

1. Raw processing of the routed $\mu S C$ signals: Entries are selected from the bank "HITS" which have parameter numbers between 6006 and 6009, corresponding to the $\mu \mathrm{SC}$ signals which were passed through a four-channel router. The router channels are assigned "port" numbers 0-3, and a unique time offset is added to each port to bring their signals into alignment. 


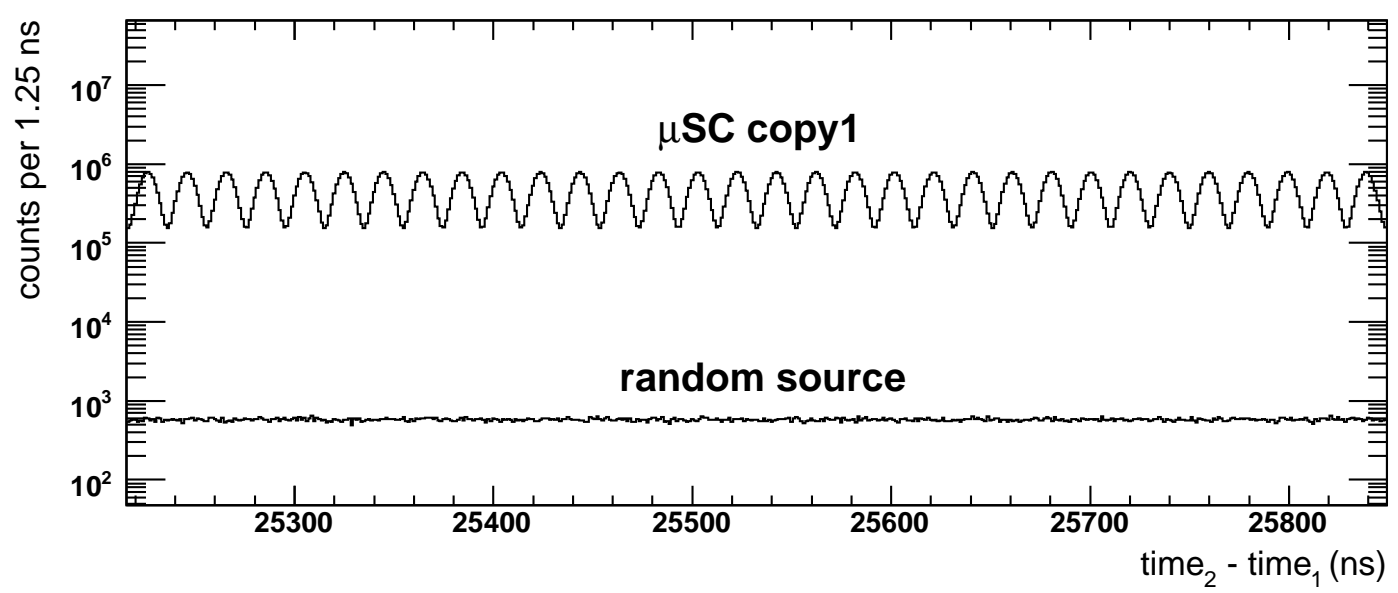

Figure 6.2: Temporal autocorrelation plots of the $\mu \mathrm{SC}$ copy 1 and random source signals. The absence of any discernible structure in the random source's signal reassuringly supports the hypothesis that the $\approx 50 \mathrm{MHz}$ oscillations in the $\mu \mathrm{SC}$ copy1 signal come from the PSI cyclotron's proton beam, not from our electronics.

2. Afterpulse clustering of the routed $\mu S C$ signals: An artificial deadtime of $29 \mathrm{~ns}$ is applied to each router port to remove any double-pulsing artifacts.

3. Merging of all $\boldsymbol{\mu} S \boldsymbol{C}$ and $\boldsymbol{\mu} \boldsymbol{S C A}$ signals: The artificial-deadtime-modified $\mu \mathrm{SC}$ copy1 and copy2 signals from the "MUSC" algorithm are merged with the routed $\mu \mathrm{SC}$ signals and the artificial-deadtime-modified $\mu \mathrm{SCA}$ signal into a single table. Any $\mu \mathrm{SC}$ copy1, copy2, routed, and $\mu \mathrm{SCA}$ hits that had previously been set aside by the skimmer are included in this union.

4. Clustering of proximate hits: The $\mu \mathrm{SC}$ signal copies may be slightly staggered in time due to cable lengths variations, etc. To prevent the signals from a single muon from cancelling each other out later on in the pileup protection operation, closelyspaced hits $( \pm 7 \mathrm{~ns})$ are clustered together. It is important to note that, although an artificial deadtime of 29 ns has been applied individually to each of the constituent $\mu \mathrm{SC}$ signals, the resulting union of signals has a much shorter effective deadtime of 


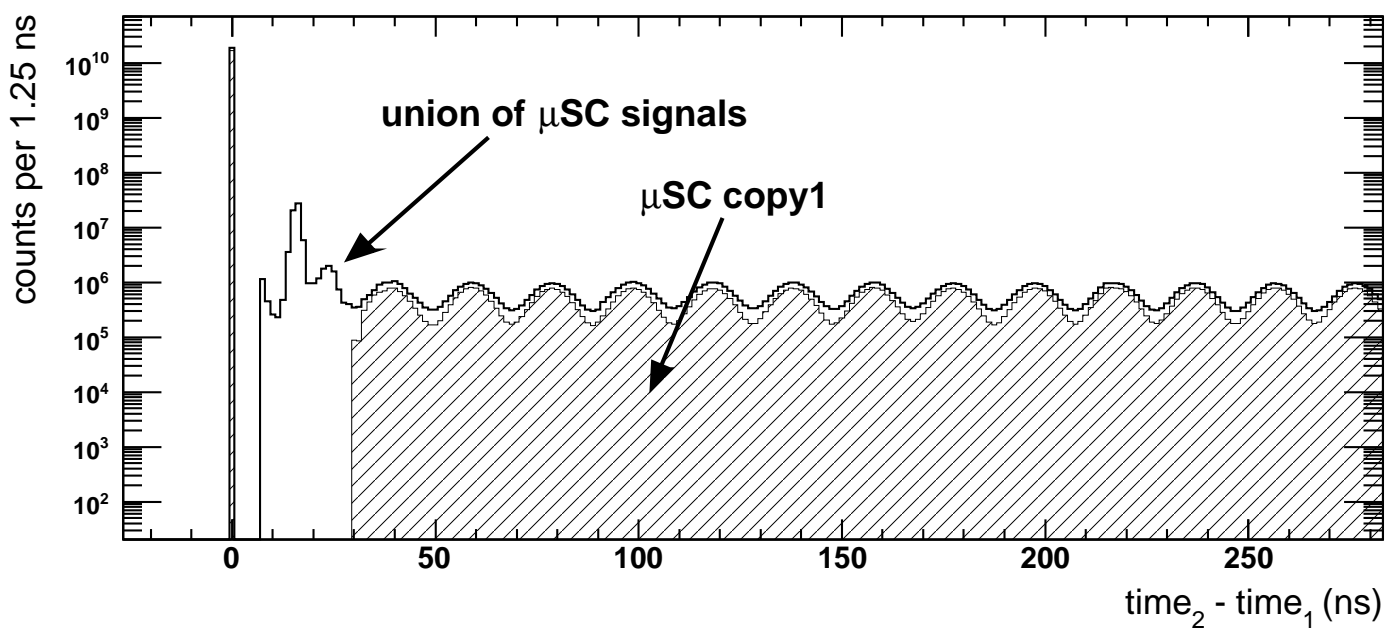

Figure 6.3: Temporal autocorrelation plots of the processed $\mu \mathrm{SC}$ copy 1 signal, and of the union of all processed $\mu \mathrm{SC}$ signals. The copy1 signal has a deadtime of $29 \mathrm{~ns}$, whereas the union has a much shorter effective deadtime of approximately $7 \mathrm{~ns}$, primarily due to the use of a four-channel router. Some minor afterpulsing is evident in the union autocorrelation, but it likely comes from a few isolated runs, since we have not seen any such behavior in the individual runs that we have inspected.

$\approx 7 \mathrm{~ns}$ (Figure 6.3), largely thanks to the router. This is advantageous as it implies that the union table will be more effective at pileup protection than the copy1 or copy2 signals alone.

5. Exported bank: The clustered union of $\mu \mathrm{SC}$ and $\mu \mathrm{SCA}$ hits is exported as bank "MUFP" for later use in pileup protection.

Some final, technical notes on the $\mu \mathrm{SC}$ analysis:

- There existed two additional $\mu \mathrm{SC}$ signals that were not utilized in the Run8 analysis: the gated $B$ and $\bar{B}$ channels, corresponding to parameters 6003 and 6004 .

- In addition to the high-threshold $\mu \mathrm{SC}$ signal which registered muon arrivals (parameter 6001), there also existed a low-threshold $\mu \mathrm{SC}$ signal which was sensitive to the arrival of beam electrons (parameter 6803). However, this latter parameter was never 
used in the 2004 data analysis.

- There was evidence of occasional double-pulsing in the $\mu \mathrm{SC}$ copy 1 signal, but the existing processing algorithm appears to handle such behavior properly.

- All of the $\mu \mathrm{SC}$ and $\mu \mathrm{SCA}$ timing offsets reside in the MIDAS online database (ODB), rather than in the Berkeley software's common header file which contains the clustering and coincidence parameters for the other detectors.

The $\mu \mathrm{SC}$ data processing marks the end of the code shared by the Berkeley and Illinois analyses. At this point the two analyses diverge, and subsequent detector processing and coincidence formation is performed according to the discretion of the individual analyzers. All of the following descriptions of detector analysis methods pertain exclusively to the Berkeley software.

\subsection{2 $\mu \mathrm{PC} 1$}

When a muon passes through the argon-ethane gas mixture inside the $\mu \mathrm{PC} 1$ detector, the muon ionizes gas particles in the vicinity of its trajectory. Electrons from this primary ionization drift to nearby anode wires under high voltage, and the resulting avalanche generates signals on the wires. The $\mu \mathrm{PC} 1$ detector contains two planes of 24 parallel anode wires, and the planes are oriented at right angles to each other in order to provide $(x, y, t)$ information about the muon's point of passage.

The $\mu \mathrm{PC} 1$ detector data is processed in the following basic manner: groups of hits generated by the passage of muons are identified in the $\mathrm{X}$ and $\mathrm{Y}$ planes separately, and then the $\mathrm{X}$ - and Y-plane information is joined together to form two-dimensional coincidences. Here is the complete sequence of operations:

1. Raw data selection: Digitized $\mu \mathrm{PC} 1$ signals in the format (wire,time) are selected 
from the bank "HITS" into tables of X-plane or Y-plane signals, according to their parameter label (see Appendix G.1 for the $\mu \mathrm{PC} 1 \leftrightarrow$ parameter correspondences). Perwire temporal autocorrelations formed from the raw data (Figure 6.4) reveal some unphysical afterpulsing, particularly in two of the X-plane channels.

2. Spark cuts: After hits from the X and Y planes have been selected into their respective tables, a search is conducted for high-multiplicity events, or "sparks." For each plane, transitive (i.e. updating) clusters are formed of hits within $\pm 1 \mu$ s of one another. If a cluster contains more than 20 hits, the earliest time in the cluster is flagged as a spark time and all $\mu \mathrm{PC} 1$ hits within the interval $[-.1,50] \mu$ s around that earliest time are cut. This step removes some extraneous noise, but it does not eliminate the egregious afterpulsing evident in Figure 6.4.

3. Per-wire afterpulse clustering: To completely eliminate afterpulsing noise, "artificialdeadtime" (AD) clustering is performed separately on each wire. That is, any hits within $260 \mathrm{~ns}$ after an initial hit are clustered together (in non-updating fashion) to simulate the effect of a deadtime. The resulting autocorrelation plots in Figure 6.5 confirm that this operation has the desired effect.

4. Clustering across wires: A passing muon typically generates signals on two or more adjacent wires in each plane. Therefore, after signals on the individual wires have been cleaned up by spark cuts and AD clustering, coincident signals on proximate wires are grouped together into clusters. This clustering of signals across wires requires temporal coincidences within a time interval $\pm 260 \mathrm{~ns}$, and spatial coincidences of the form $\left(\mid\right.$ Xwire $\left._{1}-\mathrm{Xwire}_{2} \mid-1\right) \leq 2$, which effectively allows for a gap of at most two non-firing wires between wires with hits. The time of a cluster is assigned as the time of the earliest hit in the cluster, and the position of the cluster is calculated from 

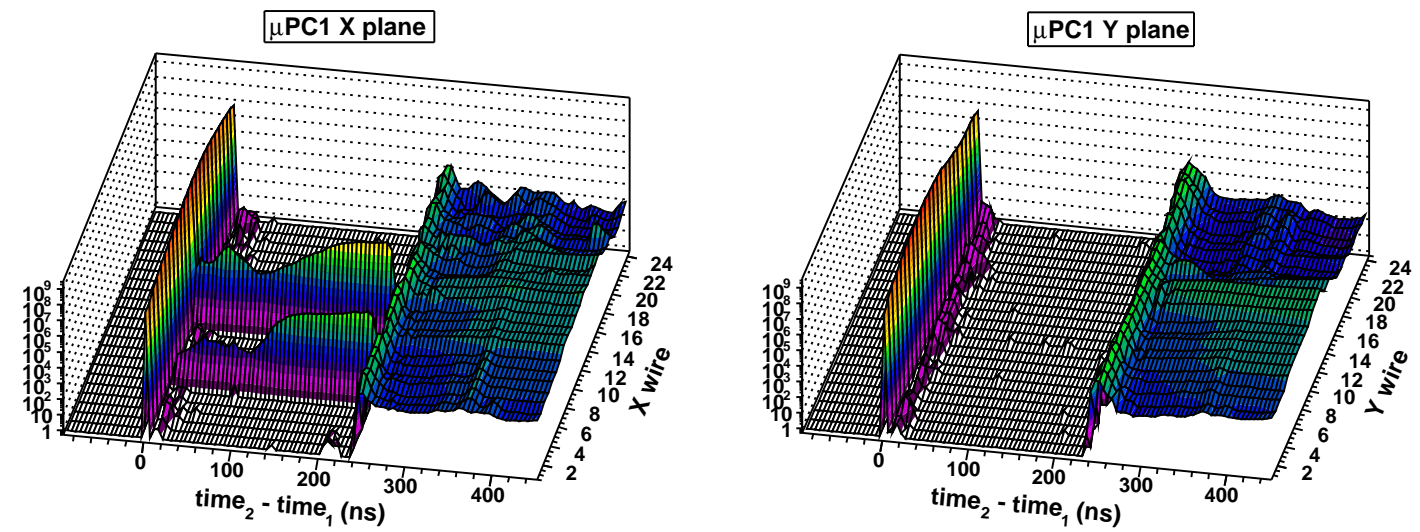

Figure 6.4: Temporal autocorrelations of raw signals from the $\mu \mathrm{PC} 1 \mathrm{X}$ and $\mathrm{Y}$ planes. Note the pronounced afterpulsing in X-plane wires 7 and 13.
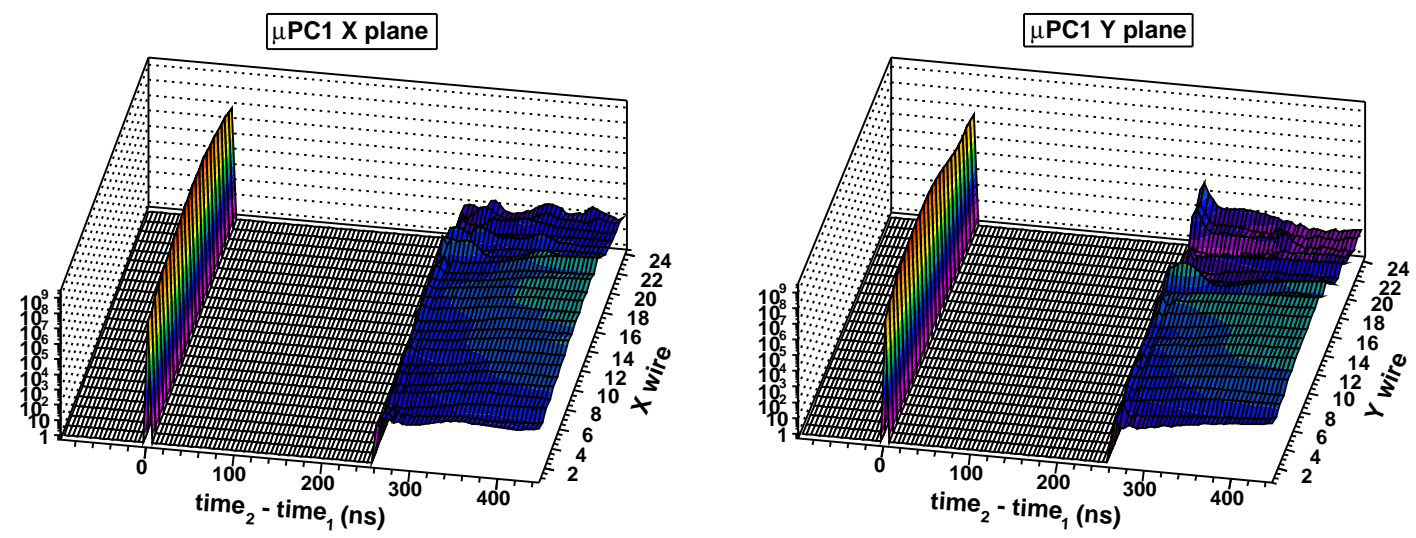

Figure 6.5: Temporal autocorrelations of signals in the $\mu \mathrm{PC} 1 \mathrm{X}$ and $\mathrm{Y}$ planes, after applying spark cuts and a per-wire artificial deadtime clustering of width 260 ns. Note that the unphysical afterpulsing noise has been eliminated.

the average of the contributing wires. The resulting profiles of hits in the $\mathrm{X}$ and $\mathrm{Y}$ planes, shown in Figure 6.6, reveal the approximately Gaussian shape of the muon beam spot.

5. Joining of $\boldsymbol{X}$ - and $\boldsymbol{Y}$-plane clusters: I first pair together X-and Y-plane clusters that are coincident within a relatively wide time interval of \pm 600 ns. Based upon 
the plot of these time differences, I chose to retain only the pairs that lie within the narrower interval $\left|T_{X}-T_{Y}\right| \leq 95 \mathrm{~ns}$, which covers the Gaussian signal coincidence peak. The resulting two-dimensional distribution of $(\mathrm{X}, \mathrm{Y})$ hits in $\mu \mathrm{PC} 1$ is plotted in Figure 6.7.

6. Exported banks: Four banks of $\mu \mathrm{PC} 1$ data are exported downstream: a table of X-plane hits, a table of Y-plane hits, a table of XY-coincident hits, and the $\mu \mathrm{PC} 1$ spark times. Here a "hit" is comprised of (wire(s),time) information.

The individual X- and Y-plane tables are later merged into a single table, " $\mu \mathrm{PC} 1 \mathrm{XY}-\mathrm{OR}, "$ while the XY-coincident table is labeled " $\mu \mathrm{PC} 1 \mathrm{XY}$-AND." These two tables provide slightly different levels of pileup protection, as will be discussed in Section 6.5.14. The $\mu \mathrm{PC} 1$ spark times are utilized later when performing spark cuts on the $\mu \mathrm{SC}$ data.

\subsubsection{TPC}

When beam muons enter the TPC volume and come to a stop in the hydrogen gas, they deposit energy along their incoming paths in a characteristic manner, illustrated in Figure 6.8. The deposited energy ionizes the nearby hydrogen gas atoms, and the resulting ionization electrons drift to the bottom of the TPC, where they generate signals in a multiwire amplifying chamber. Because the TPC operates in proportional mode, the muon-generated signals are proportional to the energy deposited per unit length.

In order to reduce the data volume, the analog TPC signals are discriminated by three tunable thresholds. The first and lowest threshold, "energy-low" (EL), is tuned such that it is reliably triggered by the ionization track preceding the muon stop. The second, intermediate "energy-high" (EH) threshold is tuned to trigger on the pronounced energy maximum, or Bragg peak, near the end of the muon's trajectory. The third and highest 

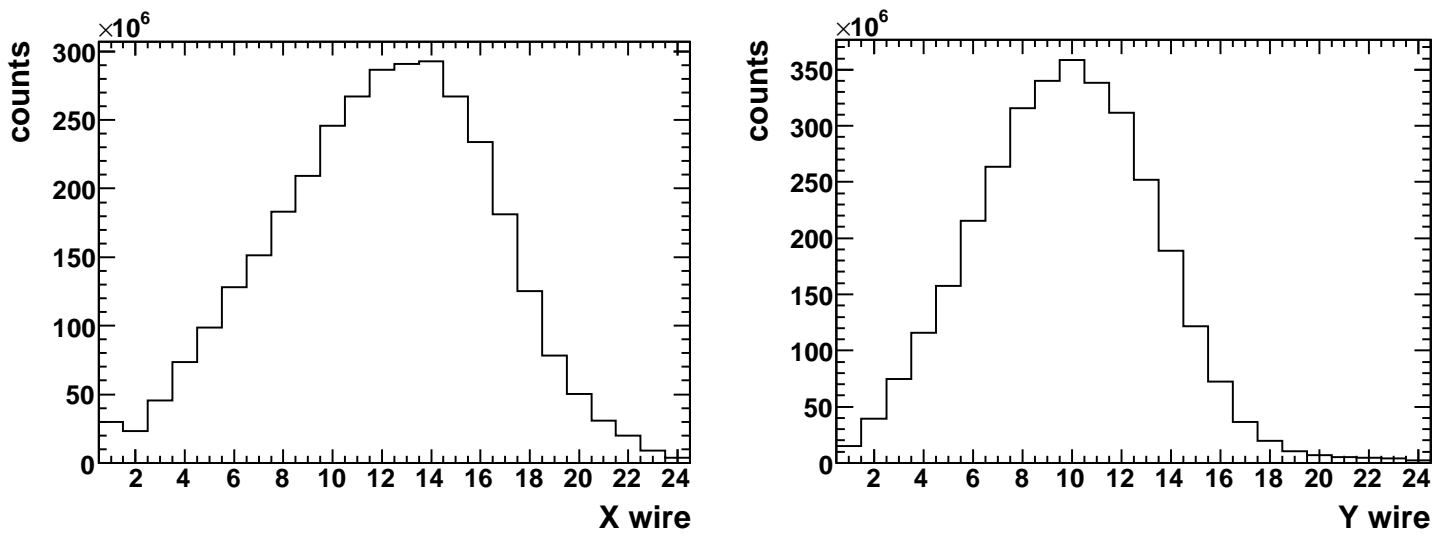

Figure 6.6: Profiles of hits in the $\mu \mathrm{PC} 1 \mathrm{X}$ and $\mathrm{Y}$ planes after clustering. The position of a hit cluster is calculated as the average of the contributing wires.

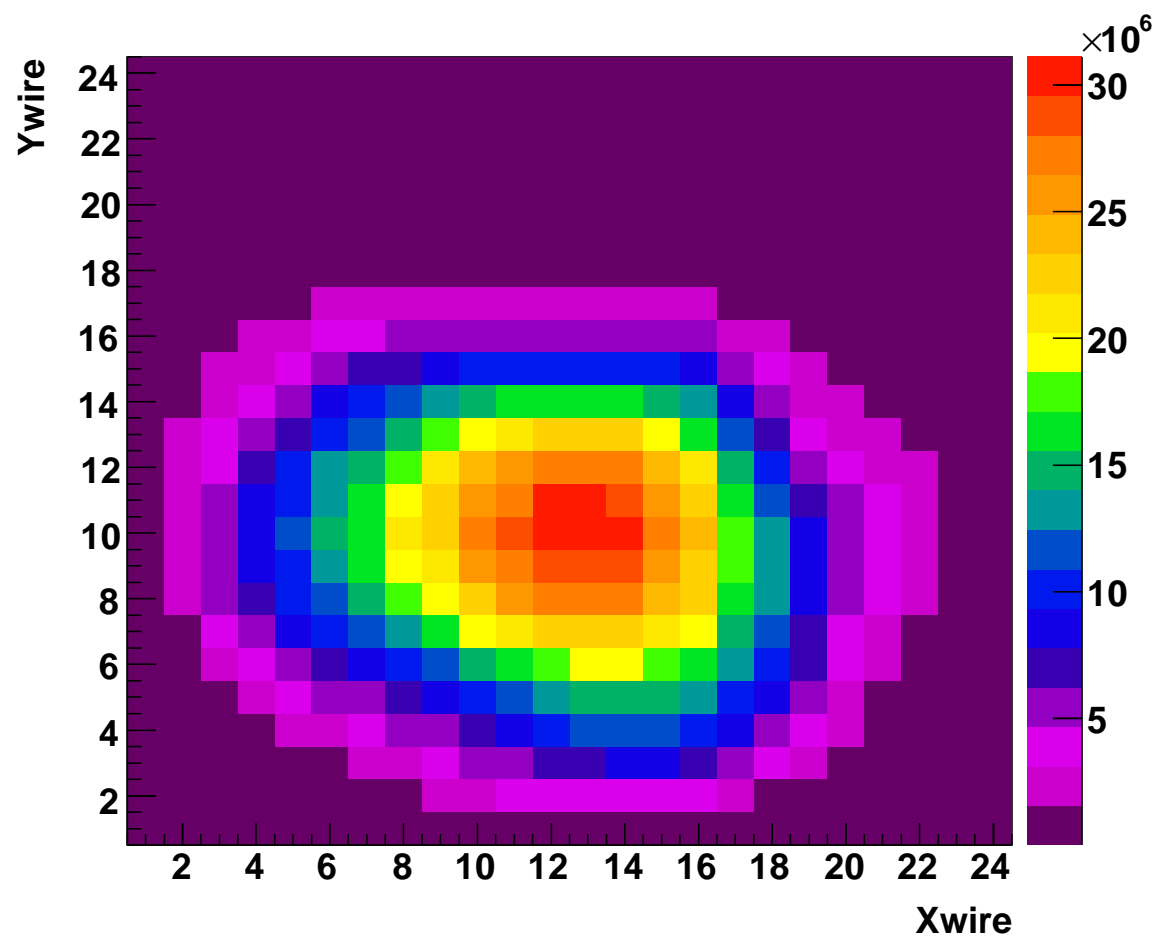

Figure 6.7: Two-dimensional distribution of hits in $\mu \mathrm{PC} 1$, formed by joining together pairs of temporally coincident X- and Y-plane clusters. This plot clearly shows the Gaussian profile of the muon beam spot at the $\mu \mathrm{PC} 1$ location. 


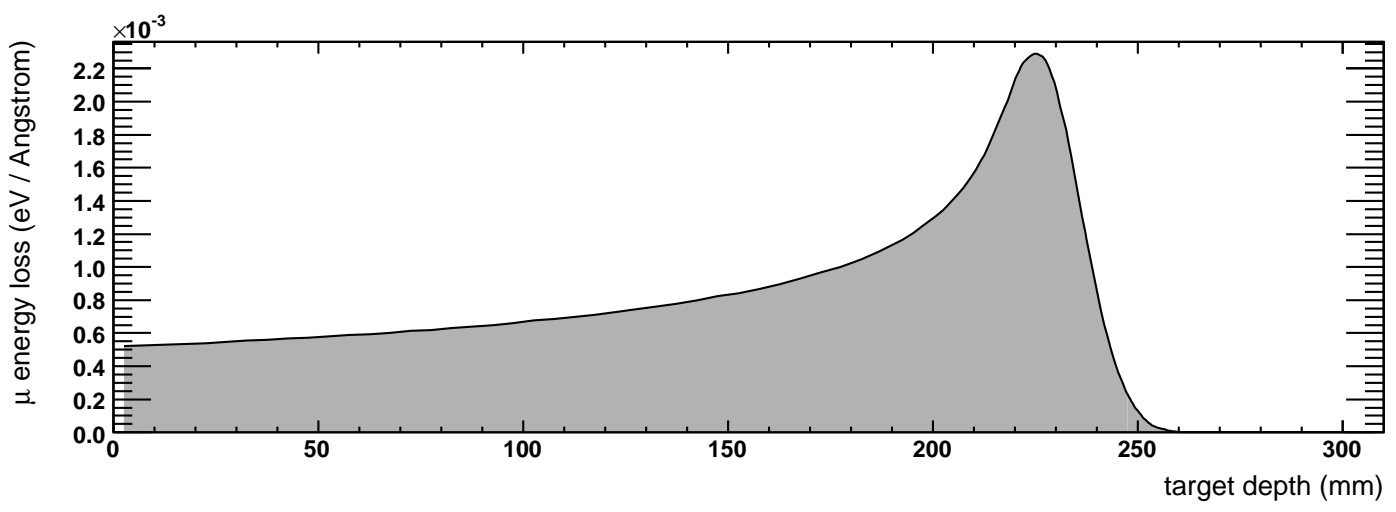

Figure 6.8: The energy deposited per unit length by muons stopping in hydrogen (a.k.a. the "Bragg curve"). As a muon decelerates to a stop, it deposits increasing amounts of energy along its path, culminating in a large maximum (the "Bragg peak") near the end of its trajectory. This plot was generated using the SRIM simulation software [202].

threshold, "energy-very-high" (EVH), is tuned to register signals from exotic, high-energy events such as nuclear recoils from captures on $Z>1$ elements, and therefore it is not used in the identification of muon stops.

In the discriminated TPC data recorded by the TDC400 electronics modules, a muon stop characteristically appears as a string of anode EL pixels terminating in one or more EH anode pixels, as shown in Figure 6.9. A similar signature should appear in the accompanying cathode data, although the signals there are not as robust as in the anodes, and the cathode thresholds were often triggered only at the end of the muon track. It is worth noting that the EL and EH thresholds were tuned by eye in Run8 using the event display, until the muon stops resembled the example shown in Figure 6.9. (Unfortunately, no comparison of the analog and digitized TPC signals had yet been implemented in the analysis, so there was no way to objectively calibrate the TPC thresholds.) The basic approach to identifying muon stop candidates in the TPC data, then, is to first search for clusters of EH pixels, and then to inspect such EH "islands" for an attached track of EL pixels. Cuts can of course be imposed on the quality and length of the track, the presence 


\section{TPC Display}

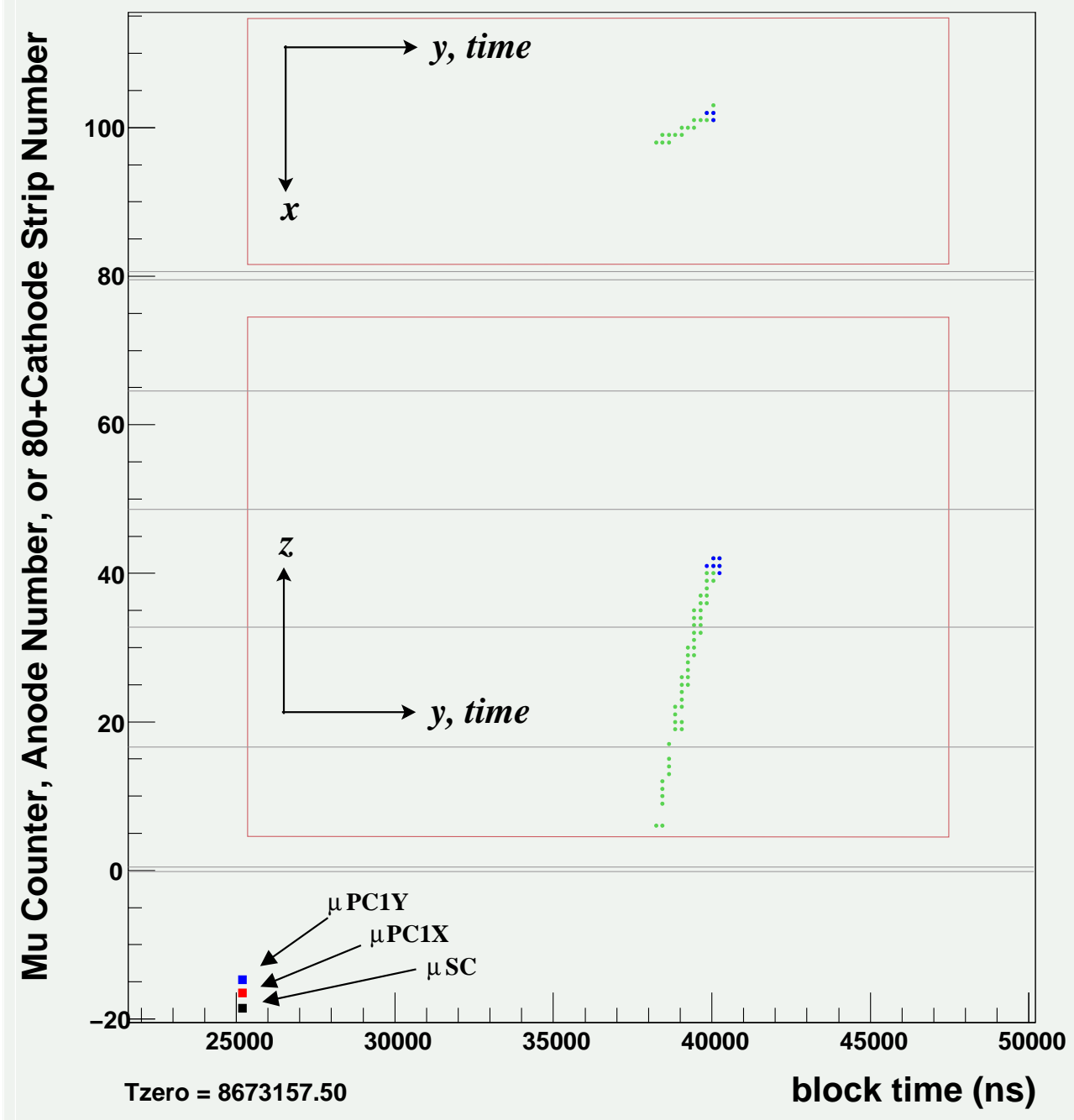

Figure 6.9: Example of a good muon stop in the TPC, as viewed in the Illinois event display developed by Steven Clayton. The arriving muon leaves behind a trail of ionization electrons which trigger the EL energy threshold of the anodes, as indicated by the long track of green pixels. When the muon stops, it deposits its remaining energy in a small area and triggers the EH energy threshold, indicated by the blue pixels at the end of the track. Note that the muon also generates a similar signature in the cathodes, which are shown at the very top of the figure. The $x, y$, and $z$ boundaries of the TPC's sensitive volume are indicated by the red box; the $y$ edges of the box are established by the muon arrival time recorded in the $\mu \mathrm{SC}$ detector. The anode numbers increase in the direction of increasing $z$, while time flows in the direction of increasing $y$. The cathode numbers, in contrast, increase in the $-x$ direction. 
of cathode pixels, etc.

In early versions of the Berkeley analysis software, some fiducial cuts were applied to TPC muon stop candidates before the TPC information was joined with the $\mu \mathrm{SC}$ and $\mu \mathrm{PC} 1$ information. However, this early, upstream application of cuts on TPC tracks had the deleterious effect of constraining subsequent analysis, by eliminating the freedom to study how different fiducial cuts affect the muon lifetime spectra. We therefore decided in August 2005 to reconfigure the software so that the TPC data processing stage only gathers information about muon stop candidates, and all fiducial cuts are postponed until just prior to the creation of lifetime histograms. The steps below thus describe the TPC information gathering; the fiducial cuts will be discussed later in Section 6.5.13.

1. Raw data selection: As mentioned earlier in Section 6.2, the raw TDC400 data is placed into an array conceptualized as a time-ordered, three-deep stack of pixel hits from the EL, EH, and EVH thresholds. TPC spark candidates are identified from the appearance of signals on non-amplifying anodes 1-4, and the times of such sparks are saved for future use.

2. Anode EH segment identification: The array of stacked TPC information is searched for groups of nearly contiguous anode EH pixels - referred to as anode EH "segments," or "islands" - where a gap of only one pixel is allowed. The boundaries of the EH segments are ascertained.

3. Gathering of information about anode EH segments: The software gathers information about the anode EL and EH pixels surrounding each anode EH segment. To collect this information, I employ the same "sniffing" algorithm used to ascertain the dimensions of the anode EH segments, but this time a gap of two pixels in either direction is allowed. The software counts up the anode EL and EH pixels and 
ascertains the extent of pixels in all directions. The software also collects concurrent cathode EL and EH information, both along the length of the track (so that muons which escape out of the TPC in the $x$ dimension can be identified) and immediately above the anode EH pixels (to get the $x$ position of the muon stop candidate).

4. Exported bank: All of the collected TPC information is passed downstream so that cuts can later be performed on the track qualities.

\subsubsection{Coincidences}

The $\mu \mathrm{SC}, \mu \mathrm{SCA}$, and $\mu \mathrm{PC} 1$ detector data is used to identify beam muon arrivals, while the $\mu \mathrm{SC}$ and TPC data is used in conjunction to identify which muon arrivals stopped in the hydrogen gas target. The efficacy of any particular identification technique must ultimately be assessed by looking at its effect on the muon lifetime histograms, as will be done in Section 6.5.14. Therefore, in this section I discuss only the technical details of how the muon detector data is joined together in coincidence.

To identify beam muon arrivals, I look for coincidences between the $\mu \mathrm{SC}$ and $\mu \mathrm{PC} 1$ detectors. I first histogram the time differences between $\mu \mathrm{SC}$ hits and $\mu \mathrm{PC} 1$ hits, as illustrated in Figure 6.10. When dealing with XY-plane-coincident $\mu \mathrm{PC} 1$ hits, I select $\mu \mathrm{SC}+\mu \mathrm{PC} 1$ events in the time region $[-145,5]$ ns containing the main coincidence peak. For the case where the $\mu \mathrm{PC} 1 \mathrm{X}$ - and Y-planes are treated as separate detectors, I perform the same procedure for each plane, but I use a slightly different coincidence interval of $[-145,50] \mathrm{ns}$, which is based upon the separate time difference plots. Good $\mu \mathrm{SC}+\mu \mathrm{PC} 1$ coincidences are considered to be events where both of the $\mu \mathrm{PC} 1$ planes fire in coincidence with a $\mu \mathrm{SC}$ hit.

Next, to identify the $(x, y, z)$ coordinates of the beam muons that stop in the active volume of the TPC, I start with the TPC EH anode clusters that were identified 


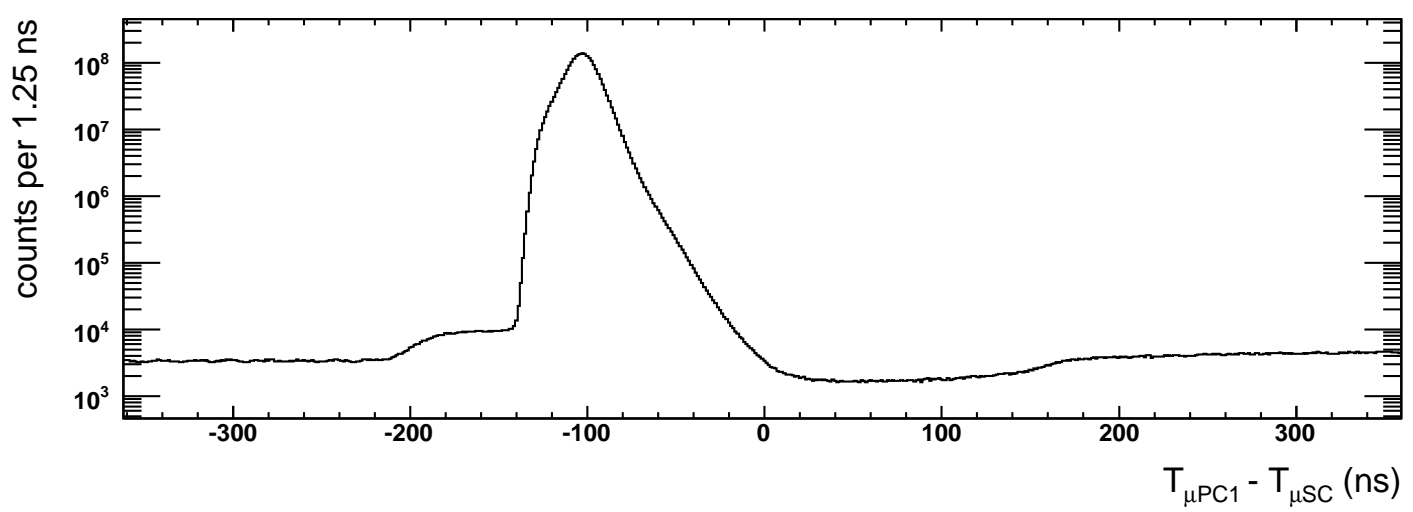

Figure 6.10: Time differences between hits in the $\mu \mathrm{SC}$ and $\mu \mathrm{PC} 1$ detectors, where the $\mu \mathrm{PC} 1$ hits are two-plane (XY) coincidences. I chose to use a $\mu \mathrm{SC} / \mu \mathrm{PC} 1$ coincidence interval of $[-145,5] \mathrm{ns}$. The hump in the background in the interval $[-200,-150]$ ns arises from the inclusion of accidental background contributions in the temporal join of the $\mu \mathrm{PC} 1 \mathrm{X}$ - and Y-planes. The trough in the region $[0,180]$ ns arises from the artifical deadtime clustering performed during the processing of hits in the $\mu \mathrm{PC} 1 \mathrm{X}$ - and Y-planes.

in Section 6.3 .3 as muon stop candidates. The $x$ and $z$ coordinates of an EH cluster are determined in straightforward fashion: the $z$ coordinate is calculated from the last anode in the EH cluster, and the $x$ coordinate is calculated from the average of the EL and EH cathode hits that are coincident with the EH anode cluster. Determining the $y$ position of an EH cluster is a more difficult proposition, because the TPC mixes space and time in the $y$ dimension. The first step in disentangling the two coordinates is to calculate the drift time of the muon stop, $\Delta t_{\text {drift }}=t_{\mathrm{TPC}}-t_{\mu \mathrm{SC}}$, where $t_{\mathrm{TPC}}$ is the earliest time at the downstream end of the $\mathrm{EH}$ cluster, and $t_{\mu \mathrm{SC}}$ is the muon arrival time supplied by the $\mu \mathrm{SC}$ detector. The resulting drift distribution of muon stops in the TPC is presented in Figure 6.11. In order to make sense of the drift distribution's features, we must recognize that the TPC is divided into two regions which have different dimensions, drift speeds, and physical properties: The multiwire proportional chamber (MWPC) has an amplifying electric field of $4.8 \mathrm{kV} / .35 \mathrm{~cm} \approx 14 \mathrm{kV} / \mathrm{cm}$, while the sensitive volume has a comparatively lower electric field of $24 \mathrm{kV} / 12 \mathrm{~cm}=2 \mathrm{kV} / \mathrm{cm}$. The two regions are illustrated in Figure 6.12. The broad 


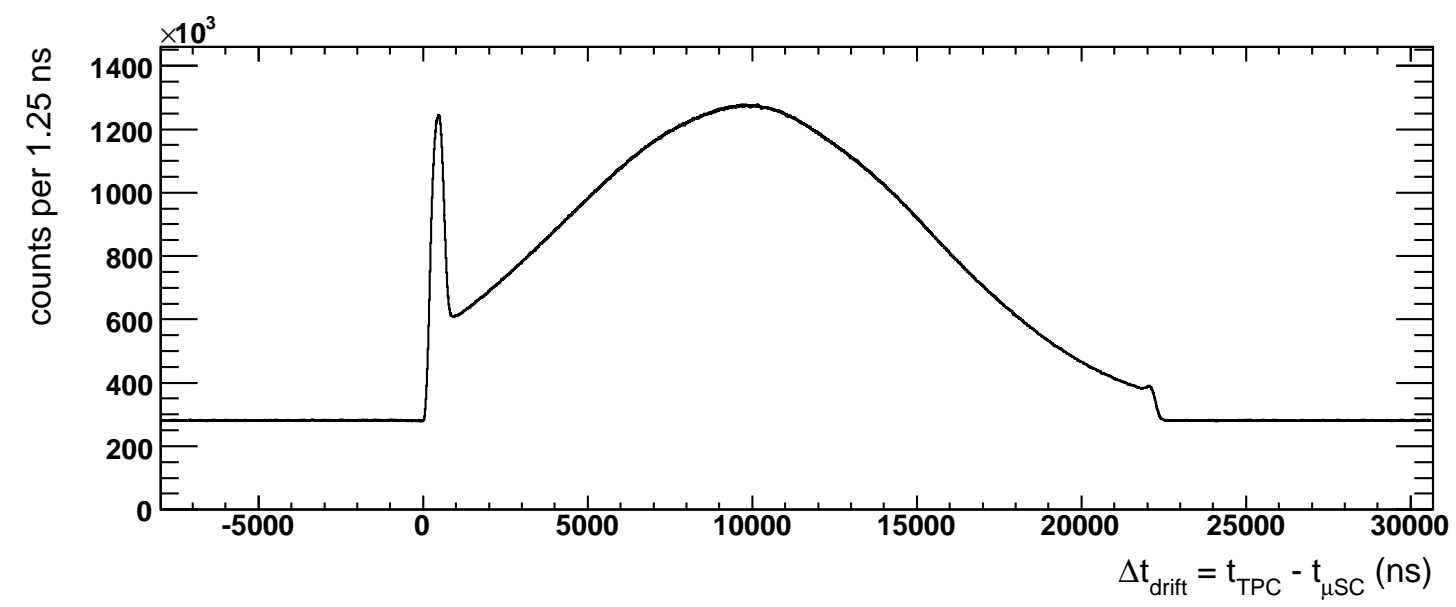

Figure 6.11: The TPC drift distribution of muon stops, created from time differences between TPC EH clusters and $\mu \mathrm{SC}$ arrival times.

central peak in the drift distribution corresponds to good muon stops in the TPC's sensitive volume, and its Gaussian profile reflects the shape of the incident muon beam. Muons that stop near the bottom of the TPC have a short drift time and lie close to $\Delta t=0 \mu \mathrm{s}$, while muons that stop near the top of the TPC have a long drift time and lie close to $\Delta t=22.5 \mu \mathrm{s}$. The sharp peak in the time region $0-1 \mu \mathrm{s}$ is the result of two effects associated with the MWPC region: First, the MWPC drift speed is $1.4 \mathrm{~cm} / \mu \mathrm{s}$, roughly three times larger than the sensitive volume's drift speed of $0.55 \mathrm{~cm} / \mu \mathrm{s}$. The larger MWPC drift speed compresses space into a smaller time interval, and thus produces an enhancement in counts relative to the central portion of the drift distribution. Second, muons that pass through the high-field MWPC region sometimes generate EH pixels that are not actually muon stops. Note that the drift distribution also contains a uniform accidental background, comprised of the time differences between uncorrelated muon arrivals and TPC stops. Ultimately we will need to suppress the accidental background which lies beneath the stopping distribution as much as possible, because the inclusion of accidentals in the fiducial muon stop selection leads to distortions in the accidental background of the muon lifetime histograms; this subtle effect 


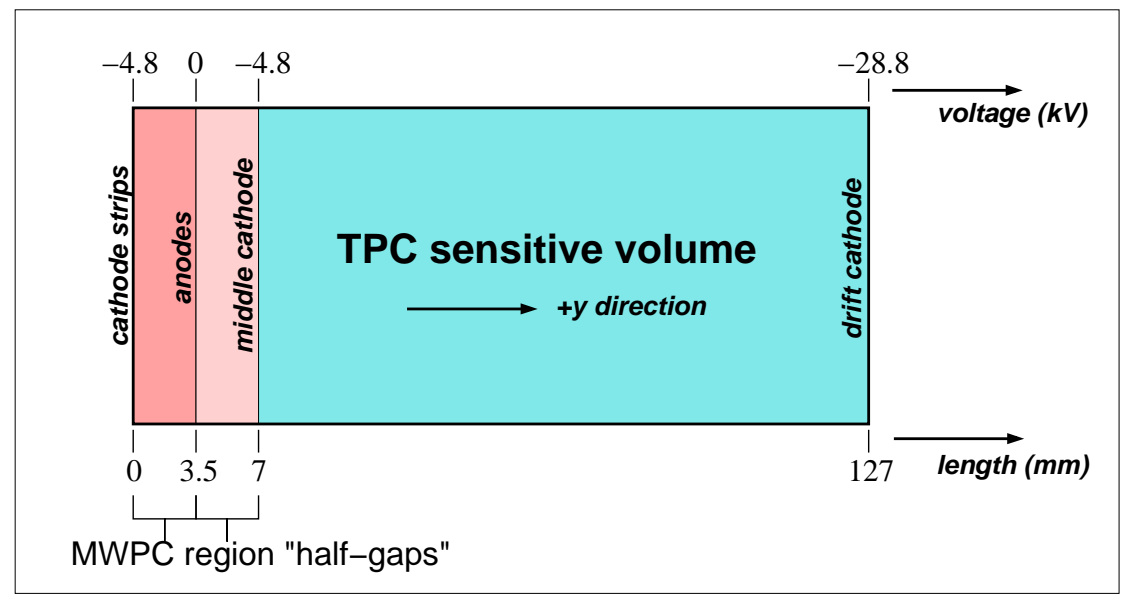

Figure 6.12: Diagram of the TPC detector regions (not to scale), viewed with the chamber lying on its side to assist in visualizing the correspondence with features of the drift distribution in Figure 6.11. The MWPC amplification region pictured at left lies on the bottom of the TPC, and the sensitive drift volume to its right is in the upper part of the TPC.

will be addressed in Section 6.5.14.

The next step is to convert the drift time into an accurate $y$ position. This is a tricky procedure because the MWPC and sensitive volumes have different dimensions and drift speeds. Based upon Dr. Peter Kammel's studies of the TPC drift properties using the GARFIELD simulation software [203,204], I concluded that the sharp peak centered at 475 ns in the TPC drift distribution in Figure 6.11 corresponds to the outer edges of the MWPC region. (It is worth mentioning that any muon track segments below the anode plane will drift up, rather than down, which gives throughgoing tracks the appearance of having been reflected at the anode plane.) Taking into account the smearing of time due to the $200 \mathrm{~ns}$ resolution of the TDC400 modules - convolution with a 200 ns-wide square pulse serves to increase the signal time by $100 \mathrm{~ns}$, on average - I determined that the two TPC regions must correspond to the following drift intervals: $\mathrm{MWPC}=[0,375] \mathrm{ns}$, and sensitive volume $(\mathrm{SV})=[375,22420] \mathrm{ns}$. That is, the earliest signals from the senstive volume arrive at a drift time of $375 \mathrm{~ns}$. The drift speed in the sensitive volume is uniform, so the formula 
for converting drift time into $y$ position is thus

$$
y\left(\Delta t_{\mathrm{drift}}\right)=\left[\frac{\Delta t_{\mathrm{drift}}-375 \mathrm{~ns}}{22420 \mathrm{~ns}-375 \mathrm{~ns}}\right] \times 120 \mathrm{~mm} .
$$

In retrospect, I should probably have used $475 \mathrm{~ns}$ instead of $375 \mathrm{~ns}$ for the edge of the MWPC region, based upon results from some fast Monte Carlo simulations I recently performed. Nonetheless, the scale of any potential inaccuracy is small, since the $100 \mathrm{~ns}$ difference corresponds to only $\approx 0.5 \mathrm{~mm}$. Moreover, the basic correctness of this procedure was confirmed by inspection of the $y$ component of the impact distribution (see Section 6.5.7), whose symmetry was vastly improved upon the introduction of the 375 ns MWPC offset. It should be acknowledged, however, that there are several features of the drift distribution that are not well understood. In particular, it is difficult to explain the width of the MWPC peak in Figure 6.11. The maximum drift length in the MWPC region is $<4 \mathrm{~mm}$, according to the MAGBOLTZ software which is used to simulate the device's properties, and any drift times over that region should be $<500 \mathrm{~ns}$, yet the MWPC peak extends to $900 \mathrm{~ns}$. My fast Monte Carlo simulations reproduced the relative height of the MWPC peak relative to the central drift distribution, but the width of the simulated MWPC was still only half that observed. Unfortunately, it is difficult to resolve this mystery because of the limited accuracy of the MAGBOLTZ software which is used to simulate device properties.

The stability of the TPC drift distribution during Run8 is a potential concern, because the TPC's amplification and drift voltages changed and/or were reset several times during the run (sometimes incorrectly), which could have affected the drift interval and hence the validity of the $y$ position reconstruction formula in Equation 6.1. We tried to maintain a voltage difference of $24 \mathrm{kV}$ across the sensitive volume so that its drift time interval remained constant, but it was not obvious during the run if we were successful in this regard. For example, we believe that there was a period of at least one day, Nov. 4, 2004, 
when the drift voltage was accidentally set to $29.8 \mathrm{kV}$ instead of the customary $28.8 \mathrm{kV}$. Fortunately, inspection of the drift distribution's width as a function of time revealed that the drift interval was stable to within one 200 ns TDC400 clocktick for the duration of Run8, which is better than $1 \%$, or $1.1 \mathrm{~mm}$.

At this point, the muon arrival time and $(x, y, z)$ stopping position information is in hand, and I am in the position to make fiducial cuts on the muon stops. The effects of fiducial cut variations on the fitted rate will be examined in Section 6.5.13.

I should note that I elected not to perform any three-dimensional matching of $\mu \mathrm{PC} 1$ hits with TPC tracks, because the tracking resolution is poor due to (1) smallangle scattering from the beryllium pressure vessel window separating the two detectors, and (2) imperfections in the "straightness" of TPC tracks. In order for such tracking to become feasible, it would be necessary to track the muon trajectory before and after the muon passes through the Be window. This would require the presence of the second muon MWPC, $\mu \mathrm{PC} 2$, between $\mu \mathrm{PC} 1$ and the Be window, which would potentially enable the matching of the $\mu \mathrm{PC} 1+\mu \mathrm{PC} 2$ vector with the $\mathrm{TPC}$ vector at a common point on the $\mathrm{Be}$ window. However, the viability of this technique has never been thoroughly studied.

\subsection{Electron detector analysis}

The purpose of the electron detectors is to measure the emission time and trajectory of outgoing decay (Michel) electrons. As described in Section 4.2, an outgoing electron passes through the electron detectors in the following sequence:

$$
e \rightarrow \mathrm{ePC} 1 \rightarrow \mathrm{ePC} 2 \rightarrow \mathrm{eSC}
$$

The Michel electron's emission time is established by the eSC detector, while its threedimensional trajectory is determined by the ePC1 and ePC2 wire chambers, operating in 
conjunction. In the following subsections I describe how the raw ePC1, ePC2, and eSC data is processed to identify Michel electron candidates in each, and then how the information from the three detectors is combined to identify good hits from throughgoing electrons. Some of the more technical, software-related details of the data processing can be found in reference [201].

\subsection{1 ePC1}

As discussed in Section 4.2, the ePC1 detector can be regarded as three detectors rolled into one: there is a central cylinder containing anode wires which run along its length, and on either side is a cylinder containing cathode strips which corkscrew around its surface (Figure 6.13). When a Michel electron passes through ePC1, it causes anode wires and cathode strips to fire in coincidence, and their point of overlap provides the $(r, \phi, z)$ coordinates of the spot where the electron struck. The inner and outer cathodes corkscrew in opposite directions, which allows for two such determinations for each electron hit, thus providing a measure of redundancy which helps to suppress background.

The raw data from each of the three ePC1 "planes" are processed in a nearly identical manner before coincidences are formed between them. Here is an outline of the general procedure:

1. Raw data selection: $\mathrm{ePC} 1$ hits are selected from the preprocessed, but otherwise raw, compressor data into three tables, one table for each detector plane.

2. Spark cuts: For each plane, I (transitively) cluster all hits within a specified time interval. If the size of a cluster exceeds a specified threshold, the event is considered to be a "spark," the earliest time in the cluster is recorded as the time of the spark, and the next $50 \mu \mathrm{s}$ of data in that plane is discarded. 


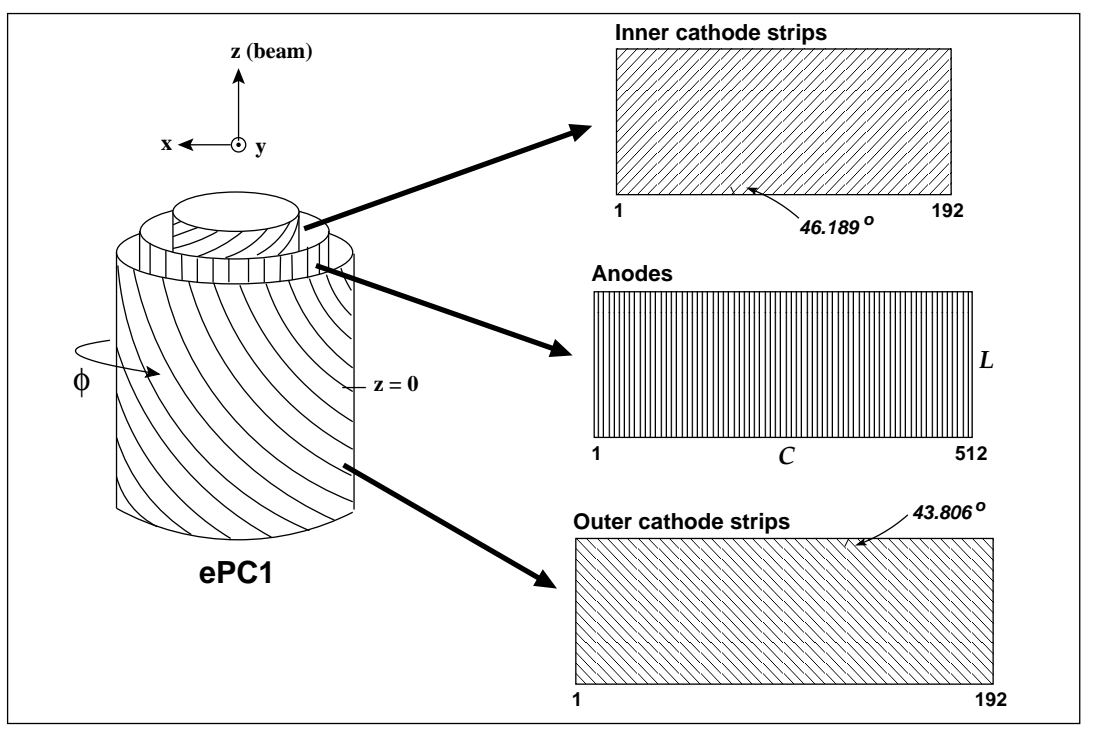

Figure 6.13: Cartoon diagram of the ePC1 detector geometry. On the left, the three ePC1 detector cylinders are drawn telescoped to illustrated the orientation of their wires; the axes indicate our conventions for the experimental coordinate system. If the cylinders were cut lengthwise between the first and last anodes/cathodes (located at approximately $-\hat{y}$, or $\phi=-\pi / 2)$ and then unrolled, the detector planes would look as shown on the right. The detector length $L=580 \mathrm{~mm}$ is the same for all ePC1 planes, while the circumference $C=$ $2 \pi d$ varies among them; see Appendix G.5 for the detector dimensions. The orientations of the cathode strips were inferred from the data analysis, by comparing the known wiring instrumentation with coincidence plots of the anode and cathode data; see reference [205] for a description of the procedure.

3. Per-wire afterpulse clustering: Even after spark cuts have been performed, there will still be some occasional afterpulsing noise. To prevent hot wires from biasing the center of gravity during the spatial clustering across wires that is performed in the next stage, I perform "artificial deadtime" clustering separately on each wire. All hits within a specified time interval following an initial hit are (non-transitively) clustered together into a single hit whose assigned time is the earliest time in the cluster.

4. Clustering across wires: A throughgoing electron can fire multiple adjacent wires in each ePC1 detector plane, so it is essential to cluster hits in space as well as time. After the signals on the individual wires have been cleaned up by afterpulse clustering, 
I group coincident hits on adjacent wires into a single hit. The time assigned to the cluster is the earliest hit in the cluster, and the position assigned to the cluster is the average of the contributing wires.

Once hits have been identified in the three detector planes separately, the hits can be joined together in coincidence. Below I outline the manner in which such coincidences are formed; a more technical description of the details of the process can be found in reference [205].

1. Joining of anode and cathode plane hits: First, the anodes are separately joined with the inner strips and outer strips in temporal coincidences, and $(\phi, z, t)$ information is assigned to each anode/inner cathode (AI) and anode/outer cathode (AO) pairing. The three ePC1 planes are brought into their true (relative) physical alignment by adjusting the cathode planes' orientations relative to the anode plane. That is, the rotational offsets of the cathode planes are tuned in the data analysis so that the $\mathrm{AI}$ and $\mathrm{AO} z$ distributions are properly centered around zero.

2. Formation of three-plane coincidences: Threefold coincidences involving data from all ePC1 planes are formed by joining together the $\mathrm{AI}$ and $\mathrm{AO}$ tables into an AIO table, where shared anode $(\phi, t)$ information serves as the bridge between the data from the two cathode planes.

3. Selection of good three-plane coincidences: Finally, a cut is made to select those three-plane AIO coincidences where the $\mathrm{AI} z$ position and the $\mathrm{AO} z$ position are within $12 \mathrm{~mm}$ of one another. The two $z$ positions are then averaged to yield a single $z$ position for the hit. The results following this operation are presented in Figure 6.14. A cut will later be performed on $z$ to eliminate the unphysical background which is visible there.

4. Exported banks: Several tables are passed downstream, each containing coincidences 


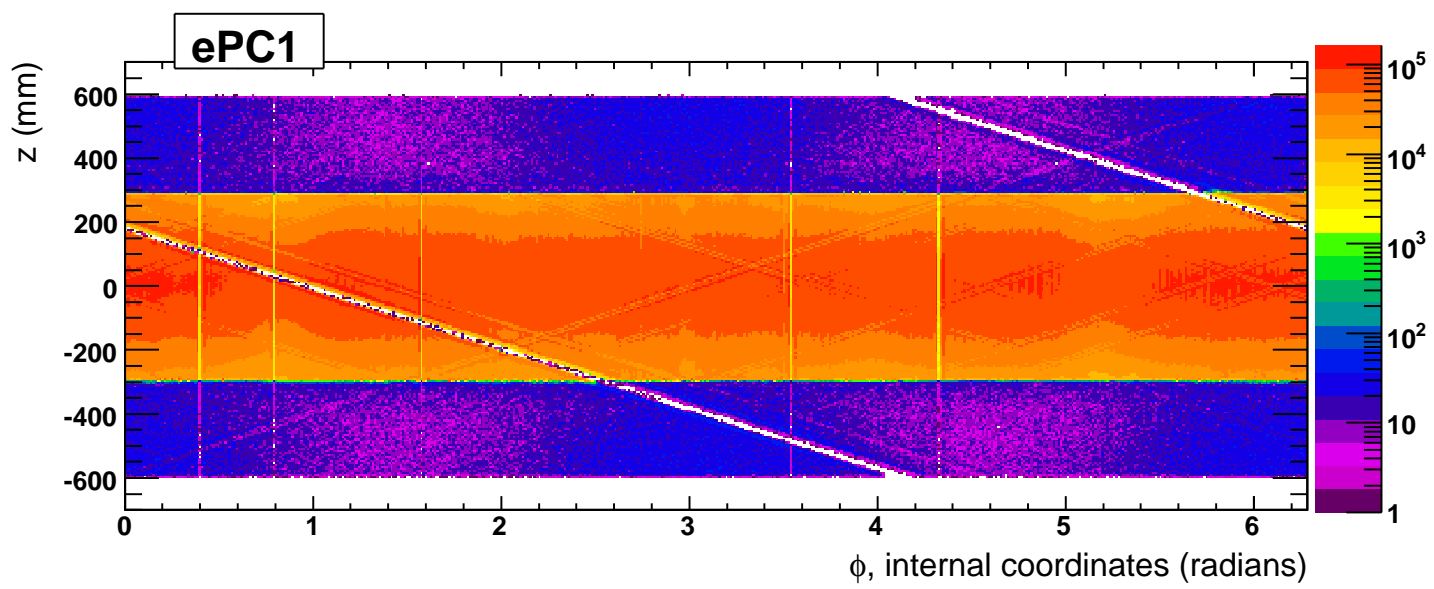

Figure 6.14: Three-plane coincidences in the ePC1 detector. The central band corresponds to hits in the physical region of ePC1, while the upper and lower background is made up of unphysical hits, constructed from anode and cathode hits which do not actually intersect. I should note that the $\phi$ coordinates here are in the "internal" coordinate space of ePC1, where anode 1 (close to $-\hat{y}$ ) is defined as $\phi=0$. An offset will later be introduced to bring ePC1 into alignment with the global experimental coordinate system. The diagonal stripe is due to a cluster of dead outer cathode channels.

for a different combination of ePC1 planes. Some of these tables are used merely for diagnostic purposes, while other tables are used for the identification of decay electrons. The electron candidates in these latter tables are represented by position and time information in the form $(r, \phi, z, t)$.

The redundancy provided by the two cathode planes offers two choices when identifying electron hit candidates: for a given anode hit, one can require a simultaneous hit in only one cathode plane, or one can require simultaneous hits in both cathode planes. The singlecathode-plane option is the minimal requirement, while the two-cathode-plane option is more stringent and should provide stronger background suppression. An assessment of the merits of these two approaches will have to await a lifetime analysis in Section 6.5.15. To enable such studies, I formed two tables of ePC1 hit candidates: I merged the anode/inner cathode (AI) and anode/outer cathode (AO) tables together to form a "cathode-OR" table, 


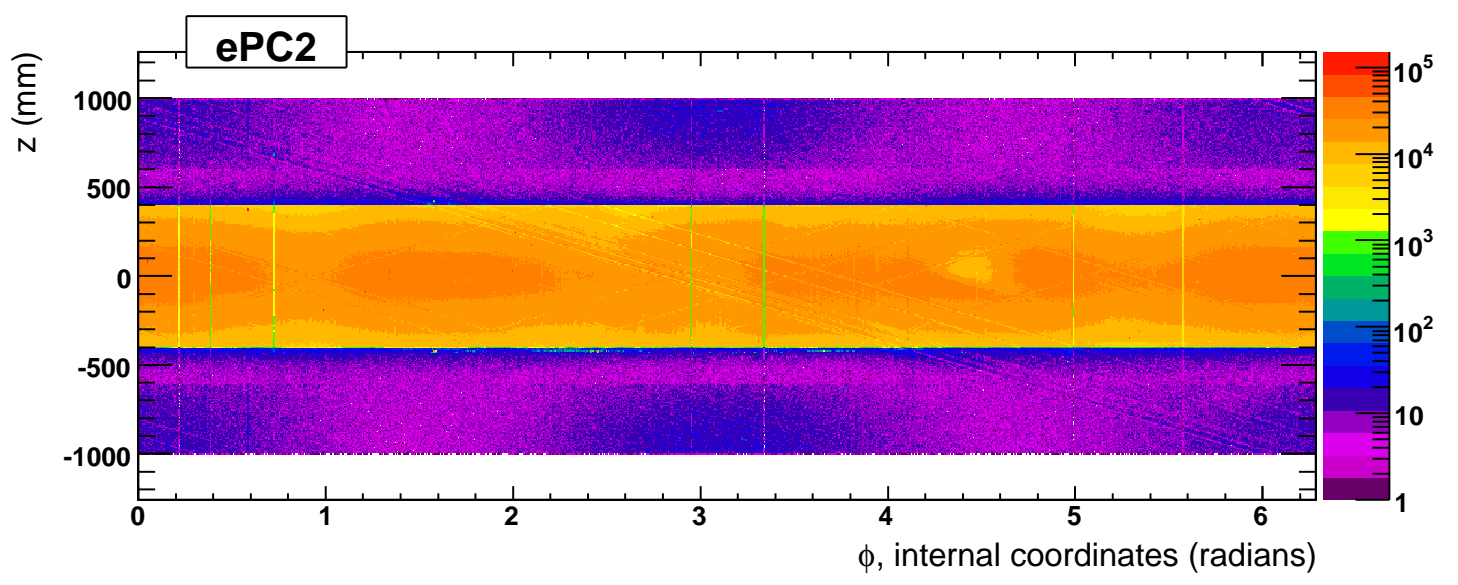

Figure 6.15: Three-plane coincidences in the ePC2 detector. As in Figure 6.14, the $\phi$ coordinates here are in the "internal" coordinate space of ePC2, where $\phi=0$ lies close to the $-\hat{y}$ direction. These local $\phi$ coordinates will be converted later into the experiment's global coordinates.

while I designated the three-plane-coincident table (AIO) as "cathode-AND."

\subsection{2 ePC2}

The ePC2 data is processed in nearly identical fashion as the ePC1 data, albeit with changes that take into account ePC2's different dimensions and number of wires. (See Appendix G.5 for a side-by-side listing of the ePC1 and ePC2 dimensions and technical specifications.) The orientation of the $\mathrm{ePC} 2$ wires is the same as the $\mathrm{ePC} 1$ geometry pictured in Figure 6.13. A plot of the three-plane coincidences in ePC2 is presented in Figure 6.15. The faint vertical bands visible in the backgrounds of Figures 6.14 and 6.15 near $\phi_{\text {relative }}=0$ and $\pi$ are likely due to the unphysical reconstructions of hits from throughgoing cosmics. That is, a throughgoing cosmic generates two strikes in the ePC, and coincidences will sometimes be formed where the cathode hits come from one strike and the anode hit comes from the other strike. The edges of these unphysical cosmics bands can be calculated from the ePC geometries: for ePC1, the edges lie at $z= \pm 313 \mathrm{~mm}$, while for ePC2 the edges lie 
at $z= \pm 606 \mathrm{~mm}$. The ePC2 edges are clearly visible Figure 6.15 , while the ePC1 edges are obscured in Figure 6.14 because of their proximity to the edges of the physical distribution. It is worth noting that both $\mathrm{ePC} 1$ and $\mathrm{ePC} 2$ were intelligently designed such that any given inner cathode and outer cathode have only one unique point of intersection, and therefore the unphysical reconstruction band does not overlap in $z$ with the physical band. Thus, as long as $z$ cuts are performed on the ePC data - as they will be later - these unphysical hits will not contribute to the lifetime histograms.

\subsection{3 eSC}

The eSC detector is comprised of sixteen scintillating segments, or "gondolas," arranged to form a cylinder (see Figure 4.7). Each gondola is made up of two layers of plastic scintillator, and each of the layers is read out at both ends by photomultipliers. Consequently, an electron typically generates four separate signals when it passes through a gondola. Thus, in order to identify electron hits in the eSC, we look for fourfold coincidences among the photomultipliers in each gondola. Below is the sequence of steps that I follow to accomplish this.

1. Raw data selection: The eSC hits are selected from the preprocessed, but otherwsie raw, CAEN data. It should be noted that copies of the eSC signals were sent to both the CAENs and COMPs, and that those two sets of signals are checked upstream (in the preprocessing stage of the data analysis) for discrepancies. If an excessive number of mismatches are encountered, the data block is cut.

2. Spark cuts: Spark events are identified from the raw data by (transitively) clustering together all eSC hits within $\pm 1 \mu$ s of each another. If any of the resulting cluster sizes exceed a prespecified threshold, the event is flagged as a spark, the earliest time in the cluster is recorded for later use, and all eSC hits within the next $50 \mu$ s are cut. 
3. Per-phototube afterpulse clustering: To eliminate afterpulsing noise, an artificial deadtime is implemented on each of the $16 \times 4=64$ gondola phototubes. All hits within 65 ns of an initial hit are non-transitively clustered together into a single hit whose assigned time is the earliest time in the cluster.

4. Identification of inner gondola coincidences: For each gondola, the inner upstream and inner downstream hits are joined together within a relatively wide, \pm 100 ns time interval. As Figure 6.16 shows, the resulting time differences $T_{\text {upstream }}-$ $T_{\text {downstream }}$ are slightly staggered due to variations in cable lengths, etc. I therefore apply a timing offset to the upstream time, $T_{\text {upstream }} \rightarrow T_{\text {upstream }}^{\prime}$, to center the time differences about zero. Finally, those coincidences within a $4 \sigma$ interval around the central peak at $\Delta T=0$ are selected as good coincidences, and assigned the time $T_{\text {inner }}=\left(T_{\text {upstream }}^{\prime}+T_{\text {downstream }}\right) / 2$, without any loss of generality.

5. Identification of outer gondola coincidences: The procedure for identifying outer gondola coincidences is identical to the procedure for identifying inner gondola coincidences.

6. Join of inner/outer gondola coincidences: For each gondola, the good inner and good outer coincidences within 100 ns of each other are joined together. In order to center the time differences $T_{\text {inner }}-T_{\text {outer }}$ for each gondola about $\Delta T=0$, yet another set of time offsets are added, in this case to the outer gondola time, $T_{\text {outer }} \rightarrow T_{\text {outer }}^{\prime}$ (Figure 6.17). Finally, the inner/outer coincidences centered around zero are selected to obtain the final set of good, fourfold gondola hits (Figure 6.18). These fourfold coincidences are assigned the time (without any loss of generality) $T_{4 \text { fold }}=\left(T_{\text {inner }}+T_{\text {outer }}^{\prime}\right) / 2$.

7. Exported bank: A table containing the fourfold eSC coincidences is passed down- 

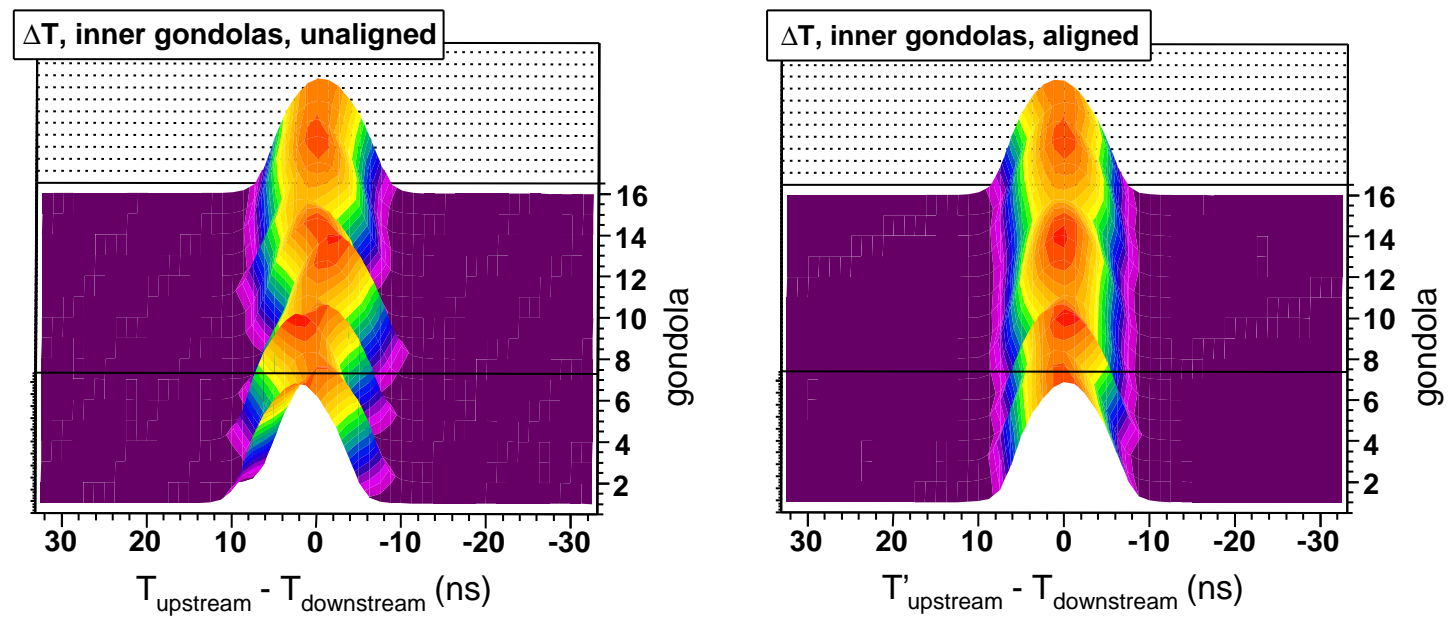

Figure 6.16: (Left) The raw time differences between upstream and downstream hits in the inner eSC gondolas. (Right) The time differences between the upstream and downstream hits in the inner gondolas after alignment about $\Delta T=0$. Note that the half-widths of the distributions are roughly consistent with the expected maximum time difference $\left.\Delta T\right|_{\max }=$ $L /(c / n)=90 \mathrm{~cm} /\left(3 \times 10^{10} \mathrm{cms}^{-1} / 1.58\right) \approx 4.7 \mathrm{~ns}$, which corresponds to the time it should take for light to traverse the entire length $L$ of the scintillator paddle; here $n=1.58$ is the index of refraction of the $\mathrm{BC}-404$ plastic scintillator material.
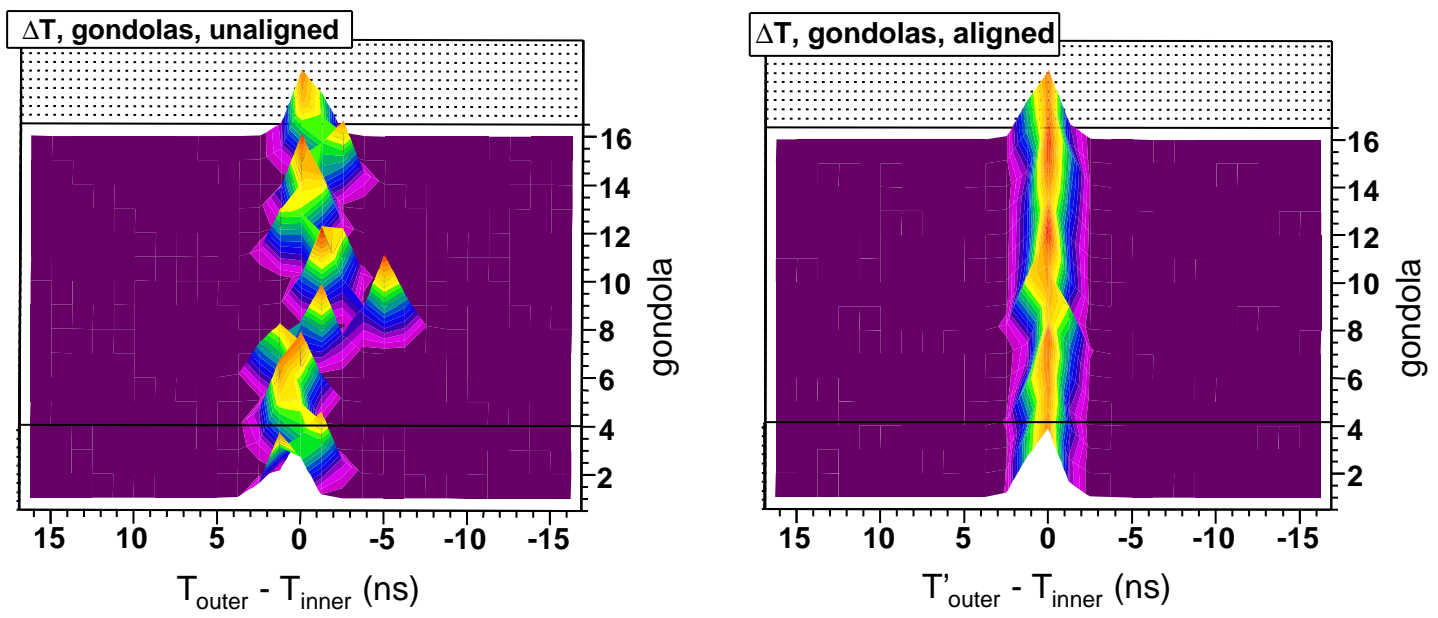

Figure 6.17: (Left) The time differences between hits in the inner and outer eSC gondolas. (Right) The same time differences after alignment about $\Delta T=0$. 


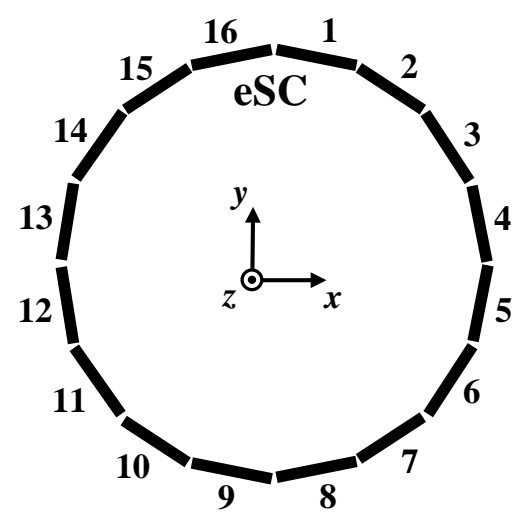

(a)

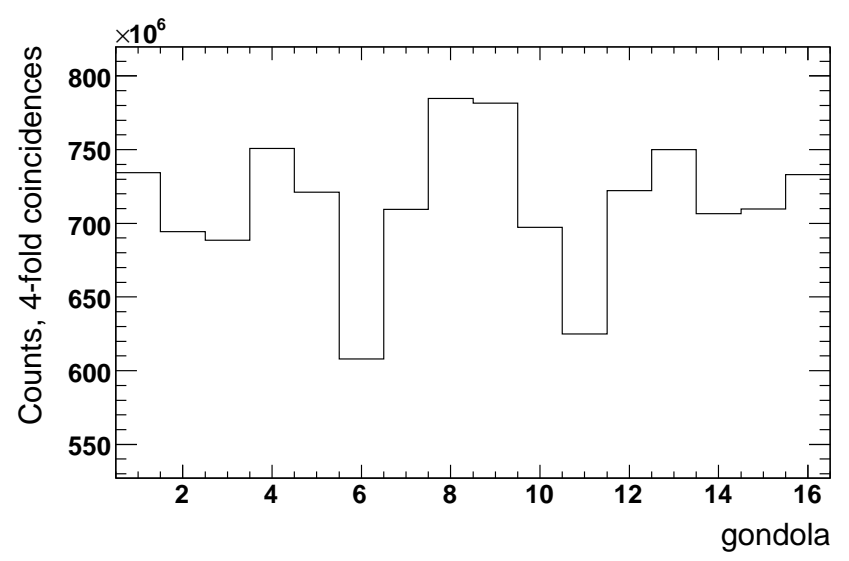

(b)

Figure 6.18: (a) The numbering scheme for the eSC gondolas. The view is looking upstream, into the oncoming muon beam. (b) The number of fourfold coincidences identified in each eSC gondola. The deficiency in counts in gondolas 6 and 11 is due to screening of Michel electrons by the TPC supports, as will be discussed in Section 6.5.12.

stream; each entry is of the form (gondola,$\left.T_{4 \text { fold }}\right)$.

I should point out that the eSC timing alignments described above only synchronize the times within an individual gondola; there may still be absolute timing offsets among the sixteen gondolas when plotted against the $\mu \mathrm{SC}$ time. Consequently, the individual lifetime spectra will need to be aligned prior to fitting, as will be done in Section 6.5.3.

\subsubsection{Coincidences}

When an outgoing Michel electron passes through all three electron detectors, its trajectory is measured by the ePCs and its time is measured by the eSC. Below I describe the algorithm that I use to join together the ePC1, ePC2, and eSC information in order to identify electron candidates. The following procedures are performed in identical fashion whether the ePC cathode-AND or the cathode-OR data set is used. 
1. Temporal join of ePC1 and ePC2 hits: Temporally coincident ePC1 and ePC2 hits are joined together.

2. Selection of $\phi$-coincident $\boldsymbol{e P C 1}+\boldsymbol{e P C 2}$ pairs: I select the subset of temporally coincident $\mathrm{ePC} 1+\mathrm{ePC} 2$ pairs where the $\phi$ values of the $\mathrm{PC} 1$ and $\mathrm{ePC} 2$ hits are within \pm 0.35 radians of each other (here $\phi$ is the cylindrical coordinate that runs around the circumference of the ePC drums). Larger values of $\Delta \phi$ are excluded because either the $\mathrm{ePC} 1+\mathrm{ePC} 2$ track does not pass through the pressure vessel (and therefore cannot have come from a muon decay in the hydrogen target), or else the ePC1 and ePC2 hits are on opposite sides of the detectors.

3. Temporal join of ePC1+ePC2 tracks with eSC hits: Temporally coincident eSC hits and $\mathrm{ePC} 1+\mathrm{ePC} 2$ pairs are joined together, where the eSC hit must be temporally coincident with both the ePC1 and the ePC2 hits in the ePC1+ePC2 pair. At this stage I also apply a cut on any $\mathrm{ePC} 1+\mathrm{ePC} 2$ pairs where an $\mathrm{ePC} z$ value lies beyond the physical extent of that ePC's active region.

4. Alignment of ePCs relative to $e \boldsymbol{S C}$ : Recall that, in order to form coincidences between an ePC's anode and cathode planes, I internally aligned each ePC's cathode planes relative to its anode plane. The ePC1 and ePC2 anode planes, however, still need to be aligned with the rest of the experimental apparatus. To accomplish this, I now tune the global $\phi$ offsets of the ePC anode planes to align them with the known locations of the eSC gondolas [205]). The precision of this approach is somewhat coarse, though, and can be fine-tuned further by observing how the impact parameter distribution - that is, the distribution of the distances between reconstructed $\mathrm{ePC} 1+\mathrm{ePC} 2$ Michel electron tracks and their parent muons' stopping points (to be discussed in Section 6.5.7) - is affected by adjustments in the global anode 
plane offsets. The optimal alignment is the one that minimizes the peak of the impact parameter distribution.

5. Selection of $\phi$-coincident ePC1+ePC2+eSC pairings: I select the subset of temporally coincident $\mathrm{ePC} 1+\mathrm{ePC} 2+\mathrm{eSC}$ groupings where the electron vector from the $\mathrm{ePC} 1+\mathrm{ePC} 2$ coincidence points in the direction of the eSC gondola. I permit some leeway in this spatial coincidence to accommodate small-angle scatters: the $\mathrm{ePC} 1+\mathrm{ePC} 2$ track can actually point into the adjacent gondola by up to, at most, $1 / 5$ of the gondola width. The final results from the ePC cathode-AND and cathodeOR data are plotted in Figure 6.19.

6. Exported ePC1+ePC2+eSC Ntuples: Once the final $\mathrm{ePC} 1+\mathrm{ePC} 2+\mathrm{eSC}$ coincidences have been formed, I write the data out as ROOT Ntuples for processing in the subsequent Ntuple-analysis stage, where they will be joined with the data from the muon detectors.

Note that I do not use $z$ information when comparing ePC1+ePC2 coincidences with eSC hits. In principle, timing differences between a gondola's upstream and downstream phototubes enables calculation of the $z$ position of hits in the eSC. However, as mentioned in the preceding section, light travels the length of a gondola in $\approx 5 \mathrm{~ns}$, while the timing resolution of the CAEN electronics is only $1.25 \mathrm{~ns}$ (and nonuniform!). Given the coarseness of this resolution, I decided that it was not worthwhile to attempt to calculate and use eSC $z$ information in forming coincidences among the detectors.

Using the eSC for normalization, the efficiencies of the ePC1 and ePC2 active regions were determined to be roughly $92 \%$ and $88 \%$, respectively. The efficiency of the eSC gondolas is difficult to determine, but is presumed to be close to $100 \%$, as inefficiencies in the eSC are overwhelmingly geometric in nature. Thus, factoring in the eSC's limiting 

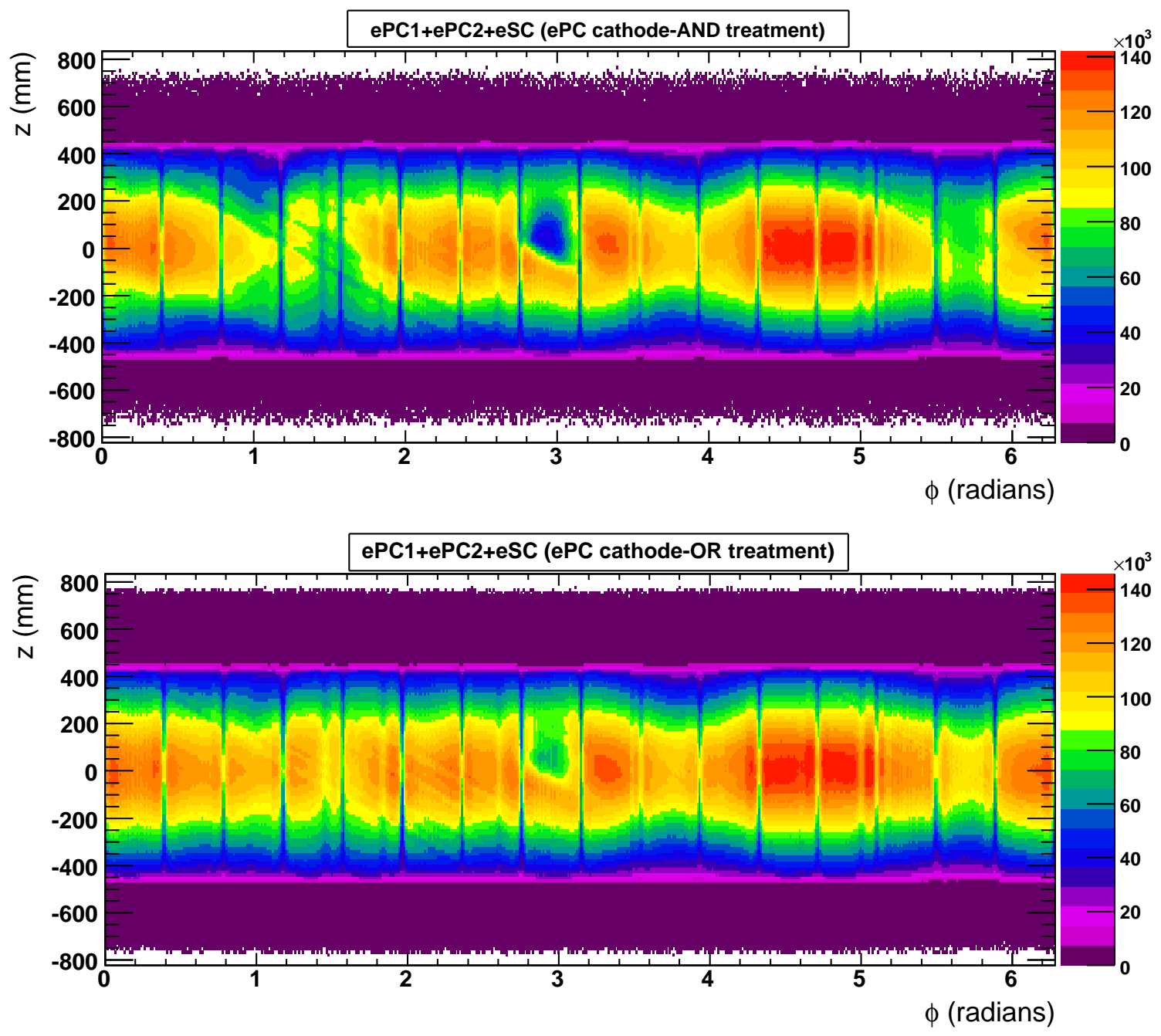

Figure 6.19: Plots of coincidences in all three electron detectors (ePC1+ePC2+eSC). The upper plot was constructed using ePC cathode-AND data, while the lower plot was constructed using ePC cathode-OR data. The $(\phi, z)$ values plotted are the points where the $\mathrm{ePC} 1+\mathrm{ePC} 2$ tracks intersect with the eSC detector's "plane;" the coordinate origin lies at the center of the electron detectors, and $\phi=0$ points along $x$ in the experimental coorindate system. Note that the contrast is greater in the upper cathode-AND plot, which involves more stringent coincidence criteria. The pronounced inefficiency at roughly 2.9 radians is due to a dead spot in ePC2, and the thin vertical lines correspond to the small gaps between the eSC gondolas. There are also some subtler vertical inefficiency stripes, e.g. at 5.7 radians, which are silhouettes of the TPC supports and frames. 
solid angle acceptance of $76 \%$ (see Section 4.2), the overall efficiency of the electron detectors can be estimated from the product

$$
\begin{aligned}
\epsilon_{\mathrm{ePC} 1+\mathrm{ePC} 2+\mathrm{eSC}} & =\epsilon_{\mathrm{ePC} 1} \cdot \epsilon_{\mathrm{ePC} 2} \cdot \epsilon_{\mathrm{eSC}} \cdot \Omega_{\mathrm{eSC}} \\
& =92 \% \cdot 88 \% \cdot 100 \% \cdot 76 \% \approx 62 \% .
\end{aligned}
$$

See reference [201] for plots of the efficiency variations across the ePCs and eSC.

\subsection{Muon lifetime studies and consistency checks}

The immediate goal of the MuCap experiment is to determine the effective $\mu^{-}$ disappearance rate in hydrogen from the time spectrum of decay electrons $N_{e}(t)$ (see Equation 1.10). In the following sections I describe how the muon lifetime spectra are constructed and analyzed, and I discuss various effects that manifest themselves in the process.

\subsubsection{The lifetime histogram}

Muon lifetime spectra are created by joining together muon and electron detector data coincident within a $[-40,40] \mu$ s time interval, making appropriate cuts, reducing the remaining data down to a unique set of $\mu \mathrm{SC}$ and eSC times, and then histogramming the

resulting time differences $\Delta t=t_{e \mathrm{SC}}-t_{\mu \mathrm{SC}}$. The lifetime spectra are generally produced by large-scale analysis passes over the Run8 data, and the histograms are written out to ROOT files for further study - namely, fitting.

In order to avoid certain systematic distortions which can arise in the background of the lifetime spectra due the TPC's inherent entanglement of time and space in the $y$ dimension, only muons that are separated in time by $\pm 25 \mu$ s from other muon arrivals are accepted. This condition, often referred to as "pileup protection," ensures that all of the ionization electrons from a muon track will have drifted to the TPC anode plane before the 


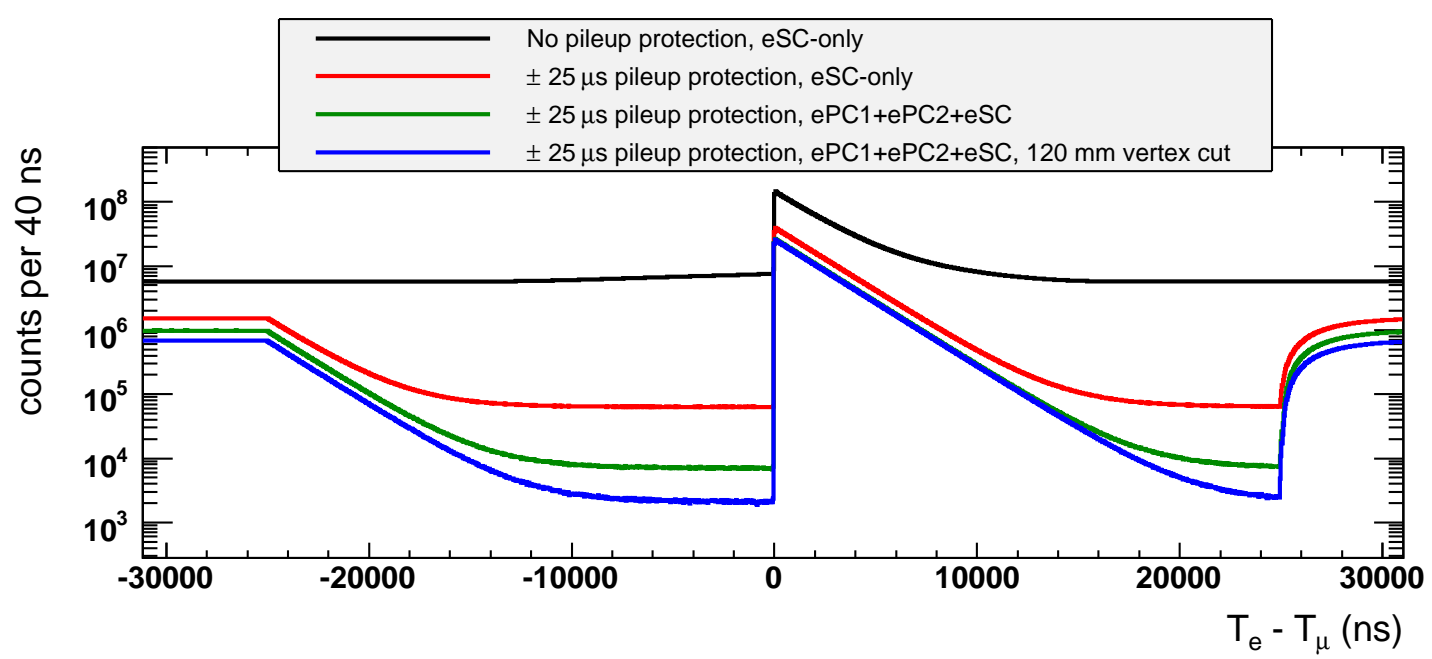

Figure 6.20: Muon lifetime spectra, all of which were created using the TPC to identify muon stops in the fiducial volume. Notice that the accidental background is not flat if no pileup protection is applied. The application of $\pm 25 \mu$ s pileup protection eliminates the background distortion, and also improves the signal-to-background ratio by "digging out" a hole from beneath the lifetime spectrum. Additional improvements are made by incorporating the $\mathrm{ePC} 1$ and $\mathrm{ePC} 2$ detector data, and by performing a $120 \mathrm{~mm}$ radial cut on the $\mu$-e vertex.

next muon arrives. (Recall that the maximum TPC drift time is $23.5 \mu \mathrm{s}$.) While pileup protection of $\pm 25 \mu$ s cuts the usable statistics by $\approx 68 \%$, it is essential in order to avoid the ambiguities that can arise when attempting to reconstruct multiple tracks in the TPC. The effectiveness of pileup protection in this regard will be assessed in Section 6.5.14. Pileup protection also has the added benefits of eliminating high-rate effects that can appear in the experimental lifetime spectrum ${ }^{2}$, and of dramatically improving the signal-to-background ratio. As Figure 6.20 shows, the signal-to-background ratio can be improved even further by incorporating the data from all electron detectors, and by performing cuts on the $\mu$-e vertex.

\footnotetext{
${ }^{2}$ High muon event rates can lead to deviations from the exponential muon disappearance law $e^{-\lambda t}$, due to the finite time resolution of the electronics and consequent inability to distinguish between decay events that are sufficiently close together in time. In particular, muon pileup can produce higher-order exponential terms of the form $e^{-2 \lambda t}, e^{-3 \lambda t}$, etc., in the experimental lifetime spectrum $[42,172,206]$.
} 
There are three identifiable components to the pileup-protected spectra in Figure 6.20:

1. The exponential signal containing muon decays.

2. An underlying accidental background (ABG) which results from binning time differences between uncorrelated muon and electron candidates.

3. The pileup background (PBG) at the edges, which receives contributions from muons outside of the $\pm 25 \mu$ s pileup protection buffer.

The ABG receives contributions from cosmics, undetected muon arrivals, and probably some degree of detector noise; its level relative to the decay signal depends largely upon electron detector and tracking cuts. For reasonable detector efficiencies the ABG should be flat. So long as this is true, and so long as the signal-to-background ratio at $\Delta t=0$ is better than $10^{-2}$ (we can achieve better than $10^{-4}$ ), the ABG does not affect the results of fits to the lifetime spectrum. The PBG also has no effect on fits, because they are generally confined to the interval 1-25 $\mu$ s. These and related issues will be discussed in more detail in the following sections.

In my analysis of the Run8 data, I employed what I call an "approval" approach towards identifying good $\mu \mathrm{SC}$ and eSC times for use in filling the lifetime spectra. Superficially, this term refers to the fact that I use the $\mu \mathrm{PC} 1$ and $\mathrm{TPC}$ data to approve certain $\mu \mathrm{SC}$ times as good muon arrivals, and I use the ePC1 and ePC2 data to approve certain eSC times as good decay electron emissions. The true meaning of the approval philosophy, however, is that I avoid making judgments about the relative quality of different combinations of detector data for any particular $\mu \mathrm{SC}$ or eSC time. For example, sometimes an eSC hit can be reasonably associated with multiple $\mathrm{ePC} 1+\mathrm{ePC} 2$ tracks, and there is simply no way of knowing which track is correct. In this case, I retain all possible combinations, instead 
of making a choice to uniquely associate the eSC time with a particular ePC1+ePC2 track. Steven Clayton did not use this approach in his implementation of the Illinois analysis. Rather, when confronted with a multiplicity of combinations, he used educated qualityof-track criteria to preferentially select one combination over all others. In principle, such judgments should not affect his fitted rate, because his track selection criteria were ostensibly time-dependent. However, it is my opinion that if we do not have any solid basis for making such judgments, they should be avoided so as not to risk inadvertantly introducing bias. In the end, the relatively small differences in our final results are a indicator of the scale of effects possibly related to our differing analysis philosophies.

I should mention that there is, in principle, an alternative to our standard $\pm 25 \mu \mathrm{s}$ "global" muon pileup protection procedure. In the early years of the experiment's development, the collaboration considered the possibility of enforcing "local" pileup protection, where each decay electron is uniquely matched with its parent muon based upon the intersection of the two particles' three-dimensional tracks (so-called "vertex matching"), and the only muons that are rejected due to pileup are those which stop in the immediate vicinity of another muon. This approach has the attractive feature of preserving a large fraction of the statistics that are rejected outright by the crude global pileup protection. However, local pileup protection is an exceedingly difficult procedure, fraught with all sorts of potential systematic distortions. There exist a variety of track overlap topologies and related ambiguities in the TPC where the presence of one muon decay event can influence the acceptance of another uncorrelated muon decay event in a time-dependent manner. Such problems are especially troublesome if the TPC voltage is sufficiently high to detect electron tracks $[207,208]$. Furthermore, local pileup protection is most likely impossible to accomplish with our existing experimental setup, because the scattering of decay electrons by the aluminum pressure vessel prevents accurate vertex matching. Due to these tracking 
limitations, local pileup protection will almost certainly never be realized in MuCap, and we will continue to rely on the more robust and viable global pileup protection.

Finally, it is worth noting that on several occasions I contemplated using the TPC data to assist in pileup protection, because the $\mu \mathrm{SC}$ and $\mu \mathrm{PC} 1$ are not perfect detectors in that regard. However, due to the TPC's entanglement of time with the $y$ dimension, the detector cannot really enforce muon pileup protection per se, but can only offer the possibility of unambiguous $\mu \mathrm{SC}+\mathrm{TPC}$ track association. In fact, one can imagine clever ways of taking advantage of the centralized muon stopping distribution in the TPC to enhance the acceptance probability of muon stops, and thereby increase the overall statistics. Unfortunately, such a procedure is so thoroughly fraught with potential systematic distortions that I abandoned any thought of using the TPC in that capacity. It is conceivable that this technique could be of use in the future, though, if carefully developed.

\subsubsection{Fitting function}

I fit the experimental $\mu^{-}$lifetime spectra with the simple exponential function

$$
f(t)=N w \lambda e^{-\lambda t}+B
$$

where the free parameters are the scaling factor $N$, the disappearance rate $\lambda$, and the constant background $B$. The parameter $\lambda$ and the fixed histogram bin width $w$ appear as coefficients of the exponential so that $N$ corresponds to the total number of decay events in the lifetime histogram. The repeated appearance of $\lambda$ also serves to reduce the off-diagonal term in the covariance matrix that links $\lambda$ and $N$, thereby improving fit convergence without affecting the fitted $\lambda$ value or its uncertainty.

I use the version of MINUIT [209] incorporated into ROOT to perform $\chi^{2}$ minimization of fits to the lifetime spectra. This procedure generates estimates for the values 
and uncertainties of parameters $N, \lambda$, and $B$, and it also affords a goodness-of-fit statistic in the quantity $\chi^{2}$, defined as

$$
\chi^{2}=\sum_{k} \frac{\left[N_{k}-f\left(t_{k}\right)\right]^{2}}{\sigma_{k}^{2}}=\sum_{k} \frac{\left[N_{k}-f\left(t_{k}\right)\right]^{2}}{f\left(t_{k}\right)},
$$

where the summation index $k$ runs over the histogram bins included in the fit range (generally between $0.1-25 \mu \mathrm{s}$, as will be discussed in Section 6.5.5), $N_{k}$ is the number of counts in the $k^{\text {th }}$ bin, and $t_{k}$ is the time at the center of the $k^{\text {th }}$ bin. Note that in the last step of Equation 6.3 it is assumed that the number of counts in each bin is sufficiently large that the bin contents are Gaussian distributed, with an error $\sigma_{k}=\sqrt{E\left(N_{k}\right)}=\sqrt{f\left(t_{k}\right)}$. For a fit over $k$ bins using the function $f(t)$ in Equation 6.2 which has three adjustable parameters, minimization of the $\chi^{2}$ — essentially a method of weighted least squares - is said to have $d=k-3$ degrees of freedom. The $\chi^{2}$ p.d.f. for $d$ degrees of freedom is

$$
f_{\chi}\left(\chi^{2}, d\right)=\frac{\left(\chi^{2}\right)^{d / 2-1} e^{-\chi^{2} / 2}}{2^{d / 2} \Gamma(d / 2)} \quad \text { for } \chi^{2} \geq 0
$$

and the statistical significance of a given fit is typically judged by the probability that a similar random data set would yield a $\chi^{2}$ value as large as or larger than the observed value,

$$
P\left(\chi^{2}, d\right)=\int_{\chi^{2}}^{\infty} f_{\chi}(z, d) d z=\frac{\Gamma\left(d / 2, \chi^{2} / 2\right)}{\Gamma(d / 2)}=Q\left(d / 2, \chi^{2} / 2\right)
$$

where $\Gamma\left(d / 2, \chi^{2} / 2\right)$ is the upper incomplete gamma function, and $Q\left(d / 2, \chi^{2} / 2\right)$ is a regularized gamma function. For large values of $d$ the $\chi^{2}$ p.d.f. tends toward normality, with the same mean $d$ and variance $2 d$ as the original distribution. In this so-called normal approximation, the "reduced chi-square" statistic, $\chi^{2} / d$, has an expectation value $E\left(\chi^{2} / d\right) \approx 1$ and standard deviation $\sigma \approx \sqrt{2 / d}$ for good fits to the data, and there is no need to compute probabilities from the integrals or gamma functions in Equation 6.5. This simplification is nearly always applicable to our lifetime spectra fits, which generally cover several hundred 
histogram bins, and it proves to be a useful quantitative tool for quickly assessing the quality of fits, since one can easily inspect the $\chi^{2} / d$ results and check for consistency with the expectation value of 1 .

ROOT also offers the possibility to use the more general method of log-likelihood minimization. As long as the statistics in each bin are sufficiently large $\left(N_{k} \gtrsim 100\right.$, which is usually the case in MuCap), Equation 6.3 remains valid and the log-likelihood and $\chi^{2}$ minimization techniques are completely equivalent. When performing fits to high-statistics lifetime spectra, the log-likelihood minimization method in ROOT actually yields a slightly smaller value $(<1 \%)$ for the error $\sigma_{\lambda}$ than does $\chi^{2}$ minimization, but the two approaches give results that are statistically consistent, and $\chi^{2}$ minimization is generally preferable because it is much faster. However, log-likelihood minimization remains applicable even when a histogram's per-bin statistics are low. We occasionally encounter situations where the lifetime histograms contain such few statistics that we must resort to using log likelihood minimization to obtain meaningful fits (e.g. the lifetime spectra of $\mu+p$ scatters in Section 6.6.2).

In reality, the experimental $\mu^{-}$lifetime spectrum is not a perfect exponential like the one described by Equation 6.2, but has a more complicated shape due to unwanted contributions from hydrogen gas impurities (both $Z>1$ elements and deuterium), $p \mu p$ molecular formation, and detector imperfections. However, the impurity concentrations are sufficiently small that their perturbations to the exponential decay rate are linear and can be corrected away. In fact, exponential fits to the clean and impurity-doped Run8 data consistently produce uniform distributions of residuals, indicating that the exponential function is a very good approximation to reality. The same basic precepts apply to the effects from detector inefficiencies and molecular formation, although molecular distortions are the largest of all these effects and, unlike the others, must be corrected after the DAQ 
clock frequency has been unblinded, because the molecular corrections are calculated using information from the literature.

It is possible to fit the lifetime spectra with a more complex function which describes the full kinetics of muon behavior in hydrogen gas containing low impurity concentrations. However, this procedure is far more elaborate than the simple exponential fit

method, and moreover it unavoidably relies in part on results from the simple exponential fits. The full kinetics fit method is therefore best regarded as a useful consistency check on the results from the more straightforward, three-parameter exponential fit approach.

Lastly, I should mention that the exponential fitting function for $\mu^{+}$is considerably more complicated than it is for $\mu^{-}$, due to the $\mu^{+}$spin precession effects discussed earlier in Section 3.5. The $\mu^{+}$data analysis will be described in Section 7.3.3.

\subsubsection{Lifetime alignment}

Each of the sixteen eSC gondolas has a unique time offset relative to the $\mu \mathrm{SC}$, primarily due to small cable length differences in the experimental apparatus. These timing variations are clearly visible in Figure 6.21(a), where I have overlaid the lifetime histograms from the sixteen gondolas, and zoomed in on the decay signals' "turn-on" region near $\Delta t=0$. The gondola timing offsets are small, with an average value of only $4 \mathrm{~ns}$ and a scatter of at most 5 ns. Nevertheless, before conducting any further analysis, all of the spectra should be aligned around $\Delta t=0$ so that they describe the underlying physics in unison, with the clock "starting" at the muon's arrival time. To accomplish this, I first determine the time offset $t_{O}$ of each gondola by fitting its lifetime histogram over the range $[-150,150]$ ns with the function

$$
f(t)=N w \lambda e^{-\lambda\left(t-t_{O}\right)}\left[1+\operatorname{erf}\left(\frac{\left(t-t_{O}\right)-\lambda \sigma^{2}}{\sqrt{2} \sigma}\right)\right]+B
$$




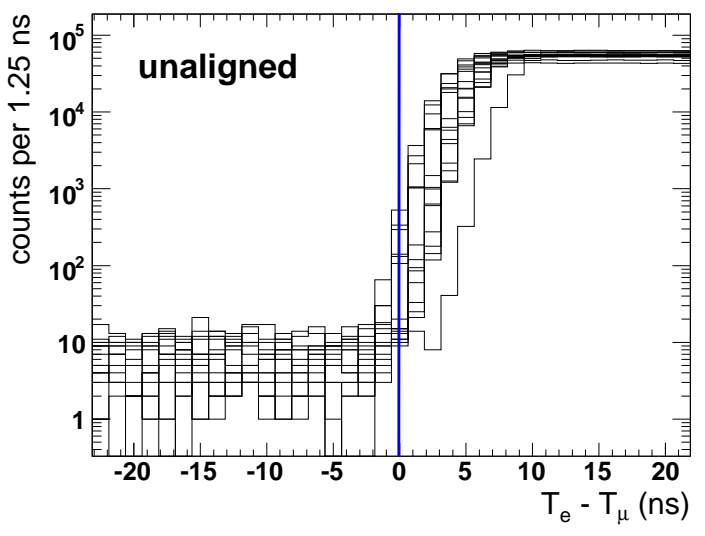

(a)

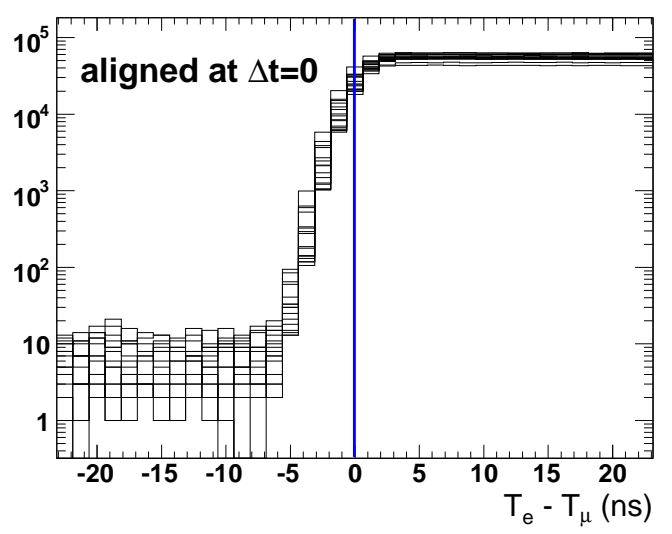

(b)

Figure 6.21: Lifetime histograms from the sixteen eSC gondolas (a) before alignment, and (b) after alignment about $\Delta t=0$. The experimental timing offsets among the gondolas are evident in the staggered "turn-on" times of the lifetime signals in (a).

which is a version of the standard fitting function (Equation 6.2) that has been modified to model the smearing of the turn-on of the decay signal by a Gaussian detector response of width $\sigma$ (see Appendix $\mathrm{H}$ for the derivation). The turn-on widths turn out to be almost uniformly $1.4 \mathrm{~ns}$ for all gondolas ${ }^{3}$. I next shift each gondola's lifetime histogram by the number of bins corresponding to its fitted $t_{O}$. The resulting aligned and centered spectra, shown in Figure 6.21(b), are added together to form a cumulative lifetime histogram which is used for all subsequent study. The alignment procedure ultimately has a small effect on the fitted rate, of the scale $\approx 0.5 \mathrm{~s}^{-1}$.

\subsubsection{Rebinning}

The lifetime histograms are created with bin widths of $1.25 \mathrm{~ns}$, which is the finest time resolution that can be achieved with our electronics. Each of the CAEN modules has a

\footnotetext{
${ }^{3}$ In fact, this finite detector response applies to the entire length of the lifetime spectrum, but does not in principle alter the fitted rate; see Appendix $\mathrm{H}$.
} 

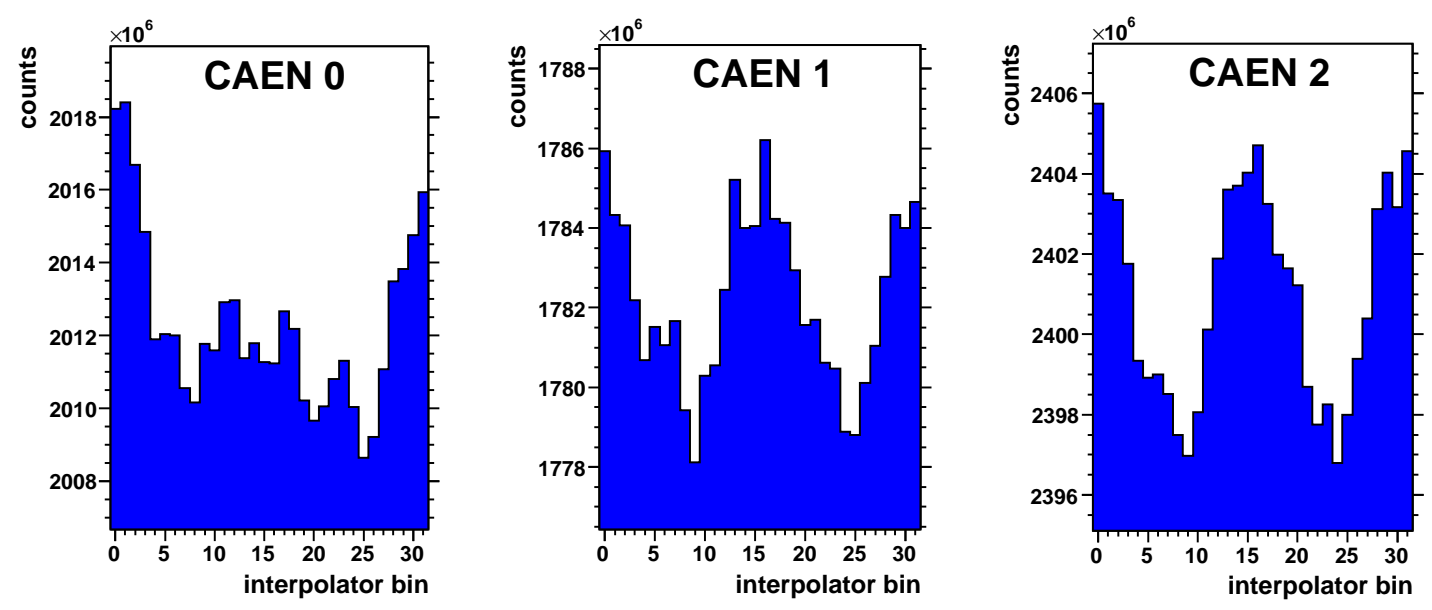

Figure 6.22: The markedly nonuniform distribution of leading edge hits (sans rollover clock signal) among the 32 interpolated time bins in each of the CAEN TDC modules. The bin count variations are far greater than what is statistically allowed, which indicates that the bins are not all exactly $1.25 \mathrm{~ns}$ wide and that there must be defects in the linearity of the interpolators. CAENs 1 and 2 in particular exhibit pronounced periodicities in their interpolated counts which could conceivably lead to $20 \mathrm{~ns}$ or $40 \mathrm{~ns}$ periodicities in the lifetime histograms. (These plots are from the Prod-50 data, which is described in Section 6.5.9.)

built-in interpolator which subdivides the externally provided 40-ns-period (25 MHz) clock signal into 32 time bins, thereby yielding an ostensible time resolution of $1.25 \mathrm{~ns}$ for the $\mu \mathrm{SC}$ and $\mathrm{eSC}$ detectors. However, the interpolator subdivision is egregiously nonuniform and exhibits variations that are far larger than what is statistically allowed, as shown in Figure 6.22. Consequently, there is some concern that the interpolators could introduce 20or 40-ns-periodic structure into the lifetime histograms. In addition, the PSI cyclotron beam $\mathrm{RF}$ is $\approx 50 \mathrm{MHz}$, which introduces an $\approx 20 \mathrm{~ns}$ periodicity in the accidental background (see Section 6.3.1). To eliminate any effects from these two sources of periodicity, I rebin the lifetime histograms by 32 - that is, I join together adjacent histogram bins in groups of 32 - to wash out any periodic behavior before performing lifetime fits. The resulting fitted decay rates are within $\approx 3 \mathrm{~s}^{-1}$ of the fitted rates from the unrebinned spectra, and the differences appear to stem entirely from the placement of the fit start time relative to the 
unrebinned spectrum's oscillations. The fits' $\chi^{2}$ values are comparable in the two cases.

In principle, the proper approach to eradicating effects from differential nonlinearities in the CAEN TDC clock subdivision circuit would be to simply ignore the interpolated clockticks from the beginning, and calculate all detector times from the CAEN clockticks modulo 32. However, this procedure is nullified by the fact that each of the eSC times is determined from a fourfold phototube coincidence within a given gondola. If the eSC data were rebinned to wash out the interpolator, then the internal coincidence window used to identify fourfold gondola scintillator hits would increase from $\approx 8 \mathrm{~ns}$ to $\approx 40 \mathrm{~ns}$, and it would become extremely difficult to resolve individual electron hits. Fortunately, Dr. Frederick Gray has performed Monte Carlo studies which reassuringly confirm that rebinning the lifetime histograms instead of the individual detector times does not have an effect on the fitted rate (see Section 7.3.2).

Having decided to rebin the lifetime histograms by 32 , the question arises where to set the rebinning boundary. This issue is intimately related to the choice of start time in the exponential fits. We generally want to start the lifetime fit $100 \mathrm{~ns}$ after the muon's arrival in order to allow complete depopulation of the $\mu p$ triplet state. Consequently, the rebin boundary should be placed such that the first 40 ns-wide bin that participates in the fit does not contain a significant amount of data from first 100 ns. To check the sensitivity of the fitted decay rate (and its corresponding reduced $\chi^{2}$ ) to the 32-rebin boundary placement, I created a custom rebinning function which allows me to specify the boundary location, and ran a fit scan over boundary locations between 30-110 ns, in $1.25 \mathrm{~ns}$ steps, with the fit range fixed at $[100,24900]$ ns. The results, plotted in Figure 6.23, reveal a relatively small, $\approx 2 \mathrm{~s}^{-1}$ variability in the fitted rate over the range of boundary locations, though there is larger volatility in the $\chi^{2} / d$ values of the fits. The exact mechanism for this behavior is unclear, though it has been reproduced in Monte Carlo simulations by Steven Clayton. 

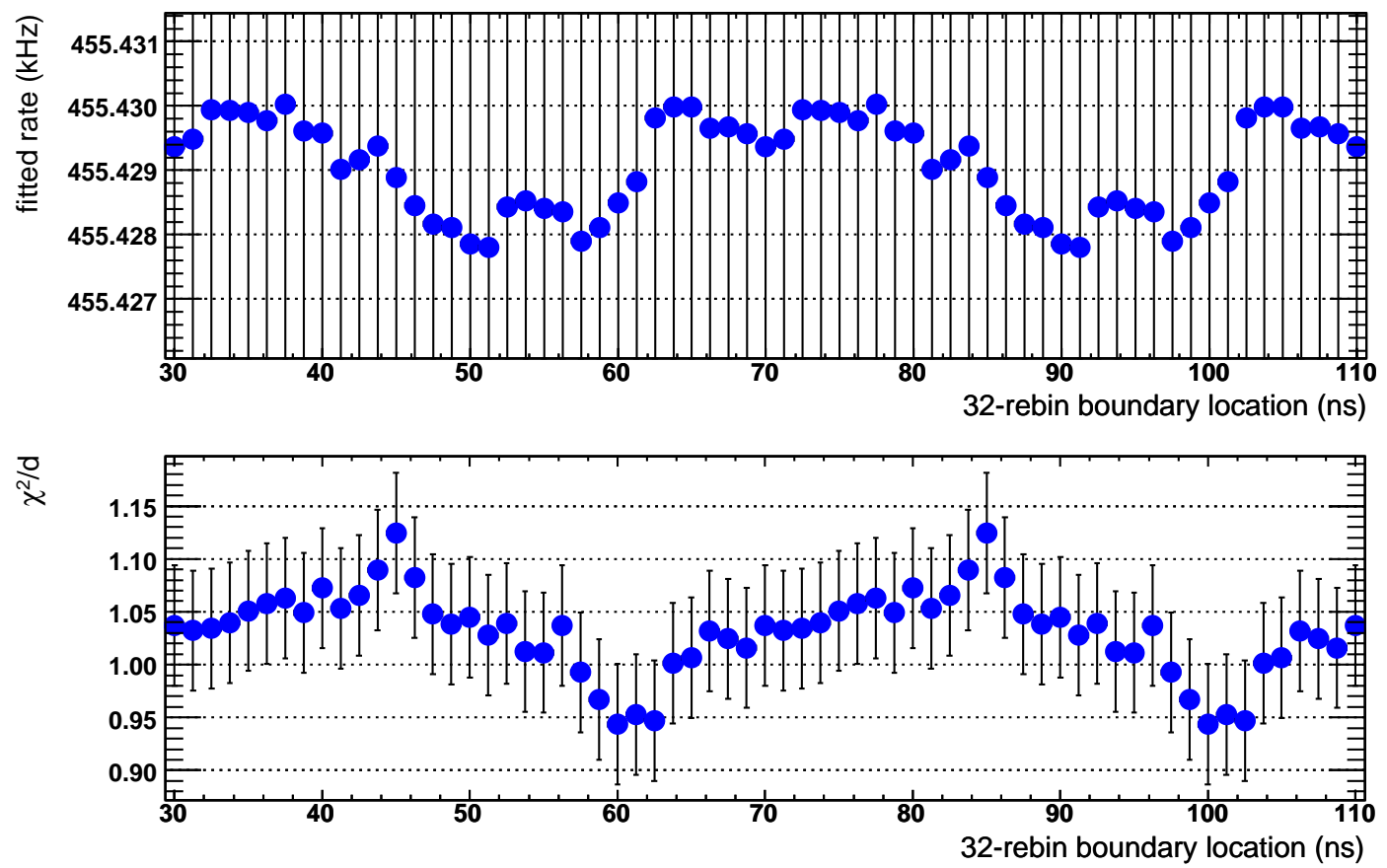

Figure 6.23: Fit results from a scan over the placement of the 32-rebin boundary in the lifetime spectra. The boundary placement varies from 30-110 ns, in $1.25 \mathrm{~ns}$ steps, and the fit range in all cases was fixed at $[100,24900]$ ns. The periodic structure exhibited in the scan is due to the fact the 32 -rebin results repeat every $40 \mathrm{~ns}$, which is the rebinned bin width. 
Nevertheless, for my standard conditions $\Delta t_{\text {rebin }}=100 \mathrm{~ns}$ and $\Delta t_{\text {start }} \geq \Delta t_{\text {rebin }}$, which are motivated by the physical reasons give above, the reduced $\chi^{2}$ is better than 1 .

For some time we were concerned about the possibility that the $\approx 25 \mathrm{MHz}$ CAEN clock frequency and the $\approx 50 \mathrm{MHz}$ PSI cyclotron $\mathrm{RF}$ could generate a beat effect in the lifetime histograms, which could in turn systematically skew the fitted rates. This subject will have to be postponed until Section 7.3.2, because an exact investigation into this possibility can only be performed after the DAQ clock frequency has been unblinded. However, it will suffice here to say that extensive Monte Carlo simulations by Dr. Frederick Gray demonstrated that any such beat effects on the fitted rate are negligible.

\subsubsection{Fit range}

Once the method for rebinning the lifetime histogram has been finalized, a fit range must be chosen. The choice of fit start and stop times is constrained by a combination of factors, including physics, the features of the lifetime histogram, and the fitting function that is used. When using the standard three-parameter exponential fitting function in Equation 6.2 , the fit start time should be delayed by at least 100 ns to allow the $\mu p$ triplet state to empty to the $10^{-5}$ level [5] (see the discussion in Section 3.1). Although the triplet depopulation rate estimates are not controversial, they are based upon theoretical calculations with little or no experimental corroboration. We do not expect this to be an issue for our measurement, but we must be sure to confirm that our fit results do not exhibit a sensitivity at early times, which if observed could indicate the presence of triplet effects. Meanwhile, the fit stop time cannot extend beyond the leading edge of the nonuniform pileup background at $\Delta t=25 \mu \mathrm{s}$ in Figure 6.20. For these reasons I chose [100,24900] ns as my standard fit range. I should note that the fitted disappearance rate would be less sensitive to molecular effects if the fit stop time were shortened to, say, $10 \mu \mathrm{s}$, well before $p \mu p$ 
molecules have time to form in significant quantities. However, in practice this abbreviated fit range is not practicable, because it prevents the fit from getting a good handle on the background level $B$.

To check the stability of fit results within my standard fit interval, it is useful to perform scans that vary the fit start time while the stop time is held fixed, and vice versa. Figures 6.24 and 6.25 show the fit parameters as a function of start and stop time; in all cases the histogram's 32-rebin boundary was fixed at 100 ns. A useful guide in evaluating the significance of the fluctuations in Figures 6.24 and 6.25 is the standard deviation of the statistical variation expected between two correlated fit results for a given parameter [210-212],

$$
\sigma_{A B}^{\text {stat. }}=\sqrt{\sigma_{B}^{2}-\sigma_{A}^{2}}
$$

where $\sigma_{A}$ and $\sigma_{B}$ are the parameter errors from separate fits over intervals $A$ and $B$. This formula is generally applicable to any fit parameter when data set $B$ is a subset of data set $A$ and the results from the two data sets are expected to be the same. The reduced $\chi^{2}$ obeys a similar formula involving the number of degrees of freedom $d_{A}$ and $d_{B}$ [213],

$$
\sigma_{A B}^{\chi^{2}}=\frac{\sqrt{2\left(d_{A}-d_{B}\right)}}{d_{B}}
$$

In each case, roughly two-thirds of the scan points should lie within the $1 \sigma_{A B}$ error band. Excessive deviations outside the error band can indicate the presence of systematic effects, while the absence of any such deviations indicates that the results are consistent within statistical error.

The $\pm 1 \sigma$ error bands described by Equations 6.7 and 6.8 are drawn in Figures 6.24 and 6.25, with the reference points fixed at the standard fit start and stop times. The data points lie mostly within or close to the error envelopes - this is especially true in the important regions close to the reference points, where the fit parameters are well constrained - so 

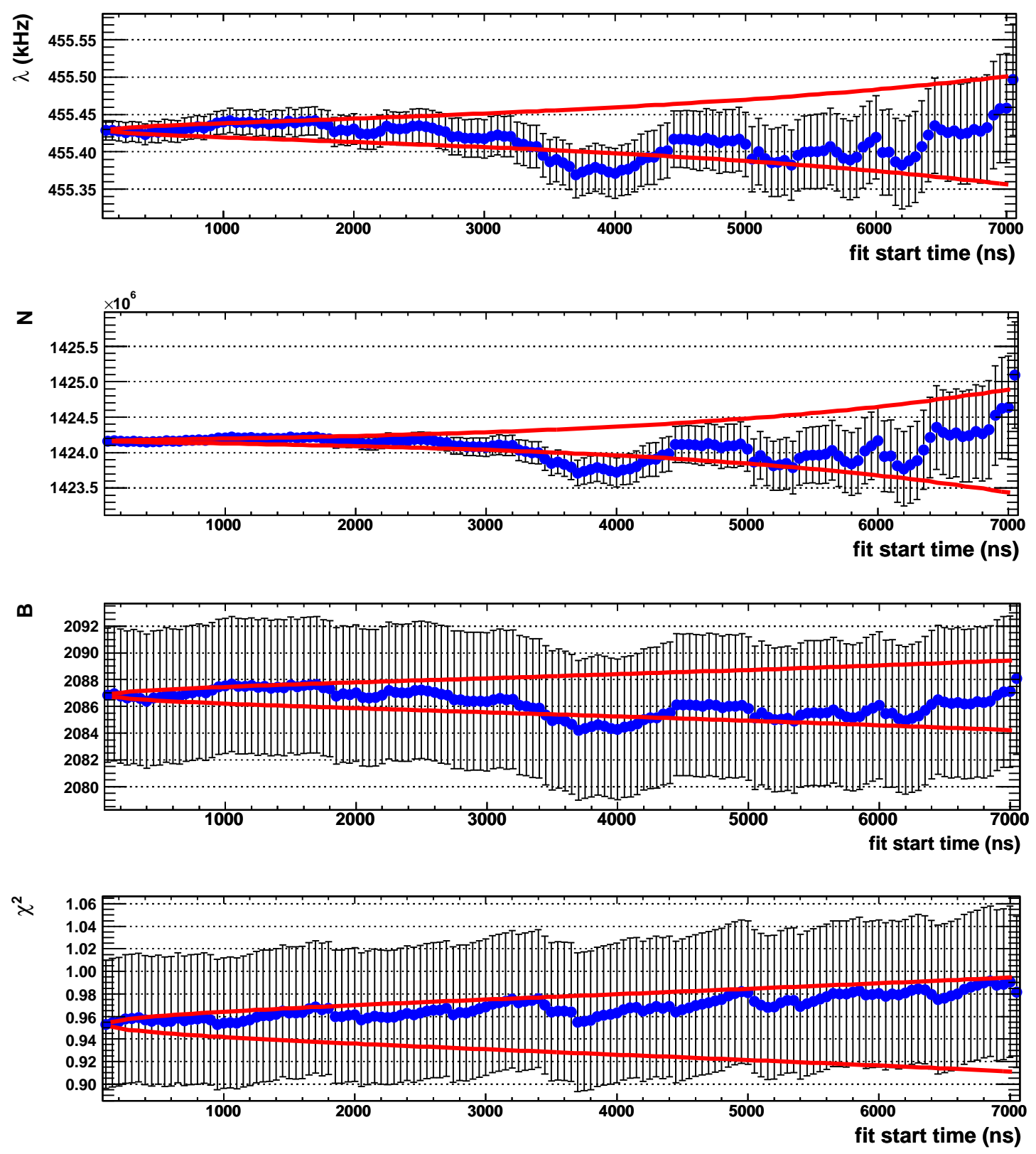

Figure 6.24: Fit results from a start time scan over the range 100-7000 ns, at 50 ns intervals, with the fit stop time fixed at 24,900 ns and the histogram 32-rebin boundary fixed at $100 \mathrm{~ns}$. All of the fit parameters remain consistent with the statistically allowed $1 \sigma$ bands indicated by the solid redlines. Closer inspection of the results in the first few hundred nanoseconds reveals that the fitted rates are quite stable at early fit start times (the results are within $\pm 3 \mathrm{~s}^{-1}$ in the first $300 \mathrm{~ns}$ ), supporting our assumption that the hyperfine triplet state quenched far earlier and is not affecting the measurement. 

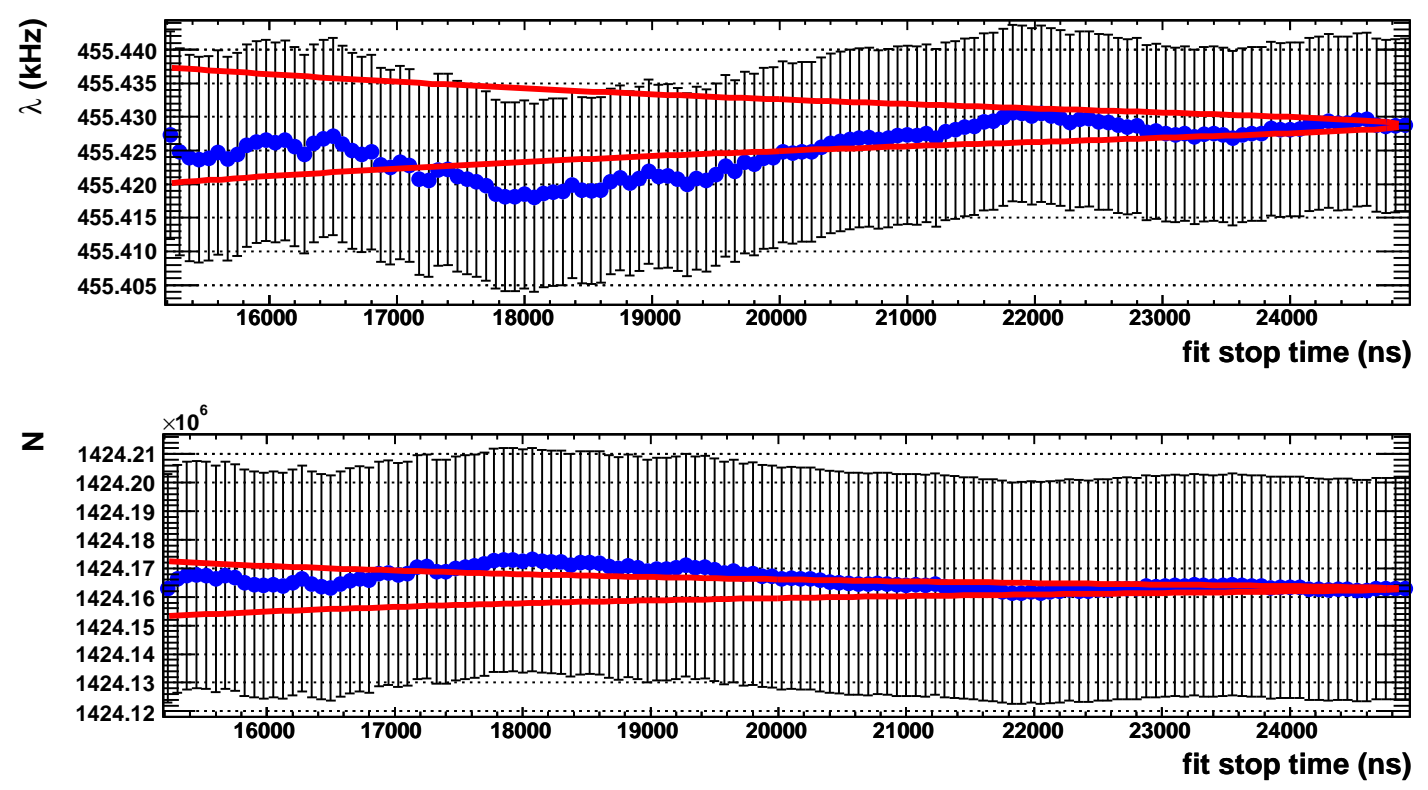

$m$
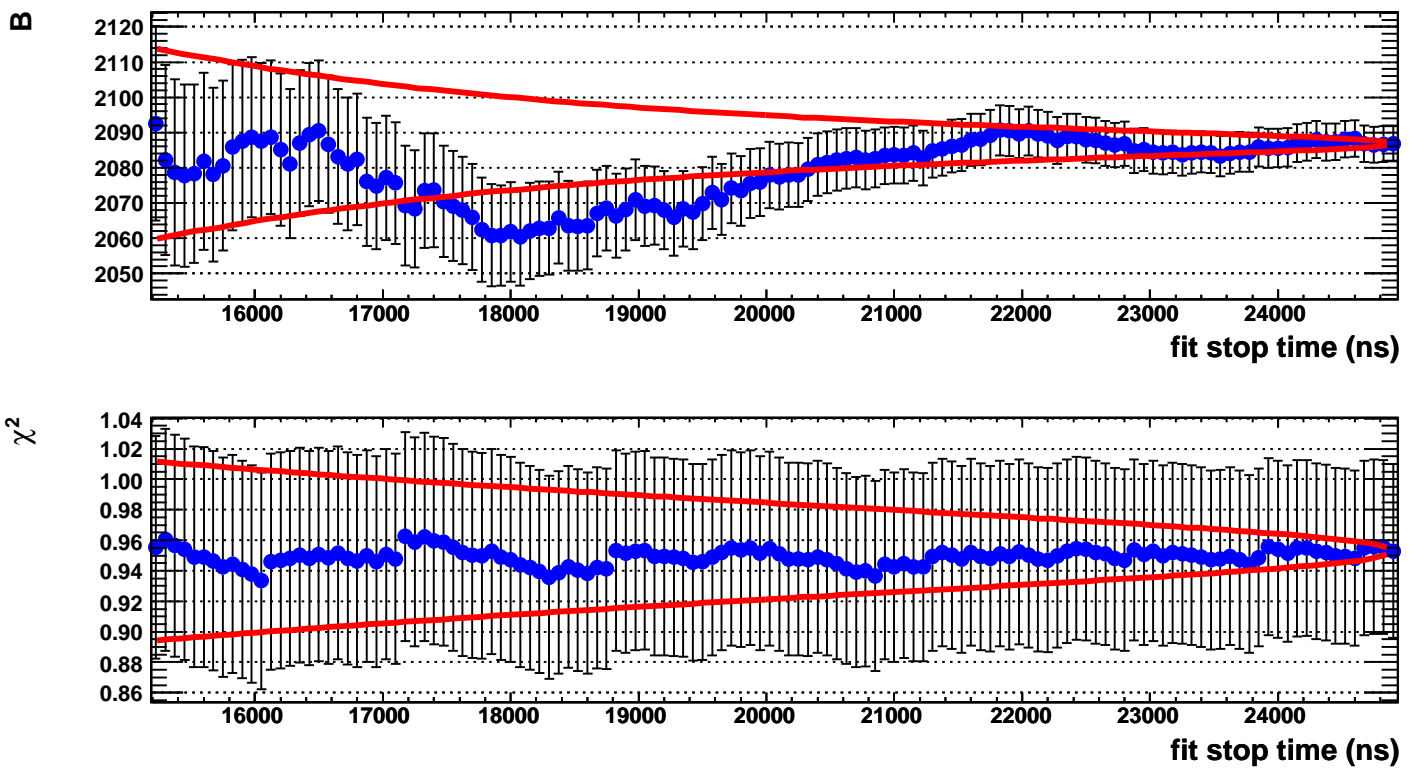

Figure 6.25: Fit results from a stop time scan over the range 15-24.9 $\mu \mathrm{s}$, at 75 ns intervals, with the fit start time and histogram 32-rebin boundary both fixed at 100 ns. All of the fit parameters remain consistent with the statistically allowed $1 \sigma$ band indicated by the solid lines. 
there is no evidence of any systematic, nonstatistical behavior. Note that the start time scans exhibit larger variations in the fit parameters than the stop time scans. This can be attributed to the simple fact that there is more data from the decay spectrum at the early times covered in the start time scan, whereas the stop time scan covers a region where the bin counts are much lower and where the flat accidental background comprises a larger fraction of the counts. Of course, these sorts of statistical considerations are already taken into account by the $1 \sigma$ error bands.

It should be noted that the ROOT fit algorithm will yield the same results for any fit start times that lie between the midpoints of two adjacent histogram bins. Thus, for a 32-rebinned lifetime spectrum whose rebin boundary is fixed at $100 \mathrm{~ns}$, any fit start time in the interval $[80,120] \mathrm{ns}$ is effectively identical. The truly important step is to have placed the 32-rebin boundary at $100 \mathrm{~ns}$ to ensure that no data from $\Delta t<100 \mathrm{~ns}$ contributes to the fit.

It should also be noted that the stable start time scan results in Figure 6.24 are not in general reproduced in fits to impurity-doped calibration data, because of the presence of either $Z>1$ capture rate components or deuterium diffusion effects in the decay spectrum. Of course, such instabilities can be mitigated or eliminated if one uses a more descriptive fit function which contains the complete muon kinetics in hydrogen (see Section 7.3.1).

Although we can mathematically describe the basic shape of the lifetime spectrum's pileup background [214], we do not generally include it in our fits. The reason is that the pileup background contains multiple $Z>1$ capture components due to muon stops in detector materials, and therefore it is not accurately described by a single, clean exponential decay rate. However, it is conceivable that the data in the lifetime histogram just before $\Delta t=0$ could provide information useful for constraining the fit parameter $B$, because the pileup background in that region has nearly disappeared and its $Z>1$ com- 
ponents have long died away. There are two possibilities for including the early-time data in the fits: (1) one could continue to use the standard three-parameter exponential fitting function in Equation 6.2 which contains a flat background $B$, and restrict the fit start time to the region immediately before $\Delta t=0$ where the pileup background is nearly zero, or (2) one could use a four-parameter fitting function of the form

$$
f(t)=N w \lambda e^{-\lambda t}+B+P e^{-\lambda(t+25000 \mathrm{~ns})},
$$

where the last additional term describes a pileup background which has a single exponential component. The four-parameter fitting function is valid over a wider portion of the $\Delta t<0$ region than the simpler three-parameter function, but the four-parameter function has the disadvantage that its pileup scaling parameter $P$ is poorly defined at early times because the pileup background is so small. Of course, when using either function one must be careful to blank out the histogram data in the time region $-10 \mathrm{~ns}<\Delta t<100 \mathrm{~ns}$ so that the signal turn-on and triplet depopulation periods do not contribute to the fits. Start time scans from the three- and four-parameter fits indicate that extending the fit range to cover early times gives essentially the same results as fitting over the standard range $[100,24900] \mathrm{ns}$. This suggests that the accidental background level $B$ is already well constrained by the data at late times in the lifetime spectrum. Moreover, these results indicate that any $Z>1$ impurity capture components in the pileup background are effectively extinct by $\Delta t=0$, and thus have no effect on the fitted disappearance rate.

\subsubsection{Adjustments for cosmics}

The accidental background of each lifetime histogram receives contributions from cosmic muons which pass through the electron detectors and are mistakenly interpreted as decay electrons. The effects of cosmics are clearly evident in the background levels observed 


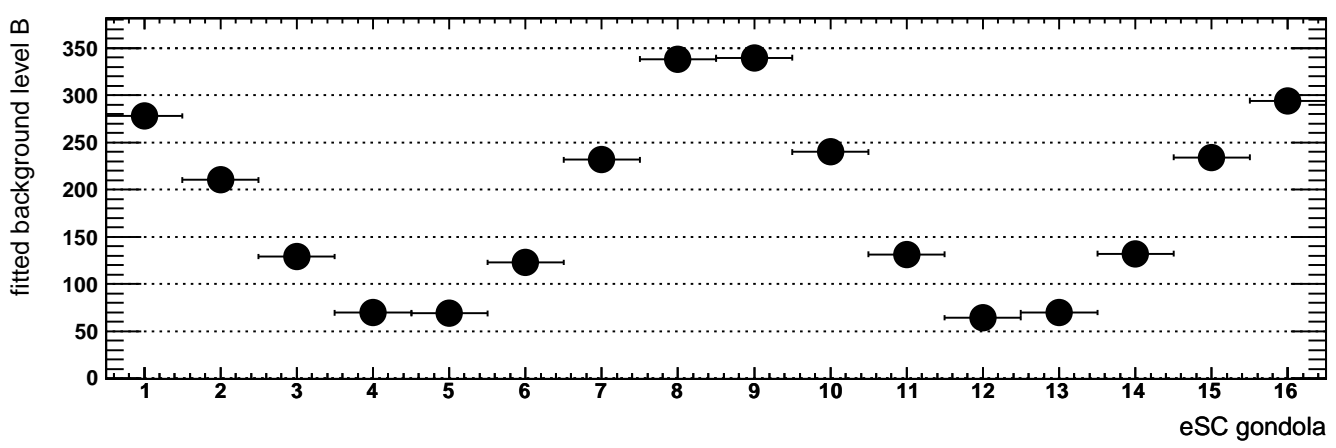

Figure 6.26: The backgrounds $B$ from fits to the individual lifetime spectra of the sixteen eSC gondolas. Cosmic muons are responsible for the sinusoidal shape of the distribution.

in fits to the lifetime spectra of the sixteen gondola segments (Figure 6.26). It is possible to improve the quality of the lifetime fits by first identifying the eSC hits that correspond to throughgoing cosmics, and then making appropriate adjustments to the lifetime histograms.

To identify throughgoing muon cosmics, I look for pairs of simultaneous electron detector tracks that are roughly colinear and oppositely directed. Here are the exact steps in my identification algorithm:

1. I join together all electron track pairs that are coincident within the time interval $|\Delta t| \leq 15 \mathrm{~ns}$, where the timing information comes from the eSC detector.

2. For each pair of temporally coincident tracks, I use the law of cosines to calculate the angle $\alpha$ between the two vectors. I then enforce a three-dimensional antiparallel condition, $\alpha \geq 3 \pi / 4$, as well as a similar two-dimensional antiparallel condition on the vectors' projection onto the $(r, \phi)$ plane of the electron detectors. The second, seemingly redundant antiparallel condition protects against rare events where the two electron tracks are oriented nearly lengthwise with respect to the electron detectors, and strike proximate anodes in a way that satisfies the first antiparallel condition, yet clearly cannot correspond to a cosmic event. 
3. I require that the distance of closest approach between the two tracks' extended lines must be $\leq 26 \mathrm{~mm}$, a number based upon the distribution of histogrammed impacts.

If a pair of tracks passes the preceding cuts, the tracks are considered part of a single cosmic event and the $(\phi, \theta)$ coordinates describing the cosmic vector are calculated from the line passing through the two tracks' ePC2 points. The resulting angular distribution of cosmic rays is plotted in Figure 6.27, and its basic features and statistics conform with expectations.

Once throughgoing cosmics have been identified, the question remains how to best use this information in the analysis of lifetime spectra. One possibility is to subtract the portion of accidental background that can be attributed to cosmics (Figure 6.28). However, I am wary of subtracting cosmic events from the decay spectra, for two reasons: (1) it isn't essential to remove cosmics, as they in principle only contribute a flat background, and (2) the distribution of identified cosmics is not perfectly uniform. As Figure 6.28(b) reveals, a small muon decay component is present in the cosmics background near $\Delta t=0$. Thus my cosmics identification algorithm is manifestly imperfect, and subtracting such events might introduce unwanted time-dependent structure.

A more attractive alternative is to enlarge the errors on the lifetime histogram bins to compensate for the double-counting produced by cosmics. That is, the statistical error on a bin with $N$ counts is generally taken to be $\sigma=\sqrt{N}$, but this value is too small because it assumes that all $N$ events are independent, whereas some of them are actually pairs of hits from a single cosmic event. In order to determine the correct bin error, let us write the number of counts $N$ in a given bin as the sum of signal and accidental background 


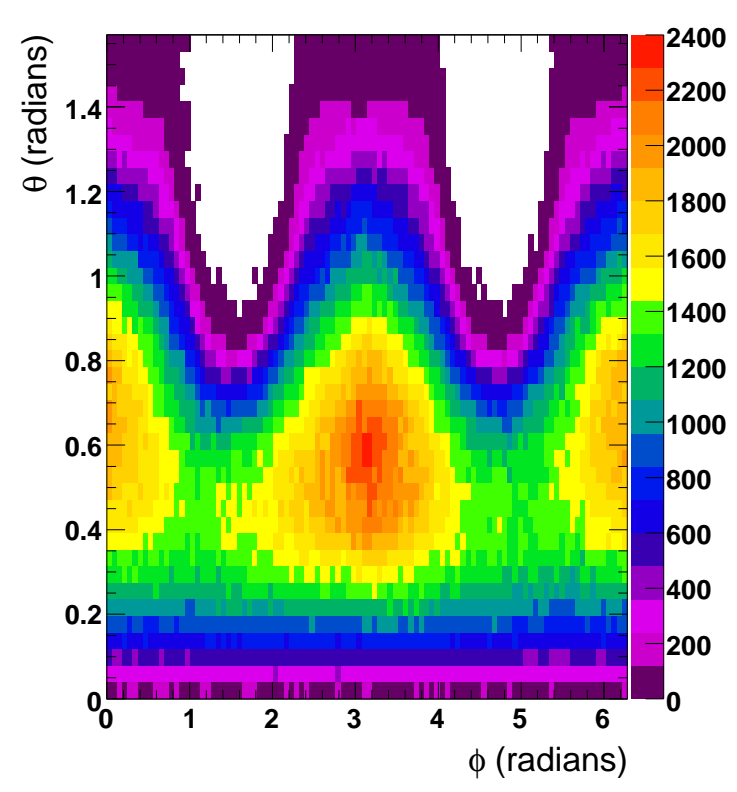

(a)

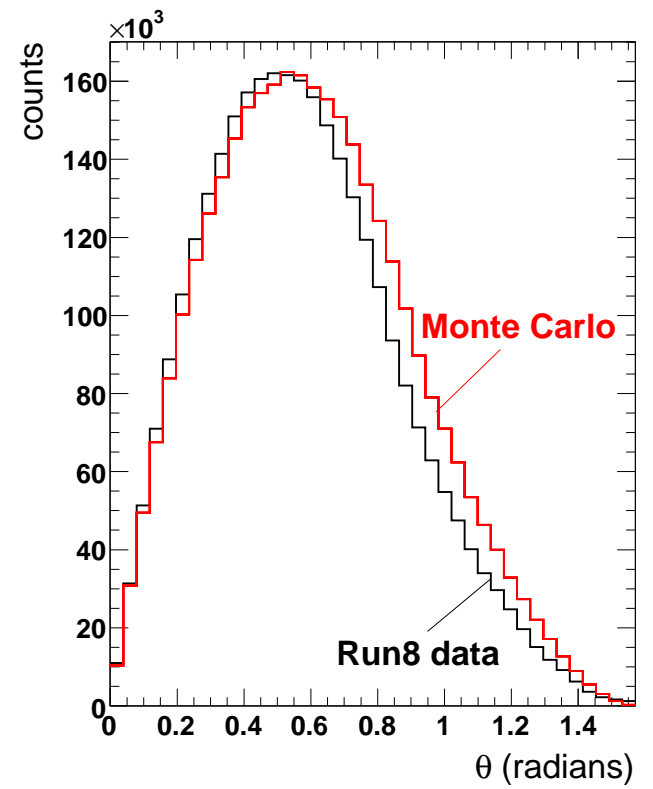

(b)

Figure 6.27: (a) The angular distribution of cosmics identified in the 2004 data, in spherical coordinates. The azimuthal angle $\phi=0$ lies along the experiment's $+x$ coordinate axis, while the zenithal angle $\theta=0$ lies along the $+y$ coordinate axis, in accordance with our standard conventions. (See Figure 4.1 for a diagram of the experimental coordinate system.) The two semicircular holes in the $(\phi, \theta)$ plot correspond to the openings at the ends of the eSC cylinder, which is the limiting detector in terms of solid angle coverage. (b) The $\theta$ projection of the experimental cosmics distribution in (a), alongside a version generated by my fast Monte Carlo software, which performs a purely geometrical simulation of the effects from a $\cos ^{1.8} \theta$ cosmics distribution incident on the cylindrical electron detectors. The real and simulated distributions are basically consistent, and the small discrepancies between them can probably be attributed to detector efficiency variations and shielding asymmetries (e.g. in the arrangement of the $\pi \mathrm{E} 3$ area's concrete, and in the site's surrounding geographical features). 


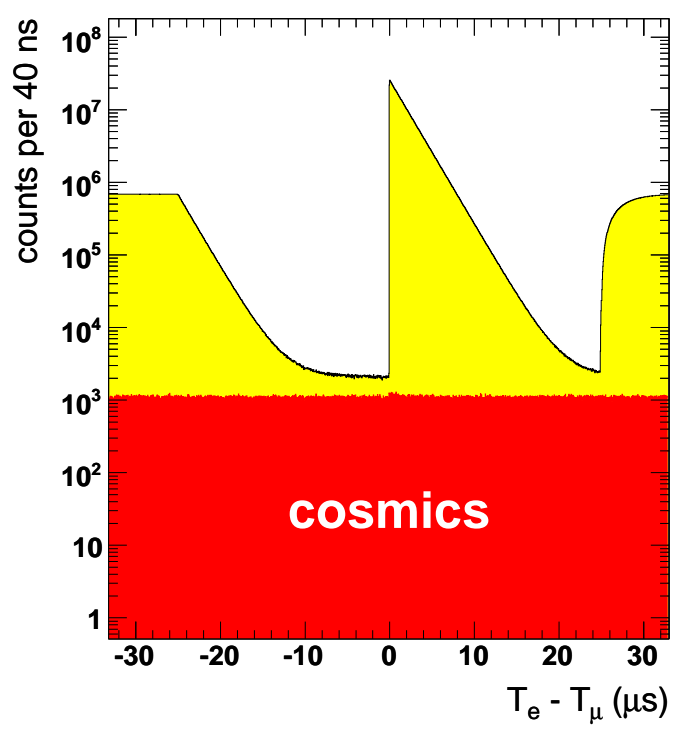

(a)

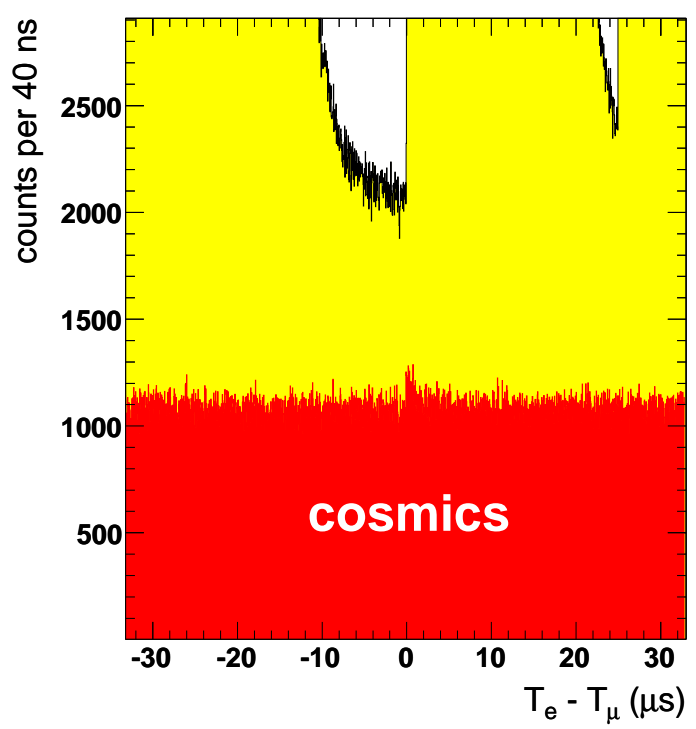

(b)

Figure 6.28: The accidental background corresponding to identified cosmic events, plotted atop the lifetime spectrum in (a) wide-view log scale, and (b) zoomed-in linear scale. It is clear from plot (b) that cosmics account for roughly half of the accidental background.

terms,

$$
\begin{aligned}
N & =S+B \\
& =S+B_{\text {cosmics }}+B_{\text {other }},
\end{aligned}
$$

where $B_{\text {cosmics }}$ is the portion of background attributable to cosmics. Since cosmic events generate pairs of hits, the corresponding error is

$$
\sigma_{B_{\text {cosmics }}}=2 \sqrt{\frac{B_{\text {cosmics }}}{2}}=\sqrt{2 B_{\text {cosmics }}}
$$

while the error for the non-cosmic background contribution is simply

$$
\sigma_{B_{\text {other }}}=\sqrt{\left(B-B_{\text {cosmics }}\right)}
$$


If we plug these formulas into the expression for the bin error, we get

$$
\begin{aligned}
\sigma_{N}^{\text {corrected }} & =\sqrt{\sigma_{S}^{2}+\sigma_{B_{\text {cosmics }}^{2}}^{2} \sigma_{B_{\text {other }}}^{2}} \\
& =\sqrt{S+\left(2 B_{\text {cosmics }}\right)+\left(B-B_{\text {cosmics }}\right)} \\
& =\sqrt{S+B+B_{\text {cosmics }}} \\
& =\sqrt{N+B_{\text {cosmics }}} .
\end{aligned}
$$

As Figure 6.28(b) shows, $B_{\text {cosmics }} \approx B / 2$. To adjust the bin errors for cosmics, I first perform an initial fit to the lifetime histogram to obtain an estimate for the total background level $B$. I then change each bin's error from $\sqrt{N} \rightarrow \sqrt{N+B / 2}$, and perform a second fit to obtain the final results. This two-stage fit procedure produces a noticeable improvement in the fit's reduced $\chi^{2}$ value, typically reducing it from $\chi^{2} / d=1.15(6)$ to $1.05(6)$ ), but has little effect on the fitted rate $\lambda$, lowering it by $0.25 \mathrm{~s}^{-1}$ at most.

With the exception of fits to the "unique electron" lifetime histograms (see Section 6.5.16), I always assume that cosmics account for half of the total accidental background and adjust the bin errors accordingly. I should note, however, that due to software complications I was only able to establish that cosmics comprise half the background for a particular set of lifetime histogram conditions. Nevertheless, it seems reasonable to assume that the cosmics fraction must be similar for other conditions, and in fact Steven Clayton has observed as much in his own analysis. In any case, the cosmics-driven adjustment of histogram bin errors serves primarily to improve the fits' $\chi^{2}$ values, and a crude cosmics estimate is sufficient.

It is worth noting that similar considerations also apply in principle to the electron multiplicities that can arise from scattering and from rare, multi-electron muon decay modes [215]. Establishing an exact numerical bound on such effects is extremely difficult, but they are believed to be negligible compared to the effects from cosmics. Indeed, the 
cosmics adjustments described above improve the $\chi^{2}$ of the lifetime fits to the point where no further action is necessary.

\subsubsection{The impact parameter}

In MuCap analysis parlance the term "impact parameter" is used to refer to the distance of closest approach between the linear trajectory of an outgoing decay electron and the stopping position of its parent muon, as measured by the detectors. This distance, typically denoted as $b$, can be easily calculated using standard geometrical formulas.

The impact parameter plays two important roles in the Run8 analysis. First, spatial acceptance cuts of the form $b \leq b_{\max }$ serve as our best means of performing vertex matches of muon/electron decay pairs, since we lack any other information about the electron's actual point of emission. (Recall that the TPC did not reach sufficient voltage to detect Michel electrons.) It is worth noting that impact cuts are applied without regard to the location of the muon stop. In some sense, this is not perfectly appropriate, because the same impact cut can in principle produce different effects at different muon stop locations, depending upon the distribution of surrounding detector materials which can scatter decay electrons. Such effects are believed to be negligible, however, and we lack any better practical alternative. Simple impact parameter cuts enable us to effectively discard many uncorrelated muon/electron pairs and reduce the accidental background in the lifetime spectra. Second, the variation in the fitted decay rate as a function of the impact cut $b$ can be used to determine the deuterium concentration ratio between different gas fills, a quantity

which is needed for the deuterium zero-extrapolation correction. That procedure will be discussed in more detail in Section 6.6.4

In Figure 6.29 I have plotted the impact parameter distribution observed in Run8, as well as its Cartesian components. The $b_{x}$ and $b_{y}$ distributions are narrower than the $b_{z}$ 

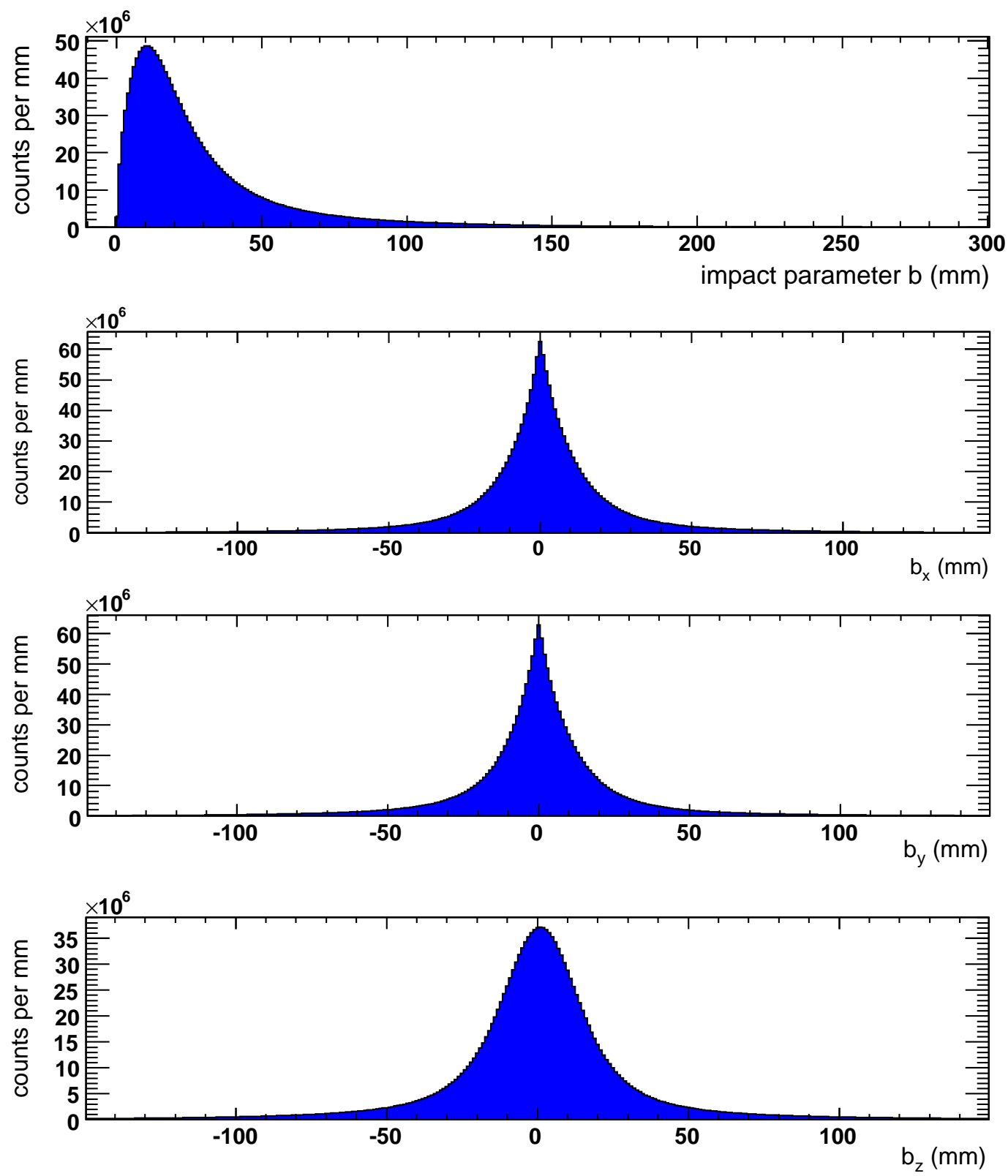

Figure 6.29: The Run8 impact parameter distribution and its $x, y$, and $z$ components. 
distribution because the $\mathrm{ePC}$ anodes are more closely spaced than the cathodes and thus provide better resolution. In order to center the $b_{y}$ and $b_{z}$ distributions, I had to add small offsets of $-2 \mathrm{~mm}$ and $8.5 \mathrm{~mm}$, respectively, to compensate for the displacement of the TPC relative to the coordinate origin of the electron detectors. I also tuned the angular $\phi$ offsets for $\mathrm{ePC} 1$ and $\mathrm{ePC} 2$, relative to the eSC, until the location of the impact distribution's peak was minimized at $\approx 10.5 \mathrm{~mm}$.

The experimental impact parameter resolution is limited primarily by electron scattering from the $4 \mathrm{~mm}$-thick $\mathrm{Al}$ pressure vessel walls, a feature confirmed by my fast Monte Carlo simulations of small-angle multiple scattering in our setup. Moreover, in 2001 we recorded data without the TPC and pressure vessel installed, instead stopping $32 \mathrm{MeV}$ muons in an array of six scintillator targets, each with a cross-sectional area of $5 \times$ $5 \mathrm{~mm}^{2}$. Steven Clayton analyzed that data and found that the electron track reconstruction resolution of the (partially instrumented) ePCs was better than $10 \mathrm{~mm}$ when no intervening materials were present to scatter the electrons.

The extended tail in the impact parameter distribution creates subtle implications for any cuts on the impact parameter. Namely, the millimeter-scale, time-dependent process of $\mu p$ diffusion is magnified by electron scattering, so performing an impact cut - even at relatively large $b$ values - increases the effective muon disappearance rate. The effect on the fitted rate varies inversely with the size of the impact parameter cut, as shown in Figure 6.30. This mechanism has been repeatedly corroborated by Monte Carlo simulations, and, as we will see in Section 6.6.4, its effects are even more pronounced for $\mu d$ diffusion, which is much faster than $\mu p$ diffusion.

We chose $b \leq 120 \mathrm{~mm}$ as our standard impact parameter cut because it lies relatively far out in the tail of the impact distribution. As such, it preserves statistics and mandates a relatively small $\mu p$-diffusion-related correction $\left(2-3 \mathrm{~s}^{-1}\right)$, while still providing 


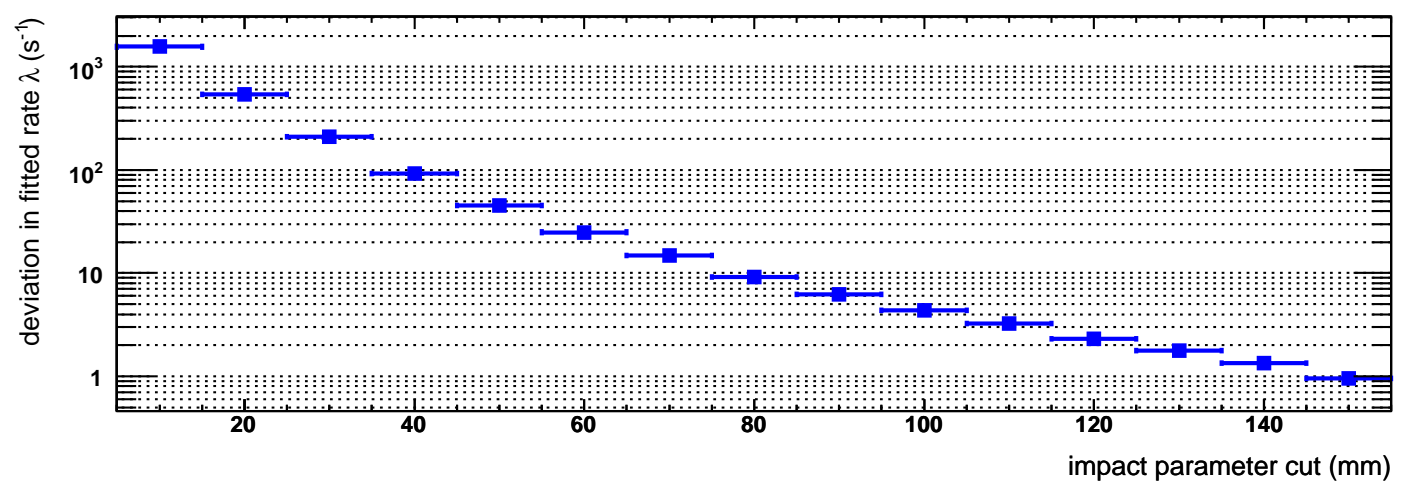

Figure 6.30: The $\mu^{-}$disappearance rate as a function of impact parameter cut. The nonuniformity in the $\lambda$ values arises from the magnification of $\mu p$ diffusion effects by electron scattering from the pressure vessel. This plot was created by Steven Clayton, who simulated the effect using my Run8 impact distribution in conjunction with a simple model of thermal $\mu p$ diffusion with constant $k=0.4875 \mathrm{~mm} / \sqrt{\mu \mathrm{S}}$ (see Appendix $\mathrm{C}$ ), extracted from his own analysis. The shape of the distribution is determined by the impact parameter resolution, while the diffusion constant $k$ determines the scaling factor-though the distribution is not very sensitive to the exact value of $k$.

some degree of background suppression. A $120 \mathrm{~mm}$ impact cut generally reduces the accidental background by a factor of $2-4$, depending upon the particular lifetime histogram conditions.

\subsubsection{Magnetic field effects on the impact parameter}

The $\mu^{+}$SR saddle coil magnet was energized during most of Run8, for the sake of maintaining stable experimental conditions during both $\mu^{+}$and $\mu^{-}$data taking. The magnet suffuses the interior of the pressure vessel with a nearly uniform 50 gauss magnetic field, intended to induce controlled precession in positive muons. (The presence of a magnetic field is irrelevant for negative muons after they have stopped in the target.) Unfortunately, the magnetic field has an undesirable side effect: it bends the trajectories of outgoing decay electrons and positrons, possibly compromising the integrity of the impact parameter. It is therefore important to ascertain the magnitude of the deflections induced by the magnetic 
field and determine if it skews the impact parameter in a significant and systematic way.

First, let us survey the underlying physics. A charged particle moving through a uniform magnetic field $B$ will follow a helical trajectory, traveling with constant speed in the direction parallel to the field while executing simple harmonic motion in the "bending plane" perpendicular to the field. The radius of the circular orbit is given by the simple formula

$$
r=\frac{p_{\perp}}{(0.3 B)}
$$

where $p_{\perp}$ is the particle's momentum component in the bending plane in $\mathrm{GeV} / \mathrm{c}, B$ is in Tesla, and $r$ is in meters. The largest deflections occur when the electron is emitted perpendicular to the $B$ field. In this case, for a typical Michel electron of energy $30 \mathrm{MeV}$ moving in a 50 Gauss, or 0.005 Tesla, magnetic field, Equation 6.10 gives a radius of curvature of $r=20 \mathrm{~m}$. This simple estimate indicates that, in the worst-case scenario, the bending radius is much larger than the size of the MuCap apparatus and thus deviations from straight line travel should be relatively small. However, there might be problems at lower electron energies.

To resolve the issue, I implemented magnetic-field-induced helical electron trajectories in the same Monte Carlo framework that I had originally designed for studying the effects of $\mu p$ and $\mu d$ diffusion and decay electron scattering on the impact distribution and lifetime spectrum. My program recreates the Run8 muon stopping distribution in the TPC; for each muon it selects a random direction for the emission of the Michel electron, and assigns the electron an energy sampled from the Michel spectrum. I next iteratively step through the electron's helical trajectory inside the pressure vessel's 50 Gauss field, which points in the $+x$ direction in our experiment's coordinate system. When the electron's path intercepts the pressure vessel wall, I calculate the electron's instantaneous velocity vector at that point. We can reasonably assume that the electron subsequently escapes from the 


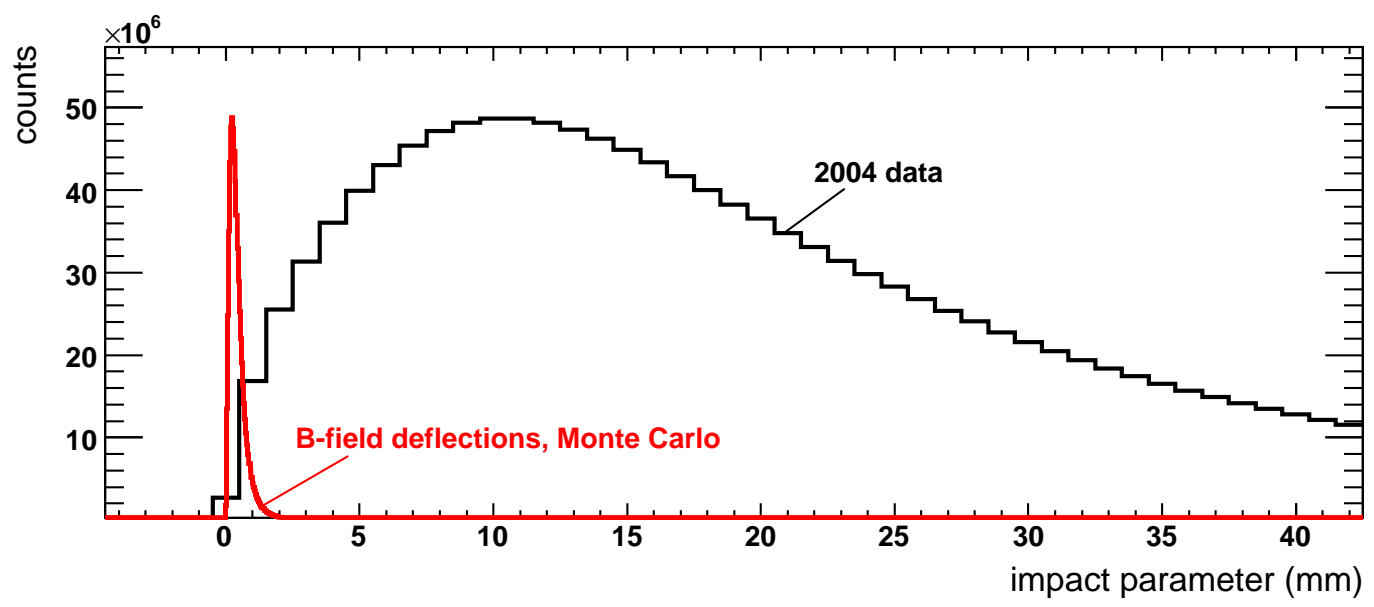

Figure 6.31: Impact parameter distributions from (1) Monte Carlo simulations of magneticfield-induced deflections of the outgoing decay electron trajectories, and (2) the 2004 experimental data, where electron scattering from the pressure vessel dominates. The magnetic field clearly has a relatively small effect on the impact parameter.

magnetic field and moves in a straight line, so the velocity vector at the pressure vessel wall determines the impact parameter. No $\mu p$ diffusion or electron scattering is involved in this particular simulation, so any nonzero impact parameter values are due entirely to magnetic field deflections.

The Monte Carlo-generated impact parameter distribution arising from magnetic field deflections is plotted in Figure 6.31 atop the impact distribution from Run8. It is clear that the magnetic field makes a tiny contribution to the experimentally observed impact distribution, which is dominated by scattering from the pressure vessel. The simulation convincingly demonstrates that we do not need to be concerned about magnetic field effects.

\subsubsection{Data selection}

During Run8 we recorded data under a wide variety of conditions. At various intervals we collected $\mu^{+}$and $\mu^{-}$decay events, and we doped the hydrogen gas with assorted levels of elemental and isotopic impurities for the purposes of calibration. We also 


\begin{tabular}{llccc}
\hline \hline $\begin{array}{l}\text { Gas fill } \\
\text { label }\end{array}$ & Description & $\begin{array}{c}\text { TPC voltage } \\
(\mathrm{V})\end{array}$ & Runs & $\begin{array}{c}\text { Statistics } \\
\left(\times 10^{9}\right)\end{array}$ \\
\hline Prod-50 & $\begin{array}{l}\text { Clean production data } \\
\left(c_{d} \approx 1 \mathrm{ppm}, c_{Z} \approx 0.01 \mathrm{ppm}\right)\end{array}$ & 5017 & $11650-14140$ & 1.5 \\
& & & \\
CalibN2 & $\mathrm{N}_{2}$-doped $\left(c_{N} \approx 11 \mathrm{ppm}\right)$ & 4817 & $14530-14648$ & .08 \\
CalibD2 & Deuterium-doped $\left(c_{d} \approx 20 \mathrm{ppm}\right)$ & 4817 & $14738-15036$ & .21 \\
CalibNat & Deuterium-doped $\left(c_{d} \approx 120 \mathrm{ppm}\right)$ & 4817 & $15072-15220$ & .06 \\
\hline \hline
\end{tabular}

Table 6.1: Run8 gas fills of interest in the $\mu^{-}$analysis, presented in chronological order. The fill labels are taken from Dr. Francoise Mulhauser's categorization of the Run8 data in reference [216]. The exact details of the $Z>1$ and deuterium contents of each fill will be examined in more detail in Section 6.6. The "Runs" column corresponds to the interval of usable run files recorded by the DAQ during the fills; each run corresponds to roughly fifteen minutes of continuous data taking. The statistics column presents the approximate number of decay events remaining after all cuts have been made, including protection against muon arrival pileup.

experienced unexpected changes in the performance of some detectors, most notably the TPC.

In February 2005, Dr. Francoise Mulhauser surveyed all of the available information on Run8 experimental conditions, as archived in the shift summaries and electronic logbooks, and she catalogued her findings in reference [216]. There, the Run8 data is categorized primarily by the gas fill and TPC voltage. The four gas fills of chief interest for the $\mu^{-}$ analysis are listed in Table 6.1. The Prod-50 clean production fill comprises the main body of data for our $\mu^{-}$measurement, and the calibration fills are used to correct for the effects of $Z>1$ and deuterium impurities on the Prod-50 $\mu^{-}$disappearance rate. The statistical requirements for the calibration fills are lower than for the production measurement, as will be explained in Section 6.6.

Note that the TPC's voltage dropped from $5.0 \mathrm{kV}$ in the Prod-50 fill to $4.8 \mathrm{kV}$ in the calibration fills. This deterioration in the stable operating voltage of the TPC occurred near the end of the clean-fill data taking, and necessitated adjustments to the TPC thresh- 
olds. An operating voltage of $4.8 \mathrm{kV}$ is believed to have little effect on the identification of good muon stops, but the decreased gain does lower the efficiency for the identification of $\mu+p$ scatter events and captures by $Z>1$ impurities. Consequently, I decided to take a conservative approach and only use the more reliable Prod-50 data, ignoring the Prod-48 data. The shortcomings of the $4.8 \mathrm{kV}$ operating voltage are of less importance in the calibration fills, where the statistics are lower and effects from impurities dominate.

I should mention that we also performed an oxygen-doped fill and a water-doped fill at the close of Run8 data taking, but these fills are not considered in this document. The reason is that the oxygen-doped fill was a failure, due to the fact that the oxygen quenched the TPC charge and rendered the data meaningless. The water-doped fill suffered from high levels of supplemental outgassing of unknown elemental composition from within the pressure vessel; as such, the experimental conditions from that particular fill were poorly determined.

The data from each gas fill are divided into numbered run files which vary in size but each of which typically corresponds to fifteen minutes of data taking. We must be careful in selecting which runs are ultimately allowed to contribute to the lifetime spectra, as not all of the data collected within a given fill's run interval are suitable for inclusion. The reason is that, although the DAQ operation was mostly continuous, we occasionally interrupted data taking to perform diagnostic tests, etc., and the apparatus also suffered from the occasional, temporary problems that occur with any experiment. The latter is especially true for the principal Prod-50 data, as there were a number of variations in experimental conditions during this period - replacement of the $\mu \mathrm{SC}$, the PSI power outages, etc.- which could affect the high-precision fitted rate. To facilitate the judicious selection of acceptable runs, the information in Dr. Mulhauser's modified Run8 shift summary was incorporated into a MySQL database used to organize and track analyses of the Run8 data. An obvious 
first cut was to only analyze runs marked as "good" in the shift summary. I also excluded a small subset of runs which exhibited detector misbehavior in the data analysis - most notably, some runs which exhibited anomolously large or small electron detector inefficiencies (see Section 6.5.15). As might be expected, a small fraction of the ostensibly "good" runs exhibited pathological problems and were never successfully processed for undiagnosed reasons. The analyzed runset also tended to vary slightly between analysis production passes, due to inevitable hiccups in the data processing.

Lastly, I should mention that Dr. Frederick Gray at one point checked the $\mu^{-}$data run-by-run for $\mu^{+} \mathrm{SR}$ oscillations, but found no evidence of misclassified $\mu^{+}$runs.

\subsubsection{Special data cuts}

In this section I discuss data cuts related to data block "bookending," detector "sparks," and misbehavior in the CAEN TDC modules.

\section{Bookending}

Detector data is recorded by the DAQ in discrete blocks of time. Muons that are located near the edges of these data blocks can generate distortions in the lifetime spectra, for two reasons: (1) the effectiveness of pileup protection on near-edge muons is compromised because of the absence of muon information beyond the block edges, and (2) the contributions of near-edge muons to the accidental background is nonuniform (sloping down as one moves away from $\Delta t=0$ ) because of the absence of electron information beyond the block edges. To avoid these problems, I implemented a "bookend" cut which removes any $\mu \mathrm{SC}$ hits within $40.5 \mu \mathrm{s}$ of either edge of each data block. The bookend width needs to be at least as large as the pileup protection interval in order to ensure effective muon pileup protection at the edges of the data block; the pileup protection interval is $\pm 25 \mu \mathrm{s}$, so this 
requirement is satisfied. The width of the bookend cut also determines how far out the accidental background remains flat, relative to $\Delta t=0$. The standard $40.5 \mu \mathrm{s}$ bookend cut ensures a flat background over the standard fit range between 0 and $25 \mu \mathrm{s}$. The $40.5 \mu \mathrm{s}$ bookend cut discards roughly $1 \%$ of the muon data.

\section{Detector sparks}

In my analysis terminology, a "spark" is an event in which an excessive number of wires fire simultaneously in a detector-for example, as a result of a sudden electric discharge. I recognize two categories of detector sparks: local and global. A local spark is a high-multiplicity event in a single detector (in the case of $\mu \mathrm{PC} 1$ and the ePCs, this applies separately to the individual detector planes), while a global spark is an occasion when "local" sparks appear in nearly every MuCap detector at the same time. The exact reasons for such occurrences remain unknown.

I have found that an effective method for identifying global sparks is to look for instances when all six ePC detector planes exhibit simultaneous local sparking (see Figures 6.32 and 6.33). When this occurs, I conservatively discard the entire data block; this resulted in a roughly $0.3 \%$ cut of the total data. The global spark cut does not eliminate all sparking-related effects, however, because most of the detectors still suffer from individual, local sparking. For example, the ePCs often spark in preamp card groups (Figure 6.34). To protect against these more frequent occurrences, I discard all data from both the guilty detector and from the $\mu \mathrm{SC}$ within a $[-5,50] \mu \mathrm{s}$ time interval around the local spark, where the long $50 \mu$ s delay is based upon the typical recovery time of an ePC chamber. It should be noted that a bookending interval of $40.5 \mu \mathrm{s}$ is added to the cut when the local spark is from an electron detector. The artificial deadtime clustering decribed in Section 6.4.1 suppresses any remaining, smaller-scale multiplicities in hot wires or channels. 

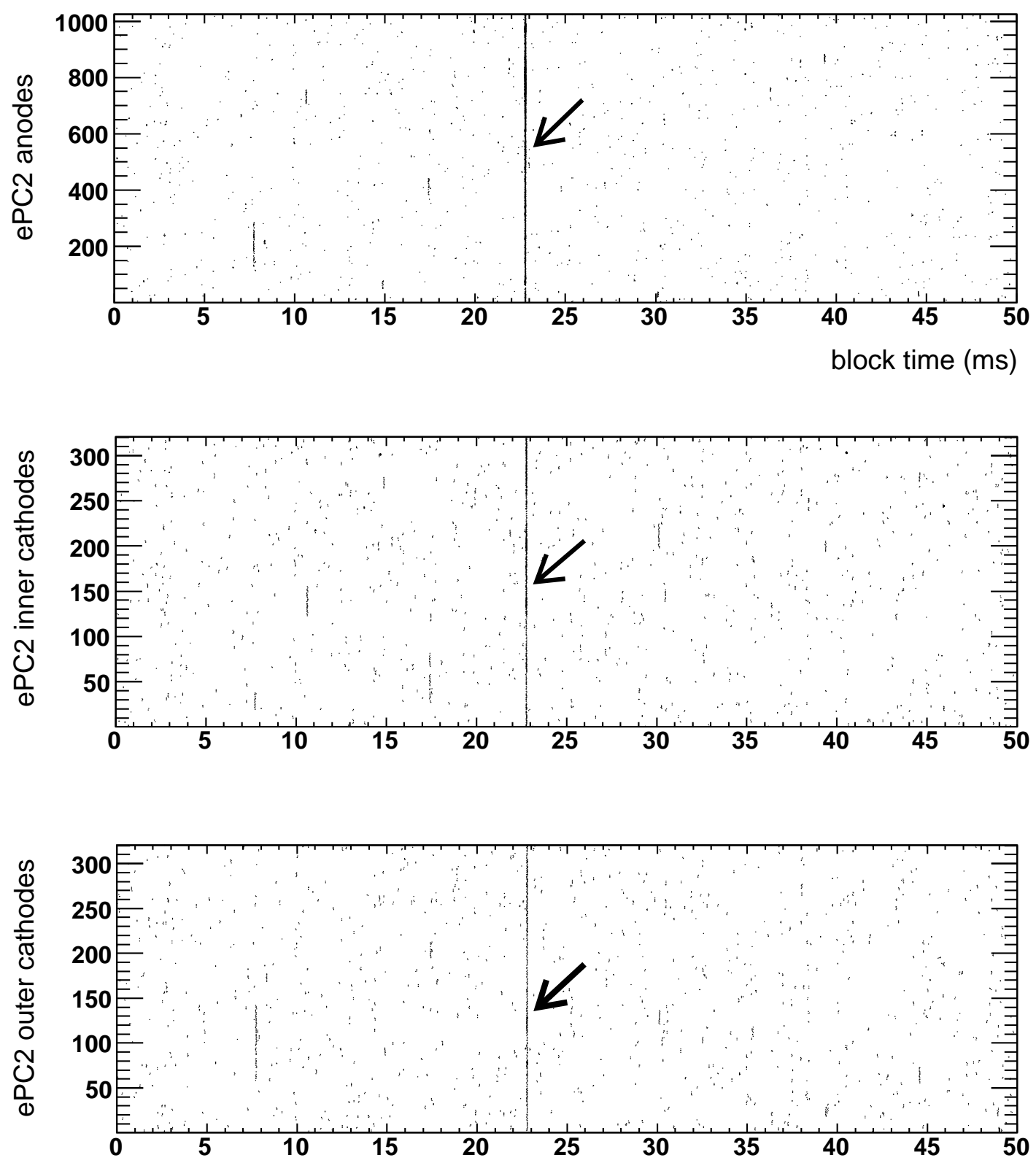

Figure 6.32: Hits in the ePC2 anode, inner cathode, and outer cathode planes during the first $50 \mathrm{~ms}$ of a data block (the time bins are $50 \mu \mathrm{s}$ in width). A chamberwide spark, indicated by the arrows, is easily recognized by the high multiplicity of nearly simultaneous hits across the $\mathrm{ePC} 2$ wires in all three planes. 

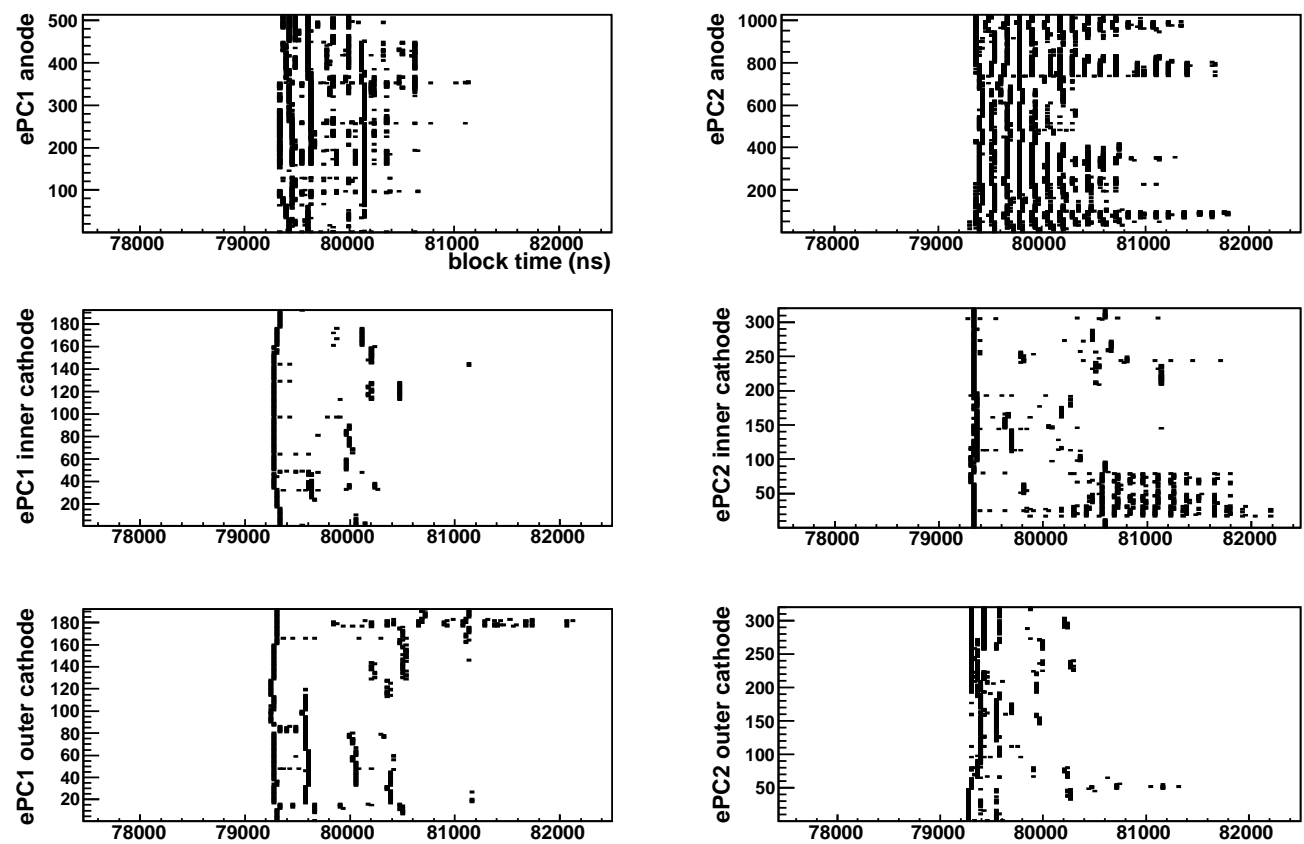

Figure 6.33: A close-up view of simultaneous chamberwide sparks in ePC1 and ePC2; notice the post-spark "ringing." When all six ePC planes exhibit sparks like this, the event is considered a "global" spark and the data block is cut. The spark pictured here is similar to that shown in Figure 6.32, but on a finer timescale.

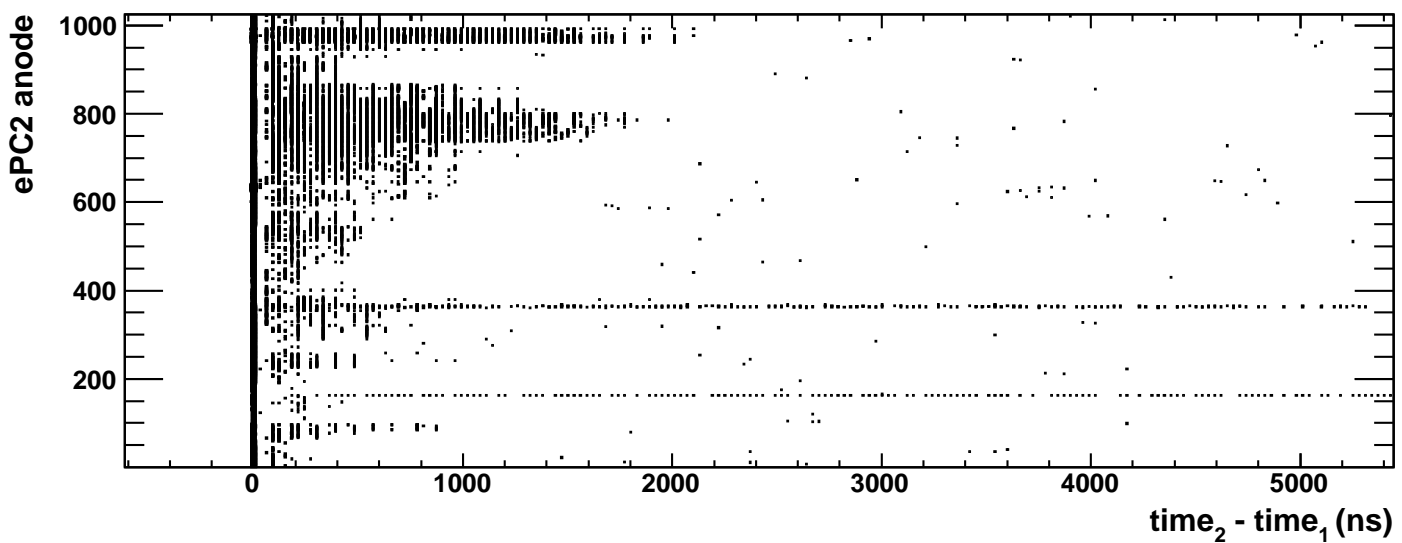

Figure 6.34: Two-dimensional autocorrelation plot of hits in the ePC2 anode plane. The plot reveals the persistence of local sparks, or afterpulsing, even after global spark cuts have been performed. Sparking/afterpulsing in the ePC2 preamp card 16-wire groups is clearly evident. 


\section{CAENs}

We have struggled for years with the CAEN V767 TDC modules [183], which have exhibited many serious deficiencies. In retrospect, we could probably not have made a worse choice of TDC for use in a precision lifetime experiment. A complete recounting of our history of CAEN problems is beyond the scope of this paper, so below I will simply describe the CAEN misbehaviors of most concern, and how we minimized their effects through a combination of preventive action during Run8 and intelligent cuts in the ensuing data analysis.

Here is a list of the most salient CAEN issues we were forced to deal with:

- Rollover: As discussed earlier in Section 6.2, the CAENs have a 20-bit time word that rolls over without any indication. To ensure accurate time reconstruction, we input $2.5 \mathrm{kHz}$ signals into each of the three CAENs. As a result, the clock rollovers never presented any problems. The $2.5 \mathrm{kHz}$ rollover inputs also enabled us to check the synchronization of the three CAENs.

- TDC desynchronization: Sometimes one of the four TDCs within a CAEN will slip behind the other three TDCs by a single external clock tick. Such an occurrence can be difficult to identify. It was first observed in the $2003 / 2004$ analysis of the Run7 data when forming fourfold coincidences in eSC gondolas, but was not observed in the Run8 data. Fortunately, it is unlikely that this misbehavior could introduce time-dependent structure into the lifetime spectra.

- Overclocking: This issue is related to the preceding issue of TDC desynchronization. In the engineering run in 2002 we observed egregious clock slips and other erratic behavior when the CAENs were run with an external clock of frequency $50 \mathrm{MHz}$, even though this was declared in the CAEN documentation to be within the acceptable 
operating range. We ultimately determined that a $25 \mathrm{MHz}$ external clock signal provided far more reliable operation.

- Memory buffer losses: The limited CAEN memory buffers will sometimes drop information if data arrives faster than it can be propagated through the internal memory queue. Due to our distribution of detector signals across many CAEN channels, the rates were sufficiently low to render this a minor issue. However, to be safe, we sent separate copies of the crucial $\mu \mathrm{SC}$ signal into different CAEN modules so that they could later be compared in the analysis. If there were too many mismatches between the $\mu \mathrm{SC}$ signal copies - whether due to desynchronization or queueing losses - the block was cut. A similar comparison of eSC CAEN and compressor (COMP) signal copies also provides some protection against CAEN data losses; if too many discrepancies are observed, the block block is cut.

- Interpolator nonlinearity: As discussed in Section 6.5.4, each CAEN has an interpolator which subdivides the external clock signal's period by 32 . Unfortunately, the interpolator subdivision is highly nonlinear. In order to avoid potential time-dependent effects, we rebin the lifetime spectra by 32 to wash out any CAENintroduced structure.

- Trailing edges: We operated the CAENs in leading-edge-only mode, in which they were supposed to only record a single digitized datum for the leading edge of each discriminated input signal. However, trailing edges still occasionally appear in the data, often in bursts on adjacent CAEN channels. Members of the MuLan experiment performed extensive studies on CAEN trailing edges in their data, and they surmised that the problem may be related to a fault in the CAEN's internal clock distribution network, whereby trailing edges can be generated when several clock bits change 
at once. In 2005, based upon these findings of the MuLan data analysis, we grew concerned that these bursts of trailing edges could lead to losses of actual data, so we implemented two types of trailing edge cuts in the raw CAEN data processing: First, if the number of trailing edges from a single CAEN in a single block exceeds 100, the entire block is cut. Otherwise, the trailing edge times are recorded, and I chose to treat them in the same way I treat local "sparks": by cutting the immediately surrounding data (see the preceding subsection), in case they were responsible for a temporary suppression in the acquisition of "real" data. In fact, I merge the CAEN trailing edge times in with the spark times, so they are all dealt with by the same local cut algorithm. (Trailing edges from muon CAENs 0 and 2 are lumped in with muon detector sparks, and the trailing edges from electron CAEN 1 are merged with electron detector sparks).

In 2006 the MuLan data analyzers reported that cuts on trailing edges could pontentially introduce time-dependent distortions in the lifetime spectra, and that the trailing edges should simply be ignored. Unfortunately, we had implemented trailing edge cuts based on the earlier 2005 assessment, before the problem had been fully characterized. However, I found that most of the trailing edge block cuts were confined to a handful of problematic run files, which together contributed very little to the final result. Moreover, a differential analysis of the lifetime results for data which included or excluded trailing edge block cuts indicated no systematic effect on the data. Finally, Steven Clayton did not implement "local" data cuts around CAEN trailing edge times as I did, and thus the proximity of our final results provides some reassurance that my local trailing edge cuts did not have any gross effects, if any.

- Cross talk: Dan Chitwood, in his analysis of the 2004 MuLan data, found evidence 
of dangerous cross talk between the signals in the four TDCs within each CAEN [215]. This cross talk can lead to serious time-dependent effects, whereby a signal in one TDC can lead to shifts in the times of subsequent signals in the other TDCs. Fortunately, in MuLan this corresponded to a relatively small, 3.7-ppm-level uncertainty in their $\mu^{+}$lifetime measurement (or, roughly $2 \mathrm{~s}^{-1}$ ). Moreover, many of the CAEN issues that are serious problems for MuLan are of far less concern in MuCap, for two reasons: (1) in MuCap, the muon and electron signals were sent to separate CAENs, and (2) the muon pileup veto in MuCap dramatically reduces signal rates in the CAEN channels and reduces possibility of correlated effects.

Of course, we also recorded several types of error flags in the CAEN data, both from the CAENs themselves and from the DAQ. If an error datum of any kind is encountered in the raw CAEN data processing, the data block is rejected. Altogether, my own CAEN-related cuts led to the rejection of $\approx 1.5 \%$ of the data blocks. A more thorough examination of CAEN V767 pathologies can be found in Dan Chitwood's 2006 report on his analysis of the 2004 data from the MuLan experiment [215].

It is worth mentioning that, early in the Run8 data analysis, Steven Clayton performed a quick analysis study in which he obtained two results for the $\mu^{-}$lifetime: one result came from using the electron times in the CAEN data, while the other result came from using the electron times in the COMP data. Mr. Clayton did not observe a statistically significant difference between the two results, indicating an absence of serious systematic CAEN effects.

Based upon my own findings, I did not deem it necessary to introduce systematic uncertainties in the $\mu^{-}$or $\mu^{+}$lifetime analysis for any of the CAEN concerns described above. The only remaining CAEN issue is the possibility for beating between the CAENbased times in the lifetime spectra and the $50 \mathrm{MHz}$ cyclotron beam structure. An accurate 
simulation of the situation requires knowledge of the DAQ clock frequency, so the error assessment will be discussed in Section 7.3.2, following the clock frequency unblinding.

\subsubsection{Lifetime vs. time}

To check the stability of the measured $\mu^{-}$disappearance rate over the duration of Run8, I subdivided the Prod-50 data files into 99 chronological run groups, each of which contained at least $1.5 \times 10^{7}$ decay events in order to ensure an even distribution of statistics. I then performed a fit to the lifetime spectra for each run group, taking care to use the log likelihood minimization method because the accidental background statistics were too low

to safely use $\chi^{2}$ minimization. As Figure 6.35 shows, the resulting distribution of fitted rates versus run group is statistically consistent with uniformity, and there is no evidence of any systematic change in the muon lifetime versus time.

\subsubsection{Lifetime vs. space}

In scrutinizing the consistency and reliability of our results, it is worth investigating whether any systematic variations appear in the $\mu^{-}$disappearance rates that are observed in different parts of the detector. One can imagine numerous ways of subdividing the detector data according to the spatial characteristics of the observed muons and electrons, although time constraints prevent the examination of all possibilities. In this subsection I consider two rather simple and natural categories: $\lambda$ vs. $z$ position in the eSC, and $\lambda$ vs. $\phi$ in the eSC. As will be shown shortly, the latter study prompted in-depth analysis and ultimately revealed some interesting physics. 


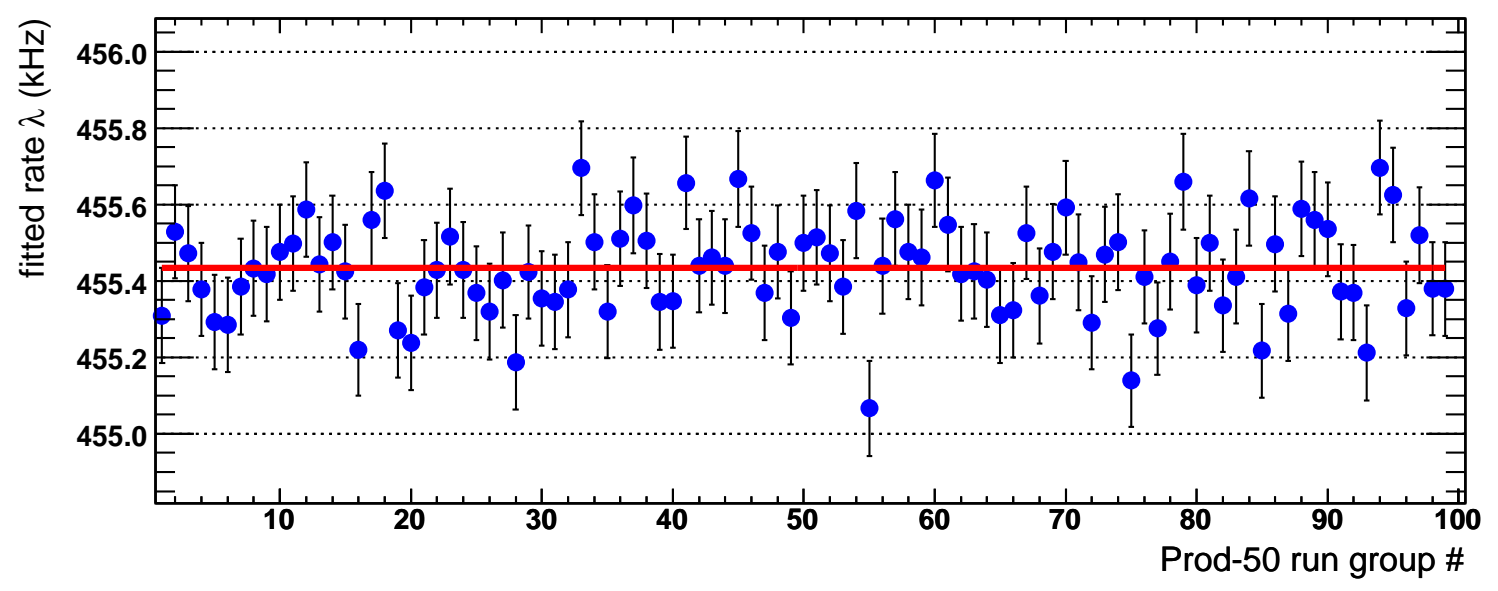

Figure 6.35: A plot of the fitted decay rate versus chronologically ordered groups of Run8 Prod-50 data files. The data was subdivided into 99 similarly-sized groups, and the points in the figure correspond to the fitted rate for each group; in this instance, the cathodeOR lifetime spectra were fit. The horizontal line is from a constant fit to the distribution of fitted rates, and it has the value $\lambda=455,437.9 \pm 12.3 \mathrm{~s}^{-1}$, which is consistent with lifetime fits to the cathode-OR summed statistics. The constant fit's reduced chi-square is $\chi^{2} / d=101.6 / 98=1.04 \pm 0.14$, so there is no evidence of any non-statistical variation in the lifetime over the course of Run8. Fits to lifetimes for other detector treatments show similar stability.

\section{Lifetime vs. $z$}

Let us examine the disappearance rate $\lambda$ as a function of $z$, where in this case the latter quantity corresponds to the $z$ position of the outgoing decay electron's intersection with the eSC cylinder, as determined from the particle's trajectory through ePC1 and ePC2. To perform this study, I subdivided the electron data into 11 bins over the $\mathrm{eSC} z$ intersection interval $[-600,600] \mathrm{mm}$; note that this range extends beyond the ends of the eSC gondolas at $\pm 450 \mathrm{~mm}$. I then created a lifetime spectrum for each of the 11 groups, and performed a fit to each. The resulting fitted disappearance rates are shown in Figure 6.36. The fitted rates are statistically consistent, and there is no evidence of any systematic variation vs. z. This has also been observed to hold true when the same study is performed on the individual gondolas. 


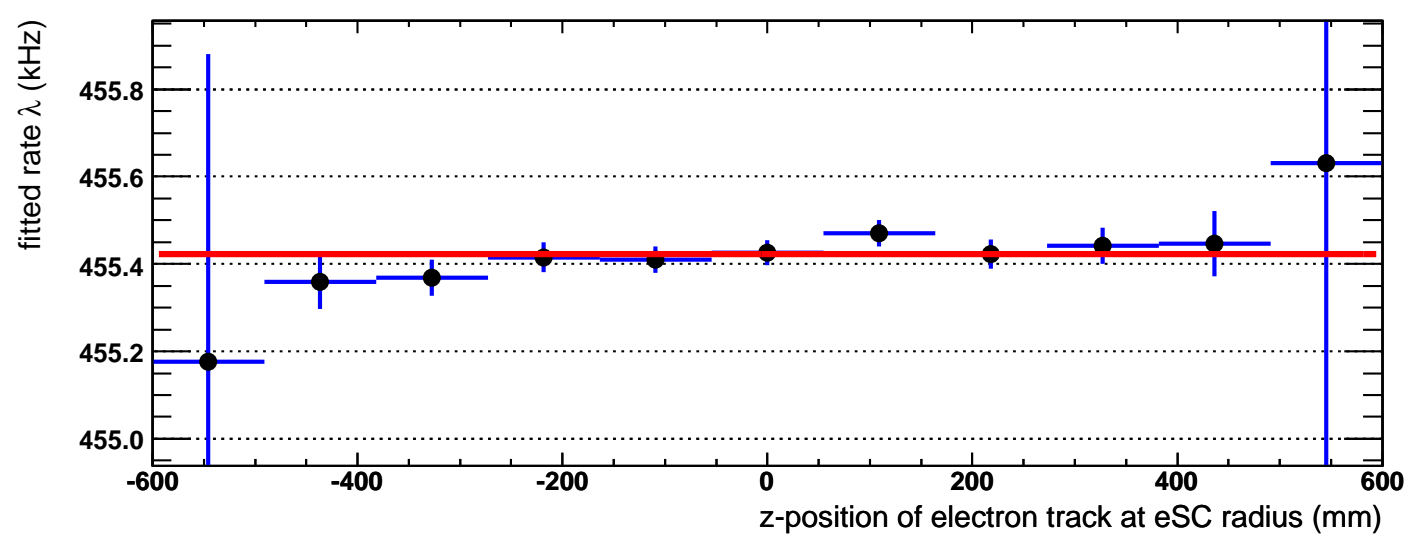

Figure 6.36: The fitted $\mu^{-}$disappearance rate vs. the $z$ position of the electron's intersection with the eSC cylinder. The low statistics for the outermost fits are to be expected, as those bins cover regions beyond the ends of the eSC detector at $\pm 450 \mathrm{~mm}$, and therefore only contain events that were originally headed to miss the eSC but then scattered back into the detector. Fitting a constant across the 11 rates as indicated yields a $\chi^{2} / d=0.60(45)$, so there is no indication of any systemaic nonuniformity.

\section{Lifetime vs. azimuthal angle $\phi$}

For a long time we observed a marked lack of uniformity in the fitted disappearance rate vs. the experiment's azimuthal angle $\phi$. This phenomenon is illustrated in Figure 6.37, where the large nonuniformity is reflected in the high $\chi^{2} / d$ values that result from fitting a constant across the disappearance rates obtained from fits to the lifetime spectra of the sixteen individual eSC elements, or "gondolas." The azimuthal nonuniformity was a source of much consternation, because in principle the variations among the fitted muon disappearance rates from the eSC elements should be purely statistical in nature, and fitting a constant across the rates should give a reduced $\chi^{2}$ value consistent with 1 . We initially labeled this mysterious behavior with the blanket term "gondola effect," since we did not understand its origins, and the issue occupied our attention for some time.

After extensive investigative effort, we determined that the gondola effect was actually the product of two distinct and subtle mechanisms: 

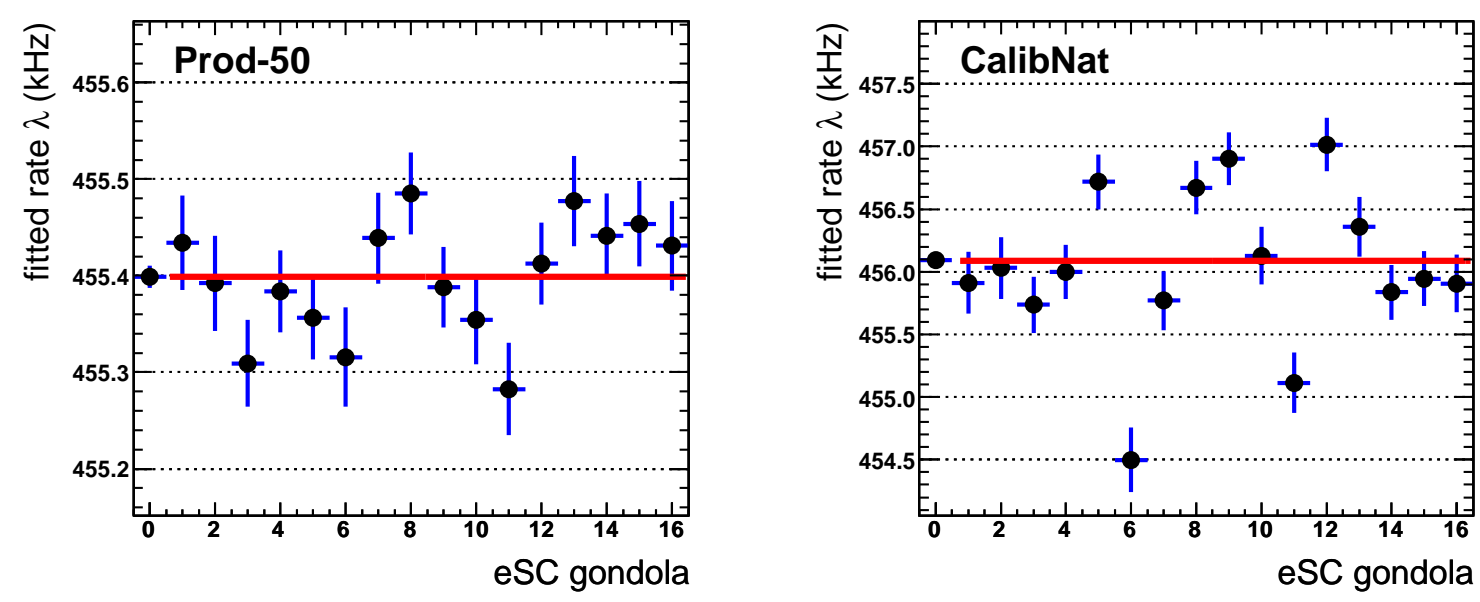

Figure 6.37: The fitted $\mu^{-}$disappearance rate vs. eSC element, or "gondola," as originally observed in the Prod-50 and CalibNat data; here gondola " 0 " refers to the lifetime histogram comprised of the sum of the individual lifetime spectra from the sixteen gondolas. In the Prod-50 distribution above, fitting a constant across the rates for gondolas 1-16 as indicated yields a $\chi^{2} / d=1.7(4)$ (where $\left.d=15\right)$, the central value of which corresponds to a likelihood of less than $5 \%$. The per-gondola nonuniformity is even worse for the CalibNat distribution $\left(\chi^{2} / d=7.5(4)\right)$, for reasons that are explained in the text.

1. The rapid diffusion of $\mu d$ atoms, combined with the scattering of decay electrons from the TPC frames and lower supports, produces a nonuniformity that scales with increasing deuterium concentration.

2. Depending upon their direction of emission, decay electrons can deposit enough energy in the TPC to trigger the EL threshold, and these EL pixels can interfere with muon stop identification in a space- and time-dependent manner.

Let us first examine the mechanism related to muon diffusion, using the illustration in Fig. 6.38 as a guide. I begin by noting that, unlike the surrounding electron detectors, the distribution of muon stops is not cylindrically symmetric in either shape or density, due to the rectangular TPC volume and the fact that the muon beam's Gaussian stopping distribution is neither centered along the experiment's $z$ axis nor symmetric around it. In 


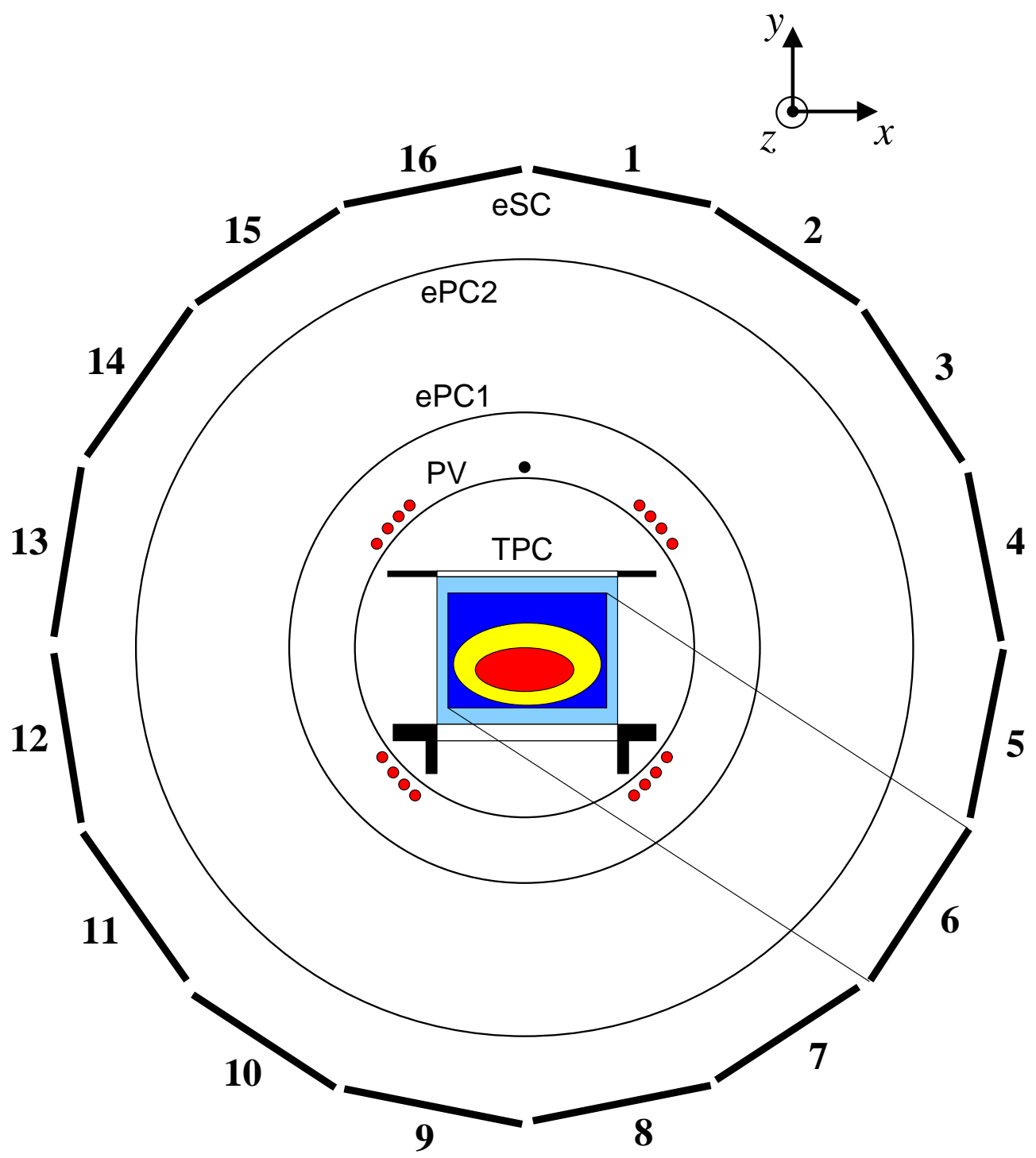

Figure 6.38: Schematic diagram (to scale) of the detectors in cross section, as viewed from downstream, looking upstream. This diagram is intended to assist in illustrating how a combination of rapid $\mu d$ diffusion and scattering from TPC materials can lead to variations in the effective muon disappearance rates observed in different eSC gondolas, as described in the text. The asymmetric muon stopping distribution is indicated by the red and yellow ovals, while the fiducial volume of the TPC is indicated by the dark blue rectangle, residing within the larger sensitive volume, indicated by the light blue rectangle. The locations of the high-density TPC scattering materials (the frames and supports) are solid black. The $\mu^{+} \mathrm{SR}$ magnet coils and the CHUPS steel return line are also depicted. Lines are drawn connecting the edges of the fiducial distribution with the edges of gondola 6 , in order to better show the central, intervening position of the TPC scattering materials. For reference, the detector radii are $r_{\mathrm{PV}}=140 \mathrm{~mm}, r_{\mathrm{ePC} 1}=192 \mathrm{~mm}, r_{\mathrm{ePC} 2}=320 \mathrm{~mm}$, and $r_{\mathrm{eSC}}=386 \mathrm{~mm}$. 
the absence of any other effects, these asymmetries would only alter the decay statistics observed in each gondola, and leave the decay rate unaffected. However, the existence of muon diffusion introduces complications. As the muon distribution expands outwards, the solid angle presented by any particular gondola - and by the electron detectors as a whole - changes with time. Since the initial muon stopping distribution is cylindrically asymmetric, muon diffusion leads to a different time-dependent structure in the lifetime spectrum observed in each gondola. Of course, if the electron detector had $4 \pi$ solid angle coverage, then any such variations would cancel out once the statistics from all of the detector components were combined. In reality, our detector has finite $(76 \%)$ solid angle coverage, though fortunately the geometric losses out of the electron detector endcaps due to $\mu p$ diffusion are negligible (as has been demonstrated by Monte Carlo simulations) and any similar $\mu d$ diffusion effects will be remedied by the zero-extrapolation deuterium correction to come (Section 6.6.4).

We believe that muon diffusion becomes a potentially serious issue because of the presence of scattering materials that are asymmetrically distributed between the muon stopping distribution and the electron detectors. Just as the solid-angle acceptance of each eSC gondola changes with time due to muon diffusion, so does the scattering profile presented by the TPC frames and supports, shrinking as the muon distribution expands. Consequently, the acceptance functions of the "screened" gondolas (e.g., gondola 6 in Figure 6.38) are suppressed at early times relative to late, thus weighting their lifetime spectra toward later times and lowering their observed disappearance rates. The effect is exacerbated by increasing the deuterium concentration (see Figure 6.37), because deuterium increases the average muon diffusion distance. It is conceivable that the nonuniformity might not affect the overall disappearance rate, because the effects should cancel out when the data from all of the gondolas are added together. Of course, this might no longer hold true if impact 
cuts are applied; in fact, it seems likely that the nonuniformity could be worsened by the application of impact parameter cuts, since these should further suppress the acceptance function of the screened gondolas at early times. However, this hypothesis is not supported by the data.

As inspection of Figures 4.3 and 4.4 suggests, and as the experimental results in Figure 6.39 confirm, electron scattering from the lower TPC frames and aluminum supports is largest because of the additional material there. It is certainly plausible that this scattering, in conjunction with muon diffusion, could be responsible for the nonuniformity in the fitted rates in Figure 6.37. But can the relationship be demonstrated, and is it large enough to account for the observed discrepancies among the fitted rates? To answer that question, we must rely on Monte Carlo simulations and special experimental studies, both of which support the diffusion+scattering hypothesis. I was able to reproduce the gondola effect in principle, though not exactly in scale, with fast Monte Carlo simulations of diffusion and scattering in the experimental setup (Figure 6.40). My Monte Carlo results confirm that the disappearance rates observed in gondolas 6 and 11, which are the gondolas most screened by the lower TPC frames and supports, should be pulled down relative to the other gondolas' rates, and that this behavior grows increasingly pronounced as the deuterium concentration increases. Note that my Monte Carlo results in Figure 6.40 also indicate that the lowering effect in gondolas 6 and 11 is offset by increases to the fitted rates in the adjacent gondolas. This makes sense, as a scattered Michel electron loss for one gondola will often correspond to a gain in a nearby gondola. The only serious shortcoming of my Monte Carlo studies was that the simulated gondola effect did not scale with deuterium as strongly as the Run8 data in Figure 6.37. The diffusion+scattering explanation is further corroborated by looking at the fitted rates vs. gondola for muon stops in different regions of the TPC. For example, Figure 6.41 shows that the rate vs. gondola distributions for the upper and lower halves of 


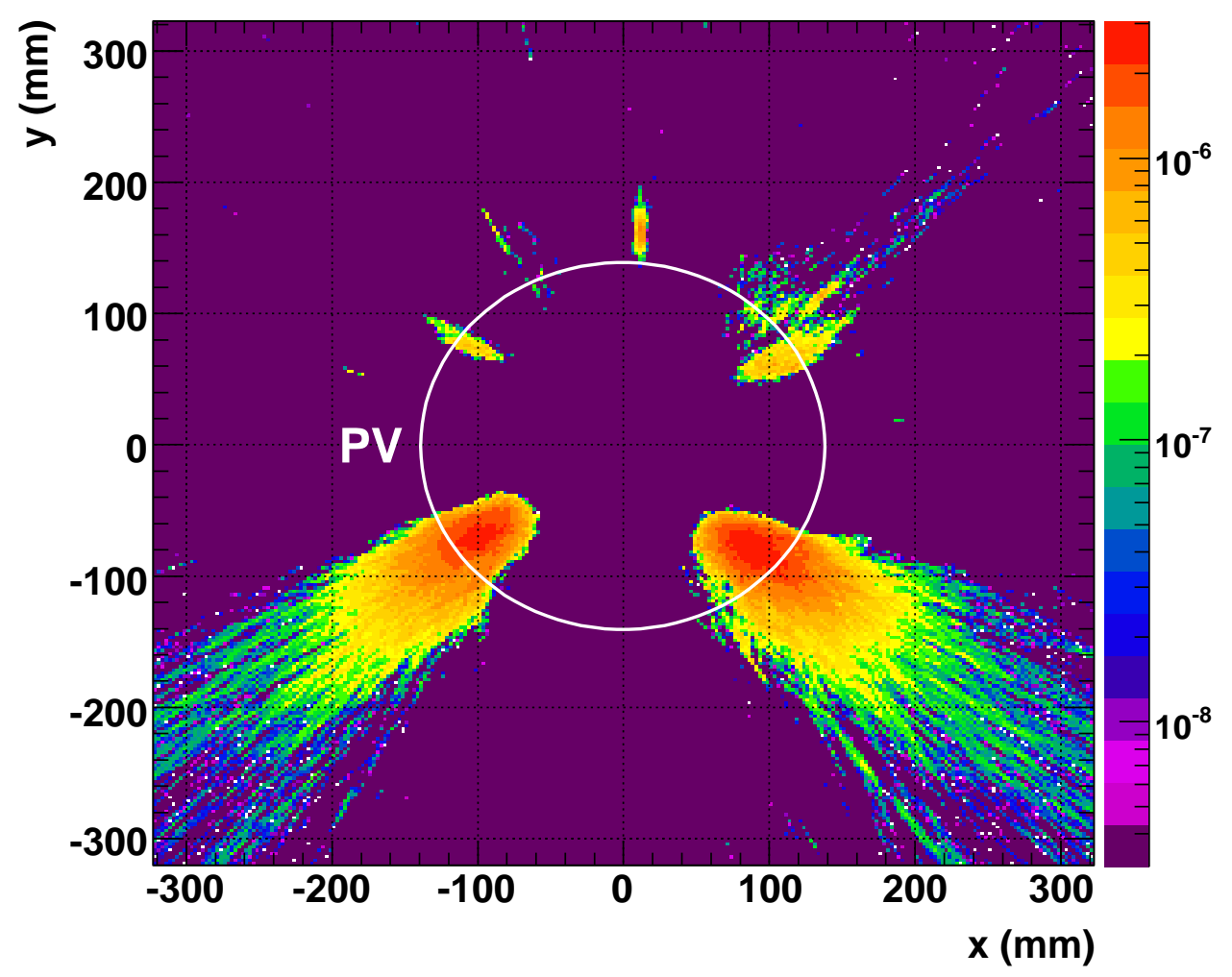

Figure 6.39: A 2D tomography plot of scattering materials in and around the pressure vessel, within the $z$ interval $[-10,10] \mathrm{mm}$ (i.e., through the center of the TPC). This plot provides empirical confirmation of the hypothesis that electrons are scattered by the TPC frames, and especially by the lower frames and supports. The cross section was created using software developed by Steven Clayton; his code fills two 3D volumes with the lines of decay electron trajectories in the Run8 data, where one volume is for electrons with an impact parameter $b<50 \mathrm{~mm}$, and the other is for electrons with an impact parameter $b>50 \mathrm{~mm}$. By normalizing the two volumes and subtracting the former from the latter, regions that have a relative dearth of electron tracks due to screening show up as areas of higher density; the $z$ scale is arbitrary. The perspective presented here - a view from downstream, looking upstream into the oncoming muon beam - is the same as in Figure 6.38, and the pressure vessel (PV) wall at radius $140 \mathrm{~mm}$ is drawn for reference. The $\mu^{+} \mathrm{SR}$ magnet is roughly in line with the TPC frames, which could perhaps explain the presence of some light, smeared scattering at $45^{\circ}$. Also visible is the stainless steel CHUPS return line, which runs along the top of the PV (see Figure 4.5). Note that the scattering in the direction of eSC gondola 6 is the largest, and that Figure 6.37 indicates that gondola 6 exhibits the largest deviation in its fitted rate in the CalibNat data. Finally, I should point out that the plot appears to suffer from a slight clockwise rotation, probably due to imperfect alignment settings, and that it is not understood why the PV walls - which are known to be responsible for a significant amount of electron scattering - do not produce a silhouette. 

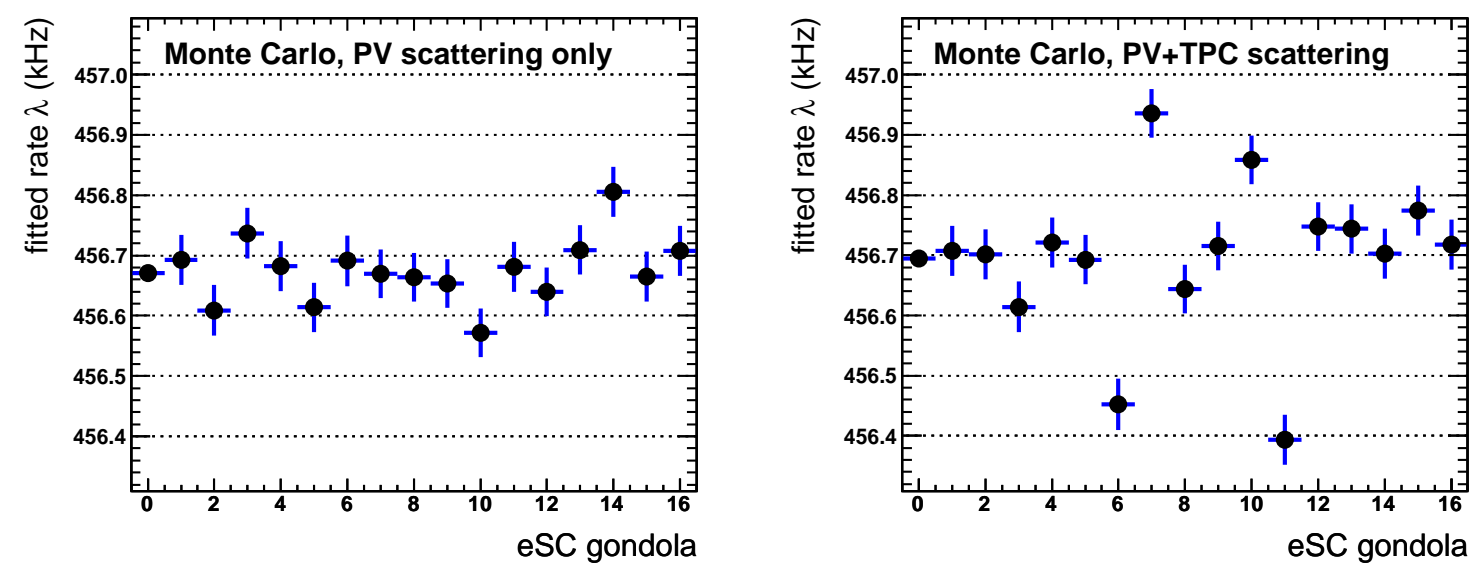

Figure 6.40: Reproduction of the $\mu d$-diffusion-driven "gondola effect," created using my fast Monte Carlo software. In the plot on the left, only scattering from the pressure vessel (PV) was simulated, and no gondola effect is apparent. In the plot on the right, a gondola effect appears once scattering from the TPC frames and supports was also included; note that the fitted rates in gondolas 6 and 11 are pulled downwards relative to the other gondolas, just as is observed in the Run8 CalibNat data in Figure 6.37. I had to use a nominal MC concentration of $c_{d}=300 \mathrm{ppm}$ to achieve results comparable to what we actually observe in the Run8 data for $c_{d} \approx 120 \mathrm{ppm}$.

the TPC exhibit different structures, as a result of the different scattering materials close to each. Decays in the lower half of the TPC are more likely to be affected by scattering from the lower TPC frames and supports, which lowers the rates in gondolas 6 and 11; similarly, some decays in the upper half of the TPC will be scattered from the upper TPC frames, which should lower the fitted rates in gondolas 3 and 14. Figure 6.41 confirms that this is indeed largely the case, although it should be noted that the fitted disappearance rate in gondola 14 does not exhibit a lowered rate as expected.

We cannot control the muon-diffusion-driven gondola effect, because it is an inescapable feature of the physics of our experimental setup. Fortunately, its effects appear to be negligible for the Prod-50 fill, which has a low deuterium concentration and is dominated by small, mm-scale $\mu p$ diffusion. The phenomenon is potentially problematic for the CalibNat fill, though, because it exhibits an exaggerated gondola effect, as seen in Figure 6.37. 

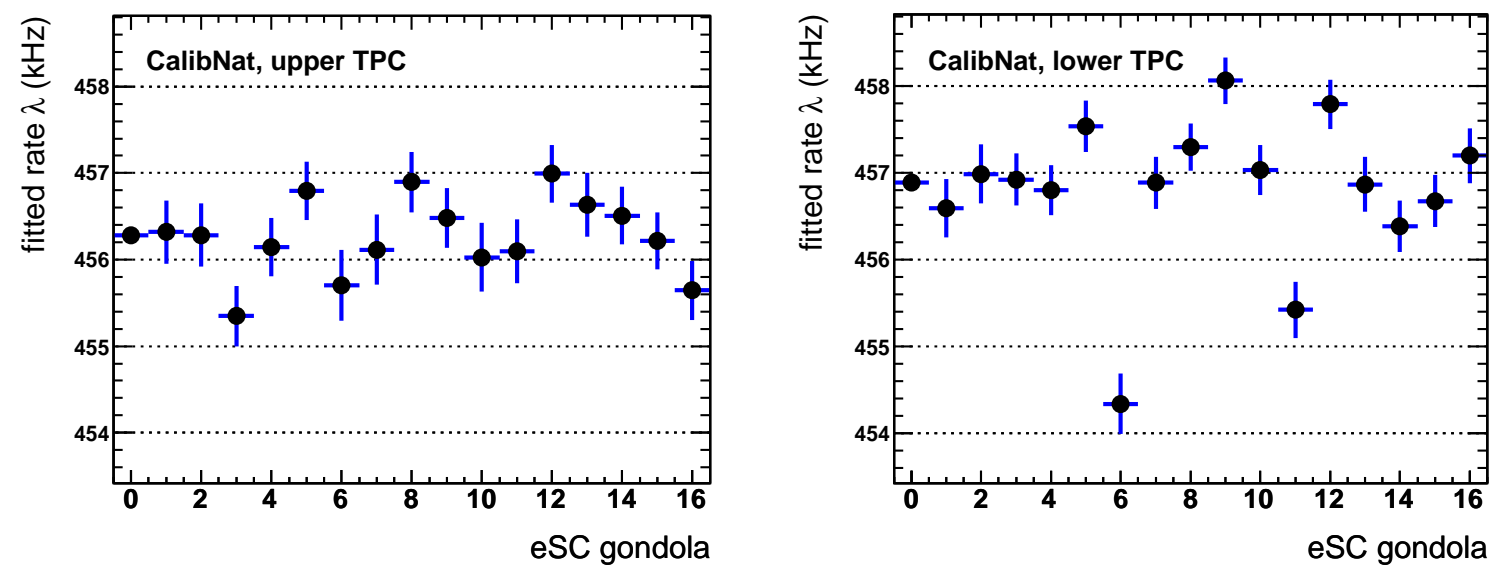

Figure 6.41: The rate vs. gondola variations observed for muon stops in the upper and lower halves of the TPC. The difference in the two distributions provides support for the hypothesis that muon diffusion and scattering combine to create a nonstatistical variation among the fitted rates in the gondolas. The CalibNat fill's results are presented here because the effect is suppressed in the Prod-50 data.

Thankfully, the gondola effect should not have a meaningful influence on the deuterium correction's zero-extrapolation procedure, for two main reasons: (1) any overall nonlinearities will be reduced by a factor commensurate with the long lever arm of the deuterium correction; (2) only two gondolas are egregiously affected, and they appear to be balanced out by opposite effects in adjacent gondolas. Although we have not rigorously demonstrated the insignificance of this particular machanism for the gondola effect, we believe that it essentially averages out once the statistics from all of the gondolas are combined, given that there is little or no absorption of decay electrons.

Let us now consider the second proposed mechanism for the "gondola effect" namely, the mechanism related to TPC performance. From the earliest days of our investigations into the gondola effect, we suspected that the phenomenon might be connected to the TPC response - or more accurately, with the identification of muon stops in the TPC. We initially observed in the low-deuterium-concentration Prod-50 data that the gondola 
effect disappeared for lifetime spectra that did not involve TPC data. By next breaking the fiducial TPC cuts down into stages, we were able to isolate which particular cuts were responsible. The most problematic cuts turned out to be those which excluded muon stop candidates that were accompanied by unattached EL pixels in the vicinity of the stop, especially to the left or right. The reasons for this remained unclear, until Steve Clayton convincingly demonstrated that it resulted from the fact that decay electrons occasionally generate EL pixels in the TPC, and that the fiducial cuts can be sensitive to the presence of these time- and space-dependent EL pixels. Unlike the muon-diffusion-driven mechanism for the gondola effect, however, the TPC-response-related mechanism is amenable to remediation. We have only to be careful to employ muon stop identification criteria that are not affected in a systematic way by the occasional appearance of time-delayed EL pixels as generated by the emission of decay electrons. This will be discussed in more detail in the next subsection on fiducial cuts.

It is difficult to disentangle the contributions from the two mechanisms that produce nonuniformities in the fitted rates across the gondolas. However, we believe that the diffusion-driven effects on the final rate are negligible, and that the fiducial-cut-related effects can be effectively eliminated through the use of judicious criteria when identifying muon stops.

\subsubsection{TPC fiducial cuts}

In order to identify which beam muons stopped in the hydrogen gas target as desired, it is necessary to perform cuts on the muon track information provided by the TPC. As discussed in Section 6.3.3, arriving muons generate a characteristic signature when they stop within the TPC's sensitive volume, so the identification of good muon stops seems as though it should be a relatively easy task. However, complications arise due to the 
wide range of physical phenomena that can affect the TPC response. For instance, decay electrons can deposit small amounts of energy which modify the appearance of the muon track, and therefore certain track cuts can introduce time-dependent effects. Also, muons occasionally scatter off protons and exit the TPC volume, while leaving behind tracks that mimic good fiducial stops. Muon escapes from the fiducial volume must be recognized in order to avoid inadvertantly including $Z>1$ capture contributions from muon stops in surrounding detector materials. Although all of these phenomena are relatively rare, they can systematically distort a precision $\mu^{-}$lifetime measurement. It is therefore essential to fully characterize the topology of muon tracks in the TPC, and to apply judicious fiducial volume cuts that select good muon stops in a time-independent manner.

First, let us examine how the dimensions and physical properties of the TPC inform basic, naïve fiducial volume cuts. The TPC sensitive volume has dimensions $\Delta x \times \Delta y \times \Delta z=$ $150 \times 120 \times 300 \mathrm{~mm}^{3}$. In the $x$ direction, the TPC is instrumented with 35 separate cathodes at $4 \mathrm{~mm}$ spacing. The outer cathodes, 1 and 35, are actually comprised of three cathode strips which are soldered together, so although the entire width of the TPC volume is effectively instrumented, the $x$ resolution at its edges is not as fine as in its interior. The full $120 \mathrm{~mm}$ height of the TPC volume is represented in the drift distribution, which is shown in Figure 6.11. The $300 \mathrm{~mm}$ length of the TPC volume is instrumented with 75 anode wires at $4 \mathrm{~mm}$ spacing, but the first four anodes and the last two anodes are made from wires that are too thick $(25-100 \mu \mathrm{m})$ to amplify the ionization signals that reach the TPC's MWPC region. As a result, the active region of the TPC is effectively $276 \mathrm{~mm}$ in length, slightly shorter than the actual sensitive volume.

Recall from Section 6.3.4 that I began with clusters of anode EH pixels as muon stop candidates, and I gathered information about the surrounding TPC data. I can now describe the cuts which are applied to that information to select good muon stops. In the $x$ 
dimension, I require hits (either EL or EH) in the central cathode interval 4-32, inclusive, in coincidence with the anode EH signals of the muon stop candidate. I also require that any cathode hits coincident with the muon track's anode EL pixels cannot extend outside of the inclusive interval 3-33, in order to protect against muon escapes out of the sides of the TPC. In the $z$ dimension, I enforce a variety of conditions: The interval of functional, amplifying anodes is 5-73, inclusive, but I require that the anode on which the muon stopped - that is, the furthest anode in an EH cluster-lie within the inclusive interval 6-67. A maximum anode of 67 is chosen to allow buffer space for the identification of $\mu+p$ scatters and escapes in the $z$ direction. I also require that the Bragg stop of EH pixels be preceded by a "leadup" of EL pixels of at least five anodes in length, thus effectively making the acceptable muon stop interval 10-67, inclusive. I require that there be no $\mathrm{EH}$ anode pixels beyond the ostensible stop, to ensure that the EH anode cluster under scrutiny is in fact a stop, and not in the middle of a track. To eliminate throughgoing muons that exit out of the back of the TPC, I require that any EL pixels downstream of the ostensible stop cannot extend all the way to the last amplifying anode, anode 73. I do not, however, enforce a cut on a maximum length of anode EL pixels extending beyond the EH Bragg stop, because EH clusters which are followed by a tail of EL pixels will be scrutinized later by the $\mu+p$ scatter identification algorithm (Section 6.6.2) and discarded if necessary.

Specifying fiducial TPC cuts in the $y$ dimension is somewhat trickier than in the other dimensions. As discussed in the preceding subsection, Steven Clayton has demonstrated that decay electrons can occasionally deposit enough energy in the TPC to trigger the EL threshold. As a result, we cannot simply require an absence of EL pixels to the immediate left and right of the anode EH cluster at the muon stop, because doing so introduces time- and space-dependent effects (i.e. the "gondola effect" discussed in Section 6.5.12). To avoid such distortions, I implemented a "double-walled" fiducial cut in the $y$ direction, as 
first proposed by Mr. Clayton. In this approach, there is an outer $y$ boundary for EL pixels from the muon track, to ensure that the muon remained within the confines of the TPC, and an inner $y$ boundary for the EH pixels of the Bragg stop. The outer EL boundaries are $5 \mathrm{~mm}$ from the top and bottom of the sensitive volume, and the inner $\mathrm{EH}$ boundaries are $15 \mathrm{~mm}$ from the edges. This separation between the two cuts dramatically minimizes the effect of the EL confinement cut on the muon stop selection.

The TPC fiducial cut boundaries in all three dimensions are illustrated in the event display example in Figure 6.42.

Recall that I postpone my TPC fiducial volume cuts until just prior to the creation of lifetime histograms. This enables me explore the effects of varying the fiducial volume cuts. For example, I performed a study where I marched the EH $y$ boundaries inwards in $2 \mathrm{~mm}$ increments, away from the EL boundaries, and created a lifetime histogram for each setting. The fit results from this scan are plotted in Figure 6.43, alongside the results from similar scans over other cut specifications. Of notable interest is the sensitivity of the uniformity of the fitted rates across the sixteen eSC gondolas to the separation between the EL and EH $y$ boundaries. When the EL and EH $y$ boundaries overlap, the distribution of fitted rates across the gondolas is highly nonuniform - the $\chi^{2} / d$ of a constant fit across the rates is close to 1.6(4) - due to the "gondola effect" from EL pixels generated by decay electrons. As the EH walls are moved inwards, away from the outer EL boundaries which are held fixed at $5 \mathrm{~mm}$ from the top and bottom of the sensitive volume, the effects of the electron-generated EL pixels attentuates, and the uniformity of the fitted rates across the gondolas improves, stabilizing around $\chi^{2} / d \approx 1.2(4)$. It is interesting to note that the fitted rates $\lambda$ to the summed gondola data do not exhibit the same sensitivity, but instead generally remain within the $1 \sigma$ statistically allowed error bands. This suggests that the decay-electron-generated nonuniformity across the gondolas' fitted rates had little effect 
TPC Display

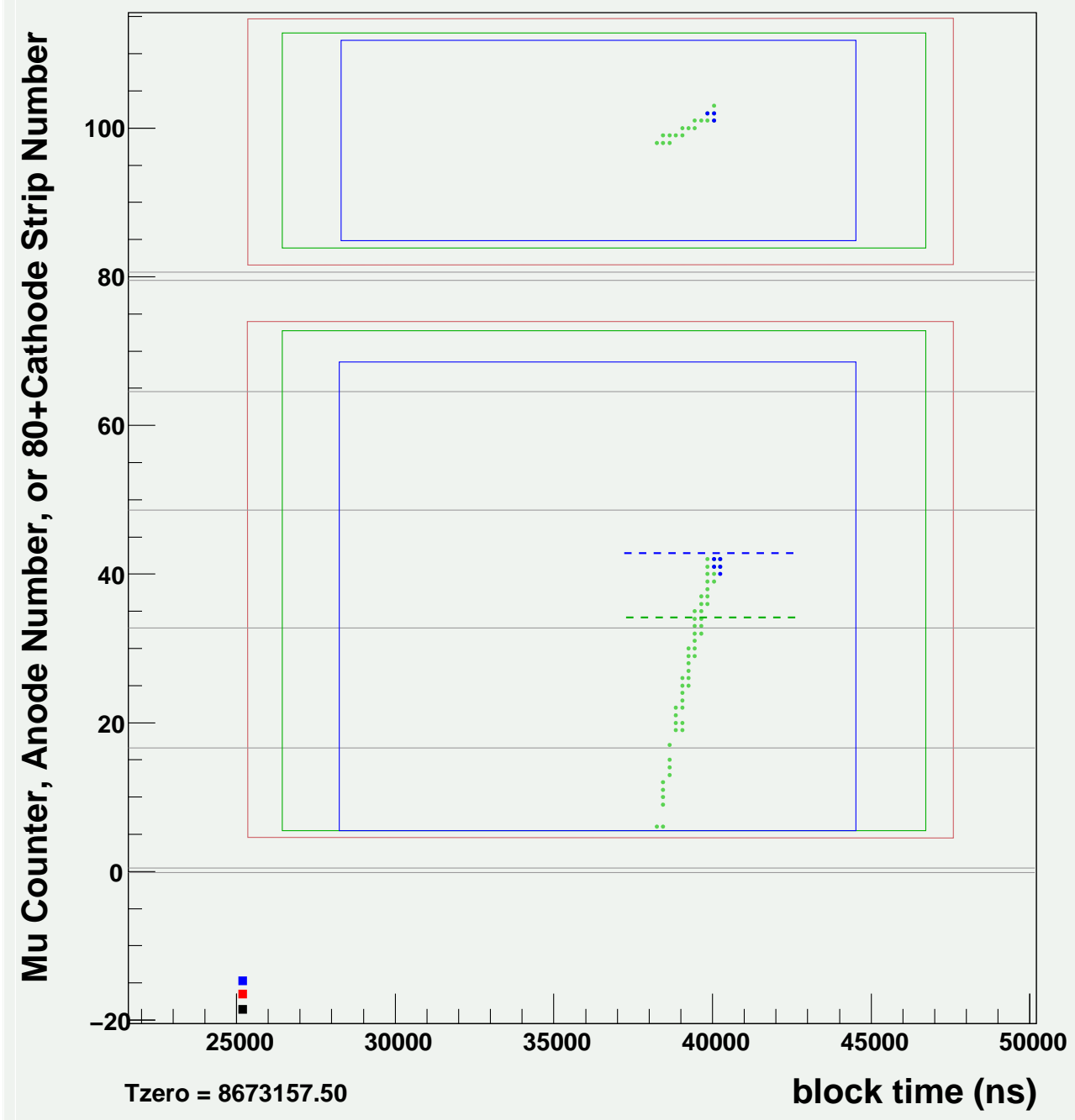

Figure 6.42: Illustration of the TPC fiducial cut boundaries. The red lines correspond to the edges of the sensitive volume, the solid green boundaries are the constraints on the EL pixels, and the solid blue boundaries are the constraints on the EH pixels. The green and blue dotted lines refer to the EL and EH constraints that are established relative to the muon stop: namely, no EH hits may lie beyond it, and there must be a lead-up of at least 5 EL pixels. Note the "double-walled" fiducial cut in $y$; the EL boundaries are at drift times $1294 \mathrm{~ns}$ and $21501 \mathrm{~ns}$, which correspond to a distance of $5 \mathrm{~mm}$ from the edges, while the EH boundaries are at drift times $3131 \mathrm{~ns}$ and $19664 \mathrm{~ns}$, which correspond to a distance of $15 \mathrm{~mm}$ from the edges. 

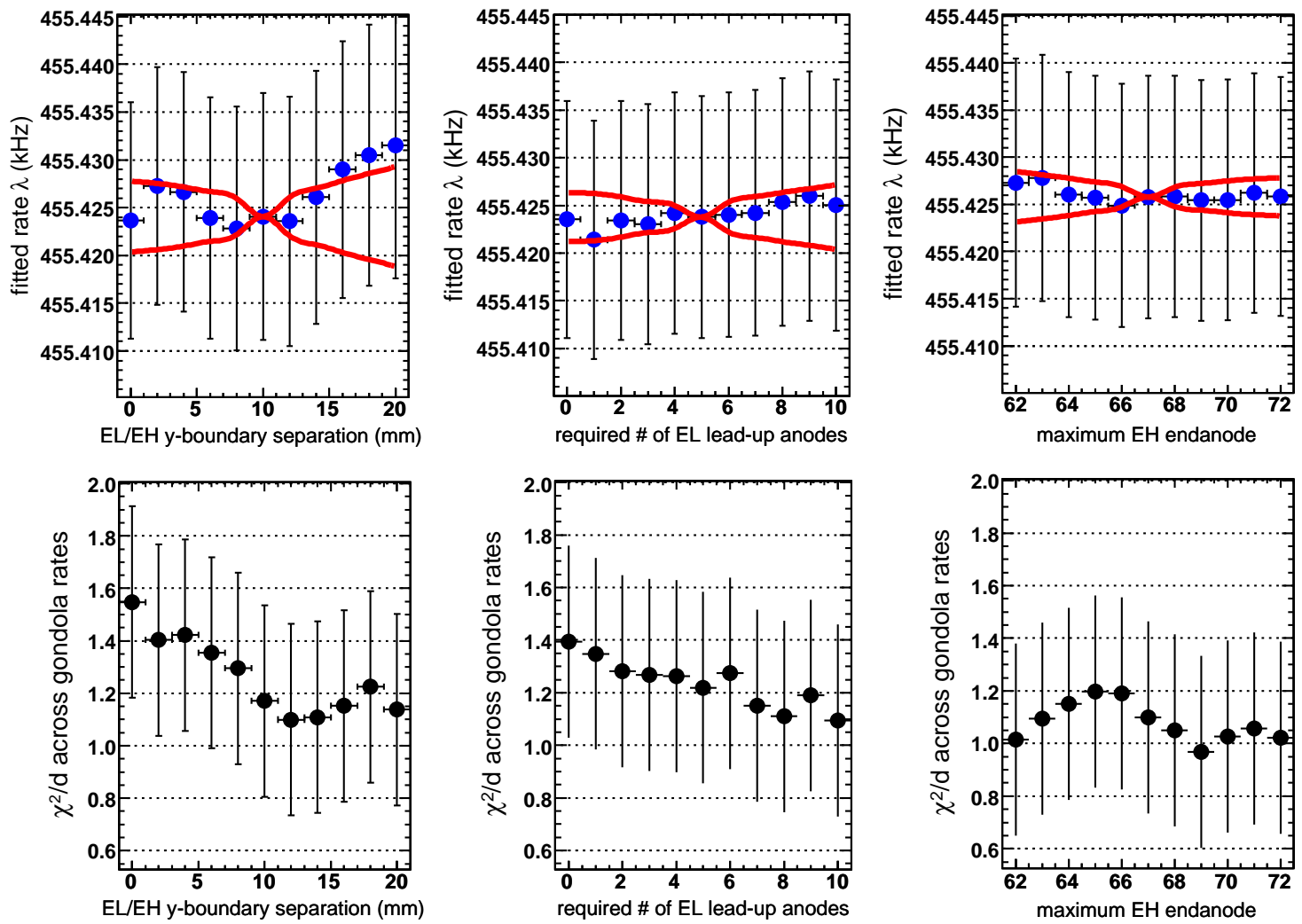

Figure 6.43: Fit results from three different scans over the TPC fiducial cut criteria. The scans were performed over (left) the separation between the EL and EH $y$ boundaries, with the EL boundary held fixed at $5 \mathrm{~mm}$ from the edges of the sensitive volume, (center) the length of the track of EL pixels leading up to the EH Bragg stop, and (right) the maximum anode allowed for muon stops. The $1 \sigma$ error bands are drawn relative to my standard cut settings. The fitted rate $\lambda$ is to the sum of the eSC gondola data, while the $\chi^{2} / d$ plots describe the uniformity of the fitted rates across the sixteen gondolas $(d=15)$. The data here is from the Prod-50 data set, and I used the cathode-AND electron table, with no impact cuts. 
on the overall observed disappearance rate. Nevertheless, after inspecting these results, I decided upon my standard EL/EH boundary separation of $\Delta y=10 \mathrm{~mm}$, which corresponds to a time difference of $\Delta t \approx 1.8 \mu \mathrm{s}$ in the TPC drift distribtion. For historical reasons, the standard EL and EH $y$ boundaries are both somewhat further in than they probably need to be, but the resulting loss in statistics is relatively small since the muon stopping distribution is centralized.

It is also worthwhile examining the stability of the fitted disappearance rate as a function of the muon stopping position. To do this, I formed lifetime spectra involving concentric shells of muon stops, where each shell's edges were equidistant from the closest surrounding TPC materials. Some examples of these shells are presented in Figure 6.44, above the fit results from all 20 shells. There it can be seen that, for the Prod-50 data, the fitted disappearance rate remains steady as the shell of muon stops moves outwards from the center of the TPC; in fact, the inner 17 points are consistent with a constant. However, when the shells approach within roughly $4 \mathrm{~mm}$ of the edges of the TPC volume, the fitted rates begin to rise. This systematic rise in the rate for the outermost shells is almost certainly due to the increased probability of diffusion of the $\mu p$ atoms into the MWPC wires at the bottom of the TPC. The fitted rates from the deuterium-doped CalibNat data begin to rise even earlier, and more significantly, because of the increased formation of $\mu d$ atoms, which have much larger diffusion distances and can easily drift into surrounding detector materials. Based on the Prod-50 results, I established standard fiducial cuts that ensure that muon stops are at least $6 \mathrm{~mm}$ away from detector materials in all directions.

Ultimately, the efficacy of my muon stop identification algorithm is evidenced in part in the stability of the fit start time scan in Figure 6.24. If my fiducial cuts were not providing adequate protection against wallstops - or if there were gross and irremediable inefficiencies in the muon detectors, such as malfunctioning TPC cathodes - then there 

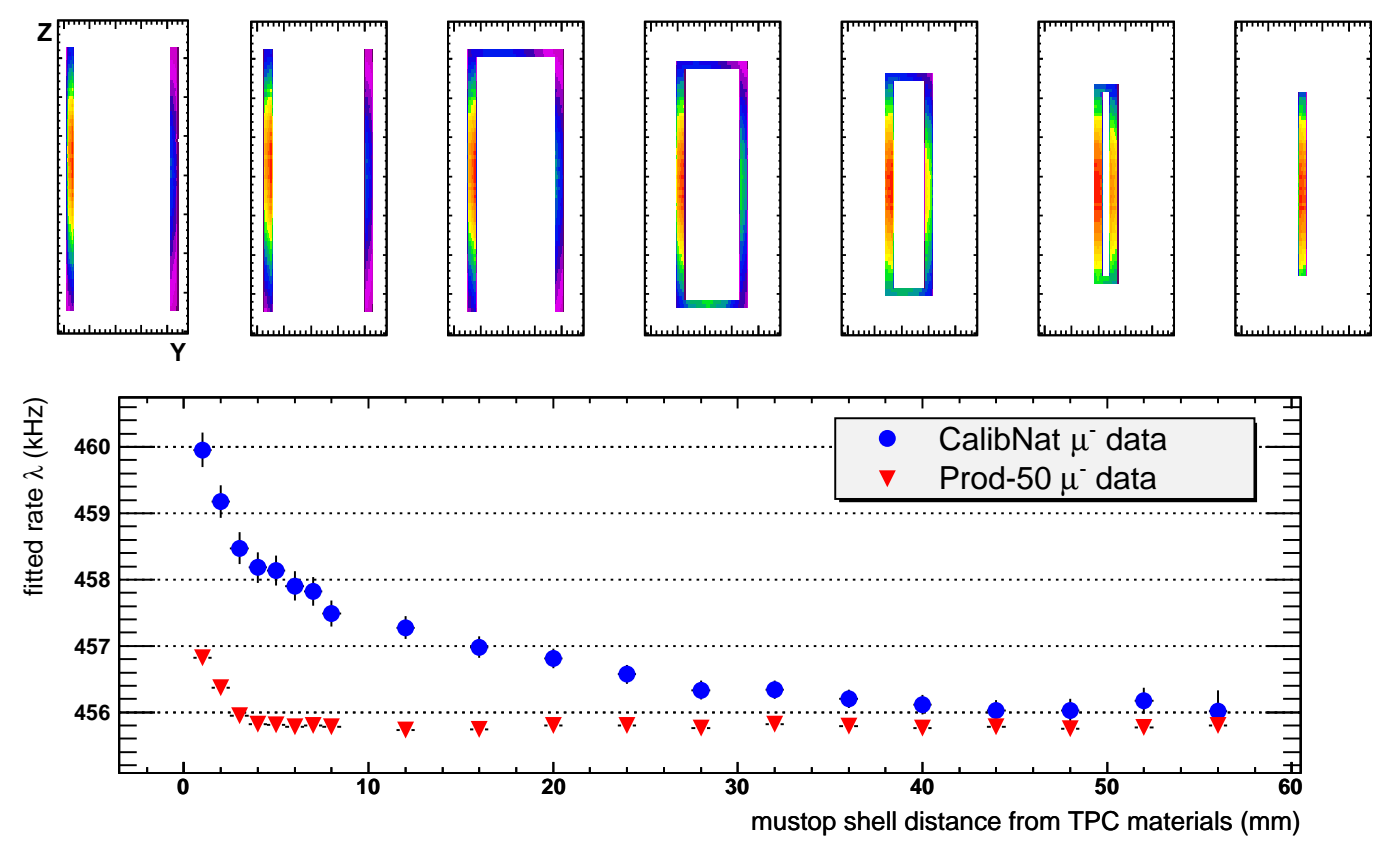

Figure 6.44: (Top) Concentric shells of muon stops in the TPC. Here I present $y z$ profiles of seven of the 20 shells, to illustrate how the shells shrink as they are moved inwards. (Bottom) The fitted rate $\lambda$ from all 20 shells of muon stops, for both the Prod-50 and CalibNat data sets. The left-to-right trend of the points parallels the $y z$ shells above.

would be obvious evidence of $Z>1$ lifetime components in the start time scans. The absence of any such effects leads me to believe that wallstop contributions are minimal. In fact, a small correction will be performed in Section 6.6.2 to compensate for the relatively few muon wallstops that result from $\mu+p$ scatter events.

Finally, it is worth mentioning that plots of the muon lifetime vs. the TPC drift time exhibited no evidence of any cross talk between the two measurements.

\subsubsection{Lifetime vs. muon entrance detector treatment (the pileup veto)}

In Section 6.5.1 I alluded to the fact that, if the TPC is used to identify good muon stops, distortions will appear in the accidental background of the resulting lifetime spectra unless pileup protection is performed on muon arrivals. The underlying reasons 

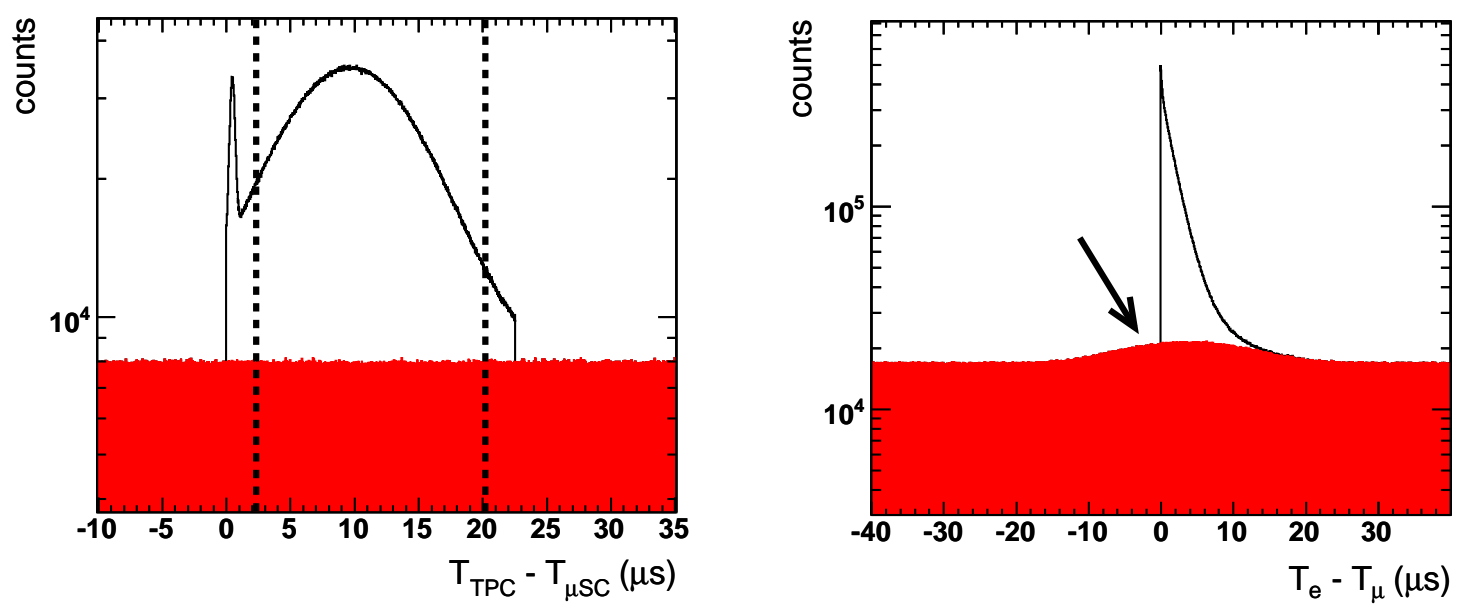

Figure 6.45: Monte-Carlo-generated reproductions of the TPC drift histogram and the muon lifetime spectrum, where muon pileup protection is not enforced. The inadvertant selection of accidental background in the TPC drift distribution when performing fiducial cuts in the $y$ dimension leads to a time-dependent distortion in the accidental background of the corresponding lifetime spectrum - namely, the bump indicated by the arrow.

for this phenomenon are illustrated in part by the Monte-Carlo-generated histograms in Figure 6.45. There, the leftmost histogram is a simulation of the non-pileup-protected TPC drift distribution, which was discussed in Section 6.3.4. A fiducial cut in the $y$ dimension is typically performed by accepting the $\mu \mathrm{SC}+\mathrm{TPC}$ coincidences within some specified interval in the central coincidence peak, as indicated by the dashed lines in drift plot in Figure 6.45. In the process, the fiducial cut unavoidably selects some of the uncorrelated coincidences in the accidental background underneath the central coincidence peak as well. This inadvertant selection of uncorrelated $\mu \mathrm{SC}+\mathrm{TPC}$ coincidences generates two "wrong-electron" accidental background components in the lifetime spectrum: an innocuous uniform background, and a wide "bump" whose peak lies beneath the lifetime spectrum. Both components are visible in the right-hand plot in Figure 6.45, and they can be described mathematically in terms of convolutions and cross-correlations [214].

A bump in the lifetime spectrum like that depicted in Figure 6.45 is problematic, 

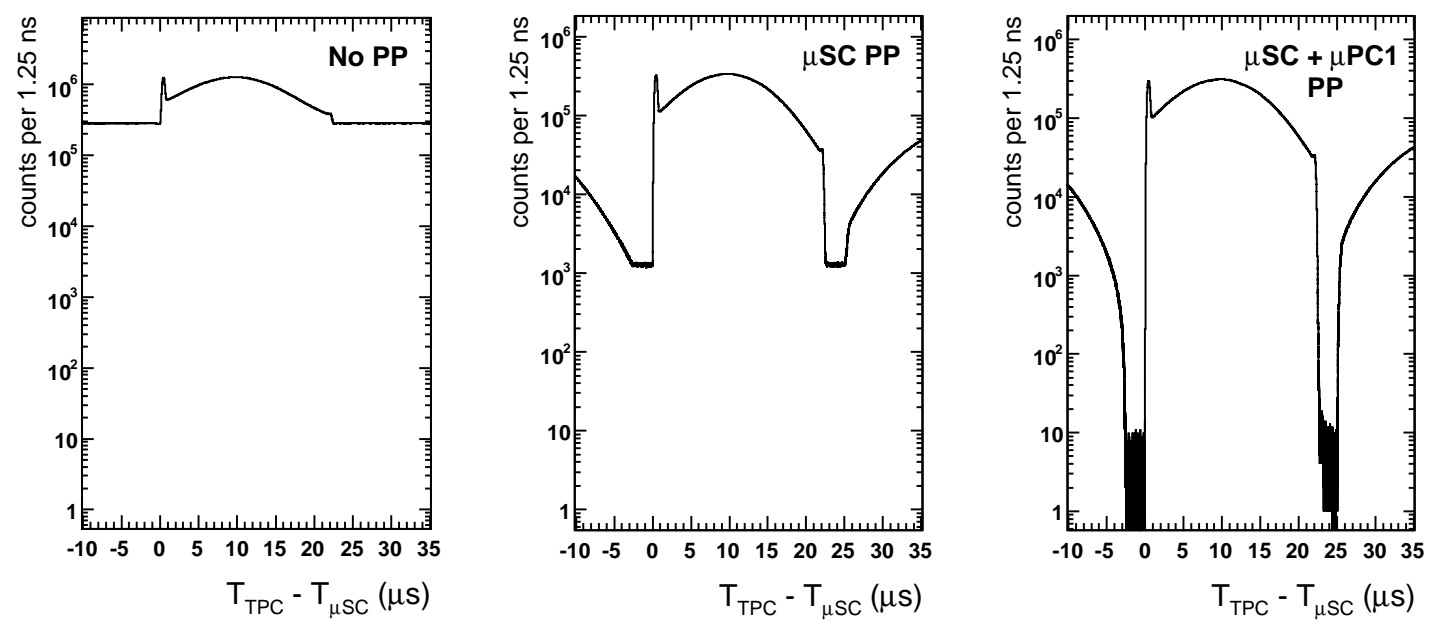

Figure 6.46: Illustration of the effects of muon pileup protection (PP) on the Prod-50 TPC drift distribution. The leftmost plot corresponds to an absence of pileup protection, just like the simulated drift histogram in Figure 6.45. The middle drift plot reveals that using the $\mu \mathrm{SC}$ alone to enforce the pileup veto removes some, but not all, of the underlying background. Finally, the right plot shows that the pileup protection is far more effective when the $\mu \mathrm{PC} 1$ detector is also used.

because it systematically lowers the fitted exponential disappearance rate. To eliminate the distortion, one must eliminate the accidental background that lies beneath the central peak in the TPC drift distribution. This is accomplished by enforcing muon pileup protection, that is, by rejecting muons that are not separated in time by at least $\pm 25 \mu$ s from other muon arrivals. The pileup protection interval is deliberately chosen larger than the maximum TPC drift time $(\approx 23.5 \mu \mathrm{s})$ in order to ensure that there can only be one muon track in the TPC in the time period immediately following a muon arrival. This eliminates the possibility for any ambiguity when associating $\mu \mathrm{SC}$ times with TPC tracks, and hence it eliminates the accidental background in the TPC drift histogram. The actual effects of pileup protection on the Run8 drift histograms are shown in Figure 6.46. There it can be seen how pileup protection "digs out" a hole from beneath the central coincidence peak. Note, however, that the $\mu \mathrm{SC}$ alone is not sufficient for the task. In order to suppress the Run8 accidental 
background to an acceptably low level, the $\mu \mathrm{PC} 1$ data must also be used to assist with pileup protection. In fact, when performing pileup protection I use the $\mu \mathrm{PC} 1 \mathrm{X}$ - and $\mathrm{Y}$ planes as independent detectors (that is, I use the $\mathrm{OR}$ of the $\mu \mathrm{PC} 1 \mathrm{X}$ - and Y-plane data) in order to maximize the pileup protection efficiency.

As Figure 6.46 makes clear, the muon pileup protection efficiency is not $100 \%$ in practice. This is due to the fact that the muon entrance detectors are not perfect at identifying muon arrivals. As a result, unwanted time-dependent structures can appear in the lifetime spectra and systematically affect the fitted disappearance rate. In the following paragraphs I examine two types of entrance detector inefficiencies - time-independent inefficiencies and time-dependent inefficiencies - and their effects on the analysis. A more detailed survey of the subject can be found in reference [214].

A time-independent inefficiency corresponds to the small but finite probability that an arriving muon will not be seen by the entrance detectors, but will nonetheless leave a track in the TPC. Such behavior produces a uniform accidental background in the TPC drift spectrum that persists even after pileup protection is applied. This residual background is visible in the middle and rightmost drift plots in Figure 6.46, in the gaps between the central coincidence peak and the pileup background on either side. The background-to-signal ratio is proportional to the inefficiency [214]. Time-independent inefficiencies generate lifetime spectrum distortions of the type depicted in Figure 6.45.

A time-dependent inefficiency is the brief period of deadtime that follows an initial hit in the muon entrance detectors. As discussed in Section 6.3.1, the effective $\mu \mathrm{SC}$ deadtime is $\approx 7 \mathrm{~ns}$ when all of the $\mu \mathrm{SC}$ signals are combined. This short deadtime is almost certainly reduced to some extent by the spatial resolution of the $\mu \mathrm{PC} 1$ detector, although the exact recovery fraction is difficult to ascertain. In Figure 6.47 I present the results of simulations of an unrealistic, $2-\mu$ s-long deadtime, in order to better illustrate the nature of deadtime 

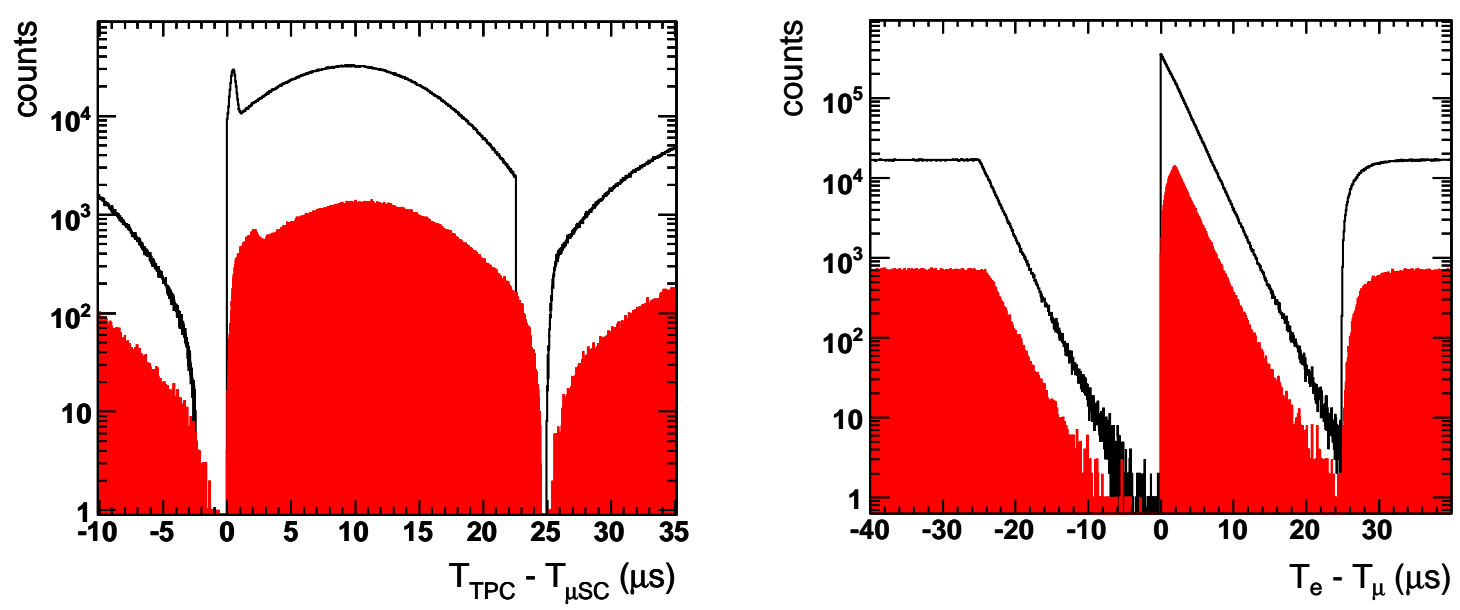

Figure 6.47: The plots above show the simulated effects of a time-dependent inefficiency (i.e. deadtime) in the muon entrance detectors on the backgrounds of the TPC drift plot (left) and the muon lifetime spectrum (right); the inefficiency-generated accidental backgrounds are indicated by solid color. The plots were created using a custom fast Monte Carlo program, and an unrealistically long deadtime of $2 \mu$ s was simulated in order to exaggerate the deadtime effects for the sake of illustration.

effects. Figure 6.47 clearly reveals how, in both the drift distribution and the muon lifetime spectrum, the deadtime produces an nonuniform accidental background which is essentially a convolution of the original distribution with a square pulse of width $T_{\text {deadtime }}$. As a result, for times $t>T_{\text {deadtime}}$, the accidental background in the lifetime histogram has the same exponential shape as the decay spectrum. If muon stops in detector materials were not a concern, then the Run8 time-dependent inefficiency would not affect the fitted disappearance rate $\lambda$, because the standard fit start time of $100 \mathrm{~ns}$ is well after the entrance detector deadtime of $\approx 10 \mathrm{~ns}$; at worst, the $\chi^{2}$ of the fit might be affected.

Muon detector inefficiencies do not only affect the shape of the accidental background in the lifetime spectra. They can also lead to unrecognized muon stops in detector materials ("wallstops"), which contribute unwanted $Z>1$ capture components to the lifetime signal and to the deadtime-generated accidental background in the lifetime histogram. 


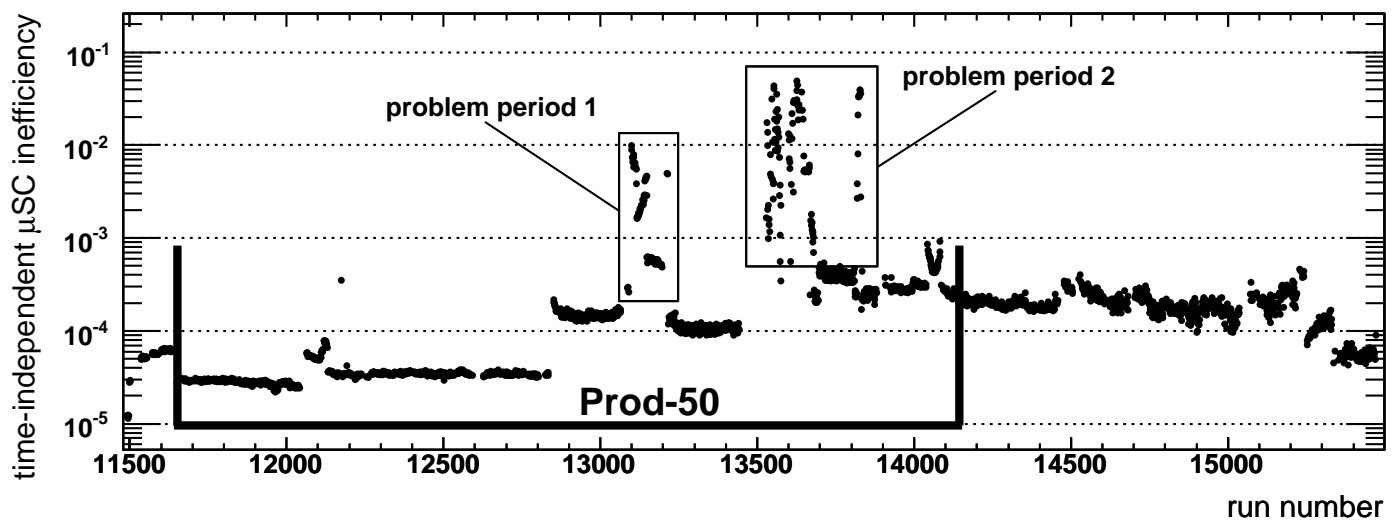

Figure 6.48: The $\mu \mathrm{SC}$ time-independent inefficiency vs. run number during Run8, as estimated from "unassociated" TPC tracks (see reference [214]). There were some technical problems with the implementation of this approach, so the inefficiencies above are rough estimates. Nevertheless, there are clearly two problematic periods during the Prod-50 fill where the $\mu \mathrm{SC}$ inefficiency rose dramatically. For the record, the $1^{\text {st }}$ and $2^{\text {nd }}$ PSI power outages occurred at runs 13067 and 13093; also, the DAQ crashed on run 13443, and numerous DAQ and electronics problems were observed in the run interval 13500-13700. The smaller rise in the inefficiency around run 12850 can be attributed to the replacement of the $500 \mu \mathrm{m}$ scintillator with the $250 \mu \mathrm{m}$ scintillator.

These $Z>1$ capture components raise the observed muon disappearance rate above its value in hydrogen, and therefore they pose a dangerous systematic threat.

In light of the potentially serious consequences of inefficiencies in the muon entrance detectors, a careful assessment of the Run8 inefficiency levels and their effects is essential. First, the time-independent inefficiency levels in the $\mu \mathrm{SC}$ detector varied significantly over the course of the run, as shown in Figure 6.48. There are two periods of particular concern during the Prod-50 fill where the $\mu \mathrm{SC}$ time-independent inefficiency levels jump dramatically. The first bad period, run interval 13099-13214, corresponds to post-power-outage misbehavior (see Section 5.4.3), while the second bad period in run interval 13528-13830 corresponds to unexplained misbehavior in the muon CAEN. Fortunately, there are two mitigating factors which help to remedy the troublesome $\mu \mathrm{SC}$ behavior. For 

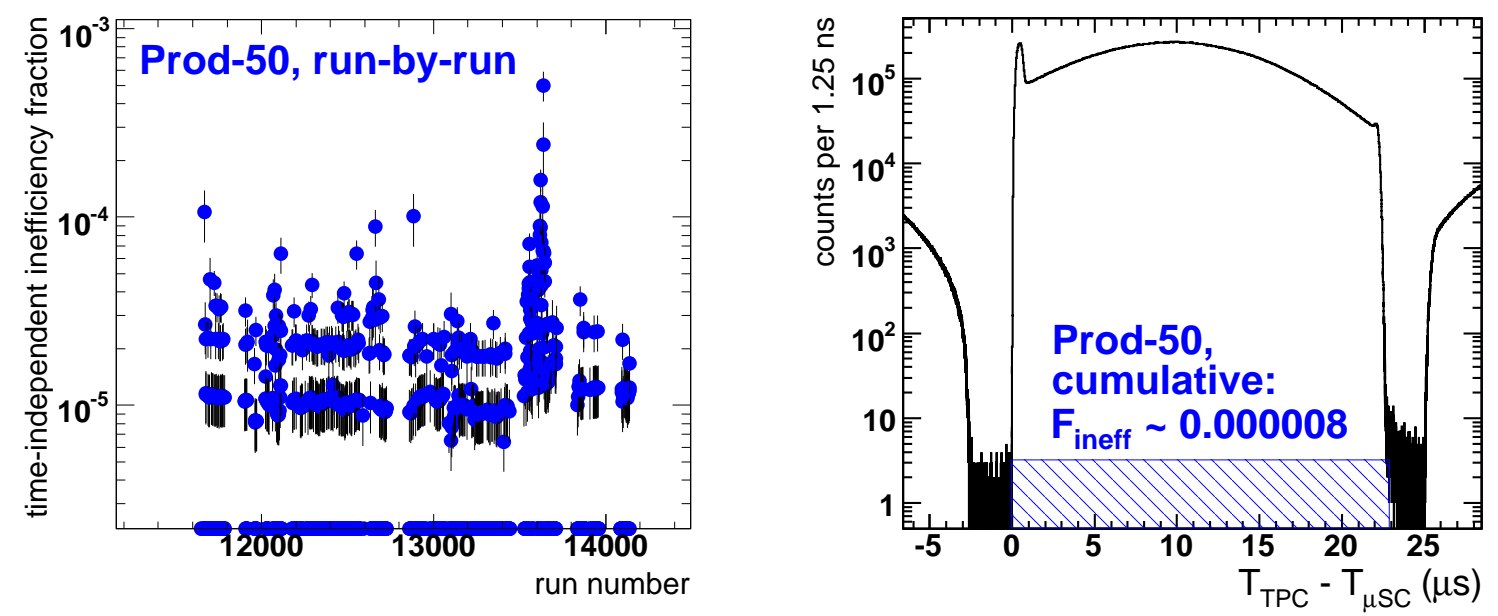

Figure 6.49: (Left) The per-run time-independent inefficiencies of the muon entrance detectors in the Prod-50 data, as estimated from the accidental background level in each run's TPC drift plot. The $\mu \mathrm{PC} 1 \mathrm{X}$ and Y planes have been used as separate detectors to enhance the pileup protection and lower the inefficiency levels. Notice that the inclusion of $\mu \mathrm{PC} 1$ improves the situation significantly, compared to Figure 6.48. The inefficiency points appear in striated bands because of the low background statistics per run; note that there are many zero points along the bottom axis of the plot. (Right) The Prod-50 cumulative TPC drift plot. The overall time-independent inefficiency indicated is the average of all of the per-run inefficiencies in the left plot.

one, it is possible that a lowered $\mu \mathrm{SC}$ inefficiency is to some extent a self-correcting problem, because under such circumstances the detector contributes fewer statistics to the lifetime spectra. More importantly, the presence of $\mu \mathrm{PC} 1$ rescues the overall pileup protection efficiency to a large extent, as was shown in the cumulative drift plots in Figure 6.46. The effect of using the $\mu \mathrm{PC} 1 \mathrm{X}$ - and Y-planes as additional, separate muon entrance detectors is shown vs. Prod-50 run number in Figure 6.49. There it is clear that the egregiously bad $\mu \mathrm{SC}$ time-independent inefficiency periods observed in Figure 6.48 have been dramatically reduced through the use of the $\mu \mathrm{PC} 1$ detector. Moreover, fits to the lifetime spectra that used both the $\mu \mathrm{SC}$ and $\mu \mathrm{PC} 1$ for pileup protection revealed that including or excluding the problematic $\mu \mathrm{SC}$ run periods does not produce any gross changes in the fitted disappear- 


\begin{tabular}{|c|c|c|c|c|}
\hline \multirow[t]{2}{*}{$\mathrm{ePC}$ treatment } & \multirow[t]{2}{*}{ Prod-50 $\mu \mathrm{SC}$ data } & \multicolumn{3}{|c|}{ Fit results } \\
\hline & & $\lambda\left(\mathrm{s}^{-1}\right)$ & $\sigma_{\lambda}\left(\mathrm{s}^{-1}\right)$ & $\chi^{2} / d$ \\
\hline \multirow{4}{*}{ cathode-AND } & all & $455,428.06$ & 12.86 & 0.964 \\
\hline & bad period 1 only & $455,415.46$ & 69.50 & 1.012 \\
\hline & bad period 2 only & $455,449.20$ & 52.30 & 0.944 \\
\hline & bad periods 1,2 excluded & $455,426.94$ & 13.51 & 0.953 \\
\hline \multirow{4}{*}{ cathode-OR } & all & $455,436.29$ & 12.33 & 0.998 \\
\hline & bad period 1 only & $455,402.63$ & 66.57 & 1.024 \\
\hline & bad period 2 only & $455,479.97$ & 50.06 & 0.959 \\
\hline & bad periods 1,2 excluded & $455,434.40$ & 12.97 & 0.981 \\
\hline
\end{tabular}

Table 6.2: Fit results to pileup-protected lifetime spectra that include or exclude the problematic $\mu \mathrm{SC}$ run periods shown in Figure 6.48. "Bad period 1" corresponds to runs in the interval 13099-13214, and "bad period 2" corresponds to runs in the interval 13528-13830. Fits to spectra from the bad run periods do not exhibit outlandish disappearance rates; that is, the rates are consistent within $1 \sigma$ with the results from spectra that excluded those runs. As their inclusion or exclusion appears to have little effect on the overall effective disappearance rate, I did not exclude the bad run periods from the data set.

ance rate (Table 6.2). Consequently, the inclusion of the potentially problematic runs does not appear to pose a threat to the final result. Furthermore, no obviously bad behavior was observed in the per-run trend plot of the fitted rate.

The time-independent inefficiency level observed in the cumulative, pileup-protected Prod-50 data is $F_{i}=8 \times 10^{-6}$, based upon the observed background in the TPC drift distribution plot (if the AND of hits in the $\mu \mathrm{PC} 1 \mathrm{X}$ and $\mathrm{Y}$ planes is used for pileup protection instead of the OR condition, the inefficiency level rises to $\left.F_{i}=16 \times 10^{-6}\right)$. The effective $\mu \mathrm{SC}$ deadtime is presumed to be roughly $7 \mathrm{~ns}$, which corresponds to an inefficiency of roughly $F_{i}=7 \mathrm{~ns} \cdot 21 \mathrm{kHz}=1.5 \times 10^{-4}$. Using these inefficiency values, and making certain assumptions about the fraction of muons that stopped in different types of detector materials (based upon fits to non-pileup-protected data), I employed the same basic Monte Carlo software that produced the plots in Figure 6.47 to estimate the effects of the Run8 inefficiencies on the data analysis [214]. Monte Carlo simulations are especially useful because they permit 
a quantitative breakdown of the different types of effects from the inefficiencies. I found that the time-independent inefficiency's contribution to the lifetime spectrum's accidental background tended to lower the fitted rate by $1-2 \mathrm{~s}^{-1}$, while wallstops in the deadtime's contribution to the accidental background tended to raise the fitted rate by $2-3 \mathrm{~s}^{-1}$. Meanwhile, wallstop contributions to the decay signal from both inefficiencies tended to raise the fitted rate by $4-5 \mathrm{~s}^{-1}$. Summing the contributions yields an overall increase in the fitted rate of approximately $5 \mathrm{~s}^{-1}$. It should be emphasized, however, that these simulation-based results are model-dependent, because I made educated guesses about the muon stopping fractions in various detector materials. The Monte Carlo results are therefore best regarded as establishing the scale of the actual effects of the Run8 inefficiencies on the fitted muon disappearance rate. Moreover, we were unable to fully reconcile the Monte Carlo results with Run8 data analysis results. Steven Clayton performed a zero-extrapolation study of deadtime effects by implementing artificially increased deadtimes, and found that the Run8 $\mu \mathrm{SC}$ deadtime appeared to have little effect on the fitted rate. We also compared the time-independent-inefficiency-generated "bumps" visible in the accidental backgrounds of the simulated and real Run8 lifetime spectra, and found discrepancies as large as a factor of three. Of course, some level of discrepancy is to be expected, because my Monte Carlo software is an imperfect model of reality and does not incorporate impact parameter cuts, the mitigating effects of $\mu \mathrm{PC} 1$, etc.

In Section 6.6.6 I will take into account all of the information available on the muon entrance detector inefficiencies - including both Monte Carlo and data analysis studies - and I will add a modest error to the fitted muon disappearance rate to cover our uncertainties regarding the exact size of the effects from these inefficiencies. 


\subsubsection{Lifetime vs. electron detector treatment}

The electron detectors operated reliably during Run8, and hence generated few acute problems in the data. A couple dozen runs were discarded outright because they exhibited exceptionally high electron data rates relative to the beamline current, but for the most part the electron detector performance was steady throughout.

One issue of serious concern is the sensitivity of the fitted $\mu^{-}$disappearance rate to the manner in which the electron detector information is treated in the data analysis. In Table 6.3 I present the disappearance rates obtained from fits to lifetime histograms that incorporated the elecron detector information in a variety of ways. The first entry, "eSC-only," refers to the lifetime spectrum in which only fourfold eSC coincidences were used to identify Michel electrons. The remaining four entries in Table 6.3 correspond to lifetime spectra in which all three electron detectors - ePC1, ePC2, and the eSC - were used to identify electrons. As described in Section 6.4.1, the "cathode-OR" condition refers to the requirement that, for each $\mathrm{ePC}$, an anode hit must be accompanied by at least one temporally coincident cathode hit - from either plane - in order to be considered a valid electron hit. The more stringent "cathode-AND" condition requires that an ePC anode hit be accompanied by both temporally coincident inner and outer cathode hits, and that the inner and outer cathodes overlap in space on the anode, in order to be considered a valid electron hit. Since both the cathode-OR and cathode-AND conditions involve the ePC1 and ePC2 detectors, they offer the additional possibility of performing a cut on the muon/electron impact parameter. In Table 6.3 I therefore give the results of not performing an impact cut, and of performing a $120 \mathrm{~mm}$ impact cut, for both the cathode-AND and cathode-OR treatments.

Unfortunately, the differences between the $\lambda$ values in Table 6.3 are much larger than expected. To appreciate the problem, first note that the "cathode-AND, $120 \mathrm{~mm}$-cut" 


\begin{tabular}{|c|c|c|c|c|c|c|c|c|}
\hline Fill & eDet treatment & Impact cut & $\begin{array}{c}N \\
\left(\times 10^{9}\right) \\
\end{array}$ & $\begin{array}{c}N / B \\
\left(\times 10^{4}\right) \\
\end{array}$ & $\lambda\left(\mathrm{s}^{-1}\right)$ & $\sigma_{\lambda}\left(\mathrm{s}^{-1}\right)$ & $\begin{array}{c}\chi^{2} / d \\
\text { fit }\end{array}$ & $\begin{array}{c}\chi^{2} / d, \\
\text { gondolas }\end{array}$ \\
\hline \multirow{4}{*}{ Prod-50 } & eSC-only & - & 1.72 & 0.06 & $455,434.46$ & 12.86 & $0.94(6)$ & $1.14(37)$ \\
\hline & cathode-AND & $120 \mathrm{~mm}$ & $\begin{array}{l}1.47 \\
1.42\end{array}$ & $\begin{array}{l}0.38 \\
1.24\end{array}$ & $\begin{array}{l}455,417.76 \\
455,428.82\end{array}$ & $\begin{array}{l}12.96 \\
12.86\end{array}$ & $\begin{array}{l}0.97(6) \\
0.95(6)\end{array}$ & $\begin{array}{l}1.36(37) \\
1.08(37)\end{array}$ \\
\hline & \multirow{2}{*}{ cathode-OR } & - & 1.60 & 0.37 & $455,426.16$ & 12.43 & $0.99(6)$ & $1.37(37)$ \\
\hline & & $120 \mathrm{~mm}$ & 1.56 & 0.96 & $455,436.90$ & 12.34 & $1.00(6)$ & $1.26(37)$ \\
\hline
\end{tabular}

Table 6.3: Results from fits to Prod-50 lifetime histograms involving different electron detector treatments; the lifetime spectra all involve standard muon pileup protection and fiducial TPC cuts, and the fits were performed using the standard procedures described in the preceding sections. The quantities $N, B$, and $\lambda$ correspond to the free parameters in the standard fit function in Equation 6.2. The quantity " $\chi^{2} / d$, fit" is the standard goodness-of-fit statistic produced by $\chi^{2}$ minimization. The quantity " $\chi^{2} / d$, gondolas" corresponds to the reduced $\chi^{2}$ that results from fitting a constant across the 16 fitted rates $\lambda$ for the eSC segments. 
data is a subset of the "cathode-AND" data, which is a subset of the "cathode-OR" data, which is a subset of the "eSC-only" data. For each pairwise relation that does not involve an impact parameter $\mathrm{cut}^{4}$, the set/subset formula in Equation 6.7 can be applied to determine the statistically allowed difference between the respective $\lambda$ values; as it turns out, the observed differences are generally far greater what is expected from random fluctuations. For example, Equation 6.7 predicts an allowed difference between the "cathode-AND" and "cathode-OR" results of $\sigma_{\lambda}=\sqrt{12.96^{2}-12.43^{2}}=3.7 \mathrm{~s}^{-1}$, yet $\Delta \lambda=8.4 \mathrm{~s}^{-1}$. It is worth noting that the consistency between any two results is not determined solely by the statistical set/subset formula: the effects of the different signal-to-background ratios in the lifetime spectra must also be taken into account, and if impact cuts are involved the comparison becomes even more complex. However, none of these considerations are sufficient to account for the size of the discrepancies between the different treatments in Table 6.3. It should be mentioned that Steven Clayton also observed large differences between his $\lambda$ values for the same basic conditions, and with the same signs as mine. However, Mr. Clayton's $\lambda$ differences were roughly half the magnitude of what I observed, and in several cases were at the edge of the statistically allowed $1 \sigma$ interval.

It was hypothesized that the large differences between the $\lambda$ values in Table 6.3 could be due to the relatively long $(1 \mu \mathrm{s})$ artificial deadtimes imposed on the ePC wires. It is known, for example, that electron detector deadtimes in principle can create background distortions very similar in nature to the distortions from muon detector deadtimes [217]. However, I found that imposing a shorter, 250 ns deadtime produced no discernible effect. Steven Clayton performed a similar study in his own analysis, implementing an even wider range of artificial deadtimes (at $300 \mathrm{~ns}, 1 \mu \mathrm{s}$, and $2 \mu \mathrm{s}$ ), and also did not observe any nonnegligible effects. The ePC deadtimes thus do not appear to be responsible for the

\footnotetext{
${ }^{4}$ Recall from Section 6.5.7 that the application of an impact parameter cut systematically elevates the fitted rate.
} 
larger-than-statistically-allowed spread in the fitted $\lambda$ values. In fact, this seems reasonable: the ePCs have a large number of wires with fine resolution, and the odds of two electron hits affecting each other are relatively small. Moreover, the muon pileup protection dramatically lowers the data rate and eliminates most deadtime-generated, time-dependent background distortions from the electron detectors.

The origins of the large discrepancies between the eSC-only, cathode-AND, and cathode-OR $\lambda$ values in Table 6.3 remain unknown. This raises the question: Which electron table(s) should be used to obtain the final result? The eSC-only condition involves fewer detectors, but the corresponding lifetime spectrum has a comparatively low signal-tobackground ratio, and the lack of electron trajectory information is a significant limitation. The cathode-AND condition provides better tracking and background suppression than the cathode-OR condition, but the cathode-AND condition is probably more susceptible to any unidentified systematic ePC effects which could responsible for skewing the results. It is clear that at least one of the cathode treatments is being skewed by some unknown effect, given the inconsistency in the results from the two approaches, but it is not clear if the $120 \mathrm{~mm}$ impact cut serves to improve the lifetime spectrum by "cleaning up" the signal, or if the cut introduces some sort of distortion. We ultimately chose to use all three electron detectors in the identification of decay electrons. Since we have no way of knowing which of the ePC cathode conditions is superior, or whether the impact cut is to be preferred or not, I will process all four electron tables in parallel. I will later have to reconcile the four approaches into a single result.

\subsubsection{Unique-electron condition}

One potentially interesting study is to form lifetime spectra where the only events accepted inside of some time interval relative to the muon arrival are those where a single 


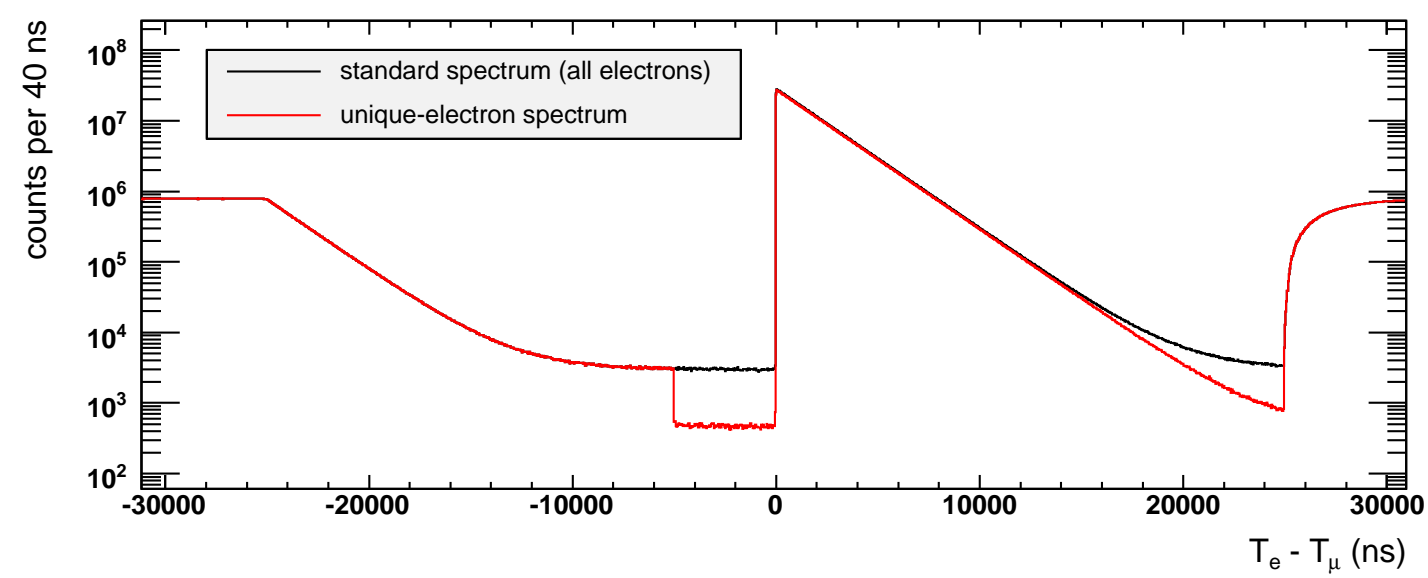

Figure 6.50: An example of a "unique-electron" lifetime spectrum, shown atop a standard lifetime spectrum for comparison. The two spectra were created under largely the same conditions: both are from the Prod-50 cathode-OR data, involve $\pm 25 \mu$ s pileup protection on muon arrivals, and utilize the impact parameter cut $b \leq 120 \mathrm{~mm}$. (It should be noted that the histograms above have been rebinned and are presented in 40 ns resolution.) The two spectra are identical outside of the time interval $[-5,25] \mu \mathrm{s}$, but inside that interval the unique-electron spectrum only contains decay events where the muon arrival can be uniquely matched to an electron. In this region the unique-electron spectrum exhibits an accidental background that is $\approx 6.5$ times lower than its counterpart, while the unique-electron decay signal is only $3 \%$ lower.

electron is observed. In other words, if two or more electron detector hits are seen within some specified time interval - which generally covers a large fraction of the muon decay period - the muon arrival is rejected. This "unique-electron" procedure obviously reduces the statistics in the lifetime spectrum, but it disproportionately affects the background data; in relative terms, the reduction in statistics is greater in the background than in the decay signal. The unique-electron condition is in principle time-independent, so its only effect should be to improve the signal-to-background ratio in the lifetime histogram. An example of a spectrum that was created by enforcing the unique-electron condition over the time interval $[-5,25] \mu \mathrm{s}$ is presented in Figure 6.50. There one can see the dramatic suppression in the background produced by the unique-electron condition. 
The results from fits to unique-electron lifetime spectra are best compared to results from fits to the cosmics-adjusted spectra, because in both cases steps have been taken to reduce contributions from the accidental background. I present the results from fits to both types of spectra side-by-side in Table 6.4. There one can see that the signal-tobackground ratios $N / B$ are significantly better in the unique-electron lifetime spectra than in the cosmics-adjusted spectra, even though the overall statistics $N$ are quite similar for the two approaches. This is likely the reason for the fact that fits to the unique-electron lifetime spectra produce somewhat better $\chi^{2} / d$ values than fits to the cosmics-adjusted spectra. Nonetheless, in spite of these differences the fitted exponential rates $\lambda$ for the two approaches are statistically consistent. As a result, I have chosen to regard the uniqueelectron fits as a reassuring consistency check, and to use the cosmics-adjusted spectra as my primary histograms, because they involve one less potentially complicating data cut.

\subsection{Determination of the (blinded) muon disappearance $\operatorname{rate}(\mathrm{s})$}

In the following subsections I describe in detail the sequence of analysis operations I performed on the 2004 data in order to obtain value(s) for the (blinded) $\mu^{-}$disappearance rate in protium gas. First, I present initial values for the disappearance rates, obtained from three-parameter exponential fits to several types of lifetime spectra produced from the cleanfill, Prod-50 production data. Next, I perform a series of corrections to the disappearance rates to compensate for the effects of both detector limitations and the presence of small amounts of impurities in the hydrogen gas. Each of the perturbations to the disappearance rates is small, less than $50 \mathrm{ppm}$, so the corrections are linear and additive, though they must be performed in a certain order. (The validity of the additive linearity of the corrections has 


\begin{tabular}{|c|c|c|c|c|c|c|c|c|}
\hline Fill & $\mathrm{ePC}$ treatment & Impact cut & Lifetime condition $^{\dagger}$ & $\begin{array}{c}N \\
\left(\times 10^{9}\right) \\
\end{array}$ & $\begin{array}{c}N / B \\
\left(\times 10^{4}\right) \\
\end{array}$ & $\lambda\left(\mathrm{s}^{-1}\right)$ & $\sigma_{\lambda}\left(\mathrm{s}^{-1}\right)$ & $\chi^{2} / d$ \\
\hline \multirow{4}{*}{ Prod-50 } & \multirow{2}{*}{ cathode-AND } & \multirow{2}{*}{$\begin{array}{c}120 \mathrm{~mm} \\
\quad,\end{array}$} & \multirow{2}{*}{$\begin{array}{l}\text { cosmics-error-adjusted } \\
\text { unique-electron }\end{array}$} & 1.42 & 1.2 & $455,425.80$ & 12.86 & $0.95(6)$ \\
\hline & & & & 1.40 & 6.4 & $455,427.42$ & 12.79 & $0.91(6)$ \\
\hline & \multirow{2}{*}{ cathode-OR } & $"$ & cosmics-error-adjusted & 1.56 & 1.0 & $455,433.78$ & 12.34 & $1.00(6)$ \\
\hline & & $"$ & unique-electron & 1.51 & 6.1 & $455,432.91$ & 12.28 & $0.90(6)$ \\
\hline
\end{tabular}

$\dagger$ The lifetime spectra here have also undergone the removal of identified $\mu+p$ scatters, a subject that will be addressed later, in Section 6.6.2.

Table 6.4: The results of fits to cosmics-error-adjusted and "unique-electron" lifetime spectra from the Prod-50 data. The fits were performed using the standard procedures described in Sections 6.5.2-6.5.5; the adjustment of the bin errors for cosmics is described in Section 6.5.6. The quantity $N$ is the total number of decays contained in the lifetime spectrum, while $N / B$ is the spectrum's signal-to-background ratio at $\Delta t=0$. The quantity $\lambda$ is the fitted exponential rate, $\sigma_{\lambda}$ is the corresponding error, and $\chi^{2} / d$ describes the quality of the fit; the number of degrees of freedom $d=617$. 
been demonstrated using Monte Carlo simulations.) It it worth noting that, because the corrections in this chapter are compensating for shortcomings in the experimental setup, in principle the corrections could be reduced, or even eliminated, with the use of a better apparatus and cleaner gas.

I should remind the reader that the disappearance rates presented in this chapter are still blinded, as indicated in this section's title. That is, the fits and calculations were performed assuming a DAQ clock frequency of exactly $100 \mathrm{MHz}$, so the results do not correspond to physical numbers. The true 2004 frequency will be unveiled at the beginning of the next chapter, and the results will be adjusted accordingly.

\subsubsection{Uncorrected rates}

To obtain initial values for the $\mu^{-}$disappearance rate in hydrogen, I performed fits to lifetime spectra that were created under the following conditions, which have been discussed in previous sections:

- The muon data came from $\mu \mathrm{SC}+\mu \mathrm{PC} 1+\mathrm{TPC}$ coincidences, with the standard $\mathrm{TPC}$ fiducial cuts applied. (Here, " $\mu \mathrm{PC} 1$ " means that hits were observed in both the $\mathrm{X}$ and Y planes, coincident with the $\mu \mathrm{SC}$ hit.) The $\mu \mathrm{SC}$ and $\mu \mathrm{PC} 1 \mathrm{XY}-\mathrm{OR}$ tables were used in conjunction to enforce $25 \mu$ s pileup protection.

- The electron data came from ePC1+ePC2+eSC coincidences. I looked at spectra that utilized the ePC cathode-AND treatment, as well as at spectra that utilized the cathode-OR treatment. The two treatments yield different results, and at present I do not have any reasons for choosing one over the other.

- Because of lingering questions about the effects of impact parameter cuts, I looked at lifetime spectra that did not involve an explicit impact cut, as well as at spectra that 
involved a $120 \mathrm{~mm}$ impact cut.

I fit the lifetime spectra under the following conditions:

- For each type of spectrum, I aligned the sixteen fine-resolution eSC lifetime histograms at $\Delta t=0$, added them together, and then rebinned the summed histogram by 32 , with the rebin boundary set at $100 \mathrm{~ns}$.

- I assumed that cosmics accounted for half of the observed background, and adjusted the bin errors accordingly.

- I used MINUIT via ROOT to perform three-parameter exponential fits over the time range $[100,24900]$ ns. In the fits I specified the available "I" option, which instructs the software to use the integral of the fit function over each histogram bin, instead of the function's value at the bin center. (This choice only has sub-Hz effects.) Although the fits were accomplished with ROOT's default $\chi^{2}$ minimization, it is possible to instead select log-likelihood (LL) minimization by specifying the "L" fit option; however, I found that the LL results were statistically consistent with the $\chi^{2}$ results, so I elected to continue using the latter. I should mention that I also tested the "M" fit option, which ostensibly provides better fit results, as well as the "E" fit option, which uses MINOS to obtain improved error estimates. In both cases the effects on the fitted rates and errors were negligible, on the scale of $1 \mathrm{mHz}$.

The numerical fit results are presented in Table 6.5. Notice that I have included there the results for both the Prod-50 and CalibNat data, so that I can later perform the deuterium zero-extrapolation correction. The results corresponding to the four types of lifetime histogram treatments - cathode-AND vs. cathode-OR, and no impact cut vs. the $120 \mathrm{~mm}$ impact cut-will be reconciled into a single number in Section 7.2.3, after the final results for $\Lambda_{S}$ have been obtained. 


\begin{tabular}{|c|c|c|c|c|c|c|c|c|}
\hline Fill & $\mathrm{ePC}$ treatment & Impact cut & $\begin{array}{c}N \\
\left(\times 10^{9}\right) \\
\end{array}$ & $\begin{array}{c}N / B \\
\left(\times 10^{4}\right) \\
\end{array}$ & $\lambda\left(\mathrm{s}^{-1}\right)$ & $\sigma_{\lambda}\left(\mathrm{s}^{-1}\right)$ & $\begin{array}{c}\chi^{2} / d \\
\text { fit }\end{array}$ & $\begin{array}{c}\chi^{2} / d, \\
\text { gondolas }\end{array}$ \\
\hline \multirow{2}{*}{ Prod-50 } & cathode-AND & $120 \mathrm{~mm}$ & $\begin{array}{l}1.47 \\
1.42\end{array}$ & $\begin{array}{l}0.4 \\
1.2\end{array}$ & $\begin{array}{l}455,417.76 \\
455,428.82\end{array}$ & $\begin{array}{l}12.96 \\
12.86\end{array}$ & $\begin{array}{l}0.97(6) \\
0.95(6)\end{array}$ & $\begin{array}{l}1.36(37) \\
1.08(37)\end{array}$ \\
\hline & cathode-OR & $120 \mathrm{~mm}$ & $\begin{array}{l}1.60 \\
1.56\end{array}$ & $\begin{array}{l}0.4 \\
1.0\end{array}$ & $\begin{array}{l}455,426.16 \\
455,436.90\end{array}$ & $\begin{array}{l}12.43 \\
12.34\end{array}$ & $\begin{array}{l}0.99(6) \\
1.00(6)\end{array}$ & $\begin{array}{l}1.37(37) \\
1.26(37)\end{array}$ \\
\hline \multirow{2}{*}{ CalibNat } & cathode-AND & $120 \mathrm{~mm}$ & $\begin{array}{l}0.06 \\
0.06\end{array}$ & $\begin{array}{l}0.4 \\
1.2\end{array}$ & $\begin{array}{l}455,947.49 \\
456,197.27\end{array}$ & $\begin{array}{l}65.03 \\
64.49\end{array}$ & $\begin{array}{l}1.05(6) \\
1.07(6)\end{array}$ & $\begin{array}{l}6.89(37) \\
7.43(37)\end{array}$ \\
\hline & cathode-OR & $120 \mathrm{~mm}$ & $\begin{array}{l}0.06 \\
0.06\end{array}$ & $\begin{array}{l}0.3 \\
0.9\end{array}$ & $\begin{array}{l}455,986.52 \\
456,184.51\end{array}$ & $\begin{array}{l}62.32 \\
61.83\end{array}$ & $\begin{array}{l}1.07(6) \\
1.09(6)\end{array}$ & $\begin{array}{l}6.82(37) \\
7.48(37)\end{array}$ \\
\hline
\end{tabular}

Table 6.5: Results from three-parameter exponential fits to several different types of lifetime spectra from the Prod-50 and CalibNat data, prior to performing any corrections. The quantity $N$ is the total number of decays contained in each lifetime spectrum, while $N / B$ is the spectrum's signal-to-background ratio at $\Delta t=0$. The quantity $\lambda$ is the fitted exponential rate, and $\sigma_{\lambda}$ is its corresponding error. The quantity " $\chi^{2} / d$, fit" is the standard goodness-of-fit statistic produced by $\chi^{2}$ minimization; in this case, the number of degrees of freedom $d=(620-3)=617$, as there are 620 40-ns bins inside the fit interval 100-24900 ns, and the fit function has three free parameters, $N, B$, and $\lambda$. Note that the " $\chi^{2} / d$, fit" values for the Prod-50 fits are roughly consistent with unity, while the values for the CalibNat fits are much worse because of the distorting effects of deuterium diffusion. The effects of deuterium are also manifested in the quantity " $\chi^{2} / d$, gondolas," which corresponds to the reduced $\chi^{2}$ that results from fitting a constant across the 16 fitted rates for the eSC segments (in this case, $d=15$ ). The " $\chi^{2} / d$, gondolas" values are far higher for the CalibNat fits for the reasons discussed in Section 6.5.12. 


\subsection{2 $\mu+p$ scatter correction}

On rare occasions, a muon enters the TPC volume and hard scatters off a proton. As illustrated in Figure 6.51, the recoiling proton sometimes deposits enough energy in the detector to trigger the $\mathrm{EH}$ threshold, and as a result the scatter event can mimic the appearance of a muon stop. The potential for misinterpreting these $\mu+p$ scatter events as muon stops is compounded by the fact that the scattered muon often does not leave behind a robust ionizing track; as a result, the scatter can easily go unrecognized by the tracking software. Such events pose a dangerous systematic threat, because they can easily be mistaken for a good muon stops in the target gas, when in fact the scattered muons might have stopped in surrounding detector materials. As a result, scatter events can introduce $Z>1$ capture components that increase the observed muon disappearance rate above the value for stops in pure hydrogen. It is therefore essential to identify $\mu+p$ scatter events so that they can be discarded from the pool of muon stop candidates, and to quantify the efficiency of the scatter identification algorithms.

I developed two methods for identifying $\mu+p$ scatter events, which I designated as "TLS" and "MWPC":

- TLS is an acronym for "Total-Least-Squares." In this method I inspect the TPC data in the region downstream of an ostensible EH Bragg stop and look for a trail of EL pixels leading away from the EH cluster-evidence of the departure of a scattered muon. If I find more than four EL pixels in the search region, I perform a total-leastsquares (as distinguished from ordinary-least-squares) fit to the points. A set of EL pixels is considered to be a scattered muon's track if (1) the spread $\sigma$ of points around the best-fit line is $<2.2 \mathrm{~mm}$, (2) the best-fit line points back towards the EH cluster (impact $<8 \mathrm{~mm}$ ), (3) the points cover a distance of at least $18 \mathrm{~mm}$, and (4) the 


\section{TPC Display}

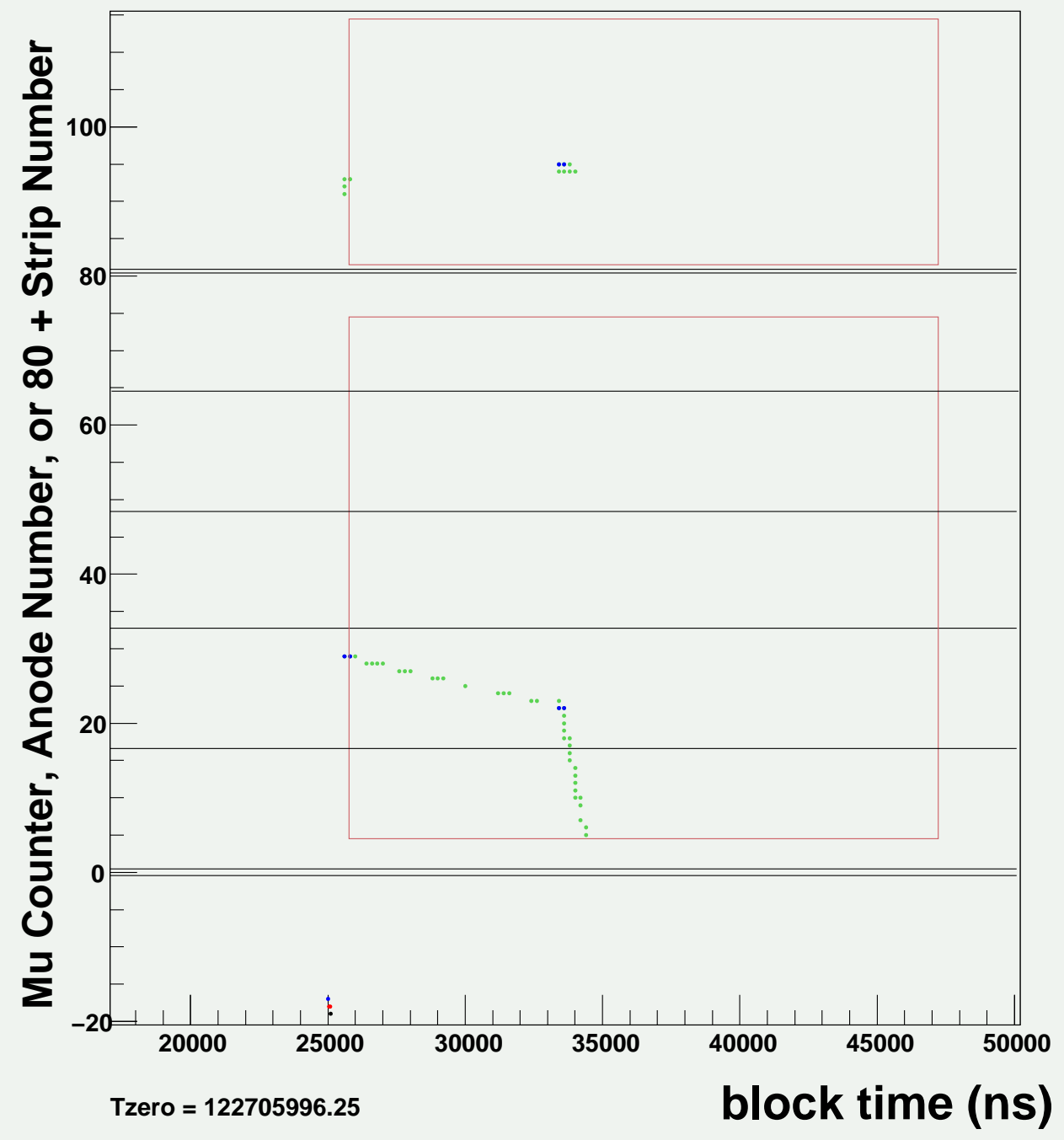

Figure 6.51: An example of an unambiguous $\mu+p$ scatter event in the Run 8 TPC data, as viewed in the event display. In this instance the muon scattered downwards through the bottom of the TPC, leaving behind two potentially useful signatures: an intermittent trail of green EL pixels leading away from the point of scatter, and a cluster of EH pixels at the point where the muon passed through the MWPC region at the bottom of the detector. 
points are sufficiently spread out along the best-fit line. Note that this algorithm, as implemented, is only capable of identifying forward scatters. I should also mention that if a trail of EL pixels is found, but leads to another EH cluster in the TPC active volume, the event is considered a safe muon stop in the gas, and is not classified as a scatter.

- MWPC is an acronym for the "MultiWire Proportional Chamber" region of the TPC. When an ionizing particle passes through the bottom of the TPC, it usually triggers the EH threshold. Thus, if a $\mu \mathrm{SC}$ hit can be associated with an apparently good TPC stop in the fiducial drift interval, but there is also an EH cluster in the time interval $[0,850]$ ns after the $\mu \mathrm{SC}$ hit, then it is likely that the muon scattered through the bottom of the TPC. Muons that scatter downward through the bottom of the TPC are especially dangerous, because they have a high likelihood of stopping in the aluminum plate on the underside of the detector (see Figure 4.4).

The $\mu+p$ scatter event shown in Figure 6.51 was recognized by both the TLS and MWPC scatter algorithms, demonstrating how the two approaches provide a certain measure of redundancy.

Statistics for the muon scatter events identified in the Prod-50 and CalibNat data are provided in Table 6.6. The majority of identified scatters come from the TLS algorithm, which accounts for $\approx 75 \%$ of total. Notice that the TLS scatter statistics for the CalibNat fill are less than half those for the Prod-50 fill. This is almost certainly attributable to the CalibNat fill's reduced TPC operating voltage (4.8 kV compared to Prod-50's $5.0 \mathrm{kV})$, which results in a deterioration of the TPC's detection sensitivity. In fact, part of the reason we chose to ignore the Prod-48 data - that is, the clean fill data collected at TPC voltage $4817 \mathrm{~V}$ - is because the reduced track sensitivity makes the identification of scatters more 


\begin{tabular}{llrc}
\hline \hline Fill & Event & Count & $\begin{array}{r}\text { Scatter fraction } \\
(\mathrm{ppm})\end{array}$ \\
\hline \multirow{4}{*}{ Prod-50 } & Muon stops & $2,378,668,731$ & - \\
& TLS scatters & 240,717 & $101.2(2)$ \\
MWPC scatters & 82,571 & $34.7(1)$ \\
Total scatters & 288,826 & $121.4(2)$ \\
& Muon stops & $95,241,158$ & - \\
& MLS scatters & 3,237 & $33.9(6)$ \\
MWPC scatters & 2,542 & $26.6(5)$ \\
Motal scatters & 5,365 & $56.3(8)$ \\
\hline
\end{tabular}

Table 6.6: Statistics for the $\mu+p$ scatter events identified in the Prod-50 and CalibNat data by my software. The category "Muon stops" refers to the number of pileup-protected muon stop candidates that were inspected for evidence of scattering. My two scatter-identification algorithms, TLS and MWPC, are described in detail in the text. The category "Total scatters" refers to the union of scatter events identified by the TLS and MWPC methods.

difficult.

The hypothesis that some of the scattered muons ultimately stop in surrounding detector materials is supported by fits to the lifetime spectra. As shown in Figure 6.52, the decay rates from fits to spectra containing only scattered muons are consistently and unambiguously higher than the rates obtained from fits to spectra containing standard fiducial muon stops. Furthermore, start time scans of the fitted disappearance rate in the scatter-only spectra (Figure 6.53) reveal the presence of high-rate transients, which is consistent with the $Z>1$ capture contributions expected if scattered muons are stopping in detector materials. A comprehensive list of numerical results of fits to lifetime spectra which include or exclude scatter events in different manners is presented in Tables 6.7 and 6.8. The results in Tables 6.7 and 6.8 indicate that, as expected, removing scatter events from the lifetime spectra tends to lower the fitted decay rate; in the case of the Prod-50 data in Table 6.7, the shift is generally $\approx 3 \mathrm{~s}^{-1}$. Moreover, differential analysis of the scatter statistics and associated decay rates enables me to place a relatively small 


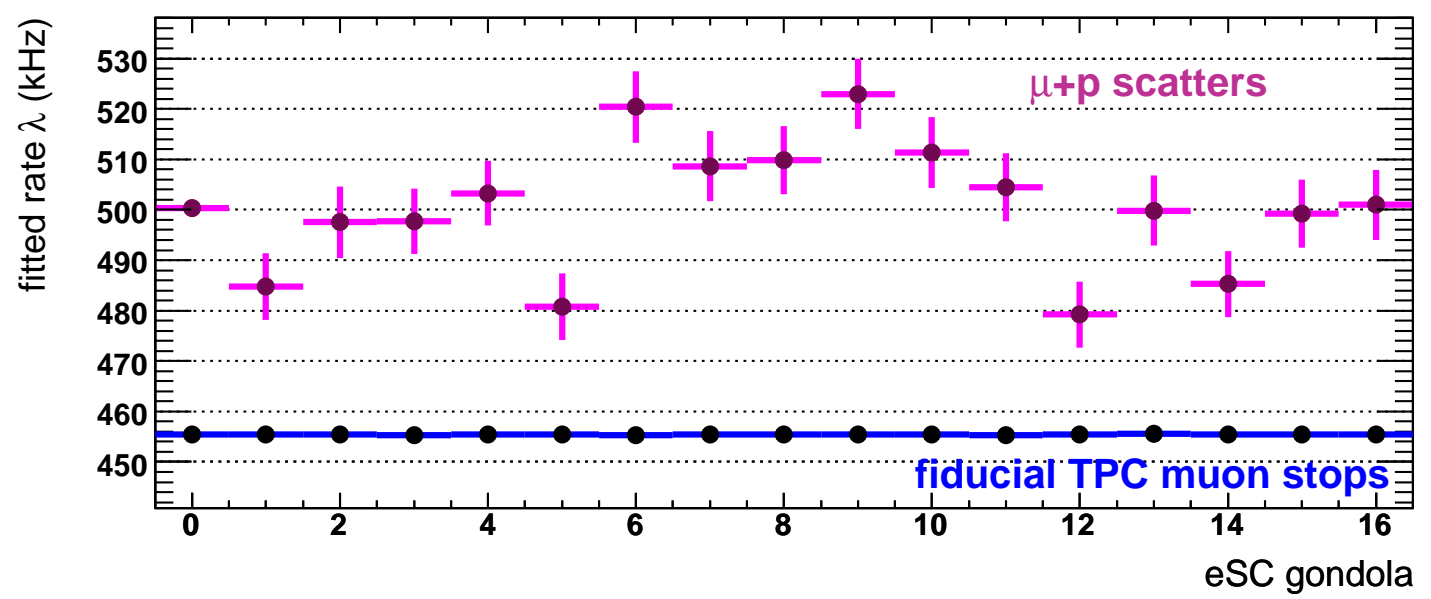

Figure 6.52: The $\mu^{-}$disappearance rate vs. eSC gondola, from fits to to lifetime spectra containing scattered muons only, as well as from fits to spectra containing normal fiducial muon stops. (In both cases, the spectra are from the Prod-50, cathode-OR, 120-mm-impactcut data.) The error bars on the $\mu+p$ scatter decay rates are comparatively large because of the far lower statistics of such events (see Table 6.6). The most important feature here is that the fitted rates for the scattered muons are systematically higher than for normal fiducial stops, which is a strong indication that the scatter spectra contain contributions from muon captures in detector materials.

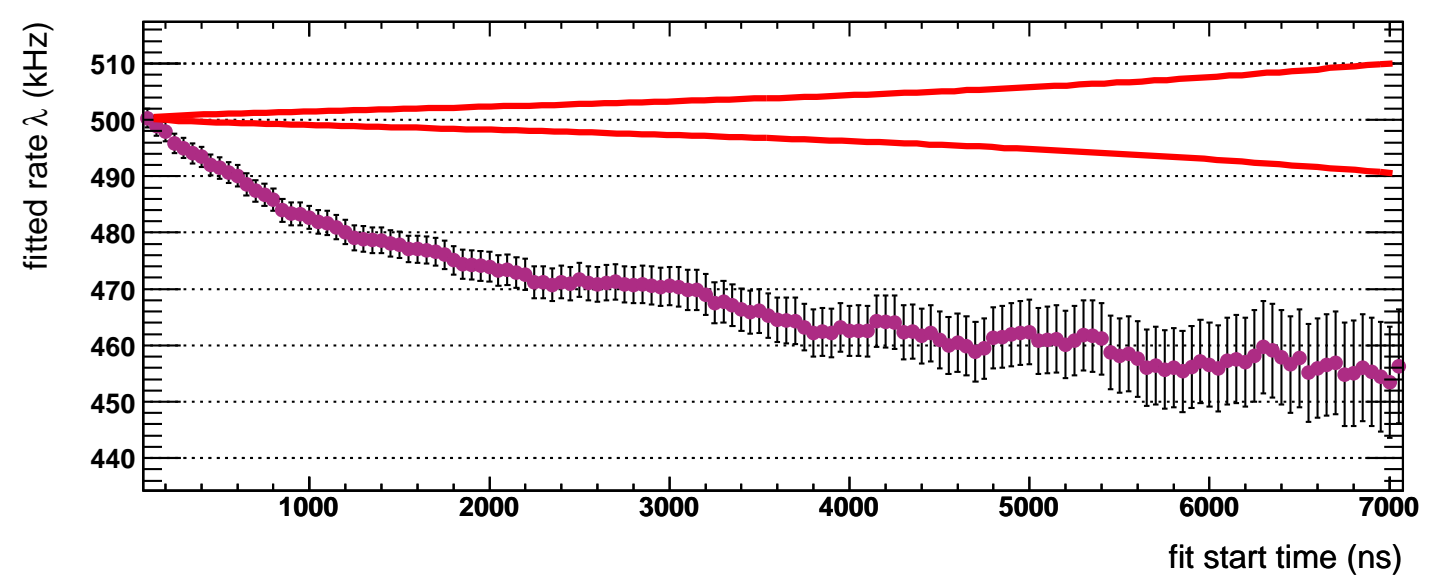

Figure 6.53: The $\mu^{-}$disappearance rates from a fit start time scan to a lifetime spectrum containing only $\mu+p$ scatters. (In this case, the scatter spectrum is from the Prod-50, cathode-OR, 120-mm-impact-cut data.) The fitted rates exhibit a pronounced downward trend with increasing fit start time, deviating far outside of the statistically allowed $1 \sigma$ band and thus providing clear evidence of $Z>1$ capture contributions from muon stops in detector materials. 


\begin{tabular}{|c|c|c|c|c|c|c|c|}
\hline Fill & $\mathrm{ePC}$ treatment & Impact cut & Condition & $\lambda\left(\mathrm{s}^{-1}\right)$ & $\sigma_{\lambda}\left(\mathrm{s}^{-1}\right)$ & $\chi^{2} / d$ & $\Delta \lambda\left(\mathrm{s}^{-1}\right)$ \\
\hline \multirow{12}{*}{ Prod-50 } & \multirow{5}{*}{ cathode-AND } & \multirow{2}{*}{ - } & scatters included & $455,417.76$ & 12.96 & $0.972(57)$ & \\
\hline & & & all scatters removed & $455,414.43$ & 12.96 & $0.972(57)$ & $-3.33(22)$ \\
\hline & & \multirow{3}{*}{$120 \mathrm{~mm}$} & scatters included & $455,428.82$ & 12.86 & $0.953(57)$ & \\
\hline & & & scatters only & $499,503.25$ & $1,767.44$ & $1.257(66)$ & \\
\hline & & & all scatters removed & $455,425.80$ & 12.86 & $0.953(57)$ & $-3.02(22)$ \\
\hline & \multirow{7}{*}{ cathode-OR } & \multirow{2}{*}{-} & scatters included & $455,426.16$ & 12.43 & $0.992(57)$ & \multirow[b]{2}{*}{$-3.35(21)$} \\
\hline & & & all scatters removed & $455,422.81$ & 12.43 & $0.992(57)$ & \\
\hline & & \multirow{5}{*}{$120 \mathrm{~mm}$} & scatters included & $455,436.90$ & 12.34 & $0.996(57)$ & \\
\hline & & & scatters only & $500,303.07$ & $1,691.66$ & $1.316(65)$ & \\
\hline & & & TLS scatters removed & $455,434.17$ & 12.34 & $0.996(57)$ & $-2.73(21)$ \\
\hline & & & MWPC scatters removed & $455,435.96$ & 12.34 & $0.996(57)$ & $-0.94(21)$ \\
\hline & & & all scatters removed & $455,433.78$ & 12.34 & $0.996(57)$ & $-3.12(21)$ \\
\hline
\end{tabular}

Table 6.7: Fitted rates to Prod-50 lifetime spectra formed according to different muon scatter inclusion/exclusion conditions. Although a $\chi^{2} / d$ value is reported for the "scatters only" lifetime spectra, they were actually fit using log-likehood minimization because their statistics are so low. The $\Delta \lambda$ values are calculated from the difference between the "scatters included" and "all scatters removed" rates, and thus describe the effect of removing scatter events. The corresponding errors $\sigma_{\Delta \lambda}$ (in parentheses) were calculated using the rates in this table and the statistics in Table 6.6, according to the prescription outlined in Appendix I. 


\begin{tabular}{|c|c|c|c|c|c|c|c|}
\hline Fill & ePC treatment & Impact cut & Condition & $\lambda\left(\mathrm{s}^{-1}\right)$ & $\sigma_{\lambda}\left(\mathrm{s}^{-1}\right)$ & $\chi^{2} / d$ & $\Delta \lambda\left(\mathrm{s}^{-1}\right)$ \\
\hline \multirow{12}{*}{ CalibNat } & \multirow{5}{*}{ cathode-AND } & \multirow{2}{*}{-} & scatters included & $455,947.49$ & 65.03 & $1.046(57)$ & \\
\hline & & & all scatters removed & $455,945.84$ & 65.03 & $1.047(57)$ & $-1.65(87)$ \\
\hline & & \multirow{3}{*}{$120 \mathrm{~mm}$} & scatters included & $456,197.27$ & 64.49 & $1.075(57)$ & \\
\hline & & & scatters only & $483,597.52$ & $15,374.57$ & $0.968(108)$ & \\
\hline & & & all scatters removed & $456,195.79$ & 64.49 & $1.076(57)$ & $-1.48(87)$ \\
\hline & \multirow{7}{*}{ cathode-OR } & \multirow{2}{*}{-} & scatters included & $455,986.52$ & 62.32 & $1.068(57)$ & \multirow{4}{*}{$-1.65(87)$} \\
\hline & & & all scatters removed & $455,984.87$ & 62.32 & $1.068(57)$ & \\
\hline & & \multirow{5}{*}{$120 \mathrm{~mm}$} & scatters included & $456,184.51$ & 61.83 & $1.087(57)$ & \\
\hline & & & scatters only & $481,362.10$ & $15,344.86$ & $0.867(107)$ & \\
\hline & & & TLS scatters removed & $456,183.54$ & 61.83 & $1.088(57)$ & $-0.97(87)$ \\
\hline & & & MWPC scatters removed & $456,183.75$ & 61.83 & $1.087(57)$ & $-0.76(87)$ \\
\hline & & & all scatters removed & $456,183.07$ & 61.83 & $1.088(57)$ & $-1.44(87)$ \\
\hline
\end{tabular}

Table 6.8: Fitted rates to CalibNat lifetime spectra formed according to different muon scatter inclusion/exclusion conditions. Although a $\chi^{2} / d$ value is reported for the "scatters only" lifetime spectra, they were actually fit using log-likehood minimization because their statistics are so low; moreover, the background parameter $B$ was held fixed at zero when fitting the CalibNat scatter-only spectra because their background statistics were basically nonexistent - otherwise, the fit would pull $B<0$. The $\Delta \lambda$ values are calculated from the difference between the "scatters included" and "all scatters removed" rates, and thus describe the effect of removing scatter events. The corresponding errors $\sigma_{\Delta \lambda}$ (in parentheses) were calculated using the rates in this table and the statistics in Table 6.6, according to the prescription outlined in Appendix I. 
error on these shifts, and thereby establish that they are not simply due to statistical fluctuations in the contents of the lifetime spectra (see Appendix I). Incidentally, the results in Tables 6.7 and 6.8 reveal that the shifts in the decay rate resulting from the removal of TLS- and MWPC-identified scatters are proportional to their respective statistics, which is to be expected if the scattering effects are small and therefore linear in nature. For example, the ratio of Prod-50 scatter fractions in Table 6.6 is $\left(f_{\mathrm{TLS}} / f_{\mathrm{MWPC}}\right) \approx 3$, and the ratio of their rate shifts in Table 6.7 is $\left(\Delta \lambda_{\mathrm{TLS}} / \Delta \lambda_{\mathrm{MWPC}}\right) \approx 3$.

At this point we must consider the fact that my analysis software is almost certainly not catching all $\mu+p$ scatter events. Indeed, the TLS algorithm is designed to search only for forward scatters; moreover, the TLS best-fit assessments can be thwarted by outlier pixels. There is also always the possibility that a muon exited the sensitive volume of the TPC without leaving behind sufficient information (that is, enough pixels) to clearly reveal its departure. Unfortunately, there's no way of knowing a priori how many scatter events are being missed - my scatter identification software could be $99 \%$ efficient, or $1 \%$ efficient. To resolve the issue, we need to somehow estimate the fraction of $\mu+p$ scatter events that deposit enough energy to mimic a muon stop. A useful resource in this regard is the SRIM simulation software [202], which enables us to simulate muon trajectories in hydrogen gas under MuCap-like conditions, and can thus provide $\mu+p$ scatter information for use as a standard for comparison with the actual Run8 data.

To start, I supplied SRIM with a list of randomly generated muon momenta and incident angles, and SRIM calculated their trajectories in hydrogen gas ${ }^{5}$. The muon beam properties were tuned so that the resulting SRIM stopping distribution resembled the Run8 stopping distribution in Figure 5.8. A potentially dangerous scatter event is one where

\footnotetext{
${ }^{5}$ Actually, SRIM only offers the possibility to simulate the stopping of ions in matter, so we were restricted to simulating $\mu^{+}$stops with "light" $\mathrm{H}$ ions. The stopping properties of positive and negative muons are essentially the same.
} 


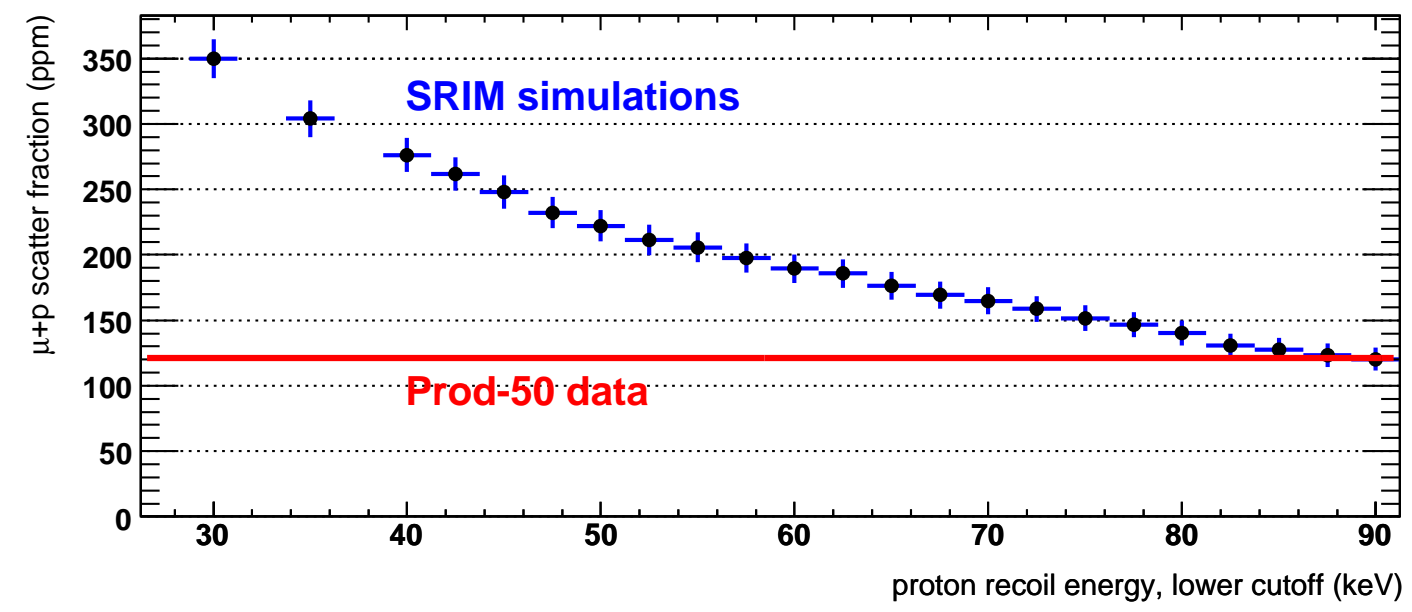

Figure 6.54: The SRIM "escape scatter" fractions as a function of a lower cutoff on the proton's recoil energy. For each cutoff value, only those $\mu+p$ scatter events whose recoiling proton deposits an amount of energy above the cutoff are included, in order to replicate the effect of the TPC EH threshold discrimination. For reference I have drawn the scatter fraction $f_{\text {scatter }}=121.4 \mathrm{ppm}$ that was identified in the Prod-50 data (see Table 6.6).

the muon collides with a hydrogen atom within the TPC's fiducial volume, but then ultimately stops outside of the TPC's active region - a so-called "escape scatter." (Scattered muons which stop inside the active region are not considered a threat, because they have stopped in gas and thus cannot skew the fitted rate.) The energy of the recoiling proton is relevant, because scatter events are only problematic if the nuclear recoil energy is large enough to trigger the TPC's EH threshold and thereby mimic a muon stop. In Figure 6.54 I have plotted the SRIM-generated "escape scatter" fractions as a function of the proton's recoil energy. The next step is to determine which lower cutoff on the proton recoil energy in SRIM corresponds to the actual Run8 EH threshold setting. Dr. Bernhard Lauss estimated that the Run8 TPC threshold settings were roughly $\mathrm{EL}=35 \mathrm{keV}, \mathrm{EH}=65 \mathrm{keV}$, and $\mathrm{EVH}=260 \mathrm{keV}$, by using GEANT to reproduce muon tracks that resembled the actual Run8 data. However, we need a better way to connect the SRIM results with the Run8 data: although the GEANT-determined settings are a helpful rule-of-thumb, we can't use one 


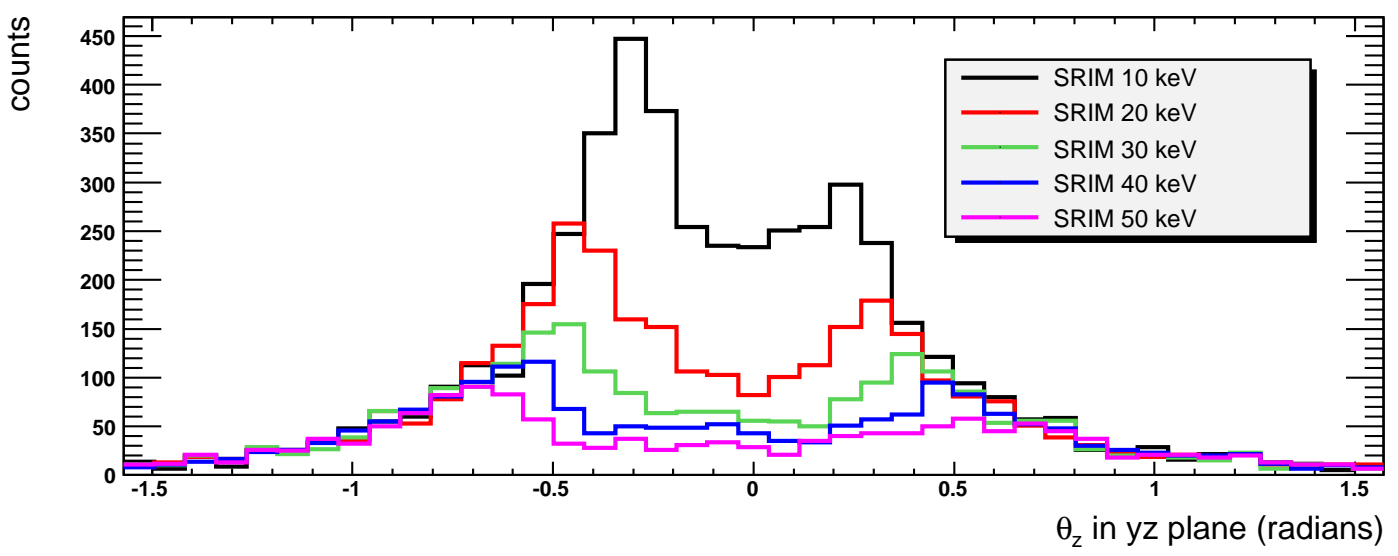

Figure 6.55: The SRIM-generated angular distributions of muon scatters in hydrogen, for several different recoil energy lower cutoffs. The quantity $\theta_{\mathrm{z}}$ is the angle between the experiment's $z$ axis and the muon scatter trajectory as projected onto the $y z$ plane; negative values of $\theta$ correspond to downward scatters (i.e. in the $-y$ direction). Note that the separation between the two scatter peaks increases as the recoil energy cutoff increases. In each plot the left peak is larger than the right peak because the SRIM muon beam was directed at a slightly downward angle, just like the Run8 muon beam.

Monte Carlo to calibrate another. Fortunately, we have recourse to two additional sources of information: (1) the angular distribution of Run8 TLS scatter events, and (2) the Run8 FADC data.

Let us first consider the distribution of angles of $\mu+p$ scatters. This observable is a function of the recoil energy cutoff, as shown in Figure 6.55, due to the fact that large-angle scatters become increasingly likely as the scatter energy increases. By comparing the angular distributions of SRIM scatters with the observed distribution of Run8 TLS scatters - in particular, by comparing their shapes and peak-to-peak spreads-we can potentially establish a correspondence that would reveal which SRIM recoil energy cutoff is closest to the Run8 EH threshold setting. Unfortunately, the SRIM peak-to-peak spread does not continue to steadily increase as the recoil energy cutoff is raised, and the comparison of the SRIM distributions with the Run8 data (Figure 6.56) is complicated by their different 

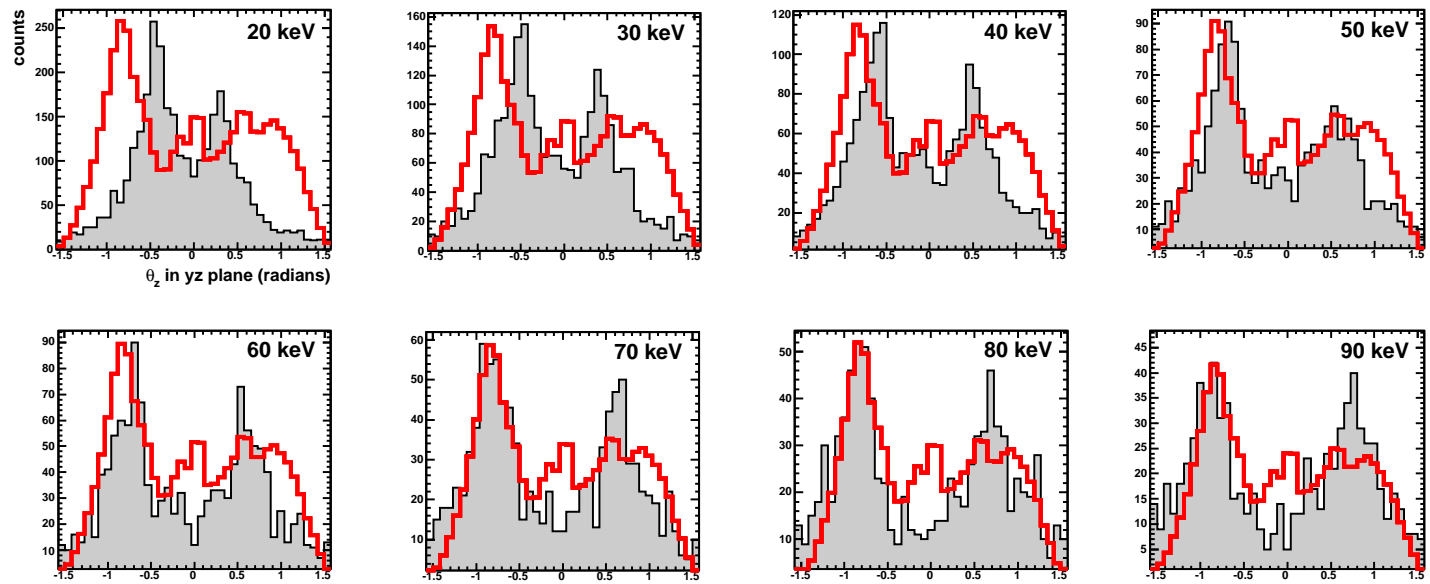

Figure 6.56: The angular distributions of $\mu+p$ scatters generated by SRIM, for different recoil energy lower cutoffs between $20-90 \mathrm{keV}$, with the (rescaled) Prod-50 TLS scattering distribution drawn atop each as a red line. It is hoped that by comparing the peak-to-peak spreads of the SRIM and Run8 distributions we can determine which SRIM recoil energy cutoff most closely corresponds to the Run8 EH threshold. In this case, it appears that the most we can conclude is that the EH threshold is above the SRIM energy of $60 \mathrm{keV}$. I should note that the small central peak in the Prod-50 scatter distribution-which accounts for roughly $5 \%$ of the total scatters - is attributable to a minor flaw in my software, whereby I mistakenly classified some otherwise good muon stops as scatters because they exhibited EH pixels preceding the Bragg peak. This mistake does not pose a systematic danger.

shapes and the statistical limitations of the SRIM results. As a result, the SRIM/Run8 comparisons in do not reveal a clear and unambiguous match, and the most we can conclude is that the Run8 EH threshold is indeed probably above the SRIM recoil energy of $60 \mathrm{keV}$. Consulting Figure 6.54, this suggests that my scatter-finding software is somewhere between $64 \%-100 \%$ efficient for the Prod-50 data.

Analysis of the Run8 FADC data yielded more promising results. By comparing the discriminated and analog TPC data, Dr. Peter Winter concluded that the Run8 TPC EH threshold was $85 \pm 5 \mathrm{keV}$, somewhat higher than Dr. Lauss's GEANT-based estimates and my own SRIM estimates. This result suggests that the appropriate SRIM scatter fraction is $f_{\text {SRIM }}(85 \mathrm{keV})=128(20) \mathrm{ppm}$, in which case the numbers in Table 6.6 indicate that 
my Prod-50 scatter identification efficiency is $95 \%$, and consistent within error with $100 \%$ (perhaps suggesting the presence of background in my identified scatter events). Meanwhile, my CalibNat scatter identification efficiency is of course somewhat lower, around $(44 \pm 9) \%$.

I should acknowledge that my SRIM simulations were fairly crude and simplistic. Steven Clayton and Dr. Peter Kammel performed more lengthy and sophisticated SRIM studies which took into account muon track lengths and energy deposition, track quality cuts, breakdown into scatter types, etc. Although under some conditions they obtained results similar to mine (e.g. Prod-50 detection efficiencies approaching 100\%), they also found that the identification efficiency is sensitive to the TPC response. For instance, if certain conservative assumptions are made about the TPC's inefficiency at detecting the most dangerous muons which scatter directly downwards into the aluminum plate, the Prod-50 identification efficiency drops to as low as $50 \%$.

In view of these circumstances, we have elected not to augment the Prod-50 $\mu+p$ scatter "corrections" beyond the direct removal of scatters presented in Table 6.7. However, we do augment the observed CalibNat scatter corrections in Table 6.8, scaling them up by the scatter fraction ratio $\left(f_{\text {Prod-50 }} / f_{\text {CalibNat }}\right)$ to compensate for the reduced TPC efficiency at $4.8 \mathrm{kV}$ during the CalibNat fill. In order to account for our uncertainty in the overall scatter identification efficiency, we concluded that it is appropriate to introduce a conservative, onesided error of $-3 \mathrm{~s}^{-1}$ to cover the possibility that we are only catching half of the scatter events. However, given the relatively small magnitude of this uncertainty, we chose to symmetrize it to $\pm 3 \mathrm{~s}^{-1}$, in order to simplify subsequent error propagation calculations. The results are presented in Table 6.9. 


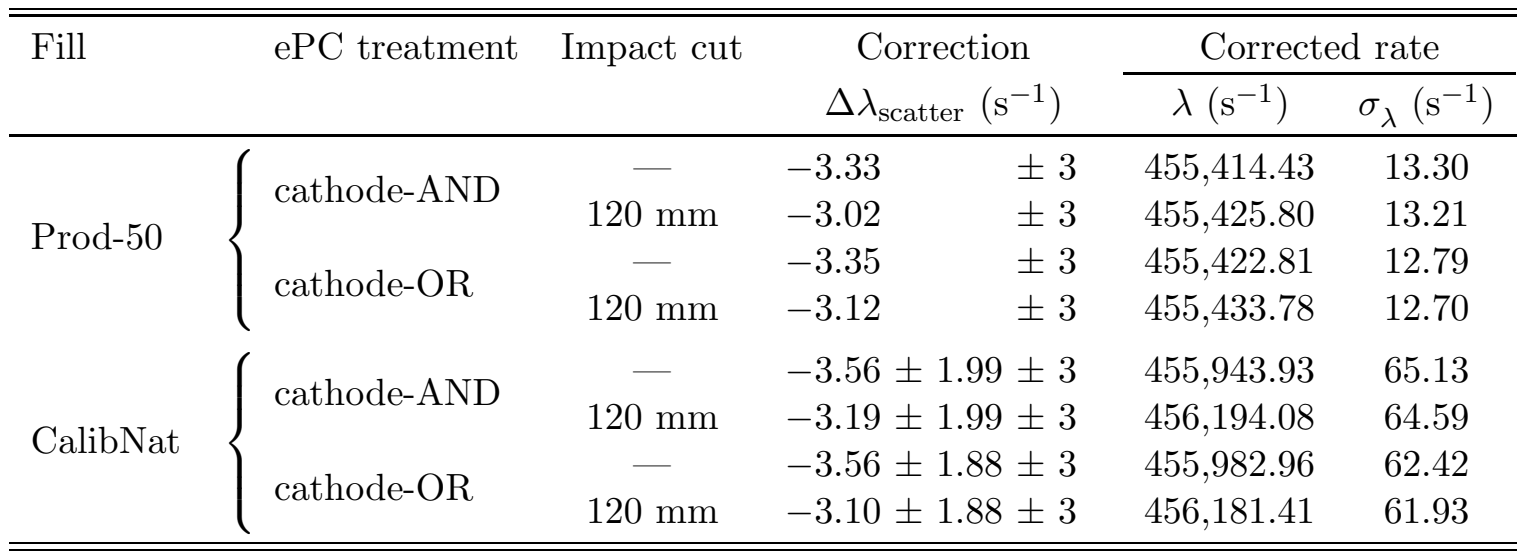

Table 6.9: The $\mu+p$ scatter corrections, performed on the rates in Table 6.5. The Prod-50 "corrections" are simply the observed shifts in the fitted rates due to the direct removal of scatters (see Table 6.7); as such, the only error associated with them is the conservative $\pm 3 \mathrm{~s}^{-1}$ error which covers any scatters we may have missed. In contrast, the CalibNat corrections in Table 6.8 have been scaled up in proportion to the scatter fractions observed for the Prod-50 and CalibNat fills, and consequently they carry an additional error term of $\approx \pm 2 \mathrm{~s}^{-1}$.

\subsection{3 $Z>1$ impurity correction}

When a $\mu p$ atom encounters an elemental impurity of $Z>1$ in the hydrogen gas, the muon will preferentially and irreversibly transfer to the heavier atom due to its stronger nuclear binding energy. This behavior is a concern because $Z>1$ atoms have high nuclear capture rates $\Lambda_{Z} \gg \Lambda_{S}$, and hence their presence raises the effective muon disappearance rate above its value in pure hydrogen. For this reason, we worked to suppress the $Z>1$ impurity levels as much as possible during Run8. Unfortunately, nonnegligible levels of contamination were still observed, and it is therefore necessary to correct the observed $\mu^{-}$ disappearance rate for the effects of the residual gas impurities. This task is not easy, however, because it has proven difficult to accurately determine the $Z>1$ impurity levels and their elemental composition. Although we have collected a wealth of information from a variety of studies and analyses, to date we have not succeeded in reconciling all of the available data into a completely consistent, definitive understanding of impurity behavior 
in the experimental setup. Consequently, the $Z>1$ correction is the most problematic of the disappearance rate corrections, and it carries a sizable uncertainty.

During Run8, we had two means of assessing the $Z>1$ impurity levels: (1) chromatography measurements of periodically obtained hydrogen gas samples, and (2) in situ monitoring of capture events in the TPC data recorded by the TDC400 modules. The latter analysis - which can also be performed offline - requires some explanation. When a muon stops in the hydrogen target and subsequently transfers to a $Z>1$ impurity and undergoes nuclear capture, the recoiling nucleus can deposit enough energy in the TPC to trigger the EH threshold and possibly the EVH threshold, depending upon the element and the recoil direction. The signature of such an event is a time-delayed cluster of EH and/or EVH pixels, located on or near the anode where the muon originally stopped (owing to the small diffusion distances of $\mu p$ and $\mu Z$ atoms); an example is shown in Figure 6.57.

In order to identify muon capture events in the TPC, I developed software that searches the TDC400 data for EH or EVH pixels in the region to the right of each 25- $\mu$ spileup-protected muon stop which is not accompanied by a decay electron candidate in the 0-20 $\mu$ s period after the muon's arrival. The capture search itself is limited to a 1-10 $\mu$ s time interval and $\mathrm{a} \pm 3$ anode interval (inclusive) around the anode on which the muon stopped. The limited time interval is necessary for a number of reasons. First, the $1 \mu$ s gap between the end of the muon track and the start of the capture search was introduced in order to avoid dealing with events where the muon stop signal and the capture signal overlap. The $10 \mu$ s time limit was implemented because of concerns about encountering subsequent muon tracks, and because of practical issues related to information management in the TPC data arrays; special studies have revealed that extending this search interval beyond $10 \mu$ s does not significantly affect the results. Because a finite time interval is searched, the maximum possible capture identification efficiency is roughly $85 \%$. In contrast, the finite 


\section{TPC Display}

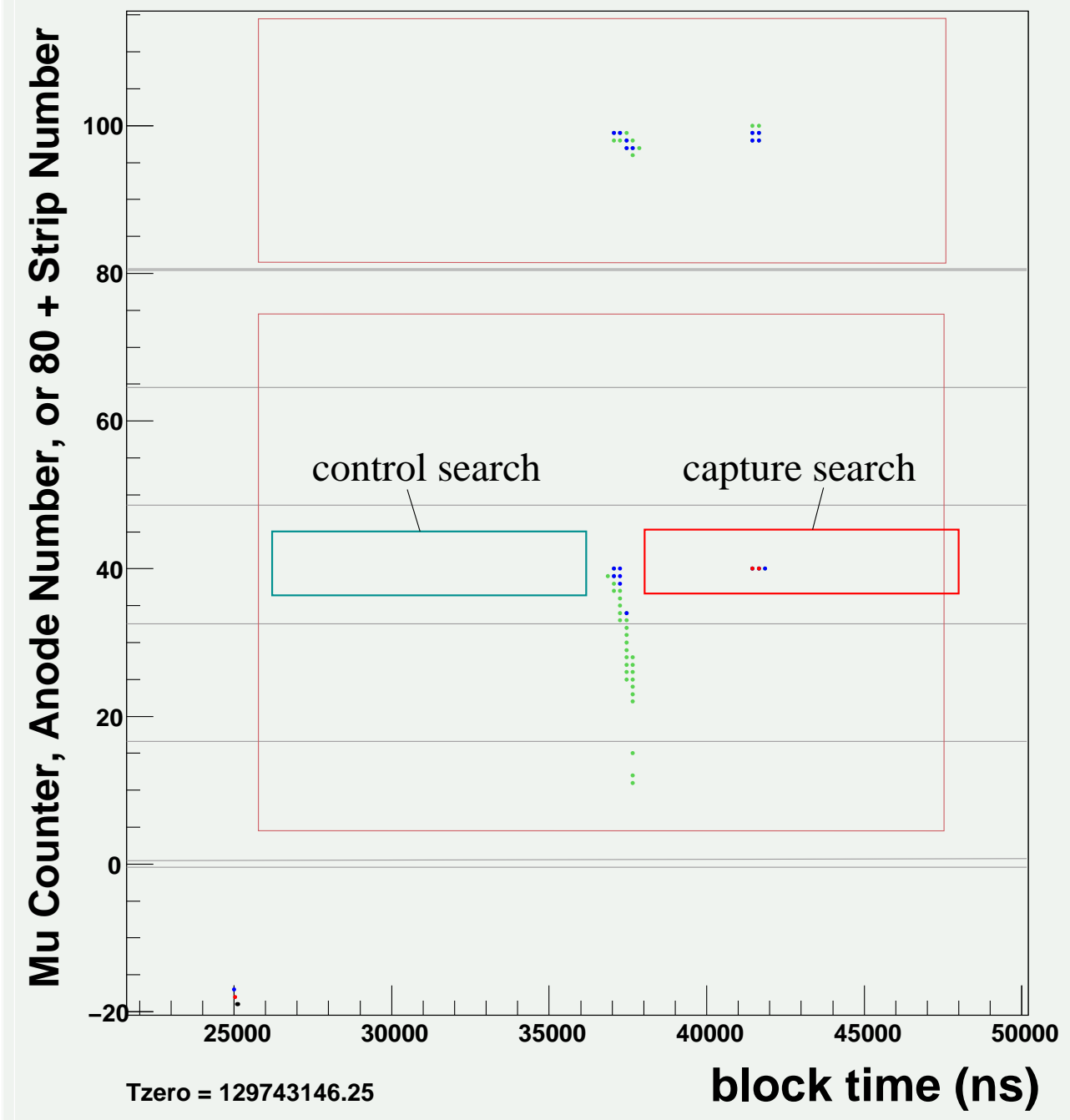

Figure 6.57: Example of a probable muon capture by a $Z>1$ impurity, as viewed in the event display. The signature of a impurity capture event is a time-delayed cluster of EH and/or EVH pixels near the same anode as the muon stop. The regions that are searched for capture events, and for control (i.e. noise) events, are indicated by the boxes on either side of the muon stop. 
spatial dimensions (in $z$ ) of the search have little effect, because the $\mu p$ and $\mu Z$ atoms do not diffuse very far from the muon's stopping point.

As a control on the capture search, a mirror search is performed in the opposite direction - that is, leftwards, in the time preceding the muon stop. A chronological plot of the $Z>1$ capture yields (yield $Y=N_{\text {captures }} / N_{\mu \text { stops }}$ ) and control yields during the entirety of Run8 is shown in Figure 6.58, alongside the results of the chromatography measurements of nitrogen concentrations. (A summary of the impurity information collected during Run8 can be found in reference [218].) Note that the impurity levels are higher in the CalibNat fill than in the Prod-50 fill, which is the reason why separate $Z>1$ corrections must be performed on the disappearance rates for each of those fills before the deuterium correction's zero-extrapolation procedure. Although not pictured, the "capture" yields from the $\mu^{+}$ data are nearly identical to the $\mu^{-}$control yields, a fact that helps to establish the veracity and reliability of the control results. Figure 6.58 clearly shows that the $\mu^{-}$capture yields remained well above the control yield background, and thus within the range of sensitivity of this particular identification technique. Moreover, the $\mu^{-}$capture yields track nicely with events that occurred during Run8: At time $t=0$ in Figure 6.58, CHUPS began cleaning $Z>1$ impurities from the hydrogen gas, as reflected in the ensuing exponential attenuation in the capture yields. During the Prod-50 data taking, we intentionally interfered with CHUPS cleaning on two notable occasions:

- Between 56 and 76 hours after its turn-on, the CHUPS cleaning was interrupted in order to study the TPC system's outgassing behavior. The resulting rise in the capture yield during this period is evident in Figure 6.58.

- At 336 hours, the CHUPS hydrogen flow rate was reduced, and then shortly afterwards increased, in order to study the equilibrium between the CHUPS cleaning and 


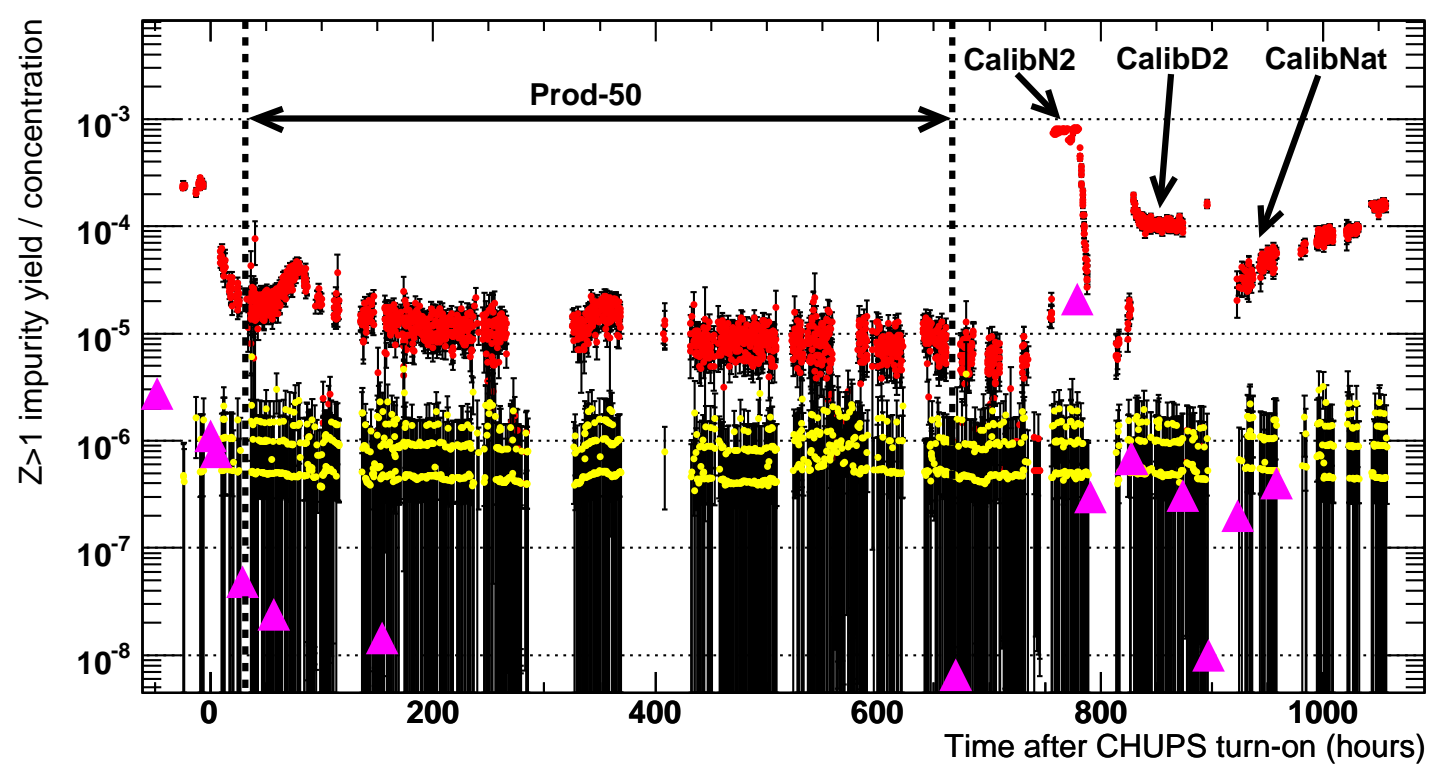

Figure 6.58: The TPC $Z>1$ capture and control yields vs. time during Run8, where $t=0$ corresponds to the time when CHUPS began cleaning impurities from the hydrogen gas. The red points are the yields of capture events containing EVH pixels, and the yellow points are the yields of control events containing EVH pixels. The control points lie in striated bands with large error bars because of the low statistics in each runfile. Note that the EVH capture yields are well above the background level indicated by the control search. The Prod-50 EVH capture points, as well as the corresponding gas chromatography measurements of the nitrogen concentration in ppm (purple triangles), track nicely with the expected exponential cleaning effect from CHUPS. We believe that the residual difference between the EVH capture and control points is due to continual outgassing from the TPC materials. The four primary gas fillings of interest are labeled; note the wide variation in $Z>1$ impurity levels among them. Many of the bumps and gaps in the capture yield points correspond to beam-down periods, $\mu^{+}$measurements, and CHUPS tests. Outgassing is clearly evident in the CalibNat data, when CHUPS was not operational. 
outgassing. These changes are evident in the capture yield.

The studies described above indicated that outgassing in the hydrogen system - which was first discovered in the 2003 run and motivated the development of CHUPS - continued to be a problem in 2004. The Prod-50 equilibrium capture yield level, which was just below 10 ppm, was somewhat higher than expected. It appears that CHUPS functioned properly, and that the cleaning and outgassing simply reached an asymptotic, terminal equilibrium.

The cumulative $Z>1$ impurity capture and control yields for the Run8 gas fills of interest are presented in Table 6.10, and the corresponding capture time distributions are plotted in Figure 6.59. Fits to the time distributions can be performed using the function

$$
f(t)=K e^{-\left(\lambda_{0}+\Lambda_{Z}\right) t}\left[e^{\left(\Lambda_{Z}-\Lambda_{S}-\phi \lambda_{\mathrm{of}}-\Lambda_{p Z}\right) t}-1\right]+B
$$

which is obtained by solving the system of muon kinetics in Equations 3.4 and 3.5, but simplifed to ignore deuterium and the detailed effects of the ortho and para $p \mu p$ states. The background $B$ is typically fixed by the corresponding time distribution of control events, and all of the parameters besides $\mathrm{K}$, and possibly $c_{Z}$ in $\Lambda_{p Z}=\phi c_{Z} \lambda_{p Z}$, are fixed to their literature values. An example of a fit to a capture time distribution is given in Figure 6.60. In this example, as in most cases, the time distribution exhibits the correct shape, and the quality of the fit is good, but the numbers extracted from the fit are wildly inconsistent with expectations. This is especially frustrating when dealing with the CalibN2 data, both because we are confident in our knowledge of the nitrogen concentration of that fill (as will be discussed shortly), and because its elevated nitrogen content should yield time distributions which give straightforward, unambiguous confirmation of the nitrogen concentration. Our best guess is that the poor fit results are due to the complexities of the TPC response - e.g., its entanglement of time and the $y$ dimension, or perhaps smearing effects from its relatively coarse time resolution - but the true reasons for this phenomenon remain a mystery. Of 


\begin{tabular}{|c|c|c|c|c|c|c|c|}
\hline \multirow[t]{3}{*}{ Fill } & \multirow[t]{3}{*}{$\mu$ stops } & \multicolumn{6}{|c|}{$Z>1$ captures } \\
\hline & & \multicolumn{3}{|c|}{$\mathrm{EH}$} & \multicolumn{3}{|c|}{$\mathrm{EVH}$} \\
\hline & & Count & Control & Yield (ppm) & Count & Control & Yield (ppm) \\
\hline Prod-50 & $1,799,056,105$ & 30,477 & 4146 & $14.64(9)$ & 24,405 & 1,208 & $12.89(8)$ \\
\hline CalibN2 & $132,859,288$ & 103,700 & 315 & $778.2(2.4)$ & 103,063 & 114 & $774.9(2.4)$ \\
\hline CalibNat & $108,492,995$ & 5,823 & 216 & $51.7(7)$ & 5,368 & 97 & $48.6(7)$ \\
\hline
\end{tabular}

Table 6.10: $Z>1$ impurity capture and control yields gleaned from the TPC's TDC400 data by the Berkeley analysis software. The capture counts are from time-delayed events to the right of the muon stop, while the control events are from a mirror image search to the left, in the time preceding the muon stop (see Figure 6.57). "EH" capture events require an EH pixel; "EVH" capture events are a subset of the EH events where at least one EVH threshold pixel is present. Both of the capture yields were calculated according to the formula Yield $=($ count - control $) /$ mustops. 

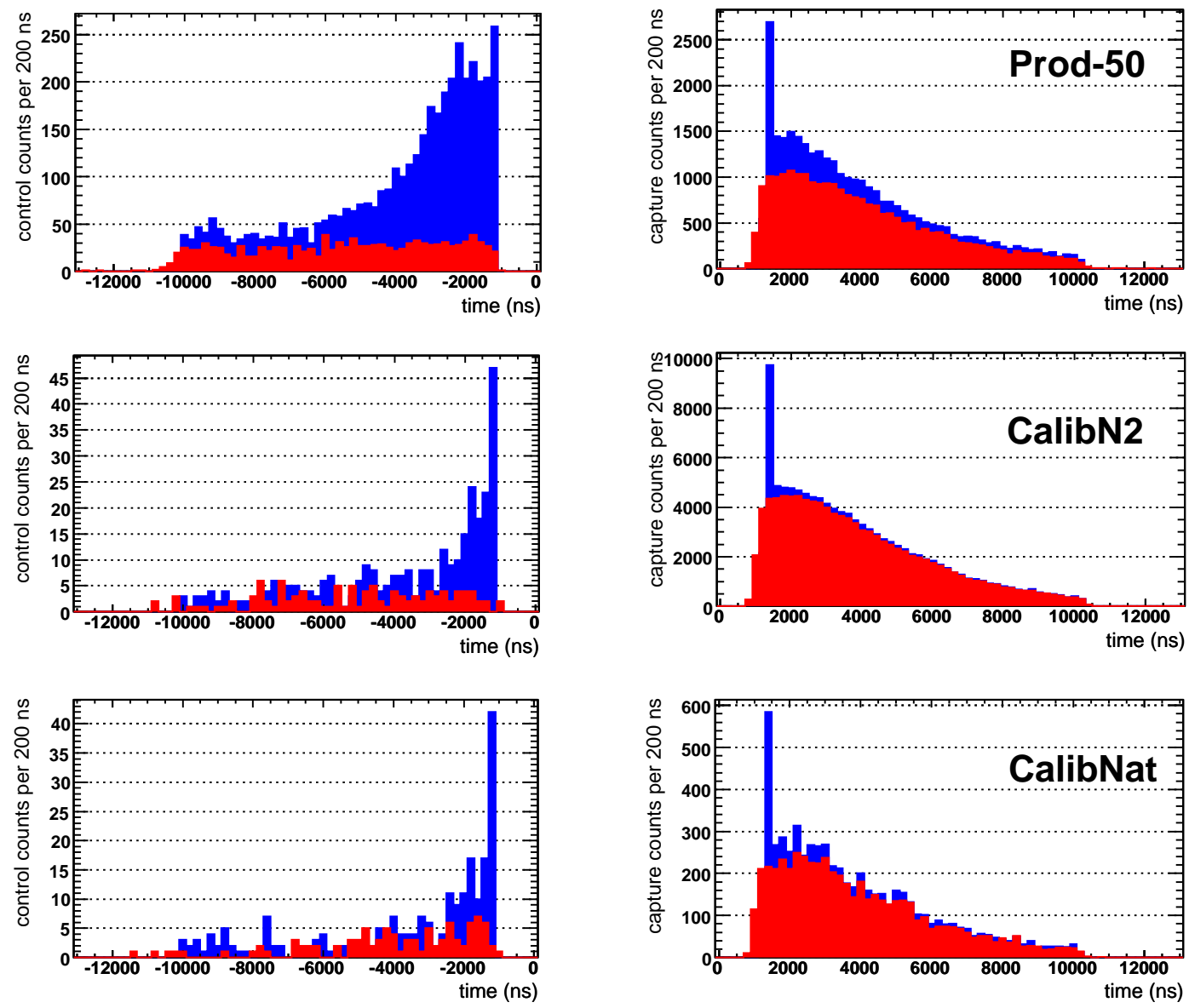

Figure 6.59: The time distributions of $Z>1$ impurity captures in the TPC data (relative to the end of the muon track) as identified by the Berkeley analysis software. Results are shown for the Prod-50, CalibN2, and CalibNat fills. The EH capture distributions are drawn in blue, and the EVH capture distributions are drawn in red. The plots in the left column are of the control events, while the plots in the right column contain the capture events. Note that the EVH distributions exhibit much lower background than the EH distributions; this is especially true of the Prod-50 data, which was more sensitive to noise because of the higher TPC voltage, but it is also evident in all of the fills at times near the muon stop, which is to be expected since muon stops trigger the EH threshold. The prompt peaks in the EH distributions were ignored when calculating the capture yield statistics in Table 6.10. Although these capture distributions all exhibit the expected shape, actual fits - such as that shown in Figure 6.60-have not yielded numerical results consistent with expectations. 


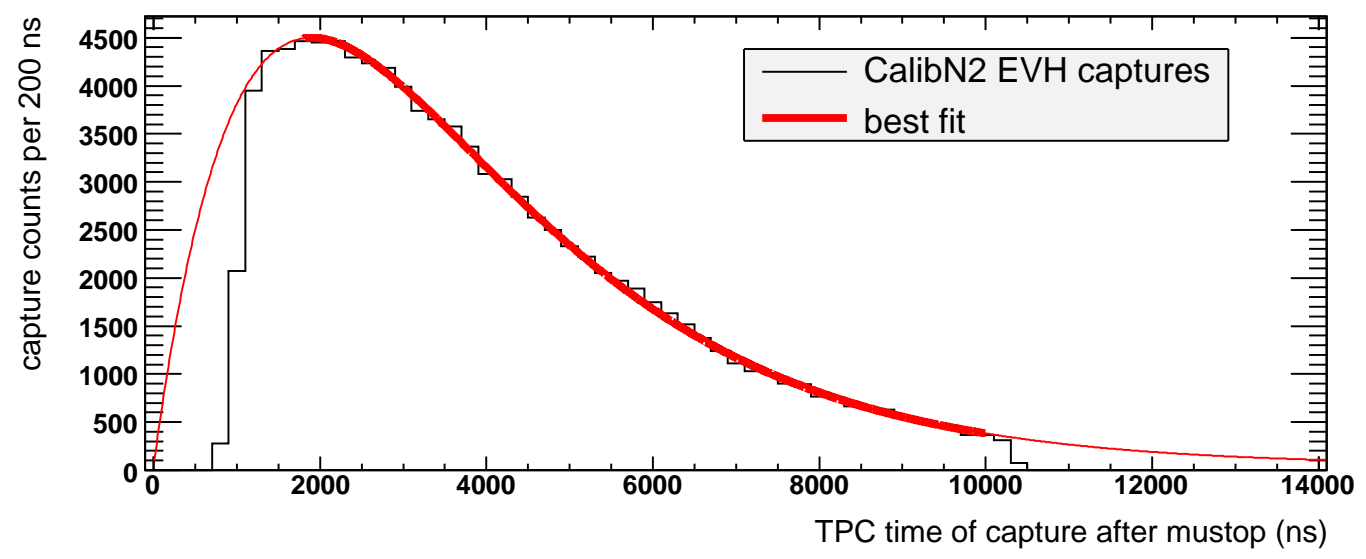

Figure 6.60: Fit to the time distribution of the EVH $Z>1$ impurity captures identified in the CalibN2 TPC data. The actual range of the fit is indicated by the thick red line; the thin red line indicates the shape of the entire fitting function. Although the fit looks fine and yields a reasonable reduced $\chi^{2}\left(\chi^{2} / d=1.01 \pm 0.22\right)$, the fit's numbers are inconsistent with expectations. The fit above yields $c_{\mathrm{N}}=70(8) \mathrm{ppm}$, whereas the ostensible doping concentration of the CalibN2 fill was $11 \mathrm{ppm}$, and this latter concentration has been corroroborated by other means; see the text.

course, it should be acknowledged that these fits are not very sensitive to the parameters of interest. Monte Carlo studies have indicated that fits to a simulated CalibN2 capture distribution only yield a $9 \mathrm{ppm}$ precision on the extracted nitrogen concentration $c_{\mathrm{N}}$. The sensitivity is further reduced by the limited time range of the fits, and by the uncertainty in the other fit parameters (e.g. the molecular rates). Although the time distributions do not provide useful numbers, they are nonetheless useful for affirming that we are in fact identifying capture events.

The software-derived impurity capture yield is the primary observable used to perform the $Z>1$ impurity corrections, while the less reliable gas chromatography measurements are used only to inform our estimates of the elemental composition of impurities in the Prod-50 fill. The capture yield is useful and appealing because (1) the TPC capture information is directly embedded in the data under study, and therefore provides a continuous, non-intrusive observable, and (2) for the low impurity concentrations observed in our 
experiment, the yield is directly proportional to the impurity concentrations and to their effect on the $\mu^{-}$diappearance rate. The latter relationship can be expressed as

$$
\Delta \lambda=Y \sum_{Z} w_{Z} \beta_{Z}^{\text {calib }}=Y \sum_{Z} w_{Z}\left(\frac{\Delta \lambda}{Y}\right)_{Z}^{\text {calib }}
$$

where $\Delta \lambda$ is the impurity-generated shift to the $\mu^{-}$disappearance rate, and the observed capture yield $Y$ is scaled by the summation term $\sum_{Z}$ which runs over all of the $Z>1$ impurity species that are present in the hydrogen gas. I generally use the EVH capture yield, $Y \equiv Y^{\mathrm{EVH}}$, because I consider it more reliable, in light of the fact that EVH captures are less sensitive to background noise than EH captures (see Figure 6.59). The quantity $w_{Z}$ is a weighting factor for each species $\left(w_{Z}=Y_{Z} / Y\right.$, so $\left.\sum_{Z} w_{Z}=1\right)$, and the ratio $\beta_{Z}^{\text {calib }}=(\Delta \lambda / Y)_{Z}^{\text {calib }}$ describe the scaling of the muon disappearance rate as a function of the TPC capture yield for each impurity $Z$. Information accumulated from Runs 8, 9, and 10 strongly indicates that nitrogen and oxygen (in the form of humidity) are the only $Z>1$ impurities present in our hydrogen target in significant levels, in which case Equation 6.12 becomes

$$
\Delta \lambda=Y\left[w_{\mathrm{N}}\left(\frac{\Delta \lambda}{Y}\right)_{\mathrm{N}}^{\mathrm{calib}}+w_{\mathrm{O}}\left(\frac{\Delta \lambda}{Y}\right)_{\mathrm{O}}^{\text {calib }}\right] .
$$

Monte Carlo simulations have demonstrated that the linear scaling relationships expressed in Equations 6.12 and 6.13 remain valid up to the highest impurity concentrations that have been encountered in MuCap. That is, we have always remained well below the concentration limits (30 ppm for nitrogen, and 14 ppm for oxygen) where the effects on the disappearance rate from impurities start to become nonlinear. We must now determine values for all of the parameters in Equation 6.13.

In theory, the $(\Delta \lambda / Y)_{Z}$ ratios are nearly equal for nitrogen and oxygen [219]. However, in practice the situation is complicated by the fact that the TPC capture yield $Y$ in the denominator is sensitive to the TPC gain and capture topology. It has already been 
mentioned that the TPC capture detection efficiency is known to be less than $100 \%$, owing to the finite search interval. Moreover, the detection efficiency is quite different for the two elements under consideration. Nitrogen captures release $\approx 300 \mathrm{keV}$ of recoil energy, while oxygen captures release $\approx 260 \mathrm{keV}[220]$. The TPC EH threshold is typically set around $65 \mathrm{keV}$, while the TPC EVH threshold setting is around $260 \mathrm{keV}$-i.e. in the vicinity of the oxygen capture energy, and just above the maximum muon stop energy of roughly $250 \mathrm{keV}$. Thus we would expect the EH and EVH yields to be similar for nitrogen, but there might be differences between the EH and EVH yields for oxygen depending upon exactly where the EVH threshold was actually set. This hypothesis is indeed supported by inspection of the Prod-50 and CalibN2 EH and EVH capture time distributions in Figure 6.59. The upshot is that the scaling factors $\beta_{Z}^{\text {calib }}=(\Delta \lambda / Y)_{Z}^{\text {calib }}$ must be established empirically, by performing calibration runs that involve the impurity species of interest. This approach obviates the need to ascertain the impurity concentrations with certainty.

It is a relatively simple task to determine the scaling ratio for nitrogen, $(\Delta \lambda / Y)_{\mathrm{N}}^{\text {calib }}$, because we conducted a nitrogen-doped calibration fill, CalibN2, during Run8. Dr. Claude Petitjean and Dr. Malte Mildebrandt added nitrogen to the hydrogen gas to achieve a concentration of $c_{\mathrm{N}}^{\text {estimate }}=11.00 \pm 0.22 \mathrm{ppm}$, according to their volumetric calculations. As nitrogen was by far the dominant impurity during this fill, we can ignore any contributions 
from oxygen and calculate ${ }^{6}$

$$
\begin{aligned}
\beta_{\mathrm{N}}^{\text {calib }}=\left(\frac{\Delta \lambda}{Y}\right)_{\mathrm{N}}^{\text {calib }} \approx \frac{\Delta \lambda}{\Delta Y} & =\frac{\lambda_{\text {CalibN2 }}-\lambda_{\text {Prod-50 }}}{Y_{\text {CalibN2 }}^{\mathrm{EVH}}-Y_{\text {Prod-50 }}^{\mathrm{EVH}}} \\
& =\frac{\left(456,424.93 \pm 55.43 \mathrm{~s}^{-1}\right)-\left(455,436.90 \pm 12.34 \mathrm{~s}^{-1}\right)}{(774.9 \pm 2.4 \mathrm{ppm})-(12.89 \pm .08 \mathrm{ppm})} \\
& =\frac{988.03 \pm 56.79 \mathrm{~s}^{-1}}{762.0 \pm 2.4 \mathrm{ppm}} \\
& =1.297 \pm 0.075 \mathrm{~s}^{-1} / \mathrm{ppm}
\end{aligned}
$$

We can test the validity of this result by checking if it corroborates the known nitrogen concentration of the CalibN2 fill. To do this, we first deduce the TPC's EVH capture detection efficiency for nitrogen by comparing Equation 6.14 with the "ideal" ratio $(\Delta \lambda / Y)_{\mathrm{N}}^{\text {lit }}$ which is calculated using values in the literature [219]:

$$
\begin{aligned}
\left(\frac{\Delta \lambda}{Y}\right)_{\mathrm{N}}^{\mathrm{calib}} & =\frac{1}{\epsilon_{\mathrm{N}}^{\mathrm{EVH}}}\left(\frac{\Delta \lambda}{Y}\right)_{\mathrm{N}}^{\mathrm{lit}} \\
\epsilon_{\mathrm{N}}^{\mathrm{EVH}} & =\frac{\left(\frac{\Delta \lambda}{Y}\right)_{\mathrm{N}}^{\mathrm{lit}}}{\left(\frac{\Delta \lambda}{Y}\right)_{\mathrm{N}}^{\mathrm{calib}}}=\frac{0.818 \pm 0.025 \mathrm{~s}^{-1} / \mathrm{ppm}}{1.297 \pm 0.075 \mathrm{~s}^{-1} / \mathrm{ppm}} \\
\epsilon_{\mathrm{N}}^{\mathrm{EVH}} & =(63 \pm 4) \% .
\end{aligned}
$$

The result above is consistent with determinations of the nitrogen detection efficiencies in other runs. Plugging the value for $\epsilon_{\mathrm{N}}^{\mathrm{EVH}}$ into a concentration formula which is completely analogous to Equation 6.15 yields the concentration:

$$
\begin{aligned}
c_{\mathrm{N}} & =\frac{\Delta Y}{\epsilon_{\mathrm{N}}^{\mathrm{EVH}}}\left(\frac{c}{Y}\right)_{\mathrm{N}}^{\mathrm{lit}} \\
c_{\mathrm{N}} & =\frac{(762.0 \pm 2.4 \mathrm{ppm})}{(0.63 \pm 0.04)}\left(\frac{11 \mathrm{ppm}}{1171.2 \mathrm{ppm}}\right) \\
c_{\mathrm{N}} & =11.4 \pm 1.0 \mathrm{ppm} .
\end{aligned}
$$

\footnotetext{
${ }^{6}$ The technical details of the results of fits to the CalibN2, cathode-OR, 120-mm-cut data are $N=$ $7.8 \times 10^{7}, N / B=9.5 \times 10^{3}, \lambda=456,424.93 \pm 55.43 \mathrm{~s}^{-1}\left(\chi^{2} / d\right)_{\text {fit }}=1.07(6),\left(\chi^{2} / d\right)_{\text {gondolas }}=0.77(37)$ (compare with the results of fits to the Prod-50 and CalibNat data in Table 6.5). The implications for $\beta_{\mathrm{N}}^{\text {calib }}$ are the same regardless of whether the cathode-AND or cathode-OR data is used, or whether impact cuts are applied.
} 
This data-analysis-based result agrees very nicely with Dr. Petitjean's and Dr. Hildebrandt's estimates of the CalibN2 nitrogen concentration, and therefore enables us to place some confidence in the value for $(\Delta \lambda / Y)_{\mathrm{N}}^{\mathrm{calib}}$.

In contrast with nitrogen, the determination of the ratio $(\Delta \lambda / Y)_{\mathrm{O}}^{\text {calib }}$ for oxygen is far more dubious. This is unfortunate, because oxygen in the form of humidity is believed to account for most impurity captures. An oxygen-doped calibration fill was in fact attempted during Run8, but the oxygen quenched the TPC charge to such an extent that the resulting data was useless. As a result, we must use information collected during Run10 in 2006. Dr. Peter Kammel surveyed the information from Runs 8 and 10-including results from the Berkeley and Illinois analyses of the data, as well as Run10 humidity sensor measurementsand arrived at a conservative estimate ${ }^{7}$ of $\beta_{\mathrm{O}}^{\text {calib }}=(\Delta \lambda / Y)_{\mathrm{O}}^{\text {calib }}=1.64 \pm 0.44 \mathrm{~s}^{-1} / \mathrm{ppm}[221]$, which corresponds to an oxygen detection efficiency of roughly $(49 \pm 13) \%$. The uncertainties in the effects of oxygen are reflected in the sizable error $\sigma_{\beta_{\mathrm{O}}^{\text {calib }}}$, which is much larger than the error for nitrogen in Equation 6.14. In fact, the error for $\beta_{\mathrm{O}}^{\text {calib }}$ will prove to be the largest source of uncertainty in the final $Z>1$ impurity correction.

To determine the weights $w_{\mathrm{N}}$ and $w_{\mathrm{O}}$ of the nitrogen and oxygen contributions to the observed capture yield $Y$, we compare the capture yields and nitrogen concentration measurements in Figure 6.58. For the low nitrogen concentrations observed in the Prod-50 fill, the constant of proportionality between yield and concentration is $(c / Y)_{\mathrm{N}}^{\text {lit }} \approx$ $105 \mathrm{ppm} / \mathrm{ppm}$, which explains why the nitrogen concentration points in Figure 6.58 lie far below the capture yields. Since we believe that the hydrogen gas only contained nitrogen and oxygen impurities, we can deduce the oxygen contribution to the capture yield, as a

\footnotetext{
${ }^{7}$ This $\beta_{\mathrm{O}}^{\text {calib }}$ value was obtained using the capture yield from the Illinois analysis of the Run10 humidity data, since no intensive Berkeley analysis was performed on that data set. However, the Illinois and Berkeley analyses observed nearly identical values for the Run8 CalibN2 fill, so I have chosen to apply $\beta_{\mathrm{O}}^{\text {calib }}$ to the Berkeley-analyzed Run8 data as is. In any case, the large uncertainty on $\beta_{\mathrm{O}}^{\text {calib }}$ should cover the scale of any variations between the two analyses.
} 
function of time, by subtracting the nitrogen yield that is predicted by the gas chromatography measurements:

$$
\begin{aligned}
Y_{\mathrm{O}}(t) & =Y(t)-Y_{\mathrm{N}}(t) \\
& =Y(t)-\left[c_{\mathrm{N}}(t)\left(\frac{c}{Y}\right)_{\mathrm{N}}^{\mathrm{lit}} \epsilon_{\mathrm{N}}\right] .
\end{aligned}
$$

By integrating the above formula over time and dividing through by the total yield $Y$, I obtained the following oxygen weight for the Prod-50 fill:

$$
w_{\mathrm{O}}^{\text {Prod-50 }}=1-w_{\mathrm{N}}^{\text {Prod-50 }}=0.94 \pm 0.05
$$

The error of $5 \%$ arises from the possibility that the precision of the lowest, asymptotic nitrogen concentration measurements might be limited by the detection sensitivity.

The determination of the weights for the CalibNat fill is more problematic, for several reasons. First, gas chromatography analysis of the earlier CalibN2 fill reported a nitrogen concentration of $22 \mathrm{ppm}$, which is twice the $11 \mathrm{ppm}$ estimated by Drs. Petitjean and Hildebrandt and corroborated by the data analysis. This discrepancy casts some suspicion on the reliability of later chromatography measurements. (The multiple Prod-50 nitrogen points are regarded as more credible because they clearly tracked with the observed TPC capture yield over time.) Second, there are only two gas chromatography measurements of the nitrogen concentration from the beginning and end of the CalibNat fill, and they are inconsistent with expectations. Although the two points support the basic theory of impurity outgassing, comparison with the TPC capture yields suggests that the impurities are comprised primarily of nitrogen! This is inconsistent with the findings from both Prod-50 and more recent runs, which have repeatedly indicated that the primary contaminant is oxygen in the form of outgassed humidity. In light of these concerns and inconsistencies, I assume that the CalibNat impurities are some combination of nitrogen and oxygen, but otherwise profess maximal ignorance regarding their relative contributions. In practice, 
this means that, when using Monte Carlo simulations to calculate the CalibNat $Z>1$ correction, I randomly sample $w_{\mathrm{N}}$ from the interval $[0,1]$, and calculate $w_{\mathrm{O}}=1-w_{\mathrm{N}}$. Fortunately, our ignorance regarding the exact CalibNat impurity composition is relatively unimportant, because any inaccuracy in the elemental composition is dwarfed by the larger deuterium-related effects in that fill.

The capture-yield-based $Z>1$ correction formula that is ultimately used is

$$
\begin{aligned}
\Delta \lambda_{(Z>1)} & =Y\left[w_{\mathrm{N}} \xi_{\mathrm{N}} \beta_{\mathrm{N}}^{\text {calib }}+w_{\mathrm{O}} \xi_{\mathrm{O}} \beta_{\mathrm{O}}^{\text {calib }}\right] \\
& =Y\left[w_{\mathrm{N}} \xi_{\mathrm{N}}\left(\frac{\Delta \lambda}{\Delta Y}\right)_{\mathrm{N}}^{\text {calib }}+w_{\mathrm{O}} \xi_{\mathrm{O}}\left(\frac{\Delta \lambda}{\Delta Y}\right)_{\mathrm{O}}^{\text {calib }}\right]
\end{aligned}
$$

which differs slightly from Equation 6.13 owing to the incorporation of additional scale factors $\xi_{Z}$, which modify the calibration-determined $\beta_{Z}^{\text {calib }}$ values according to experimental conditions - namely, the $\beta_{Z}$ values are affected by the deuterium concentration of the hydrogen gas. The Prod-50 and CalibNat fills require separate $Z>1$ corrections because of their unique impurity circumstances. For the Prod-50 correction I used the numbers:

$$
\begin{aligned}
& Y=12.89 \pm 0.08 \mathrm{ppm} \\
& w_{\mathrm{N}}=0.06 \pm 0.05 \\
& \xi_{\mathrm{N}}=\frac{(\Delta \lambda / Y)_{\operatorname{Prod}-50}^{\mathrm{lit}}}{(\Delta \lambda / Y)_{\text {CalibN2 }}^{\mathrm{lit}}}=\frac{0.819 \pm 0.025 \mathrm{~s}^{-1} / \mathrm{ppm}}{0.818 \pm 0.025 \mathrm{~s}^{-1} / \mathrm{ppm}} \\
& \beta_{\mathrm{N}}=\left(\frac{\Delta \lambda}{\Delta Y}\right)_{\mathrm{N}}^{\text {Run8,CalibN2 }}=\frac{988.03 \pm 56.79 \mathrm{~s}^{-1}}{762.0 \pm 2.4 \mathrm{ppm}^{-}} \\
& w_{\mathrm{O}}=1-w_{\mathrm{N}}=0.94 \pm 0.05 \\
& \xi_{\mathrm{O}} \equiv 1 \\
& \beta_{\mathrm{O}}=1.64 \pm 0.44 \mathrm{~s}^{-1} / \mathrm{ppm}
\end{aligned}
$$




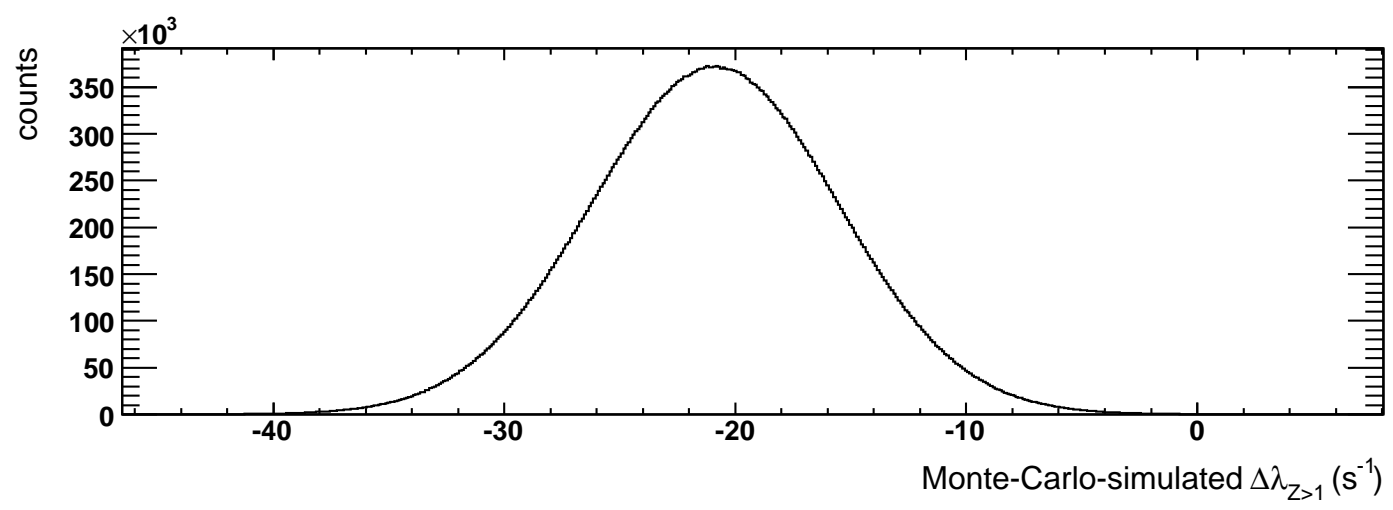

Figure 6.61: The $Z>1$ impurity correction distribution for the Prod-50 fill, as generated by my custom Monte Carlo software.

The parameters used in the CalibNat $Z>1$ correction are

$$
\begin{aligned}
Y & =48.6 \pm 0.7 \mathrm{ppm} \\
w_{\mathrm{N}} & =\text { Randomly selected from interval }[0,1] \\
\xi_{\mathrm{N}} & =\frac{(\Delta \lambda / Y)_{\text {CalibNat }}^{\mathrm{lit}}}{(\Delta \lambda / Y)_{\text {CalibN2 }}^{\text {lit }}}=\frac{0.992 \pm 0.030 \mathrm{~s}^{-1} / \mathrm{ppm}}{0.818 \pm 0.025 \mathrm{~s}^{-1} / \mathrm{ppm}} \\
\beta_{\mathrm{N}} & =\left(\frac{\Delta \lambda}{\Delta Y}\right)_{\mathrm{N}}^{\text {Run} 8, \text { CalibN2 }}=\frac{988.03 \pm 56.79 \mathrm{~s}^{-1}}{762.0 \pm 2.4 \mathrm{ppm}} \\
w_{\mathrm{O}} & =1-w_{\mathrm{N}} \\
\xi_{\mathrm{O}} & =\frac{(\Delta \lambda / Y)_{\text {CalibNat }}^{\mathrm{lit}}}{(\Delta \lambda / Y)_{\text {Prod-50 }}^{\mathrm{iit}}}=\frac{0.813 \pm 0.025 \mathrm{~s}^{-1} / \mathrm{ppm}}{0.797 \pm 0.025 \mathrm{~s}^{-1} / \mathrm{ppm}} \\
\beta_{\mathrm{O}} & =1.64 \pm 0.44 \mathrm{~s}^{-1} / \mathrm{ppm}
\end{aligned}
$$

A ROOT macro calculates the central value of each $Z>1$ correction by plugging the appropriate set of numbers above into Equation 6.18, albeit with the errors set to zero. I then use Monte Carlo simulations to determine the error of the corrections. The input errors are restored, and the macro repeatedly samples from the input values to generate a correction distribution (Figure 6.61). This distribution has a slightly asymmetric shape due to the presence of uncertainties in the denominators of the terms in Equation 6.18, so 


\begin{tabular}{|c|c|c|c|c|c|}
\hline \multirow[t]{2}{*}{ Fill } & \multirow[t]{2}{*}{ ePC treatment } & \multirow[t]{2}{*}{ Impact cut } & \multirow{2}{*}{$\begin{array}{c}\text { Correction } \\
\Delta \lambda_{(Z>1)}\left(\mathrm{s}^{-1}\right)\end{array}$} & \multicolumn{2}{|c|}{ Corrected rate } \\
\hline & & & & $\lambda\left(\mathrm{s}^{-1}\right)$ & $\sigma_{\lambda}\left(\mathrm{s}^{-1}\right)$ \\
\hline \multirow{4}{*}{ Prod-50 } & \multirow{2}{*}{ cathode-AND } & - & \multirow{2}{*}{$-20.9 \pm 5.4$} & $455,393.53$ & 14.35 \\
\hline & & $120 \mathrm{~mm}$ & & $455,404.90$ & 14.27 \\
\hline & \multirow{2}{*}{ cathode-OR } & - & $"$ & $455,401.91$ & 13.88 \\
\hline & & $120 \mathrm{~mm}$ & $"$ & $455,412.88$ & 13.80 \\
\hline \multirow{4}{*}{ CalibNat } & \multirow{2}{*}{ cathode-AND } & & \multirow{2}{*}{$-78.9 \pm 12.3$} & $455,865.03$ & 66.28 \\
\hline & & $120 \mathrm{~mm}$ & & $456,115.18$ & 65.75 \\
\hline & \multirow{2}{*}{ cathode-OR } & - & $"$ & $455,904.06$ & 63.62 \\
\hline & & $120 \mathrm{~mm}$ & $"$ & $456,102.51$ & 63.14 \\
\hline
\end{tabular}

Table 6.11: The $Z>1$ impurity corrections, performed on the rates in Table 6.9. The corrections and their associated errors were determined from Monte Carlo simulations involving Equation 6.18 and using the input parameters given in the text.

I determine each correction's Gaussian error from the larger shoulder of its distributionspecifically, from the $1 \sigma$ location (i.e. the point inside of which $68.26 \%$ of the shoulder's statistics are contained). The results are presented in Table 6.11.

\subsubsection{Deuterium correction}

The presence of deuterium in the hydrogen gas target raises the $\mu^{-}$disappearance rate above the value that would be observed in pure protium, due to the $\mu d$-diffusionrelated effects described in Section 3.3.2. In order to suppress these undesirable effects in the 2004 run, we filled the pressure vessel with deuterium-depleted hydrogen $\left(c_{d} \approx 1 \mathrm{ppm}\right)$. To correct for any effects from residual deuterium contamination, we must perform a zero extrapolation according to the simple formula

$$
\lambda=\frac{c \lambda_{1}-\lambda_{2}}{c-1}
$$

where $\lambda_{1}$ corresponds to the "clean" (i.e. $c_{d} \approx 1 \mathrm{ppm}$ ) measurement with high statistics, $\lambda_{2}$ corresponds to a deuterium-doped calibration measurement of shorter duration and hence 


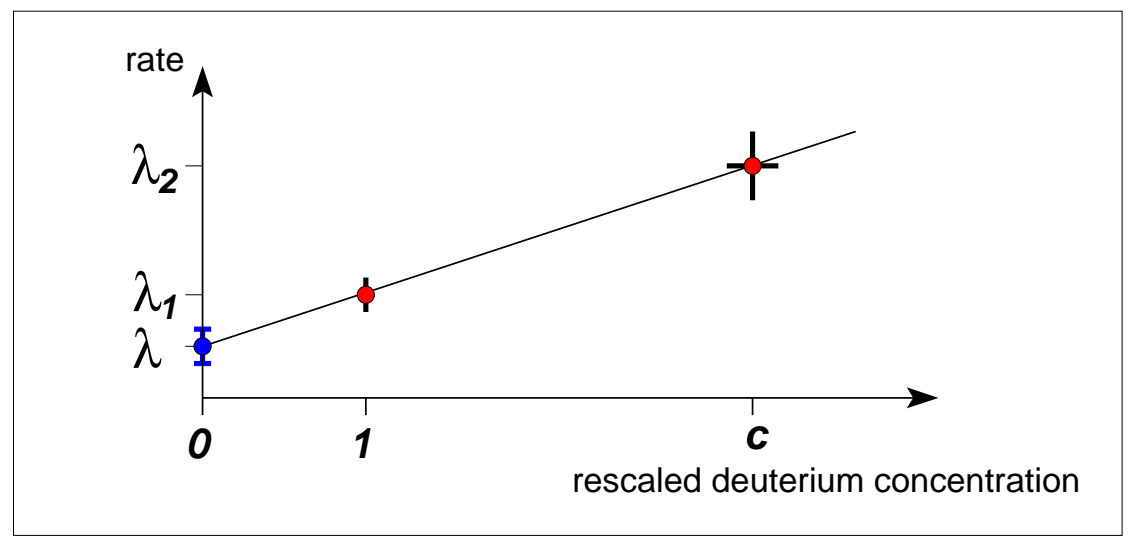

Figure 6.62: Schematic illustration of the zero-extrapolation procedure for the deuterium correction. Point $\left(1, \lambda_{1}\right)$ corresponds to the high-statistics clean fill, while point $\left(c, \lambda_{2}\right)$ corresponds to a low-statistics, deuterium-doped calibration fill. (The $x$ axis has been rescaled so that the deuterium concentration of the clean fill is unity.) The corrected result $\lambda$ is simply the $y$ intercept of the best-fit line passing through the two experimental data points. The uncertainty in $\lambda$ is determined by the uncertainties in $\lambda_{1}, \lambda_{2}$, and $c$.

lower statistics, and $c$ is the ratio of the deuterium concentrations of the two fills, $c=$ $\left(c_{d 2} / c_{d 1}\right)>1$. The zero-extrapolation procedure is illustrated schematically in Figure 6.62.

Applying the general error propagation law to Equation 6.19 gives

$$
\begin{aligned}
\sigma_{\lambda}^{2} & =E_{\lambda_{1}}^{2}+E_{\lambda_{2}}^{2}+E_{c}^{2}+E_{\lambda_{2} c}^{2} \\
& =\sigma_{\lambda_{1}}^{2}\left(\frac{c}{c-1}\right)^{2}+\sigma_{\lambda_{2}}^{2} \frac{1}{(c-1)^{2}}+\sigma_{c}^{2} \frac{\left(\lambda_{2}-\lambda_{1}\right)^{2}}{(c-1)^{4}}+2 \sigma_{\lambda_{2} c}^{2} \frac{\left(\lambda_{1}-\lambda_{2}\right)}{(c-1)^{3}}
\end{aligned}
$$

The errors $\sigma_{\lambda_{1}}$ and $\sigma_{\lambda_{2}}$, which are represented by vertical error bars in Figure 6.62, come primarily from statistics but also receive contributions from the preceding corrections in Sections 6.6.2 and 6.6.3. The quantity $\sigma_{c}$, depicted as a horizontal error bar on the point $\left(c, \lambda_{2}\right)$, corresponds to the error in our estimation of $c$, which will be discussed below. Finally, $\sigma_{\lambda_{2} c}$ is a covariant error which accounts for any correlations between $\lambda_{2}$ and $c$. In principle, this term is nonzero if we use the data from the $\lambda_{2}$ measurement to determine the value of $c$. Note, however, that the covariant error has a negative sign, which means that omitting it gives a conservative upper bound on the error. 
Having established the basic deuterium correction formalism, let us turn our attention towards applying it to the 2004 data. During Run8 we performed two deuteriumdoped calibration measurements: "CalibD2" with $c_{d} \approx 20 \mathrm{ppm}$, and "CalibNat" with $c_{d} \approx 120 \mathrm{ppm}$, where the "Nat" label indicates that this fill came closest to the natural deuterium concentration in hydrogen. (In fact, both fillings were accomplished using the same bottle of natural hydrogen gas with $\left.c_{d} \approx 150 \mathrm{ppm}.\right)$ Both of the deuterium calibration fills suffered from elevated $Z>1$ impurity levels, and also from a reduced TPC operating voltage of $4.8 \mathrm{kV}$. The CalibD2 statistics are larger than the CalibNat statistics, $N_{\text {CalibD2 }} \approx 21.3 \times 10^{7}$ vs. $N_{\text {CalibNat }} \approx 6.2 \times 10^{7}$, but the CalibNat fill is more attractive because (1) it exhibits a lower $Z>1$ capture yield, and (2) its larger deuterium concentration gives it a longer lever arm in the zero-extrapolation operation, which yields $\mathrm{a} \approx 1 \mathrm{~s}^{-1}$ smaller error on the corrected result.

Having decided to use the CalibNat data for the high- $c_{d}$ point in the zero extrapolation, the next step is to determine the deuterium concentrations in the CalibNat and Prod-50 gas fills. There are several conceivable ways to do so (see Section 3.3.2), but only two methods proved feasible: (1) external, or outsourced, mass spectrometry measurements of the deuterium concentrations in Run8 hydrogen samples, and (2) data analysis, by studying how the fitted decay rate varies with impact parameter cuts. The results from these two approaches are discussed in turn below.

In the time following the conclusion of the 2004 run, Dr. Claude Petitjean arranged for several mass spectrometry measurements of the deuterium concentrations in samples of liquid and gaseous hydrogen preserved from the run. He approached two groups, who had the following devices capable of performing such measurements:

1. A conventional mass spectrometer (MS) at the PSI Laboratory of Atmospheric Chemistry, operating under the direction of Dr. M. Saurer and designed for measuring iso- 
topic differences in atmospheric precipitation. This instrument ionizes the hydrogen gas sample into $\mathrm{H}_{2}^{+}, \mathrm{HD}^{+}$, etc., under UHV conditions, and measures the resulting ion flux intensities to determine the relative abundance of deuterium. Unfortunately, the spectrometer also creates $\mathrm{H}_{3}^{+}$ions - the amount depends upon the pressure in the ionizer - which cannot be distinguished from $\mathrm{HD}^{+}$in the mass analyzer. In order for the device to measure near-zero deuterium concentrations, it is necessary to extrapolate from an isotopic standard - in our case, the Vienna Standard Mean Ocean Water (VSMOW) standard of $\mathrm{D} / \mathrm{H}=155.75 \mathrm{ppm}$ - but the aforementioned sizable $\mathrm{H}_{3}^{+}$background introduces uncertainties regarding the zero point of $\mathrm{HD}^{+}$. As a result, the MS measurement of the low Prod-50 deuterium concentration is not reliable on the ppm level. Furthemore, relatively large $(\approx 5 \mathrm{ppm})$ variations were observed in the MS measurements of the CalibNat deuterium concentration.

2. The Mini Radiocarbon Dating System (MICADAS) [222,223], a new, compact, tabletop radiocarbon accelerator mass spectrometer (AMS) at the PSI/ETH Laboratory for Ion Beam Physics at ETH Zürich, developed by Dr. H.-A. Synal under the direction of Prof. M. Suter. The MICADAS system was recently commissioned after several years of methodical development at the ETH Zürich Tandem accelerator laboratory, and the instrument has proved to be ideally suited for measuring near-zero deuterium admixtures, due to its extreme background suppression and good zero point determination.

In MICADAS, hydrogen gas from the sample is guided onto the surface of an aluminum sputter cathode (specially designed for MuCap) which is bombarded by a beam of $9 \mathrm{keV} \mathrm{Cs}^{+}$ions. Negatively charged hydrogen ions and molecules such as $\mathrm{H}^{-}$, $\mathrm{D}^{-}, \mathrm{H}_{2}^{-}, \mathrm{HD}^{-}$, etc., are ejected from the cathode surface, magnetically separated in 
a mass spectrometer, and then injected into the small $200 \mathrm{keV}$ Tandem accelerator's argon-gas-filled canal (mass 1 and mass 2 particles are injected in sequence). The negative ions are stripped of all electrons at the accelerator's high voltage terminal, and the positively charged beam is accelerated towards ground to a total energy of $400 \mathrm{keV}$. Collisions in the gas efficiently break up any molecular ions in the beam, and the resulting fragments - that is, protons and deuterons - are separated by a second mass spectrometer. The proton beam current is determined by a Faraday cup at the entrance of the accelerator, while the deuteron current is measured by a cup behind the second mass spectrometer. The deuterium concentration is then calculated from the ratio of the two ion currents, which are each measured with fA sensitivity. The transmission of protons and deuterons through the system differs, so the proton and neutron currents must be normalized to a standard - in our case, the same VSMOW standard as used in the PSI MS measurements. However, the MICADAS result is much more precise because the AMS method suppresses deuteron-mimicking molecular backgrounds by up to ten orders of magnitude. Nevertheless, in principle a possible background from residual gas in the AMS ion source needs to be deducted. The deuterium current without gas flow was therefore measured and subtracted from the current measured with gas flow. It should be noted that the custom Al cathode was specially developed to suppress background contributions, and in fact its background was found to be very low, and in some cases, negligible.

Dr. M. Döbeli of ETH Zürich used MICADAS to measure the deuterium concentration of a sample of hydrogen gas from the MuCap Prod-50 clean fill to $\approx 100 \mathrm{ppb}$.

The mass spectrometry findings are collected in Table 6.12. To determine the Run8 deuterium concentration ratio $c$ from these measurements, I use Döbeli's AMS value $c_{d 1}=$ 


\begin{tabular}{|c|c|c|c|c|}
\hline Analyzed gas & Investigator & Method & $\begin{array}{c}c_{d} \\
(\mathrm{ppm})\end{array}$ & $\begin{array}{c}\text { Date } \\
(\mathrm{m} / \mathrm{d} / \mathrm{y})\end{array}$ \\
\hline Prod-50 sample $(11 / 04 / 04)$ & $\begin{array}{l}\text { Saurer (PSI) } \\
\text { Döbeli (ETH) }\end{array}$ & $\begin{array}{l}\text { MS } \\
\text { AMS }\end{array}$ & $\begin{array}{l}1.58 \pm \mathrm{n} / \mathrm{a} \\
1.44 \pm 0.13\end{array}$ & $\begin{array}{l}1 / 26 / 05 \\
5 / 17 / 06\end{array}$ \\
\hline CalibNat filling bottle & $\begin{array}{l}\text { Saurer (PSI) } \\
\text { Saurer (PSI) }\end{array}$ & $\begin{array}{l}\text { MS } \\
\text { MS }\end{array}$ & $\begin{array}{l}117.6 \pm \mathrm{n} / \mathrm{a} \\
126.7 \pm \mathrm{n} / \mathrm{a}\end{array}$ & $\begin{array}{l}1 / 26 / 05 \\
4 / 4 / 06\end{array}$ \\
\hline
\end{tabular}

Table 6.12: Mass spectrometer (MS) and atomic mass spectrometer (AMS) measurements of the deuterium concentrations $c_{d}$ of the Prod-50 and CalibNat hydrogen gas fills. Precise error bars are not available for the Saurer MS measurements, and the reasons for the difference in the CalibNat results remain unknown; the spread suggests an uncertainty of the order $5 \mathrm{ppm}$. It should be noted that the deuterium concentration for the CalibD2 fill can be inferred from the results in the table, because the same bottle was used to dope the hydrogen gas in the CalibD2 and CalibNat calibration fills. Using volumetric arguments, Dr. Petitjean has calculated that $c_{d}($ CalibD2 $)=17.75 \pm 1.9 \mathrm{ppm}$.

$c_{d}($ Prod-50) $=1.44 \pm 0.13 \mathrm{ppm}$, and I average the two Saurer CalibNat measurements to get $c_{d 2}=c_{d}($ CalibNat $)=122.15 \pm 4.55 \mathrm{ppm}$. Together these numbers give

$$
c=\left(\frac{122.15 \pm 4.55 \mathrm{ppm}}{1.44 \pm 0.13 \mathrm{ppm}}\right)=84.8 \pm 8.3
$$

The alternative method for determining the deuterium concentration ratio relies upon the fact that the fitted decay rate vs. impact cut scales with deuterium concentration, as illustrated in Figure 6.63. The shape of those distributions arises because, for smaller impact cuts, the $\mu d$ atom is more likely to have diffused outside the cut radius as time progresses, thereby mimicking capture and increasing the observed disappearance rate. The magnitude of the effect thus grows with increasing deuterium concentration, as more and more $\mu d$ atoms are formed. Unfortunately, the extraction of deuterium concentration ratios from the distributions in Figure 6.63 is far from straightforward, because each distribution also contains a small component generated by $\mu p$ diffusion (see Figure 6.30, and the accompanying discussion in Section 6.5.7). Steven Clayton performed an extensive study of the subject and, using "annulus" impact parameter cuts $\left(b_{\text {inner }}<b<b_{\text {outer }}\right)$ instead of 


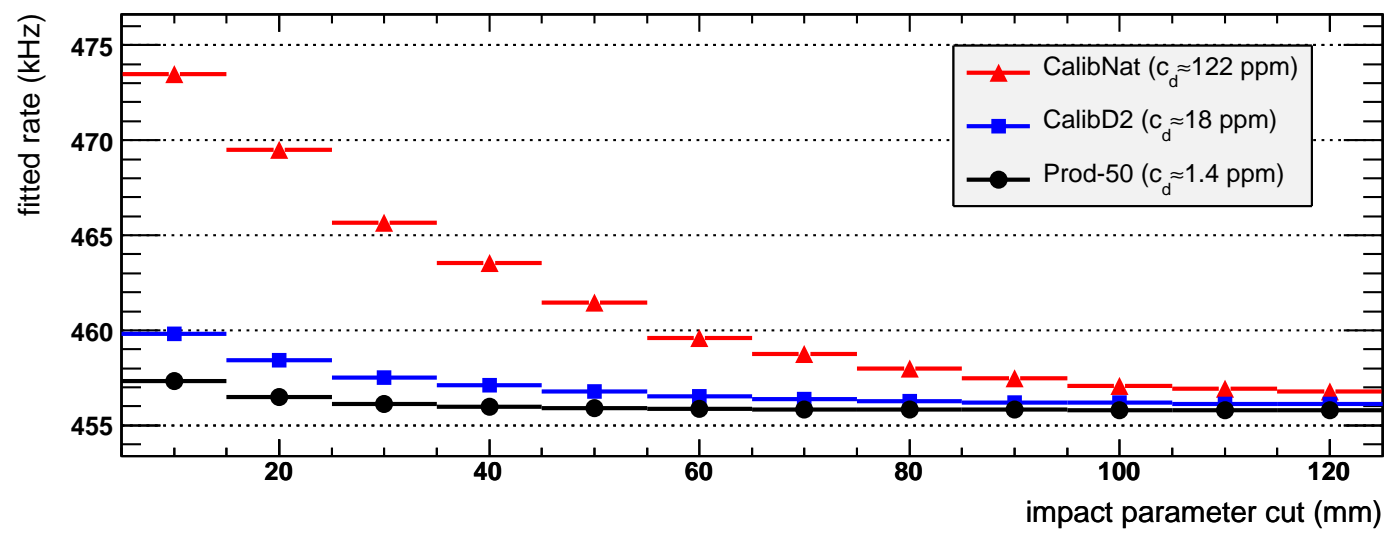

Figure 6.63: The fitted decay rate as a function of impact parameter cut, for three different Run8 hydrogen target deuterium concentrations. Each of the distributions has roughly the same shape, which scales up with increasing deuterium concentration. The structure in the Prod-50 distribution, however, is due almost entirely to $\mu p$ diffusion (see Section 6.5.7). A similar $\mu p$-generated contribution also underlies the CalibD2 and CalibNat distributions, but is obscured by the larger $\mu d$-generated effects.

conventional "disc" impact cuts $\left(b<b_{\text {outer }}\right)$, along with a standard model of isotropic $\mu p$ diffusion like that described in Appendix $\mathrm{C}$, he was able to extract deuterium ratios $c$ from the data itself. He obtained the value [224]

$$
\begin{aligned}
c^{-1} & =\frac{c_{d}(\text { Prod }-50)}{c_{d}(\text { CalibNat })} \\
& =0.0122 \pm 0.0010,
\end{aligned}
$$

or equivalently,

$$
c=82.0 \pm 7.3
$$

which is in excellent agreement with the value in Equation 6.21 from mass spectrometry measurements.

For the zero extrapolation I have elected to use the $c$ value from the mass spectrometry measurements, and to regard the results from Mr. Clayton's data analysis as a corroboration of the spectrometry results. To perform the deuterium correction, I plug 
the $c$ information from Equation 6.21 and the $\lambda$ information in Table 6.11 into software (a ROOT macro) that uses Monte Carlo simulations to generate distributions according to the zero-extrapolation formula in Equation 6.19. Just as with the $Z>1$ corrections, the deuterium correction distributions are asymmetric, in this case because of the uncertainty in $c$ which appears in the denominator of Equation 6.19. Consequently, the Gaussian error of the deuterium correction is determined by the $1 \sigma$ point in the larger shoulder of the simulated distribution, and the central value of the correction is calculated by setting the errors on the inputs to zero. The resulting deuterium-corrected rates are presented in Table 6.13. Note that the Monte Carlo approach obviates the need to employ the analytic error expression in Equation 6.20. Furthermore, the covariant error term in Equation 6.20 can be ignored because I chose to use the $c$ value from the spectrometry measurements, which are completely uncorrelated with the data analysis. I should point out that I have added an uncertainty of $\pm 0.5 \mathrm{~s}^{-1}$ to each of the deuterium corrections in order to account for possible deviations from linearity in the zero extrapolation. This number comes from Monte Carlo studies of $\mu d$ diffusion, conducted by Dr. Peter Kammel and Brendan Kiburg, which suggest that the zero extrapolation has a slight deviation from linearity of $<0.5 \mathrm{~s}^{-1}$ when the calibration point has a high deuterium concentration, like the $c_{d}=122 \mathrm{ppm}$ of the CalibNat fill [153]. Finally, I should emphasize that the zero-extrapolation correction encompasses all deuterium-related time-dependent effects, including those from the unique $\mu d$ nuclear capture rate, $\mu d$ diffusion outside of the impact cut radius, $\mu d$ diffusion into $Z>1$ detector materials, and even $p \mu d$ fusion.

\subsubsection{Impact cut correction}

As discussed in Section 6.5.7, a purely geometric cut on the impact parameter $b$ inadvertantly introduces a time-dependent effect into the muon lifetime histograms, because 


\begin{tabular}{lcccc}
\hline \hline ePC treatment & \multirow{2}{*}{ Impact cut } & Correction & \multicolumn{2}{c}{ Corrected rate } \\
\cline { 4 - 6 } & & $\Delta \lambda_{\text {deuterium }}\left(\mathrm{s}^{-1}\right)$ & $\lambda\left(\mathrm{s}^{-1}\right)$ & $\sigma_{\lambda}\left(\mathrm{s}^{-1}\right)$ \\
\hline \multirow{2}{*}{ cathode-AND } & - & $-5.63 \pm 1.08 \pm 0.5$ & $455,387.90$ & 14.40 \\
& $120 \mathrm{~mm}$ & $-8.48 \pm 1.31 \pm 0.5$ & $455,396.42$ & 14.34 \\
\multirow{2}{*}{ cathode-OR } & - & $-5.99 \pm 1.09 \pm 0.5$ & $455,395.92$ & 13.93 \\
& $120 \mathrm{~mm}$ & $-8.23 \pm 1.27 \pm 0.5$ & $455,404.65$ & 13.87 \\
\hline \hline
\end{tabular}

Table 6.13: The deuterium-related corrections to the $\mu^{-}$disappearance rates. The corrections were determined by applying the zero-extrapolation procedure in Equation 6.19 to the rates in Table 6.11, using the mass spectrometry-based value $c=84.8 \pm 8.3$. The corrections' errors were determined by repeated Monte Carlo simulations of the zero extrapolation, with the input values sampled from their respective dsitributions. As expected, the corrections are slightly larger for the rates involving a $120 \mathrm{~mm}$ impact cut, because impact cuts enhance the time-dependent loss of $\mu d$ atoms to diffusion. Note that I have added a $\pm 0.5 \mathrm{~s}^{-1}$ error to each correction to account for possible deviations from linearity in the zero-extrapolation procedure, as suggested by Monte Carlo studies.

the small-scale ( $\mathrm{mm}$ ) process of $\mu p$ diffusion is magnified by the scattering of Michel electrons as they pass through the pressure vessel walls. A similar effect also applies to $\mu d$ atoms, but was implicitly addressed by the deuterium correction performed in the preceding section. Here I must perform an explicit correction specifically for the $\mu p$ effect.

Steven Clayton devised a method to calculate the magnitude of $\mu p$-diffusion-related rate shifts $\Delta \lambda_{\mu p}(b)$ as a function of impact parameter cut, using the data itself [224]. This procedure played an integral role in his efforts to determine the deuterium concentration ratios of the Run8 fills via data analysis alone. Assuming only a model of isotropic, thermal $\mu p$ diffusion from a point source, Mr. Clayton first extracts an effective diffusion constant $k$ from the distribution of the $\mu^{-}$disappearance rate vs. impact parameter. Both "clean" and deuterium-doped data are necessary for this step, in order to disentangle the $\mu p$ and $\mu d$ drift contributions to the histograms plotted in Figure 6.63. He then calculates the rate shifts $\Delta \lambda_{\mu p}(b)$ by performing a series of complicated integrals which involve the diffusion formula and the experimentally observed impact parameter distribution-preferably the 
impact distribution comprised of muon decays in the time interval $0-1 \mu \mathrm{s}$, because the early, undiffused impact distribution gives the best approximation of the true experimental vertex resolution.

From his own data, Mr. Clayton calculates an effective diffusion constant of $k=$ $0.4875 \mathrm{~mm} / \sqrt{\mu \mathrm{s}}$. This value for $k$ is roughly half that predicted by the naïve calculations in Appendix C, but the discrepancy is certainly not egregious and most likely reflects detector limitations. Unfortunately, Mr. Clayton was unable to calculate a reliable estimate for $k$ from my data, due to technical difficulties related to the incompatibility of our analysis histograms. Nevertheless, using his $k$ value and my impact parameter distribution, he calculated the following $\mu p$-diffusion-related rate increase, corresponding to the standard $120 \mathrm{~mm}$ impact cut:

$$
\Delta \lambda_{\mu p}(b \leq 120 \mathrm{~mm})=2.32 \pm 0.07 \mathrm{~s}^{-1}
$$

The rate offsets for impact parameter cuts in the range $10 \mathrm{~mm}-150 \mathrm{~mm}$ were also calculated; those results were presented graphically in Figure 6.30. It should be mentioned that for his own analysis, Mr. Clayton calculates a slightly larger rate shift of $\Delta \lambda_{\mu p}(b \leq 120 \mathrm{~mm})=$ $3.15 \pm 0.09 \mathrm{~s}^{-1}$ for the $120 \mathrm{~mm}$ impact cut. The difference between his value and Equation 6.23 is consistent with the fact that the tail of his impact distribution at $120 \mathrm{~mm}$ is slightly higher than mine. Moreover, these rate shifts are quite stable and relatively insensitive to the exact decay time interval from which the impact distribution is drawn, or to the value of the diffusion constant $k$. Nevertheless, I have decided to be conservative and enlarge the error on Equation 6.23 to $\pm 0.83 \mathrm{~s}^{-1}$, in order to cover Mr. Clayton's value. The results of the correction are presented in Table 6.14 . 


\begin{tabular}{lcccc}
\hline \hline ePC treatment & Impact cut & Correction & \multicolumn{2}{c}{ Corrected rate } \\
\cline { 4 - 5 } & & $\Delta \lambda_{\mu p}\left(\mathrm{~s}^{-1}\right)$ & $\lambda\left(\mathrm{s}^{-1}\right)$ & $\sigma_{\lambda}\left(\mathrm{s}^{-1}\right)$ \\
\hline \multirow{2}{*}{ cathode-AND } & - & - & $455,387.90$ & 14.40 \\
& $120 \mathrm{~mm}$ & $-2.32 \pm 0.83$ & $455,394.10$ & 14.36 \\
cathode-OR & - & - & $455,395.92$ & 13.93 \\
& $120 \mathrm{~mm}$ & $-2.32 \pm 0.83$ & $455,402.33$ & 13.89 \\
\hline \hline
\end{tabular}

Table 6.14: The impact cut correction, performed on the rates in Table 6.13. The magnitude of the correction was calculated by Steven Clayton using histograms from my data analysis. I have enlarged the error of the correction substantially, from 0.07 to 0.83 , in order to cover Mr. Clayton's own correction of $-3.15 \mathrm{~s}^{-1}$.

\subsubsection{Uncertainty from muon entrance detector inefficiencies}

The final act prior to unblinding the DAQ clock frequency is to introduce an error that accounts for our uncertainty in the effects of inefficiencies in the entrance muon detectors. As discussed in Section 6.5.14, such inefficiencies can lead to distortions in the accidental background of the lifetime spectrum, as well as to $Z>1$ contributions in the decay spectrum due to unrecognized wallstops. My Monte Carlo studies of the subject indicated that the background distortion from the time-independent inefficiency observed in the Run8 Prod-50 data should lower the fitted rate by approximately $2 \mathrm{~s}^{-1}$, while the wallstop contributions from both types of inefficiencies should raise the fitted rate anywhere between $4-7 \mathrm{~s}^{-1}[214]$. However, I made extremely conservative assumptions in my simulation software about the wallstop fractions, and I did not include certain mitigating factors such as impact parameter cuts, redundancy in the entrance detectors, TPC noise, etc. Moreover, Steven Clayton performed zero-extrapolation-style analyses of both timeindependent and time-dependent inefficiencies in the Run8 data, and he observed sub- $\mathrm{Hz}$ effects in both cases.

Unfortunately, it has proven difficult to reconcile Mr. Clayton's Run8 data analysis 


\begin{tabular}{ccccc}
\hline \hline ePC treatment & Impact cut & $\sigma_{\text {muDet }}\left(\mathrm{s}^{-1}\right)$ & $\lambda\left(\mathrm{s}^{-1}\right)$ & $\sigma_{\lambda}\left(\mathrm{s}^{-1}\right)$ \\
\hline \multirow{2}{*}{ cathode-AND } & - & \pm 3 & $455,387.90$ & 14.71 \\
& $120 \mathrm{~mm}$ & $"$ & $455,394.10$ & 14.67 \\
\multirow{2}{*}{ cathode-OR } & - & $"$ & $455,395.92$ & 14.25 \\
& $120 \mathrm{~mm}$ & $"$ & $455,402.33$ & 14.21 \\
\hline \hline
\end{tabular}

Table 6.15: The $\mu^{-}$disappearance rates from Table 6.14 , but with an additional $\pm 3 \mathrm{~s}^{-1}$ error to cover our uncertainties surrounding the effects of inefficiencies in the entrance muon detectors.

studies and my Monte Carlo simulations into a consistent picture of the scale of effects of muon entrance detector inefficiencies. There are indications that my software is not a perfectly accurate model of reality; for example, using a value for the time-independent inefficiency derived from the Run8 $\mu$ SC-pileup-protection-only TPC drift histograms, my program produces a distortion in the lifetime spectrum that is a factor of $2-3$ smaller than what is actually observed in the data. Yet, the same simulations predict effects on the fitted disappearance rate that are larger than what Mr. Clayton has observed in his Run8 data analysis. Of course, these data analysis studies suffer from an inherent limitation that does not afflict the Monte Carlo: it can be extremely difficult or impossible to disentangle small systematic effects on the fitted rate from normal statistical variations in the results.

In light of these uncertainties, I have decided that there is no justification for performing a correction for the effects of muon entrance detector inefficiencies. Instead, I have decided to add a $\pm 3 \mathrm{~s}^{-1}$ error to all of the rates in Table 6.14 to conservatively cover the scale of the effects from such inefficiencies. The $\pm 3 \mathrm{~s}^{-1}$ error is a compromise: it is slightly smaller than the highly conservative $5 \mathrm{~s}^{-1}$ scale of effects that I observed in my Monte Carlo simulations, but considerably larger than the negligible effects Mr. Clayton reported from his Run8 data analysis. The results of the $\pm 3 \mathrm{~s}^{-1}$ error enlargement are presented in Table 6.15 . 


\section{Chapter 7}

\section{Determination of the muon}

\section{capture rate $\Lambda_{S}$}

In this chapter I describe how the $\mu p$ hyperfine singlet capture rate $\Lambda_{S}$ is extracted from the measured $\mu^{-}$disappearance rates in pure hydrogen. With the exception of the $Z>1$ impurity correction in Section 6.6.3, my analysis up to this point has relied primarily on MuCap measurements. However, to arrive at final precision results for $\Lambda_{S}$, I will have to rely on published results for the properties of $p \mu p$ molecules and the free muon decay rate $\lambda_{0} \equiv \lambda_{\mu^{+}}$. As such, I must first unblind the experimental results from the previous chapter.

\subsection{Unblinding of the DAQ clock frequency}

The formula for converting our blinded disappearance rates into proper time is

$$
\lambda^{\text {unblinded }}=\lambda^{\text {blinded }}\left(\frac{f_{\mathrm{DAQ}}}{100 \mathrm{MHz}}\right)
$$

where $f_{\mathrm{DAQ}}$ is the Run8 DAQ clock frequency which was set and kept confidential by Dr. Malte Hildebrandt of PSI and Genna Petrov of PNPI (see Section 6.1.1). The error 
propagation formula corresponding to Equation 7.1 is

$$
\sigma_{\lambda_{\text {unblinded }}}=\sqrt{\sigma_{\lambda^{\text {blinded }}}^{2}\left(\frac{f_{\mathrm{DAQ}}}{100 \mathrm{MHz}}\right)^{2}+\sigma_{f_{\mathrm{DAQ}}}^{2}\left(\frac{\lambda^{\text {blinded }}}{100 \mathrm{MHz}}\right)^{2}} .
$$

The first group of terms under the square root in Equation 7.2 describes a simple rescaling of the blinded error, while the second group of terms incorporates the uncertainty in $f_{\text {DAQ }}$ arising from the inherent limitations of the Agilent E4400B frequency generator. The E4400B module we used belongs to the MuLan Collaboration, who purchased the device in 2003 and chose the model with the "1E5 High-Stability Time-Base Option." According to its technical specifications [225], this version of the E4400B has a frequency stability of

$$
\begin{aligned}
& < \pm 0.1 \mathrm{ppm} / \text { year, or }< \pm 0.0005 \mathrm{ppm} / \text { day after } 45 \text { days, due to aging; } \\
& < \pm 0.05 \mathrm{ppm} \text { due to temp. variations when operated in the range } 0-55^{\circ} \mathrm{C} ; \\
& < \pm 0.002 \mathrm{ppm} \text { due to line voltage. }
\end{aligned}
$$

In the years since the purchase of the E4400B module, MuLan has routinely performed a variety of empirical tests that have confirmed its signal stability is at the level of 0.01-0.02 ppm under typical experimental conditions, including after repeated power-cycling [226-229].

We must also evaluate the accuracy of $f_{\mathrm{DAQ}}$. In February 2005, five months after the commencement of Run8 and three months after its conclusion, Dr. Francoise Mulhauser used a Tektronix TDS 644B oscilloscope to compare the E4400B's output against a $60 \mathrm{MHz}$ reference signal generated by a far more precise $\left(10^{-11}\right)$ Quartzlock Model 10A-R rubidium atomic clock owned by the FAST experiment at PSI. She observed a frequency variation of only [230, 231]

$$
\delta f_{\mathrm{E} 4400 \mathrm{~B}}=\frac{f_{\mathrm{FAST}}-f_{\mathrm{E} 4400 \mathrm{~B}}}{f_{\mathrm{FAST}}}=\frac{60 \mathrm{MHz}-59.99999878(1) \mathrm{MHz}}{60 \mathrm{MHz}} \approx 2 \times 10^{-8}
$$

and annual calibrations of the E4400B by MuLan have obtained similar results [228, 229]. If we regard Dr. Mulhauser's 2005 comparison with the FAST rubidium frequency reference as a calibration of the E4400B to within $\approx 0.02 \mathrm{ppm}$, and consider that the E4400B signal 


\begin{tabular}{|c|c|c|c|c|}
\hline \multirow[t]{2}{*}{ ePC treatment } & \multirow[t]{2}{*}{ Impact cut } & \multirow{2}{*}{$\begin{array}{c}\lambda \text { rescaling } \\
\text { factor }\end{array}$} & \multicolumn{2}{|c|}{ Unblinded rate } \\
\hline & & & $\lambda\left(\mathrm{s}^{-1}\right)$ & $\sigma_{\lambda}\left(\mathrm{s}^{-1}\right)$ \\
\hline \multirow{2}{*}{ cathode-AND } & - & $\times(100.1 / 100)$ & $455,843.29$ & 14.72 \\
\hline & $120 \mathrm{~mm}$ & $"$ & $455,849.49$ & 14.68 \\
\hline \multirow{2}{*}{ cathode-OR } & - & $"$ & $455,851.32$ & 14.26 \\
\hline & $120 \mathrm{~mm}$ & $"$ & $455,857.73$ & 14.22 \\
\hline
\end{tabular}

Table 7.1: The $\mu^{-}$disappearance rates and errors after the unblinding of the Run8 DAQ clock frequency. The rates from Table 6.15 have simply been rescaled according to Equation 7.1. The tiny increase in the errors is due entirely to the same rescaling; the error contribution from the $0.1 \mathrm{ppm}$ uncertainty in the DAQ clock frequency - the second term in the quadrature sum in Equation 7.2 - is negligible.

stability has been demonstrated to reside at the same level, then $\delta \sigma_{f_{\mathrm{DAQ}}}=0.1 \mathrm{ppm}$ can be taken as a safe, conservative estimate for the overall fractional uncertainty in the frequency.

The decision to unblind the Run8 DAQ clock frequency was made at an October 2006 analysis meeting at UIUC, after Steven Clayton and I formally presented our preliminary results for the blinded muon disappearance rate in hydrogen to the collaboration. Following a lengthy deliberation, the meeting participants decided that the rigor of the analyses and the robustness of the results had been demonstrated to their satisfaction, and they authorized the unblinding to proceed. We therefore opened the sealed envelope containing the Run8 DAQ frequency to reveal

$$
f_{\mathrm{DAQ}}=100.1 \mathrm{MHz},
$$

which in turn implies that

$$
\sigma_{f_{\mathrm{DAQ}}}=0.1 \mathrm{ppm} \cdot f_{\mathrm{DAQ}}=10 \mathrm{~Hz}
$$

The unblinded muon disappearance rates and errors are presented in Table 7.1. 


\subsection{Self-consistent determination of $\Lambda_{S}$}

At this juncture, two steps remain in determining $\Lambda_{S}$ : I must correct for the effects of $p \mu p$ molecules, and I must subtract the muon decay rate $\lambda_{0}$ from the $\mu^{-}$disappearance rate(s). These procedures are complicated by a self-consistency dilemma: the size of the molecular correction $\Delta \lambda_{p \mu p}$ depends upon the value of $\Lambda_{S}$ (because the $p \mu p$ nuclear capture rates are functions of $\Lambda_{S}$ ), yet the result for $\Lambda_{S}$ depends upon the size of $\Delta \lambda_{p \mu p}$. This chicken-and-egg situation can be formally expressed as

$$
\Lambda_{S}=\left(\lambda^{\exp }+\Delta \lambda_{p \mu p}\left(\Lambda_{S}, \lambda_{\mathrm{of}}, \lambda_{\mathrm{op}}\right)\right)-\left(\lambda_{0}+\Delta \lambda_{\mu p}\right)
$$

where $\lambda^{\exp }$ is an unblinded $\mu^{-}$disappearance rate from Table $7.1, \Delta \lambda_{p \mu p}$ is a function in part of $\Lambda_{S}$ and the molecular kinetics parameters $\lambda_{\text {of }}$ and $\lambda_{\mathrm{op}}, \lambda_{0}$ is the free muon decay rate, and $\Delta \lambda_{\mu p}$ is the $\mu p$ bound-state modification to $\lambda_{0}$ (see Section 3.1). In order to obtain a self-consistent value for $\Lambda_{S}$, I conduct the following iterative procedure:

1. Assume an initial value for $\Lambda_{S}$;

2. Calculate $\Delta \lambda_{p \mu p}$ using the value for $\Lambda_{S}$;

3. Plug the values for $\lambda^{\exp }, \Delta \lambda_{p \mu p}, \lambda_{0}$, and $\Delta \lambda_{\mu p}$ into Equation 7.4 to obtain a new value for $\Lambda_{S}$;

4. Repeat steps $2-3$ until the value for $\Lambda_{S}$ converges.

This algorithm is repeatedly carried out for each of the disapperance rates $\lambda^{\exp }$ in Table 7.1, using a Monte Carlo program. With each pass, the program randomly samples values for $\lambda^{\exp }, \lambda_{0}$, and the $p \mu p$ kinetics, based upon their values and errors, and then calculates a selfconsistent value for $\Lambda_{S}$ using the iterative procedure above. The repeated passes produce a distribution of $\Lambda_{S}$ values, and the final error for $\Lambda_{S}$ is determined from the Gaussian width 
of the larger shoulder. I should note that the central value for $\Lambda_{S}$ is determined using the same algorithm, but with the input parameter errors set to zero, just as in the $Z>1$ and deuterium corrections.

A subtle and unexpected effect manifests itself in the final error for $\Lambda_{S}$. To illustrate, let us rewrite Equation 7.4 as

$$
\Lambda_{S} \approx\left(\lambda^{\exp }+\frac{\Lambda_{S}}{\Lambda_{S}^{0}} \cdot \Delta \lambda_{p \mu p}\left(\Lambda_{S}^{0}\right)\right)-\left(\lambda_{0}+\Delta \lambda_{\mu p}\right)
$$

Here I have taken advantage of the fact that $\Delta \lambda_{p \mu p}$ is a slowly varying function of $\Lambda_{S}$, which allows me to evaluate $\Delta \lambda_{p \mu p}$ for some reasonable guess $\Lambda_{S}^{0}$, and then scale the correction by the ratio $\Lambda_{S} / \Lambda_{S}^{0}$. This trick yields a closed formula for $\Lambda_{S}$,

$$
\Lambda_{S}=\frac{\left(\lambda^{\exp }-\lambda_{0}-\Delta \lambda_{\mu p}\right)}{\left(1-\frac{\Delta \lambda_{p \mu p}\left(\Lambda_{S}^{0}\right)}{\Lambda_{S}^{0}}\right)}
$$

and applying the error propagation formula gives

$$
\begin{aligned}
\sigma_{\Lambda_{S}}^{2} & =\frac{\left(\sigma_{\lambda^{\exp }}^{2}+\sigma_{\lambda_{0}}^{2}\right)}{\left(1-\frac{\Delta \lambda_{p \mu p}\left(\Lambda_{S}^{0}\right)}{\Lambda_{S}^{0}}\right)^{2}}+\sigma_{\Delta \lambda_{p \mu p}}^{2}\left[\frac{\left(\lambda^{\exp }-\lambda_{0}-\Delta \lambda_{\mu p}\right) / \Lambda_{S}^{0}}{\left(1-\frac{\Delta \lambda_{p \mu p}\left(\Lambda_{S}^{0}\right)}{\Lambda_{S}^{0}}\right)^{2}}\right]^{2} \\
\sigma_{\Lambda_{S}} & \approx \frac{1}{\left(1-\frac{\Delta \lambda_{p \mu p}\left(\Lambda_{S}^{0}\right)}{\Lambda_{S}^{0}}\right)} \sqrt{\sigma_{\lambda^{\exp }}^{2}+\sigma_{\lambda_{0}}^{2}+\sigma_{\Delta \lambda_{p \mu p}}^{2}} \\
\sigma_{\Lambda_{S}} & \approx 1.033 \sqrt{\sigma_{\lambda^{\exp }}^{2}+\sigma_{\lambda_{0}}^{2}+\sigma_{\Delta \lambda_{p \mu p}}^{2}} .
\end{aligned}
$$

Equation 7.6 reveals that the final error for $\Lambda_{S}$ is not simply the quadrature sum of the errors for $\lambda^{\exp }, \lambda_{0}$, and $\Delta \lambda_{p \mu p}$ as might be expected, but instead involves an overall multiplicative scale factor $\left[1-\Delta \lambda_{p \mu p}\left(\Lambda_{S}^{0}\right) / \Lambda_{S}^{0}\right]^{-1} \approx 1.033$. This scale factor-whose exact value depends upon the input parameters used - is a consequence of the existence of molecular capture, which reduces the sensitivity of our experimental result to $\Lambda_{S}$. Even if we could eliminate the molecular uncertainty $\sigma_{\Delta \lambda_{p \mu p}}$, the multiplicative scale factor would persist, since it would reduce to unity only if muon capture proceeded exclusively from the $\mu p$ atomic state. 
In the following subsections I present three separate tables leading to the final values and errors for $\Lambda_{S}$ : the first table contains the $p \mu p$ corrections to the $\mu^{-}$disappearance rates, the second table depicts the subtraction of $\lambda_{0}$, and the third table enlarges the error on $\Lambda_{S}$ by the "sensitivity" scale factor presented in Equation 7.6. In reality, these three steps are carried out simultaneously as part of the iterative self-consistent algorithm, so the entries in the $p \mu p$ and $\lambda_{0}$ tables have been inferred a posteriori from the $\Lambda_{S}$ values and errors in the third and last table.

\subsection{1 p $\mu p$ correction}

A correction for $p \mu p$ effects is necessary for two reasons. First, although we create conditions that suppress $p \mu p$ formation, it remains an unavoidable physical process that occurs to some extent in any hydrogen target. In this sense, the $p \mu p$ "correction" is fundamentally different from the earlier corrections in Section 6.6, which in principle could be reduced or eliminated with cleaner gas and better detectors. The $p \mu p$ correction turns out to be the largest of all of the rate corrections, as will be demonstrated shortly. Second, the effective muon disappearance rates were originally obtained in Section 6.6.1 using a threeparameter exponential function, and are thus influenced by the existence of muon capture in $p \mu p$ molecules. In contrast, an explicit molecular correction would be unnecessary if the fits were performed using a function that incorporated the full kinetics. In this case, the molecular effects would already be integrated into the fit results. Such an approach will be examined in Section 7.3.1.

To determine the magnitude of the $p \mu p$ correction for a given value of $\Lambda_{S}$, I use the formula

$$
\Delta \lambda_{p \mu p}=\left(\lambda_{0}+\Delta \lambda_{\mu p}+\Lambda_{S}\right)-\lambda^{\text {moment }}
$$

where $\lambda^{\text {moment }}$ is calculated from Equation 3.9, the analytic formula for the first moment of 
the muon lifetime spectrum. The coefficients $\lambda_{i}$ and $c_{i}$ in the first moment formula come from Equations 3.5 and 3.8, respectively, with the impurity concentrations $c_{Z}$ and $c_{d}$ set to zero so that Equation 3.9 is restricted to a description of the three-state $\left(\mu p, p \mu p_{\text {ortho }}\right.$, $\left.p \mu p_{\text {para }}\right)$ system. For the molecular kinetics parameters I use the values

$$
\begin{aligned}
\phi & =0.01123 \pm 0.00013 \\
\lambda_{\text {of }} & =(2.3 \pm 0.5) \times 10^{6} \mathrm{~s}^{-1} \\
\lambda_{\mathrm{pf}} & =7.4 \times 10^{3} \mathrm{~s}^{-1} \\
\lambda_{\mathrm{op}} & =(6.9 \pm 4.3) \times 10^{4} \mathrm{~s}^{-1} .
\end{aligned}
$$

The Run8 gas density $\phi$ is calculated in Appendix D, while the $p \mu p$ formation and transition rates come from reference [232], written by Dr. Peter Kammel. In that document he employs a so-called "maximum average" approach, whereby the mean value of each quantity is calculated from multiple data points according to the weighted average

$$
\bar{x}=\frac{\sum_{i}\left(\frac{x_{i}}{\sigma_{i}^{2}}\right)}{\sum_{i}\left(\frac{1}{\sigma_{i}^{2}}\right)},
$$

and the associated error $\sigma_{\bar{x}}$ is taken as the distance from $\bar{x}$ to the farthest contributing data point. The average ortho state formation rate $\left(\lambda_{\text {of }}\right)$ was computed using the experimental liquid and gas data in Table 3.2, although the uncertainty was increased to cover the theoretical prediction. The average ortho-to-para transition rate $\left(\lambda_{\mathrm{op}}\right)$ was computed from the single theoretical result and the two experimental results available in Table 3.2. Finally, the nuclear muon capture rates from the ortho and para $p \mu p$ states were calculated from the formulas first presented in Table 3.1,

$$
\begin{aligned}
& \Lambda_{\mathrm{om}}=2 \gamma_{\mathrm{o}}\left[(3 / 4) \Lambda_{S}+(1 / 4) \Lambda_{T}\right] \\
& \Lambda_{\mathrm{pm}}=2 \gamma_{\mathrm{p}}\left[(1 / 4) \Lambda_{S}+(3 / 4) \Lambda_{T}\right]
\end{aligned}
$$




\begin{tabular}{lcccc}
\hline \hline ePC treatment & Impact cut & Correction & \multicolumn{2}{c}{ Corrected rate } \\
\cline { 4 - 5 } & & $\Delta \lambda_{p \mu p}\left(\mathrm{~s}^{-1}\right)$ & $\lambda\left(\mathrm{s}^{-1}\right)$ & $\sigma_{\lambda}\left(\mathrm{s}^{-1}\right)$ \\
\hline \multirow{2}{*}{ cathode-AND } & - & $23.64 \pm 6.62$ & $455,866.93$ & 16.14 \\
& $120 \mathrm{~mm}$ & $23.87 \pm 6.68$ & $455,873.36$ & 16.13 \\
cathode-OR & - & $23.93 \pm 6.64$ & $455,875.25$ & 15.73 \\
& $120 \mathrm{~mm}$ & $24.17 \pm 6.82$ & $455,881.90$ & 15.77 \\
\hline \hline
\end{tabular}

Table 7.2: The $\mu^{-}$disappearance rates and errors after applying $p \mu p$ corrections to the rates in Table 7.1. The $p \mu p$ corrections differ in size among the four treatments because $\Delta \lambda_{p \mu p}$ is determined concurrently with $\Lambda_{S}$ through iterative application of Equation 7.4, and thus the size of $\Delta \lambda_{p \mu p}$ depends upon the size of the disappearance rates in Table 7.1.

using the values

$$
\begin{aligned}
& \Lambda_{T}=12 \mathrm{~s}^{-1} \\
& \gamma_{\mathrm{o}}=1.009 / 2 \\
& \gamma_{\mathrm{p}}=1.143 / 2 .
\end{aligned}
$$

One must be cautious about naïvely applying the first-moment-based $p \mu p$ correction $\Delta \lambda_{p \mu p}$ from Equation 7.7 to the experimental rates in Table 7.1, which were originally obtained from three-parameter exponential best-fits over a limited time range. Indeed, Monte Carlo simulations reveal that the first moment formula tends to slightly overestimate the magnitude of the $p \mu p$ correction, compared to the value obtained from best fits. To compensate for this discrepancy, I subtracted $0.99 \pm 0.02 \mathrm{~s}^{-1}$ from the first-moment-based values for $\Delta \lambda_{p \mu p}$ that were obtained from application of Equation 7.7.

The $p \mu p$ corrections and the resulting muon disappearance rates in the $\mu p$ system are presented in Table 7.2. The corrections are all $\approx 24 \mathrm{~s}^{-1}$ in magnitude, of which roughly $17 \pm 5 \mathrm{~s}^{-1}$ comes from the formation of ortho states (i.e. from $\lambda_{\mathrm{of}}$ ), and $7 \pm 5 \mathrm{~s}^{-1}$ comes from the formation of para states, primarily via ortho $\rightarrow$ para transitions (i.e. from $\lambda_{\mathrm{op}}$ ). Note that the $p \mu p$ corrections are positive in sign, unlike the previous corrections in Section 6.6. 
This is due to the fact that we are compensating for the influence of the $p \mu p$ nuclear capture rates $\Lambda_{\mathrm{om}}$ and $\Lambda_{\mathrm{pm}}$, which are smaller than the $\mu p$ capture rate $\Lambda_{S}$ and therefore tend to pull down the $\mu^{-}$disappearance rate from that which would be observed for the $\mu p$ state only.

\subsubsection{Subtraction of the free muon decay rate $\lambda_{\mu^{+}}$}

Having corrected for $p \mu p$ effects, the next step in determining $\Lambda_{S}$ is to subtract the muon decay rate $\lambda_{0} \equiv \lambda_{\mu^{+}}$from the muon disappearance rates in Table 7.2. Recall from Section 3.1 that the $\mu^{-}$decay rate is altered by its participation in the $\mu p$ bound state. Consequently, the correct formula for calculating $\Lambda_{S}$ from the muon disappearance rate in the $\mu p$ system is

$$
\begin{aligned}
\Lambda_{S} & =\lambda-\lambda_{0}^{\prime} \\
& =\lambda-\left(\lambda_{0}+\Delta \lambda_{\mu p}\right),
\end{aligned}
$$

where the free muon decay rate, as determined from measurements of $\mu^{+}$decay, has the value $[49]$

$$
\lambda_{0}=\frac{1}{2.197019(21) \mathrm{s}}=455,162.2 \pm 4.4 \mathrm{~s}^{-1}
$$

and the Uberall bound-state modification is

$$
\begin{aligned}
\Delta \lambda_{\mu p} & =-\lambda_{0}(Z \alpha)^{2}\left[\frac{1}{2}+0.06\left(\frac{m_{\mu}}{m_{N}}\right)\right] \\
& =-\lambda_{0}\left(\frac{1}{137.03599911}\right)^{2}\left[\frac{1}{2}+0.06\left(\frac{105.7 \mathrm{MeV}}{939 \mathrm{MeV}}\right)\right] \\
& =-12.28 \mathrm{~s}^{-1}
\end{aligned}
$$

It should be noted that this value of $\Delta \lambda_{\mu p}$ is specific to $\mu p$ atoms, and in principle a different bound-state modification should be applied to those muons that form $p \mu p$ molecules. 


\begin{tabular}{lcccc}
\hline \hline ePC treatment & Impact cut & $-\lambda_{0}^{\prime}\left(\mathrm{s}^{-1}\right)$ & $\Lambda_{S}\left(\mathrm{~s}^{-1}\right)$ & $\sigma_{\Lambda_{S}}\left(\mathrm{~s}^{-1}\right)$ \\
\hline \multirow{2}{*}{ cathode-AND } & - & $-455,149.92 \pm 4.40$ & 717.01 & 16.73 \\
& $120 \mathrm{~mm}$ & $"$ & 723.44 & 16.72 \\
\multirow{2}{*}{ cathode-OR } & - & $"$ & 725.33 & 16.33 \\
& $120 \mathrm{~mm}$ & $"$ & 731.98 & 16.37 \\
\hline \hline
\end{tabular}

Table 7.3: The $\Lambda_{S}$ values obtained by subtracting the $\mu^{-}$decay rate in the $\mu p$ system from the disappearance rates in Table 7.2.

\begin{tabular}{ccccc}
\hline \hline ePC treatment & Impact cut & Scaling of $\sigma_{\Lambda_{S}}$ & $\Lambda_{S}\left(\mathrm{~s}^{-1}\right)$ & $\sigma_{\Lambda_{S}}\left(\mathrm{~s}^{-1}\right)$ \\
\hline \multirow{2}{*}{ cathode-AND } & - & $1.033 \times \sigma_{\Lambda_{S}}$ & 717.01 & 17.28 \\
& $120 \mathrm{~mm}$ & $"$ & 723.44 & 17.27 \\
\multirow{2}{*}{ cathode-OR } & - & $"$ & 725.33 & 16.87 \\
& $120 \mathrm{~mm}$ & $"$ & 731.98 & 16.91 \\
\hline \hline
\end{tabular}

Table 7.4: The final values and errors for $\Lambda_{S}$, after enlarging the uncertainties $\sigma_{\Lambda_{S}}$ in Table 7.3 by the "sensitivity" scale factor from Equation 7.6. The central values of $\Lambda_{S}$ are unaffected by this step.

However, the difference in bound-state decay rates for the two systems is assumed to be negligible, so only $\Delta \lambda_{\mu p}$ is applied. The resulting values for $\Lambda_{S}$ are presented in Table 7.3.

\subsubsection{Result for $\Lambda_{S}$}

The last step is to enlarge the errors $\sigma_{\Lambda_{S}}$ in Table 7.3 by the "sensitivity" scale factor 1.033, presented in Equation 7.6. The final results for $\Lambda_{S}$ are presented in Table 7.4.

At this point I must address an issue that has been postponed: I need to decide how to reconcile the four $\Lambda_{S}$ numbers in Table 7.4 into a single result. In fact, the decision was made some time ago, at our October 2006 analysis meeting. There, before the collaboration "opened the box" and unblinded the Run8 DAQ clock frequency, we agreed to the following prescription for arriving at a final result for $\Lambda_{S}$ : the two 120-mm-impact- 
cut rates from the cathode-AND and cathode-OR treatments should be averaged, and the error should be enlarged to account for the inconsistencies among the various approachesperhaps including the no-cut rates. In calculating these error augmentations, the statistical correlations between the rates should be be taken into account. The 120-mm-impact-cut results were deemed preferable to the no-cut results because of the lower background and higher signal-to-noise ratio in the former. However, it should be emphasized that this judgement was somewhat subjective, and that the superiority of the 120-mm-impact-cut results has not been rigorously demonstrated.

In accordance with the decided-upon policy, I find that the 120-mm-impact-cut rates in Table 7.4 average to

$$
\Lambda_{S}=\frac{\left(723.44 \mathrm{~s}^{-1}+731.98 \mathrm{~s}^{-1}\right)}{2}=727.71 \mathrm{~s}^{-1}
$$

To calculate the corresponding error, I start with the larger of the two associated uncertainties (cathode-AND) and I enlarge it by an amount that encompasses the larger-thanstatistically-expected difference between the cathode-AND and cathode-OR rates, using the formula in Equation 6.7:

$$
\begin{aligned}
\sigma_{\Lambda_{S}}(120 \mathrm{~mm} \text { cut }) & =\sqrt{\left(\operatorname{MAX}\left(\sigma_{\mathrm{AND}}, \sigma_{\mathrm{OR}}\right)\right)^{2}+\left(\Delta \lambda_{\mathrm{AND} / \mathrm{OR}}-\Delta \lambda_{\text {allowed }}\right)^{2}} \\
& =\sqrt{\sigma_{\mathrm{AND}}^{2}+\left(\left(\lambda_{\mathrm{OR}}-\lambda_{\mathrm{AND}}\right)-\sqrt{\sigma_{\mathrm{AND}}^{2}-\sigma_{\mathrm{OR}}^{2}}\right)^{2}} \\
& =\sqrt{\left(17.27 \mathrm{~s}^{-1}\right)^{2}+\left(731.98 \mathrm{~s}^{-1}-723.44 \mathrm{~s}^{-1}-\sqrt{\left(12.86 \mathrm{~s}^{-1}\right)^{2}-\left(12.34 \mathrm{~s}^{-1}\right)^{2}}\right)^{2}} \\
& =17.96 \mathrm{~s}^{-1}
\end{aligned}
$$

My final result from analysis of the Run8 MuCap data is thus:

$$
\Lambda_{S}^{\text {MuCap,Berkeley }}=727.71 \pm 17.96 \mathrm{~s}^{-1}=727.71 \pm 13.59_{\text {stat }} \pm 11.74_{\text {syst }} \mathrm{s}^{-1}
$$

I have included the uncertainty from $\lambda_{0}$ in the statistical uncertainty in $\Lambda_{S}$ above. 
In the time since the unblinding meeting, we have revised our approach to the averaging procedure. In order to obtain a final collaboration result for $\Lambda_{S}$ suitable for publication, Dr. Peter Kammel decided to average the two UCB 120-mm-cut results and the two UIUC 120-mm-cut results, and then calculate a "consistency uncertainty" by summing in quadrature the deviations of the four contributing results from this average. This procedure, whose result will be presented in Section 8.1, avoids the awkward situation of "half-using" the no-cut results (i.e. in such a way that they only contribute via the error), but they are effectively covered within the generous error anyways.

\subsubsection{Error summary}

In Table 7.5 I summarize the statistical errors, systematic errors, and corrections involved in my determination of $\Lambda_{S}$. I should point out that the numbers in the table are only general, since there are small variations among the four different electron detector and impact cut treatments.

\subsection{Final consistency checks}

In the following three sections I present three consistency checks on my analysis results. These particular checks are presented at this juncture because they require knowledge of the true Run8 clock frequency.

\subsubsection{Lifetime fits using the full kinetics function}

Up to this point, I have only used the exponential function in Equation 6.2,

$$
f(t)=N w \lambda e^{-\lambda t}+B
$$




\begin{tabular}{|c|c|c|c|c|c|}
\hline \multirow[t]{2}{*}{ Stage } & \multirow[t]{2}{*}{ Source } & \multicolumn{2}{|c|}{ Correction } & \multicolumn{2}{|c|}{ Error } \\
\hline & & $\left(\mathrm{ppm}\right.$ of $\left.\lambda_{0}\right)$ & $\left(\mathrm{s}^{-1}\right)$ & $\left(\mathrm{ppm}\right.$ of $\left.\lambda_{0}\right)$ & $\left(\mathrm{s}^{-1}\right)$ \\
\hline \multirow{6}{*}{ Pre-unblinding } & Statistics & & & 27.9 & 12.9 \\
\hline & $\mu+p$ scatter & -7.0 & -3.2 & 6.6 & 3.0 \\
\hline & High- $Z$ impurities & -45.9 & -20.9 & 11.9 & 5.4 \\
\hline & Deuterium & -18.5 & -8.4 & 2.9 & 1.3 \\
\hline & Impact cut & -5.1 & -2.3 & 1.8 & 0.8 \\
\hline & $\mu$ Detector inefficiencies & & & 6.6 & 3.0 \\
\hline \multirow[t]{3}{*}{ Unblinding } & Rescaling of error & & & "1.3" & $“ .6 "$ \\
\hline & $p \mu p$ formation & 52.3 & 23.8 & 18.0 & 6.7 \\
\hline & $\lambda_{0}$ & & $-455,160$ & 17.6 & 4.4 \\
\hline \multirow[t]{3}{*}{ Post-unblinding } & $\Delta \lambda_{\mu p}$ & & 12.28 & & \\
\hline & Sensitivity multiplicative factor 1.033 & & & & "4.3" \\
\hline & eDetector treatments & & & & 4.9 \\
\hline Total & & & & & 18.0 \\
\hline
\end{tabular}

Table 7.5: Tabulation of corrections and errors, both statistical and systematic, from my determination of $\Lambda_{S}$ from the Run8 data. The PDG value for $\lambda_{0} \equiv 455,160 \mathrm{~s}^{-1}$ was used to calculate the table entries in ppm where relevant (i.e. prior to the subtraction of $\left.\lambda_{0}\right)$; note that to convert from ppm to Hertz one must multiply by the factor $\left(455,160 \mathrm{~s}^{-1} /\left(1 \times 10^{6}\right)\right) \approx 1 / 2$. The error in the "eDetectors treatments" entry comes from the averaging procedure described in Section 7.2.3. The unblinding's "Rescaling of error" entry and the post-unblinding's "Sensitivity" entry are enclosed in quotes because they are technically multiplicative operations, but I have written them as additive error contributions so that the magnitude of their effects can be more easily compared with the uncertainties from the other procedures. 
to fit $\mu^{-}$lifetime spectra. Although this fitting function has the advantage of simplicity, it does not accurately reflect the level of our understanding of the details of muon processes in hydrogen. For instance, in Section 3.4 I presented differential equations describing the kinetics of negative muons in a hydrogen target containing small amounts of impurities. Solving the system yields an analytic function (Equation 3.7) which describes the $\mu^{-}$population over time, and by extension, the lifetime spectrum observed from decay electrons. Although the full kinetics function is complicated, in principle it can be used to fit experimental data. Because the function contains a more complete description of muon physics in hydrogen, it naturally incorporates $p \mu p$ processes in the fit, and therefore obviates the need for a special $p \mu p$ correction like that performed in Section 7.2.1. For similar reasons, a full kinetics fitting function can avoid the instabilites which often appear in fit range scans when fitting impurity-doped data with the simple exponential function. However, in order to perform meaningful fits with the full kinetics function, it is necessary to work in true (unblinded) time, so that published knowledge about $p \mu p$ kinetics parameters can be applied. Therefore we did not have the option of performing full kinetics fits in the twoyear-long Run8 data analysis that was conducted prior to the October 2006 DAQ frequency unblinding. It should be emphasized that the full kinetics approach does not provide any new information, but merely offers an alternative, complementary method of analyzing the data, and therefore serves as a consistency check on the corrected exponential fit results.

Ideally, one would use the muon kinetics treatment in Section 3.4 to obtain a fitting function

$$
f(t)=f\left(t ; \lambda_{0}, Q, \Lambda_{S}, \Lambda_{T}, \lambda_{\mathrm{of}}, \lambda_{\mathrm{pf}}, \lambda_{\mathrm{op}}, c_{d}, \lambda_{p d}, \Lambda_{d}, c_{Z}, \lambda_{p Z}, \lambda_{d Z}, \Lambda_{Z}\right)+B
$$

which describes the effects of the $\mu p, p \mu p, \mu d$, and (possibly multiple) $\mu Z$ systems on the muon decay spectrum. In reality this approach is not practicable - not because the formula 
is unwieldy, but because kinetics alone do not describe all the phenomena that affect the experimental lifetime spectrum. In particular, the formula does not account for the effects of $\mu d$ diffusion on the reconstruction of muon decays, described in Section 6.6.4. To remedy this shortcoming, Dr. Peter Kammel proposed an alternative approach [153] which employs a fit function that analytically describes the $\mu p, p \mu p$, and $\mu Z$ kinetics, but empirically describes the complex $\mu d$ effects:

$$
f(t)=m(t)\{1-\gamma[R(t, b)-1]\}+B
$$

Here the function

$$
m(t)=m\left(t ; \lambda_{0}, Q, \Lambda_{S}, \Lambda_{T}, \lambda_{\text {of }}, \lambda_{\mathrm{pf}}, \lambda_{\mathrm{op}}, c_{Z}, \lambda_{p Z}, \lambda_{d Z}, \Lambda_{Z}\right)
$$

describes all non- $\mu d$ kinetics, and is calculated from Equations 3.7, 3.5, and 3.8, with the impurity concentration $c_{d}$ set to zero. The multiplicative factor

$$
\{1-\gamma[R(t, b)-1]\}
$$

describes the effects of $\mu d$ atoms, and its terms are established by carefully comparing the Prod-50 and CalibNat lifetime spectra, according to the prescription outlined in reference [153].

I adopted an intermediate approach: I performed fits using a function that describes only $\mu p$ and $p \mu p$ kinetics, and I corrected the fit results with the exponential-fitbased $\mu Z$ and $\mu d$ corrections presented earlier in Tables 6.11 and 6.13. I chose not to incorporate $\mu Z$ kinetics into the fitting function because of the sizable uncertainties surrounding $\mu Z$ effects. I was mildly successful at obtaining values for the $\mu d$ multiplicative factor in Equation 7.11, although the ensuing fitted results were consistently $1-2 \mathrm{~s}^{-1}$ lower than what I obtained if I simply applied the $\mu d$ corrections from Table 6.13. Given the subtlety and complexity of the procedure for determining the terms in the empirical $\mu d$ 


\begin{tabular}{cccc}
\hline \hline ePC treatment & Impact cut & $\Lambda_{S}\left(\mathrm{~s}^{-1}\right)$ & $\sigma_{\Lambda_{S}}\left(\mathrm{~s}^{-1}\right)$ \\
\hline \multirow{2}{*}{ cathode-AND } & - & 716.33 & 16.77 \\
& $120 \mathrm{~mm}$ & 723.65 & 16.92 \\
cathode-OR & - & 724.51 & 16.38 \\
& $120 \mathrm{~mm}$ & 731.93 & 16.35 \\
\hline \hline
\end{tabular}

Table 7.6: The $\Lambda_{S}$ results obtained using a fitting function that incorporates $p \mu p$ kinetics. As expected, the central values are close $\left(<1 \mathrm{~s}^{-1}\right)$ to the exponential-fit-based results in Table 7.4. However, the $\sigma_{\Lambda_{S}}$ errors above are somewhat smaller, for unknown reasons.

correction factor, it is likely that these small discrepancies could be eliminated with further study, but unfortunately I did not have the luxury of time to do so. I should note that it is still necessary to explicitly correct the full kinetics fit results for $\mu p$ diffusion effects in the the 120-mm-impact-cut spectra, just as was done in Section 6.6.5. Similarly, one must remember to include the assorted uncertainties from Tables 6.9 and 6.15, which are also applicable here.

The $\Lambda_{S}$ results from my more comprehensive kinetics fits to the Prod-50 data are presented in Table 7.6. I used the same Run8 lifetime spectra as in Table 6.9-namely, the spectra in which identified $\mu+p$ scatter events were removed. When fitting, however, the time axis of the lifetime spectra must be rescaled, $t \rightarrow t \times(100.0 / 100.1)$, in order to convert the blinded time into true time. The errors from the uncertainties in the $p \mu p$ kinetics parameters were obtained through repeated Monte Carlo sampling of the parameter values and fits, for each lifetime spectrum. The central values for $\Lambda_{S}$ were obtained by setting the errors in the $p \mu p$ kinetics parameters to zero.

As expected, the $\Lambda_{S}$ values in Table 7.6 are consistent with the three-parameter exponential best-fit results in Table 7.4, and appear to confirm the basic validity of the exponential fit approach with regard to molecular effects. It should be noted, however, that the final errors $\sigma_{\Lambda_{S}}$ from the kinetics-inclusive fits are somewhat smaller than from 
the exponential fits. At present, the exact reasons for this discrepancy are not clear, although it appears to be related to the "sensitivity" of $\Lambda_{S}$ to the existence of capture in $p \mu p$ molecules, as discussed in Section 7.2. In particular, it seems that the sensitivity-related uncertainty that is naturally produced by the more descriptive kinetics fit is slightly smaller in magnitude than the error applied to the exponential fit results in Table 7.4. It should be emphasized, however, that the amount of time spent studying the more comprehensive kinetics fits was much less than the time spent on the exponential fits, due to the fact that the clock blinding was in effect for most of the duration of the data analysis. A more thorough examination of the full kinetics approach would be useful and potentially informative, although improvements in the sophistication of this method are not expected to reveal any shortcomings in the exponential fit approach.

\subsubsection{CAEN clock and beam RF beating studies}

In Section 6.5.4 I described how we rebin the fine-resolution lifetime spectra in order to wash out any structure arising from the nonuniformities in the CAEN interpolator distributions (Figure 6.22). We still had some concerns, however, that a systematic bias might arise from beating effects between the $\approx 25 \mathrm{MHz}$ CAEN clock frequency and the $\approx 50 \mathrm{MHz}$ PSI cyclotron RF, because the two frequencies are not derived from the same source and therefore are not perfect multiples of each other. In particular, it was conceivable that the CAEN interpolator nonuniformities could combine with the RF-modulated arrival of beam muons (Figure 6.2) to produce time-dependent effects in the lifetime spectrum. However, an accurate examination of this possibility could only be performed after the DAQ clock frequency had been unblinded, because the exact value of the CAEN frequency is needed. In October 2006 we finally learned that the primary DAQ clock frequency was 
100.1 MHz (see Section 7.1), which meant that

$$
\begin{aligned}
f_{\mathrm{CAEN}} & =\frac{1}{4} \cdot f_{\mathrm{DAQ}}=25.025 \mathrm{MHz} \\
f_{\mathrm{RF}} & =50.582 \mathrm{MHz} .
\end{aligned}
$$

Note that the difference frequency of $0.532 \mathrm{MHz}$ corresponds to a period of $1.9 \mu \mathrm{s}$, which is comparable to the muon lifetime. Although this situation seems vaguely worrisome, it should be emphasized that no clear mechanism was ever proposed to explain how this difference frequency might affect the disappearance rates extracted from the rebinned spectra. Nevertheless, Dr. Frederick Gray wrote some simple Monte Carlo software to empirically test for any beating effects. He faithfully replicated the experimental sequence, generating lifetime spectra by simulating individual muon arrival and decay events, rebinning the resulting 1.25-ns-resolution lifetime histograms by 32 , and fitting the rebinned spectra. The times between stochastic muon arrivals were obtained by sampling the experimental distribution of arrival time differences, which is an exponential (see Equation 5.1) modulated by $f_{\mathrm{RF}}=50.582 \mathrm{MHz}$. The CAEN time bin corresponding to each 25 - $\mu$ s-pileupprotected muon arrival was determined using the CAEN interpolator distributions from the Run8 data (see Figure 6.22). Each muon's decay time was sampled from an exponential distribution, and the corresponding CAEN time of the decay electron's detection was computed in a manner that simulated the fourfold gondola coincidence requirement of the experiment: a Gaussian perturbation of $\mathrm{rms}=0.9 \mathrm{~ns}$ was applied four times to the decay electron time, a CAEN bin was determined for each perturbed electron time, and the four CAEN times were averaged. The differences between the muon's and electron's CAEN times were filled into lifetime histograms with 1.25-ns-wide bins.

Dr. Gray generated several high-statistics lifetime distributions in this manner, and for each he magnified the CAEN interpolator nonuniformity by a different scale factor, 


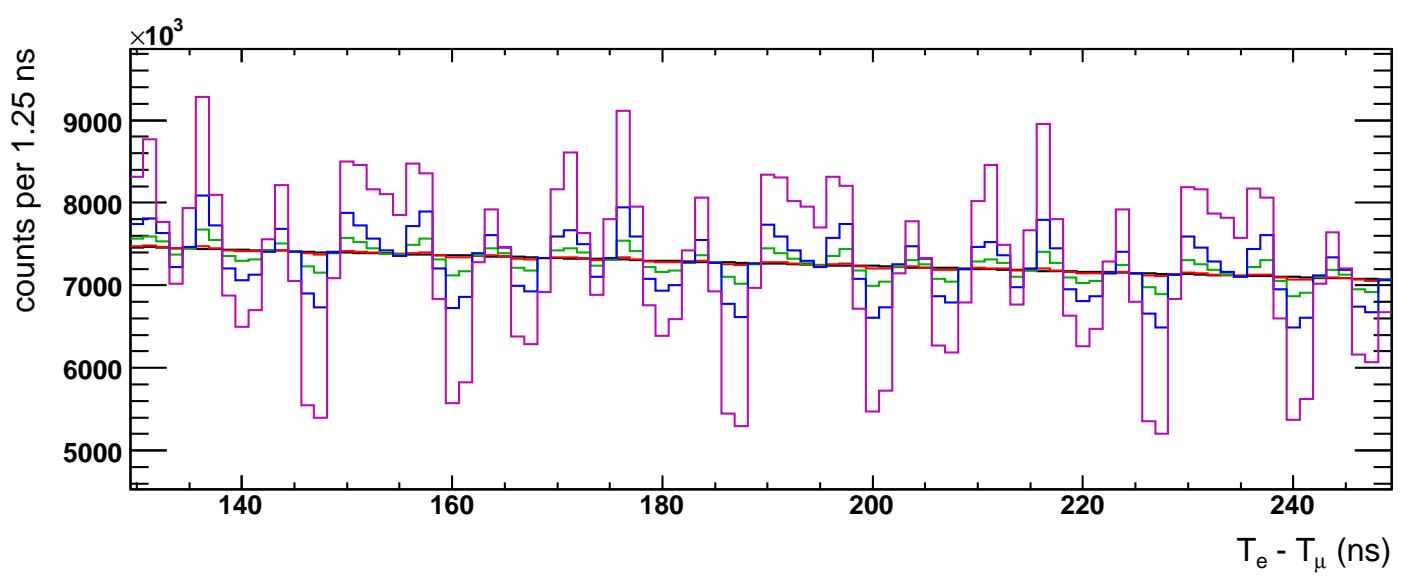

Figure 7.1: Close-up view of simulated lifetime spectra, which were created using different magnifications of the CAEN interpolator nonuniformities. These spectra were generated by a Monte Carlo program written by Dr. Frederick Gray to study the combined effects on the lifetime spectrum of the PSI cyclotron beam RF and the CAEN interpolator nonuniformities. The plots above correspond to interpolator nonuniformity magnifications of 0 (that is, perfectly uniform), 20,60, 100, and 200. For these fine-resolution spectra with $1.25 \mathrm{~ns}$ bins, the distortions clearly grow in size as the CAEN interpolator nonuniformities are magnified.

including 0, 20, 60, 100, and 200 (see Figure 7.1). The disappearance rates obtained from fits to the rebinned versions of these simulated lifetime spectra were all statistically consistent (Figure 7.2), and exaggerating the CAEN interpolator nonuniformities did not produce any apparent effect. We therefore concluded that there is no reason to suspect that any systematic bias exists from beating effects between the beam RF and CAEN clock frequencies. The only phenomenon of real concern - the CAEN interpolator nonuniformities - is already handled properly by the rebinning procedure.

\subsection{3 $\mu^{+}$lifetime measurement}

Previous $\mu^{-}$lifetime-based capture rate measurements have relied on simple scintillators, not slower and more complex wire chambers as used in MuCap, so a complementary measurement of the $\mu^{+}$lifetime serves as an important check on instrumental systematics. 


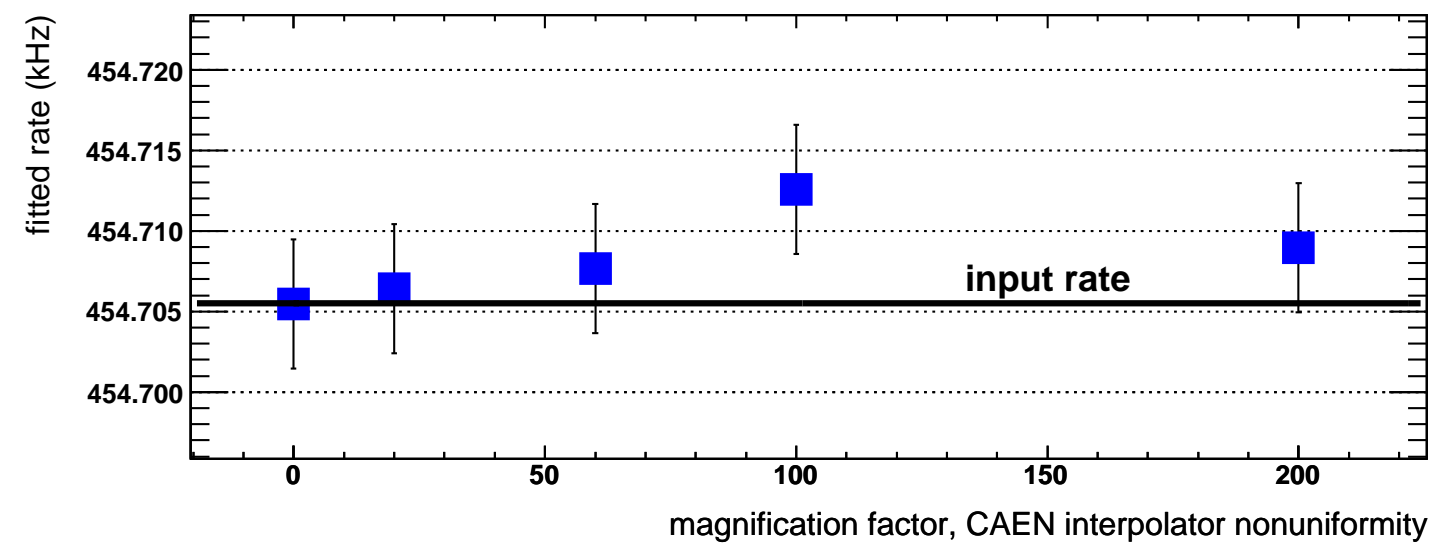

Figure 7.2: The rates obtained from fits to the simulated lifetime spectra in Figure 7.1, the creation of which involved magnification of the effects of the CAEN interpolator nonuniformities. The fitted rates above are statistically consistent, and thus do not indicate the existence of any systematic bias from interference between the CAEN clock and cyclotron beam RF frequencies. I should note that the indicated MC input rate was $454,705.5 \mathrm{~s}^{-1}=455,159.9 \mathrm{~s}^{-1} /(100.1 \mathrm{~Hz} / 100.0 \mathrm{~Hz})$; that is, the input vacuum muon decay rate was subjected to the global clock blinding in reverse, as part of the effort to simulate the MuCap experimental procedure.

At several intervals during Run8, we switched the polarity of the muon beam and collected $\mu^{+}$data using the same experimental setup used to collect $\mu^{-}$data. Altogether we recorded approximately $0.5 \times 10^{9}$ good $\mu^{+}$decay events in 2004 . Like the $\mu^{-}$data in Section 6.5.9, the $\mu^{+}$data was categorized according to the gas and detector conditions at the time the data was recorded [216]. Three sets of $\mu^{+}$data were ultimately used to obtain a lifetime result: Prod-50, Prod-48, and CalibD2-48, where "Prod" refers to the production data collection period when the TPC was filled with clean hydrogen gas, "CalibD2" refers to the $\approx 22$ ppm-deuterium-doped hydrogen gas fill, and the labels " 50 " and " 48 " refer to TPC voltages of $5.0 \mathrm{kV}$ and $4.8 \mathrm{kV}$, respectively. In the data analysis, the Prod-48 and CalibD2-48 data sets were combined into a single group, "All-48," which was studied separately from the Prod-50 data. The two lifetime results were then combined, as will be discussed below.

Before describing the analysis of the Run $8 \mu^{+}$data, it is worth examining the 
behavior of positive muons in the experimental setup. The arriving beam of positive muons was (partially) polarized in the direction of propagation, in contrast to the negative muons, which were (partially) polarized opposite to their momentum vector. As mentioned earlier in Section 3.5, a significant fraction of the $\mu^{+}$retain their initial polarization after stopping in the hydrogen target. Consequently, the $\mu^{+}$spins will proceed to precess if an ambient magnetic field is present, and this behavior can introduce time-dependent effects that distort the lifetime measurement. In order to exert some control over the $\mu^{+}$precession, we installed a saddle-coil magnet (see Section 4.1) that suffused the interior of the pressure vessel with a nearly uniform, $\approx 50$ Gauss magnetic field, oriented transverse to the $\mu^{+}$spins. The magnetic field induced an $\approx 1.5 \mu$ s-period spin rotation $\left(\mu^{+} \mathrm{SR}\right)$ in the "free" $\mu^{+}$that did not form muonium $(\mathrm{Mu})$. The orientations of the $\mu^{+}$spins and the $\vec{B}$ field in the experimental setup are illustrated in Figure 7.3.

As described earlier in Section 3.5, a muon's decay electron is preferentially emitted in the direction of the muon's spin. As a result, the free $\mu^{+}$spin rotation depicted in Figure 7.3 creates a "lighthouse" effect, that is, a sinusoidal modulation of the lifetime spectrum observed in any particular eSC segment. The effect is most pronounced in the eSC gondolas at the top and bottom of the detector, which are oriented perpendicular to the spin rotation plane.

The Run8 $\mu^{+}$data was analyzed primarily by Dr. Frederick Gray, in the following manner:

1. Positive muon lifetime spectra were created using essentially the same criteria as for the $\mu^{-}$lifetimes (see Section 6.6.1), but with some notable exceptions. First, only the ePC cathode-OR spectra were considered in the $\mu^{+}$analysis, because there was no evidence of any systematic difference in the electron detector treatments. Second, the standard, rectangular fiducial TPC cut proved to be unacceptable, due to the sizable 


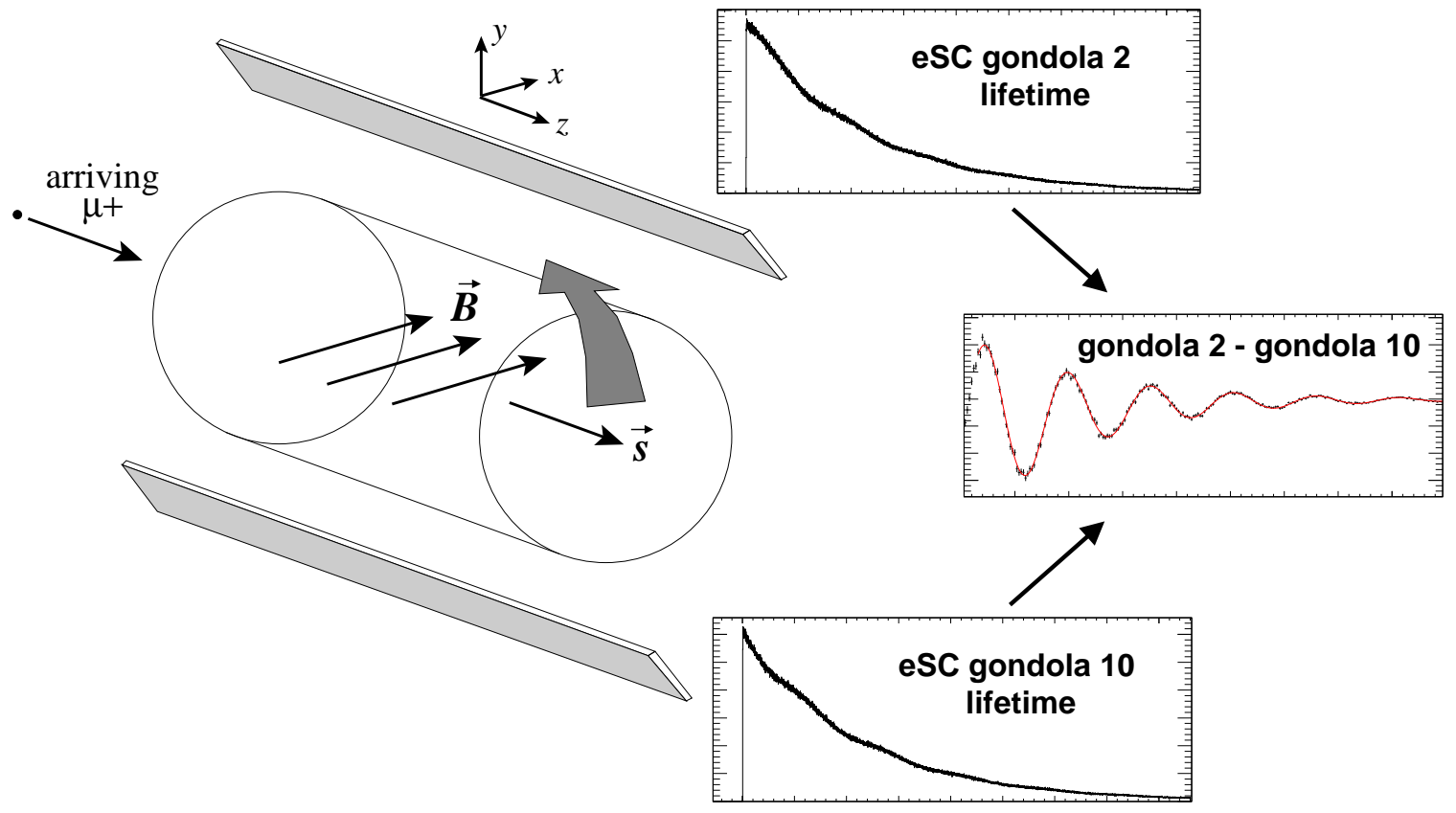

Figure 7.3: Diagram of $\mu^{+}$spin rotation geometries in the MuCap apparatus. When a muon stops inside the pressure vessel, its spin $\vec{s}$ experiences a torque $\vec{\tau}=\vec{\mu} \times \vec{B}$, where $\vec{\mu}=$ $\left(\mathrm{ge} / 2 m_{\mu}\right) \vec{s}$ is the muon's magnetic moment and $\vec{B}$ is the uniform magnetic field generated by the saddle-coil magnet. For a positive muon, $g$ and $e$ are both greater than zero, so $\vec{\tau} \propto \vec{s} \times \vec{B}$. Using this relationship, combined with (1) the knowledge that the arriving $\mu^{+}$beam is polarized in the downstream direction, and (2) the $\mu^{+} \mathrm{SR}$ oscillation phases observed in the upper and lower eSC gondolas, we can deduce that our applied, transverse, $\approx 50$ Gauss magnetic field pointed in the ${ }^{+} x$ direction, and that the spin precession direction is as indicated above. It is worth noting that the data confirm the precession frequency calculations in Appendix F, which predict that the $\mathrm{Mu}$ triplet and the free $\mu^{+}$rotations are in opposite directions; compare, for example, the free $\mu^{+}$phase in gondola 10 above with the $\mathrm{Mu}$ triplet phase in the neighboring gondola 9 in Figure 3.7. 


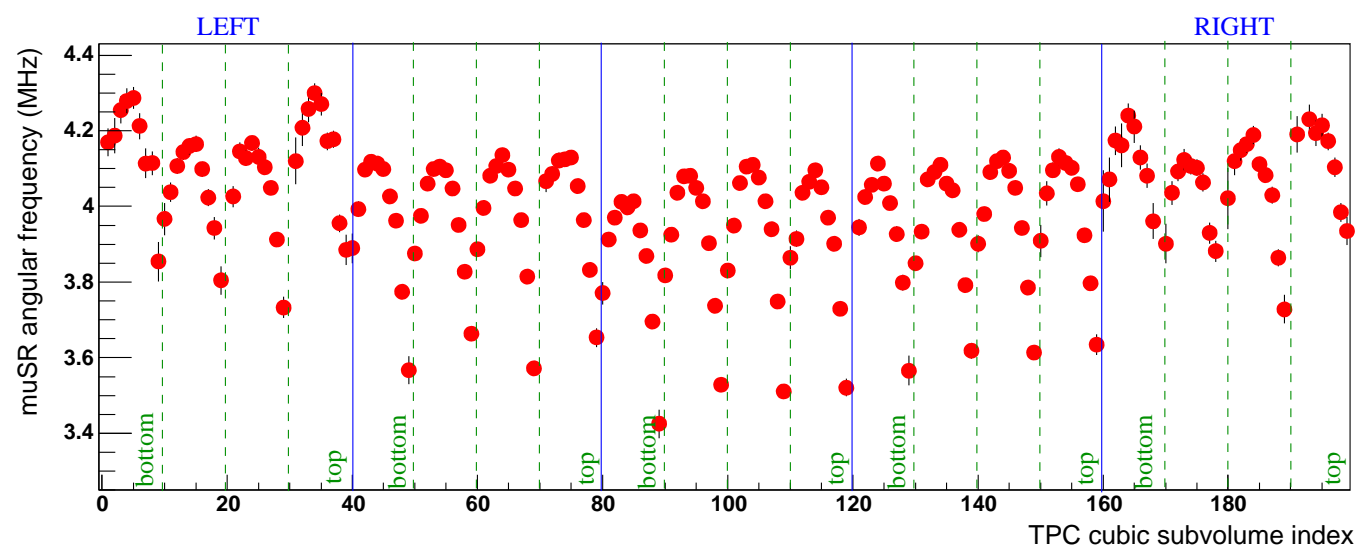

Figure 7.4: The $\mu^{+} \mathrm{SR}$ angular frequencies observed for $\mu^{+}$stops in different regions of the TPC. For this study, the TPC volume was divided into $200(30 \mathrm{~mm})^{3}$ cubic subvolumes, which corresponds to five divisions in the $x$ dimension, four divisions in the $y$ dimension, and ten divisions in the $z$ dimension. In the plot above, the bottom axis corresponds to the cubic subvolume index, which varies fastest in $z$, then $y$, then $x$. The $\mu^{+} \mathrm{SR}$ frequencies vary significantly across the TPC volume due to nonuniformities in the magnetic field generated by the saddle-coil magnet.

variation in the magnitude of the magnetic field over the TPC volume, especially in the $z$ direction (Figure 7.4). These magnetic field nonuniformities produce variations in the $\mu^{+} \mathrm{SR}$ frequencies, which in turn can lead to instabilities in the overall fitted rate. To avoid this problem, Dr. Gray implemented a cylindrical cut on the muon stopping point, consisting of a fiducial volume of dimensions $r=\sqrt{x^{2}+y^{2}}<60 \mathrm{~mm}$ and $60 \mathrm{~mm}<z<120 \mathrm{~mm}$, where the $z$ coordinate here is defined relative to the upstream end of the TPC's 300-mm-length sensitive volume. The radial cut excludes the problematic edges which contain the outer magnetic field regions, and thereby reduces the field nonuniformity from $\approx 15 \%$ to $\approx 3 \%$.

2. For each diametrically opposed pair of eSC gondolas (e.g. gondolas 1 and 9, 2 and 10, etc.), Dr. Gray formed the difference between the two gondolas' lifetime spectra. This subtraction eliminates most of the underlying exponential structure from decay, 
and thereby largely isolates the $\mu^{+} \mathrm{SR}$ signals (see Figure 7.3).

3. Each of the eight differential lifetime spectra was fit with the function

$$
f(t)=N \lambda e^{-\lambda t}\left\{1-P(t)\left[A_{\mathrm{c}} \cos \left(\omega_{\mu \mathrm{SR}}\right)+A_{\mathrm{s}} \sin \left(\omega_{\mu \mathrm{SR}}\right)\right]\right\}+B
$$

which is similar to the simple exponential function in Equation 6.2 that is used to fit $\mu^{-}$spectra, but is here modified to describe $\mu^{+} \mathrm{SR}$ modulations to the decay signal. The parameters in square brackets describe the sinusoidal $\mu^{+} \mathrm{SR}$ behavior, while the function $P(t)$ describes the attenuation in the $\mu^{+}$polarization. The form of $P(t)$ depends in large part on the uniformity of the surrounding magnetic environment, and Dr. Gray found that both exponential and Gaussian envelopes, $P_{\exp }(t)=e^{-t / \tau_{\mu \mathrm{SR}}}$ and $P_{\text {Gauss }}(t)=e^{-t^{2} / 2 T_{\mu \mathrm{SR}}^{2}}$, serve as good approximations. All of the following results were obtained using the Gaussian form because it provided slightly more stable results, but the choice of parameterization ultimately proved to have little effect on the final $\mu^{+}$ lifetime measurement.

Equation 7.12 was first applied to extract the spin-related parameters $\omega_{\mu \mathrm{SR}}$ and $T_{\mu \mathrm{SR}}$ from the difference spectrum of each opposing gondola pair. In this case, the lifetime $\lambda$ was held fixed at a reasonable value, and the fit range was restricted to the interval $[320,15000]$ ns. The delayed fit start time is necessary to avoid fit instabilities arising from the fast muonium oscillations which exist at early times (see Figure 3.7). The fits yielded free $\mu^{+}$angular precession frequencies in the vicinity of $\omega_{\mu \mathrm{SR}}=4.08 \mathrm{MHz}$, or $\nu_{\mu \mathrm{SR}}=\omega_{\mu \mathrm{SR}} / 2 \pi \approx 650 \mathrm{kHz}$, which is in good agreement with the frequency expected in our 50 Gauss magnetic field (see Appendix F).

4. Weighted averages for the $\mu^{+} \mathrm{SR}$ parameters, $\omega_{\mu \mathrm{SR}}$ and $T_{\mu \mathrm{SR}}$, were obtained by fitting a constant to the eight lifetime fit results from the preceding step. The gondolas at the top and bottom of the eSC provide better constraints on the $\mu^{+} \mathrm{SR}$ parameters 
than the gondolas at the sides, due to the fact that the spin rotation plane is vertically oriented.

5. The $\mu^{+} \mathrm{SR}$ frequency $\omega_{\mu \mathrm{SR}}$ and the envelope time constant $T_{\mu \mathrm{SR}}$ were then fixed at their averaged values, and each of the sixteen eSC lifetime spectra were fit with the function in Equation 7.12, thus yielding sixteen values for $\lambda=\lambda_{\mu^{+}}$. The fit range used was $[320,24500] \mathrm{ns}$, which is slightly narrower than the standard $\mu^{-}$fit range described in Section 6.5.5, due to the need to avoid the fast muonium oscillations at early times; the resulting loss in statistical precision is minor.

6. The sixteen $\lambda_{\mu^{+}}$values were fit with a constant to obtain a single, effective lifetime.

7. The effective $\mu^{+}$lifetimes from the Prod-50 and the All-48 data sets were combined into a single lifetime using a weighted average. It is worth mentioning that the lifetimes of the Prod-50 and All-48 data sets were statistically consistent.

8. Errors were added to the final result to cover systematic uncertainties.

Dr. Gray's analysis of $0.5 \times 10^{9} \mu^{+}$total decay events in the Run8 data yielded [233]

$$
\lambda_{\mu^{+}}^{\mathrm{MuCap}}=455,164 \pm 25_{\text {stat }} \pm 13_{\text {syst }} \mathrm{s}^{-1}
$$

This result is consistent with the latest world average for the $\mu^{+}$lifetime, $455,162.2 \pm 4.4 \mathrm{~s}^{-1}[49]$, and therefore provides some reassurance regarding the trustworthiness of our $\mu^{-}$measurement. The systematic error in Equation 7.13 is dominated by uncertainties related to the accidental background of the $\mu^{+}$lifetime spectra. First, the $120 \mathrm{~mm}$ impact cut reduced the background levels by a factor of three, and lowered the fitted rate $\lambda$ by $10-15 \mathrm{~s}^{-1}$, which is larger than what is statistically allowed. Second, the fitted background $B$ exhibited a marked time-dependent structure in fit stop time scans to the individual gondolas' lifetime spectra. However, the background structure disappears if 


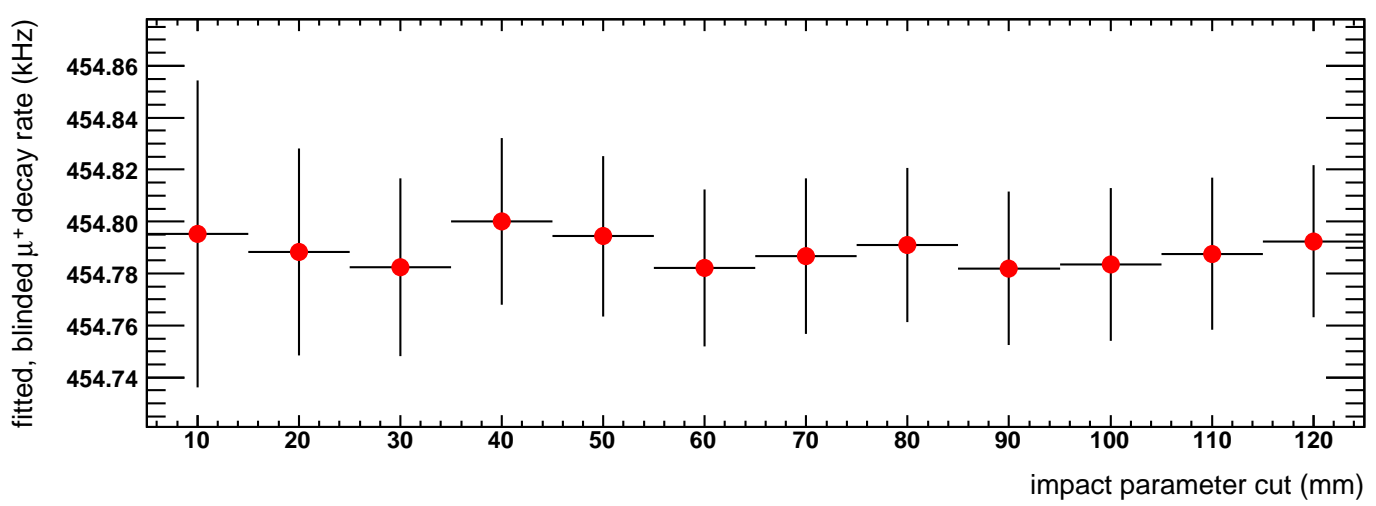

Figure 7.5: Fitted $\mu^{+}$decay rate vs. impact parameter cut. The $\lambda_{\mu^{+}}$values here are doubly blinded by the DAQ clock offset and Dr. Frederick Gray's own secret offset, which is why they differ in magnitude from the result in Equation 7.13. The most important feature here is that the distribution of points is flat, and does not exhibit the structure observed in similar plots involving negative muons (see Figures 6.30 and 6.63).

the sixteen eSC spectra are combined prior to fitting (the fitted rate is unaffected). Nevertheless, we chose to retain the sizable background-related systematic error in Equation 7.13 to conservatively encompass any other $\mu^{+}$uncertainties, a detailed breakdown of which was obviated by the large statistical error.

It is worth mentioning that the $\mu^{+}$decay rate does not exhibit the same impact parameter dependence that appears in the $\mu^{-}$disappearance rates (see Figure 6.63). Instead, the $\mu^{+}$decay rate is a uniform function of impact parameter cut, as illustrated in Figure 7.5. The lack of any apparent structure in Figure 7.5 supports our hypothesis that the diffusion of free $\mu^{+}$in the TPC drift field is small in comparison to $\mu p$ diffusion (see Section 3.5). If there is in fact any diffusion-related structure in Figure 7.5, it is obscured by the statistical error. It should be noted that, given the absence of any impact-cut-generated structure in the $\mu^{+}$decay rates, we cannot explain why imposing the $120 \mathrm{~mm}$ impact cut significantly lowers the $\mu^{+}$decay rate from its no-cut value. 


\section{Chapter 8}

\section{Conclusions}

In this chapter I describe how the $\Lambda_{S}$ values obtained by the Berkeley and Illinois analyses of the 2004 data are reconciled into a single result. I also describe the ensuing extraction of the nucleon's pseudoscalar coupling $g_{P}$, and I discuss how its value compares to previous theoretical and experimental determinations. I conclude by listing several outstanding analysis issues, and by examining what the future holds for the MuCap experiment.

\subsection{Comparison of Berkeley and Illinois results for $\Lambda_{S}$}

The $\Lambda_{S}$ values obtained from the 2004 data by the Berkeley and Illinois analysesthat is, by myself and Steven Clayton, respectively - are presented in Table 8.1. There I include our 120-mm-impact-cut results only, in accordance with the policy decided upon by the collaboration at the October 2006 unblinding meeting (see Section 7.2.3).

Table 8.1 shows that Mr. Clayton and I both observe variations in our $\Lambda_{S}$ values according to the electron detector treatment method, although the difference between my values is somewhat larger than his. Moreover, our average $\Lambda_{S}$ values are different: my

numbers average to $727.7 \mathrm{~s}^{-1}$, while Mr. Clayton's average to $722.3 \mathrm{~s}^{-1}$. The exact reasons 


\begin{tabular}{llccc}
\hline \hline Analysis & ePC treatment & Impact cut & $\Lambda_{S}\left(\mathrm{~s}^{-1}\right)$ & $\sigma_{\Lambda_{S}}\left(\mathrm{~s}^{-1}\right)$ \\
\hline \multirow{2}{*}{ Berkeley } & cathode-AND & $120 \mathrm{~mm}$ & 723.44 & 17.27 \\
& cathode-OR & $"$ & 731.98 & 16.91 \\
\multirow{2}{*}{ Illinois } & cathode-AND & $"$ & 719.09 & 16.19 \\
& cathode-OR & $"$ & 725.42 & 15.89 \\
\hline \hline
\end{tabular}

Table 8.1: The $\Lambda_{S}$ results obtained from the Berkeley and Illinois analyses of the 2004 data.

for these discrepancies remain unknown. The differences in our $\Lambda_{S}$ values are somewhat larger than the statistically allowed deviation of $\approx 2 \mathrm{~s}^{-1}$ that comes from naïvely applying Equation 6.7 to our fitted disappearance rates $\lambda$. Given the sizable overlap in our data sets, the culprit(s) likely lie somewhere in the details of our respective analyses. We know of a few potential candidates, though the following list is by no means exhaustive:

- When clustering the raw data from the ePC planes, Mr. Clayton employs an updating artificial deadtime, whereas I employ a non-updating deadtime.

- Mr. Clayton uses a lower threshold than I do for identifying ePC clusters as "spark" events: his threshold is $\approx 10$ wires, whereas mine is $\approx 100$.

- I perform spark searches using the raw ePC data, whereas Mr. Clayton performs his spark search after clustering the data in the ePC planes.

- Mr. Clayton dynamically masks "hot" ePC wires; that is, if the number of hits on a wire within a certain time interval exceeds some threshold, he masks the data from the offending wire for the remainder of the run file.

- Following a spark event in the detectors, I impose an artifical deadtime over the interval $[-5,+50] \mu$ s relative to the spark event, whereas Mr. Clayton imposes an artifical deadtime of $\pm 25 \mu \mathrm{s}$. 
- Mr. Clayton incorporates $z$ information from the eSC into his electron identification algorithm; I do not.

- As discussed in Section 6.5.1, I adopt an "approval" approach towards identifying decay electrons, in which I do not make any judgments about the quality of certain electron tracks compared to others involving the same data. In contrast, when presented with multiple possible pairings from the same electron detector data, Mr. Clayton selects one to the exclusion of all others, based upon subjective criteria.

- Mr. Clayton also applies a subjective selection further upstream when processing the electron detector data, namely, when forming three-plane ePC coincidences.

In some sense, it is reassuring that the Berkeley and Illinois results for $\Lambda_{S}$ are as close as they are, given the numerous differences in the details of our analyses; it suggests that the results are relatively robust. Unfortunately, it is extremely difficult to ascertain what effects the analysis differences listed above might have on the final results. To do so would require significant effort, which is not really called-for given the relatively small scale of the $\Lambda_{S}$ differences.

We must somehow reconcile the four slightly inconsistent $\Lambda_{S}$ results in Table 8.1 into a single number suitable for publication by the collaboration. To obtain a central value, we simply average the $\Lambda_{S}$ values to get $725.0 \mathrm{~s}^{-1}$. Determining the corresponding error $\sigma_{\Lambda_{S}}$ is somewhat trickier, both because of the different paths leading to the four results, and because the errors are largely correlated owing to the common data set. To arrive at an "average" error, Dr. Peter Kammel computed an average $\sigma$ for each step in the analyses leading to the final results [234]. Furthermore, he introduced an additional error term, $\sigma_{\text {consistency }}$, to account for the uncertainty due to the analysis-related variations in Table 8.1. To determine $\sigma_{\text {consistency }}$, he first averaged the four disappearance rates $\lambda$ leading 
to the $\Lambda_{S}$ values in Table 8.1, but just prior to the unblinding stage. He next calculated the $\lambda$ deviations from that average, $\delta_{i}=\left|\lambda_{i}-\lambda^{\text {avg }}\right|$, and then calculated $\sigma_{\text {consistency }}$ according to

$$
\sigma_{\text {consistency }}=\sqrt{\frac{\delta_{1}^{2}+\delta_{2}^{2}+\delta_{3}^{2}+\delta_{4}^{2}}{3}}=5.0 \mathrm{~s}^{-1}
$$

This procedure avoids the awkward protocol that was originally proposed at the unblinding meeting, which was to partially use the no-impact-cut $\Lambda_{S}$ results by only allowing them to contribute to the final error. Moreover, although this procedure does not explicitly incorporate the no-impact-cut numbers into the final result ${ }^{1}$, they are nevertheless partially covered by the generous $\sigma_{\text {consistency }}$ error. Adding the consistency error in quadrature with the averaged step-by-step errors, Dr. Kammel ultimately calculated a final uncertainty of $\sigma_{\Lambda_{S}}=17.4 \mathrm{~s}^{-1}[234]$.

The MuCap Collaboration's final result for $\Lambda_{S}$ from the 2004 data set is therefore

$$
\Lambda_{S}^{\mathrm{MuCap}}=725.0 \pm 17.4 \mathrm{~s}^{-1}=725.0 \pm 13.7_{\text {stat }} \pm 10.7_{\text {syst }} \mathrm{s}^{-1}
$$

A report on this measurement has been accepted for publication in Physical Review Letters [7]. To compare Equation 8.1 with theory, we average the two relatively recent NNLO chiral perturbation theory calculations of $\Lambda_{S}, 687.4 \mathrm{~s}^{-1}$ [82] and $695 \mathrm{~s}^{-1}$ [67], to obtain $691.2 \mathrm{~s}^{-1}$. Applying the very recently calculated multiplicative radiative correction factor of 1.028 [8] yields $\Lambda_{S}^{\text {theory }}=710.6 \mathrm{~s}^{-1}$. This modified chiral prediction is close to the radiative-corrected phenomenological result $713 \pm 4 \mathrm{~s}^{-1}$ obtained in reference [8]. Our experimental result for $\Lambda_{S}$ in Equation 8.1 is thus consistent within $1 \sigma$ with theoretical predictions, provided that the latest radiative corrections in [8] are applied.

\footnotetext{
${ }^{1}$ It is worth noting that including the no-impact-cut numbers would lower the $\Lambda_{S}$ average by $\approx 2.5 \mathrm{~s}^{-1}$.
} 


\subsection{Determination of $g_{P}$}

The extraction of the pseudoscalar coupling $g_{P}$ from the MuCap result for $\Lambda_{S}$ in Equation 8.1 depends to some extent upon the model which is used and its input values, just as is the case with the extraction of $\Lambda_{S}$ from the $\mu^{-}$disappearance rate in hydrogen. We elected to perform a linear expansion around the value for $g_{P}$ predicted by chiral perturbation theory,

$$
g_{P}^{\text {MuCap }}=g_{P}^{\text {theory }}+\frac{\partial g_{P}}{\partial \Lambda_{S}}\left(\Lambda_{S}^{\text {MuCap }}-\Lambda_{S}^{\text {theory }}\right)=7.3 \pm 1.1
$$

where $g_{P}^{\text {theory }}=8.26[23], \frac{\partial g_{P}}{\partial \Lambda_{S}}=-0.065 \mathrm{~s}[9], \Lambda_{S}^{\text {theory }}=710.6 \mathrm{~s}^{-1}$ (as calculated at the end of the preceding section), and only the experimental uncertainty in $\Lambda_{S}^{\mathrm{MuCap}}$ from Equation 8.1 is propagated. The linear approximation in Equation 8.2 is valid because of the relatively small difference between $\Lambda_{S}^{\mathrm{MuCap}}$ and $\Lambda_{S}^{\text {theory }}$. There has been recent, unpublished speculation that $\frac{\partial g_{P}}{\partial \Lambda_{S}}$ is actually closer to $-0.070 \mathrm{~s}$ [235], in which case the extracted value of $g_{P}$ would drop slightly to 7.2. It should be mentioned that the authors of reference [8] extracted the same value for $g_{P}$ from $\Lambda_{S}^{\mathrm{MuCap}}$ as obtained in Equation 8.2, but using a phenomenological formula for muon capture, and with the slightly different input values $g_{P}^{\text {theory }}=8.2$ and $\Lambda_{S}^{\text {theory }}=711.5 \mathrm{~s}^{-1}$.

We have deliberately tried to sidestep the ongoing theoretical debate surrounding $g_{P}$ as much as possible by presenting a transparent determination of $g_{P}$ which makes use of noncontroversial numbers. The result in Equation 8.2 might need to be refined once further theoretical work clarifies the present $1 \%$ difference between the chiral calculations in [67] and [82] and perhaps resolves the outstanding dispute regarding the compatibility of the chiral and phenomenological approaches [23]. It is hoped that all sources of theoretical uncertainty will soon be quantified at the sub-percent level, in which case theory would become competitive with the anticipated precision of future MuCap results. 


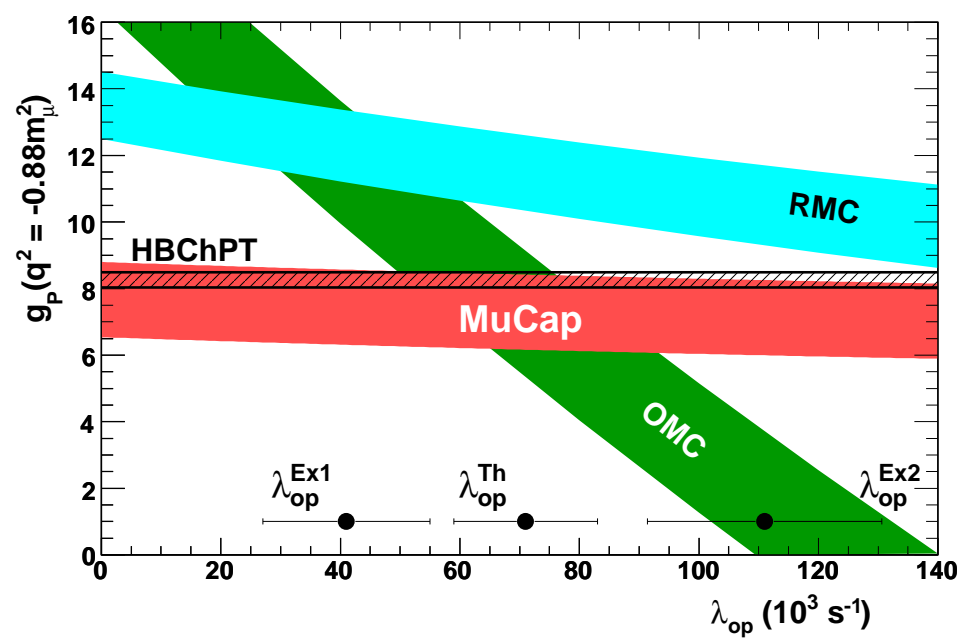

Figure 8.1: Experimental and theoretical determinations of $g_{P}$, presented vs. the ortho-para transition rate $\lambda_{\text {op }}$ in the $p \mu p$ molecule. The most precise previous OMC experiment [42] and the lone RMC experiment [44] both depend significantly on the value of $\lambda_{\mathrm{op}}$, which itself is poorly known due to mutually inconsistent experimental $\left(\lambda_{\mathrm{op}}^{\operatorname{Ex} 1}[43], \lambda_{\mathrm{op}}^{\operatorname{Ex} 2}\right.$ [51] $)$ and theoretical $\left(\lambda_{\mathrm{op}}^{\mathrm{Th}}[52]\right)$ results. In contrast, the MuCap result for $g_{P}$ is nearly independent of molecular effects. In principle, the half-width of the MuCap error band above should be smaller than the error on the $g_{P}$ determination in Equation 8.2, since the latter number is essentially a projection of the band onto the $y$ axis. However, both errors are given as 1.1, because the result in Equation 8.2 has been rounded down from 1.13.

The current information on $g_{P}$ is summarized in Figure 8.1. There we have updated the constraints [5] from the Saclay OMC experiment [42] to reflect the larger $\Lambda_{S}$ which includes the latest radiative corrections [8]. The situation prior to MuCap was inconclusive, due to the existence of mutually inconsistent theoretical predictions and experimental determinations of both $g_{P}$ and $\lambda_{\mathrm{op}}$. The low gas density in MuCap renders our result relatively insensitive to the ortho-para transition rate $\lambda_{\text {op }}$ in the $p \mu p$ molecule and thus avoids most model dependence, enabling us to report the first precise, unambiguous determination of $g_{P}$. The experimental result in Equation 8.2 agrees with present theory to within $1 \sigma$ and does not support the dramatic deviation from the chiral prediction that the RMC result originally had implied. 


\subsection{Remaining questions and possibilities}

Below is a list of unresolved issues from the Run8 analysis, in roughly decreasing order of importance.

- We have accumulated a wealth of experimental information about $Z>1$ impurities in our hydrogen gas, but much of the data is inconsistent. Despite our best efforts, it has proven difficult to reconcile the fits to the lifetime spectra, the fits to the capture time distributions, the TPC capture yields, the external gas chromatography measurements, and the humidity sensor measurements into a coherent picture. If some of these mysteries could be resolved, the uncertainty of the $Z>1$ correction could almost certainly be reduced.

- The reasons for the larger-than-statistical differences between my fitted rates for the eSC-only, cathode-AND, and cathode-OR electron detector treatments remain unknown. (See Section 6.5.15.)

- The reasons for the larger-than-expected difference between the 120-mm-impact-cut fitted rates and the no-impact-cut fitted rates remain unknown. It is not clear if the $120 \mathrm{~mm}$ impact cut cleans up and improves the decay signal, or if it introduces some as-yet unknown effect.

- The Berkeley and Illinois analyses report significantly different fitted rates and $Z>1$ capture yields for the Run8 CalibN2 and CalibNat gas fills, which in turn affects the agreement of our corrected Prod-50 rates. We know that there is a small difference in our uncorrected Prod-50 fitted disappearance rates because of the fact that I included data from the period when CHUPS was turned off and outgassing was observed in the TPC system, whereas Steven Clayton omitted this data; however, this difference is 
likely rectified by my larger $Z>1$ correction. It is possible that the difference in our CalibNat rates could be due in part to a similar effect: namely, Mr. Clayton included files from near the end of that data collection period, when the TPC exhibited a reduced operating voltage of $4.6 \mathrm{kV}$ ("CalibNat-46"). It is also conceivable that some fraction of the observed discrepancies are simply related to differences in our analysis choices, and are therefore resolved in a self-consistent manner through the impurity corrections. However, these mechanisms seem insufficient in explaining the size of our discrepancies, as described in reference [201].

- The analysis could benefit from a better understanding and characterization of the entrance muon detector inefficiencies and the resulting effects.

- There has been recent speculation that the EH pixel signature of $\mu+p$ scatter events might be distinguishable from the EH pixel signature of good muon stops. Whether or not such discrimination is practicable depends upon the TPC threshold settings and the topological overlap between the two types of events in the TPC. A differential lifetime analysis of the EH topologies is needed to empirically establish or disprove the viability of such discrimination.

- A better understanding of free $\mu^{+}$drift in the TPC and resulting effects is desirable.

- Further development of the method for fitting with a more descriptive function is warranted. Ultimately, the sophistication of the procedure should be comparable to the well-studied and established exponential fit approach.

- It has been observed that the $Z>1$ capture yield varies in roughly linear fashion as a function of $y$ position in the TPC. In fact, the capture yield at the bottom of the $\mathrm{TPC}$ is $\approx 25 \%$ higher than at the top of the TPC. We do not know what is reponsible 
for the nonuniformity.

- The TPC drift distribution exhibits two features that are not well understood: First, the sharp peak corresponding to the MWPC region is twice as wide as expected (see Section 6.3.3). Second, there is a small tail in the accidental background on the right-hand side of the pileup-protected TPC drift distribution, between the central coincidence peak and the pileup background (see the rightmost plot in Figure 6.46.)

- We have demonstrated that throughgoing cosmics are responsible for roughly half of the accidental background observed in the lifetime spectra (see Section 6.5.6), but we do not know exactly what comprises the other half. Noise, inefficiencies in the muon entrance detectors, and radioactivity are probable contributors.

- A better understanding of the magnitude and phase of the $50 \mathrm{MHz}$ oscillations in the lifetime spectra is desirable. There is some indication that the oscillations in the pileup background and signal are slightly different from the oscillations in the accidental background, perhaps due to separate effects from the CAENs and the beam RF?

- It would be reassuring if we could more conclusively demonstrate the absence of any time-dependent CAEN effects.

It should be noted that, in Berkeley's case, future analyses would benefit greatly from improvements to our infrastructure for large-scale data analysis. The existing arrangement, in which data is analyzed on computers at PSI and managed remotely from California, suffers from numerous limitations which hinder analysis progress: resources are limited, data access is slow and unwieldy, and remote troubleshooting is inherently difficult. A more powerful and accessible setup would provide faster turnaround time for analysis results, and thus enable more rapid and efficient exploration of the remaining analysis questions. 


\subsection{Outlook}

At the time of this writing, the data collected in Run9 (winter 2005) and Run10 (summer 2006) is being analyzed by Brendan Kiburg of UIUC. Altogether, more than $10^{10}$ pileup-protected $\mu^{-}$decay events were accumulated during those two runs, under cleaner hydrogen gas conditions than in Run8, and with a higher TPC MWPC voltage of $5.35 \mathrm{kV}$. As a result of these respective improvements, the effects of impurities should be reduced, and $\mu+p$ scatter events should be more readily identifiable - although it should be noted that with increased TPC sensitivity comes an increased threat in time-dependent effects from decay electrons.

The accumulation of high statistics in Runs 9 and 10 was facilitated by the introduction of an electrostatic muon "kicker" into the beamline. When operated in MuonOn-REquest (MORE) mode, the kicker offers the capability of injecting muons into the experimental apparatus one at a time. This is a more efficient method of obtaining pileupprotected muons, and thus enables the rapid accumulation of usable data. The effective accumulation rate of good, pileup-protected decay events in Runs 9 and 10 was roughly $5.5 \mathrm{kHz}$, more than a factor-of-two improvement over the effective Run8 event rate of $2.5 \mathrm{kHz}[236]$.

In Run9, roughly $2 \times 10^{9}$ events were collected in hydrogen gas which had the same $1.5 \mathrm{ppm}$ deuterium content as in Run8, but improvements to CHUPS produced a slightly lower equilibirum $Z>1$ capture yield of $\approx 7 \mathrm{ppm}$, down from $\approx 13 \mathrm{ppm}$ in Run8. In Run10, approximately $8.5 \times 10^{9}$ decay events were collected in gas containing sub-100ppb deuterium concentration, which was made possible by the introduction of an isotopic separation column; the Run10 equilibrium $Z>1$ capture yield was comparable to that in Run9. Because $Z>1$ impurities continue to be a source of concern, extensive calibration 
studies were performed in Runs 9 and 10 in an effort to better characterize the composition and effects of the residual elemental impurities. In particular, a humidity sensor installed in Run9 confirmed that the dominant impurity was oxygen in the form of water, and numerous studies were performed in Run10 to study the correlation between the humidity level and the TPC capture yield.

At present, there are plans to conduct a final physics run, Run11, in fall 2007. It will be devoted to additional impurity calibration studies, and possibly to measurements of the molecular rates $\lambda_{\text {of }}$ and $\lambda_{\mathrm{op}}$, in order to reduce the large systematic error related to $p \mu p$ effects.

The statistics collected in Runs 9 and 10 should result in a more than twofold reduction in the statistical error for $\Lambda_{S}$. It is hoped that a similar reduction in the systematic uncertainty will result from continued analysis of the existing and forthcoming data, as we gain a better understanding of the systematics and perhaps resolve some of the outstanding analysis questions listed in Section 8.3. The final determination of $\Lambda_{S}$ is expected to be within the design goal of $1 \%$ precision. 


\section{Appendix A}

\section{Muon capture kinematics}

In order to calculate the characteristic momentum transfer $q_{0}$ of ordinary nuclear muon capture, $\mu+p \rightarrow n+\nu_{\mu}$, it is necessary to consider momentum and energy conservation in turn. First, we can regard the $\mu p$ bound system as being at rest, in which case $\vec{p}_{i}=\vec{p}_{f}=0$. Consequently, the momenta of the final-state particles must be equal and oppositely directed, $\vec{p}_{n}=-\vec{p}_{\nu}$. Since the neutrino is effectively massless, $\left|\vec{p}_{n}\right|=\left|\vec{p}_{\nu}\right|=E_{\nu}$.

Applying the results above to energy conservation gives:

$$
\begin{aligned}
E_{i} & =E_{f} \\
E_{\mu}+E_{p} & =E_{n}+E_{\nu} \\
m_{\mu}+m_{p} & =\sqrt{m_{n}^{2}+\vec{p}_{n}^{2}}+E_{\nu} \\
m_{\mu}+m_{p}-E_{\nu} & =\sqrt{m_{n}^{2}+E_{\nu}^{2}} \\
\left(m_{\mu}+m_{p}-E_{\nu}\right)^{2} & =m_{n}^{2}+E_{\nu}^{2} \\
2 E_{\nu}\left(m_{\mu}+m_{p}\right) & =\left(m_{\mu}+m_{p}\right)^{2}-m_{n}^{2} \\
E_{\nu} & =m_{\mu}\left[\frac{\left(m_{\mu}+m_{p}\right)^{2}-m_{n}^{2}}{2\left(m_{\mu}^{2}+m_{\mu} m_{p}\right)}\right] \\
& \approx m_{\mu}\left[1-\frac{m_{\mu}}{2 m_{p}}\right] .
\end{aligned}
$$


Plugging in the particle masses $m_{\mu}=105.658 \mathrm{MeV}, m_{p}=938.272 \mathrm{MeV}$, and $m_{n}=$ 939.566 MeV, we find

$$
E_{\nu} \approx m_{\mu}(0.938379)
$$

Thus, in order to conserve momentum, the neutrino receives most of the muon mass energy which is liberated by capture. From this result we can calculate the characteristic momentum transfer, which is illustrated in the Feynman diagram in Figure 1.1:

$$
\begin{aligned}
q_{0} & =p_{\mu}-p_{\nu}\left(=p_{n}-p_{p}\right) \\
q_{0}^{2} & =p_{\mu}^{2}+p_{\nu}^{2}-2 p_{\mu} \cdot p_{\nu} \\
& \approx p_{\mu}^{2}-2 p_{\mu} \cdot p_{\nu} \\
& =m_{\mu}^{2}-2 m_{\mu} E_{\nu} \\
& =m_{\mu}^{2}(1-2(0.938379)) \\
& \approx-0.88 m_{\mu}^{2} .
\end{aligned}
$$

Because the momentum transfer in ordinary muon capture is fixed, the outgoing neutrons are monoenergetic with a kinetic energy of $\varepsilon_{n} \approx 5.2 \mathrm{MeV}$, which is an important fact for experiments that measure the capture rate by detecting final-state neutrons. It is straightforward to calculate $\varepsilon_{n}$ from the relativistic kinematics:

$$
\begin{aligned}
\epsilon_{n} & =E_{n}-E_{n}^{\mathrm{rest}} \\
& =\sqrt{m_{n}^{2}+\vec{p}_{n}^{2}}-m_{n} \\
& =\sqrt{m_{n}^{2}+E_{\nu}^{2}}-m_{n} \\
& \approx 5.2 \mathrm{MeV} .
\end{aligned}
$$




\section{Appendix B}

\section{Muon capture calculations}

Out of a desire to better understand the underlying theory of muon capture in hydrogen, I calculated a phenomenological expression for the $\mu p$ hyperfine singlet capture rate $\Lambda_{S}=\Lambda_{S}\left(g_{V}, g_{M}, g_{A}, g_{P}\right)$ from first principles, using time-dependent perturbation theory. My work, described in detail in reference [78], was guided in large part by the calculations outlined by Dr. E. D. Commins in references [237] and [238]. Due to space considerations I will not reproduce my own calculations here in full. Instead, I present only my final expression for $\Lambda_{S}$,

$$
\begin{aligned}
\Lambda_{S}=\frac{G_{F}^{2} V_{u d}^{2} C \hbar^{2} E_{\nu}^{2}}{2 c^{5} \pi^{2} a_{\mu}^{3}\left(1+E_{\nu} / \sqrt{m_{n}^{2}+E_{\nu}^{2}}\right)}\left[g_{V}\left(1+3 \frac{E_{\nu}}{2 m_{N}}\right)+g_{M}\left(2 E_{\nu}\right)\right. \\
\left.+g_{A}\left(3+\frac{E_{\nu}}{2 m_{N}}\right)+g_{P}\left(\frac{E_{\nu}}{2 m_{N}}\right)\right]^{2},
\end{aligned}
$$

which corresponds to a tree-level treatment of muon capture (Figure 1.1) that does not include radiative corrections (see Section 2.4). Many of the quantities in Equation B.1 are either self-evident or have already been defined in Section 1.1 or Appendix A, but others require some explanation. The quantity $a_{\mu}$ is the Bohr radius of the ground state of muonic 
hydrogen, and is proportional to the Bohr radius of electronic hydrogen:

$$
a_{\mu}=a_{e}\left(\frac{m_{e}}{m^{\prime}}\right)=\left(\frac{\hbar^{2}}{m_{e} e^{2}}\right)\left(\frac{m_{e}}{\left(\frac{m_{\mu}+m_{p}}{m_{\mu} m_{p}}\right)}\right) .
$$

The quantity $C=0.9956$ is the overlap reduction factor, a correction which accounts for the "finite spatial extent of the [proton] charge distribution, [the] bound state character of the [...] muonic atom, [and] possible relativistic corrections" [9]. When applying Equation B.1, the form factors must be evaluated at the characteristic momentum transfer of muon capture, $q_{0}^{2}=-0.88 m_{\mu}^{2}$ :

$$
\begin{aligned}
g_{V}\left(q_{0}^{2}\right) & =0.976(1) \\
2 m_{N} g_{M}\left(q_{0}^{2}\right) & =3.583(3) \\
g_{A}\left(q_{0}^{2}\right) & =1.270(3) .
\end{aligned}
$$

Equation B.1 yields reasonable numerical results, though I have been unable to fully reconcile the expression with similar formulations in references [8] and [9]. It is worth noting that the authors of reference [23], who used ChPT techniques to obtain predictions for the singlet and triplet $\mathrm{OMC}$ rates in hydrogen, claim that capture rate expressions like Equation B.1 are inaccurate, and "though very appealing, should not be used." The reasons for their objections are unclear, however, and the approach is regarded by many as well-established. 


\section{Appendix C}

\section{$\mu p$ diffusion in hydrogen gas}

Thermal diffusion of $\mu p$ atoms away from the muon's stopping point is described by the partial differential equation

$$
\frac{\partial}{\partial t} \rho(\vec{r}, t)=D \nabla^{2} \rho(\vec{r}, t)-\lambda \rho(\vec{r}, t)
$$

where $\rho(\vec{r}, t)$ is the probability density of finding the $\mu p$ atom at position $\vec{r}$ at time $t, \lambda$ is the muon's effective decay rate (e.g., in protium, $\lambda=\lambda_{0}+\Lambda_{S}$ at early times), and $D$ is the coefficient of diffusion. Since diffusion is an isotropic process, it is convenient to rewrite Equation C.1 in spherical coordinates

$$
\frac{\partial}{\partial t} \rho(r, t)=D\left[\frac{2}{r} \frac{\partial}{\partial r}+\frac{\partial^{2}}{\partial r^{2}}\right] \rho(r, t)-\lambda \rho(r, t)
$$

The solution to Equation C.2 is a product of the normalized functions which separately describe diffusion and exponential decay,

$$
\begin{aligned}
\rho(r, t) & =G(r, t) \cdot N(t) \\
& =\left[\left(\frac{1}{4 \pi D t}\right)^{3 / 2} e^{-r^{2} / 4 D t}\right] \cdot\left[\lambda e^{-\lambda t}\right] \\
& =\left(\frac{1}{4 \pi D t}\right)^{3 / 2} \lambda e^{-\left(\lambda t+r^{2} / 4 D t\right)} .
\end{aligned}
$$


The typical diffusion distance for a muon that survives until time $t$ is customarily estimated by the root-mean-square value of the radial distance $r$,

$$
r_{\mathrm{rms}}=\sqrt{\left\langle r^{2}\right\rangle}=\left[\int_{0}^{\infty} r^{2} \cdot G(r, t) \cdot 4 \pi r^{2} d r\right]^{1 / 2}=\sqrt{6 D t}
$$

According to the kinetic theory of gases, the diffusion coefficient is given by [239]

$$
D=\frac{l \bar{v}}{3}
$$

where $l$ is the mean free path of the $\mu p$ atom and $\bar{v}$ is its mean speed. The mean free path can be expressed in terms of the gas density $n$, the scattering cross section $\sigma$, the mean speed $\bar{v}$, and the mean relative speed $\bar{V}$ between scattering particles,

$$
l=\left(\frac{1}{n \sigma}\right) \frac{\bar{v}}{\bar{V}}
$$

The mean speed of a particle with mass $m$ at temperature $T$ is

$$
\bar{v}=\sqrt{\frac{8 k_{B} T}{\pi m}} .
$$

The MuCap hydrogen target is maintained at a temperature of $\approx 300 \mathrm{~K}$, or $k_{B} T \approx 1 / 40 \mathrm{eV}$, so the mean speeds for $\mu p$ atoms and $\mathrm{H}_{2}$ moelcules are

$$
\begin{aligned}
& \bar{v}_{\mu p}=\sqrt{\frac{8 k_{B} T}{\pi\left(m_{\mu}+m_{p}\right)}}=\sqrt{\frac{8(1 / 40 \mathrm{eV})\left(3 \times 10^{8} \mathrm{~m} / \mathrm{s}\right)^{2}}{\pi(105.6 \mathrm{MeV}+938.3 \mathrm{MeV})}} \approx 2.34 \mathrm{~mm} / \mu \mathrm{s} \\
& \bar{v}_{\mathrm{H}_{2}}=\sqrt{\frac{8 k_{B} T}{\pi\left(2 m_{p}\right)}}=\sqrt{\frac{8(1 / 40 \mathrm{eV})\left(3 \times 10^{8} \mathrm{~m} / \mathrm{s}\right)^{2}}{\pi(2 \cdot 938.3 \mathrm{MeV})}} \approx 1.75 \mathrm{~mm} / \mu \mathrm{s} .
\end{aligned}
$$

The mean relative speed between a $\mu p$ atom and an $\mathrm{H}_{2}$ molecule can be approximated as

$$
\bar{V} \approx \sqrt{\bar{v}_{\mu p}^{2}+\bar{v}_{\mathrm{H}_{2}}^{2}} \approx 2.92 \mathrm{~mm} / \mu \mathrm{s}
$$

The gas density can easily be calculated from the ideal gas law

$$
\begin{aligned}
n & =\frac{\bar{p}}{k_{B} T}=\frac{10 \mathrm{bar}}{(1 / 40) \mathrm{eV}} \cdot \frac{\left[10^{5} \mathrm{~Pa} / \mathrm{bar}\right]\left[\frac{1 \mathrm{~m}}{1000 \mathrm{~mm}}\right]^{3}}{\left[1.602 \times 10^{-19} \mathrm{~J} / \mathrm{eV}\right]} \\
& \approx 2.5 \times 10^{17} \mathrm{~mm}^{-3}
\end{aligned}
$$


and the so-called "transport" cross section [103,104],

$$
\sigma \approx 2.08 \times 10^{-19} \mathrm{~cm}^{2}
$$

is an average over the differential cross sections [240] for scattering of thermal $\mu p$ atoms by target $\mathrm{H}_{2}$ molecules at 10 bar pressure and $300 \mathrm{~K}$ that takes into account angular dependencies. It is important to employ molecular cross sections because "nuclear" scattering (i.e. $\mu p+p$ scattering) cross sections [241-244] do not include molecular binding and electron screening effects, and therefore are only valid at high, non-thermal scattering energies $\varepsilon>10 \mathrm{eV}$ where such effects are minimal (see Figure 3.4(a)). Moreover, the molecular transport cross-section incorporates information about the highly anisotropic nature of low-energy $\mu p+\mathrm{H}_{2}$ scattering, whereas the nuclear cross sections are isotropic. Consequently, Monte Carlo simulations have demonstrated [104] that the less accurate nuclear cross sections predict diffusion radii twice as large as molecular cross sections.

By combining Equations C.4, C.5, and C.6 and plugging in the preceding numbers, we can obtain an estimate of the diffusion distance as a function of time:

$$
\begin{aligned}
r_{\mathrm{rms}}(t) & =\sqrt{\frac{2 \bar{v}_{\mu p}^{2} t}{\bar{V} n \sigma}} \\
& \approx \sqrt{\frac{2(2.34 \mathrm{~mm} / \mu \mathrm{s})^{2} t}{(2.92 \mathrm{~mm} / \mu \mathrm{s})\left(2.5 \times 10^{17} \mathrm{~mm}^{-3}\right)\left(2.08 \times 10^{-17} \mathrm{~mm}^{2}\right)}} \\
& \approx 0.85 \sqrt{t} \frac{\mathrm{mm}}{\mu \mathrm{s}^{1 / 2}} .
\end{aligned}
$$

Because most muons disappear via decay, the muon lifetime $\tau_{0}=1 / \lambda_{0} \approx 2.2 \mu$ s sets the diffusion timescale, and consequently the distance scale, $r_{\mathrm{rms}}\left(\tau_{0}\right)=1.26 \mathrm{~mm}$. This crude estimate is somewhat larger than the results produced by Monte Carlo simulations of thermal $\mu p$ diffusion, which are presented in Figure C.1. Of course, the average diffusion distance is in fact slightly enlarged by the occasional epithermal effects which occur before the $\mu p$ atom has completely thermalized. The typical epithermal diffusion range is also on 


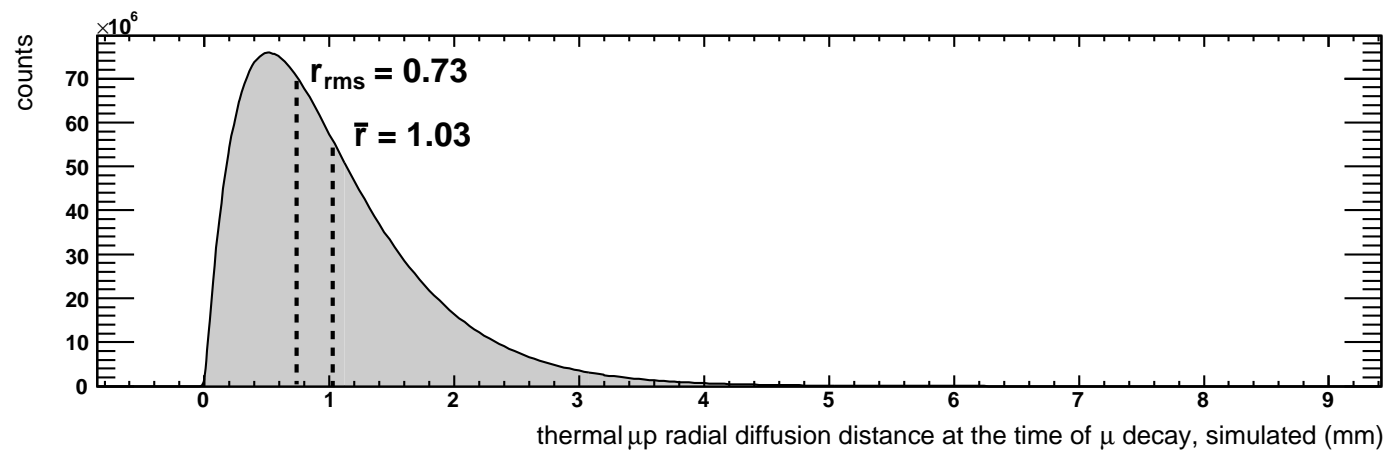

Figure C.1: Thermal $\mu p$ radial diffusion distances at the time of $\mu$ decay, generated by Monte Carlo simulations.

the order of $1 \mathrm{~mm}$ and must be added to the thermal diffusion distance in quadrature.

A calculation very similar to that given above can be found in reference [104]. There, the authors use simple analytical estimates to confirm the results of more elaborate Monte Carlo simulations. 


\section{Appendix D}

\section{Calculation of the hydrogen gas}

\section{density in Run8}

Knowledge of the exact hydrogen gas density inside the pressure vessel is important, because the density determines in part the rate of formation of $p \mu p$ molecules. Hydrogen density is customarily described by the quantity $\phi$, defined as the atomic density $n$ relative to that of liquid hydrogen $\left(\mathrm{LH}_{2}\right)$,

$$
\phi=\frac{n}{n_{\mathrm{LH}_{2}}},
$$

where $n_{\mathrm{LH}_{2}} \equiv 4.25 \times 10^{22} \mathrm{H} / \mathrm{cm}^{3}$. Assuming for the moment that the gas temperature during Run8 was held at $27^{\circ} \mathrm{C}=300.15 \mathrm{~K}$, and that CHUPS maintained a constant pressure of exactly 10.0 bar $=1 \mathrm{MPa}$, we can make use of the ideal gas law to estimate the value of $\phi$ under the 2004 experimental conditions:

$$
\begin{aligned}
\left.\phi\right|_{\text {ideal gas }} & =\frac{\left(p / k_{B} T\right) \times\left(2 \mathrm{H} / \mathrm{H}_{2}\right)}{4.25 \times 10^{22} \mathrm{H} / \mathrm{cm}^{3}} \\
& =\frac{\left(1 \mathrm{MPa} /\left[\left(1.3807 \times 10^{-23} \mathrm{~J} / \mathrm{K}\right) \cdot(300.15 \mathrm{~K})\right]\right) \times\left(2 \mathrm{H} / \mathrm{H}_{2}\right)}{\left(4.25 \times 10^{22} \mathrm{H} / \mathrm{cm}^{3}\right) \cdot\left(1 \times 10^{6} \mathrm{~cm}^{3} / \mathrm{m}^{3}\right)} \\
& =0.01136 .
\end{aligned}
$$


This calculation is slightly inaccurate, however, because the compressibility of hydrogen reduces with increasing pressure, and therefore the hydrogen density does not scale linearly with pressure as assumed in the ideal gas law. Parahydrogen density at 10 bar pressure is $0.52 \%$ less than the value predicted by the ideal gas law [245]; making the reasonable assumption that normal hydrogen has similar properties gives

$$
\phi=\left.0.9948 \cdot \phi\right|_{\text {ideal gas }}=0.01130
$$

This result is corroborated by another approach, which is to use the hydrogen density provided by a NIST website that is devoted to computing the thermophysical properties of fluids under various conditions [246]. The NIST website reports a hydrogen atomic density of

$$
n\left(27^{\circ} \mathrm{C}, 10 \mathrm{bar}\right)=0.00080296 \mathrm{~g} / \mathrm{cm}^{3},
$$

which in turn yields

$$
\begin{aligned}
\left.\phi\right|_{\mathrm{NIST}} & =\frac{0.00080296 \mathrm{~g} / \mathrm{cm}^{3} \cdot\left[\left(1.66054 \times 10^{-24} \mathrm{~g} / \mathrm{amu}\right)(1.007825 \mathrm{amu} / \mathrm{H})\right]^{-1}}{4.25 \times 10^{22} \mathrm{H} / \mathrm{cm}^{3}} \\
& =0.01129
\end{aligned}
$$

in excellent agreement with the result in Equation D.1.

Finally, let us calculate a value for the Run8 gas density that incorporates the actual experimental pressure and temperature readings and their uncertainties. Dr. Peter Kravtsov has reported that the CHUPS system measured a pressure of $10.00 \pm 0.02$ bar for the duration of the run. However, the pressure sensors had an inherent $\pm 0.5 \%$ absolute calibration uncertainty, so we can conservatively claim that

$$
\left.p\right|_{\text {Run8 }}=10.00 \pm 0.05 \text { bar, }
$$


where the uncertainty in the pressure is calibration-limited, rather than stability-limited. The gas temperature was determined by Bernhard Lauss, who looked at Run8 temperature readings from an external sensor that was attached to the top of the pressure vessel and provides the best approximation we have of the actual gas temperature. He reported $T=$ $28.8 \pm 1.8^{\circ} \mathrm{C}$, but we decided to enlarge to the error to $\pm 1 \%$ (of the temperature in $\mathrm{K}$ ) in order to conservatively cover any calibration uncertainties, giving

$$
\left.T\right|_{\text {Run8 }}=28.8 \pm 3^{\circ} \mathrm{C}=302 \pm 3 \mathrm{~K} \text {. }
$$

Plugging the numbers from Equations D.3 and D.4 into the modified ideal gas formalism that led to Equation D.1 gives a conservative Run8 density estimate of

$$
\left.\phi\right|_{\text {Run8 }}=0.01123 \pm 0.00013 .
$$




\section{Appendix E}

\section{Effects of $\mu d$ diffusion on the}

\section{lifetime spectrum}

In this appendix I derive Equation 3.3, which describes how $\mu d$ diffusion outside of an electron cut radius can distort the lifetime spectrum. I start with the first-order, ordinary, linear, homogeneous differential equations for a simplified $(\mu p, \mu d)$ system,

$$
\begin{aligned}
& \frac{d N_{\mu p}}{d t}=-\left(\lambda_{0}+\Lambda_{p d}\right) N_{\mu p} \\
& \frac{d N_{\mu d}}{d t}=\Lambda_{p d} N_{\mu p}-\lambda_{0} N_{\mu d},
\end{aligned}
$$

where for simplicity I have ignored the isotopic nuclear capture rates $\Lambda_{S}$ and $\Lambda_{d}$ and assumed that muons can only disappear via decay at constant rate $\lambda_{0}$. The solutions to the above system of differential equations are

$$
\begin{aligned}
& N_{\mu p}(t)=A e^{-\left(\lambda_{0}+\Lambda_{p d}\right) t} \\
& N_{\mu d}(t)=B e^{-\lambda_{0} t}+e^{-\lambda_{0} t} \int_{0}^{t} e^{\lambda_{0} t^{\prime}} \cdot \Lambda_{p d} N_{\mu p}\left(t^{\prime}\right) d t^{\prime} .
\end{aligned}
$$


For ppm-level deuterium concentrations, I can safely assume that $N_{\mu p}(0)=1$ and $N_{\mu d}(0)=$ 0 , in which case $A \rightarrow 1$ and $B \rightarrow 0$, giving

$$
\begin{aligned}
& N_{\mu p}(t)=e^{-\left(\lambda_{0}+\Lambda_{p d}\right) t} \\
& N_{\mu d}(t)=e^{-\lambda_{0} t} \int_{0}^{t} \Lambda_{p d} e^{-\Lambda_{p d} t^{\prime}} d t^{\prime} .
\end{aligned}
$$

The total (normalized) muon population is thus

$$
\begin{aligned}
N_{\mu}(t) & =N_{\mu p}(t)+N_{\mu d}(t) \\
& =e^{-\left(\lambda_{0}+\Lambda_{p d}\right) t}+e^{-\lambda_{0} t} \int_{0}^{t} \Lambda_{p d} e^{-\Lambda_{p d} t^{\prime}} d t^{\prime} \\
& =e^{-\left(\lambda_{0}+\Lambda_{p d}\right) t}\left[1+e^{\Lambda_{p d} t} \int_{0}^{t} \Lambda_{p d} e^{-\Lambda_{p d} t^{\prime}} d t^{\prime}\right] .
\end{aligned}
$$

At this point I perform an ad hoc insertion of the function $p\left(r, t-t^{\prime}\right)$ which describes the probability that the muon transferred to the deuteron at time $t^{\prime}$ but remains within a radius $r$ of the muon stop at decay time $t$,

$$
N_{\mu}(t)=e^{-\left(\lambda_{0}+\Lambda_{p d}\right) t}\left[1+\Lambda_{p d} e^{\Lambda_{p d} t} \int_{0}^{t} p\left(r, t-t^{\prime}\right) e^{-\Lambda_{p d} t^{\prime}} d t^{\prime}\right]
$$

here I have ignored the comparatively smaller effects of $\mu p$ diffusion. Notice that $p$ is a function of the elapsed $\mu d$ diffusion time interval $\Delta t=t-t^{\prime}$. If I now perform the change of variables $\left(t-t^{\prime}\right) \rightarrow t^{\prime}$, noting that $d t=0$ in this case because $t$ is being treated as a constant in the $d t^{\prime}$ integral, then I obtain

$$
\begin{aligned}
N_{\mu}(t) & =e^{-\left(\lambda_{0}+\Lambda_{p d}\right) t}\left[1+\Lambda_{p d} \int_{0}^{t} p\left(r, t-t^{\prime}\right) e^{\Lambda_{p d}\left(t-t^{\prime}\right)}\left(-d\left(t-t^{\prime}\right)\right)\right] \\
& =e^{-\left(\lambda_{0}+\Lambda_{p d}\right) t}\left[1+\Lambda_{p d} \int_{t}^{0} p\left(r, t^{\prime}\right) e^{\Lambda_{p d} t^{\prime}}\left(-d t^{\prime}\right)\right] \\
& =e^{-\left(\lambda_{0}+\Lambda_{p d}\right) t}\left[1+\Lambda_{p d} \int_{0}^{t} p\left(r, t^{\prime}\right) e^{\Lambda_{p d} t^{\prime}} d t^{\prime}\right] .
\end{aligned}
$$




\section{Appendix F}

\section{$\boldsymbol{\mu}^{+}$precession frequencies in hydrogen gas}

When polarized $\mu^{+}$are stopped in hydrogen gas and subjected to a weak, transverse, external magnetic field $\vec{B}$, the spins of the stopped muons exhibit three characteristic precession frequencies: $13.5 \mathrm{kHz} /$ gauss for free muons, and $1.4 \mathrm{MHz} /$ gauss and $4.5 \mathrm{GHz}$ for muons that have formed muonium $\left(\mu^{+} e^{-}\right)$. These rotation frequencies are derived below using crude semiclassical arguments; rigorous quantum mechanical treatments yield essentially the same results [163].

\section{F.1 Free $\boldsymbol{\mu}^{+}$}

Every muon possesses an inherent magnetic moment $\vec{\mu}$ which is proportional to its spin $\vec{s}$

$$
\vec{\mu}=\left(\frac{g e}{2 m_{\mu}}\right) \vec{s}=\gamma_{\mu} \vec{s},
$$

where the constant of proportionality $\gamma_{\mu}$ is commonly referred to as the muon's "gyromagnetic ratio." When placed in a transverse magnetic field, a free muon experiences a 
torque

$$
\begin{gathered}
\vec{\tau}=\vec{\mu} \times \vec{B} \\
\frac{d \vec{s}}{d t}=\gamma_{\mu} \vec{s} \times \vec{B},
\end{gathered}
$$

and the expectation value of the muon spin rotates in straightforward fashion according to the classical Larmor formula

$$
\vec{\omega}_{\mu}=-\gamma_{\mu} \vec{B}
$$

We can safely assume that the muon's g-factor is exactly 2, and therefore the angular precession frequency per unit magnetic field for a positive muon is

$$
\begin{aligned}
\frac{\omega_{\mu^{+}}}{B} & =\left|\gamma_{\mu}\right|=\frac{|e|}{m_{\mu}}=\frac{1.602 \times 10^{-19} \mathrm{C}}{105.6 \mathrm{MeV} / c^{2}} \\
& =\frac{\left(1.602 \times 10^{-19} \mathrm{C}\right)\left(3 \times 10^{8} \mathrm{~m} / \mathrm{s}\right)^{2}}{(105.6 \mathrm{MeV})} \cdot \frac{\left[\frac{\mathrm{As}}{\mathrm{C}}\right]\left[\frac{\mathrm{kg} / \mathrm{As}^{2}}{10^{4} \mathrm{gauss}}\right]}{\left[\frac{1.602 \times 10^{-19} \mathrm{~J}}{1 \mathrm{eV}}\right]\left[\frac{\mathrm{kg} \mathrm{m} / \mathrm{s}^{2}}{\mathrm{~J}}\right]} \\
& \approx 8.5 \times 10^{4} \mathrm{rad} / \text { gauss } \cdot \mathrm{s} .
\end{aligned}
$$

This corresponds to a frequency

$$
\frac{\nu_{\mu^{+}}}{B}=\left(\frac{1}{2 \pi}\right) \frac{\omega_{\mu^{+}}}{B} \approx 13.5 \mathrm{kHz} / \text { gauss }
$$

\section{F.2 Muonium "triplet"}

In the muonium spin-1 "triplet" system $(\uparrow \uparrow)$, the muon and electron spins can be crudely regarded as locked together by the hyperfine interaction. The combined system rotates in a weak transverse $\vec{B}$ field at the frequency [163]

$$
\begin{aligned}
\vec{\omega}_{\uparrow \uparrow} & =\frac{1}{2}\left(\gamma_{\mu}+\gamma_{e}\right) \vec{B} \\
& =\frac{1}{2}\left(\frac{|e|}{m_{\mu}}-\frac{|e|}{m_{e}}\right) \vec{B} .
\end{aligned}
$$


Note that, because of the electron's smaller mass and hence larger magnetic moment, the magnetic moment of the $\mathrm{Mu}$ triplet points opposite to the muon spin. As a result, the triplet's Larmor precession is in the opposite direction as for free $\mu^{+}$, with an angular frequency per unit magnetic field given by

$$
\begin{aligned}
\frac{\omega_{\uparrow \uparrow}}{B} & =\frac{1}{2}\left(1-\frac{m_{\mu}}{m_{e}}\right) \frac{\omega_{\mu^{+}}}{B} \\
& \approx-103 \frac{\omega_{\mu^{+}}}{B} \\
& =-8.8 \times 10^{6} \mathrm{rad} / \mathrm{gauss} \cdot \mathrm{s},
\end{aligned}
$$

which corresponds to a frequency

$$
\frac{\nu_{\uparrow \uparrow}}{B} \approx-103 \frac{\nu_{\mu^{+}}}{B}=-1.4 \mathrm{MHz} / \text { gauss }
$$

\section{F.3 Muonium "singlet"}

In the muonium spin-0 "singlet" system $(\uparrow \downarrow)$, the muon spin can be viewed as oscillating in the magnetic field produced by the electron's dipole moment, independently of any external magnetic fields. The hyperfine energy correction for the spherically-symmetric $s$ state of muonium is $[247,248]$

$$
\begin{aligned}
\Delta E=\hbar \omega_{0} & =-\frac{\mu_{0}}{4 \pi} \frac{8 \pi}{3}\left|\psi_{e}(0)\right|^{2}\left\langle\vec{\mu}_{\mu} \cdot \vec{\mu}_{e}\right\rangle \\
& =-\frac{2 \mu_{0}}{3}\left|\frac{1}{\sqrt{\pi a^{3}}}\right|^{2}\left(\frac{e \hbar}{m_{\mu}}\right)\left(\frac{e \hbar}{m_{e}}\right)\left\langle\vec{s}_{\mu} \cdot \vec{s}_{e}\right\rangle \\
& =\frac{8 \hbar^{4}}{3 c^{2} m_{\mu} m_{e}^{2} a^{4}}\left\langle\vec{s}_{\mu} \cdot \vec{s}_{e}\right\rangle,
\end{aligned}
$$

where I have made use of SI formulas for the speed of light $c=\left(\mu_{0} \epsilon_{0}\right)^{-1 / 2}$, and the Bohr radius $a=4 \pi \epsilon_{0} \hbar^{2} / m_{e} e^{2}$. The angular frequency of the muonium singlet's precession is determined by the hyperfine energy splitting, which is the coefficient of $\left\langle\vec{s}_{\mu} \cdot \vec{s}_{e}\right\rangle$ in Equa- 
tion F.3:

$$
\begin{aligned}
\omega_{0} & =\frac{8 \hbar^{3}}{3 c^{2} m_{\mu} m_{e}^{2} a^{4}} \\
& =\frac{8\left(1.055 \times 10^{-34} \mathrm{Js}\right)^{3}}{3 c^{2}\left(105.6 \mathrm{MeV} / c^{2}\right)\left(0.511 \mathrm{MeV} / c^{2}\right)^{2}\left(5.29 \times 10^{-11} \mathrm{~m}\right)^{4}} \\
& =\frac{8\left(1.055 \times 10^{-34} \mathrm{Js}\right)^{3}\left(3 \times 10^{8} \mathrm{~m} / \mathrm{s}\right)^{4}}{3(105.6 \mathrm{MeV})(0.511 \mathrm{MeV})^{2}\left(5.29 \times 10^{-11} \mathrm{~m}\right)^{4}} \cdot \frac{1}{\left[\frac{1.602 \times 10^{-19} \mathrm{~J}}{1 \mathrm{eV}}\right]^{3}} \\
& =2.86 \times 10^{10} \mathrm{rad} / \mathrm{s} .
\end{aligned}
$$

The corresponding frequency is then

$$
\nu_{\uparrow \downarrow}=\frac{\omega_{0}}{2 \pi} \approx 4.5 \mathrm{GHz} .
$$




\section{Appendix G}

\section{Technical specifications: detectors,}

\section{electronics, and software}

In the following sections I provide some technical details about several components of our experimental apparatus, such as the detectors and DAQ electronics. I also provide a listing of the software modules used for the Berkeley data analysis.

\section{G.1 General detector channel / parameter assignments}

Table G.1: The famous "wiring.map," which depicts the parameter numbers that are assigned to the Run8 detector channels. In the eSC entries, "IU"= "inner upstream," "ID" = "inner downstream," "OU"= "outer upstream," and "OD"="outer downstream," referring to the locations of the four phototubes that are present in each of the sixteen eSC segments.

\begin{tabular}{llc}
\hline \hline Parameter nos. & Detector channels & Elec. module \\
\hline $1-512$ & ePC1 anode wires 1-512 & COMP \\
\hline & & (Continued on next page)
\end{tabular}


Table G.1 (continued)

\begin{tabular}{|c|c|c|}
\hline Parameter nos. & Detector channels & Elec. module \\
\hline $701-892$ & ePC1 inner strips 1-192 & COMP \\
\hline 1001-1192 & ePC1 outer strips $1-192$ & COMP \\
\hline $2001-3024$ & ePC2 anode wires $1-1024$ & COMP \\
\hline $3101-3420$ & ePC2 inner strips $1-320$ & COMP \\
\hline $3601-3920$ & ePC2 outer strips $1-320$ & COMP \\
\hline $4001-4024$ & $\mu \mathrm{PC} 1$ vertical $(\mathrm{X})$ wires $1-24$ & CAEN 0 \\
\hline $4051-4074$ & $\mu \mathrm{PC} 1$ horizontal $(\mathrm{Y})$ wires $1-24$ & CAEN 0 \\
\hline $4101-4124$ & $\mu \mathrm{PC} 2$ vertical $(\mathrm{X})$ wires $1-24$ & CAEN 0 \\
\hline $4151-4174$ & $\mu \mathrm{PC} 2$ horizontal $(\mathrm{Y})$ wires $1-24$ & CAEN 0 \\
\hline $5001-5075$ & TPC anodes 1-75, EL threshold & TDC400 \\
\hline $5101-5175$ & TPC anodes $1-75, \mathrm{EH}$ threshold & TDC400 \\
\hline $5201-5275$ & TPC anodes $1-75$, EVH threshold & TDC400 \\
\hline $5301-5338$ & TPC cathodes $1-38$, EL threshold & TDC400 \\
\hline $5401-5438$ & TPC cathodes $1-38$, EH threshold & TDC400 \\
\hline 6001 & $\mu \mathrm{SC}$, copy 1 & CAEN 2 \\
\hline 6002 & $\mu \mathrm{SCA}$ & CAEN 2 \\
\hline 6003 & $\mu \mathrm{SC} \mathrm{B}$, start pileup gate signal & CAEN 2 \\
\hline 6004 & $\mu \mathrm{SC} \overline{\mathrm{B}}$, end pileup gate signal (updating) & CAEN 2 \\
\hline 6006 & $\mu \mathrm{SC}$ router signal 1 & CAEN 2 \\
\hline 6007 & $\mu \mathrm{SC}$ router signal 2 & CAEN 2 \\
\hline 6008 & $\mu \mathrm{SC}$ router signal 3 & CAEN 2 \\
\hline 6009 & $\mu \mathrm{SC}$ router signal 4 & CAEN 2 \\
\hline 6011 & $\mu \mathrm{SC}$, copy 2 & CAEN 0 \\
\hline 6101 & ADC gate 1 (eSC IU/OU 1-6) & CAEN 1 \\
\hline 6102 & ADC gate 2 (eSC IU/OU 7-12) & CAEN 1 \\
\hline 6103 & ADC gate 3 (eSC IU/OU 13-16) & CAEN 1 \\
\hline 6104 & ADC gate 4 (eSC ID/OD 1-6) & CAEN 1 \\
\hline 6105 & ADC gate 5 (eSC ID/OD 7-12) & CAEN 1 \\
\hline 6106 & ADC gaet 6 (eSC ID/OD 13-16) & CAEN 1 \\
\hline 6500 & rollover clock & CAEN 0 \\
\hline 6501 & rollover clock & CAEN 1 \\
\hline 6502 & rollover clock & CAEN 2 \\
\hline
\end{tabular}

(Continued on next page) 
Table G.1 (continued)

\begin{tabular}{|c|c|c|}
\hline Parameter nos. & Detector channels & Elec. module \\
\hline 6701 & FADC trigger: TR_( EVH | EHEH | MTPCK) & CAEN 1 \\
\hline 6702 & FADC trigger: TR_EVH & CAEN 1 \\
\hline 6703 & FADC trigger: TR_EHEH & CAEN 1 \\
\hline 6704 & FADC trigger: TR_MTPCK & CAEN 1 \\
\hline 6801 & proton current scaler (freq. $[\mathrm{Hz}]=\operatorname{current}[\mu \mathrm{A}]$ ) & CAEN 1 \\
\hline 6802 & TPC spark & CAEN 1, CAMAC \\
\hline 6803 & $\mu \mathrm{SC} \_\mathrm{L}$ (low), ! $\mu \mathrm{SC}$ (electron-only muSC counter) & CAEN 2 \\
\hline 6804 & $\mu \mathrm{SC} \_\mathrm{L}(\mathrm{low}), ! \mu \mathrm{SC}(\mathrm{ADC}$ gate) & CAEN 2 \\
\hline 6805 & random source & CAEN 1 \\
\hline 6806 & prescaled $50 \mathrm{MHz}$ PSI clock & CAEN 0 \\
\hline 6807 & kicker signal & CAEN 2 \\
\hline $7001-7016$ & eSC IU 1-16 (discriminated) & CAEN 1 \\
\hline $7021-7036$ & eSC ID 1-16 (discriminated) & CAEN 1 \\
\hline $7041-7056$ & eSC OU 1-16 (discriminated) & CAEN 1 \\
\hline $7061-7076$ & eSC OD 1-16 (discriminated) & CAEN 1 \\
\hline $7101-7116$ & eSC IU 1-16 & COMP \\
\hline $7121-7136$ & eSC ID $1-16$ & COMP \\
\hline $7141-7156$ & eSC OU 1-16 & COMP \\
\hline $7161-7176$ & eSC OD 1-16 & COMP \\
\hline $7201-7216$ & eSC IU 1-16 & CAMAC ADC \\
\hline $7221-7236$ & eSC ID $1-16$ & CAMAC ADC \\
\hline $7241-7256$ & eSC OU 1-16 & CAMAC ADC \\
\hline $7261-7276$ & eSC OD 1-16 & CAMAC ADC \\
\hline
\end{tabular}

\section{G.2 TDC400 wiring map}

The routing of the TPC channels into the TDC400 modules during Run8 is depicted in the schematic diagram in Table G.2. Since each of the TDC400 connector plugs contains sixteen input channels, we naturally divided the signals from the TPC anodes into 


\begin{tabular}{|c|c|c|c|c|c|c|c|c|}
\hline Word & Plug & TDC1 & TDC2 & TDC3 & TDC4 & TDC5 & TDC6 & TDC7 \\
\hline \multirow[t]{2}{*}{4} & \multirow[t]{2}{*}{3} & \multirow[t]{2}{*}{ A:EL } & \multirow[t]{2}{*}{ B:EL } & \multirow[t]{2}{*}{ C:EL } & \multirow[t]{2}{*}{ D:EL } & $\begin{array}{c}\text { cath:EL } \\
33-35\end{array}$ & \multirow[t]{2}{*}{$\begin{array}{c}\text { cath:EL } \\
17-32\end{array}$} & \multirow[t]{2}{*}{$\begin{array}{c}\text { cath:EH } \\
17-32\end{array}$} \\
\hline & & & & & & $\mathrm{E}: \mathrm{EL}$ & & \\
\hline \multirow[t]{2}{*}{3} & \multirow[t]{2}{*}{2} & \multirow[t]{2}{*}{ A:EH } & \multirow[t]{2}{*}{ B:EH } & \multirow[t]{2}{*}{$\mathrm{C}: \mathrm{EH}$} & \multirow[t]{2}{*}{ D:EH } & $\begin{array}{c}\text { cath:EH } \\
33-35\end{array}$ & \multirow[t]{2}{*}{$\begin{array}{c}\text { cath:EL } \\
1-16\end{array}$} & \multirow[t]{2}{*}{$\begin{array}{c}\text { cath:EH } \\
1-16\end{array}$} \\
\hline & & & & & & $\mathrm{E}: \mathrm{EH}$ & & \\
\hline 2 & 1 & A:EVH & B:EVH & $\mathrm{C}: \mathrm{EVH}$ & $\mathrm{D}: \mathrm{EVH}$ & $\mathrm{E}: \mathrm{EVH}$ & & \\
\hline 1 & 0 & clock & clock & clock & clock & clock & clock & clock \\
\hline
\end{tabular}

Table G.2: Schematic diagram of the arrangement of TPC channel inputs to the TDC400 modules during Run8.

five sectors, A-E, each of which corresponds to a group of sixteen contiguous wires; i.e. $\mathrm{A}=1-16, \mathrm{~B}=17-32, \mathrm{C}=33-48, \mathrm{D}=49-64, \mathrm{E}=65-75$. Most of the plugs are entirely occupied by contiguous TPC wires, and the TDC400 wire number increases in the same direction as the TPC wire number, for example,

$$
\mathrm{B}: \mathrm{EH}=\text { anodes } 17-32, \text { threshold } \mathrm{EH}=\text { word 3, bits } 1-16 .
$$

However, sector E's EL and EH anodes each shared a TDC400 \#5 plug with cathode signals. In this case, anodes $65-76$ were assigned to bits $1-12$, and cathodes $33-35$ were assigned to bits 14-16; bit 13 was empty. One unfortunate consequence of using a shared plug for both anode and cathode signals is that the thresholds for cathodes 33-35 were constrained by the threshold setting for the more important anode signals, and therefore the cathode 33-35 thresholds could not be tuned independently to match those for the other cathodes. It is worth noting that the actual physical locations of the TDC400 modules in the crate differed from the sequential representation in Table G.2. 
The "Word" labels in Table G.2 refer to the correspondence of the input signals with the components of the TDC400 output data words, while the "Plug" labels refer to the physical location of inputs on the front of the TDC400 panel. The "Plug" labels were used in the MIDAS ODB to specify the input signal locations, because of concerns about the potentital for byte-swapping-related confusion with the "Word" labels.

\section{G.3 $\mu \mathrm{SC}$ logic}

A schematic diagram of the electronic logic implemented by Dr. Bernhard Lauss for processing the $\mu \mathrm{SC}$ signals during Run8 is presented in Figure G.1.

\section{G.4 TPC}

A sideview drawing of the TPC and pressure vessel is presented in Figure G.2.

A brief technical note on the design and baking of the TPC: Our design goal was to solder gold-plated tungsten wires onto glass frames, which preserved the mechanical tension in the wires up to a temperature of $150^{\circ} \mathrm{C}$. However, since tests showed that heating systems would produce occasional temperature fluctuations above this limit, a conservative limit of $130^{\circ} \mathrm{C}$ for long baking periods was established. In fact, we have grown increasingly conservative with the baking temperature over the years, and now usually set it at $110 \pm 5^{\circ} \mathrm{C}$. In general we consider it important to minimize the number of heating cycles, because in the original prototype we found that some wire solderings began to loosen from the glass plate after repeated heatings. 


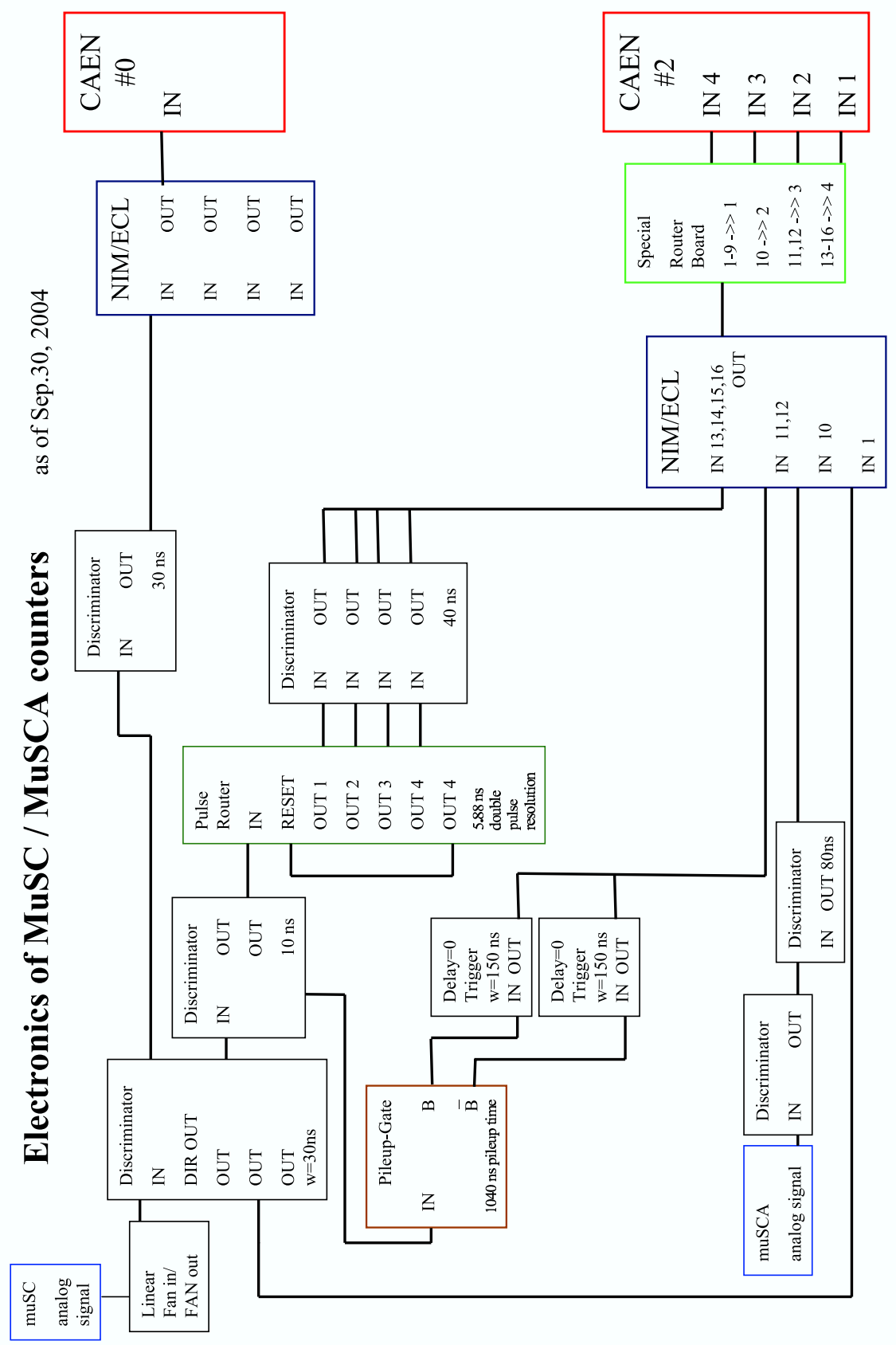

Figure G.1: Schematic diagram of the Run8 $\mu \mathrm{SC}$ electronics setup, as designed and built by Dr. Bernhard Lauss. The "Special Router Board" rectangle refers to a module that was specially designed to channel NIM/ECL signals onto a ribbon cable in a manner compatible with the Robinson Nugent connectors that plug into the CAENs; the special router board should not be confused with the "Pulse Router" which processed the $\mu \mathrm{SC}$ signals and yielded a shorter effective detector deadtime. 


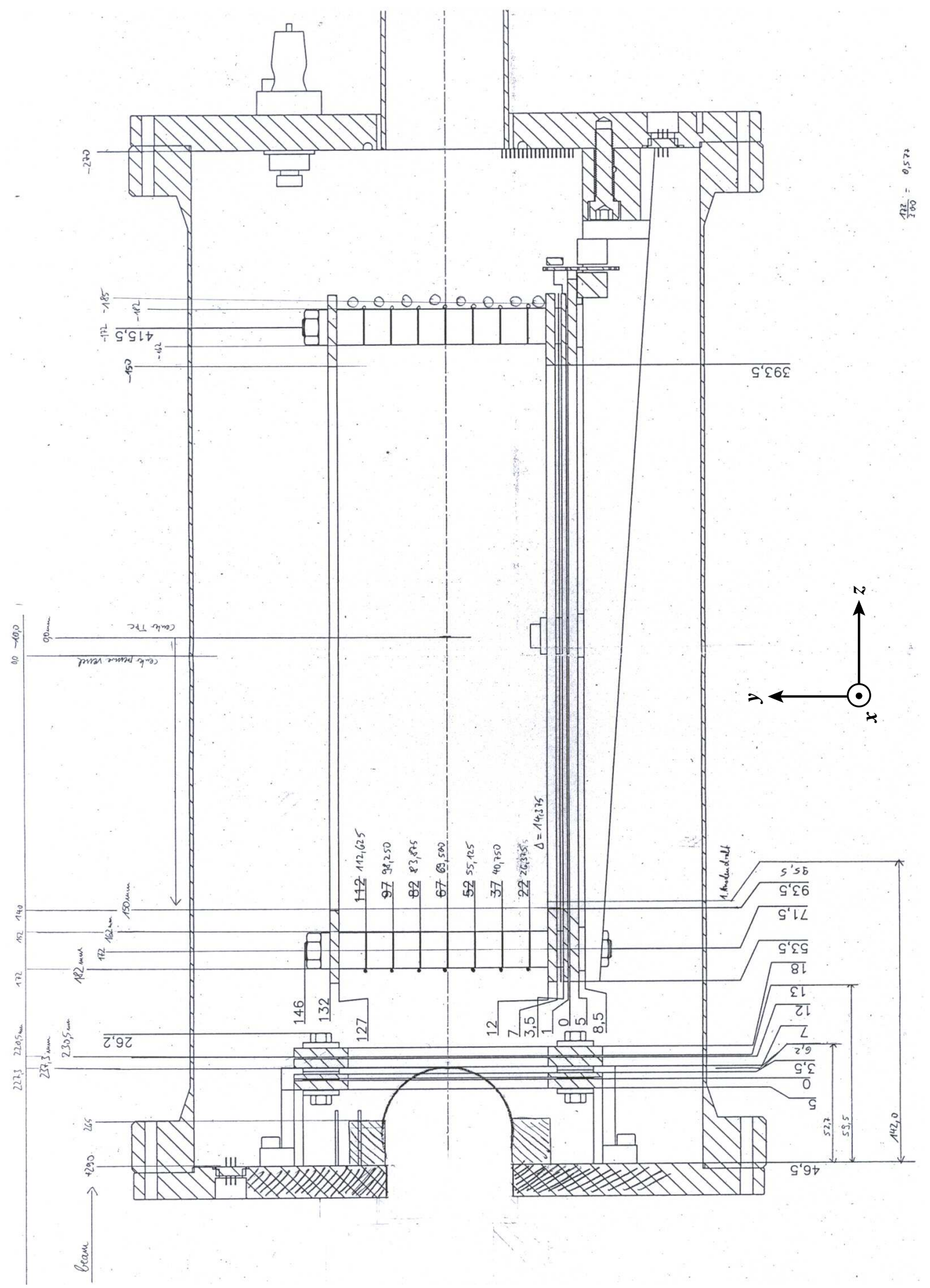

Figure G.2: Schematic drawing of the TPC and pressure vessel in sideview. 


\section{G.5 ePCs}

The dimensions and technical specifications for ePC1 and $\mathrm{ePC} 2$ are provided in Table G.3. A few additional aspects of the ePC setup are worth mentioning:

- During Run8, the ePC1 voltage was $\approx 2600 \mathrm{~V}$, and the ePC2 voltage was $\approx 2800 \mathrm{~V}$. The resulting gas gain was $\approx 5 \times 10^{4}$.

- The gas used in the ePCs was the same as used in $\mu \mathrm{PC} 1$ : a mixture of $49.9 \%$ argon, $49.9 \%$ ethane $\left(\mathrm{C}_{2} \mathrm{H}_{6}\right)$, and $0.2 \%$ Freon.

- During Run8, there were three bronze grounding meshes installed between the ePCs: the first was between ePC1 and the pressure vessel, the second was on the inner surface of ePC2, and the third was on the outer surface of ePC2. Each grounding mesh was made of $240 \mu \mathrm{m}$ bronze mesh soldered to $200 \mu \mathrm{m}$ bronze foil endcaps - which were outside of the active length - for rigidity. The two meshes surrounding ePC2 also had a central, circumferential foil strip for support.

- The cathode strips are glued onto self-supporting Rohacell 51 foam which forms the structure of the inner and outer cylinders.

- The term "half-gap," which is occasionally encountered in ePC chamber documentation, refers to the radial separation between the anode and cathode cylinders. According to the chamber dimensions given in Table G.3, the half-gap is $4 \mathrm{~mm}$ in all cases.

\section{G.6 eSC}

The eSC hodoscope is comprised of sixteen separate scintillating detector pairs, arranged to form a cylinder of active length $900 \mathrm{~mm}$ and diameter $772 \mathrm{~mm}$. The eSC 


\begin{tabular}{|c|c|c|}
\hline Detector geometry & ePC1 & $\mathrm{ePC} 2$ \\
\hline $\begin{array}{l}\text { diameter, inner support ring } \\
\text { diameter, inner shielding } \\
\text { diameter, inner cathode cylinder } \\
\text { diameter, anode cylinder } \\
\text { diameter, outer cathode cylinder } \\
\text { diameter, outer shielding } \\
\text { diameter, outer support ring } \\
\text { length, detector } \\
\text { length, cylinders } \\
\text { length, cylinders, active } \\
\text { radiation length, detector } \\
\text { thickness, detector, fraction of rad. length } \\
\text { material, shielding foil } \\
\text { thickness, shielding foil } \\
\text { coating, shielding foil }\end{array}$ & $\begin{array}{r}359 \mathrm{~mm} \\
373 \mathrm{~mm} \\
376 \mathrm{~mm} \\
384 \mathrm{~mm} \\
392 \mathrm{~mm} \\
395 \mathrm{~mm} \\
423 \mathrm{~mm} \\
690 \mathrm{~mm} \\
640 \mathrm{~mm} \\
580 \mathrm{~mm} \\
37 \mathrm{mg} / \mathrm{cm}^{2} \\
0.978 \times 10^{-3} \\
\mathrm{Kapton} \\
25 \mu \mathrm{m} \\
\mathrm{Al}\end{array}$ & $\begin{array}{r}615 \mathrm{~mm} \\
628 \mathrm{~mm} \\
632 \mathrm{~mm} \\
640 \mathrm{~mm} \\
648 \mathrm{~mm} \\
652 \mathrm{~mm} \\
662 \mathrm{~mm} \\
910 \mathrm{~mm} \\
860 \mathrm{~mm} \\
800 \mathrm{~mm} \\
64 \mathrm{mg} / \mathrm{cm}^{2} \\
1.758 \times 10^{-3} \\
\mathrm{Kapton} \\
50 \mu \mathrm{m} \\
\mathrm{Al}\end{array}$ \\
\hline \multicolumn{3}{|l|}{ Anodes } \\
\hline $\begin{array}{l}\text { number } \\
\text { spacing } \\
\text { wire material } \\
\text { wire diameter } \\
\text { mechanical wire tension }\end{array}$ & $\begin{array}{r}512 \\
2.356 \mathrm{~mm} \\
\text { Au-plated W } \\
20 \mu \mathrm{m} \\
0.6 \mathrm{~N}\end{array}$ & $\begin{array}{r}1024 \\
1.963 \mathrm{~mm} \\
\text { Au-plated W } \\
20 \mu \mathrm{m} \\
0.6 \mathrm{~N}\end{array}$ \\
\hline \multicolumn{3}{|l|}{ Cathodes (a.k.a. "strips") } \\
\hline $\begin{array}{l}\text { number, inner cathodes } \\
\text { number, outer cathodes } \\
\text { pitch (perpendicular spacing) } \\
\text { angle, inner cathodes (right-hand screw) } \\
\text { angle, outer cathodes (left-hand screw) } \\
\text { material, cathode foils } \\
\text { thickness, cathode foils } \\
\text { coating }(\approx 0.03 \mu \mathrm{m}), \text { cathode foils }\end{array}$ & $\begin{array}{r}192 \\
192 \\
4.444 \mathrm{~mm} \\
46.189^{\circ} \\
43.806^{\circ} \\
\text { Kapton } \\
50 \mu \mathrm{m} \\
\mathrm{Au}\end{array}$ & $\begin{array}{r}320 \\
320 \\
4.444 \mathrm{~mm} \\
45.738^{\circ} \\
44.308^{\circ} \\
\text { Kapton } \\
50 \mu \mathrm{m} \\
\mathrm{Al}\end{array}$ \\
\hline \multicolumn{3}{|l|}{ Bronze grounding mesh } \\
\hline $\begin{array}{l}\text { composition (wt\%) } \\
\text { radiation length } \\
\text { thickness, physical } \\
\text { thickness, fraction of rad. length }\end{array}$ & $\mathrm{Cu}(89)$ & $\begin{array}{r}\mathrm{Zn}(9), \mathrm{Pb}(2) \\
8.82 \mathrm{~g} / \mathrm{cm}^{2} \\
240 \mu \mathrm{m} \\
0.007\end{array}$ \\
\hline
\end{tabular}

Table G.3: Technical specifications for the ePC chambers. 
phototube voltages were generally set in the vicinity of $1500 \mathrm{~V}$. The phototube voltages were individually tuned so that the minimum ionizing particle signals were well separated from the noise, and the thresholds were set accordingly. Unfortunately, however, the LeCroy discriminators we used enabled us to set only a single, common threshold for all sixteen hodoscope elements. 


\section{G.7 Berkeley analysis modules-listing}

Below is a list of the software modules that were processed by the MIDAS Analyzer in the three stages of the Berkeley Run8 data analysis (see Sections 6.1.4 and 6.1.3).

Stage 1: Skimming (From file mu/work.skim/MODULES)

$\begin{array}{ll}\text { MUnCompressRawData } & \text { MMuSCAnalysisMQL } \\ \text { MUnDuplicator } & \text { MMUSCAnalysisC } \\ \text { MCaenCompProcessRaw } & \text { MeSCCaenCompCheckMQL } \\ \text { MRolloverCheckMQL } & \text { MeSCCaenCompCheckC } \\ \text { MRolloverCheckC } & \text { MSkimmerMQL } \\ \text { MTDC400ProcessRaw } & \text { MSkimmerC }\end{array}$

Stage 2: Ntuple production (From file mu/work.ana-skim/MODULES)

$\begin{array}{ll}\text { MUnCompressRawData } & \text { MePC2AnalysisC } \\ \text { MFadcProcessRaw } & \text { MePC2AnalysisMQL } \\ \text { MCamacAdcProcessRaw } & \text { MePC2AnalysisMQL_copy2 } \\ \text { MMuSCAnalysisMQL } & \text { MeSCAnalysisC } \\ \text { MMuSCAnalysisC } & \text { MeSCAnalysisMQL } \\ \text { MMuPC1AnalysisC } & \text { MGlobalSparkCut } \\ \text { MMuPC1AnalysisMQL } & \text { MRates } \\ \text { MTPCSegmentSniffer } & \text { MeDetCoincidenceCalcs } \\ \text { MTPCTrackAnalysisC } & \text { MCoincidenceCalcs } \\ \text { MTPCTrackAnalysisMQL } & \text { MTPCImpurityCaptureSearch } \\ \text { MePC1AnalysisC } & \text { MFadcAnalysisC } \\ \text { MePC1AnalysisMQL } & \text { MCamacAdcC } \\ \text { MePC1AnalysisMQL_copy2 } & \text { MCamacAdcMQL } \\ & \text { MThresholdCalc }\end{array}$

Stage 3: Ntuple analysis (From file mu/work.ana-ntuple/MODULES)

MNtupleAnalysisMQL

MNtupleAnalysisC 


\section{Appendix $\mathbf{H}$}

\section{Smearing of the lifetime spectrum}

\section{due to detector response}

The experimentally observed muon lifetime spectrum can be described as the result

of a convolution

$$
f(t)=g(t) \otimes s(t)
$$

of a Gaussian describing the finite detector response,

$$
g(t)=\frac{1}{\sqrt{2 \pi} \sigma} e^{-t^{2} / 2 \sigma^{2}},
$$

with an exponential describing muon decay,

$$
s(t)=M \lambda e^{-\lambda t} \cdot H(t) ;
$$

here $H(t)$ is the Heaviside step function

$$
H(t)=\left\{\begin{array}{ll}
0 & t<0 \\
1 & 0 \leq t
\end{array} .\right.
$$


Carrying out the convolution gives

$$
\begin{aligned}
f(t) & =g(t) \otimes s(t) \\
& =\int_{-\infty}^{\infty}\left(\frac{1}{\sqrt{2 \pi} \sigma} e^{-u^{2} / 2 \sigma^{2}}\right)\left(M \lambda e^{-\lambda(t-u)} \cdot H(t-u)\right) d u \\
& =\int_{-\infty}^{t}\left(\frac{1}{\sqrt{2 \pi} \sigma} e^{-u^{2} / 2 \sigma^{2}}\right)\left(M \lambda e^{-\lambda(t-u)}\right) d u \\
& =\frac{M \lambda e^{-\lambda t}}{\sqrt{2 \pi} \sigma} \int_{-\infty}^{t} e^{-u^{2} / 2 \sigma^{2}+\lambda u} d u \\
& =\frac{M \lambda e^{-\lambda t}}{\sqrt{2 \pi} \sigma} \int_{-\infty}^{t} e^{-\left[\left(\frac{u}{\sqrt{2} \sigma}-\frac{\lambda \sigma}{\sqrt{2}}\right)^{2}-\frac{\lambda^{2} \sigma^{2}}{2}\right]} d u \\
& =\frac{M \lambda e^{\lambda^{2} \sigma^{2} / 2-\lambda t}}{\sqrt{2 \pi} \sigma} \int_{-\infty}^{t} e^{-\left(\frac{u-\lambda \sigma^{2}}{\sqrt{2} \sigma}\right)^{2}} d u .
\end{aligned}
$$

If we now introduce the change of variables

$$
z=\frac{u-\lambda \sigma^{2}}{\sqrt{2} \sigma}
$$

we can rewrite the integral in Equation H.4 as

$$
\begin{aligned}
f(t) & =\frac{M \lambda e^{\lambda^{2} \sigma^{2} / 2-\lambda t}}{\sqrt{2 \pi} \sigma} \cdot \sqrt{2} \sigma \int_{-\infty}^{\left(\frac{t-\lambda \sigma^{2}}{\sqrt{2} \sigma}\right)} e^{-z^{2}} d z \\
& =\left(M e^{\lambda^{2} \sigma^{2} / 2}\right) \lambda e^{-\lambda t} \cdot \frac{1}{2}\left[\frac{2}{\sqrt{\pi}} \int_{-\infty}^{0} e^{-z^{2}} d z+\frac{2}{\sqrt{\pi}} \int_{0}^{\left(\frac{t-\lambda \sigma^{2}}{\sqrt{2} \sigma}\right)} e^{-z^{2}} d z\right] \\
& =N \lambda e^{-\lambda t} \cdot \frac{1}{2}\left[1+\operatorname{erf}\left(\frac{t-\lambda \sigma^{2}}{\sqrt{2} \sigma}\right)\right]
\end{aligned}
$$

which is the basis for the fit function in Equation 6.6 in the text. The term

$$
T(t)=\frac{1}{2}\left[1+\operatorname{erf}\left(\frac{t-\lambda \sigma^{2}}{\sqrt{2} \sigma}\right)\right]
$$

varies between 0 and 1 as illustrated in Figure H.1, and describes the "turn-on" of the muon decay signal. Equation H.6 reveals that the fitted exponential disappearance rate $\lambda$ is not affected by the process of Gaussian smearing, provided that sufficient time has elapsed (i.e., several $\sigma$ ) such that $T(t) \rightarrow 1$. This is true for our experiment, since $\sigma \approx 1.4$ ns while the 


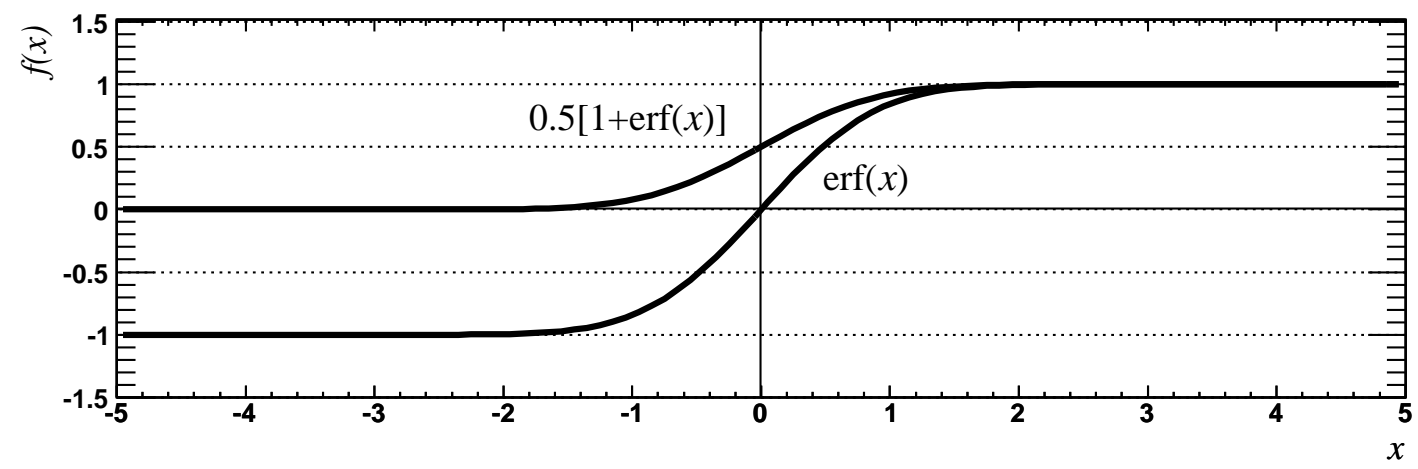

Figure H.1: Plot of the error function (erf) and the basic lifetime "turn-on" function $0.5[1+\operatorname{erf}(x)]$, the latter of which describes the effects of the Gaussian detector response on the experimental lifetime spectrum. The actual argument of the turn-on function has a offset describing the time delayed response, and the argument is also scaled by a constant; i.e. $x=k\left(t-t_{0}\right)$ (see Equation H.7).

standard fit start time is $100 \mathrm{~ns}$; for these values, the argument of the $\operatorname{erf}(x)$ function in Figure H.7 is $x \approx 50$. 


\section{Appendix I}

\section{Error calculations for muon}

\section{disappearance rate shifts}

\section{associated with $\mu+p$ scatters}

I have found that when identified $\mu+p$ scatter events are removed from the Prod-50 lifetime histograms, the fitted decay rate decreases from $\lambda^{\prime}$ to $\lambda$ by

$$
\Delta \lambda=\lambda-\lambda^{\prime} \approx-3 \mathrm{~s}^{-1}
$$

Although this downward shift is to be expected (see Section 6.6.2), it is not obvious what the corresponding error $\sigma_{\Delta \lambda}$ should be, as the shift itself is smaller than the statistical error $\sigma_{\lambda} \approx 12 \mathrm{~s}^{-1}$. The following calculations address this problem.

Scatter events are relatively rare, $\sim 100$ ppm of muon stop candidates, so we can assume that their effects on the decay rate scale linearly ${ }^{1}$. If we let $N$ be the number of good TPC muon stops and $N_{s}$ be the number of $\mu+p$ scatters, then we can be describe

\footnotetext{
${ }^{1}$ This assumption is empirically supported by the data in Tables $6.6,6.7$, and 6.8 .
} 
the situation in the following manner:

$$
\begin{aligned}
N \lambda+N_{s} \lambda_{s} & =\left(N+N_{s}\right) \lambda^{\prime} \\
N \lambda+N_{s}\left(\lambda+\Delta \lambda_{s}\right) & =\left(N+N_{s}\right) \lambda^{\prime} \\
\left(N+N_{s}\right) \lambda+N_{s} \Delta \lambda_{s} & =\left(N+N_{s}\right) \lambda^{\prime} \\
\lambda+f_{s} \Delta \lambda_{s} & =\lambda^{\prime} \\
\Delta \lambda & =-f_{s} \Delta \lambda_{s} .
\end{aligned}
$$

The error on $\Delta \lambda$ is thus

$$
\sigma_{\Delta \lambda}=\sqrt{f_{s}^{2} \sigma_{\Delta \lambda_{s}}^{2}+\left(\Delta \lambda_{s}\right)^{2} \sigma_{f_{s}}^{2}}
$$

We can make the approximation $\sigma_{\Delta \lambda_{s}} \approx \sigma_{\lambda_{s}}$ because the error on the quantity $\Delta \lambda_{s} \equiv$ $\left(\lambda_{s}-\lambda\right)$ is dominated by the scatters, owing to their far smaller statistics and correspondingly larger error.

As a demonstration, let us calculate the error $\sigma_{\Delta \lambda}$ for the Prod-50, cathode-AND, 120-mm-impact-cut muon scatters; the errors for the other Prod-50 analysis conditions are nearly identical. The relevant numbers from Tables 6.6 and 6.7 are

$$
\begin{aligned}
f_{s} & =121.4 \mathrm{ppm} \\
\sigma_{f_{s}} & =0.2 \mathrm{ppm} \\
\Delta \lambda_{s} & =499,503 \mathrm{~s}^{-1}-455,426 \mathrm{~s}^{-1}=44,077 \mathrm{~s}^{-1} \\
\sigma_{\Delta \lambda_{s}} & =1,767 \mathrm{~s}^{-1},
\end{aligned}
$$

and plugging them into Equation I.3 yields

$$
\begin{aligned}
\sigma_{\Delta \lambda} & =\sqrt{\left(121.4 \times 10^{-6}\right)^{2}\left(1,767 \mathrm{~s}^{-1}\right)^{2}+\left(44,077 \mathrm{~s}^{-1}\right)^{2}\left(0.2 \times 10^{-6}\right)^{2}} \\
& \approx 0.22 \mathrm{~s}^{-1}
\end{aligned}
$$


I should point out that $\sigma_{\Delta \lambda}$ is determined almost entirely by the first term under the square root in Equation I.3, as the contribution from the second term is negligible. 


\section{Bibliography}

[1] P. Kammel et al., Hyperfine Interactions 138, 435 (2001).

[2] D. V. Balin et al., PSI Proposal R-97-05 (1996).

[3] V. A. Andreev et al., PSI Proposal R-97-05.2 .

[4] D. Binosi and L. Theussl, Comput. Phys. Commun. 161, 76 (2004), [hep-ph/0309015].

[5] T. Gorringe and H. W. Fearing, Rev. Mod. Phys. 76, 31 (2004), [nucl-th/0206039].

[6] N. C. Mukhopadhyay, Phys. Rept. 30, 1 (1977).

[7] MuCap Collaboration, V. A. Andreev et al., Phys. Rev. Lett. 99, 032002 (2007), [arXiv:0704.2072 [nucl-ex]].

[8] A. Czarnecki, W. J. Marciano and A. Sirlin, Phys. Rev. Lett. 99, 032003 (2007), [arXiv:0704.3968 [hep-ph]].

[9] J. Govaerts and J.-L. Lucio-Martinez, Nucl. Phys. A678, 110 (2000), [nuclth/0004056].

[10] C. W. Kim and H. Primakoff, Phys. Rev. 140, B566 (1965).

[11] CHARM Collaboration, J. Dorenbosch et al., Phys. Lett. B180, 303 (1986).

[12] P. Mergell, U.-G. Meissner and D. Drechsel, Nucl. Phys. A596, 367 (1996), [hep$\mathrm{ph} / 9506375]$.

[13] J. C. Hardy and I. S. Towner, Phys. Rev. C71, 055501 (2005).

[14] Y. K. Lee, L. W. Mo and C. S. Wu, Phys. Rev. Lett. 10, 253 (1963).

[15] C. S. Wu, Rev. Mod. Phys. 36, 618 (1964).

[16] T. D. Lee and C. S. Wu, Ann. Rev. Nucl. Part. Sci. 15, 381 (1965).

[17] T. D. Lee and C. S. Wu, Ann. Rev. Nucl. Part. Sci. 17, 513 (1967).

[18] Particle Data Group, W. M. Yao et al., J. Phys. G33, 1 (2006).

[19] L. A. Ahrens et al., Phys. Lett. B202, 284 (1988). 
[20] V. Bernard, N. Kaiser and U.-G. Meissner, Phys. Rev. Lett. 69, 1877 (1992).

[21] S. Choi et al., Phys. Rev. Lett. 71, 3927 (1993).

[22] A1 Collaboration, A. Liesenfeld et al., Phys. Lett. B468, 20 (1999), [nucl-ex/9911003].

[23] V. Bernard, L. Elouadrhiri and U.-G. Meissner, J. Phys. G28, R1 (2002), [hep$\mathrm{ph} / 0107088]$.

[24] S. Weinberg, Phys. Rev. 112, 1375 (1958).

[25] L. Grenacs, Ann. Rev. Nucl. Part. Sci. 35, 455 (1985).

[26] H. Shiomi, J. Korean Phys. Soc. 29, S378 (1996).

[27] D. H. Wilkinson, Eur. Phys. J. A7, 307 (2000).

[28] D. H. Wilkinson, Nucl. Instrum. Meth. A455, 656 (2000).

[29] K. Minamisono et al., Phys. Rev. C65, 015501 (2002).

[30] M. L. Goldberger, Rev. Mod. Phys. 31, 797 (1959).

[31] H. Primakoff, Rev. Mod. Phys. 31, 802 (1959).

[32] E. Zavattini, in Muon Physics, Vol. II: Weak Interactions (Academic Press, New York, 1975).

[33] N. C. Mukhopadhyay, Nucl. Phys. A335, 111 (1980).

[34] D. F. Measday, Phys. Rept. 354, 243 (2001).

[35] R. H. Hildebrand, Phys. Rev. Lett. 8, 34 (1962).

[36] R. H. Hildebrand and J. H. Doede, (1962).

[37] E. Bertolini et al., (1962).

[38] E. Bleser et al., Phys. Rev. Lett. 8, 288 (1962).

[39] J. E. Rothberg et al., Phys. Rev. 132, 2664 (1963).

[40] A. Alberigi Quaranta et al., Phys. Rev. 177, 2118 (1969).

[41] V. M. Bystritsky et al., Zh. Eksp. Teor. Fiz. 66, 43 (1974), [Sov. Phys. JETP 39, 19 (1974)].

[42] G. Bardin et al., Nucl. Phys. A352, 365 (1981).

[43] G. Bardin et al., Phys. Lett. B104, 320 (1981).

[44] D. H. Wright et al., Phys. Rev. C57, 373 (1998). 
[45] G. I. Opat, Phys. Rev. B134, 428 (1964).

[46] P. Ackerbauer et al., Phys. Lett. B417, 224 (1998), [hep-ph/9708487].

[47] J. G. Congleton and H. W. Fearing, Nuclear Physics A552, 534 (1993).

[48] L. E. Marcucci, R. Schiavilla, S. Rosati, A. Kievsky and M. Viviani, Phys. Rev. C66, 054003 (2002), [nucl-th/0112008].

[49] MuLan Collaboration, D. B. Chitwood et al., Phys. Rev. Lett. 99, 032001 (2007), [arXiv:0704.1981 [hep-ex]].

[50] S.-i. Ando, F. Myhrer and K. Kubodera, Phys. Rev. C65, 048501 (2002), [nuclth/0109068].

[51] J. H. D. Clark et al., Phys. Rev. Lett. 96, 073401 (2006), [arXiv:nucl-ex/0509025].

[52] D. D. Bakalov, M. P. Faifman, L. I. Ponomarev and S. I. Vinitsky, Nucl. Phys. A384, $302(1982)$.

[53] M. L. Goldberger and S. B. Treiman, Phys. Rev. 111, 354 (1958).

[54] M. L. Goldberger and S. B. Treiman, Phys. Rev. 110, 1178 (1958).

[55] S. L. Adler, National Academy of Sciences, Biographical Memoirs 80 (2001).

[56] M. Gell-Mann and M. Levy, Nuovo Cimento 16, 705 (1960).

[57] Y. Nambu, Phys. Rev. Lett. 4, 380 (1960).

[58] S. L. Adler and Y. Dothan, Phys. Rev. 151, 1267 (1966).

[59] L. Wolfenstein, in High Energy Physics and Nuclear Structure (Plenum, New York, 1970).

[60] R. P. Feynman and M. Gell-Mann, Phys. Rev. 109, 193 (1958).

[61] M. Gell-Mann, Phys. Rev. 111, 362 (1958).

[62] H. W. Fearing, R. Lewis, N. Mobed and S. Scherer, Phys. Rev. D56, 1783 (1997), [hep-ph/9702394].

[63] J. Gasser and H. Leutwyler, Nucl. Phys. B250, 465 (1985).

[64] E. Jenkins and A. V. Manohar, Phys. Lett. B255, 558 (1991).

[65] V. Bernard, N. Kaiser and U.-G. Meissner, Phys. Rev. D50, 6899 (1994), [hep$\mathrm{ph} / 9403351]$.

[66] N. Kaiser, Phys. Rev. C67, 027002 (2003), [nucl-th/0301034].

[67] S.-i. Ando, F. Myhrer and K. Kubodera, Phys. Rev. C63, 015203 (2001), [nuclth/0008003]. 
[68] T. R. Hemmert, B. R. Holstein and J. Kambor, J. Phys. G: Nucl. Part. Phys. 24, 1831 (1998).

[69] M. R. Schindler and S. Scherer, hep-ph/0608325.

[70] M. R. Schindler, T. Fuchs, J. Gegelia and S. Scherer, Phys. Rev. C75, 025202 (2007), [nucl-th/0611083].

[71] T. Becher and H. Leutwyler, Eur. Phys. J. C9, 643 (1999), [hep-ph/9901384].

[72] T. Fuchs, J. Gegelia, G. Japaridze and S. Scherer, Phys. Rev. D68, 056005 (2003), [hep-ph/0302117].

[73] J. Gasser, M. E. Sainio and A. Svarc, Nucl. Phys. B307, 779 (1988).

[74] V. Bernard, arXiv:0706.0312 [hep-ph].

[75] V. Bernard, H. W. Fearing, T. R. Hemmert and U.-G. Meissner, Nucl. Phys. A635, 121 (1998), [hep-ph/9801297].

[76] V. Bernard, H. W. Fearing, T. R. Hemmert and U.-G. Meissner, Nucl. Phys. A642, 563 (1998).

[77] A. Fujii and H. Primakoff, Nuovo Cimento 12, 327 (1959).

[78] T. I. Banks, MuCap Internal Note No. 37, 2005.

[79] J. Bernstein, T. D. Lee, C.-N. Yang and H. Primakoff, Phys. Rev. 111, 313 (1958).

[80] A. Santisteban and R. Pascual, Nucl. Phys. A260, 392 (1976).

[81] S.-i. Ando and H. W. Fearing, Phys. Rev. D75, 014025 (2007), [hep-ph/0608195].

[82] V. Bernard, T. R. Hemmert and U.-G. Meissner, Nucl. Phys. A686, 290 (2001), [nucl-th/0001052].

[83] A. Sirlin, Nucl. Phys. B71, 29 (1974).

[84] A. Sirlin, Rev. Mod. Phys. 50, 573 (1978).

[85] W. J. Marciano and A. Sirlin, Phys. Rev. Lett. 56, 22 (1986).

[86] A. Czarnecki, W. J. Marciano and A. Sirlin, Phys. Rev. D70, 093006 (2004), [hep$\mathrm{ph} / 0406324]$.

[87] W. J. Marciano and A. Sirlin, Phys. Rev. Lett. 96, 032002 (2006), [hep-ph/0510099].

[88] M. R. Goldman, Nucl. Phys. B49, 621 (1972).

[89] H. Primakoff, in Nuclear and Particle Physics at Intermediate Energies (Plenum, New York, 1975). 
[90] B. R. Holstein, Phys. Rev. C29, 623 (1984).

[91] W. H. Breunlich, P. Kammel, J. S. Cohen and M. Leon, Ann. Rev. Nucl. Part. Sci. 39, 311 (1989).

[92] E. Fermi and E. Teller, Phys. Rev. 72, 399 (1947).

[93] J. S. Cohen, Phys. Rev. A27, 167 (1983).

[94] V. V. Balashov et al., Muon Cat. Fusion 2, 105 (1988).

[95] J. S. Cohen, in Electromagnetic Cascade and Chemistry of Exotic Atoms (Plenum, New York, 1990).

[96] G. Y. Korenman, V. P. Popov and G. A. Fesenkoothers, Muon Cat. Fusion 7, 179 (1992).

[97] H. Anderhub et al., Phys. Lett. B101, 151 (1981).

[98] P. Hauser et al., in Muonic Atoms and Molecules (Birkhauser, Basel, 1993), Proceedings, Monte Verita, Ascona, Italy, April 5-9, 1992.

[99] F. Kottmann, in Muonic Atoms and Molecules (Birkhauser, Basel, 1993), Proceedings, Monte Verita, Ascona, Italy, April 5-9, 1992.

[100] M. Leon and H. A. Bethe, Phys. Rev. 127, 636 (1962).

[101] V. E. Markushin, in Electromagnetic Cascade and Chemistry of Exotic Atoms (Plenum, New York, 1990).

[102] V. E. Markushin, Phys. Rev. A50, 1137 (1994).

[103] A. Adamczak, Atomic Data and Nuclear Data Tables 62, 255 (1996).

[104] A. Adamczak and J. Gronowski, European Physical Journal D (2006), 10.1140/epjd/e2006-00252-6.

[105] D. J. Abbott et al., Phys. Rev. A55, 214 (1997).

[106] A. Werthmüller et al., Hyperfine Interactions 103, 147 (1996).

[107] A. Adamczak, V. I. Korobov and V. S. Melezhik, Hyperfine Interactions 96 (1), 277 (1995).

[108] V. M. Bystritsky, Nukleonika 40, 37 (1995).

[109] J. S. Cohen, Phys. Rev. A43, 4668 (1991).

[110] A. Igarashi, M. P. Faifman and I. Shimamura, Hyperfine Interactions 138, 77 (2001).

[111] E. Rusjan and P. F. Zweifel, Phys. Rev. A38, 4162 (1988). 
[112] A. Adamczak, Hyperfine Interactions 101/102, 113 (1996).

[113] A. Adamczak, Muonic Hydrogen Atoms and Molecules in Condensed Matter, Ph.D. thesis, Institute of Nuclear Physics PAN, 2003.

[114] V. S. Evseev, in Muon Physics, Vol. III: Chemistry and Solids (Academic Press, New York, 1975).

[115] A. P. Bukhvostov and N. P. Popov, Zh. Eksp. Teor. Fiz. 82, 23 (1982), [Sov. Phys. $55,13(1982)]$.

[116] C. E. Porter and H. Primakoff, Phys. Rev. 83, 849 (1951).

[117] R. W. Huff, Ann. Phys. (N. Y.) 16, 288 (1961).

[118] H. Überall, Phys. Rev. 119, 365 (1960).

[119] H. C. von Baeyer and D. Leiter, Phys. Rev. A19, 1371 (1979).

[120] G. Bardin et al., Nucl. Phys. A453, 591 (1986).

[121] S. Weinberg, Phys. Rev. Lett. 4, 575 (1960).

[122] A. Halpern, Phys. Rev. Lett. 13, 660 (1964).

[123] W. R. Wessel and P. Phillipson, Phys. Rev. Lett. 13, 23 (1964).

[124] P. K. Kabir, Physics Letters 14, 257 (1965).

[125] S. I. Vinitsky et al., Zh. Eksp. Teor. Fiz. 79, 698 (1980), [Sov. Phys. JETP 52, 353 (1980)].

[126] D. Bakalov, in Muonic Atoms and Molecules (Birkhauser, Basel, 1993), Proceedings, Monte Verita, Ascona, Italy, April 5-9, 1992.

[127] F. Mulhauser et al., Phys. Rev. A53, 3069 (1996).

[128] M. P. Faifman, Muon Cat. Fusion 4, 341 (1989).

[129] L. I. Ponomarev, in Muonic Atoms and Molecules (Birkhauser, Basel, 1993), Proceedings, Monte Verita, Ascona, Italy, April 5-9, 1992.

[130] E. J. Bleser, Phys. Rev. 132, 2679 (1963).

[131] G. Conforto, C. Rubbia, E. Zavattini and S. Focardi, Nuovo Cimento 33, 1001 (1964).

[132] V. M. Bystritsky et al., Sov. Phys. JETP 43, 606 (1976).

[133] H. Schneuwly, in Electromagnetic Cascade and Chemistry of Exotic Atoms (Plenum, New York, 1990).

[134] L. Schellenberg, Muon Catalyzed Fusion 5/6, 73 (1990/91). 
[135] S. S. Gershtein, in Muonic Atoms and Molecules (Birkhauser, Basel, 1993), Proceedings, Monte Verita, Ascona, Italy, April 5-9, 1992.

[136] H. Schneuwly, in Muonic Atoms and Molecules (Birkhauser, Basel, 1993), Proceedings, Monte Verita, Ascona, Italy, April 5-9, 1992.

[137] A. Werthmüller et al., Hyperfine Interactions 116, 1 (1998).

[138] T. Suzuki, D. F. Measday and J. P. Roalsvig, Phys. Rev. C35, 2212 (1987).

[139] L. Schellenberg et al., in Muonic Atoms and Molecules (Birkhauser, Basel, 1993), Proceedings, Monte Verita, Ascona, Italy, April 5-9, 1992.

[140] A. Dupays, Phys. Rev. Lett. 93, 043401 (2004).

[141] R. Jacot-Guillarmod et al., in Electromagnetic Cascade and Chemistry of Exotic Atoms (Plenum, New York, 1990).

[142] A.-T. Le and C. D. Lin, Phys. Rev. A71, 022507 (2005).

[143] P. Kammel, MuCap Run8 ELOG \#694 (2004).

[144] A. Bertin, M. Bruno and A. Vitale, Lett. Nuovo Cim. 4S2, 449 (1972).

[145] A. Adamczak et al., Phys. Lett. B285, 319 (1992).

[146] M. Cargnelli et al., in Proceedings of the XXIII Yamada Conf. on Nuclear Weak Processes and Nuclear Structure, Osaka (World Scientific, 1989).

[147] N. Tatara, Y. Kohyama and K. Kubodera, Phys. Rev. C42, 1694 (1990).

[148] S. Ando, T. S. Park, K. Kubodera and F. Myhrer, Phys. Lett. B533, 25 (2002), [nucl-th/0109053].

[149] J.-W. Chen, T. Inoue, X.-d. Ji and Y.-c. Li, Phys. Rev. C72, 061001 (2005), [nuclth/0506001].

[150] S. S. Gershtein and L. I. Ponomarev, in Muon Physics, Vol. III: Chemistry and Solids (Academic Press, New York, 1975).

[151] F. Mulhauser et al., Phys. Rev. A73, 034501 (2006).

[152] B. Kiburg, unpublished Monte Carlo studies (2007).

[153] S. M. Clayton, P. Kammel and B. Kiburg, MuCap Internal Note No. 45, 2006.

[154] V. M. Bystritsky et al., Zh. Eksp. Teor. Fiz. 71, 1680 (1976), [Sov. Phys. - JETP 44, 881 (1976)].

[155] C. Petitjean et al., Invited paper given at Int. Conf. on Muon Catalyzed Fusion, Vienna, Austria, May 27 - Jun 1, 1990. 
[156] B. Lauss, P. Kammel and A. Vasilyev, MuCap Internal Note No. 30, 2005.

[157] P. Kammel, MuCap Internal Note No. 26, 2002.

[158] P. Kammel, MuCap Internal Note No. 29, 2003.

[159] Y.-A. Thalmann et al., Phys. Rev. A57, 1713 (1998).

[160] F. Mulhauser and H. Schneuwly, J. Phys. B26, 4307 (1993).

[161] A. Werthmüller et al., Hyperfine Interactions 101-102, 271 (1996).

[162] R. Peierls, Proc. Roy. Soc. Lond. A149, 467 (1935).

[163] J. H. Brewer, K. M. Crowe, F. N. Gygax and A. Schenck, in Muon Physics, Vol. III: Chemistry and Solids (Academic Press, New York, 1975).

[164] D. G. Fleming, R. J. Mikula and D. M. Garner, Phys. Rev. A26, 2527 (1982).

[165] M. Hildebrandt, private communication (2007).

[166] A. Czarnecki, G. P. Lepage and W. J. Marciano, Phys. Rev. D61, 073001 (2000), [hep-ph/9908439].

[167] M. P. Balandin, V. M. Grebenyuk, V. G. Zinov, A. D. Konin and A. N. Ponomarev, Zh. Eksp. Teor. Fiz. (1974), [Sov. Phys. JETP 40, 811 (1974)].

[168] G. Bardin et al., Phys. Lett. B137, 135 (1984).

[169] K. L. Giovanetti et al., Phys. Rev. D29, 343 (1984).

[170] RIKEN RAL R77 Collaboration, D. Tomono, AIP Conf. Proc. 842, 906 (2006).

[171] F. R. Cavallo et al., PSI Proposal R-99-06 .

[172] R. M. Carey et al., PSI Proposal R-99-07 (1999), also archived as MuLan Internal Note \#1.

[173] J. H. Brewer and K. M. Crowe, Ann. Rev. Nucl. Part. Sci. 28, 239 (1978).

[174] J. Egger, D. Fahrni, L. Meier and C. Petitjean, "Test of the hydrogen wire chamber PC2 developed for the MUCAP experiment," (August 16, 2002).

[175] E. M. Maev et al., Nucl. Instrum. Meth. A515, 288 (2003).

[176] F. Sauli, CERN-77-09.

[177] J. L. Friar, B. F. Gibson, H. C. Jean and G. L. Payne, Phys. Rev. Lett. 66, 1827 (1991).

[178] L. W. Alvarez et al., Phys. Rev. 105, 1127 (1957).

[179] SINDRUM Collaboration, W. Bertl et al., Nucl. Phys. B260, 1 (1985). 
[180] C. Petitjean, "The PSI protium gas filling system," presentation given at the MuCap Collaboration meeting in Gatchina, Russia, June 15, 2004.

[181] V. A. Ganzha et al., arXiv:0705.1473 [nucl-ex].

[182] R. Prieels et al., (2002).

[183] CAEN, (2003).

[184] Agilent Technologies, E4400B ESG-A Series Analog RF Signal Generator, $250 \mathrm{kHz}$ to $1 \mathrm{GHz}$ (2003-2006), http://www.home.agilent.com.

[185] S. Ritt et al., http://midas.psi.ch/.

[186] D. A. Huffman, Proceedings of the IRE 40, 1098 (1952).

[187] T. H. Cormen, C. E. Leiserson, R. L. Rivest and C. Stein, Introduction to Algorithms, 2nd ed. (MIT Press and McGraw-Hill, 2001).

[188] V. A. Andreev et al., (2005).

[189] P. Kammel and S. M. Clayton, MuCap Internal Note No. 28, 2003.

[190] http://ait.web.psi.ch/services/linux/hpc/merlin/.

[191] D. R. Price, http://www.nongnu.org/cvs/.

[192] http://kaon.physics.berkeley.edu/cgi-bin/viewcvs.cgi/\#dirlist.

[193] http://weak0.physics.berkeley.edu/weakint/.

[194] S. Ritt et al., http://midas.psi.ch/htmldoc/analyzer_struct.html.

[195] T. I. Banks and F. E. Gray, [mucap]/mu/doc.

[196] S. M. Clayton, [mucap]/mu/src/uiuc/doc.

[197] [mucap]/mu/mql/doc.

[198] R. Brun and F. Rademakers, Nucl. Instrum. Meth. A389, 81 (1997).

[199] http://root.cern.ch/.

[200] http://www.gnu.org/software/m4/.

[201] T. I. Banks, "MuCap Run8 Analysis Report — UC Berkeley" (2006).

[202] http://www.srim.org/.

[203] P. Kammel, MuCap Internal Note No. 21, 1999.

[204] P. Kammel, "Edge Tracks: Quick Garfield study," internal MuCap memo (2005). 
[205] T. I. Banks, MuCap Internal Note No. 36, 2004.

[206] K. L. Lynch, MuLan No. 16, 2005.

[207] D. V. Balin et al., (1997).

[208] V. A. Andreev et al., (2000).

[209] F. James and M. Roos, Comput. Phys. Commun. 10, 343 (1975).

[210] D. Kawall, Muon $g-2$ Internal Note No. 322, 1998.

[211] S. Redin, Muon $g-2$ Internal Note No. 387, 2001.

[212] S. Redin, Paper prepared for PHYSTAT2003: Statistical Problems in Particle Physics, Astrophysics, and Cosmology, Menlo Park, California, 8-11 Sep 2003.

[213] S. Redin, Muon $g-2$ Internal Note No. 410, 2002.

[214] T. I. Banks, MuCap Internal Note No. 39, 2006.

[215] D. B. Chitwood, Analysis Report for the MuLan 2004 Data Set (2006).

[216] F. Mulhauser, MuCap Internal Note No. 38, 2005, (see also the modified shift summary that was produced in conjunction with this note).

[217] P. Kammel, "Electron detector correlations" (2005).

[218] T. I. Banks and B. Lauss, MuCap Internal Note No. 34, 2004.

[219] T. I. Banks and P. Kammel, MuCap Internal Note No. 43, 2006.

[220] D. Measday, private communication (2000).

[221] P. Kammel and B. Kiburg, MuCap Internal Note No. 48, 2007.

[222] H.-A. Synal, M. Döbeli, S. Jacob, M. Stocker and M. Suter, Nucl. Instrum. Meth. B223-224, 339 (2004).

[223] H.-A. Synal, M. Stocker and M. Suter, Nucl. Instrum. Meth. B259, 7 (2007), Unfortunately, this paper does not describe the MICADAS configuration that was used for the H-D mass analysis of the Run8 MuCap hydrogen gas sample. That information is expected to be presented by Dr. M. Döbeli in a September 2007 conference whose proceedings will appear in NIM B.

[224] S. M. Clayton, MuCap Internal Note No. 40, 2006.

[225] Agilent Technologies, Agilent ESG-A and ESG-D RF Signal Generators: Data Sheet (2003-2006).

[226] K. L. Lynch and Y. Matus, MuLan No. 9, 2003. 
[227] K. L. Lynch, private communication (2007).

[228] K. L. Lynch, http://www.npl.uiuc.edu/elog/mulan/Summer2006/105.

[229] K. L. Lynch, http://www.npl.uiuc.edu/elog/mulan/Summer2006/1158.

[230] F. Mulhauser, http://www.npl.uiuc.edu/elog/mulan/Analysis2004Data/144.

[231] F. Mulhauser, http://www.npl.uiuc.edu/elog/mulan/Analysis2004Data/151.

[232] P. Kammel, MuCap Internal Note No. 46, 2007.

[233] F. E. Gray, MuCap Internal Note No. 47, 2006, The $\mu^{+}$decay rates presented in Dr. Gray's report are doubly blinded, by both the DAQ clock frequency and by a secret offset of Dr. Gray's choosing. The final, unblinded result for $\lambda_{\mu^{+}}$presented in this dissertation came from communications with Dr. Gray.

[234] T. I. Banks, S. M. Clayton and P. Kammel, MuCap Internal Note No. 44, 2007, see also the accompanying spreadsheet final_new.xls.

[235] S.-i. Ando, F. Myhrer and K. Kubodera, private communication (2007).

[236] V. A. Andreev et al., (2006).

[237] E. D. Commins, New York, USA: McGraw-Hill, (1973).

[238] E. D. Commins and P. H. Bucksbaum, Cambridge, USA: Univ. Pr. (1983) 473p.

[239] F. Reif, Fundamentals of Statistical and Thermal Physics (McGraw-Hill, 1965).

[240] A. Adamczak, Phys. Rev. A74, 042718 (2006).

[241] L. Bracci et al., Muon Catalyzed Fusion 4, 247 (1989).

[242] L. Bracci et al., Phys. Lett. A134, 435 (1989).

[243] L. Bracci et al., Phys. Lett. A149, 463 (1990).

[244] C. Chiccoli et al., Muon Catalyzed Fusion 7, 87 (1992).

[245] R. D. McCarty, (1975), NASA-SP-3089.

[246] http://webbook.nist.gov/chemistry/fluid/.

[247] D. J. Griffiths, Introduction to Quantum Mechanics (John Wiley \& Sons, New York, 1995).

[248] J. D. Jackson, Classical Electrodynamics, 3rd ed. (John Wiley \& Sons, New York, 1999). 